Universitat Politècnica de València

Departamento de Máquinas y Motores Térmicos

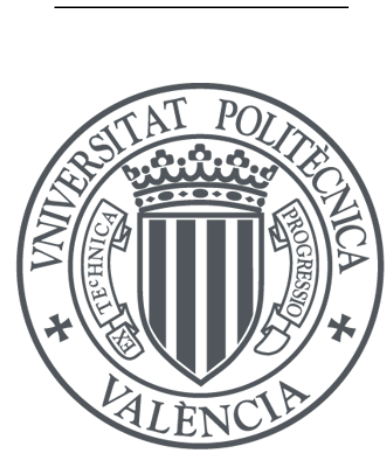

A Contribution to the Global Modeling of Heat Transfer Processes in Diesel Engines

Doctoral Thesis

Presented by:

Josep Salvador Iborra

Supervised by:

Prof. Pablo César Olmeda González

Valencia, January 2020 

Doctoral Thesis

\title{
A Contribution to the Global Modeling of Heat Transfer Processes in Diesel Engines
}

Presented by: Josep Salvador Iborra

Supervised by: Prof. Pablo César Olmeda González

\author{
EXAMINING BOARD \\ President: Prof. José Manuel Luján Martínez \\ Secretary: Prof. Octavio Armas Vergel \\ Examiner: Dr. Carlo Beatrice
}

REVIEWING BOARD

Prof. Angelo Onorati

Dr. Arantzazu Gómez Esteban

Dr. Carlo Beatrice

Valencia, January 2020 



\begin{abstract}
Current challenges in research and development of powertrains demand new computational tools capable of simulating vehicle operation under very diverse conditions. This is due, among other reasons, to new homologation standards in the automotive sector requiring compliance of exhaust emissions regulations under any possible driving condition on the road. Global engine or vehicle models provide many advantages to engineers because they allow to reproduce the entire system under study, considering the physical processes that take place in different components and the interactions among them. This thesis aims to enable the modeling of heat transfer processes in a complete engine simulation tool developed at CMT-Motores Térmicos research institute. This 0D/1D simulation tool is called Virtual Engine Model (VEMOD).

The development of heat transfer models comprises the engine block and the ancillary systems. The model of heat transfer in the engine block deals with the central problem of in-cylinder convection by means of a combination of experimental research, CFD simulation and multizone 0D modeling. The other thermal processes present in the engine block are examined in order to implement suitable submodels. Once the model is complete, it undergoes a validation with experimental transient tests. Afterwards, the ancillary systems for engine thermal management are brought into focus. These systems are considered by means of two new models: a model of heat exchangers and a model of thermo-hydraulic circuits. The development of those models is reported in detail.

Lastly, with the referred thermal models integrated in the global simulation tool, a validation study is undertaken. The goal is to validate the ability of the Virtual Engine Model to capture the thermal response of a real engine under various operating conditions. To achieve that, an experimental campaign combining tests under steady-state operation, under transient operation and at different temperatures is conducted in parallel to the corresponding simulation campaign. The capacity of the global engine simulations to replicate the measured thermal evolution is finally demonstrated.
\end{abstract}




\section{Resum}

Els reptes actuals en la recerca i el desenvolupament de trens de potència demanden noves eines computacionals capaces de simular el funcionament d'un vehicle en condicions molt diverses. Açò es deu, entre altres raons, a que els nous estàndards d'homologació al sector de l'automoció obliguen al compliment de les regulacions d'emissions en qualsevol condició possible de conducció en carretera. Els models globals de motor o de vehicle proporcionen molts avantatges als enginyers perquè permeten reproduir el sistema sencer a estudiar, considerant els processos físics que tenen lloc als distints components i les interaccions entre ells. Aquesta tesi pretén fer possible el modelat dels processos de transmissió de calor en una completa eina de simulació de motor desenvolupada a l'institut de recerca CMT-Motores Térmicos. Aquesta eina de simulació 0D/1D s'anomena Motor Virtual o Virtual Engine Model (VEMOD).

El desenvolupament de models de transmissió de calor comprén el bloc motor i els sistemes auxiliars. El model de transmissió de calor al bloc motor aborda el problema central de la convecció a l'interior del cilindre mitjançant una combinació de recerca experimental, simulació CFD i modelat $0 \mathrm{D}$ multizona. La resta de processos tèrmics presents al bloc motor són examinats per a poder implementar submodels adequats. Una vegada el model està acabat, es fa una validació amb assajos experimentals en règim transitori. A continuació, el focus d'atenció passa als sistemes auxiliars de gestió tèrmica. Aquests sistemes es prenen en consideració per mitjà de dos nous models: un model d'intercanviadors de calor i un model de circuits termohidràulics. El desenvolupament dels models s'explica en detall en aquesta tesi.

Per últim, amb els referits models integrats al Motor Virtual, es porta a terme un estudi de validació. L'objectiu és validar la capacitat del Motor Virtual per a reproduir la resposta tèrmica d'un motor real en diverses condicions de funcionament. Per a assolir-ho, es realitza una campanya experimental que combina assajos en règim estacionari, en règim transitori i a diferents temperatures, en paral-lel a la campanya de simulació corresponent. La capacitat de les simulacions globals de motor per a replicar l'evolució tèrmica observada experimentalment queda finalment demostrada. 


\section{Resumen}

Los retos actuales en la investigación y desarrollo de trenes de potencia demandan nuevas herramientas computacionales capaces de simular el funcionamento de un vehículo en condiciones muy diversas. Esto se debe, entre otras razones, a que los nuevos estándares de homologación en el sector de la automoción obligan al cumplimiento de las regulaciones de emisiones en cualquier condición posible de conducción en carretera. Los modelos globales de motor o de vehículo proporcionan muchas ventajas a los ingenieros porque permiten reproducir el sistema entero a estudiar, considerando los procesos físicos que tienen lugar en los distintos componentes y las interacciones entre ellos. Esta tesis pretende hacer posible el modelado de los procesos de transmisión de calor en una completa herramienta de simulación de motor desarrollada en el instituto de investigación CMT-Motores Térmicos. Esta herramienta de simulación 0D/1D se denomina Motor Virtual o Virtual Engine Model (VEMOD).

El desarrollo de modelos de transmisión de calor comprende el bloque motor y los sistemas auxiliares. El modelo de transmisión de calor en el bloque motor aborda el problema central de la convección en el interior del cilindro mediante una combinación de investigación experimental, simulación CFD y modelado 0D multizona. El resto de procesos térmicos presentes en el bloque motor son examinados para poder implementar submodelos adecuados. Una vez el modelo está terminado, se realiza una validación con ensayos experimentales en régimen transitorio. A continuación, el foco de atención pasa a los sistemas auxiliares de gestión térmica. Estos sistemas se toman en consideración por medio de dos nuevos modelos: un modelo de intercambiadores de calor y un modelo de circuitos termohidráulicos. El desarrollo de los modelos se explica en detalle en esta tesis.

Por último, con los citados modelos integrados en el Motor Virtual, se lleva a cabo un estudio de validación. El objetivo es validar la capacidad del Motor Virtual para reproducir la respuesta térmica de un motor real en varias condiciones de funcionamento. Para conseguirlo, se realiza una campaña experimental que combina ensayos en régimen estacionario, en régimen transitorio y a diferentes temperaturas, en paralelo a la campaña de simulación correspondiente. La capacidad de las simulaciones globales de motor para replicar la evolución térmica medida experimentalmente queda finalmente demostrada. 

As you set out for Ithaka hope the voyage is a long one, full of adventure, full of discovery.

Cavafy 



\section{Acknowledgments}

A $\mathrm{PhD}$ is a long voyage of discovery and personal growth. Far from being a solitary journey, there are many people who intervene to make possible that all efforts reach a good conclusion. Having reached this point, I wish to acknowledge all people and institutions that have contributed to this thesis by one means or another.

First of all, I want to thank my PhD supervisor, Prof. Pablo Olmeda, for his teachings and support over these years. I'm also grateful to Dr. Antonio García for sharing with me his invaluable knowledge about research communication. Another important figure in this period has been Dr. Jaime Martín, from whom I have learned a lot by collaborating in research projects. Also thanks to Dr. Francisco Arnau, for his compromise in building the Virtual Engine Model and for providing me with continued development support.

I want to extend my gratitude to all people who have dedicated time and effort to review my research and have helped me to improve my methods. Needless to say, transferring best practices is one of the pillars of scientific research.

This research has been facilitated by various institutions. General Motors Global R\&D supplied an engine employed in several investigations of this thesis. In this regard, I'm thankful to Dr. Alok Warey for supporting our research. The second engine used to conduct the investigations accounted in this thesis was provided by Renault. I want to acknowl- 
edge their interest in supporting the development of the Virtual Engine Model. In the same direction, European funds received in the framework of Horizon 2020's DiePeR project have contributed to the validation and improvement of the Virtual Engine Model. My own dedication has been funded by Universitat Politècnica de València through the predoctoral contract FPI-S2-2016-1357 of "Programa de Apoyo para la Investigación y Desarrollo (PAID-01-16)". I do not forget to express my gratitude to AVL for receiving me during my research stay in Graz, which became the door to the present stage in my life. Especially, I want to thank Jürgen Schneider and Wolfgang Baier for the trust placed in me.

Several students have assisted me during their internships and final degree projects: Alicia, Victor, Jorge, Yannis, Nacho, Shubham and Gonzalo. With them I have learned the art of mentoring. Especially, I want to acknowledge Jaume Monfort for his helpful assistance during the Virtual Engine Model development. In addition, I wish to say thanks to the staff at CMT for all their help and professionalism. Finally, thanks to my friends and family because they bring the joy and meaning to my life that I need to move forward. 


\section{Contents}

\section{List of figures}

\section{List of tables}

\section{List of abbreviations}

\section{List of publications}

1 Introduction 1

1.1 Background ................... 1

1.2 Objectives and scope . . . . . . . . . . . . . 9 9

1.3 Outline ................... 10

2 Previous work and literature review 13

2.1 Origin of the Virtual Engine Model . . . . . . . . . . . . . 13

2.2 Engine heat transfer . . . . . . . . . . . . . . . 15

2.2.1 Convective heat transfer . . . . . . . . . . . . 17

2.2.2 Measurement of engine heat transfer . . . . . . . . 32

2.2.3 Lumped heat transfer . . . . . . . . . . . . 40

2.2.4 Multizone heat transfer . . . . . . . . . . . . 51

2.3 Modeling of ancillary thermal systems . . . . . . . . . . . . 62

2.4 Thermal codes integrated into global engine models . . . . . 68 
3 Modeling tools $\quad \mathbf{7 5}$

3.1 Virtual Engine Model (VEMOD) . . . . . . . . . . 75

3.2 CALMEC: Combustion diagnosis . . . . . . . . . . . . 77

3.3 Initial model of heat transfer in the engine block . . . . . . 80

3.4 Heat transfer in the turbocharger . . . . . . . . . . . 89

3.5 Heat transfer in gas ducts . . . . . . . . . . . . . . 91

4 Heat transfer in the engine block 93

4.1 Qualitative evaluation of the initial model . . . . . . . . . 94

4.2 Quantitative evaluation of the initial model . . . . . . . . 97

4.2.1 Methods .................. . 97

4.2.2 Results ................... . . 125

4.3 Multizone model of in-cilinder convection . . . . . . . . . . 141

4.3.1 In-cylinder gas motion . . . . . . . . . . . . . . . 143

4.3.2 Thermal analysis . . . . . . . . . . . . . . . 170

4.4 Heat rejection to coolant and lubricant . . . . . . . . . . 188

4.5 Transient behavior . . . . . . . . . . . . . . . 193

4.6 Adaptation for integration into VEMOD . . . . . . . . . . 201

5 Heat transfer in ancillary systems 211

5.1 Model of heat exchangers . . . . . . . . . . . . . . 212

5.1.1 Model structure . . . . . . . . . . . . . 213

5.1.2 Effectiveness-NTU method . . . . . . . . . . . 215

5.1.3 Definition of heat exchangers . . . . . . . . . 218

5.2 Thermo-hydraulic model . . . . . . . . . . . . . 221

5.2.1 Description of objects ............... 223

5.2 .2 General workflow . . . . . . . . . . . . . 227

5.2 .3 Circuit definition . . . . . . . . . . . . . . . 229

5.2.4 Circuit creation . . . . . . . . . . . . . . 231

5.2.5 Hydraulic network analysis . . . . . . . . . . 242

5.2.6 Thermal state of the circuit . . . . . . . . . . 255

6 Heat transfer in the global engine model 273

6.1 Validation methods . . . . . . . . . . . . . . . 274

6.1.1 Experimental tests . . . . . . . . . . . 275

6.1.2 Simulation methods . . . . . . . . . . . . 279

6.2 Validation results . . . . . . . . . . . . . . . 287

6.2.1 Validation in steady state . . . . . . . . . . . . 287

6.2.2 Validation in WLTP driving cycle . . . . . . . . . . 288

6.3 Concluding remarks ................. 295 
$\begin{array}{lll}7 & \text { Conclusions and future work } & 297\end{array}$ 7.1 Main contributions . . . . . . . . . . . . . . . . . . . 298

7.2 Suggestions for future work . . . . . . . . . . . . 302

$\begin{array}{ll}\text { Bibliography } & 307\end{array}$ 



\section{List of figures}

1.1 Generation of greenhouse gases by sectors [12]. . . . . . . 2

1.2 Evolution of $\mathrm{CO}_{2}$ emissions from light-duty vehicles in different countries [13]. . . . . . . . . . . . 2

1.3 Evolution of emissions limits of $\mathrm{HC}$, organic gas and $\mathrm{NOx}$ from light-duty vehicles in US, EU and China [14]. . . . . 3

1.4 Limits of NOx and PM emissions in Euro standards [29]. . . 4

1.5 Exhaust emissions compliance of different diesel cars during real driving emissions tests [30]. . . . . . . . . . . . 4

1.6 Implementation dates of new emissions regulations in the European Union [34]. . . . . . . . . . . . . . . . . 5

1.7 Energy flows in the global energy balance of a diesel engine. 7

2.1 Results of Taylor and Toong [79]. . . . . . . . . . . . . 20

2.2 Results of Annand and Ma [80]. . . . . . . . . . . . . . 21

2.3 Experimental setup used by Woschni [83]. . . . . . . . . . 23

2.4 Results of Sihling and Woschni [85]. . . . . . . . . . . . . 24

2.5 Comparison of Hohenberg and Woschni correlations [86]. . . 25

2.6 Results of Dent and Suliaman [88]. . . . . . . . . . . 25

2.7 Spherical bomb used by Nusselt [83] . . . . . . . . . . . 26

2.8 Relation between heat flux and temperature difference [90]. 28

2.9 Comparison of different heat flux correlations [91]. . . . . . 29

2.10 Contribution of pressure and convection terms in energy equation [98]. . . . . . . . . . . . . . . . . . . 30

2.11 Measured heat flux at various locations [91] . . . . . . . . 31 
2.12 Measured heat flux under different assumptions [100]. . . . 32

2.13 Heat flux curve obtained from in-cylinder pressure signal. . 34

2.14 Slow-response thermocouple installation in cylinder-head [104] . . . . . . . . . . . . . . . . 35

2.15 Slow-response thermocouple installation in engine block [105]. 35

2.16 Coaxial type thermocouple $[102] \ldots \ldots \ldots$. . . . . 36

2.17 Pair-wire thermocouple $[107] \ldots \ldots \ldots$. . . . . . . 37

2.18 Eroding ribbon thermocouple $[100] \ldots \ldots \ldots \ldots$

2.19 Film temperature sensor $[108] . \ldots \ldots \ldots$

2.20 Linkage system to measure piston temperature [102] . . . . 39

2.21 Example of lumped element analysis in engine applications $[117] \ldots \ldots \ldots \ldots \ldots$. . . . . . . . . . . . 4 . . . . . . .

2.22 Example thermal system. . . . . . . . . . . . . . 46

2.23 Lumped models of Shayler et al. [127] (left) and Veshagh and Chen [128] (right). . . . . . . . . . . . . . 47

2.24 Lumped model of Bohac et al. [40]. . . . . . . . . . . . . 49

2.25 Lumped model of Jarrier et al. [117]. . . . . . . . . . . . . 50

2.26 Lumped model proposed by Wagner et al. [130]. . . . . . . 50

2.27 Velocity distributions on the firedeck [86] . . . . . . . . . 51

2.28 Temperature distributions in piston and cylinder-head [46]. 52

2.29 Heat flux distribution on the piston [131]. . . . . . . . 53

2.30 Distributions of velocity and heat transfer coefficient on the piston [131] . . . . . . . . . . . . . . . . . 53

2.31 Flow regions in a multizone model [132] . . . . . . . . . 54

2.32 Wall surfaces in a multizone model [136]. . . . . . . . . . 55

2.33 Example of a two-zone model considering combustion. . . . 57

2.34 Swirl regions considered by Murakami et al. [138]. . . . . . 58

2.35 Flowchart of Hansen's model [141]. . . . . . . . . . . . . 59

2.36 Flowchart of the model of Eiglmeier et al. [132]. . . . . . . 60

2.37 Model of Komninos and Kosmadakis [143]. . . . . . . . . . 61

2.38 Model of Park et al. [153]. . . . . . . . . . . . . . . . . . 64

2.39 Control system of Luptowski et al. [67]. . . . . . . . . . . 64

2.40 Calculation flowchart in the study of Torregrosa et al. [154]. 65

2.41 Calculation flowchart and cylinder heat transfer in the study of Caresana et al. [155]. . . . . . . . . . . . . . 66

2.42 Lumped elements in the cooling circuit studied by Millo et al. [42]. . . . . . . . . . . . . . . . 6 67

2.43 Co-simulation of Samhaber et al. [160]. . . . . . . . . 70

2.44 Model of Kunze et al. [162]. . . . . . . . . . . . . . . . . 71 
2.45 Co-simulation of Banjac et al. [43] . . . . . . . . . . . 72

2.46 Co-simulation of Chalet et al. [44] . . . . . . . . . 73

3.1 Flow-chart of the Virtual Engine Model (VEMOD). . . . . 76

3.2 General workflow of CALMEC . . . . . . . . . . . 78

3.3 3D reconstruction of cylinder liner model. . . . . . . . . . 81

3.4 3D reconstruction of cylinder-head model. Full (left). Enlarged (right). . . . . . . . . . . . . . . . . 82

3.5 Cross section of cylinder-head model (left). Bottom view of firedeck (right). . . . . . . . . . . . . . 82

3.6 3D reconstruction of piston model (left). Cross section (right). 83

3.7 Gas nodes in contact with liner. . . . . . . . . . . . . 87

3.8 Cross section of the complete Nodal Model (half). . . . . . 88

3.9 Simplification of turbocharger geometry in heat transfer model [191] . . . . . . . . . . . . . . . . . . 89

3.10 Lumped heat transfer model of the turbocharger [190]. . . . 90

4.1 Photograph of Engine 1 in test facility. . . . . . . . . . . 98

4.2 Photograph of a swirl valve of Engine 1 [144] . . . . . . . 100

4.3 Temperature conditioning of fluid flows in Engine 1. . . . . 101

4.4 Drill holes and thermocouples in a section of the cylinderhead. . . . . . . . . . . . . . . . . 102

4.5 Location of thermocouples in the firedeck of cylinder 1. . . 103

4.6 Location of thermocouples in the firedeck of cylinder 2. . . 104

4.7 Location of thermocouples in the firedeck of cylinder 3. . . 104

4.8 Location of thermocouples in the firedeck of cylinder 4. . . 105

4.9 Location of thermocouples in the liner of cylinder 1. . . . 105

4.10 Location of thermocouples in the liner of cylinder $2 . ~ \ldots 106$

4.11 Location of thermocouples in the liner of cylinder 3. . . . 106

4.12 Location of thermocouples in the liner of cylinder 4. . . . 107

4.13 Operating points (keypoints). . . . . . . . . . 108

4.14 Procedure to measure the test at maximum swirl ratio. . . . 111

4.15 Procedure to measure the rest of the swirl sweep. . . . . . 112

4.16 Workflow for studies with CALMEC. . . . . . . . . . . . 114

4.17 Configuration of nodes in the firedeck. . . . . . . . . . 115

4.18 View from above of some elements of the new cylinder-head model . . . . . . . . . . . . . . . . 116

4.19 Thickness of firedeck nodes in the adapted model . . . . . 118

4.20 Lateral view of some elements in the new cylinder-head model118

4.21 Study of number of axial nodes in the liner. . . . . . . . 120 
4.22 View of the piston's 3D reconstruction. . . . . . . . . . 120

4.23 Obtaining mean wall temperature in firedeck [144] . . . . . 121

4.24 Notation used in Eq. 4.2. . . . . . . . . . . . . . . . . . 122

4.25 Obtaining mean wall temperature in cylinder liner. . . . . . 123

4.26 Comparison of predicted and measured mean cylinder-head temperatures classified by keypoint. . . . . . . . . . . 126

4.27 Comparison of predicted and measured mean liner temperatures classified by keypoint. . . . . . . . . . . . . . 127

4.28 Comparison of predicted and measured mean firedeck temperatures in the swirl sweep. . . . . . . . . . . . . 128

4.29 Comparison of predicted and measured mean liner temperatures in the swirl sweep. . . . . . . . . . . . . . . . 129

4.30 Measured cylinder-head temperature in the swirl sweep. . . 130

4.31 Measured cylinder liner temperature in the swirl sweep. . . 131

4.32 Heat flux at different swirl ratios estimated from in-cylinder measurements. . . . . . . . . . . . . . . 133

4.33 Temperature distribution in the firedeck. . . . . . . . . . 134

4.34 Temperature distribution in the liner. . . . . . . . . . 135

4.35 Prediction of local temperature at measurement locations. . 136

4.36 Prediction map of the firedeck. . . . . . . . . . . . 137

4.37 Prediction map of the liner. . . . . . . . . . . . . . . 139

4.38 Influence of swirl on a temperature near the center of the firedeck. . . . . . . . . . . . . . . . . . 140

4.39 Influence of swirl on a temperature away from the center of the firedeck. . . . . . . . . . . . . . . . . . 140

4.40 Influence of swirl on a temperature of the liner. . . . . . . 141

4.41 Split of flow regions and surfaces in the cylinder. . . . . . 146

4.42 Calibration of CFD model with pressure curve. . . . . . . . 148

4.43 Gas velocities from CFD simulation. . . . . . . . . . . . 149

4.44 Mesh independence study of axial velocity. . . . . . . . . . 151

4.45 Mesh independence study of radial velocity. . . . . . . . . 151

4.46 Mesh independence study of swirl velocity. . . . . . . . . . . 152

4.47 Mesh independence study of turbulent velocity. . . . . . . . 152

4.48 Heat flux with different laws of the wall. . . . . . . . . . 153

4.49 Heat flux using Han \& Reitz model at different swirl ratios. 154

4.50 Gas velocities with different laws of the wall. . . . . . . . 154

4.51 Control volumes for radial squish $[139]$. . . . . . . . 156

4.52 Control volumes for axial squish [139]. . . . . . . . . 158

4.53 Adjustment of axial velocity component. . . . . . . . . . 167 
4.54 Adjustment of radial velocity component. . . . . . . . . . 168

4.55 Adjustment of swirl velocity component. . . . . . . . . . 168

4.56 Adjustment of turbulent velocity component. . . . . . . . 169

4.57 Comparison of heat flux obtained with different methods: experiment, global model and multizone model. . . . . . . . 174

4.58 Temperature error comparison of a multizone and a global in-cylinder convection model in motored operation. . . . . . 177

4.59 Heat transfer coefficient for the different surfaces and swirl ratios. . . . . . . . . . . . . . . 180

4.60 Prediction at low swirl in the firedeck of cylinder $1 \ldots \ldots 181$

4.61 Prediction at low swirl in the firedeck of cylinder $3 \ldots$. . . 182

4.62 Prediction at high swirl in the firedeck of cylinder 1. . . . 182

4.63 Prediction at high swirl in the firedeck of cylinder 3. . . . 183

4.64 Prediction at low swirl in the liner of cylinder 4. . . . . . 184

4.65 Prediction at high swirl in the liner of cylinder 4 . . . . 185

4.66 Flow past a single row of tubes. . . . . . . . . . . . 189

4.67 Convection between solid and fluid in the old configuration. 191

4.68 Convection between solid and fluid in the new configuration. 192

4.69 Example thermal system including capacitances. . . . . . . 195

4.70 Finite-difference schemes. . . . . . . . . . . . . . . 196

4.71 Comparison of different schemes. . . . . . . . . . . . . 198

4.72 Transient validation: load change. . . . . . . . . . . . 199

4.73 Transient validation: warm-up of structure, coolant and oil. 200

4.74 Grey-box diagram of the Nodal Model integrated in VEMOD.202

4.75 Structure of the XML code that contains the input data of the engine heat transfer model. . . . . . . . . . . . . 203

$4.763 \mathrm{D}$ cut of cylinder cooling galleries. . . . . . . . . . 207

4.77 3D cut of exhaust port. . . . . . . . . . . 208

5.1 Structure of the model of heat exchangers. . . . . . . . . . 213

5.2 Maximum temperature difference in counterflow heat exchanger. . . . . . . . . . . . . . . 216

5.3 Structure of the XML code that contains the input data of the model of heat exchangers. . . . . . . . . . . . . 219

5.4 Global workflow of the thermo-hydraulic model. . . . . . . . 228

5.5 Flowchart of variables in the thermo-hydraulic model. . . . 229

5.6 Structure of the XML code that contains the input data of the thermo-hydraulic model. . . . . . . . . . . . . 230

5.7 Example graph and associated adjacency matrix. . . . . . 233 
5.8 Depth-first search algorithm to find all possible paths from the top. . . . . . . . . . . . . . . . 234

5.9 Path search. Step 1. . . . . . . . . . . . . . . . . 234

5.10 Path search. Step 2. . . . . . . . . . . . . . . . . . 234

5.11 Path search. Step 3. . . . . . . . . . . . . . . . . 235

5.12 Path search. Step 4. . . . . . . . . . . . . . . . 235

5.13 Path search. Step 5. . . . . . . . . . . . . . . . 236

5.14 Path search. Step 6. . . . . . . . . . . . . . . 237

5.15 Path search. Step 7. . . . . . . . . . . . . . . . . 237

5.16 Split of a path into an open and a closed loop. . . . . . . 238

5.17 Split of a path into three branches. . . . . . . . . . . . 239

5.18 Relation between meshes and branches. . . . . . . . . . 240

5.19 Union of two split branches. . . . . . . . . . . . . . . . 241

5.20 Simplification of series circuit. . . . . . . . . . . . . 244

5.21 Simplification of parallel circuit. . . . . . . . . . . . . 244

5.22 Kirchhoff's first law. . . . . . . . . . . . . . . . . . . 247

5.23 Kirchhoff's second law. . . . . . . . . . . . . . . . . . 247

5.24 Algebraic solver. . . . . . . . . . . . . . . . . . . 248

5.25 Formulation of node equations for the system solver. . . . . 250

5.26 Selection of mesh equations for the system solver. . . . . . . 251

5.27 Formulation of mesh equations for the system solver. . . . . 252

5.28 Comparison of Taxi-cab and Powell solving methods. . . . . 254

5.29 Example vector pairs defining the thermal states of branches. 256

5.30 InsertVolume, Action 1. . . . . . . . . . . . . . . . . 258

5.31 Procedure to model heat transfer in a heat exchanger. "Slow flow" case. . . . . . . . . . . . . . . . . 260

5.32 Parcel displacement inside and outside a branch. . . . . . . 260

5.33 Procedure to model heat transfer in a heat exchanger. "Fast flow" case. . . . . . . . . . . . . . . . . 262

5.34 Mass and heat distribution among branches. Example circuit.263

5.35 Mass and heat distribution among branches. Initial state. . 264

5.36 Mass and heat distribution among branches. State 1. . . . 265

5.37 Mass and heat distribution among branches. State 2. . . . 265

5.38 Mass and heat distribution among branches. State 3. . . . 266

5.39 Mass and heat distribution among branches. Final state. . . 267

5.40 Calculation of inlet temperature of heat exchanger. "Slow flow" case. . . . . . . . . . . . . . . . . 268

5.41 Calculation of inlet temperature of heat exchanger. "Fast flow" case. Heat exchanger's branch. . . . . . . . . . . . . 268 
5.42 Calculation of inlet temperature of heat exchanger. "Fast flow" case. Previous branches. . . . . . . . . . . . . . 269

5.43 Simplification of parcels distribution by merging. . . . . . . 270

5.44 Summary of the procedure for thermal state determination. 271

6.1 Photograph of Engine 2 in test facility. . . . . . . . . . . 276

6.2 Photograph of room conditioning system. . . . . . . . . 276

6.3 Steady-state operating points for validation and WLTC trace. 278

6.4 Diagram of cooling circuit model. . . . . . . . . . . . . 280

6.5 Diagram of lubrication circuit model. . . . . . . . . . . . . 281

6.6 Coolant flow rate and oil pressure after calibration of engine and thermostat hydraulic resistances. . . . . . . . . . 284

6.7 Steady-state validation. . . . . . . . . . . . . . . 287

6.8 Engine speed and torque during WLTP driving cycle. . . . 289

6.9 Coolant temperature during WLTC starting after warm-up. 289

6.10 Oil temperature during WLTC starting after warm-up. . . . 290

6.11 Coolant temperature during WLTC starting at $20^{\circ} \mathrm{C}$. . . . 291

6.12 Oil temperature during WLTC starting at $20^{\circ} \mathrm{C}$. . . . . . . 292

6.13 Coolant temperature during WLTC starting at $-7^{\circ} \mathrm{C}$. . . . 293

6.14 Oil temperature during WLTC starting at $-7^{\circ} \mathrm{C}$. . . . . . 294 



\section{List of tables}

4.1 Main specifications of Engine 1. . . . . . . . . . . . . . 99

4.2 Main instrumentation used in Engine 1. . . . . . . . . . . 99

4.3 Test plan. . . . . . . . . . . . . . . . . . . 108

4.4 Common settings for all operating points. . . . . . . . . 109

4.5 Pressure settings for each operating point. . . . . . . . . 110

4.6 Influence of swirl ratio on wall temperature. . . . . . . . . 132

4.7 Motoring tests. . . . . . . . . . . . . . . . . 143

4.8 Adjusted constants for the two models and swirl ratios. . . 186

5.1 Outputs of the thermo-hydraulic model. . . . . . . . . . . 231

6.1 Main specifications of Engine 2. . . . . . . . . . . . . 277

6.2 Main instrumentation used in Engine 2. . . . . . . . . . 277

6.3 Steady-state operating points for validation. . . . . . . . 278 



\section{List of abbreviations}

\section{Latin letters}

\begin{tabular}{|c|c|c|c|}
\hline$A$ & area & $G r$ & Grashof number \\
\hline$C$ & heat capacity rate & $h$ & heat transfer coefficient \\
\hline$c_{m}$ & mean piston speed & $H$ & $\begin{array}{l}\text { hydraulic head differ- } \\
\text { ence }\end{array}$ \\
\hline$C_{p}$ & $\begin{array}{l}\text { specific heat capacity at } \\
\text { constant pressure }\end{array}$ & $H C$ & hydrocarbons \\
\hline cad & crank angle degree & $I$ & moment of inertia \\
\hline $\mathrm{CO}$ & carbon monoxide & $k$ & thermal conductivity \\
\hline $\mathrm{CO}_{2}$ & carbon dioxide & $K$ & thermal conductance \\
\hline$D$ & diameter & $L$ & length \\
\hline$E C U$ & engine control unit & & mass \\
\hline$E G R$ & $\begin{array}{l}\text { exhaust gas recircula- } \\
\text { tion }\end{array}$ & $\begin{array}{l}\dot{m} \\
m . c . f .\end{array}$ & $\begin{array}{l}\text { mass flow rate } \\
\text { meter column fluid } \\
\text { (unit of hydr. head) }\end{array}$ \\
\hline$f$ & friction factor & $N E D C$ & New European Driving \\
\hline$F$ & view factor & & Cycle \\
\hline$a$ & gravity acceleration & $N O x$ & nitrogen oxides \\
\hline
\end{tabular}




$\begin{array}{ll}N u & \text { Nusselt number } \\ N T U & \text { number of transfer units } \\ p & \text { pressure } \\ P r & \text { Prandtl number } \\ q & \text { heat density } \\ \dot{q} & \text { heat flux density } \\ Q & \text { heat (energy) } \\ \dot{Q} & \text { heat flux or heat rate } \\ R & \text { (power) } \\ R & \text { radius } \\ R & \text { hydraulic resistance } \\ R a & \text { Rayleigh number } \\ R e & \text { Reynolds number } \\ s & \text { piston displacement } \\ S R & \text { swirl ratio }\end{array}$

St Stanton number

$t \quad$ time

$T$ temperature

$T D C$ top dead center

$T K E$ turbulent kinetic energy

$U A$ heat exchanger overall conductance

$v \quad$ velocity

$V \quad$ volume

$\dot{V} \quad$ volume flow rate

WLTC Worldwide Harmonized Light-Duty Vehicles Test Cycle

WLTP Worldwide Harmonized Light-Duty Vehicles Test Procedures 


\section{Greek letters}

$\Delta \quad$ increment

$\nabla \quad$ gradient

$\varepsilon \quad$ turbulence dissipation term

$\epsilon \quad$ heat exchanger effectiveness

$\eta \quad$ efficiency

$\mu \quad$ dynamic viscosity

$\rho \quad$ density

$\sigma \quad$ Stefan-Boltzmann constant

$\tau \quad$ angular momentum flux

$v \quad$ kinematic viscosity

$\omega \quad$ angular velocity 



\section{List of publications}

1. Broatch, A., Olmeda, P., García, A., Salvador-Iborra, J., Warey, A., Impact of swirl on in-cylinder heat transfer in a light-duty diesel engine, Energy 119 (2017) 1010-1023, doi:10.1016/j.energy.2016.11.040

2. Torregrosa, A.J., Broatch, A., Olmeda, P., Salvador-Iborra, J., Warey, A., Experimental study of the influence of exhaust gas recirculation on heat transfer in the firedeck of a direct injection diesel engine, Energy Conversion and Management 153 (2017) 304-312, doi:10.1016/j.enconman.2017.10 .003

3. Tanov, S., Salvador-Iborra, J., Andersson, Ö., Olmeda, P., García, A., Influence of the number of injections on piston heat rejection under low temperature combustion conditions in an optical compression-ignition engine, Energy Conversion and Management 153 (2017) 335-345, doi:10.1016/ j.enconman.2017.10.015

4. Broatch, A., Olmeda, P., Martín, J., Salvador-Iborra, J., Development and Validation of a Submodel for Thermal Exchanges in the Hydraulic Circuits of a Global Engine Model, SAE Technical Paper 2018-01-0160 (2018), doi:10.4271/2018-01-0160

Copyright notice. Some illustrations of publication 1 have been reproduced in this thesis as indicated by citations in figures' captions. Some illustrations of publication 4 have been republished in this thesis with permission of SAE International; permission conveyed through Copyright Clearance Center, Inc. 



\section{Introduction}

1.1 Background . . . . . . . . . . . . . . . . . . 1

1.2 Objectives and scope . . . . . . . . . . . . . . 9

1.3 Outline ....................... 10

\section{$1.1 \quad$ Background}

Atmospheric pollution and climate change are two major concerns for society in the $21^{\text {st }}$ century. Transportation is one of the main contributors, as it is a primary source of pollutants and greenhouse gases, as shown in Fig. 1.1. Globally, transportation systems rely on the internal combustion engine, which at present is the most successful technology for mobility [1].

To reduce the environmental impact of engine-based transportation, governments have issued regulations on exhaust emissions of vehicles [2] [3]. Over the years, regulations have effectively driven the development of cleaner and increasingly efficient internal combustion engines [4], as can be seen in Figs. 1.2, 1.3 and 1.4. But despite regulations and technological improvements, on-road vehicle emissions can be above the legal limits [5] [6]. In the past, homologation tests were conducted only in laboratories. Moreover, testing cycles were scarcely representative of real driving profiles. On-road experiments with portable emissions measurement systems demonstrated that real-world pollutant production is much higher than the values shown by laboratory measurements [7] [8] [9] [10] [11], as shown in Fig. 1.5. This is most critical for the problematic emissions of nitrogen 


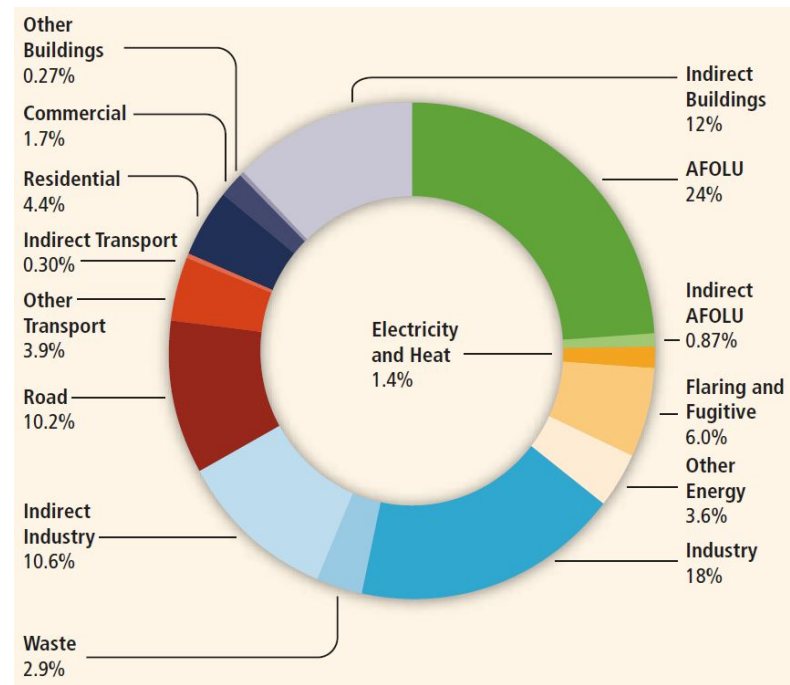

Figure 1.1: Generation of greenhouse gases by sectors [12].

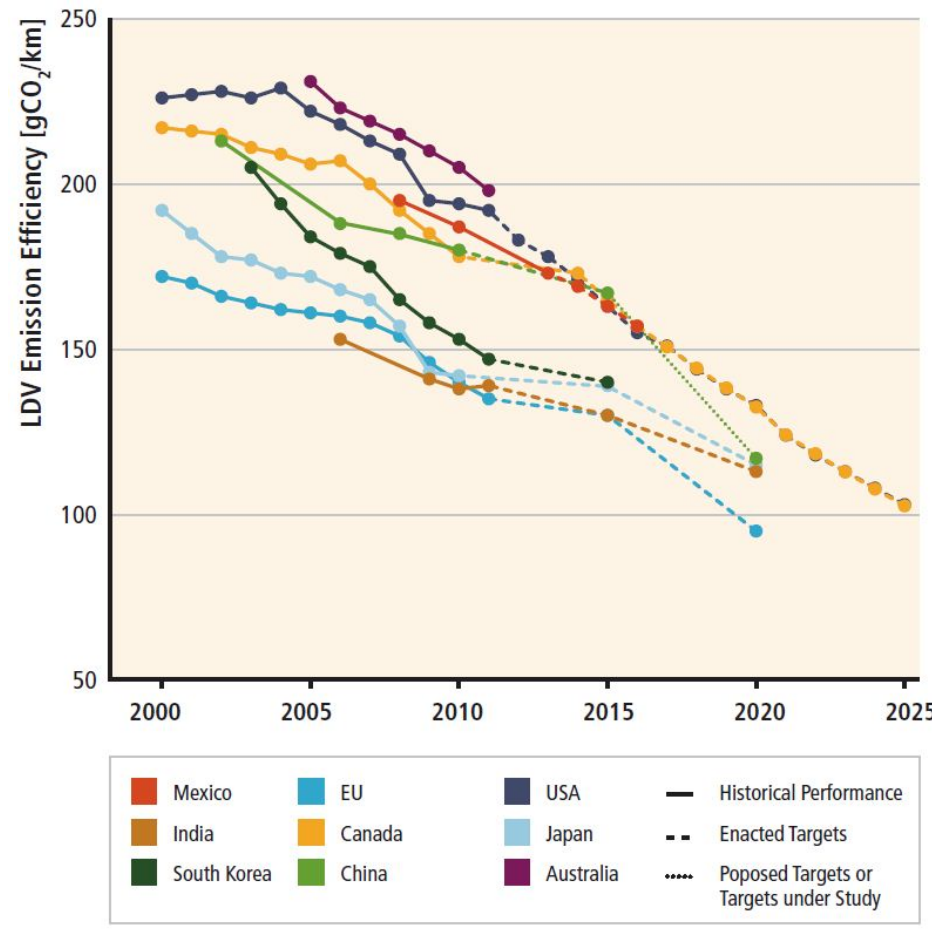

Figure 1.2: Evolution of $\mathrm{CO}_{2}$ emissions from light-duty vehicles in different countries [13]. 


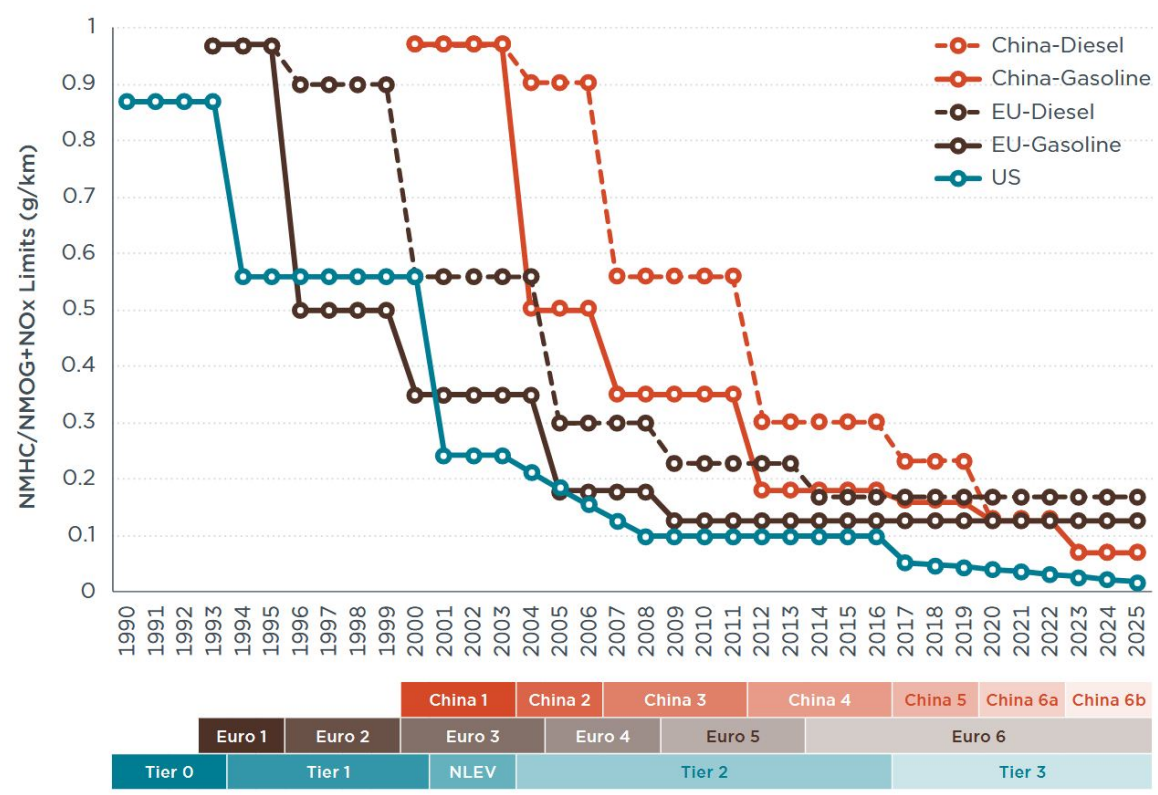

Figure 1.3: Evolution of emissions limits of $\mathrm{HC}$, organic gas and NOx from light-duty vehicles in US, EU and China [14].

oxides (NOx) and particulate matter (PM) from diesel engines [15] [16] [17]. In 2015, a scandal known as Dieselgate shook the automotive industry. It was discovered that one of the principal vehicle manufacturers was installing devices that detected when a diesel car was undergoing a standard emissions test and then modified engine operation to comply with emissions limits during the test [18] [19]. Even in cars without fraudulent devices, researches have found that the employ of defeat strategies tailored for the New European Driving Cycle (NEDC) is widespread [20].

Such problems have triggered the announcement of more stringent emissions standards [21] [22]. In addition to lower emissions limits, new regulations introduce new homologation procedures [23], see Fig. 1.6. In the European Union, the Worldwide harmonized Light vehicles Test Procedure (WLTP) [24] has replaced the NEDC, which was less realistic and less dynamic [25] [26] [27]. In addition, Real Driving Emissions (RDE) tests with portable emissions measurement systems have been incorporated to the homologation procedures. RDE procedures entail that vehicle emissions have to be below the limits for any possible trip on the road [28]. Coincidentally, emissions must not soar under conditions of low 


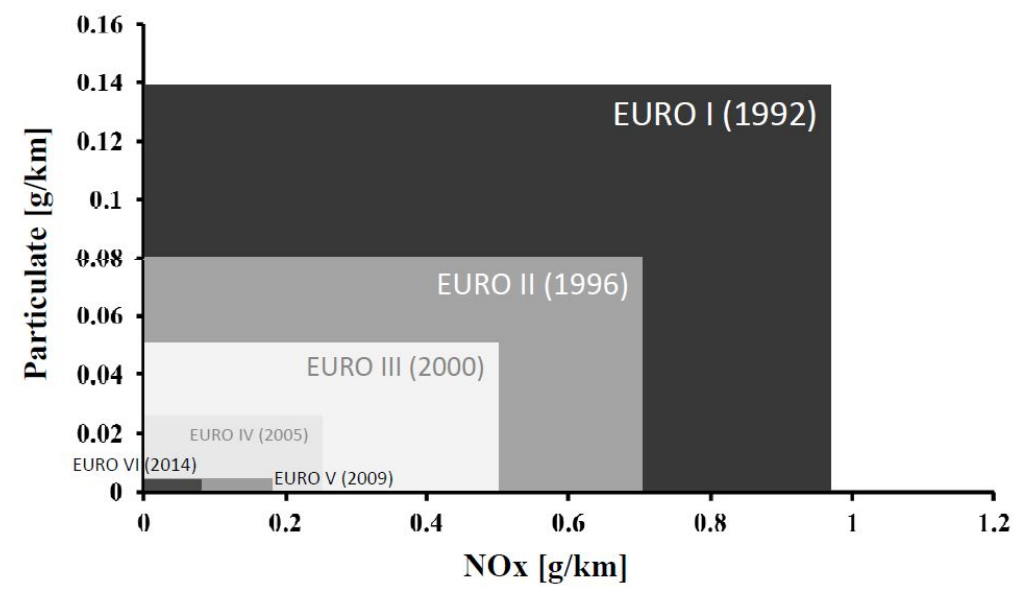

Figure 1.4: Limits of NOx and PM emissions in Euro standards [29].

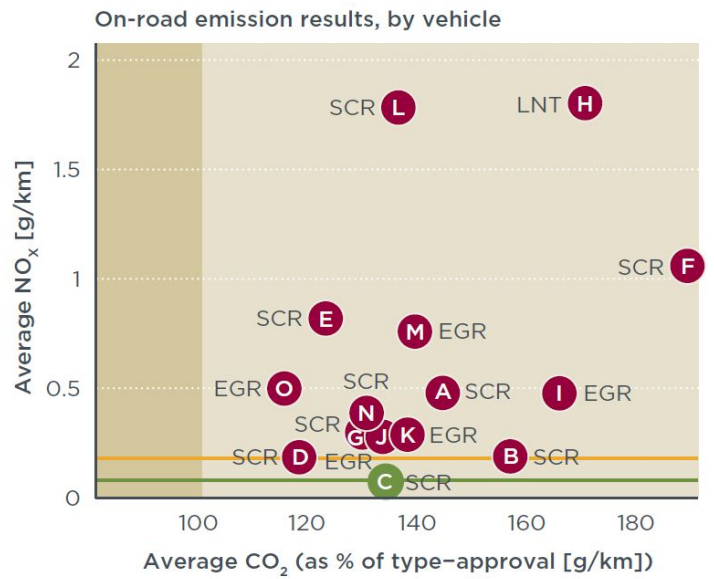

Euro 6 limit

15 test vehicles in total ( 6 manufacturers), with different $\mathrm{NO}_{x}$ control technologies:

- 10 selective catalytic reduction (SCR)

- 4 exhaust gas recirculation (EGR)

- 1 lean $\mathrm{NO}_{\mathrm{x}}$ trap (LNT)

Average Euro $6 \mathrm{NO}_{x}$ conformity factors (ratio of on-road emissions to legal limits): - all cars: 7.1

- best performer (Vehicle C, SCR): 1.0

- bad performer (Vehicle H, LNT): 24.3

- worst performer (Vehicle L, SCR): 25.4

Figure 1.5: Exhaust emissions compliance of different diesel cars during real driving emissions tests [30]. 
ambient temperature or high altitude [31] [32] [33].

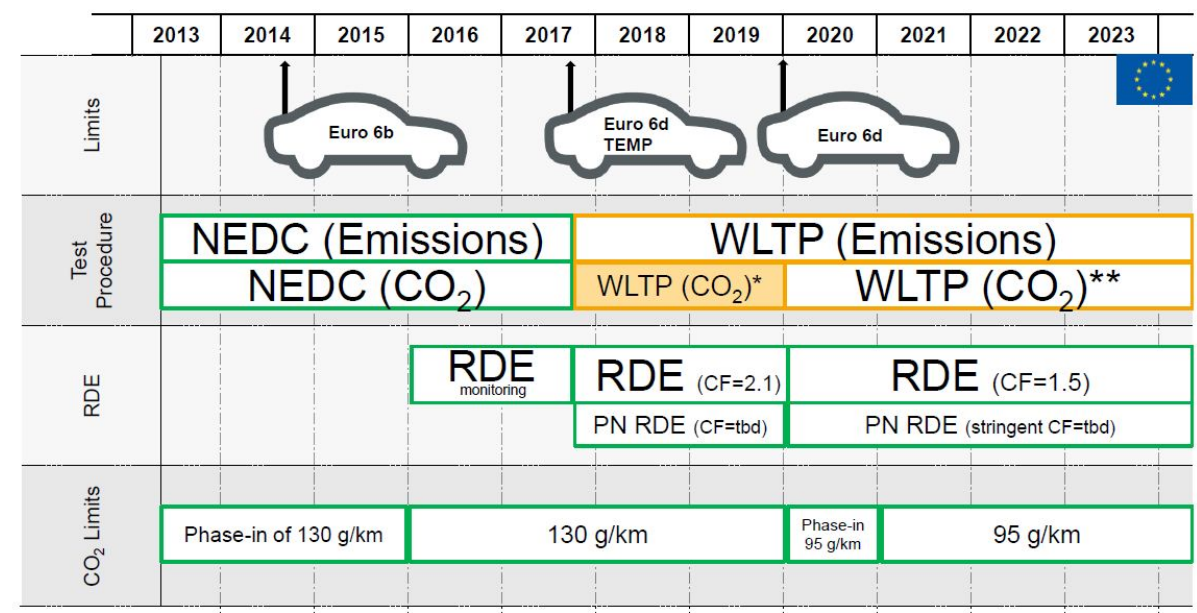

* WLTP $\mathrm{CO}_{2}$ converted (CO2MPAS or double testing) to NEDC, NEDC value for compliance ${ }^{\star *}$ Compliance checked against WLTP based targets

Figure 1.6: Implementation dates of new emissions regulations in the European Union [34].

This context demands tools that are capable of assessing engine performance and emissions under any operating condition. Experimental research is fundamental in internal combustion engines, but it has limitations. Experimental test campaigns are costly. For engine development, test facilities equipped with expensive instrumentation are required. In addition, on-road validation is necessary. If a larger number of operating, driving and environmental conditions have to be evaluated, the costs in terms of labor, time and complexity escalate. For experimental engine research, complex and expensive setups are usually needed. Moreover, at the current level of engine development, small-scale and stochastic processes are being studied [35] [36]. Such processes are very sensitive to changes in engine conditions. To accurately measure those processes, invasive methods or simplified setups are required [37]. Eventually, the system under test is significantly different from the production engine that is being designed. Thus, experimental research may lose representativeness.

Computational research and development by means of modeling and simulation can overcome limitations of experimental methods and cut costs. With the increase of computational power in vehicles, models can 
also be used on board, for diagnosis or for engine management. Different deterministic approaches can be used to model engine processes [38]. The fastest option in computational terms is to use look-up tables. The values in the tables may come from experimental measurements or from simulations of more complex models. Related to this, ad-hoc mathematical models can be adjusted basing on experimental or simulation data. However, the lack of physical foundation of these data driven models deters extrapolations beyond the tested conditions [39]. Moreover, the models are rarely applicable to transient conditions. Another disadvantage is that substantial experimental effort is usually needed to fill in look-up tables or to calibrate coefficients of mathematical models. As such, data driven models are best suited for simulating large systems in which accuracy of the processes going on in different components is not critical.

The next set of computational tools are the semiempirical models. They are based on correlations that provide physical foundation and are generally fast to evaluate. They can work stand-alone or can be integrated in $0 \mathrm{D}$ or even $1 \mathrm{D}$ codes. 0D models have time dependency, but no spatial dependency [40]. They are expressed as sets of ordinary differential equations. 1D models, in contrast, have spatial dependency in one direction, together with time dependency [41]. They are formed by systems of partial differential equations with time and a spatial variable as independent variables. 0D/1D modeling offers a balance between calculation time and accuracy. In addition, extrapolation results are reliable as long as the physical framework does not change. These models require a certain amount of calibration to correct for the three-dimensional nature of real processes [42]. In general, 0D/1D modeling is useful to simulate complex systems with multiple parts that interact with each other, assuming that a certain physical base is required. This is usually the case of global engine models [43] [44] [45].

At the end of the highest computational demand, 3D numerical models simulate physical phenomena with great accuracy and resolution [46]. They are usually employed for component design [47] [48]. 3D modeling is also useful for research of complex physical phenomena, but in that case it is necessary to bear in mind the simplifying hypotheses and boundary conditions under which the model was tested [49]. An advantage of 3D simulation is that calibration effort is smaller, whenever boundary conditions are accurate, because physical processes can be simulated in detail.

Of course, the referred computational approaches can be combined in multidomain models [50]. In internal combustion engines, many complex 
processes take place, and they significantly influence each other. Since the physical nature of each engine process can be different, multiphysics simulation is essential. Only with a physics-based approach is it possible to simulate dynamic interactions of transient processes. To face the current challenges of engine development, an integral approach that includes all engine subsystems is necessary to procure the whole picture of engine operation. Engine and vehicle simulations enable the virtualization of technological development. Through model-based development, the size of experimental tests campaigns in laboratories and on the road can be effectively shrunk. The variety of conditions that can be efficiently tested by means of simulations is enormous, which is a big advantage to anticipate real driving emissions [51]. Moreover, virtual models have intrinsic benefits in terms of flexibility, portability and manipulation when compared to physical prototypes. Finally, virtualization allows several tasks to be performed in earlier stages (front-loading), shortening the product cycle. With this in mind, a new engine simulation tool called Virtual Engine Model (VEMOD) has been developed at CMT-Motores Térmicos research institute. This thesis is a contribution to the construction of this tool. Particularly, this thesis covers the development of heat transfer models.

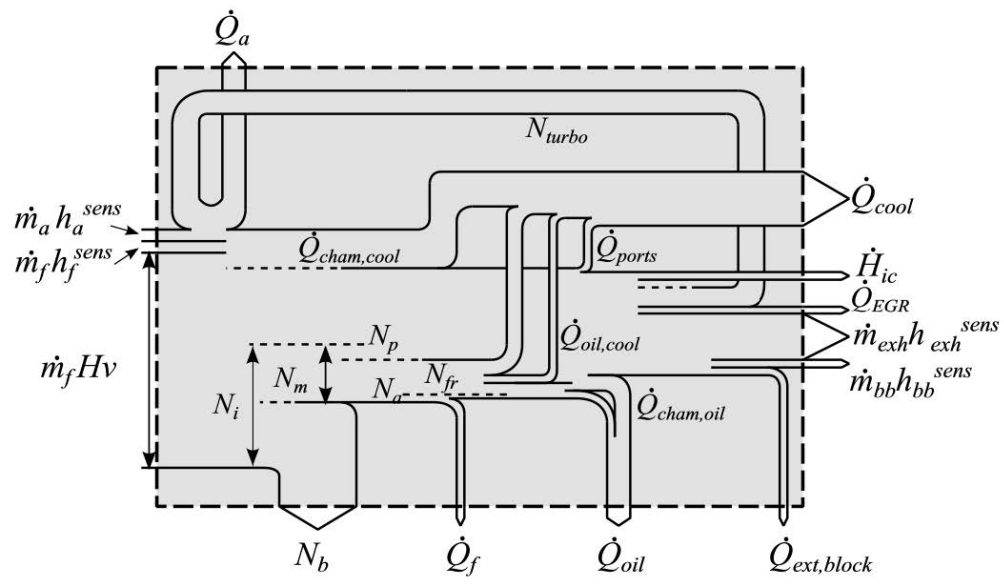

Figure 1.7: Energy flows in the global energy balance of a diesel engine.

The present thesis focuses on engine heat transfer, which is an important phenomenon in internal combustion engines. During the thermodynamic cycle, a considerable amount of energy is lost through the cylinder 
walls and delivered to the cooling medium. This energy loss is around $30 \%$ of the energy released by fuel combustion [52], as can be observed in Fig. $1.7\left(\dot{Q}_{\text {cool }}\right)$. Therefore, heat loss has a significant influence on indicated engine efficiency. If heat loss is reduced, more energy remains inside the combustion chamber. Part of this extra energy can be converted into additional mechanical work. The higher the indicated efficiency, the lower the fuel consumption and thereby the $\mathrm{CO}_{2}$ emissions. Apart from in-cylinder heat losses, heat transfer is important in internal combustion engines for other reasons [53]. In the intake ports, heat transfer from walls to air increases charge temperature, diminishing volumetric efficiency. In the exhaust ports, heat rejection from gas to walls reduces the amount of energy that can be recovered by the turbocharger. In addition, heat transfer is linked to combustion anomalies such as flame quenching and to lubrication problems due to oil degradation at high temperatures. Heat transfer is also relevant because it affects gas and wall temperatures, which have an important influence on the formation of pollutants like NOx [54], carbon monoxide (CO) [55] or unburned hydrocarbons (HC) [56]. Finally, heat transfer processes determine temperatures in the engine structure and are thus important for safe engine operation.

In addition to engine heat transfer, this thesis delves into the whole picture of engine thermal management. Adequate engine thermal management is accomplished by ancillary systems. The mission of thermal management is to maintain temperatures of different engine components within the nominal operation range. That includes dissipating excess heat, preventing degradation of lubricant properties or actively cooling the intake system to increase volumetric efficiency, among other tasks. Due to their ubiquity, thermal management systems interact with most engine subsystems. Thermal management can directly cut fuel consumption and pollutant emissions by shortening the warm-up process. If nominal engine temperatures are reached earlier, $\mathrm{HC}$ and $\mathrm{CO}$ emissions are lowered [55], the catalyst reaches light-off temperature earlier [57], friction is reduced [58] and excessive heat loss is limited [59]. Several innovations in the field of thermal management promise to accelerate the warm-up process or improve engine performance by other means. Optimizing the cooling architecture can deliver reductions in $\mathrm{HC}$ [60], NOx [61] and CO [62] emissions and shorten warm-up [63]. New concepts aim to transport energy from where it is excessive to where it is needed [64]. Electrovalves and electrical pumps allow to control the coolant flow, tailoring cooling to actual needs; thus preventing excessive heat rejection and also parasitic mechan- 
ical losses from pumps, fans or alternators [65] [66] [67]. Other approaches propose to reduce thermal mass, store heat or actively heat the coolant or lubricant [68]. The study of such technological innovations demands testing different hardware, architectures or control strategies. Consequently, to undertake the research and development of thermal management systems, simulation tools are key [42]. Ideally, computational research of thermal management systems must be conducted with simulations of complete vehicle models.

\subsection{Objectives and scope}

The aim of this thesis is to enable modeling heat transfer processes in internal combustion engines in full. As discussed in the background section, the best way to simulate engine operation is by means of a global engine model that contains all relevant submodels. The global engine model used in this research is called Virtual Engine Model (VEMOD). VEMOD is an in-house tool developed at CMT-Motores Térmicos research institute. It is based on $0 \mathrm{D} / 1 \mathrm{D}$ simulation and semiempirical correlations, which provide a balance between accuracy and computation speed. This thesis addresses VEMOD's need to include a heat transfer model of the engine block, as main heat source in the engine, and models of heat transfer in ancillary systems. Once the models are ready and integrated in VEMOD, the engine model has to demonstrate the ability to capture the thermal evolution of a real engine under different operating conditions.

In order to fulfill the research goal, three specific objectives are proposed:

1. Modeling heat transfer processes in the engine block.

2. Modeling heat transfer processes in ancillary systems of the engine.

3. Validating the thermal behavior of a global engine model.

The scope of the research was defined attending to the objectives and to the state-of-the-art. The problem of the optimal method to predict in-cylinder heat rejection remains unresolved to this day after decades of research efforts. This is the most important thermal process of the engine: convective heat transfer between the working gas and the cylinder walls [53]. The most promising approaches for engine heat transfer were reviewed and assessed, with special focus on in-cylinder convection. 
However, multidimensional modeling was excluded because VEMOD is a full 0D/1D tool. Considering 1D models, 0D models and semiempirical correlations, researchers have focused heavily on the latter two. The reason is that engine heat transfer is not a one-dimensional process. As a consequence, 1D models provide unclear enhancements at the cost of significantly increasing calculation time. Only in studies of internally insulated engines, 1D models have been employed [41], but that was not the case of this research. Therefore, 1D approaches were discarded too.

On the subject of ancillary systems, research efforts were devoted to the development of two models. The first one had to provide a common framework for all heat exchange processes in the engine, to ensure that all models can interact thermally. Not limited to this, the model had to implement a customizable element where fluids inside circuits can exchange heat. The second model had to simulate mass and heat transfer in closed hydraulic circuits. In most engines, hydraulic circuits are essential for thermal management. Other ancillary systems of the engine undergo heat transfer processes but they are not considered in this thesis. They are the turbocharger and the gas ducts. Still, these models exist in VEMOD. They had been previously developed in CMT-Motores Térmicos, based on experience gained over the years. When a VEMOD simulation is run, all models come into play together.

Finally, it was decided to limit the research to diesel engines, due to the broad experience of the research group in that kind of engines. Subsequently, many resources were available for this research: a deep knowledge base, experimental equipment, applied know-how and the existence of theoretical models for multiple phenomena present in diesel engines. Nevertheless, whenever possible, the work was developed with the intention of being general and flexible enough to be applicable to any engine, and even to diverse thermal systems.

\subsection{Outline}

Having established the problem to be studied, the first task was to get acquainted with the state of the art. In Chapter 2, the relevant literature is surveyed. First, the antecedents that led to the development of a global engine model at CMT-Motores Térmicos are explained. Second, classical and advanced approaches to model thermal processes in engines are reviewed. The most promising non-dimensional approaches for modeling 
in-cylinder heat transfer are discussed. Moreover, methods to measure and simulate different thermal processes taking place in the engine are examined. Finally, the most remarkable efforts to simulate thermal processes in multisystem and global engine models are summarized. This review provided the necessary theoretical background to study heat transfer processes in the cylinders and in the ancillary systems of the engine.

In Chapter 3, the modeling tools that were employed to fulfill the thesis' works are reported. Among them, the most important are the Virtual Engine Model (VEMOD) and an existing engine heat transfer model. Special emphasis is placed on heat transfer submodels and correlations.

After these preparatory chapters, the objectives of the thesis can be dealt with. The research to meet each specific objective is reported in a different chapter.

Chapter 4 accounts the research carried out to obtain a model capable of predicting heat transfer in the engine block. An existing model was upgraded to that end. First, a qualitative and quantitative evaluation of the model was carried out. After evaluation, some aspects to improve were identified. A campaign of experimental engine tests provided the groundwork for evaluation and for supporting model development. Throughout the chapter, the work to expand the model capabilities is explained. Finally, the implemented improvements were validated on the basis of dedicated engine tests.

Chapter 5 focuses on the models that allow to simulate heat transfer processes in ancillary systems of the engine. Two models were developed from the ground up because no precedents were available in the research group. One is a model of heat exchangers and the other one is a model of thermo-hydraulic circuits. Together, they contribute to simulate heat and mass transfer processes in order to complete the virtual engine. The models are described in depth in this chapter.

In Chapter 6, a joint validation of the virtual engine's thermal behavior is presented. The goal of this validation was to evaluate the reliability of the Virtual Engine Model (VEMOD) for conducting thermal management studies of internal combustion engines. A dedicated campaign of experimental engine tests was undertaken under various operating situations. The experimental tests were replicated by simulations of the Virtual Engine Model. In addition to the tests, the simulation procedure and the model calibration are also described in detail in this chapter. Finally, the 
simulation outcomes are compared with measurements from the experimental campaign.

Chapter 7 gives a final overview of the work done and summarizes the most relevant contributions. To conclude, some ideas for continuing this research in the future are suggested. 


\section{Previous work and literature review}

2.1 Origin of the Virtual Engine Model . . . . . . . . . . . . 13

2.2 Engine heat transfer . . . . . . . . . . . . . . 15

2.2.1 Convective heat transfer . . . . . . . . . . . 17

2.2.2 Measurement of engine heat transfer . . . . . . . . 32

2.2.3 Lumped heat transfer . . . . . . . . . . . . . . 40

2.2.4 Multizone heat transfer . . . . . . . . . . . . 51

2.3 Modeling of ancillary thermal systems . . . . . . . . . 62

2.4 Thermal codes integrated into global engine models . . . . . 68

\subsection{Origin of the Virtual Engine Model}

As explained in the Introduction, engineering offers today different approaches to deal with modeling problems. 0D/1D modeling attains a compromise between accuracy and computation time. Over the years, several 0D and 1D models have been developed at CMT-Motores Térmicos to simulate the diverse processes that are found in internal combustion engines. The common characteristic of all relevant models is that they are based on physics, not on bare numerical data. The correct description of physical systems through the applicable equations allows to model the real system behavior. In contrast to look-up tables, physical modeling achieves reasonable accuracy even under operating conditions that are far away from the operating points used to calibrate the model. In parallel with development, there has been a trend of integration. Putting different 
models to work together has many benefits: models can exchange information to provide better boundary conditions to each other, more realistic simulations can be undertaken, interaction effects that cannot be recreated by individual models can be captured when additional elements are added to the simulation, etc. Eventually, this process of integration led to the development of the Virtual Engine Model (VEMOD).

The core of VEMOD is the gas dynamics model. That is a $1 \mathrm{D}$ code that simulates the mass and energy transfer processes in the gas path. Before the existence of VEMOD, this code was known as OpenWAM ${ }^{\mathrm{TM}}$. WAM stands for Wave Action Model. OpenWAM ${ }^{\mathrm{TM}}$ had been developed and extended during more than 30 years. It was capable of simulating the different aspects of compressible flow, tracking the composition of chemical species along the gas path and considering heat transfer in gas ducts. The model included many components found in thermal engines, such as pipes, volume elements, different kinds of valves, compressors, turbines, bifurcations, etc. An important asset of OpenWAM ${ }^{\mathrm{TM}}$ was the detailed turbocharger model. Main heat transfer submodels of turbochargers and gas ducts are reviewed later in this dissertation, in Sections 3.4 and 3.5. OpenWAM ${ }^{\mathrm{TM}}$ code was significantly improved during its transformation into VEMOD. OpenWAM ${ }^{\mathrm{TM}}$ was solved with a finite differences method, while VEMOD uses a finite volume method. Numerical schemes are different: OpenWAM ${ }^{\mathrm{TM}}$ allowed to choose among Lax-Wendroff, Sweby TVD and CE-SE; VEMOD allows Godunov and MUSCL schemes. Moreover, VEMOD allows a higher variety of species and calculation of physical properties was also improved.

Another precursor of VEMOD is siCiclo. siCiclo is a 0D model of the engine cycle able to calculate in-cylinder thermodynamics [69] [70]. The complementary tool of siCiclo is CALMEC. CALMEC is a combustion diagnosis tool. It can be said that siCiclo and CALMEC work in 'opposite' directions: the main output of siCiclo is the pressure trace and the main input is the combustion law; in contrast, the main output of CALMEC is the combustion law and the main input is the pressure trace. Over the years, CALMEC has incorporated many submodels. The most relevant among them are a simple emptying and filling model for gas management, a heat transfer model of the engine block and a mechanical losses model. This features allowed CALMEC to become a suitable tool for analyzing the energy balance of the engine. The standalone version of CALMEC has a significant role in this thesis and, as such, more details are given during the description of modeling tools, in Section 3.2. 
None of the above referenced models contained a combustion model. When needed, combustion was modeled by means of Wiebe laws, a very common approach in engine research [69]. Wiebe laws are ad-hoc mathematical models of the process of energy release from fuel. For VEMOD to be a complete $0 \mathrm{D} / 1 \mathrm{D}$ model, a $0 \mathrm{D}$ or $1 \mathrm{D}$ combustion model had to be implemented. The suitable in-house tool was a 0D code called ACT [71]. ACT stands for Aparent Combustion Time, the name of a fundamental parameter of the model. The mixing submodel considers two zones in the combustion chamber and five fuel elements in each spray. The existence of several fuel elements provides local information about temperature and chemical composition. Ignition, premixed combustion and diffusive combustion models are based on a simplification and parameterization of chemical kinetics. The model takes into account transient effects through calibrated parameters.

One of the main motivations of having a global tool as VEMOD was to ensure that the models would be kept updated with the latest research innovations, including in-house findings. This is why the constituents of VEMOD are up-to-date physical models. The gas dynamics model is onedimensional while other important models are zero-dimensional. In many cases, the 0D and 1D models are assisted by well-established semiempirical correlations; for instance, to determine concentration of some pollutants or heat transfer in some components. Altogether, the code has solid physical foundations. This makes possible to trust the model results over a broad operation range. Through a combination of 1D, 0D and correlations, VEMOD becomes a multidomain tool, that devotes more computational resources where higher accuracy is required. It is noted that the most computationally demanding models allow to select the solving method. A simplified method (0D instead of 1D, mathematical instead of physical) can be selected when speed is more important than accuracy. This way, the user can decide to some extent where the computational capacity is invested during the simulation according to particular needs.

\subsection{Engine heat transfer}

Multiple heat transfer processes take place in internal combustion engines. Three are the fundamental heat transfer mechanisms: conduction, convection and radiation. Next, the equations that describe these fundamental mechanisms are introduced. 
Fourier's law for unidirectional heat transfer is Eq. 2.1. It applies to heat conduction under the assumptions of unidirectionality, steady state, absence of heat generation and uniformity of properties. Fourier's law implies that heat flux density is proportional to temperature gradient. The proportionality constant or transport property of conductive heat transfer, $k$, is called thermal conductivity. The negative sign indicates that heat flows from bodies at higher temperature to bodies at lower temperature. Alternatively, the equation can be expressed in terms of heat flux as 2.2, because $q=\frac{Q}{A}$. $A$ is cross-sectional area normal to the direction of heat flux. Temperature distribution, $T(x)$, has to be determined for each geometry through integration of the unidirectional heat equation, Eq. 2.3.

$$
\begin{gathered}
\frac{d q}{d t}=\dot{q}=-k \nabla T=-k \frac{d T(x)}{d x} \\
\frac{d Q}{d t}=\dot{Q}=-k A \frac{d T(x)}{d x} \\
\frac{d}{d x}\left(k \frac{d T}{d x}\right)=0
\end{gathered}
$$

Convection is heat transfer between a fluid and a solid surface. Fundamentally, that is the combination of heat conduction (energy transfer) and advection (mass transfer). Convection is modeled by Newton's law of cooling, Eq. 2.4. Newton's law of cooling states that heat flux density is proportional to the temperature difference between fluid and surface. In convection, $h$ is the transport property. It is called convective heat transfer coefficient. Alternatively, the equation can be formulated in terms of heat flux as Eq. 2.5, taking into account surface area $A$.

$$
\begin{gathered}
\dot{q}=h\left(T_{\text {fluid }}-T_{\text {wall }}\right) \\
\dot{Q}=h A\left(T_{\text {fluid }}-T_{\text {wall }}\right)
\end{gathered}
$$

The third kind of heat transfer is radiation. Radiation is determined by Eq. 2.6, where $\varepsilon$ is emissivity, $\sigma$ is the Stefan-Boltzmann constant and $F$ is a view factor between two bodies. Eq. 2.7 shows the expression considering heat flux. Radiative heat transfer does not need a physical medium to exist.

$$
\begin{gathered}
\dot{q}=\varepsilon \sigma F\left(T_{1}^{4}-T_{2}^{4}\right) \\
\dot{Q}=\varepsilon \sigma F A\left(T_{1}^{4}-T_{2}^{4}\right)
\end{gathered}
$$

Among the three mechanisms, convection is the one that concentrates more engine research. Though thermal conduction has a relevant role in 
engines, the most critical processes are dominated by convection. Among those convective processes, the most important one is heat transfer between in-cylinder gas and cylinder walls. Radiation has lower significance in the engine heat transfer processes.

\subsubsection{Convective heat transfer}

Commonly, the type of convective heat transfer processes in engines is considered forced convection in internal turbulent flows. To predict this kind of heat transfer process, the customary approach is to use correlations that can be derived from the Colburn analogy [72]. Analogies among heat, mass and momentum assume that the transport mechanisms are equivalent for those phenomena. The Colburn analogy relates momentum transfer between a solid surface and a fluid flow with heat transfer between them. An empirical factor is defined in Eq. 2.8.

$$
j_{H}=\frac{h}{\rho C_{p} v} \operatorname{Pr}^{2 / 3}=\frac{f}{2}
$$

Alternatively, Eq. 2.8 can be expressed in terms of the Stanton number (Eq. 2.9).

$$
j_{H}=S t \operatorname{Pr}^{2} / 3
$$

Dimensionless numbers definitions are given in Eqs. 2.10 - 2.13.

$$
\begin{aligned}
& S t=\frac{N u}{R e P r} \\
& R e=\frac{\rho v L}{\mu}
\end{aligned}
$$$$
N u=\frac{h L}{k}
$$

$$
\operatorname{Pr}=\frac{C_{p} \mu}{k}
$$

It is important to note that properties of St are evaluated at the fluid bulk temperature while properties of $P r$ are calculated at film temperature (mean of bulk and wall temperatures). The analogy is valid for values of Re higher than 10000 and values of $\operatorname{Pr}$ between 0.7 and 160 .

Applying the analogy to engines presents difficulties. In-cylinder heat rejection transgresses some of the restrictions that make heat and momentum transfer analogies valid [73]. First, in an engine cylinder the velocity 
profile is neither constant nor uniform like e.g. in long pipes. In addition to wall friction, there are momentum and mass transfer mechanisms due to both pressure and thermal gradients as well as engine dynamics. Separation of the boundary layer occurs. Finally, gas-wall convection coexists with other mechanisms of heat transfer, being the most important heat generation by the combustion process.

Reordering Eq. 2.8, a correlation for the heat transfer coefficient can be directly derived, Eq. 2.14 [74].

$$
h=\rho C_{p} v \operatorname{Pr}^{-2 / 3} \frac{f}{2}
$$

The widely known Dittus-Boelter correlation [75] [76] can be inferred from the Colburn analogy. The second law of Blasius provides a simple expression for the turbulent Fanning friction factor, Eq. 2.15, avoiding to solve the implicit Colebrook-White equation.

$$
f=0.046 R e^{-0.2}
$$

This is accurate for Re between 5000 and 200000. Properties of Re are obtained at film temperature. Combining previous equations 2.8, 2.9, 2.10 and 2.15, Dittus-Boelter correlation is obtained, Eq. 2.16.

$$
\begin{aligned}
& \text { St } \operatorname{Pr}^{2 / 3}=0.023 \operatorname{Re}^{-0.2} \\
& N u=0.023 \operatorname{Re}^{0.8} \operatorname{Pr}^{1 / 3}
\end{aligned}
$$

It must be noted that this deduction neglects the fact that $R e$ of the friction factor and Re of the Stanton number have their properties evaluated at different temperatures and therefore are not equal. Dittus-Boelter equation is generally considered applicable to fully developed flows inside smooth tubes with a ratio between length and diameter higher than 60 . Recommended exponent for $\mathrm{Pr}$ is 0.4 when fluid temperature is lower than wall temperature (fluid is heated up) and 0.3 when heat transfer goes from the fluid to the solid. Properties are calculated at the bulk temperature of the fluid.

A related correlation for internal turbulent flow in ducts is the SiederTate equation, Eq. 2.17 [77]. For smooth tubes, its form is very similar to Dittus-Boelter correlation. However, it includes a multiplicative term with the ratio of viscosities at bulk and wall temperatures. This is a correction 
to overcome the requirement of using two different exponents for $\mathrm{Pr}$ in the Dittus-Boelter equation. When the fluid is receiving heat, viscosity decreases and $N u$ increases. When it is cooling, viscosity near the wall increases and heat transfer is hindered. The larger the difference between fluid and wall temperature, the more significant this effect is.

$$
N u=0.023 \operatorname{Re}^{0.8} \operatorname{Pr}^{1 / 3}\left(\frac{\mu}{\mu_{\text {wall }}}\right)^{0.14}
$$

All properties are evaluated at bulk temperature except $\mu_{\text {wall }}$, which is evaluated at wall temperature. Ranges of validity for $\operatorname{Re}$ and $\operatorname{Pr}$ are the same as those of the Colburn analogy.

A newer correlation for smooth tubes is due to Gnielinski, Eq. 2.18 [78]. According to Gnielinski, an equation of general validity can be derived by making use of the fundamental form developed by Prandtl on the basis of momentum theory.

$$
N u=\frac{f}{2} \frac{(R e-1000) P r}{1+12.7\left(\operatorname{Pr}^{2} / 3-1\right) \sqrt{\frac{f}{2}}}
$$

$f$ is the Fanning friction factor, Eq. 2.15. This equation is valid for $R e$ between 3000 and $510^{6}$ and $\operatorname{Pr}$ between 0.5 and 2000. Physical properties are evaluated at bulk temperature. Heat transfer conditions are assumed to be fully developed. Therefore, ducts should still fulfill the requirement of having a length 60 times larger than its diameter. In contrast, engine cylinders have stroke-to-bore ratios between 0.5 and 3 .

Up to now, several applicability problems were encountered. Many flow assumptions are not fulfilled in the kind of processes found in internal combustion engines, in particular inside the cylinder. In addition, the presented correlations are valid for an ideal system, ducts where there are no distortions of the velocity profile. When these correlations are applied to internal combustion engines, they provide a certain of physical base, but it is unrealistic to expect the same degree of accuracy as in long tubes. Due to the highly dynamic flow conditions found in engines, usually it is more desirable to use a correlation that can be applied over a wide range of operating conditions than a correlation that is very accurate under a single set of very particular conditions. 


\section{In-cylinder convection}

Historically, despite the applicability issues, many authors have proposed correlations between $N u$ and $R e$ to deal with the problem of in-cylinder heat transfer, basing on the Colburn analogy. A common assumption has been that variation of $\mathrm{Pr}$ of the working gas is small during the thermodynamic cycle (between 0.6 and 0.8 ) and thus $\operatorname{Pr}$ is deemed constant.

$$
N u=a R e^{b}
$$

Exponent $b$ usually takes values between 0.7 and 0.8 . Since $N u$ is related with the heat transfer coefficient through Eq. 2.11, the following expression may be written, Eq. 2.20.

$$
h=a \operatorname{Re}^{b} \frac{k}{L}
$$

The main differences between author proposals are the variables used as characteristic length $L$ and velocity for $R e$.

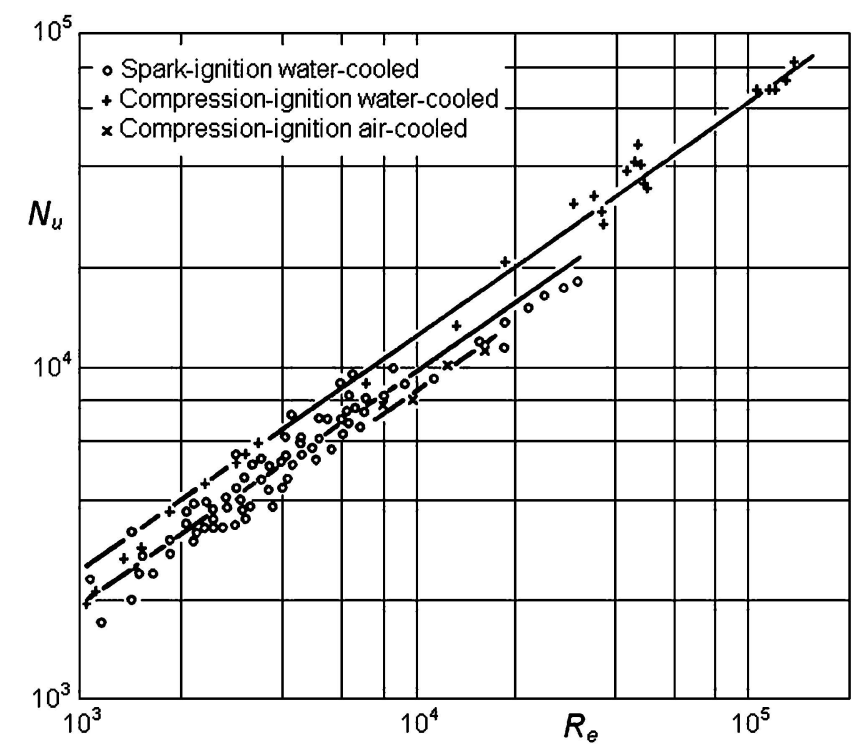

Figure 2.1: Results of Taylor and Toong [79].

Taylor and Toong were the first researchers to apply this approach [79]. They measured mean heat rejection to coolant in 19 engines of different 
types and in various operating conditions. Correlation 2.19 was used along with the following expressions of $N u$ (Eq. 2.21) and Re (Eq. 2.22).

$$
\begin{gathered}
N u=\frac{\dot{Q} D_{\text {cyl }}}{k A_{\text {piston }}\left(T_{\text {gas }}-T_{\text {coolant }}\right)} \\
R e=\frac{\dot{m} D_{\text {cyl }}}{\mu A_{\text {piston }}}
\end{gathered}
$$

$\dot{Q}$ is the measured heat rejection to coolant and $\dot{m}$ is the mean gas mass flow. Constant $b$ was fixed at 0.75 , while three different values of $a$ were adjusted, each one for a distinct engine type. Correlation agreement can be assessed in Fig. 2.1.

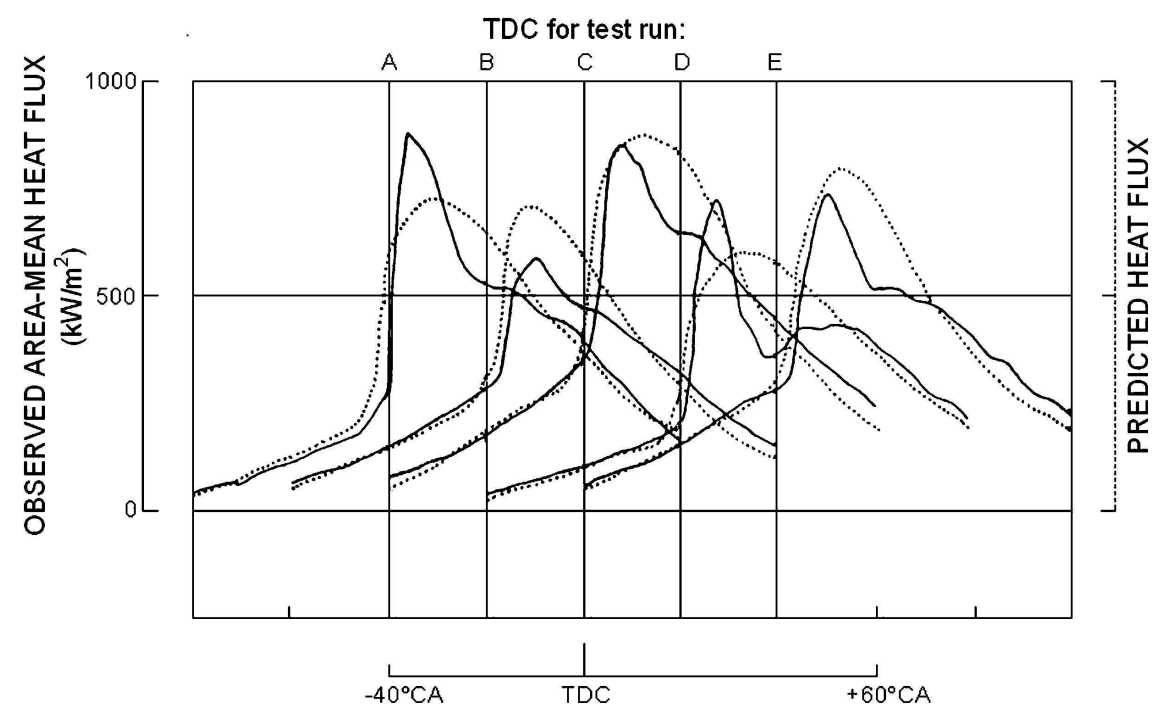

Figure 2.2: Results of Annand and Ma [80].

Other authors aimed to determine the instantaneous heat transfer curve during an engine cycle. Among them, Annand proposed a correlation that remains popular to this day, Eq. 2.23 [80]. The first term on the right side is, directly, Eq. 2.20. The second term is an unsteady term. It attempts to model the phase shift between heat flux and $\left(T_{\text {gas }}-T_{\text {wall }}\right)$. As it can be seen, Annand and Ma propose that this phase shift is inversely proportional to engine speed, $\omega$, in $\mathrm{rad} / \mathrm{s}$. The subject of unsteady heat transfer is discussed later. The final term account for radiative heat transfer, coming from 2.6. $\sigma$ is Stefan-Boltzmann constant. Some comments 
about in-cylinder radiation in engines are given in Section 2.2.3.

$$
\begin{aligned}
\dot{q}= & 0.12 R e^{0.7} \frac{k}{D_{\text {cyl }}}\left(T_{\text {gas }}-T_{\text {wall }}\right)-0.2 \frac{R e^{0.7}}{\omega} \frac{k}{D_{\text {cyl }}} \frac{d T_{\text {gas }}}{d t}+ \\
& +1.5 \sigma\left(T_{\text {gas }}^{4}-T_{\text {wall }}^{4}\right)
\end{aligned}
$$

Results obtained with this correlation in an indirect injection diesel engine are shown in Fig. 2.2. Measured curve is plotted in solid line and predicted curve in dashed line. A particularity of Annand's formula is the velocity to evaluate $R e$. The velocity was calculated from a balance of mean kinetic energies, as proposed by Knight [81]. Annand and Ma considered the inflowing and outflowing kinetic energy through valves as well as the kinetic energy of the prechamber flow. The version of the correlation that is cited here comes from a work by Annand and Ma published in 1970, but a previous version without unsteady term was published earlier, in 1963, by Annand [82].

Perhaps the most widely known correlation is the one due to Woschni [83] [84]. It can be deduced from Eq. 2.20, as can be seen in Eq. 2.24. The new constant is $C_{0}$, different from $a$. Woschni proposed $C_{0}=110$ and $b=0.8$ after tests with the experimental setup shown in Fig. 2.3.

$$
h=a \operatorname{Re}^{b} \frac{k}{L}
$$

For a circular tube, $L=D_{c y l}$

$$
h=a\left(\frac{\rho D_{c y l} v}{\mu}\right)^{b} \frac{k}{D_{c y l}}
$$

Woschni assumptions:

$$
\begin{aligned}
& k \propto T^{0.75} \\
& \mu \propto T^{0.62} \\
& \rho \propto \frac{p}{T} \text { (ideal gas) }
\end{aligned}
$$

Rearranging,

$$
h=C_{0} D_{c y l}^{b-1} p^{b} T^{(0.75-1.62 b)} v^{b}
$$

$v$ is gas velocity, which is calculated with Eq. 2.25. In Eq. 2.25, velocity is the sum of three terms. The first one stands for the contribution of piston motion, as $c_{m}$ is mean piston speed. $C_{W 1}$ is 6.18 for the scavenging process and 2.28 during compression and expansion. The second term 


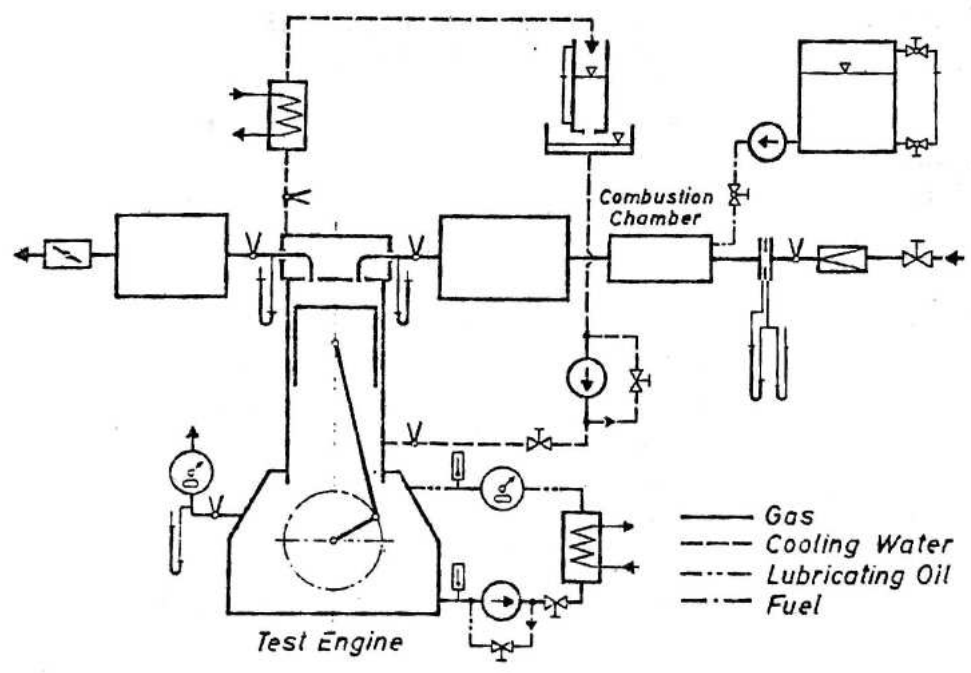

Figure 2.3: Experimental setup used by Woschni [83].

is the contribution of the tangential velocity or swirl motion. $\omega$ is rotational gas speed around the cylinder axis. $C_{W 2}$ has the value 0.417 during scavenging and 0.308 during compression and expansion. The third term on the right is the combustion velocity term. In this term, IVC stands for intake valve closure conditions, $V_{d}$ is displaced volume, $V_{d}=\pi \frac{D_{c y l}^{2}}{4} s$, where $s$ is piston stroke, and $p_{0}$ is pressure under motoring conditions. The factor $\left(p-p_{0}\right)$ is used because combustion has a very direct influence on pressure. $\frac{T_{I V C}}{p_{I V C} V_{I V C}}$ accounts for in-cylinder mass. Recommended value for $C_{2}$ is $3.2410^{-3} \frac{\mathrm{m}}{\mathrm{s}^{\circ} \mathrm{C}}$ during the expansion stroke and 0 for the rest of the engine cycle. The effect of heat radiation is considered in lumped form by the combustion term. Woschni warned that constants had to be calibrated for every engine design.

$$
v=C_{W 1} c_{m}+C_{W 2} \frac{D_{c y l}}{2} \omega+C_{2} \frac{V_{d} T_{I V C}}{p_{I V C} V_{I V C}}\left(p-p_{0}\right)
$$

Sihling and Woschni published a study [85] summarized in Fig. 2.4, in which the prediction of this correlation was compared with measurements at three different cylinder locations.

Hohenberg [86] conducted an experimental campaign using various measurement techniques in different direct injection diesel engines. His findings led him to propose a correlation similar to Woschni's, Eq. 2.26. 


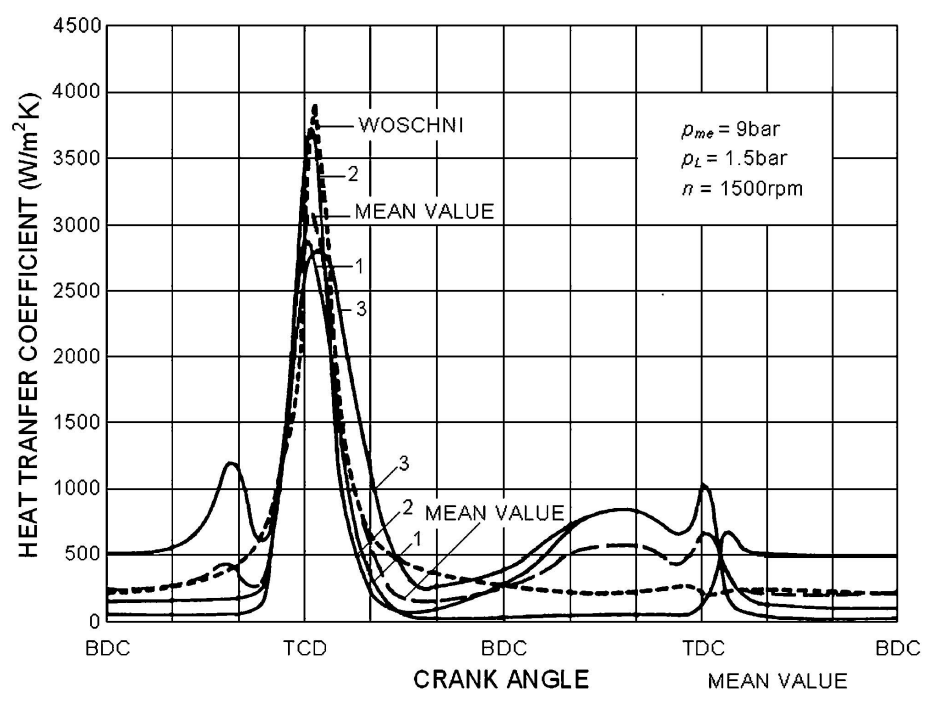

Figure 2.4: Results of Sihling and Woschni [85].

$V$ is not the instantaneous cylinder volume but a characteristic volume given by $V=\frac{\pi}{6} D_{s p h}^{3}$, where $D_{s p h}$ is the diameter of a sphere that has the same instantaneous volume as the engine cylinder.

$$
h=130 V^{-0.06} p^{0.8} T^{-0.4}\left(c_{m}+1.4\right)^{0.8}
$$

Hohenberg claimed that some limitations of Woschni's approach, like the overestimation of the heat peak and the underestimation of heat transfer during scavenging, were overcome by his formula. A comparison between the two correlations can be seen in Fig. 2.5.

An approach that attempted to provide higher spatial resolution was developed by LeFeuvre et al. [87] and by Dent and Suliaman [88]. LeFeuvre based his formula in the Colburn analogy but did not assume a constant $\mathrm{Pr}$, as can be seen in Eq. 2.27. However, the most important innovation of his equation was to use the local radius $r$ as characteristic length, instead of a fixed value. This means that the heat transfer coefficient presents a radial gradient in piston and cylinder head. In addition, the $R e$ formulation was different, with rotational gas speed $\omega$ as characteristic velocity. Gas properties were evaluated at film temperature.

$$
h=0.047\left(\frac{\rho r^{2} \omega}{\mu}\right)^{0.8} \operatorname{Pr}^{1 / 3} \frac{k}{r}
$$




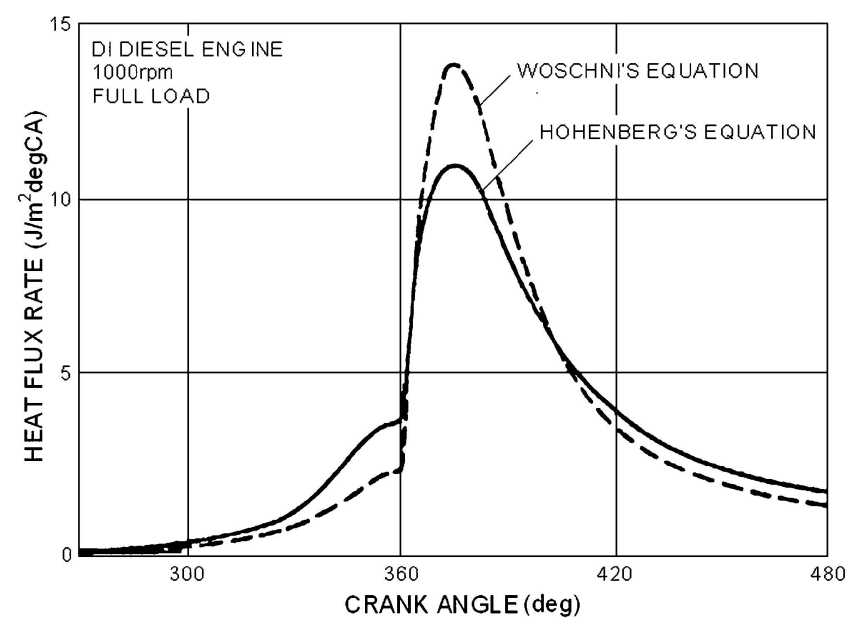

Figure 2.5: Comparison of Hohenberg and Woschni correlations [86].

LeFeuvre's research was conducted in a direct injection diesel engine. The correlation was calibrated under motoring conditions. However, when it was applied to firing operation, it displayed limited prediction capacity.

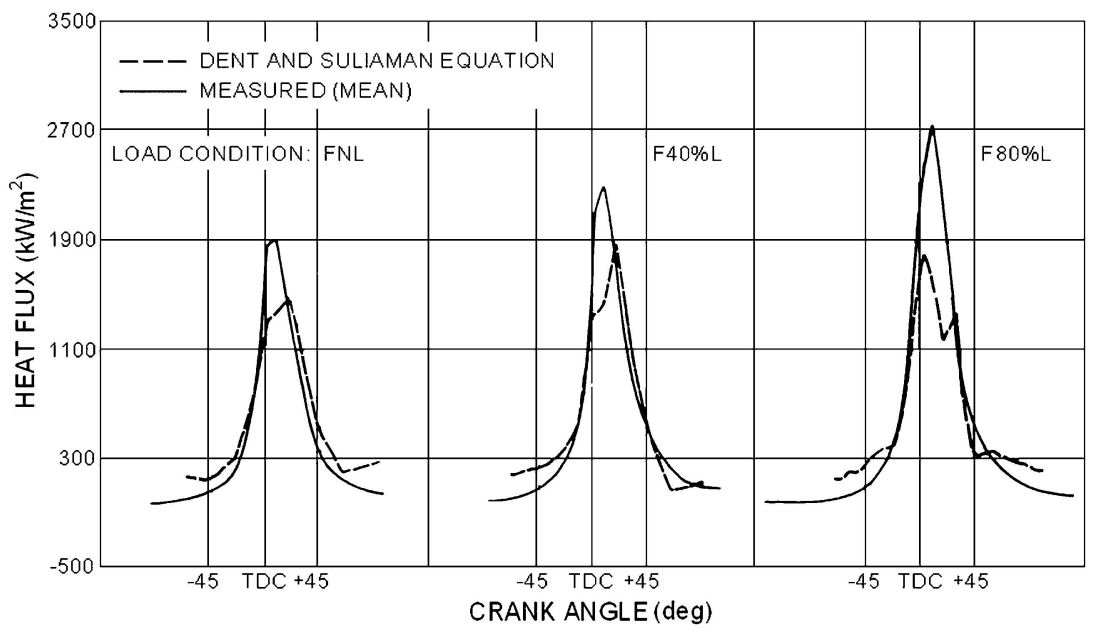

Figure 2.6: Results of Dent and Suliaman [88].

An analogous correlation was employed by Dent and Suliaman, Eq. 2.28. They considered $\operatorname{Pr}$ constant and evaluated gas properties at bulk temperature. Their correlation was considered suitable only for motored operation. Results of mean heat flux on the piston crown at different loads 
are presented in Fig. 2.6.

$$
h=0.023\left(\frac{\rho r^{2} \omega}{\mu}\right)^{0.8} \frac{k}{r}
$$

This approach, using the local radius as characteristic length, is a simplistic way to achieve non-uniform heat transfer. More physical alternatives for providing spatial resolution to in-cylinder convection will be examined in Sections 2.2.3 and 2.2.4. They are non-dimensional approaches known as lumped element modeling and multizone heat transfer modeling.

At the beginning of engine heat transfer research, other approaches were used. Nusselt, Eichelberg and Pflaum used correlations that were not based on internal forced convection but on free convection. They are briefly reviewed here due to its historical relevance.

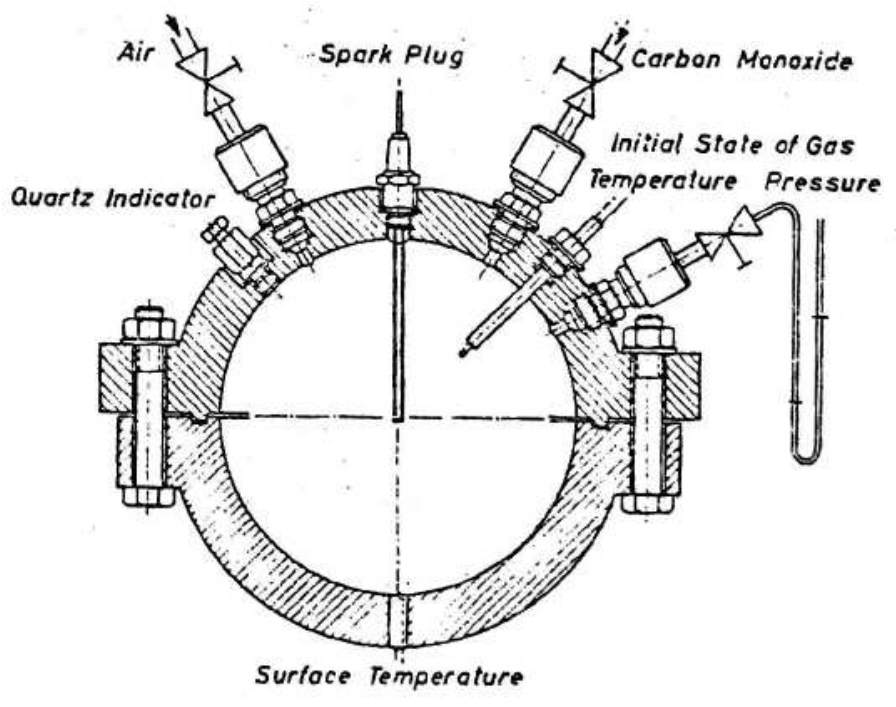

Figure 2.7: Spherical bomb used by Nusselt [83].

Nusselt did experiments in a spherical bomb under quiescent combustion conditions [89]. An image of the bomb can be found in Fig. 2.7. Through a combination of measurements and the application of the first law of thermodynamics, he managed to formulate Eq. 2.29 in 1923. The equation is displayed here as reported by Woschni [83]. The term $\left(1+1.24 c_{m}\right)$, where $c_{m}$ is mean piston speed, was added to the basic formula to take the influence of piston motion into account. One downside of 
Nusselt's experimental approach was that it could not be applied during combustion.

$$
h=0.99\left(1+1.24 c_{m}\right) \sqrt[3]{p^{2} T}
$$

Eichelberg made measurements in a reciprocating engine. He adapted Nusselt's formula to include the influence of heat transfer by radiation in a lumped form.

$$
h=2.43 \sqrt[3]{c_{m}} \sqrt{p T}
$$

The free convection approach has been considered unsuitable for engine heat transfer since correlations of forced convection in turbulent flow started to be employed.

\section{Unsteady heat transfer}

Measurements of convective heat flux reveal that this magnitude is not directly proportional to the difference of temperature of the fluid and the wall, as enunciated by Eq. 2.4. Instead, there is a phase shift between heat flux and temperature difference. This has been observed under highly dynamic or cyclic conditions, like the ones found in internal combustion engines. Some of the first researchers to study this effect were Annand and Ma [80] and Lawton [90]. The phase shift can cause the direction of heat flux to be opposite to the direction of the temperature gradient. For example, when wall temperature is higher than fluid temperature, heat can unexpectedly flow from fluid to wall temporarily. This effect is called heat flux reversal and is illustrated in in Fig. 2.8. Over time, if boundary conditions are stable, flow and heat transfer start stabilizing, directions of heat flux and temperature gradient realign and, eventually, a steady state is reached. That is why the phase shift is known as an unsteady effect. In a cyclic system such as a closed reciprocating machine, directions of heat flux and temperature gradient can align, dealign and realign over and over, cyclically. In a simulation code, these effects cannot be simulated under quasi-steady assumptions of fluid flow and heat flux as Newton's law of cooling, Eq. 2.4.

In the literature, different approaches have been proposed to model unsteady heat transfer. They can be divided into four categories:

1. Unsteady term. A term that depends on the time-derivative of a thermodynamic variable is added in the correlation of heat flux or heat transfer coefficient. Annand and Ma used the derivative of gas temperature, as was seen in Eq. 2.23 [80]. The amelioration of heat 


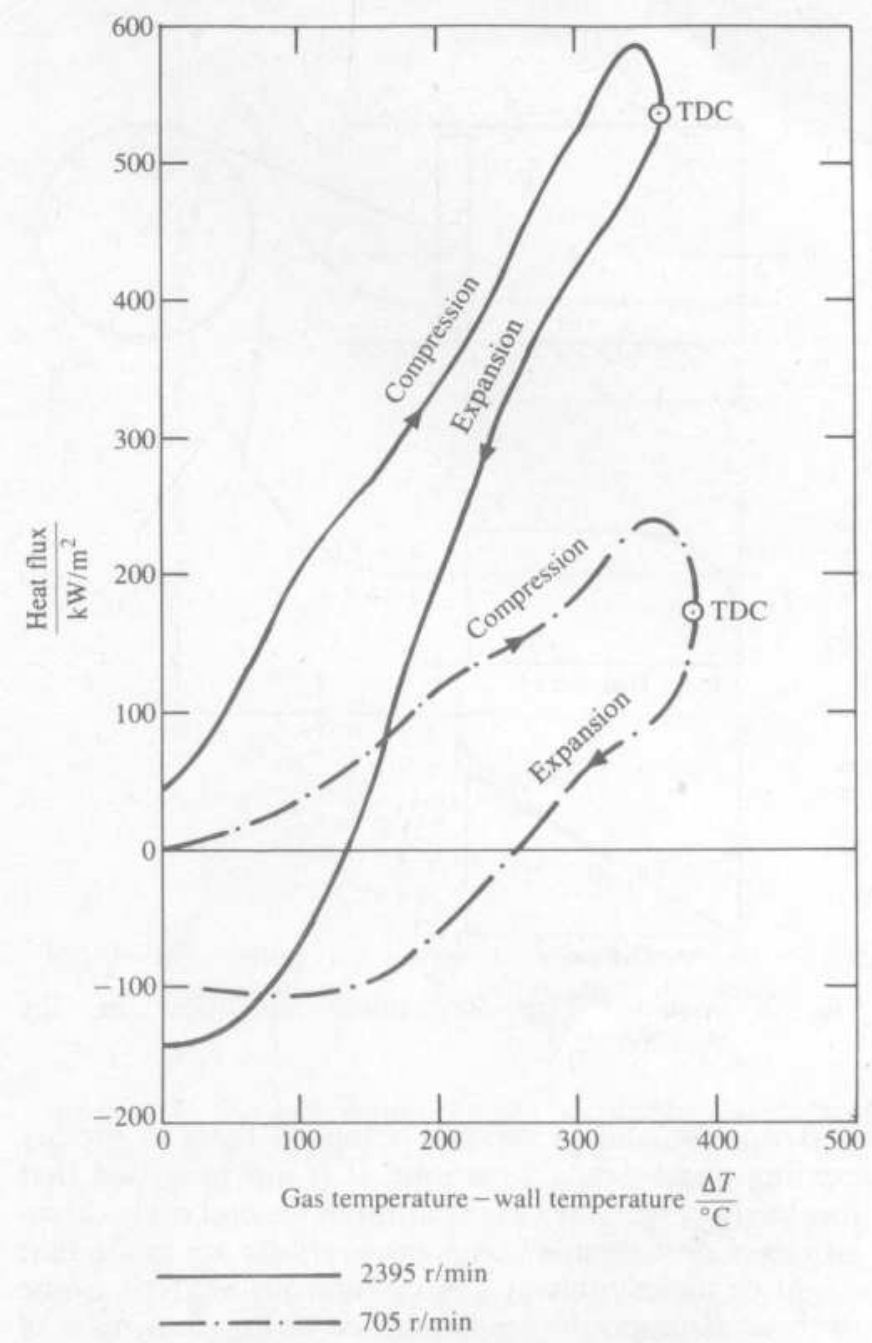

Figure 2.8: Relation between heat flux and temperature difference [90]. 
flux phase prediction in contrast with Woschni and Hohenberg correlations is shown in Fig. 2.9. In contrast, Lawton, after a throughout study, considered more appropriate to use the derivative of the cylinder volume [90]. His correlation was able to model the phase shift at different engine speeds under motoring conditions.

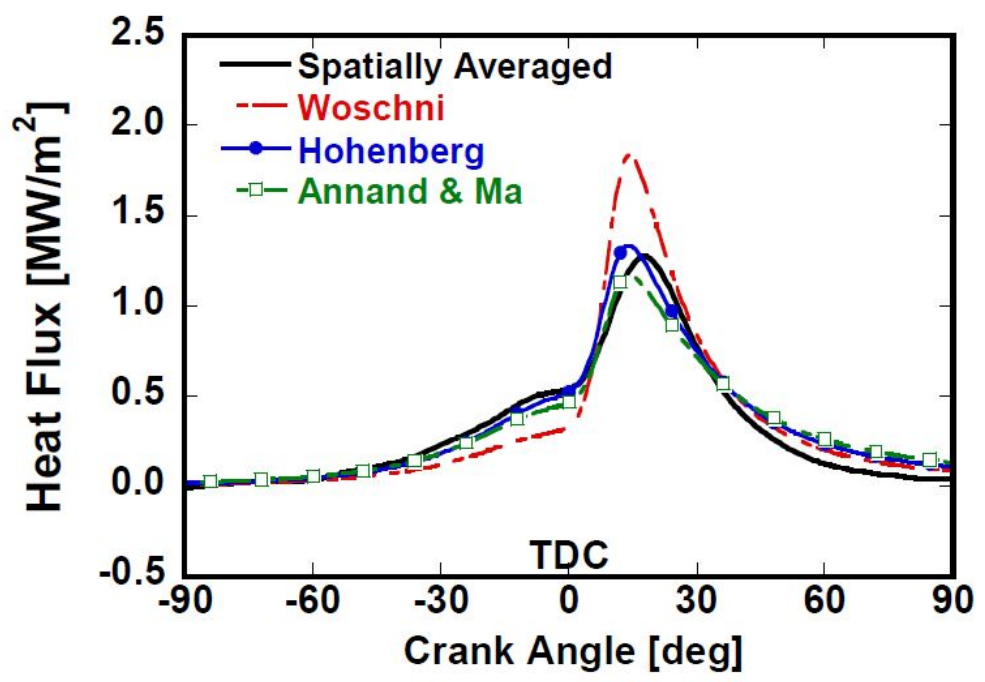

Figure 2.9: Comparison of different heat flux correlations [91].

2. Complex Nusselt. The overall evolution of heat rejection in a closed reciprocating machine can be theoretically formulated considering that heat flux has a real component and an imaginary component. This can be modeled as a complex Nusselt number of the form of Eq. 2.31. The real part, $N u_{R}$, is proportional to the temperature difference while the imaginary part, $N u_{I}$, is proportional to the product of the inverse of rotational speed and the derivative of temperature difference. Research works that applied this concept to gas springs were the one of Kornhauser and Smith [92] and the more recent study of Mathie et al. [93]. A related work was the numerical study of Catto and Prata [94], also focused on a gas spring. They found that both the correlation of Lawton and the correlation of Kornhauser and Smith captured the simulated trends for various fluids and operating conditions.

$$
\dot{q}=\frac{k}{L}\left[N u_{R}\left(T_{\text {gas }}-T_{\text {wall }}\right)+N u_{I} \frac{1}{\omega} \frac{d\left(T_{\text {gas }}-T_{\text {wall }}\right)}{d t}\right]
$$


3. Turbulent conductivity. Several authors have considered a turbulent thermal conductivity to model the unsteady thermal boundary layer, instead of using a time-derivative [95] [96] [97]. The turbulent thermal conductivity was obtained from a turbulent Prandtl number and a turbulent viscosity. This could provide better physical foundation.

4. Pressure work. Oude Nijeweme et al. [98] found contradictions in the usage of turbulent Prandtl numbers and turbulent viscosities. Maintaining the physics-based approach, they found that phasing is dominated by the pressure work term. Therefore, they proposed to add pressure work and axial convection terms to solve the energy equation in the boundary layer. The influence of these terms is shown in Fig. 2.10.

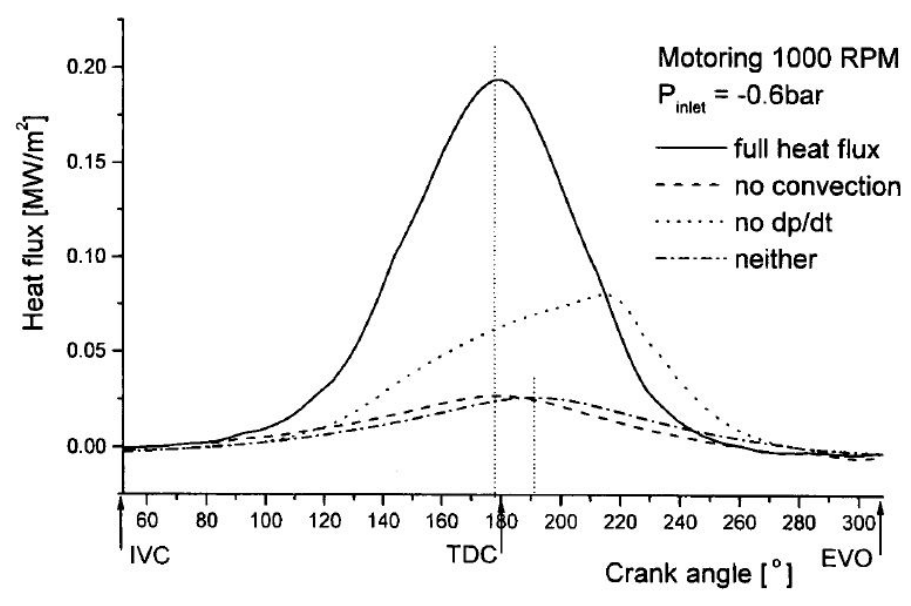

Figure 2.10: Contribution of pressure and convection terms in energy equation [98].

In summary, today there is no universal agreement on how to model the unsteady aspect of heat transfer by means of correlations or nondimensional models. Also, no proposed procedures to calibrate the unsteady term in heat flux correlations have been found in the literature, except direct comparison with experiment. Chang et al. [91] were able to empirically fit a modified Woschni correlation to experimental measurements, without using an unsteady term. First, they modified the temperature exponent, claiming that conductivity and viscosity of the gas 
should be considered constant with temperature. Second, they used the instantaneous chamber height as characteristic length, instead of the constant bore diameter. Finally, they reduced the weight of the combustion velocity term to make it six times smaller.

What is more, the phase shift and the reversal of heat flux have been called into question. Rakopoulos et al. [99] made measurements in the cylinder-head of a direct injection diesel engine. The curves of indicated gas pressure and gas-to-wall heat flux were almost in phase, while wall temperature on the firedeck surface presented a 10 cad phase delay. This delay was attributed to inertia. Clearly, inertia of the instrument and inertia of the wall material can introduce a delay. Also, if gas temperature is measured in a location that is far away from the heat flux probe, the measured gas temperature may be different from the local temperature. Heat flux phasing varies widely depending on location, as shown in Fig. 2.11 .

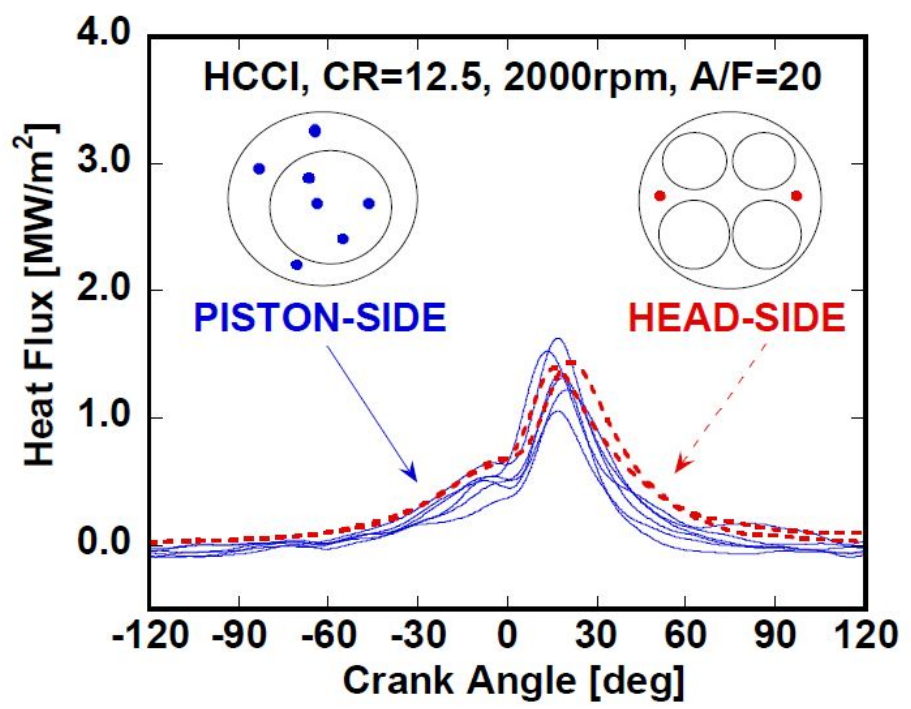

Figure 2.11: Measured heat flux at various locations [91].

Besides, several researchers have demonstrated that the experimental measurement of instantaneous heat flux itself is far from trustworthy. In particular, the assumption of one-dimensional conduction in heat flux probes has been disputed both by experimental and by numerical studies. Moreover, insulation and homogeneity of the inner medium are not ideal. 
All this can make conduction in heat flux probes three-dimensional. Along these lines, Buttsworth et al. [100] did not observe heat flux reversal when they applied a method that did not make 1D and 2D assumptions. However, when the assumptions were applied, flux reversal appeared, as can be seen in Fig. 2.12. Thus, they suggested that flux reversal could be the product of wrong $1 \mathrm{D}$ and $2 \mathrm{D}$ approaches for measuring heat flux.

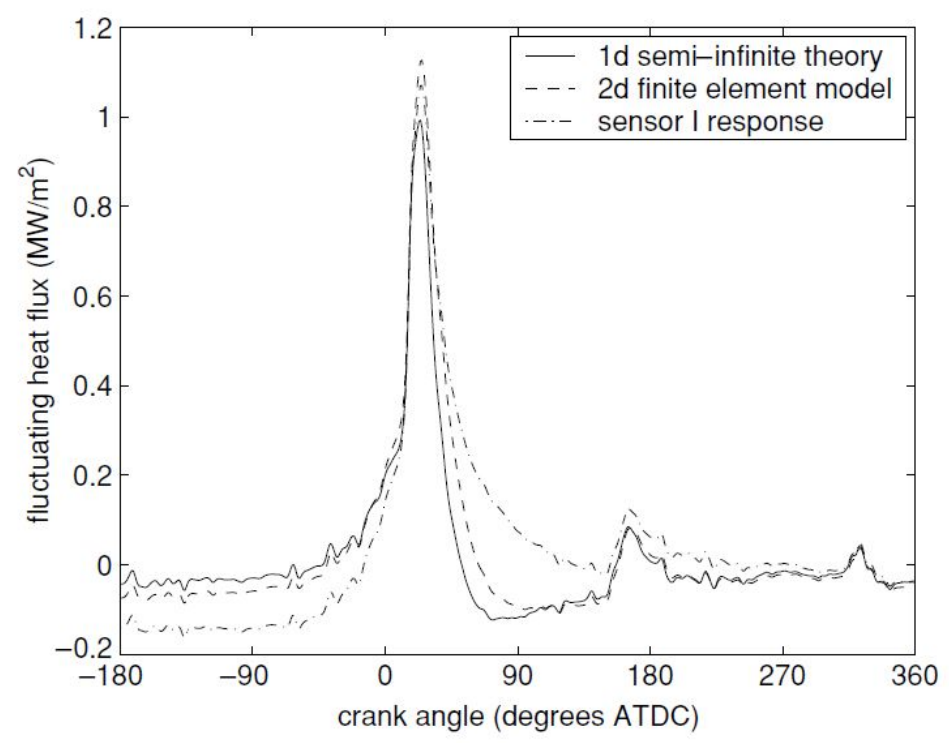

Figure 2.12: Measured heat flux under different assumptions [100].

After this review, it can be concluded that unsteady heat transfer is not yet fully understood. Therefore, more research is needed to clarify this matter. In this thesis, research was focused on quasi-steady heat transfer.

\subsubsection{Measurement of engine heat transfer}

A brief overview of the available techniques to measure heat transfer in engines is provided in this section, with special focus on in-cylinder heat rejection. This review is intended to provide a glimpse of the capabilities and limitations of the different techniques, as well as the implementation pitfalls. 


\section{Energy balance}

The first and less precise technique was used by Nusselt in his research [89]. It consists in a balance of the energy flows present in the system, e.g. the internal combustion engine. To apply this principle to the combustion chamber, the first law of thermodynamics is used. This requires the measurement of in-cylinder pressure. This technique is still employed today with a high degree of sophistication [101]: consideration of all heat energy flows in the system, extensive instrumentation, more accurate sensors and equipment, combination with models and correlations, etc. The limitation of this method is that in-cylinder heat flux is measured indirectly and therefore there are many uncertainties associated to the result.

Under motoring operation, it is possible to estimate the instantaneous heat rejection to the cylinder walls using the pressure trace, the curve of cylinder volume and the first law of thermodynamics for closed systems, see Eq. 2.32, where $n$ is the polytropic index and $\gamma$ is the adiabatic index, also called specific heat ratio.

$$
\begin{array}{r}
d Q=\frac{n-\gamma}{\gamma-1} p d V \\
\text { where, } n=-\frac{d p}{p} \frac{V}{d V}
\end{array}
$$

There are two problems associated to this procedure. The first one is to determine the relative phasing of pressure and volume curves. The second problem is that the derivative of the pressure trace amplifies signal noise. A result of this procedure is plotted in Fig. 2.13, where heat flux is sampled every $0.5 \mathrm{cad}$. It is important to note that the method can only be applied during the part of the cycle in which valves are closed, i.e. when the cylinder is a closed volume. In this thesis, this method is employed to provide reference heat flux curves for calibrating correlation constants and also to provide insights during the analyses.

\section{Slow-response temperature sensors}

A common technique for thermal research is to drill the engine structure and insert thermocouples, thermistors or resistance temperature detectors (RTD) to measure metal temperature at different locations. Slow-response sensors measure temperature with low temporal resolution (mean temperature). Nevertheless, this resolution can be enough for many studies. Different experimental studies showed that the maximum temperature swing 


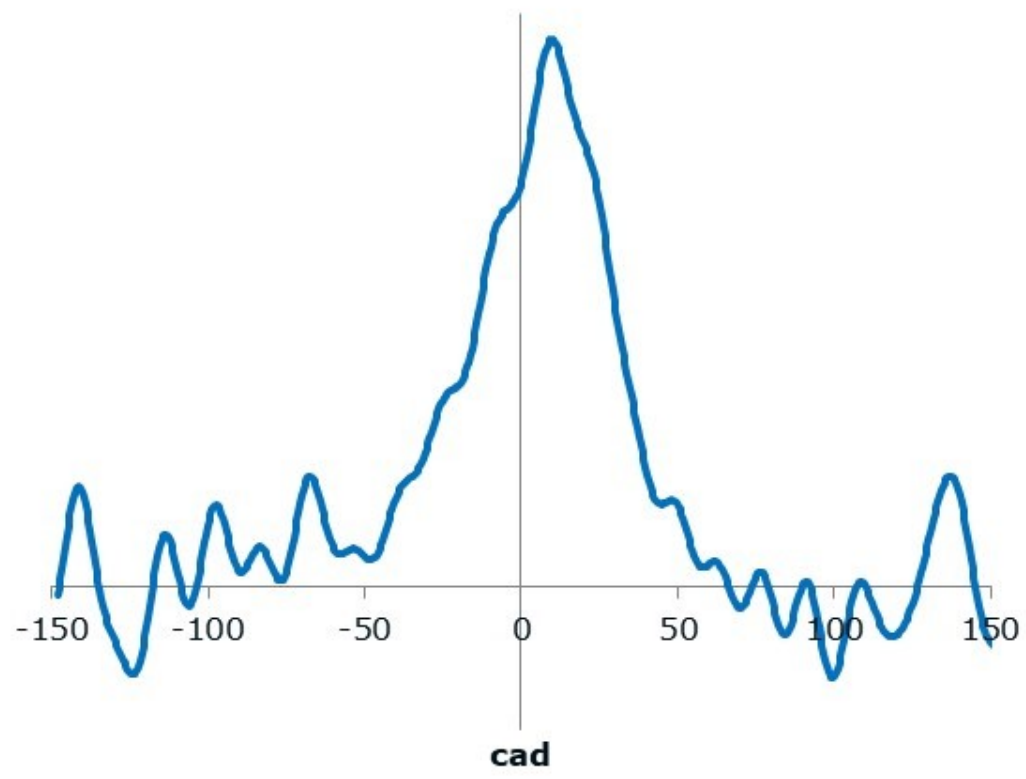

Figure 2.13: Heat flux curve obtained from in-cylinder pressure signal.

on the metallic surface of the cylinder wall is in the order of a few tens of ${ }^{\circ} \mathrm{C}$, while instantaneous temperature swing in the gas under firing operation is in the order of hundreds of ${ }^{\circ} \mathrm{C}$ [99] [91] [102] [103]. Today, slow-response thermocouples are commonly installed inside the structure, away from the surface, where temperature swing is greatly attenuated. This is a reliable and simple technique that can provide a broad spatial resolution. An installation of slow-response temperature sensors is useful to study mean trends of heat transfer. When the conditions in the surroundings of the engine cylinder are maintained constant (cooling, lubrication, ancillary systems), structure temperatures depend only on in-cylinder conditions. On the other hand, when in-cylinder conditions are maintained constant, the influence of ancillary systems on engine heat transfer can be studied. In addition, slow-response temperature sensors are usually employed to measure temperature and heat rejection in secondary flows as the cooling or the oil circuits. Finally, if the heat flux curve has been estimated by the above mentioned procedure and a mean temperature has been measured on the cylinder surface, it is possible to calculate the heat transfer coefficient with Eq. 2.4 under certain assumptions: ideal gas, quasi-steady heat 
transfer and constant surface temperature.
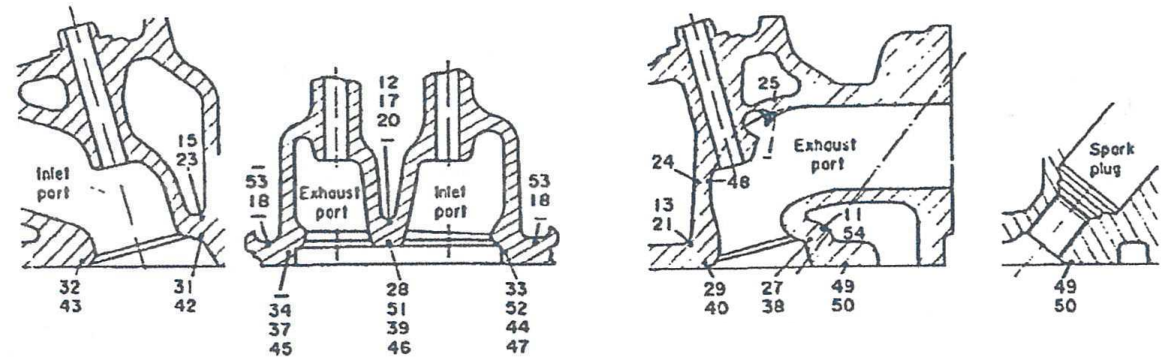

Figure 2.14: Slow-response thermocouple installation in cylinder-head [104].

Installations of slow-response thermocouples have been employed in many studies. Finlay et al. [104] applied this technique in the cylinderhead of a spark ignition engine to study precision cooling. A partial view of the installation is shown in Fig. 2.14. The research led them to conclude that precision cooling reduced engine temperatures up to $50{ }^{\circ} \mathrm{C}$, thus preventing nucleate boiling and allowing to reduce coolant flow rate by $40 \%$.

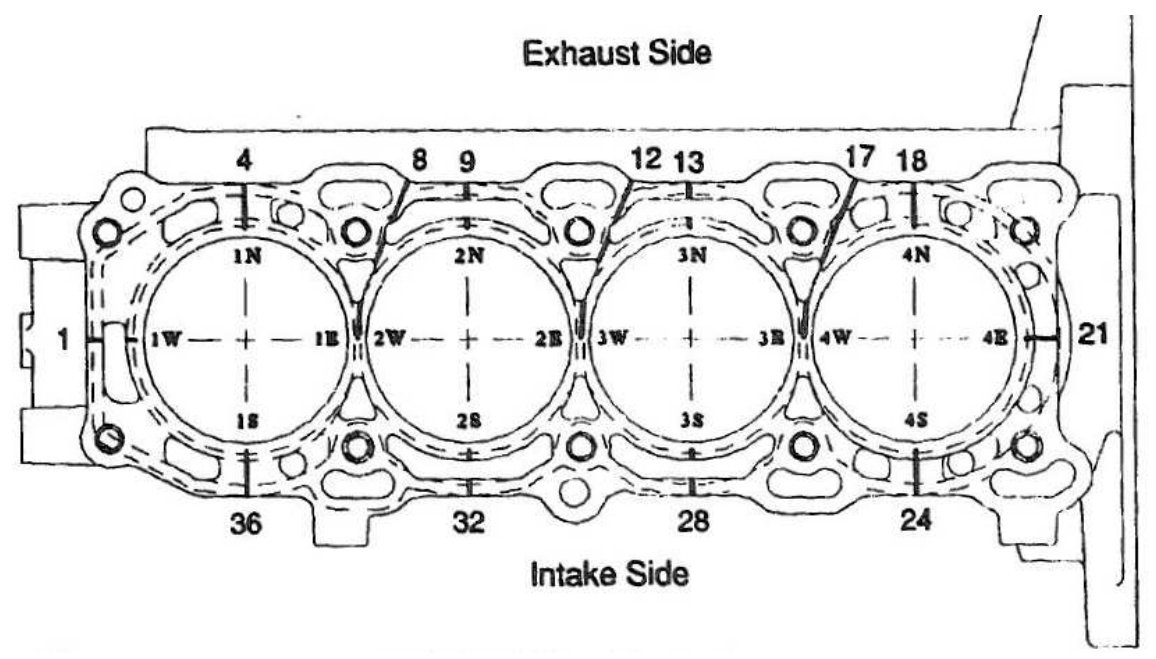

Figure 2.15: Slow-response thermocouple installation in engine block [105].

Alkidas [105] used an installation with 38 thermocouples in the engine block, as shown in Fig. 2.15, and 30 thermocouples in the cylinder- 
head. He also measured temperatures of coolant and oil. Alkidas observed widespread temperature non-uniformities in the engine structure. He was able to report findings regarding the influence of engine operational parameters and coolant conditions on mean structural temperatures and on heat rejection to coolant and oil.

In this thesis, an installation of slow-response thermocouples was used to measure temperatures of the engine structure near the cylinder surfaces. Measured data was used for evaluation and validation of engine heat transfer models.

\section{Fast-response temperature sensors and heat flux probes}

Fast-response temperature sensors are employed when a high temporal resolution is needed [106]. Engine cycles typically have a duration in the order of tens or a few hundreds of milliseconds. In order to obtain an appropriate temporal resolution during a cycle, sampling frequency has to reach the order of tens of microseconds, at least. There are thermocouples and RTD that offer this capability. Fast-response sensors are extensively used to measure instantaneous temperatures on the surface of cylinder walls. The technique has been refined to account for the thermal inertia of the instrument, which attenuates high frequency fluctuations.

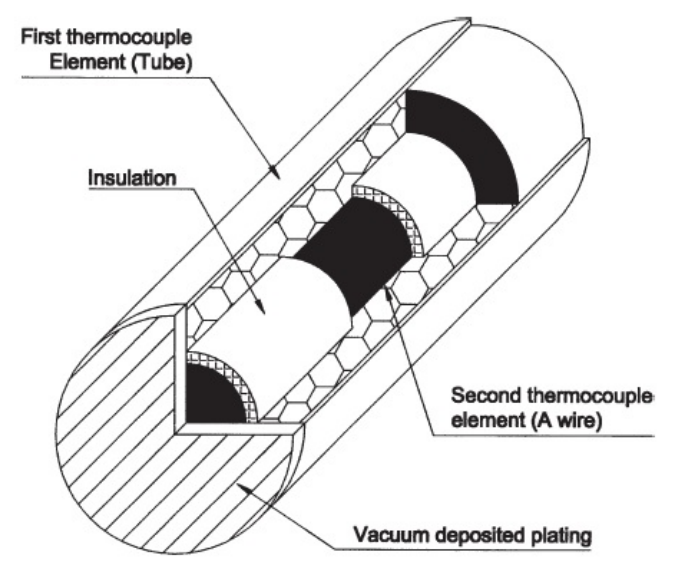

Figure 2.16: Coaxial type thermocouple [102].

A surface temperature measurement can be combined with another temperature measurement to calculate heat flux. The first sensor is flush 
to the surface in contact with the fluid. Regarding the second temperature measurement, there are two possibilities:

1. Temperature in the engine structure, away from the surface. In this case, heat flux can be calculated under the assumption of onedimensional unsteady heat conduction. To ensure that heat flux is one-dimensional, probes are usually employed. Heat flux probes consist of two temperature sensors placed at the two ends of a conductive medium, preferably of the same material as the engine structure. Heat flows one-dimensionally between both sensors through the homogeneous medium of known thermal conductivity. An example of heat flux measurement with a probe can be found in [103].

2. Bulk gas temperature. The assumption to obtain the heat flux curve from the measurements is zero heat flux when bulk gas temperature is equal to wall temperature. This is a quasi-steady assumption. As seen in Section 2.2.1, this assumption is compromised by the postulated phase shift between heat flux and temperature difference.

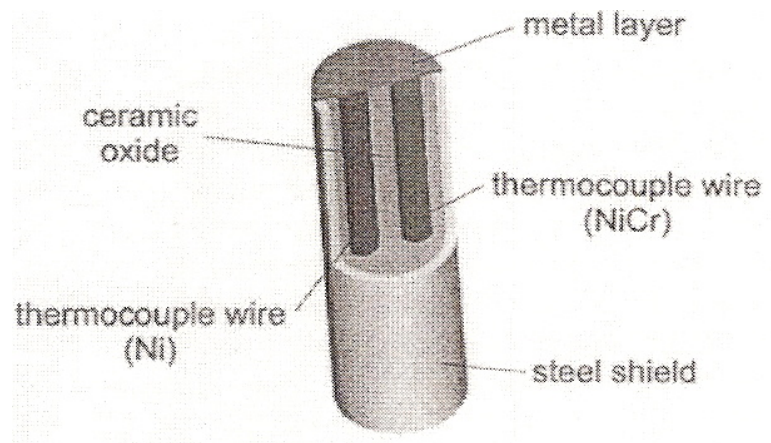

Figure 2.17: Pair-wire thermocouple [107].

The surface temperature sensor can be classified in three groups:

- Coaxial type. One of the metals is a tube that forms the external wall of the thermocouple. The other metal is a wire located in the center. An example is shown in Fig. 2.16.

- Pair-wire type. Both metals are wires, inserted in a passive tube, see Fig. 2.17. A derivation of this type is the eroding ribbon thermocouple, Fig. 2.18. An eroding ribbon thermocouple can provide higher durability because the junction can be remade through erosion. 
- Film type. It is formed by thin layers of different materials. It can be a thermocouple or a RTD. An example can be seen in Fig. 2.19. A drawback of this type of sensor is size.

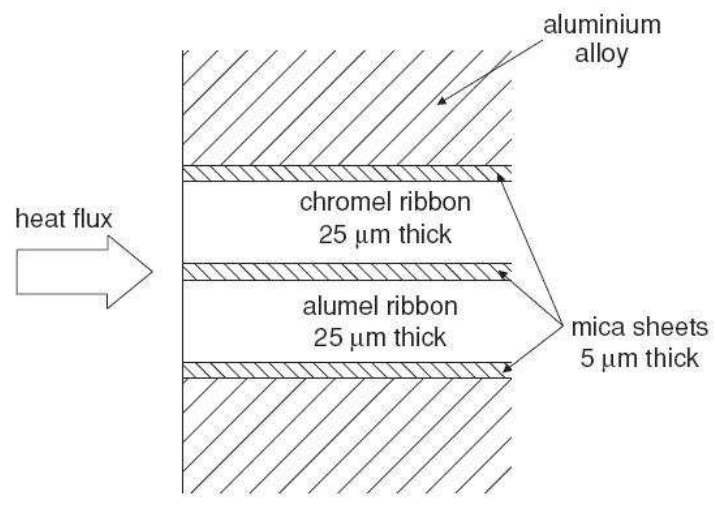

Figure 2.18: Eroding ribbon thermocouple [100].

Wimmer et al. [107] compared the different types of sensors using the second method of the above mentioned (bulk gas temperature measurement) and found that film type sensors were more accurate than coaxial and pair-wire thermocouples. Demuynck [108], in his recent doctoral research, also compared different sensors. He concluded that thin film sensors were more accurate than eroding ribbon thermocouples. In that thesis, many interesting pitfalls of temperature sensor usage were reported.

Another pertinent topic is piston temperature measurement. Placing sensors in the piston has the additional problem that the piston is a moving component. The researchers have used special setups to install temperature sensors in pistons. An early experience of piston temperature measurement was reported in [109]. Chang et al. [91] and Cho et al. [102] used a two-bar linkage mechanism to lead the signals to the exterior through a continuous wire, as shown in Fig. 2.20. A different solution was employed by Hendricks et al. [110]. They used a wireless telemetry system with a microwave transmitter in the piston. Moreover, they calculated heat flux through an inverse method combined with first order regularization. These techniques for placing temperature sensors in the piston can of course be applied to any sensor, not just fast-response thermocouples. A very recent work presented a system that can be used 


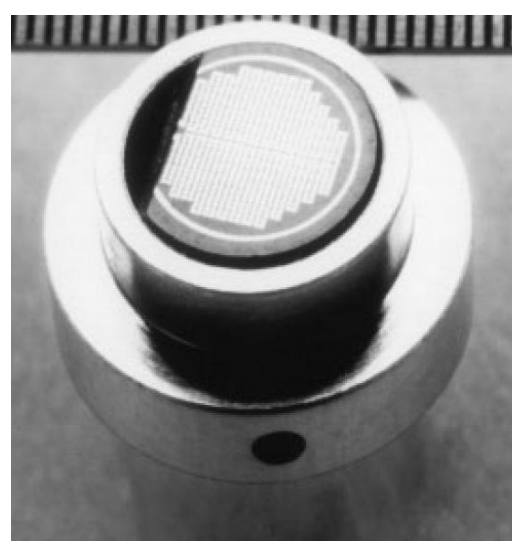

Figure 2.19: Film temperature sensor [108].

for measurements with low temporal resolution [111]. The temperature signal arrives to two pins in the piston. The pins move together with the piston but, once per revolution, at top dead center, the pins make contact with two stationary plates bound to the structure. This allows to record the temperature measurement once per crank revolution. The system was used to study the warm-up process of an optical engine.
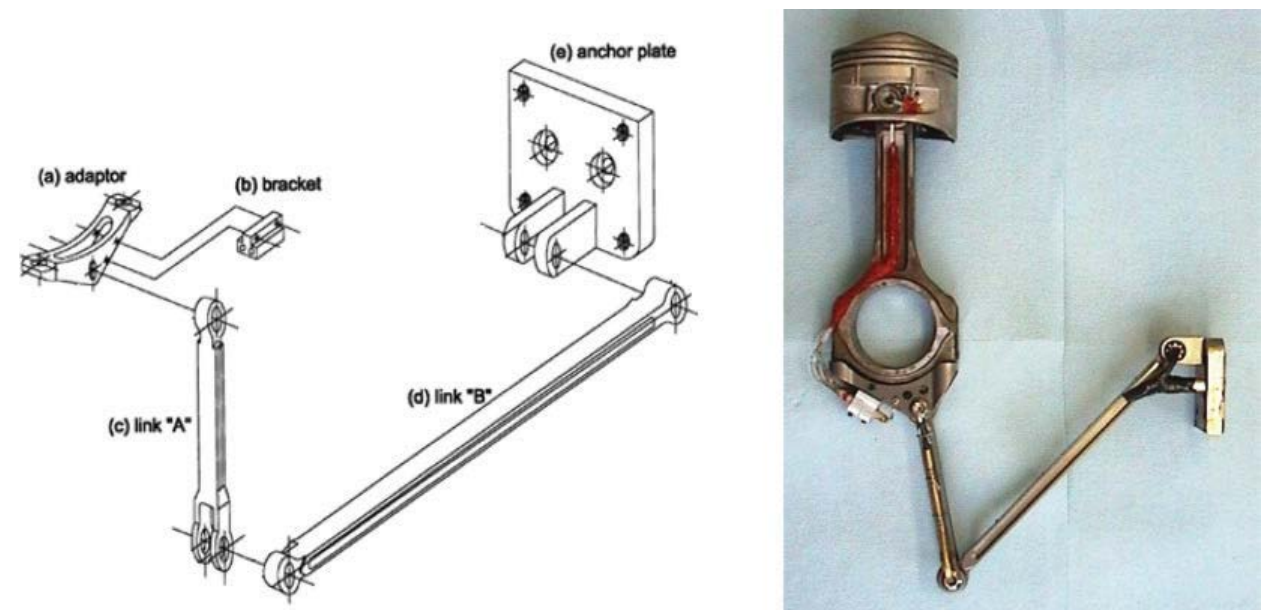

Figure 2.20: Linkage system to measure piston temperature [102].

Disadvantages of heat flux probes are that they are sizable and they have to be mounted with a small inclination with respect to the normal direction of the surface. That conditions the locations where it can be 
installed and restricts the number of probes. As a consequence, spatial resolution tends to be low.

More importantly, the accuracy of heat flux probes has been called into question by several researchers. For instance, Buttsworth et al. [100] conducted experiments with eroding ribbon thermocouples and concluded that the assumption of one-dimensional unsteady heat conduction led to wrong results. They measured the impulse response of various thermocouples. The first relevant finding was that the response varied substantially from sensor to sensor. Then they used the impulse response to derive the surface heat flux curve in the case of in-cylinder convection in a spark ignition engine. This curve was compared with the curves obtained from the application of a 1D and a 2D model. They were very different, as was seen in Fig. 2.12.

In addition to this problems, Borman and Nishiwaki also noted that the presence of the probe disturbed the temperature field in the engine structure [53]. Finally, a disadvantage of fast-response sensors is the fragility of the instrument.

Even though this technique is popular among researchers, the mentioned drawbacks discouraged its employment in this thesis' research.

\section{Other techniques}

In the literature, it is possible to find other methods to measure temperatures in internal combustion engines. Some are optical techniques such as Coherent anti-Stokes Raman Spectroscopy (CARS) [112], laser-induced fluorescence imaging [113] or phosphor thermometry [114]. Acoustic techniques have also been proposed and tested [115]. All these techniques require the development of complex, specialized facilities, something that was out of the scope of this thesis.

\subsubsection{Lumped heat transfer}

Lumped heat transfer is a modeling approach that provides spatial resolution while considering the different thermal phenomena: conduction, convection, radiation, flow enthalpy variation, thermal inertia and heat generation. That is therefore a very useful approach to model complex systems as an engine. The lumped model approach was described by Holman [116]. 


\section{Description}

A lumped element model is a thermal system made up of 'lumps', or nodes. The nodes are connected forming a network of thermal relations. Other names given to these model are: lumped parameter model, lumped component model, lumped capacitance model, resistor-capacitor network and lumped system analysis. The fundamental assumption of the lumped element model is that the attributes of the system are concentrated in the nodes or in the relations. In particular, each node has a determined temperature, and it is assumed that temperature difference inside each node is negligible; nodes are isothermal. The property of temporal variation of temperature, named capacitance, is located in the node as well. The properties of heat transfer throughout the system, named conductances, are concentrated in the relations among nodes. Conductances and capacitances are examined in more detail later. The advantage of the lumped element model is that network theory applies. Through this, partial differential equations can be simplified to energy balances in the nodes using the concentrated properties.

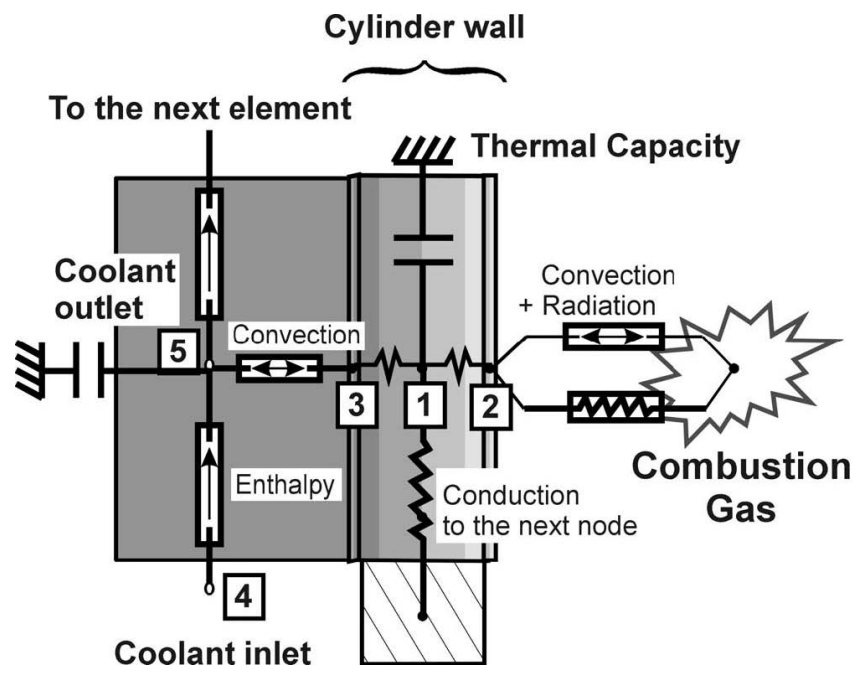

Figure 2.21: Example of lumped element analysis in engine applications $[117]$.

The prerequisite to apply the lumped element approach is that heat transfer within a node is much faster than heat transfer across the boundary of the node. This validates the approximation of spatially uniform temperature within the node, because the relatively low internal resis- 
tance allows for a fast energy distribution in the node. In comparison, external thermal resistances must be much higher.

\section{Formulation}

When the heat equation is integrated in systems where heat flows unidirectionally (plane slabs, cylinders or spheres), the form of the temperature distribution is found. This temperature distribution is formed by the sum of two terms. The first is the temperature at one of the locations used as integration limit. The other is the temperature difference between the integration limits, $\Delta T$, multiplied by an expression that contains the spatial variable, $x$ or $r$. Temperature distributions for slabs, cylinders and spheres are provided in Eqs. 2.33, 2.34 and 2.35, respectively [118].

$$
\begin{gathered}
\text { Plane slab: } T(x)=T_{1}-\Delta T \frac{x}{L} \\
\text { Cylinder: } T(r)=T_{2}+\Delta T \frac{\ln \left(r / r_{2}\right)}{\ln \left(r_{1} / r_{2}\right)} \\
\text { Sphere: } T(r)=T_{1}-\Delta T \frac{1-\ln \left(r_{1} / r\right)}{1-\ln \left(r_{1} / r_{2}\right)}
\end{gathered}
$$

When the temperature distribution, $T(x)$ or $T(r)$, is entered into Fourier's law, Eq. 2.2, it is differentiated over the spatial variable, $x$ or $r$. In all cases, this variable disappears and it is obtained that heat flux is proportional to the temperature difference between the integration limits. Therefore it is possible to define a thermal conductance, $K$, as shown in Eq. 2.36. The inverse of the conductance is called thermal resistance.

$$
K=\frac{\dot{Q}}{\Delta T}
$$

In a lumped heat transfer model, the locations used as integration limits of the heat equation are nodes. Therefore, $\Delta T$ is the temperature difference between two nodes. The conductance depends on the thermal properties and the geometry of nodes. In the case of plane slabs, conductive conductance is calculated with Eq. 2.37. $A$ is cross section for heat flux and $d$ is distance between nodes.

$$
K=\frac{k A}{d}
$$


Eq. 2.38 is applied to obtain the conductance in the case of axisymmetric, cylindrical conduction. $L$ is the system's size (height or length) in axial direction and $R_{1}$ and $R_{2}$ are the respective radii of each node.

$$
K=\frac{2 \pi L k}{\ln \frac{R_{2}}{R_{1}}}
$$

For spherical conduction, the thermal conductance has the form of Eq. 2.39 .

$$
K=\frac{4 \pi k}{\frac{1}{R_{1}}-\frac{1}{R_{2}}}
$$

For convection, the heat transfer equation is Newton's cooling law, Eq. 2.5. According to this law, heat flux is proportional to temperature difference, as in unidirectional conduction. Therefore, the convective conductance can be directly obtained, Eq. 2.40. A is the area of the solid surface in contact with the fluid.

$$
K=h A
$$

In radiation, heat flux is proportional to the difference of temperatures raised to the fourth power. Thus, it is not a linear process, unlike unidirectional heat conduction or convection, in which heat flux is proportional to temperature difference. Nevertheless, heat radiation can be considered in different ways. If making the system of equations not linear is not a problem, Eq. 2.6 can be entered along with the other equations. In such case, non-linear methods must be used to solve the system of equations. If a linear system is required, Eq. 2.7 can be linearized to obtain a radiation conductance.Alternatively, radiation can be solved separately and entered as a heat source.

Heat radiation has been a topic of interest to engine researchers for decades. In the past, it was proposed that up to $40 \%$ of in-cylinder heat rejection during combustion was due to radiation [119]. Considering the total amount of energy released by the fuel, up to $10 \%$ could be lost through radiative heat transfer radiation according to some authors [120]. However, other studies pointed to a much smaller role of thermal radiation [121]. It was found that radiation was lower in spark ignition engines than in compression ignition engines. Experiments allowed to determine that the main source of thermal radiation in an engine are soot particles, which are more abundant in diesel engines. Over time, different methods were developed to study radiative heat flux [122] [123] [124]. Recently, 
experiments using modern optical techniques in diesel engines revealed that radiation has a low influence on in-cylinder heat rejection. Radiative heat fluxes amounting to just $0.1 \%$ up to $0.75 \%$ of the fuel energy were reported [125].

Another thermal process that can be modeled with a conductance is the enthalpy variation of a flowing fluid between an inlet and an outlet location. When there is a temperature change in the fluid, there is also heat transfer. The variation of the enthalpy flow, $\Delta \dot{H}$ is proportional to the difference of temperatures at the inlet and outlet of the control volume, $\Delta T$. The conductance is calculated as shown in Eq. 2.41. $\dot{m}$ is mass flow, $C_{p}$ is specific heat capacity of the fluid at constant pressure. In the literature, this conductance is called flow conductance.

$$
\begin{aligned}
\Delta \dot{H} & =\dot{m} C_{p} \Delta T \\
K & =\dot{m} C_{p}
\end{aligned}
$$

The phenomenon of thermal inertia happens when heat is stored in a body or liberated from it in order for the body to change its temperature. Assuming that temperature changes linearly between two time steps, it is possible to define a proportionality parameter between heat flux and temperature difference for thermal inertia. This parameter is called capacitance, $C$, and it is calculated as shown in Eq. 2.42. $m$ is mass and $\Delta t$ is the time step. Capacitances are used in calculations solved over time, not spatially.

$$
\begin{aligned}
\dot{Q} & =m C_{p} \frac{d T}{d t} \\
C & =\frac{\rho V C_{p}}{\Delta t}
\end{aligned}
$$

After these examinations, it is clear that a thermal system can be seen as a set of conductances and capacitances that represent the processes of heat conduction, heat convection, thermal inertia and enthalphy variation. Heat generation has to be included as a heat source to complete the heat balance.

The heat balance emerges from the principle of energy conservation. In each node, the balance has the form of Eq. 2.43. Subscript $i$ refers to the solid node where the balance is done, $j$ refers to a neighboring solid node, $f$ 
to a contacting fluid, gen to heat generation, $c d$ stands for conduction and $c v$ for convection. Variable $t$ is the current time, therefore $t-1$ denotes the previous time step. Here it is considered that conductances and capacities change over time because thermal properties change. Every node of the network produces one equation. All equations are then assembled as a system of equations that represents the thermal behavior of the system. The system of equations can be solved using matrix methods. It must be noted that the equations are defined here using an implicit approach. That determines whether each variable is evaluated at time $t$ or $t-1$. Later in this thesis, solving methods are discussed with more detail but, at the moment, the implicit scheme is used for illustrating purposes due to its simplicity and due to the relevancy it has in this thesis.

$$
\begin{gathered}
\dot{Q}_{g e n}+\dot{Q}_{c d}+\dot{Q}_{c v}-\dot{Q}_{a c c u m}=\dot{Q}_{g e n, i}(t)+K_{c d}(t)\left(T_{j}(t)-T_{i}(t)\right)+ \\
+K_{c v}(t)\left(T_{f}(t)-T_{i}(t)\right)-C_{i}(t)\left[T_{i}(t)-T_{i}(t-1)\right]=0
\end{gathered}
$$

In Eq. 2.44, a complete system of 4 nodes and 4 equations is shown as example. Nodes $i$ and $j$ are solid nodes in thermal contact. $f$ is a fluid node is in contact only with node $i$. The fluid $f$ undergoes an enthalpy change, modeled by $K_{f l}$. Inlet temperature of the fluid flow is considered as a boundary condition equal to $T_{i n}^{*}(t)$. The system is illustrated in Fig. 2.22 .

$$
\begin{gathered}
\text { (I) } \dot{Q}_{g e n, i}(t)+K_{c d}(t)\left[T_{j}(t)-T_{i}(t)\right]+K_{c v}(t)\left[T_{f}(t)-T_{i}(t)\right]- \\
\quad-C_{i}(t)\left[T_{i}(t)-T_{i}(t-1)\right]=0 \\
\text { (II) } K_{c d}(t)\left[T_{i}(t)-T_{j}(t)\right]-C_{j}(t)\left[T_{j}(t)-T_{j}(t-1)\right]=0 \\
\text { (III) } K_{c v}(t)\left[T_{i}(t)-T_{f}(t)\right]-K_{f l}(t)\left[T_{f}(t)-T_{i n}(t)\right]- \\
\quad-C_{f}(t)\left[T_{f}(t)-T_{f}(t-1)\right]=0 \\
\text { (IV) } T_{i n}(t)-T_{i n}^{*}(t)=0
\end{gathered}
$$

The system is assembled in matrix form in Eq. 2.45. First matrix contains the conductances and multiplies the vector of current temperatures to be calculated. Second matrix contains the capacitances that multiply the current temperatures. Third matrix contains the capacitances that multiply the temperatures of the previous time step, which are known. Last vector contains boundary conditions and source terms, which are also known. 


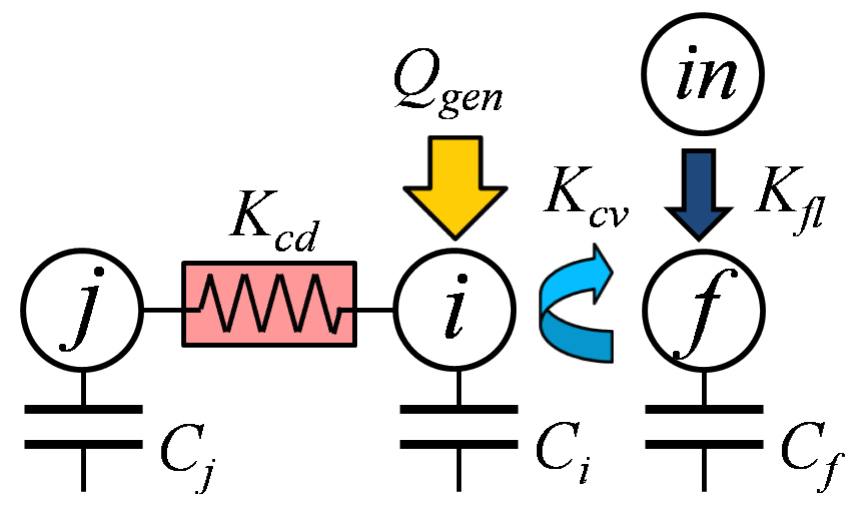

Figure 2.22: Example thermal system.

$$
\begin{aligned}
& {\left[\begin{array}{cccc}
-\left(K_{c d}+K_{c v}\right) & K_{c d} & K_{c v} & 0 \\
K_{c d} & -K_{c d} & 0 & 0 \\
K_{c v} & 0 & -\left(K_{c v}+K_{f l}\right) & K_{f l} \\
0 & 0 & 0 & 1
\end{array}\right]\left[\begin{array}{c}
T_{i}(t) \\
T_{j}(t) \\
T_{f}(t) \\
T_{i n}(t)
\end{array}\right]-} \\
& -\left[\begin{array}{cccc}
C_{i} & 0 & 0 & 0 \\
0 & C_{j} & 0 & 0 \\
0 & 0 & C_{f} & 0 \\
0 & 0 & 0 & 0
\end{array}\right]\left[\begin{array}{c}
T_{i}(t) \\
T_{j}(t) \\
T_{f}(t) \\
T_{i n}(t)
\end{array}\right]+\left[\begin{array}{cccc}
C_{i} & 0 & 0 & 0 \\
0 & C_{j} & 0 & 0 \\
0 & 0 & C_{f} & 0 \\
0 & 0 & 0 & 0
\end{array}\right]\left[\begin{array}{c}
T_{i}(t-1) \\
T_{j}(t-1) \\
T_{f}(t-1) \\
T_{i n}(t-1)
\end{array}\right]+\left[\begin{array}{c}
Q_{g e n, i} \\
0 \\
0 \\
-T_{i n}^{*}(t)
\end{array}\right]=0
\end{aligned}
$$

This is a linear system that can be arranged to be solved as $[K-$ $C][T(t)]+[C \times T(t-1)+B C]=0$, where $K$ represents the conductance matrix, $C$ the capacitance matrix, $B C$ the vector of boundary conditions and source terms, $T(t)$ is the vector of temperatures, which is the only unknown to be solved, and $T(t-1)$ is the vector of temperatures in the previous time step.

\section{Previous research}

One of the first works that combined a lumped element system and convective correlations as those of Section 2.2.1 to represent an engine was a study of Kaplan and Heywood [126]. In that ambitious study, they attempted to predict hydrocarbon emissions. The heat transfer model provided information about exhaust temperature and about the contraction 
of the crevice between piston and liner during engine warm-up. In order to achieve this, they increased the number of nodes in the exhaust port and in the crevice region to improve calculation resolution. In addition, they were able to report some insights comparing the warm-up process at different engine locations. For in-cylinder heat transfer, they data generated by equations of the form of Eq. 2.19 to adjust ad-hoc mathematical correlations. In contrast, a corrected Dittus-Boelter correlation, Eq. 2.16, was employed in the exhaust port directly.

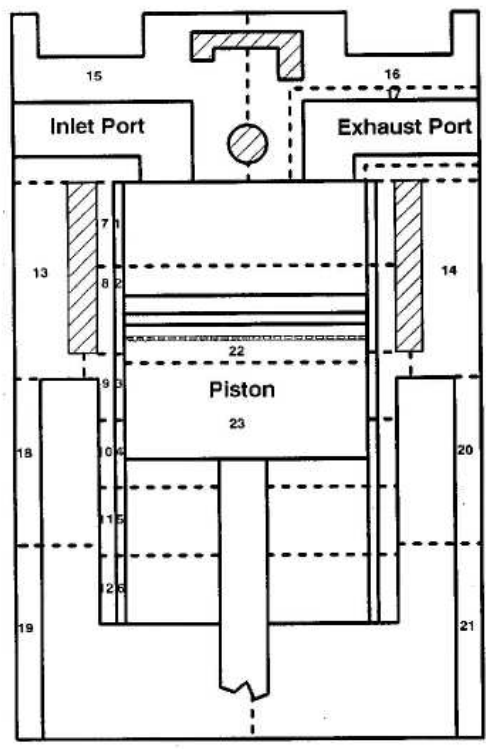

Coolant Passage

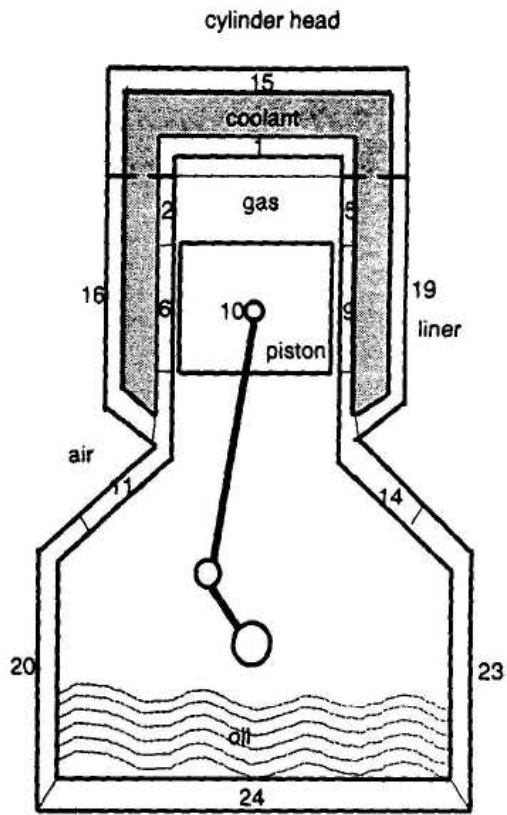

Figure 2.23: Lumped models of Shayler et al. [127] (left) and Veshagh and Chen [128] (right).

Shayler et al. [127] used a lumped model made up of 23 nodes (see Fig. 2.23, left) and simulated the same system in a finite element code as well. Calculation time decreased dramatically with respect to the finite element model, but mean trends matched. They modeled mean heat transfer using a correlation for heat rejection to coolant obtained from the work of Taylor and Toong [79]. In the same conference, Veshagh and Chen [128] presented a detailed description of a similar lumped heat transfer code. The model had 24 nodes and is shown on the right side of Fig. 
2.23. They used Sieder-Tate equation for internal convection on the gas side, Eq. 2.17. For coolant, Veshagh and Chen used a correlation similar to Dittus-Boelter correlation and considered nucleate boiling. With the model, they conducted various parametric studies of engine warm-up. Finally, the authors included an interesting analysis relating temperature uncertainty and Biot number.

Bohac et al. [40] made a complete lumped element model of an engine. It can be seen in Fig. 2.24. They considered the 0D lumped element approach to be quasi-dimensional. They used another quasi-dimensional simulation of the thermodynamic cycle to provide in-cylinder boundary conditions. Combustion was modeled with a two-zone (burned and unburned), turbulent entrainment model. In-cylinder heat transfer was modeled with correlations of the form of Dittus-Boelter equation and was consistent with the two zone model. To determine velocity for $R e$, mean piston speed, turbulent intensity and mean flow velocity were taken into account. They considered heat rejection to ambient, including radiation with the linearized conductance method. In total, each cylinder was formed by 115 nodes and 217 thermal connections. Combining the model and experimental tests, relevant investigations concerning various aspects were undertaken: nucleate boiling, head gasket conductance, split cooling, modeling of oil influence and a sensitivity analysis of heat transfer. In a later study [129], this lumped heat transfer model was reduced and integrated into a powertrain model. The powertrain model was then used to simulate a driving cycle. This work is reviewed in Section 2.4 because it is an early example of multiphysics powertrain model.

Jarrier et al. [117] made measurements in an engine enclosed in a calorimeter. They studied the distribution of heat during warm-up experimentally and then built up a lumped model to replicate the engine's thermal response. The model had 59 nodes. A 3D reconstruction is shown in Fig. 2.25. For in-cylinder convection, they used Woschni's formula, Eq. 2.24. Friction and convection processes were calibrated in steady state, assuming that the calibration would be valid for transient operation as long as fluid properties were temperature dependent. Then they used the model to assess the potential of different thermal management strategies.

Wagner et al. [130] comprehensively described the procedure of making lumped models intended for air cooled (see Fig. 2.26) and liquid cooled spark ignition engines. The models were non-linear because radiative conductances were not linearized. For convection, they used fixed 

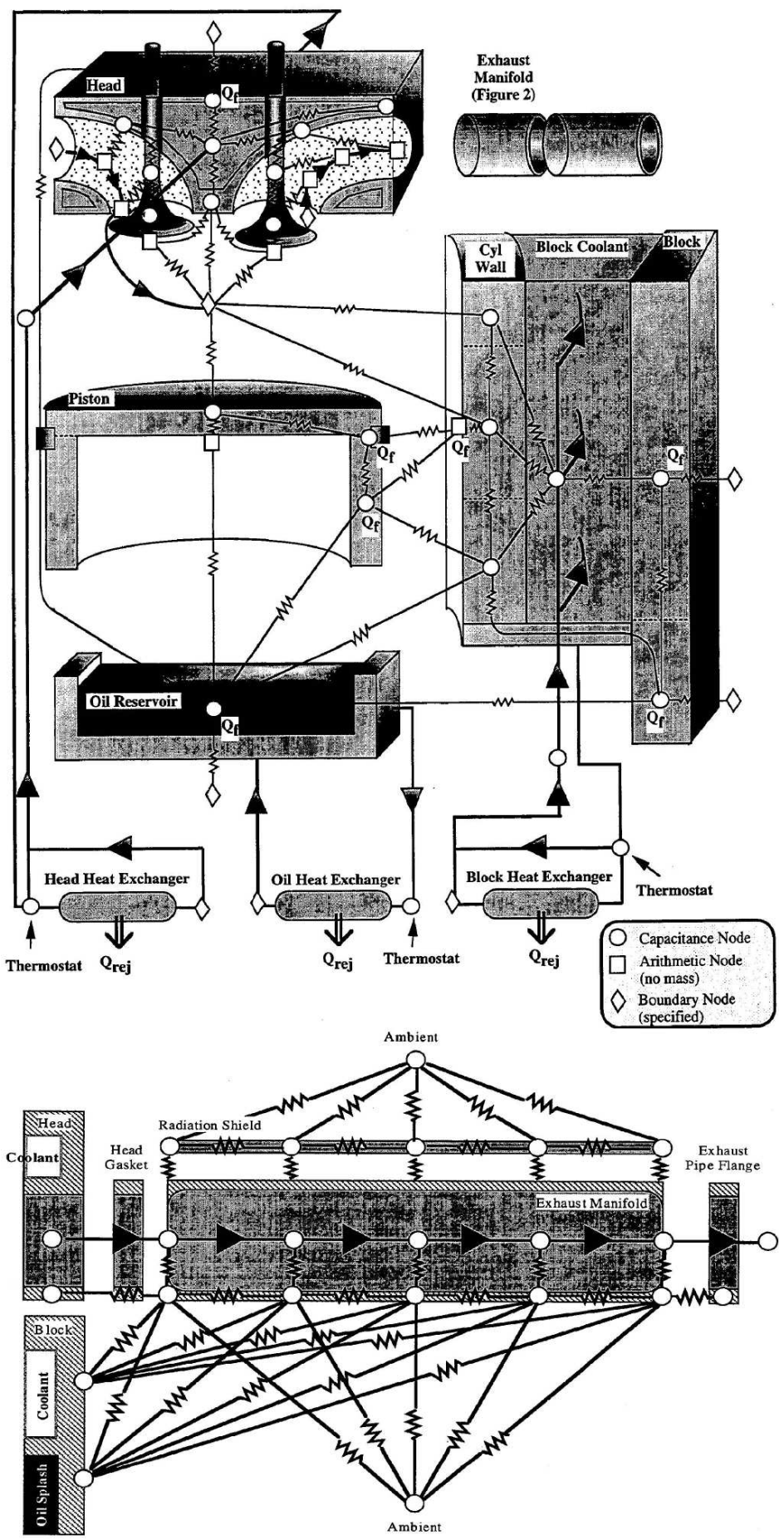

Figure 2.24: Lumped model of Bohac et al. [40]. 


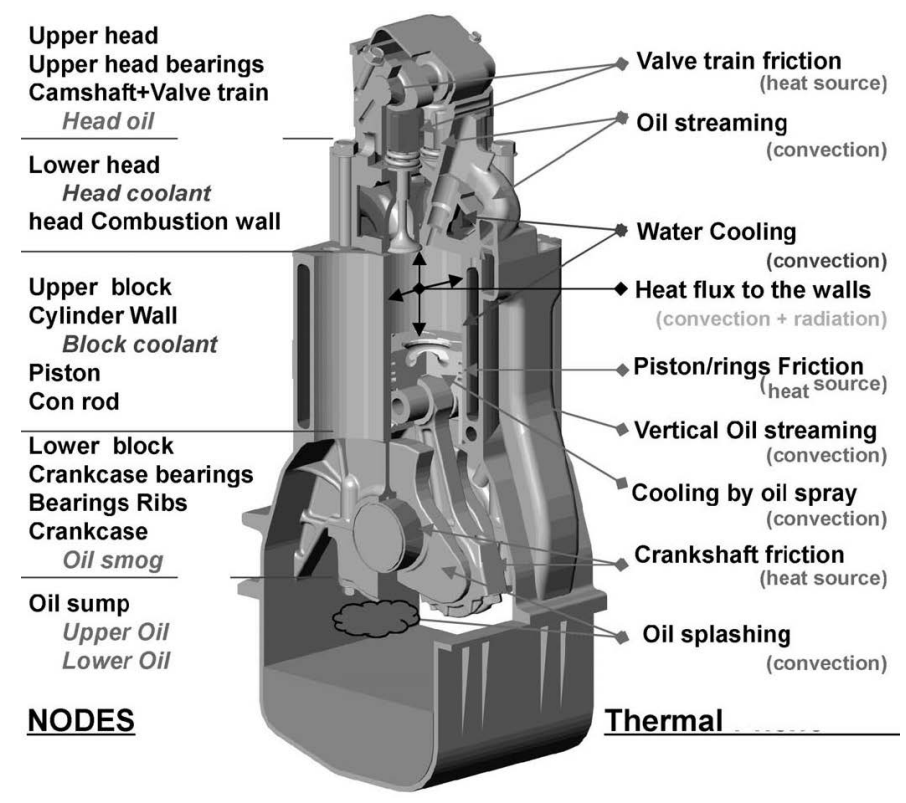

Figure 2.25: Lumped model of Jarrier et al. [117].

heat transfer coefficients, which decreased accuracy. The authors stressed the convenience of real-time calculation capability to integrate a lumped model in the ECU (engine control unit). This would allow on-line estimation of engine temperatures and other thermal variables. In this regard, the authors discussed the impact of the on-line application on diverse aspects of thermal management.
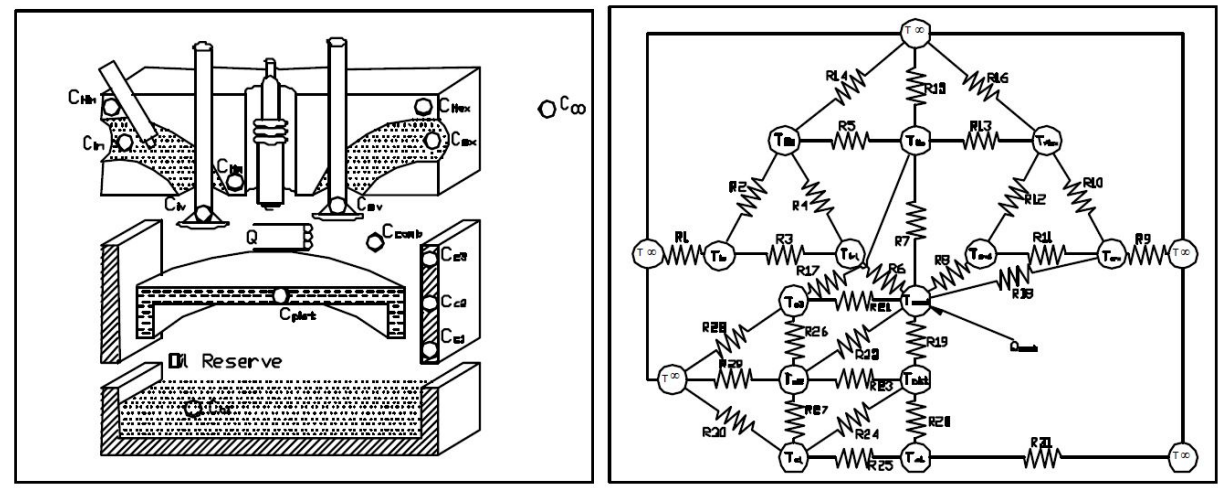

Figure 2.26: Lumped model proposed by Wagner et al. [130]. 
Later lumped models that are mentioned in the literature are coupled with other models that simulate other engine or vehicle components. Such integrated approaches are reviewed in Sections2.3 and 2.4. By now, from the research works surveyed in the present section, it can be concluded that lumped element models are an efficient way to gain spatial resolution and to take into account the different heat transfer mechanisms in one single model. It has been shown throughout this review that lumped models have several advantages: computational demand is relatively low when compared to finite element methods, they can include semi-empirical correlations or be coupled to other models, they can provide an accurate account of boundary conditions and they allow scalability of complexity. CMT-Motores Térmicos has a lumped model to simulate engine heat transfer. It is thoroughly described in Section 3.3. The advancement of this model is one of the cornerstones of this thesis.

\subsubsection{Multizone heat transfer}

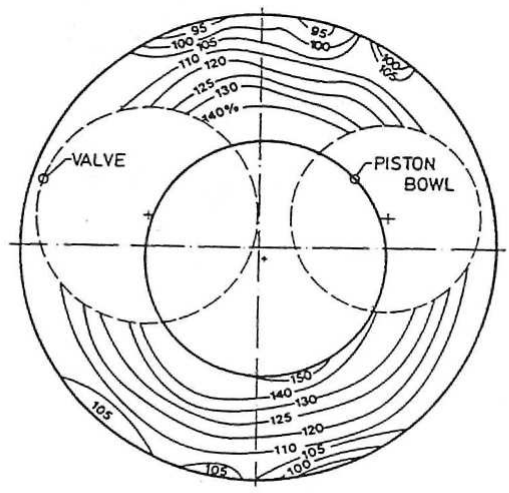

$100 \%=$ VELOCITY FOR FLAT PISTON

Fig. 4 - Relative iso-velocity diagram for a bowl and a flat piston

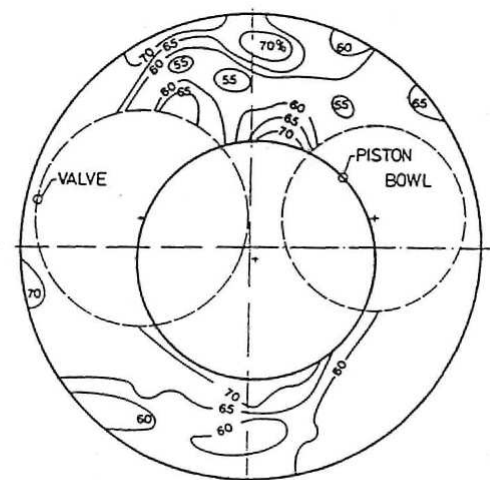

$100 \%=$ VELOCITY FOR HIGH SWIRL

Fig. 6 - Relative iso-velocity diagram for high and low swirl

Figure 2.27: Velocity distributions on the firedeck [86].

Lumped heat transfer models like those reviewed previously can provide spatial resolution to calculations, but it can be insufficient to capture the real local distribution of in-cylinder heat transfer. The limitation of the reviewed lumped models is that they assumed that heat transfer properties were uniform all over the cylinder. That is to say, gas temperature and 
heat transfer coefficient were considered uniform or were surface-averaged to obtain single values that were applied to all surfaces of the combustion chamber. However, several experiments confirm that in-cylinder conditions are far from uniform, specially in modern diesel engines. In a study on this matter, Hohenberg [86] made surface temperature measurements at 72 locations of the firedeck. He assumed that temperature differences on the firedeck were caused by differences in the heat transfer coefficient, assumed to be determined locally by gas velocity. Therefore, to Hohenberg, temperature distribution was caused by gas velocity distribution. In Fig. 2.27, results are shown in the form of velocity contours. Hohenberg reported that, for a flat piston, distribution was uniform. For a bowl piston, in contrast, temperature and velocity distribution in the firedeck varied between $95 \%$ to $150 \%$ with respect to the distribution using a flat piston (see Fig. 2.27, left). On the right side of the figure, distributions using two levels of swirl motion were reported. These experiments revealed that temperature and heat transfer are not uniform throughout the combustion chamber, and the manner in which in-cylinder conditions influence the temperature distribution is not uniform either. Over time, this was confirmed by CFD simulations as well [46]. Some examples are given in Figs. 2.28 and 2.29. Regarding Hohenberg's assumption, Noori and Rashidi obtained distributions of velocity and heat transfer coefficient to determine how similar they were [131]. Though distributions were not exactly equal, the similarity indicated strong ties between gas velocity and heat transfer (see Fig. 2.29).
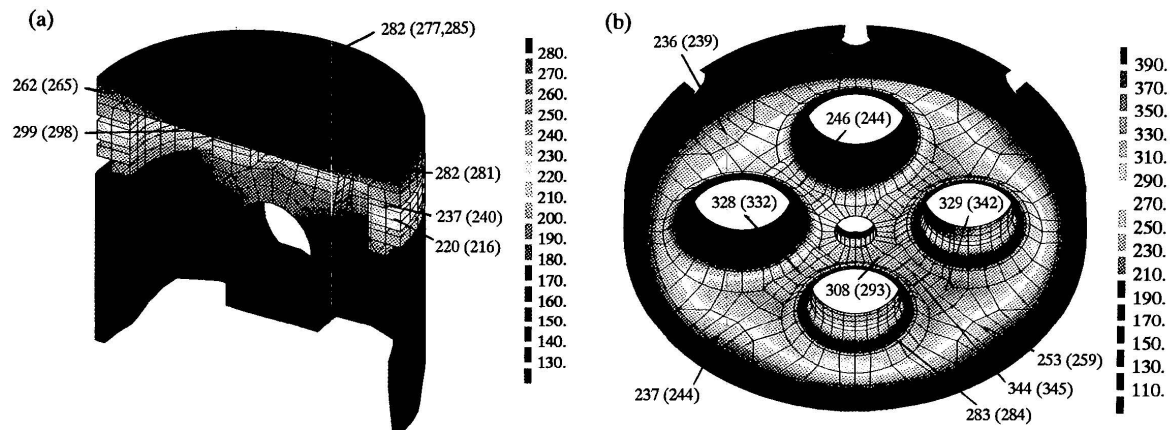

Figure 2.28: Temperature distributions in piston and cylinder-head [46].

After this introduction, it is clear that the approach of single-zone thermal state and single heat transfer coefficient has limited capability 


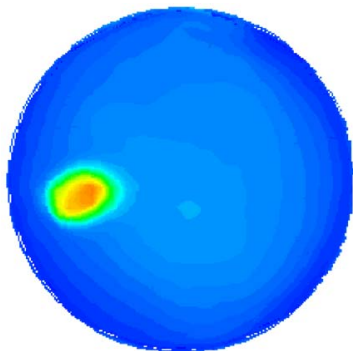

(a) $10^{\circ} \mathrm{BTDC}$

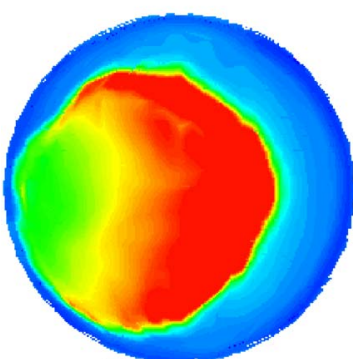

(c) $5^{\circ}$ ATDC

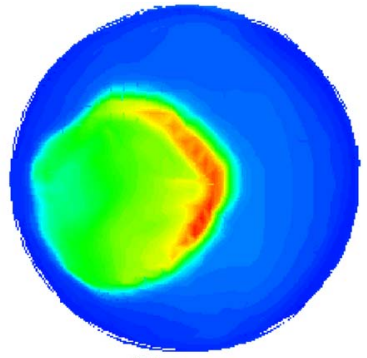

(b) TDC

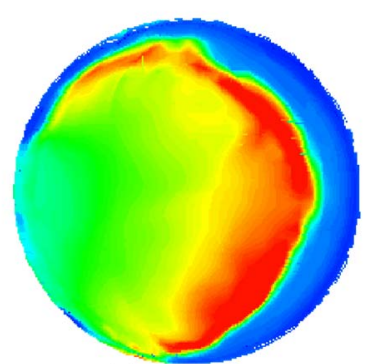

(d) $10^{\circ} \mathrm{ATDC}$

Figure 2.29: Heat flux distribution on the piston [131].

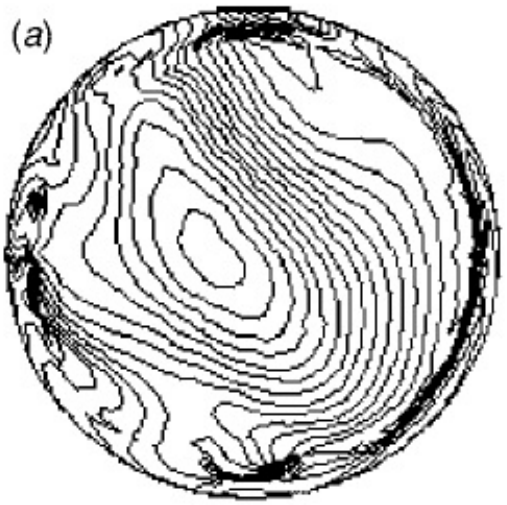

Velocity distribution

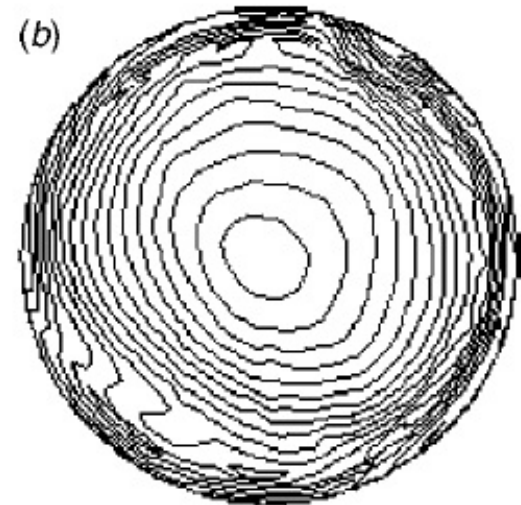

Heat transfer coefficient distribution

Figure 2.30: Distributions of velocity and heat transfer coefficient on the piston [131]. 
to capture local trends of heat transfer. It is also clear that in-cylinder heat transfer is strongly tied to gas velocity. A 0D model that provides reliable space-resolved results has to divide the combustion chamber in different zones with uniform properties. In each zone, a different heat transfer coefficient for convection between gas and wall has to be obtained. Moreover, the heat transfer coefficient must take into account the local influence of gas motion.

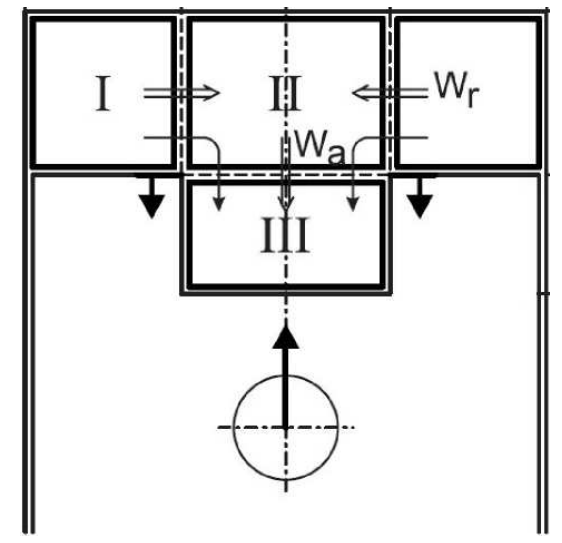

Figure 2.31: Flow regions in a multizone model [132].

The work of Morel et al. [133] [134] [135] provided key foundations to multizone heat transfer models. They recognized that the correlations that modeled more thoroughly the convective heat transfer process in the cylinder used instantaneous characteristic velocities. They recognized also that gas motion was not uniform throughout the cylinder. Therefore, they proposed to divide the cylinder volume into three flow regions, shown in Fig. 2.31: the squish area above the piston crown, the piston bowl volume and the region above the bowl lip. Coherently, the cylinder walls were divided into six surfaces, which are shown in Fig. 2.32. On each surface, three components of gas velocity were used to calculate the characteristic velocity of the heat transfer coefficient, as expressed by Eq. 2.46: two orthogonal components parallel to the surface and a turbulent component. In Eq. 2.46, TKE is the turbulent kinetic energy and $\mathrm{t}$ is time. The relevant gas motions were the following:

$$
v_{\text {char }}(t)=\sqrt{v_{x}^{2}(t)+v_{y}^{2}(t)+2 T K E(t)}
$$




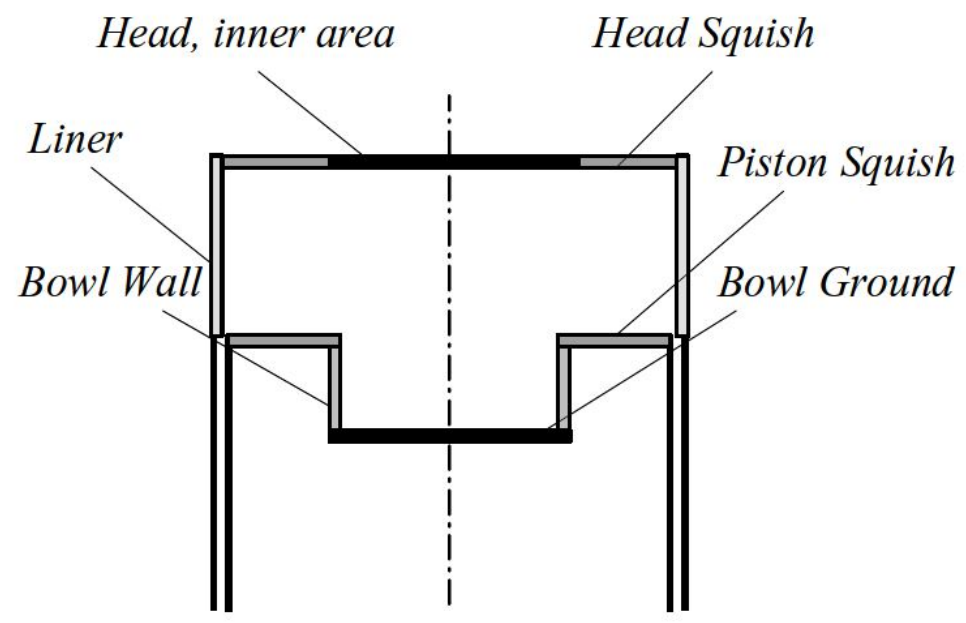

Figure 2.32: Wall surfaces in a multizone model [136].

- Swirl motion. Swirl velocity is parallel to all surfaces. For modeling, the assumption of rigid body rotation was made in each one of the three flow regions. That is to say, flow spins with uniform angular velocity within a region. The temporal evolution of swirl was obtained from the solution of a differential equation for angular momentum in each of the three flow regions. The equation of angular momentum flux included the influence of mass transfer due to squish, the influence of viscous shear among fluid regions (which can also be seen as momentum diffusion due to turbulence), the influence of wall friction and the influence of intake and exhaust flows.

- Reciprocating axial motion. It is related to two mechanisms: piston kinematics and squish. For the first mechanism, piston motion relative to the liner was considered. For squish, the mass conservation principle was applied taking into account the varying volume of the three flow regions. Axial velocity is parallel to the vertical surfaces.

- Radial velocity. It was assumed that radial motion is only caused by squish. Through mass conservation, both axial and radial velocities could be obtained. Radial motion is parallel to horizontal surfaces.

- Turbulence. A $k-\epsilon$ model was employed. This means that, in each flow region, sources and sinks of turbulence were identified. In a $k-\epsilon$ model, turbulence generation, convection and dissipation 
are represented by two partial differential equations per region. Regarding turbulence generation, Morel et al. included the effects of the mean strain field, compression, squish, intake flow, injection and swirl gradients. Apart from this, turbulence convection among zones and turbulence dissipation through viscous decay were considered. According to the authors, the turbulent velocity term also considered three-dimensional effects of mass and momentum transfer in a lumped form.

The heat transfer coefficient was obtained with Eq. 2.14, a correlation that comes directly from the Colburn analogy. For the friction factor, Morel et al. used Eq. 2.47. The value of 0.0565 is the mean between 0.046 , the constant for boundary layers on flat plates, and 0.067, the constant for fully developed turbulent flow in pipes. The expression is similar to that of Blasius, Eq. 2.15. The authors demonstrated the accuracy of this formulation using data available in the literature. In particular, they focused on the applicability of this formulation to engines with swirl motion.

$$
f=0.0565 R e^{-1 / 4}
$$

The final form of the heat transfer coefficient is Eq. 2.48. Fluid properties were evaluated at film temperature, the mean between bulk gas temperature and wall temperature.

$$
\begin{aligned}
h & =\frac{0.0565}{2} \rho C_{p} v_{\text {char }} \operatorname{Re}^{-1 / 4} \operatorname{Pr}^{-2 / 3} \\
& =0.02825 \rho C_{p} v_{\text {char }}\left(\frac{\rho L_{\text {char }} v_{\text {char }}}{\mu}\right)^{-1 / 4}\left(\frac{C_{p} \mu}{k}\right)^{-2 / 3}
\end{aligned}
$$

Notably, Morel et al. also proposed approximations for the boundary layer thickness on the different surfaces in order to use this magnitude as characteristic length $L_{\text {char }}$. The expressions are given in equations from 2.49 to 2.54 . In the equations, $H_{\text {bowl }}$ is the bowl depth, $d$ is the instantaneous distance between the piston top and the firedeck and $D_{\text {rim }}$ is the bowl diameter at the bowl rim. On the vertical surfaces, characteristic length is constant, while on the horizontal surfaces, it varies instantaneously. Lengths on the piston crown, Eq. 2.50, and on the outer firedeck, Eq. 2.53, are the same. Lengths on the bowl ground, Eq. 2.49, and on the inner firedeck, Eq. 2.54, are related.

$$
\text { Bowl ground: } L_{\text {char }}=0.05\left(H_{\text {bowl }}+d\left(\frac{D_{\text {rim }}}{D_{\text {bowl }}}\right)^{2}\right)
$$


Bowl lateral: $L_{\text {char }}=0.05 D_{\text {bowl }}$

Piston crown: $L_{\text {char }}=0.05 d$

Liner: $L_{\text {char }}=0.05 D_{\text {cyl }}$

Outer firedeck: $L_{\text {char }}=0.05 d$

Inner firedeck: $L_{\text {char }}=0.05\left(d+H_{\text {bowl }}\left(\frac{D_{\text {rim }}}{D_{\text {bowl }}}\right)^{2}\right)$

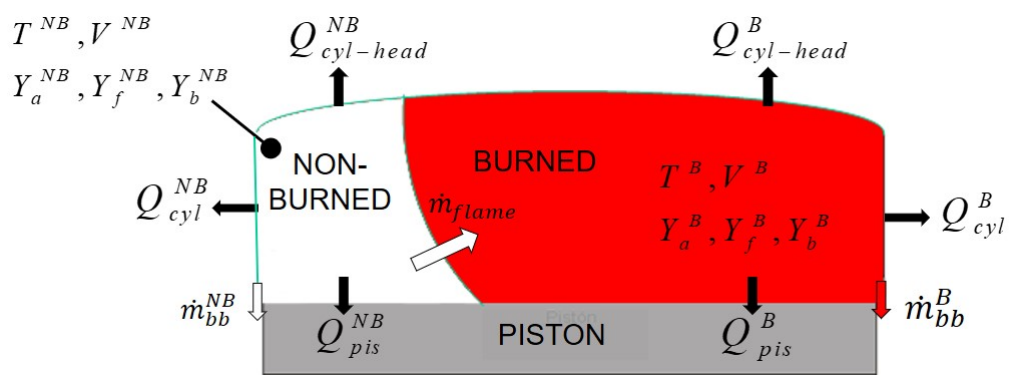

Figure 2.33: Example of a two-zone model considering combustion.

The multizone model for convective heat transfer was completed with a two-zone model that distinguished between a burned zone and an unburned zone, as shown in Fig. 2.33.

In this research, the authors also included radiation. As a first approximation, a quasi-steady model was implemented. Still, the model took into account the instantaneous extent of the burned zone. Only soot contribution to heat radiation was considered because it is the largest one. Later, a zonal model was developed and used as a predictive tool [137].

Regarding heat conduction in the solid, it must be taken into account that Morel et al. were interested on studying thermally insulated cylinders. In that case, the temperature swing close to the wall surface becomes significant, as wall temperature responds to the fluctuation of gas temperature. However, at short distance below the surface, the swing is so dampened that temperature in the material behaves quasi-steadily. To model the transient temperature swing near the surface, the authors included a model of one-dimensional conduction in their research [41]. In contrast, heat conduction in the solid structure away from the surface was modeled with a steady-state finite element model made up of 150 elements. 


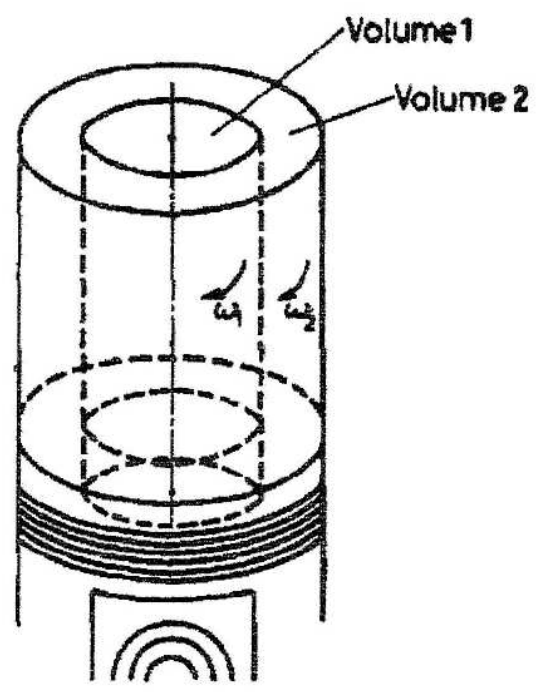

Figure 2.34: Swirl regions considered by Murakami et al. [138].

Several authors developed further the multizone model method. Murakami et al. studied swirl motion in depth [138]. They made laser doppler velocimetry measurements in a motored engine. Four different cylinderheads were used to induce different levels of swirl ratio. Four different pistons with different bowl depths were tested. Along with the hardware modifications, three different compression ratios and three different engine speeds were examined. The extensive test campaign allowed the researchers to obtain many insights about the swirling flow distribution and, afterwards, to validate the swirl model. For modeling, they proposed an analogous approach to Morel et al., but they found appropriate to use two volumes with different swirl velocity, instead of three. One volume represented the inner zone above the piston bowl and the other was the outer annular area above the piston crown, see Fig. 2.34. The model description was very thorough, focusing on the different terms of the differential equations.

Hansen published a comprehensive description of methods to model squish, swirl and turbulence [139] [140] and then assembled a multizone model. For swirl, he chose the approach of Murakami et al. Regarding turbulence, Hansen discussed previous works and proposed a $k-\epsilon$ model, consisting of two coupled differential equations in each one of the two vol- 


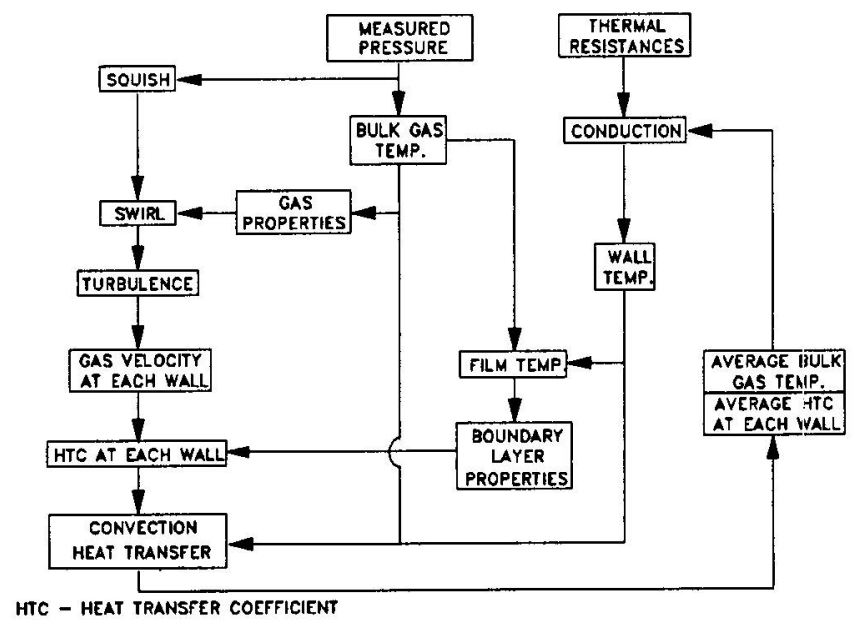

Figure 2.35: Flowchart of Hansen's model [141].

umes considered in the swirl model. The model was valid for the period of the engine in which valves were closed. He took into account the effects of compression, squish and viscous dissipation. The model did not consider turbulence generation due to shear at the interface of the inner and outer volumes, nor the turbulence production due to shear at the walls. With the help of the model, he found that the contribution of turbulence to gas motion was significant but far from dominant. Hansen noted that the roots of the turbulence model lie in the Navier-Stokes equations. In the final paper of the series [141], Hansen included the previously developed gas motion models into a multizone heat transfer model. The aim of the study was to validate the predictive capability of the model under motored engine operation. A flowchart depicting the calculation procedure can be seen in Fig. 2.35. The heat transfer coefficient correlation was the same used by Morel et al. Hansen completed the multizone convection model with a one-dimensional steady state heat conduction model in the liner and a lumped element network to model heat transfer in the rest of the engine structure. During validation, difficulties were met due to the disparity between the idealized geometry considered in the model and the complexity of the real cylinder. Also, aggressive corrections of the squish influence were needed to fit the predicted and measured curves of heat flux. Moreover, predictions during the late expansion stroke and at different engine speeds left room for improvement. Finally, Hansen applied 
the multizone model to fired operation [142]. In order to achieve that, he added a radiation model and a two-zone combustion model. Upon testing, it was concluded that additional work was required to better capture injection and combustion effects on heat transfer.

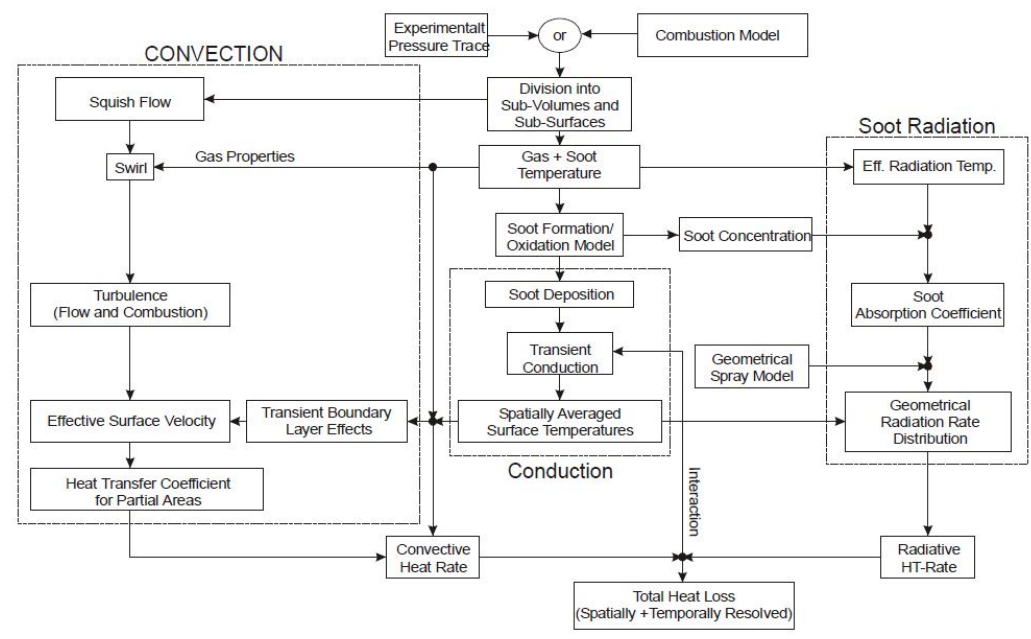

Figure 2.36: Flowchart of the model of Eiglmeier et al. [132].

Eiglmeier et al. [132] built a complete multizone heat transfer model. The flow models were similar to Hansen's proposal. The most interesting innovation of this work was the inclusion of a soot deposition model. It was based on the modeling of thermophoresis and oxidation processes. Soot deposition was relevant for heat transfer because the insulation effect of the soot layer on the cylinder walls was considered. As a consequence, transient heat conduction through the layer was assumed. Making use of the detailed calculation of soot concentration, a heat radiation model based on the thermal emission from soot was set up. Another particularity of the research of Eiglmeier et al. was the calculation method of the boundary layer thickness. This magnitude, which was used as characteristic length of the heat transfer coefficient, was obtained from the 1D thermal energy equation. The validation of the final model was undertaken in a singlecylinder diesel engine under different operating conditions and at various locations all over the cylinder walls. In general, very good agreement with experiment was obtained. The calculation flowchart is shown in Fig. 2.36.

Schubert et al. [136] proposed simplifications to the previous models 
backed by 3D-CFD analysis. In particular, the turbulence model was simplified to a single differential equation that used several approximations. For the friction coefficient, the authors used $f=0.0592 R e^{-0.2}$. They also included a radiation model which took into consideration the processes of soot formation and oxidation. Soot concentration was validated experimentally. The multizone model was validated with experimental data from 11 different engines. Schubert et al. reported improved predictions of heat flux and other engine parameters in comparison with Woschni's correlation.

Komninos and Kosmadakis [143] studied a multizone model for HCCI engines with a different configuration from the one of the previous models. Their model was formed by an arbitrary number of nested zones in the cylinder volume plus one zone in the crevices between the piston and the liner, as shown in Fig. 2.37. The piston head was flat. Mass conservation and the first law of thermodynamics for open systems were applied to each zone, in order to take into consideration species and enthalpy transfer. Heat diffusion among zones was calculated as one-directional conduction taking into account both laminar and turbulent conductivity. The characteristic velocity was proportional to engine speed. For wall heat flux, the authors derived a correlation dependent on gas temperatures in the different zones, assuming that the temperature gradient near the wall followed a geometric progression.

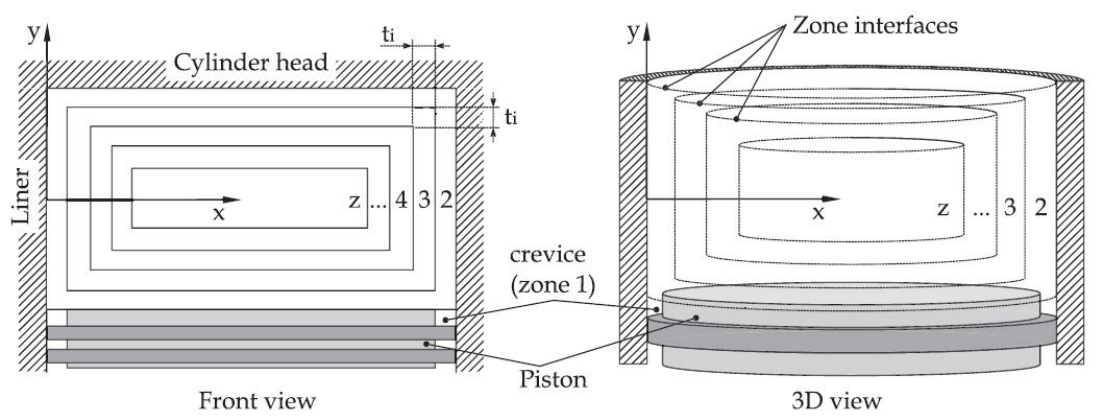

Figure 2.37: Model of Komninos and Kosmadakis [143].

In the framework of this thesis, two studies related to the topics covered in the present section were published. In the first one [144], the influence of swirl motion on heat transfer was experimentally measured. It was found that swirl plays a decisive role on the amount of heat losses. Thus, 
to correctly simulate in-cylinder heat transfer, it is important to model swirl motion with accurate approaches. The second study [145] constituted an exploration of the benefits that could be obtained by replacing modeled gas velocities by measured velocities in a multizone model. The proposed approach allowed to use velocity data measured with the particle image velocimetry (PIV) technique to study heat transfer under partially premixed combustion (PPC) conditions.

To sum up, a multizone model is the most advanced method for quasidimensional heat transfer modeling found in the literature. This kind of model can provide physical meaning, temporal resolution and spatial resolution. A key factor is that zonal models take into account the influence of gas velocity on convective heat in detail. In different research works, multizone models have demonstrated to be compatible with almost any kind model, but specially with correlations and lumped element models. This advantage makes zonal models an appealing add-in for integrated engine models. It was also shown that the zonal approach has been extensively developed for diesel engines with swirl motion and bowl in piston, like the engines studied in the present thesis. Considering all mentioned benefits, it could be concluded that a multizone lumped model was a promising tool to be included in a strict 0D/1D code like VEMOD.

For higher accuracy, only 3D codes are suitable [146]. Certainly, 3D simulations provide spatial resolution along with accurate geometry to capture local effects. In addition, physical and chemical processes can be modeled in 3D codes with further detail. This includes the simulation of flame brush or the accurate flow structure, including accurate turbulence models [147] [148] [149]. Unsteady heat transfer effects can be modeled too when local thermodynamics are simulated. Pressure work and convection terms can be included in the equations to model the phenomenon of phase shift physically [150]. Top detail can be obtained by means of DNS (direct numerical simulation) technology, that solves the Navier-Stokes equations numerically. DNS can even simulate the thermal and the velocity boundary layers accurately, at the cost of a very high computational demand [151].

\subsection{Modeling of ancillary thermal systems}

This section surveys models that were developed to simulate the ancillary systems that are subjected to thermal processes. Those ancillary systems 
are circuits that undergo thermal exchanges either if that is their primary purpose, as is the case with the cooling circuit, or as a collateral effect, as with the lubricating circuit. Since these systems are relatively large and play a secondary role in engine operation, 0D/1D or even simpler approaches have been preferred for simulations over computationally demanding CFD models. The models of ancillary systems reported in the literature are usually linked to other models, such as cylinder heat transfer models, or at least they include look-up maps or correlations to define boundary conditions. Often, the look-up maps are generated from experimental or simulation data before the simulation of the ancillary systems. Under this decoupled approach, crossed effects between the main and the ancillary engine components are neglected. Even when the different engine elements are simulated simultaneously, engine heat rejection tends to be modeled by means of simplified approaches. The models of ancillary systems have received considerable attention from researchers because they are necessary to study engine thermal management. Therefore, many reference works on the topic of ancillary circuits modeling could be found. Next, some relevant studies are reported in chronological order.

Zoz et al. [152] developed a model of the lubricating circuit of a gasoline engine for design purposes. The thermal and hydraulic aspects of the circuit were modeled in FLOWMASTER ${ }^{\mathrm{TM}}$. Heat from combustion was calculated with a correlation, while heat from friction in the piston rings was a constant value estimated in motoring tests. Heat from friction in the bearings was obtained from an external tool and entered in the circuit model as a temperature-dependent curve. The authors conducted a validation with experimental data and later used the model to make sensitivity studies of oil temperature to various parameters.

Park et al. [153] linked different components at the system level. The joint model included cylinder, cooling system, radiator and thermostat. Combustion was modeled with a simple, cosine-shaped burning law. The cylinder model considered two zones (burned and unburned) and used the Woschni correlation for heat transfer. Convective heat transfer in the cooling circuit, was modeled with Dittus-Boelter equation. The thermal state of the coolant was solved iteratively. The heat exchanger model was based on the effectiveness-NTU method. Iterations of the global model were undertaken until flows in the system fulfilled energy and mass conservation. A successful validation of the model was achieved under diverse steady-state conditions. In Fig. 2.38, the model's structure is depicted. 


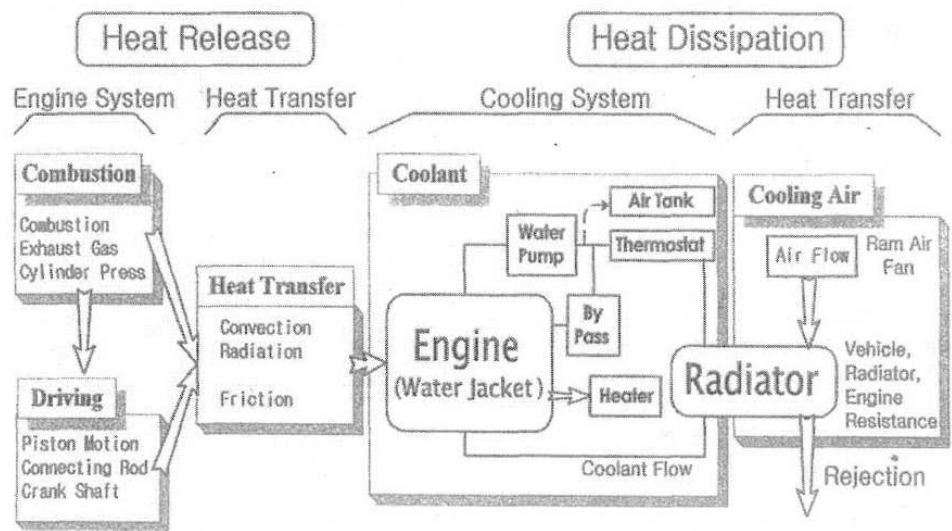

Figure 2.38: Model of Park et al. [153].

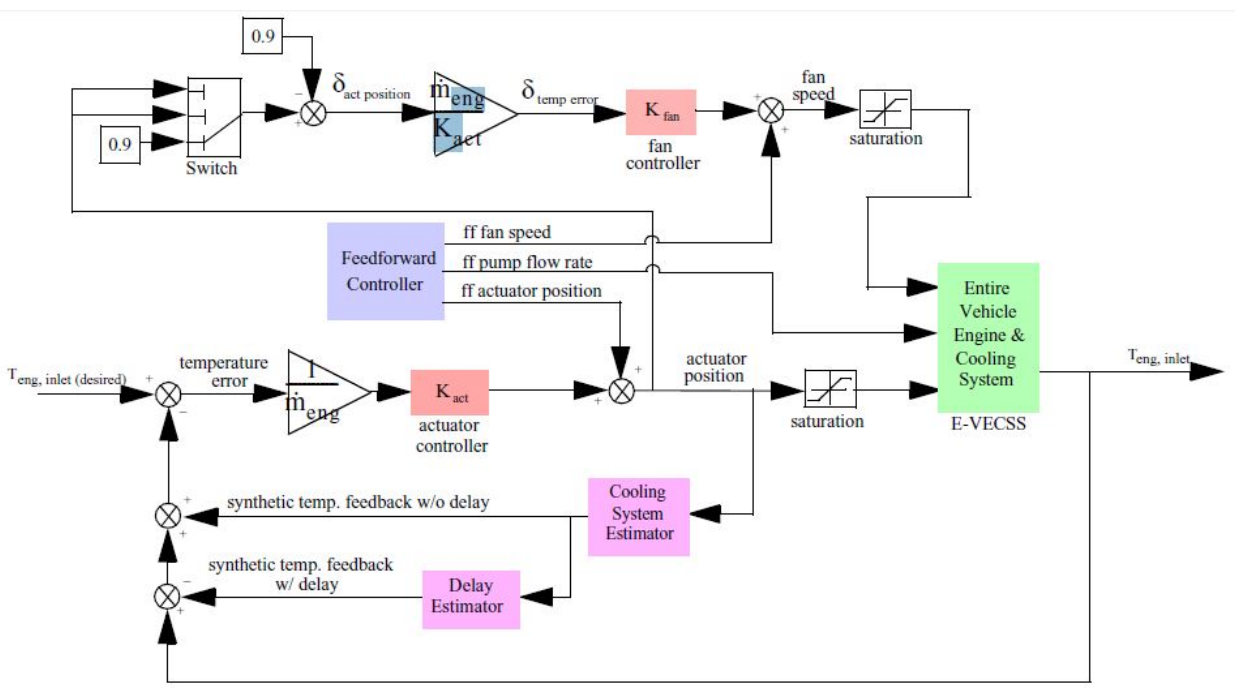

Figure 2.39: Control system of Luptowski et al. [67]. 
Luptowski et al. [67] combined an in-house model implemented in Simulink ${ }^{\circledR}$ and GT-Power simulations of the engine cycle. The in-house model allowed to simulate an actively controlled electric cooling system that included several heat exchangers. A combination of methods was employed to calculate heat transfer in the different elements: empirical Colburn correlations, the effectiveness-NTU method and the NewtonRaphson method. The cooling circuit and the engine model were linked to exchange data. Among other variables, the engine supplied the cooling circuit with heat rejection from cylinder block, cylinder head and piston to coolant and oil. In exchange, the cooling circuit returned temperatures and heat transfer coefficients of fluids to the engine. Moreover, the authors added a system to control the pump and the fan. It is shown in Fig. 2.39. They found that active thermal management greatly reduced power consumption of pump and fan and it also reduced temperature fluctuations at the cylinder wall.

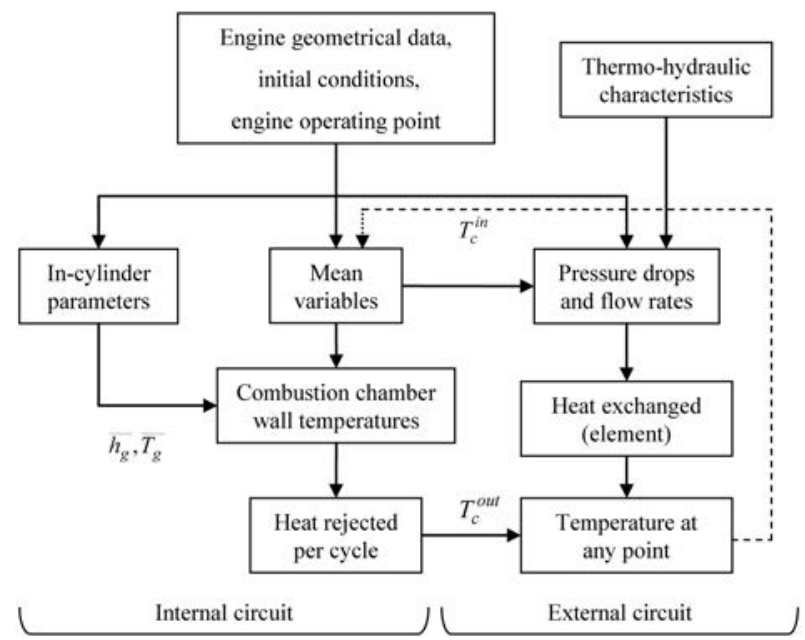

Figure 2.40: Calculation flowchart in the study of Torregrosa et al. [154].

Torregrosa et al. [154] used a thermo-hydraulic model of an engine as an auxiliary tool to find the most interesting configuration to be studied experimentally. They distinguished between an internal and an external part of the cooling circuit which were interconnected, as shown in Fig. 2.40. The internal part, in turn, was divided into three zones, the innermost of which was the engine heat transfer model described in Section 3.3. The hydraulic resistance of the cooling circuit was determined from exper- 
imental tests under steady-state conditions. Those tests were employed to calibrate the heat transfer models as well. The circuit included several heat exchangers that were modeled with the effectiveness-NTU method. Moreover, transient tests were conducted to find the sensible mass of the system and measurements in a NEDC were employed for validation and as baseline case. Afterwards, two valves were added in the theoretical model and five different configurations were studied. The configuration that offered the faster warm-up was reproduced experimentally. Measurements showed significant reductions of fuel consumption and emissions with respect to the baseline case.
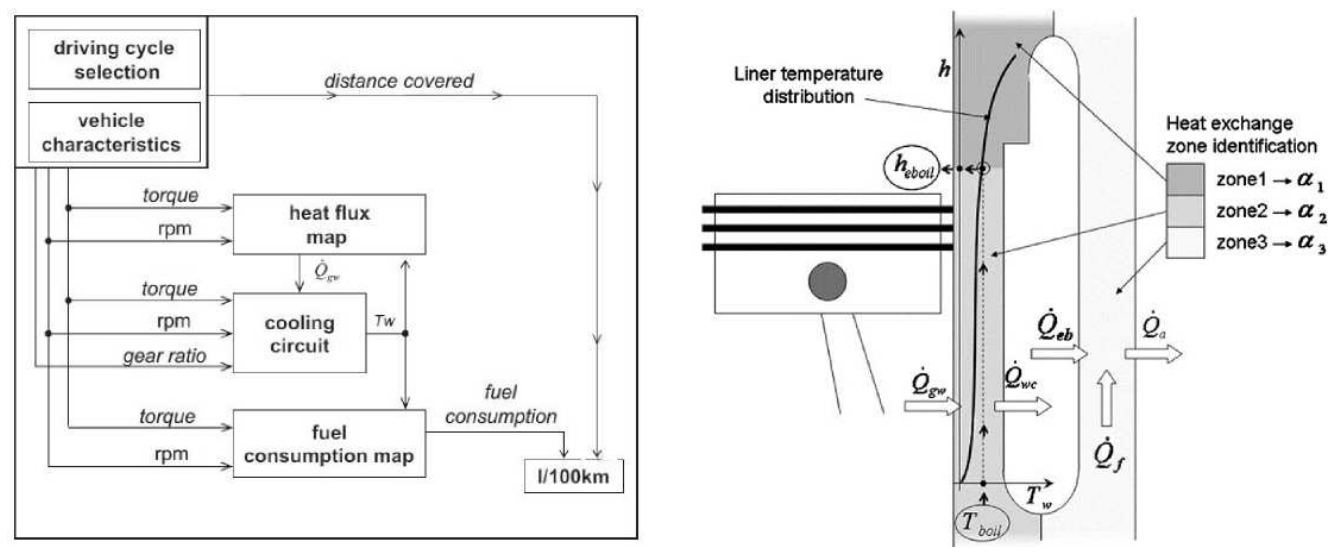

Figure 2.41: Calculation flowchart and cylinder heat transfer in the study of Caresana et al. [155].

Caresana et al. [155] presented a study to assess the imperfections of a conventional cooling system with respect to hypothetical idealized systems. They ran a set of engine simulations in AVL BOOST ${ }^{\mathrm{TM}}$ with different temperatures of the engine structure. The simulations allowed to generate maps of fuel consumption and heat rejection. The maps were linked to a Simulink ${ }^{\circledR}$ code that included models of the cooling system, the drive train and the vehicle aerodynamics. The cooling circuit passed through engine and radiator. Circuit elements were modeled with a combination of methods: correlations for convection, nucleate boiling and friction, experimental measurements of liner temperature, a thermostat model and heat balance equations. The study evaluated fuel consumption in seven driving cycles with one conventional and two idealized cooling circuits. A schema of the calculation flow and a sketch of the cylinder thermal model 
are shown in Fig. 2.41.

Cipollone et al. [156] [157] developed a flexible model of the cooling system and linked it to an engine heat transfer model. The engine heat transfer model was a lumped element model with four nodes per cylinder. ECU maps supplied the values of the operating parameters to the model. The coolant circuits were defined by the equations that described the different components: pumps, thermostats, heat exchangers and tanks. All models were implemented in Simulink ${ }^{\circledR}$. The authors used the thermal model to study the potential of split-cooling in the New European Driving Cycle (NEDC).

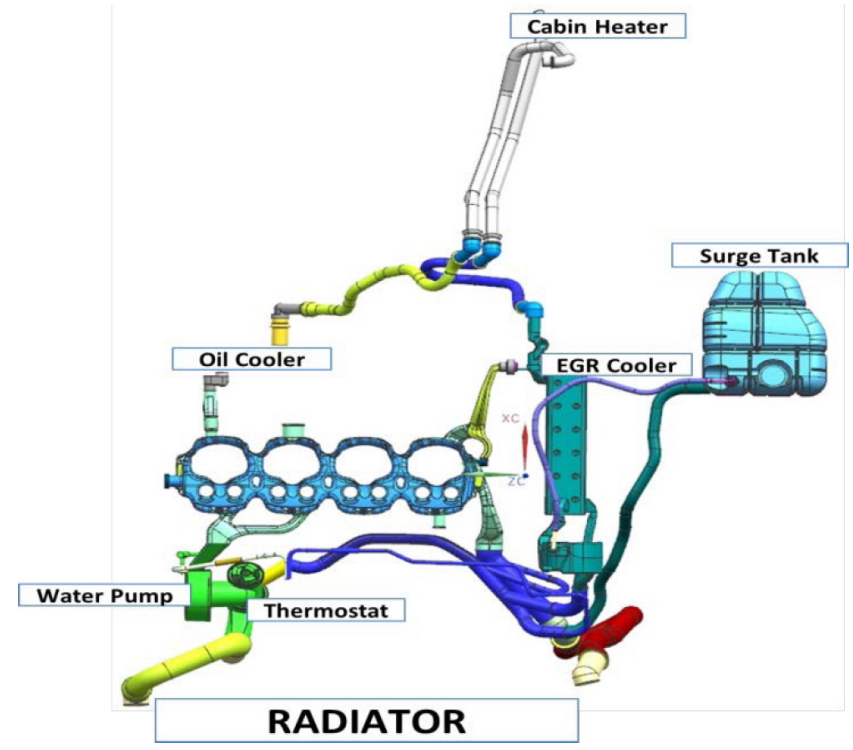

Figure 2.42: Lumped elements in the cooling circuit studied by Millo et al. [42].

More recently, Millo et al. [42] employed a 3D model of an engine and its cooling circuit to create an equivalent lumped element model. Oil volumes in the cylinder-head and cylinder block were modeled as lumped elements too. Heat transfer in the engine block was determined using look-up maps that had been populated with data from 3D simulations. The cooling circuit included various heat exchangers, a thermostat and a switchable coolant pump actuated with an electromechanical clutch, as can be seen in Fig. 2.42. The operation of the thermostat and the pump was defined by characteristic curves. Heat exchanged in the oil 
cooler and the turbocharger as well as friction inputs were obtained from maps populated with experimental data. That limited the validity of the model to temperatures higher than $23^{\circ} \mathrm{C}$, the lowest temperature at which measurements were made. The radiator model used the method of the logarithmic mean temperature difference. Data from steady-state experiments was used to adjust the radiator heat transfer correlations. Upon calibration, parametric studies regarding the operation of the switchable coolant pump were conducted.

\subsection{Thermal codes integrated into global engine models}

This section covers those studies that combined the simulation of different physical processes of the engine. That is accomplished when models of the different processes are coupled together to interact in multi-physics co-simulations. This introduces the challenge of selecting the appropriate solving time scale for the simulations. Many of the simulations included models of the drivetrain to reproduce driving conditions. Due to the existence of standard driving cycles to test the compliance of vehicle regulations, there has been substantial interest in developing complete models that can simulate engine and vehicle operation during driving cycles. That contributes to reduce the required number of expensive experimental tests by replacing them with simulations.

One of the earliest works about multi-physics simulations in internal combustion engines was authored by Baker and Assanis [158]. They combined a thermal model of the piston and liner and a thermo-fluid dynamic model of the gas path. The thermal model was 2D-FEM and axisymmetric. The gas dynamics code comprised quasi-steady models of turbocharger, manifolds, intercooler and cylinders. In this code, mass and energy transfer were simulated, considering energy conversion (e.g., thermal to work) and 1D heat transfer [159]. The coupling between the two models occurred only on the interface. Convection was solved as a quasisteady state process, using the boundary conditions provided by both models. An iterative process achieved the consistency of temperatures and heat fluxes on both sides. Upon convergence, the transient processes on the gas side and on the solid side were solved separately.

The first work that could be found in which a thermal model was coupled to a powertrain model was published by Murrell et al. [129]. The aim 
of the research was to build a model capable to predict engine performance and emissions. The authors used embedded maps of fuel consumption and emissions that depended on engine speed and torque. Torque and engine speed were determined by a drivetrain model that followed a FTP urban driving cycle. From the maps, values valid under fully warm operation were obtained. Those values were then multiplied with temperature-based correction factors. The correction factors depended on temperatures calculated by the thermal model. The thermal simulation was undertaken with a reduced version of the lumped model of Bohac et al. [40], which was reviewed in Section 2.2.3. Thus, in this work a decoupled approach was used instead of proper co-simulation. To finish, the researchers discussed the application of their approach to a hybrid vehicle.

Samhaber et al. [160] presented a procedure to realize a complete co-simulation focused on thermal aspects. The structure is depicted in Fig. 2.43. They used a vehicle and drivetrain model implemented in AVL CRUISE ${ }^{\mathrm{TM}}$ that considered aerodynamic and rolling resistances, road loads, system inertia and mechanical friction. The engine gas flow and the cylinder cycle would be solved with the 1D code AVL BOOST ${ }^{\mathrm{TM}}$. On the contrary, fuel consumption, friction and emissions were characterized by look-up maps. Engine heat transfer was evaluated at a lower solving frequency with a lumped element model. The model was formed by 21 nodes and 34 thermal conduction conductances. In-cylinder data of gas temperature and heat transfer coefficient was received from the engine model. The authors noted that, ideally, this should be done by means of coupling the cylinder and the thermal simulation but for their study they used look-up maps instead. Moreover, the heat flux was corrected for the engine temperature. The thermal model also took into account friction. The convective heat transfer coefficient on the oil side was given by a look-up table dependent on engine speed. On the coolant side, the Nusselt analogy was applied. In both circuits, the thermal and hydraulic aspects were solved with mass and energy conservation equations. The thermal response of the solid and the cooling and lubrication circuits were modeled together in AVL CRUISE ${ }^{\mathrm{TM}}$. With the global model, the authors conducted simulations of the NEDC in which they assessed different thermal management measures from the point of view of the transient response and the thermal balance of the engine.

Kitanoski et al. [161] combined different tools to model the vehicle components. For the gas path, including the cylinder, AVL BOOST ${ }^{\mathrm{TM}}$ was used. Heat release was simulated by means of Wiebe laws and heat 


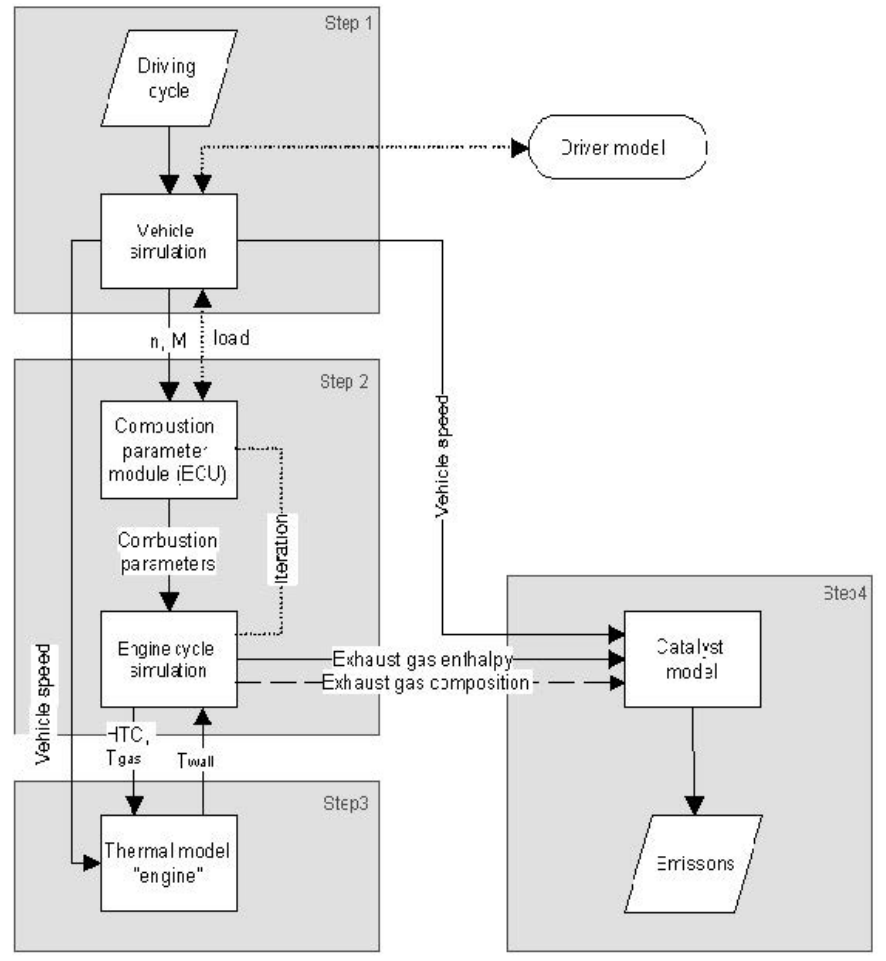

Figure 2.43: Co-simulation of Samhaber et al. [160]. 
rejection to cylinder walls was modeled with Woschni's correlation. The engine heat transfer model was a lumped model with 9 elements. This model was made in MATLAB ${ }^{\circledR}$ and Simulink ${ }^{\circledR}$. In fact, conductances and capacitances were implemented as Simulink ${ }^{\circledR}$ modules. The cooling and oil circuits were implemented in KULI. The cylinder-head and the block were the heat sources for the circuits, which had no thermal inertia. Finally, AVL CRUISE ${ }^{\mathrm{TM}}$ was employed to set up the drive train, which generated the load signal. All the different models were integrated with an in-house platform that managed the data exchange. Once the vehicle model was set up, the authors simulated a warm-up at constant vehicle speed and a NEDC cycle, both at an ambient temperature of $-20^{\circ} \mathrm{C}$.

Kunze et al. [162] conducted a study in which they evaluated preheating strategies with the aim of reducing fuel consumption. Their vehicle model is depicted in Fig. 2.44. It included engine, gearbox, coolant, motor oil and gearbox oil in the thermal analysis. The reference test was the NEDC. The authors validated the model with experimental data and then carried out simulations to assess the potential of two strategies: preheating different components before the start of the cycle and heating different fluid circuits during the NEDC. A final investigation of the impact of preheating the engine fluids was conducted with the model and experimentally to check the prediction accuracy.

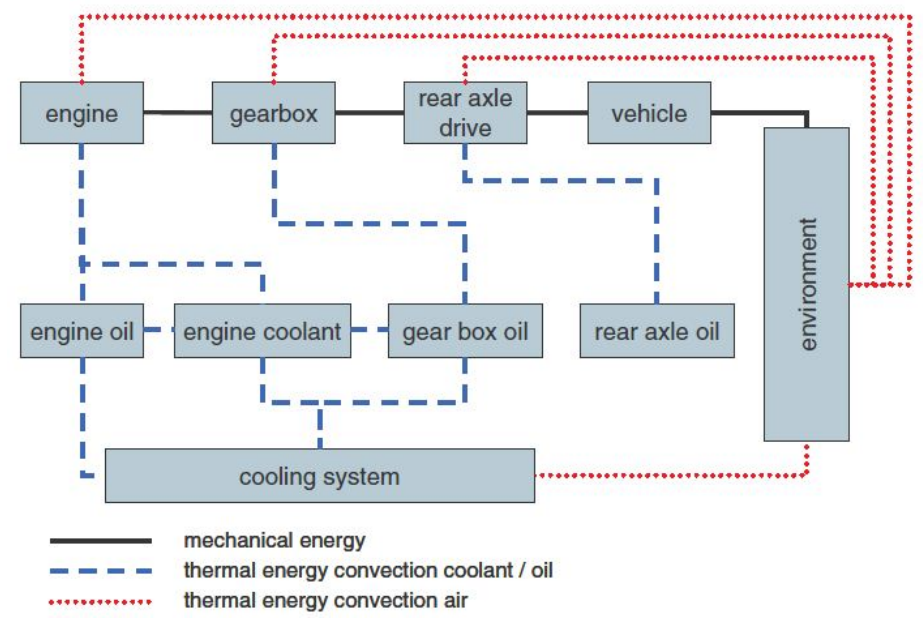

Figure 2.44: Model of Kunze et al. [162].

Banjac et al. [43] presented a complete simulation of the engine, the 


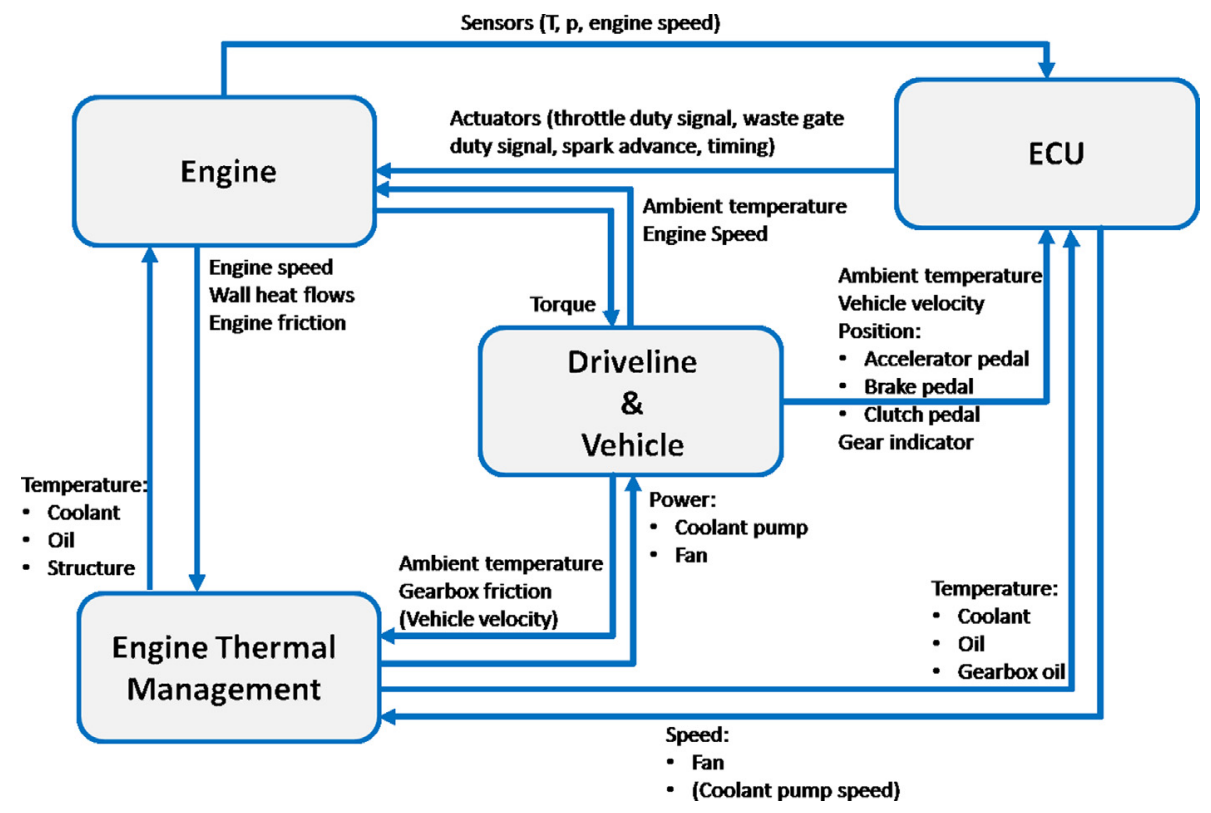

Figure 2.45: Co-simulation of Banjac et al. [43].

vehicle and the thermal management system. Driving conditions were simulated through a model of vehicle dynamics and a detailed mechanical model of the drivetrain. The engine block model was angle resolved. Combustion was modeled with Wiebe laws. Heat transfer was modeled with lumped elements and correlations. Other systems were evaluated with lower temporal resolution. The gas path model was $0 \mathrm{D}$ and heat rejection to gas was resolved once per cycle. The thermo-hydraulic model for the cooling and lubricating circuits combined two time scales. The shorter time scale was employed to solve the incompressible flow distribution. In this regard, mass and momentum equations were solved quasi-steadily basing on the electric analogy described by Kirchhoff's laws. The longer time scale of the thermo-hydraulic model was used to solve the energy equations that defined the transient thermal response of the fluid in a circuit. In addition to this, an ECU model controlled all aspects of vehicle and engine operation. An overview of the system is shown in Fig. 2.45. In order to solve the entire system, the common solver had to manage different time scales and different numerical techniques to provide a suitable coupling among the various physical domains. This implied optimizing computational speed and ensuring numerical robustness. Upon validating 
the model with a mechanically driven coolant pump, the authors analyzed the convenience of replacing it by an electrically driven pump. Two driving cycles, JC08 and Artemis, were simulated. Fuel saving was found to be around 1\%, derived from the lowered power required to propel the pump and from the faster warm-up which reduced friction. Also related to the faster warm-up, higher turbine outlet temperatures were observed, which could result in faster catalyst heat-up.

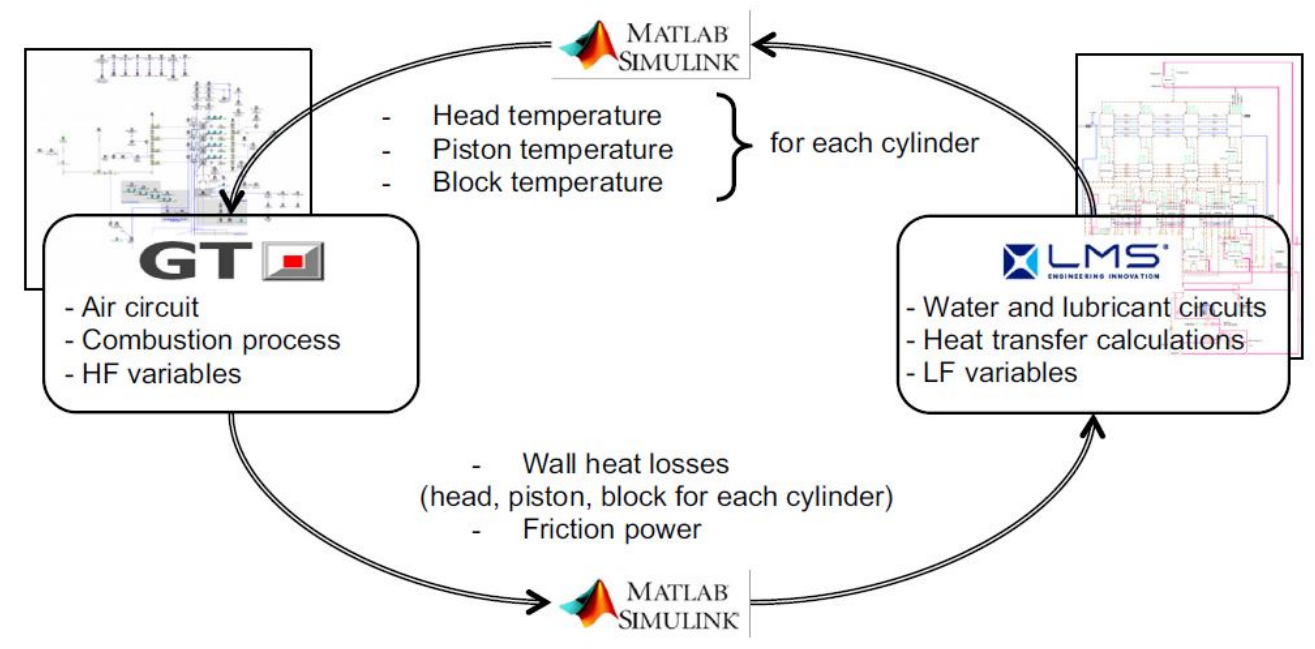

Figure 2.46: Co-simulation of Chalet et al. [44].

Recently, Chalet et al. [44] reported an engine co-simulation that included a detailed thermal model of the engine block. Gas path, injection, combustion, friction and wall heat rejection were computed at high frequency with a GT-Suite model. This model and its look-up maps were calibrated with experimental tests. In particular, Wiebe laws to model the heat release process were obtained. Due to the experimental calibration, the validity of the GT-Suite model was constrained to the tested conditions. Engine heat transfer and thermo-hydraulic circuits (oil and coolant) were modeled in LMS AMESim ${ }^{\circledR}$. This part of the simulation was run at low frequency. The engine thermal model was a lumped element model with 91 nodes representing solid masses and fluid volumes. Convective heat transfer to oil and coolant was determined through SiederTate correlation, Eq. 2.17. The vehicle, driver and ambient data was also included in the AMESim ${ }^{\circledR}$ low-frequency model. The co-simulation was accomplished by means of a Simulink ${ }^{\circledR}$ module that made possible the 
data exchange between the two codes, as shown in Fig 2.46. The authors conducted a validation in three engine operation points. Later, a NEDC cycle in a motored vehicle was measured and simulated. The correct prediction of oil and coolant temperatures was confirmed. Finally, the model was employed in a study to reduce the metal mass at different locations of the engine block.

Overall, the field of co-simulation in global engine or vehicle models is under development and it is providing many valuable insights. Global engine models are a primary tool for engine development. As reported in this section and in Section 2.3, various approaches can be applied, but the most sound and promising one is the co-simulation of physics-based models dedicated to the particular processes occurring in the different subsystems of the engine. It has been shown that the modeling of thermal aspects is indispensable in this kind of simulations. Such considerations encourage the development of thermal models for VEMOD, the main objective of this thesis. 
3.1 Virtual Engine Model (VEMOD) . . . . . . . . . . . 75

3.2 CALMEC: Combustion diagnosis . . . . . . . . . . . . 77

3.3 Initial model of heat transfer in the engine block . . . . . . 80

3.4 Heat transfer in the turbocharger . . . . . . . . . . . . 89

3.5 Heat transfer in gas ducts . . . . . . . . . . . . . . 91

\subsection{Virtual Engine Model (VEMOD)}

VEMOD is a tool for engine modeling which covers the calculation of several physical processes, as sketched in Fig. 3.1. Air management is computed by means of a 1D gas dynamics model [45]. The model deals with flow properties transport along the intake and the exhaust systems as well as the high and low pressure EGR paths [163]. Specific submodels are considered for the boosting system, i.e. compressor [164] and turbine [165] models, air-charge and EGR coolers, throttle valves, heat transfer including gas-to-wall heat exchange and wall temperature prediction, etc. The gas dynamics model is coupled to a cylinder model whose main function is the prediction of in-cylinder conditions based on the combustion process [69] [71]. A detailed heat transfer model is used to obtain the heat rejection to chamber walls, coolant and oil [166]. A mechanical losses model [167] allows obtaining the brake power. An emission submodel is coupled to the combustion process to predict raw $\mathrm{CO}, \mathrm{HC}$, NOx, and soot emissions as a function of the engine operating conditions. Different exhaust aftertreatment systems, such as DOC, DPF and deNOx systems, 
e.g. LNT or SCR, can be considered. Aftertreatment submodels combine thermo-and fluid-dynamic with chemical modelling in order to assess the tailpipe emissions [168] [169].

The engine model is coupled to additional models providing the capability to simulate driving cycles. On one hand, a control system model emulates the electronic control unit (ECU) of the engine. The ECU model manages different engine actuators, such as throttle position, EGR valves or variable geometry turbine depending on information from engine sensors. The vehicle and driver models calculate the vehicle response according to the driving cycle being simulated. Engine speed is the main input to the engine model. The engine model is compiled as a dynamic link library (.dll) compatible with other codes.

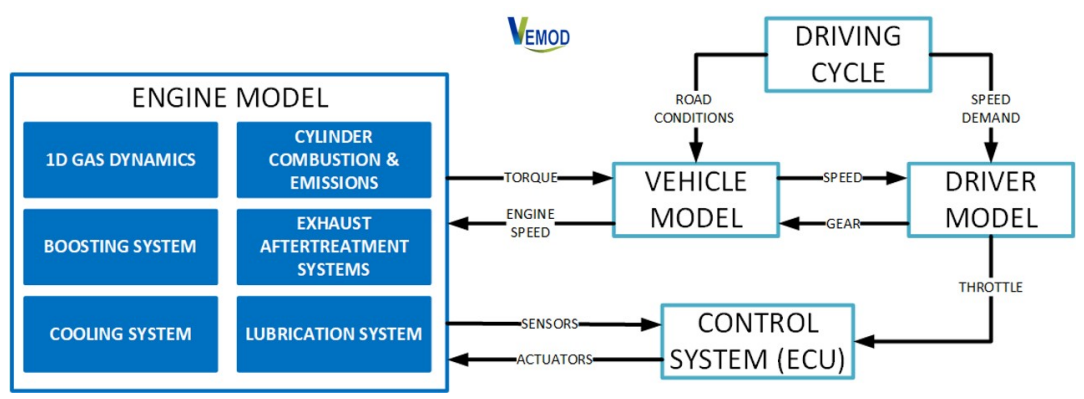

Figure 3.1: Flow-chart of the Virtual Engine Model (VEMOD).

VEMOD uses four different time scales to run the different engine submodels, since the involved physical processes are very dissimilar. Gas dynamics and in-cylinder thermodynamics are calculated with a timestep which varies to ensure numerical stability. Injection, combustion and emissions formation may require smaller time steps. In such case, the current time step of the gas-dynamics model is divided into smaller parts. Thermal evolution of the engine block and the liquids is solved once per cycle. Control system, vehicle and driver model are integrated with a fixed time step of $20 \mathrm{~ms}$. Many processes are executed in parallel, benefiting from multithreading.

The thermal models developed in this thesis were integrated into VEMOD. During the research reported in Chapter 6, a fully assembled virtual engine model was deployed along with experimental data to validate the modeled thermal response. 


\subsection{CALMEC: Combustion diagnosis}

CALMEC is a tool for combustion diagnosis. It is mentioned in Section 2.1 as one of the predecessors of the Virtual Engine Model. In addition to this, CALMEC was used as an auxiliary tool during the research of this thesis. One of the roles of CALMEC was to supply boundary conditions to the engine heat transfer model in Chapter 4. In the same chapter, CALMEC provided reference curves of in-cylinder heat rejection for a study. Another application was the calibration of some submodels of the Virtual Engine Model (VEMOD) in Chapter 6. These tasks are described in-depth in the sections of the respective studies.

The main input of CALMEC is the in-cylinder pressure signal [170]. In-cylinder pressure is measured with a piezoelectric transducer. Data from a number of engine cycles is collected, filtered and averaged to obtain a representative pressure trace. The number of engine cycles sampled in every study of this thesis was 25. Then, the first law of thermodynamics can be applied in the closed cycle, between intake valve closing (IVC) and exhaust valve opening (EVO), exploiting the fact that the cylinder is a quasi-closed system. The Rate of Heat Release (RoHR) can then be calculated as the primary outcome [171] of CALMEC, Eq. 3.1.

$$
R o H R=m C_{V} \frac{d T}{d \alpha}+\frac{d Q}{d \alpha}+p \frac{d V}{d \alpha}-\left(h_{f, i n j}-u_{f, g}\right) \frac{d m_{f, e v}}{d \alpha}+R T \frac{d m_{b b}}{d \alpha}
$$

where $\alpha$ is crank angle degree, $m$ is mass in the cylinder, $T$ is gas temperature, $V$ is instantaneous cylinder volume, $Q$ is heat rejection to the walls, $C_{V}$ is specific heat at constant volume, $m_{f, e v}$ is mass of evaporated fuel, $h_{f, i n j}$ is enthalpy of injected fuel, $u_{f, g}$ is internal energy of the evaporated fuel, $R$ is the ideal gas constant and $m_{b b}$ is blow-by mass leak.

Main hypotheses and features of CALMEC are:

- Gas pressure and temperature are assumed to be spatially uniform inside the cylinder.

- The ideal gas law is used to calculate instantaneous gas temperature from pressure signal. According to Lapuerta et al. [172], employing the ideal gas equation in combustion flow instead of a more complex correlation yields small and localized overestimations up to $2.1 \%$.

- Three species are considered: air, fuel and stoichiometric combustion products. 
- Specific heat of the gas depends on instanteneous temperature and composition [173].

- Trapped mass is calculated by means of a filling and emptying model [174].

- Blow-by is equated with an isentropic process [69].

- Volume variation due to mechanical deformation induced by pressure and inertia is considered by means of a simple model [175].

- Wall temperatures and heat fluxes are calculated by the lumped conductance model that is described in Section 3.3 as one of the subjects of this thesis.

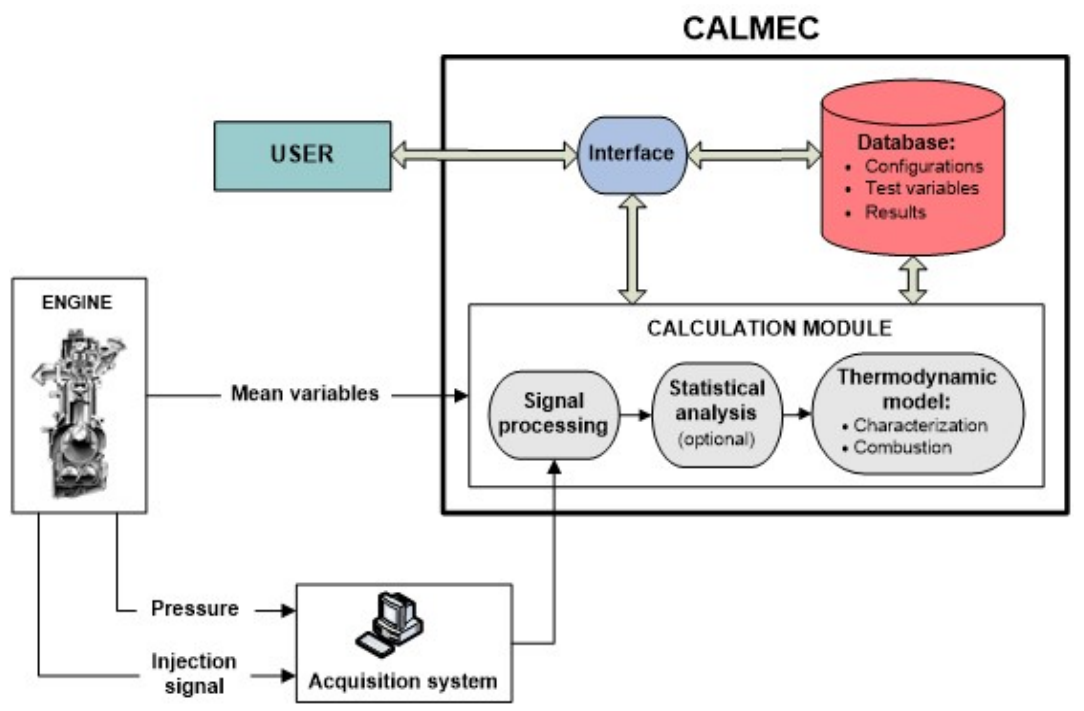

Figure 3.2: General workflow of CALMEC.

CALMEC has been thoroughly examined in earlier research works. Methodological foundations were laid by Armas [176]. In that work, the experimental combustion diagnosis procedure was developed, the thermodynamic model was created and experimental and theoretical uncertainties were analyzed. Martín [177] undertook in-depth studies about CALMEC's performance. This prompted the stablishment of procedures and recommendations for CALMEC usage. Furthermore, some submodels were improved. In a more recent work [101], Carreño focused on the application 
of CALMEC to calculate the engine's global energy balance. A detailed analysis of the global energy balance and the capabilities of CALMEC allowed to propose comprehensive methods and to improve the model. Of particular interest for this work is that the calibration procedure was critically improved.

As a consequence of using submodels (blow-by, deformation, heat transfer, etc.) to improve the accuracy of Eq. 3.1, several calibration constants are introduced in the calculation. The values of these constants are a source of uncertainty. The calibration procedure of CALMEC attributes the uncertainty to four sources [178]:

- Compression ratio. When the piston is in the top dead center position, in-cylinder volume is very small and thus it can be difficult to determine with enough accuracy [179]. Calculation of the thermodynamic process is very sensitive to small errors in volume estimation [178].

- Mechanical deformation model. Related to the model's tuning constant. The influence of this uncertainty on the in-cylinder volume is smaller than the influence of compression ratio [175].

- Heat transfer model. As discussed in Section 2.2, semiempirical convective heat transfer correlations use calibration constants. The model used in CALMEC is described in Section 3.3.

- Thermodynamic shift. The instant of maximum pressure occurs before the top dead center. This is related to heat and mass losses. For combustion diagnosis, it is necessary to determine the shift between maximum pressure and minimum volume, in order to relate both curves. CALMEC uses a formula proposed by Hohenberg [180]. The expression is given in Eq. 3.2 to showcase the relation between thermodynamic shift, $\Delta \alpha$, and heat rate, $\dot{Q}$. Because of such relation, there are crossed effects between the calibration of both magnitudes. In Eq. 3.2, $\Delta \alpha_{Q}$ is the shift due to heat loss and $\Delta \alpha_{m}$ is the shift due to mass leak, which is much smaller [181]. $R$ is the ideal gas constant, $n$ is engine speed, $D$ is bore, $s$ is stroke, $R_{c}$ is crankshaft radius and $L$ is connecting rod length.

$$
\Delta \alpha=\Delta \alpha_{Q}+\Delta \alpha_{m}=\frac{4 R \dot{Q}}{2 \pi^{2} n C_{p} p_{\max } D^{2} s\left(1+\frac{R_{c}}{L}\right)}+\Delta \alpha_{m}
$$


The calibration is conducted using data from motored engine tests. During calibration, the four commented uncertainties are simultaneously tuned until Eq. 3.1 satisfies that heat release from fuel is zero all over the engine cycle [178].

\subsection{Initial model of heat transfer in the engine block}

The output of the engine is mechanical power, produced in the engine block through a thermal process. This makes the engine block the main source of heat in the system. The importance of the heat transfer processes in the engine block motivates the necessity of a dedicated model.

At the beginning of this thesis' research, a model of heat transfer in the engine block already existed [182]. This 0D model was able to calculate temperatures in various parts of the engine block, as well as heat fluxes. It was based on the lumped system analysis, which was explained in Section 2.2.3. Because of this, it was known as Nodal Model. The Nodal Model was able to take into account the different thermal processes of conduction, convection and heat generation. Radiation required an external radiation model to supply the heat fluxes, which could then be considered as heat sources. An advantage of the model was that nodes had a physical meaning because they were a representation of parts of the engine block or specific elements of the engine, such as valves or pipes. On the other hand, a limitation of the model was that the thermal network was formed only by conductances, without capacitances, and therefore it did not consider transient effects. This model had been extensively tested over the years.

The first published work related to the Nodal Model explained the development, calibration and validation in steady state [183]. The experimental work was carried out in two diesel engines. The validation demonstrated that the model could predict engine temperatures under varying operation conditions: engine speed, load, coolant and oil temperatures and intake pressure. A subsequent study improved the model and extended the validation to two additional engines [166]. One of the engines was instrumented internally and allowed to expand the steady-state validation and to undertake a preliminary transient validation. In the other engine, the Nodal Model could successfully predict the temperature evolution of coolant and oil in a New European Driving Cycle. Transient 
studies were conducted without regard to thermal capacity. Instead, a quasi-steady approach in discrete time instants was applied.

Thermal conductances are calculated with Eq. 2.37 and Eq. 2.38 for conduction and Eq. 2.40 for convection. Therefore, the model needs thermal properties, like conductivity or heat transfer coefficients, and geometrical magnitudes, like contact areas or distance between nodes. Geometry is automatically generated by the model in order to be a representation of a real engine block. It can be divided into three elements: cylinder liner, cylinder-head and piston. Next, the geometry of each of the three elements is described.

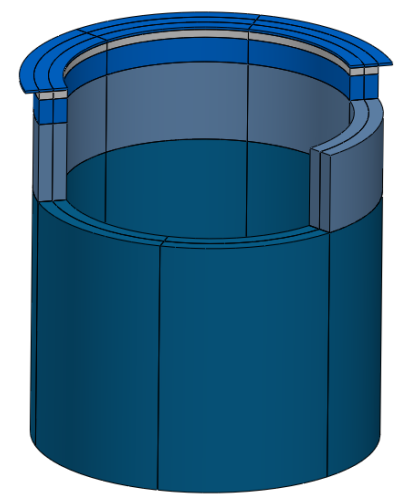

Figure 3.3: 3D reconstruction of cylinder liner model.

The cylinder liner model is displayed in Fig. 3.3. Geometry is automatically generated using three inputs: bore, stroke and squish height. The number of nodes in axial and radial direction can be specified by the user, to provide the desired degree of discretization. In circumferential direction, there are 6 nodes. Among the uppermost nodes, there is one additional, external ring of nodes. Therefore, the total number of nodes is $n_{a x} \cdot n_{\text {rad }} \cdot 6+6$. Height of upper nodes is equal to the squish height and the rest follow a geometric progression in axial direction. In general, inner cylinder surfaces are in contact with gas and outer surfaces are in contact with coolant. An exception is the area between cylinders in case of multicylinder engines. Another exception is the top external ring of nodes. The coolant gallery starts below it, and thus only the underside of those nodes is in contact with coolant. Conductive conductance among nodes in radial direction is calculated with Eq. 2.38. In the other directions, Eq. 2.37 is used. 

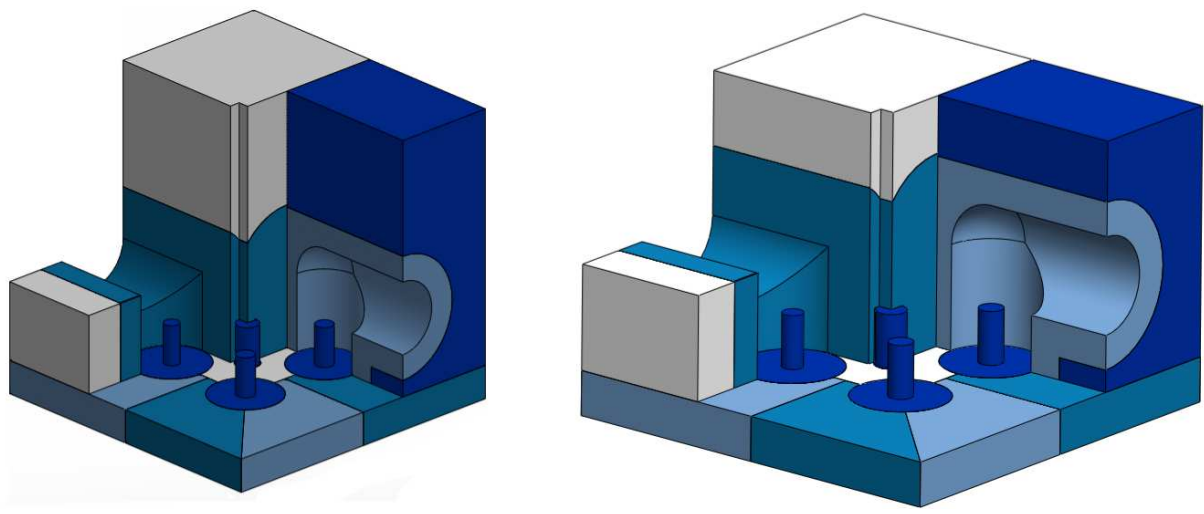

Figure 3.4: 3D reconstruction of cylinder-head model. Full (left). Enlarged (right).
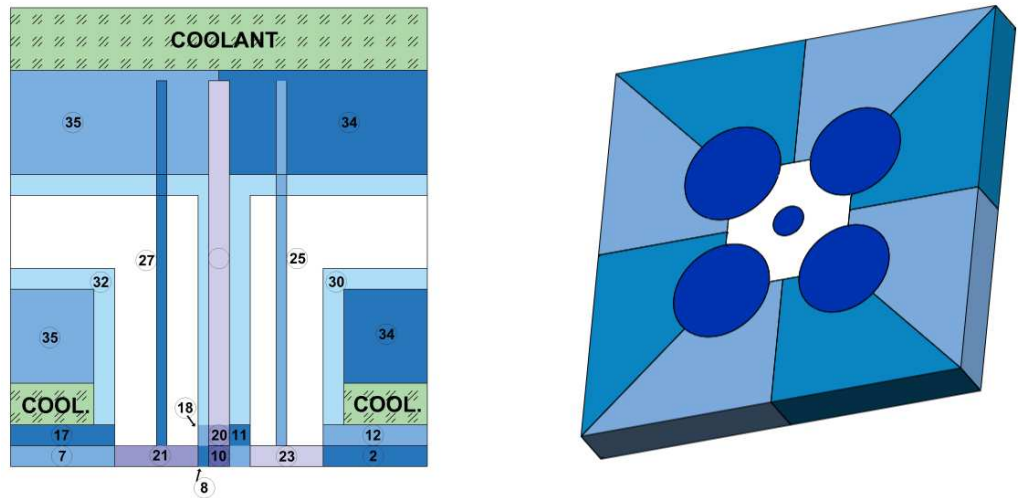

Figure 3.5: Cross section of cylinder-head model (left). Bottom view of firedeck (right). 
The cylinder-head is the most complex model, see Fig. 3.4. Still, the entire geometry is generated using as inputs the bore, the diameters of the intake and exhaust valves and, optionally, the surface areas of intake and exhaust ports. Node sizes are scaled proportionally to these inputs. Relative sizes and shapes of nodes are in accordance to typical dimensions of four-valve cylinder-heads [184]. A cross section of the model is shown on the left side in Fig. 3.5. In total, there are 35 nodes:

- 18 nodes in the firedeck, some of them in contact with in-cylinder gas. A bottom view of these nodes can be seen on the right side of Fig. 3.5.

- For each valve, one node for the plug and one node for the stem. The underside of the plug is in contact with in-cylinder gas. The upper side of the plug and the stem are in contact with gas in the intake or exhaust port.

- One node for each of the four ports, representing the walls in contact with intake or exhaust gas.

- Three nodes for the injector. The undermost node is in contact with in-cylinder gas.

- Two nodes for the bulk of the cylinder-head, one on the side of the intake ports and one on the side of the exhaust ports. They are in contact with the coolant galleries.

Eq. 2.37 is employed to calculate all thermal conductances except those of the valve seats, where $K=0.56 \cdot 3000 A[118]$.
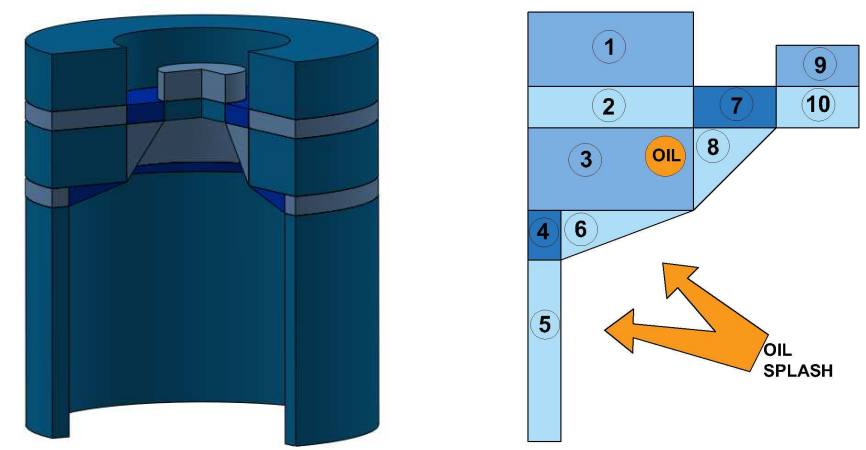

Figure 3.6: 3D reconstruction of piston model (left). Cross section (right).

The piston model is shown in Fig. 3.6, left. It is an axisymmetrical model formed by 10 nodes. A cut view of one half is provided on the right side of the same figure. It is built using the bore, the bowl diameter and, 
optionally, the upper surface area as inputs. Sizes and shapes are based on common proportions of engine pistons. Upper surfaces are in contact with in-cylinder gas. Node number 3 is in contact with oil, simulating that it is crossed by an oil gallery. The underside surfaces, where oil is assumed to splash from the pan, are in contact with this fluid as well (nodes 5, 6, 8 and 10 of Fig. 3.6, right). Conductive conductances in radial direction (for instance, between nodes 2 and 7) are calculated with Eq. 2.38. For conductances in axial direction (for example, between nodes 2 and 3), Eq. 2.37 is used. Thermal conduction between piston and cylinder liner through piston rings is considered in this model. Contact area between piston and liner nodes changes over time due to piston movement. Radial conductive conductances are obtained taking the precaution of averaging the varying contact areas between nodes over the calculation period.

Apart from geometrical measurements, the other required inputs to the Nodal Model are mean temperatures of the following fluids: in-cylinder gas, intake gas, exhaust gas, oil, coolant in liner galleries and coolant in cylinder-head galleries. These temperatures act as boundary conditions. They can be measured or can be provided by an external model.

In the engine block there are different processes of thermal convection (fluid - solid), each one using a different correlation for the heat transfer coefficient, $h$. They are detailed next.

The heat transfer coefficient from in-cylinder gas to walls is the most important. In this model, it is modeled with a modified Woschni correlation, Eq. 3.3. It is reminded that the original Woschni correlation was introduced in Eq. 2.24 and Eq. 2.25.

$$
\begin{aligned}
h(\alpha)= & 0.012 D_{c y l}^{-0.2} p(\alpha)^{0.8} T(\alpha)^{-0.55} \\
& \cdot\left[C_{W 1} c_{m}+C_{W 2} c_{u}(\alpha)+C_{2} \frac{V_{d} T_{I V C}}{p_{I V C} V_{I V C}}\left(p(\alpha)-p_{0}(\alpha)\right)\right]^{0.8}
\end{aligned}
$$

$\alpha$ is the crank angle degree. $p_{0}$ is given by $p_{0}(\alpha)=p_{I V C}\left(\frac{V_{I V C}}{V(\alpha)}\right)^{1.36}$, thus assuming a polytropic process. $C_{2}$ is equal to 0 in the absence of combustion or when $p<p_{0}$. Otherwise, $C_{2}$ is given a value which is adjusted for every engine. $c_{u}$ is calculated in CALMEC according to Eq. 3.4 during the closed part cycle and according to Eq. 3.5 during the open part. This was motivated by the research published in [185]. In the equations, $n$ is engine speed in $\mathrm{rpm}$ and $S R$ is nominal swirl ratio by cylinder-head design. 
$c_{u}(\alpha)=\left(\frac{D_{\text {bowl }}}{D_{c y l}}\right)^{2} \frac{D_{\text {bowl }}}{2} K_{\text {no-dis }} n \operatorname{SR} x(\alpha)$

where,

$$
K_{n o-d i s}=e^{-0.200679 S R^{0.431262}}
$$

If $K_{n o-d i s}$ is higher than 1 , it is made equal to 1

Moreover,

$$
\begin{aligned}
& x(\alpha)=r+\frac{1}{\left[\cosh \left(\frac{\alpha}{100}\right)\right]^{40}+\frac{r}{1-r}} \\
& r=\left[\left(\frac{D_{\text {bowl }}}{D_{\text {cyl }}}\right)^{2} K_{\text {no-dis }}\right]^{-1}
\end{aligned}
$$

Constants $C_{W 1}$ and $C_{W 2}$ are adjusted for each engine under study. During the closed part of the cycle, adjustment of $C_{W 1}$ was done in combination of other constants as explained in Section 3.2. Adjustment of $C_{W 2}$ is done by trial and error under the criterion of balancing the global energy balance of the engine over tests with different swirl ratios. If no tests with different swirl ratios are available, $C_{W 2}$ is calculated as $C_{W 2}=0.59 * C_{W 1}$, a typical ratio observed in previous experiments [177]. Also under the criterion of energy balance consistency, values of $C_{W 1}$ and $C_{W 2}$ during the the open part of the cycle are adjusted from Woschni's reference values of 6.18 and 0.417 . In this model, the same heat transfer coefficient is used for all surfaces inside the cylinder.

$$
\begin{aligned}
& c_{u}=0.75 n \operatorname{SR} r\left(\frac{D_{c y l}}{2 r}\right)^{2} \\
& \text { where, } r=0.5 \sqrt{D_{b o w l}^{2} D_{c y l}^{2} \frac{D_{c y l}}{s}\left(1-\frac{D_{c y l}}{s}\right)}
\end{aligned}
$$

The heat transfer coefficient is necessary to obtain the gas temperature used in the calculation of heat flux between in-cylinder gas and walls. This is demonstrated in Eq. 3.6, where $i$ is the wall node, $Q_{i}$ is the amount of heat received by a node over time, $t$ is the time instant, $n$ is the number of instants, $\Delta t$ is the time period, $A$ is the exposed area and an overline means time-averaged. The demonstration shows that this procedure guarantees the agreement among heat flux, thermal conductances and node temperatures [40]. 
On the one hand,

$$
\begin{aligned}
Q_{i} & =\sum_{t} h_{t} A_{i, t}\left(T_{\text {gas }, t}-T_{\text {wall }, i}\right) \Delta t_{t} \\
Q_{i} & =\sum_{t} h_{t} A_{i, t} T_{\text {gas }, t} \Delta t_{t}-\sum_{t} h_{t} A_{i, t} T_{\text {wall }, i} \Delta t_{t}
\end{aligned}
$$

Neglecting changes of $T_{\text {wall }, i}$ during an engine cycle,

$$
Q_{i}=\sum_{t} h_{t} A_{i, t} T_{g a s, t} \Delta t_{t}-T_{w a l l, i} \sum_{t} h_{t} A_{i, t} \Delta t_{t}
$$

On the other hand,

$$
\begin{aligned}
& Q_{i}=\Delta t{\overline{Q_{i}}}_{i}=\Delta t \overline{h A}_{i}\left(\bar{T}_{\text {gas }}-T_{\text {wall }, i}\right) \\
& Q_{i}=\Delta t \frac{\sum_{t}\left(h_{t} A_{i, t} \Delta t_{t}\right)}{\Delta t}\left(\bar{T}_{\text {gas }}-T_{\text {wall }, i}\right) \\
& Q_{i}=\sum_{t}\left(h_{t} A_{i, t} \Delta t_{t}\right)\left(\bar{T}_{\text {gas }}-T_{\text {wall }, i}\right) \\
& \sum_{t} h_{t} A_{i, t} T_{\text {gas }, t} \Delta t_{t}-T_{\text {wall }, i} \sum_{t} h_{t} A_{i, t} \Delta t_{t}=\sum_{t}\left(h_{t} A_{i, t} \Delta t_{t}\right)\left(\bar{T}_{\text {gas }}-T_{\text {wall }, i}\right) \\
& \frac{\sum_{t} h_{t} A_{i, t} T_{\text {gas }, t} \Delta t_{t}}{\sum_{t} h_{t} A_{i, t} \Delta t_{t}}-T_{\text {wall }, i}=\bar{T}_{\text {gas }}-T_{\text {wall }, i}
\end{aligned}
$$

Results in,

$$
\bar{T}_{g a s}=\frac{\sum_{t} h_{t} A_{i, t} T_{g a s, t} \Delta t_{t}}{\sum_{t} h_{t} A_{i, t} \Delta t_{t}}
$$

If contact area between the wall node and the cylinder gas is constant over time, as it is in most nodes, then $A_{i, t}=A_{i}$ and Eq. 3.6 is simplified to Eq. 3.7. Only the exposed area of the nodes in the liner wall changes over time because of piston movement, as shown in Fig. 3.7. For these nodes it is necessary to use Eq. 3.6.

$$
\bar{T}_{g a s}=\frac{\sum_{t} h_{t} T_{g a s, t} \Delta t_{t}}{\sum_{t} h_{t} \Delta t_{t}}
$$

Convective heat transfer in intake and exhaust ports in modeled by means of correlations for turbulent flow inside tubes. For the intake ports, a correlation from Sleicher and Rouse is used, Eq. 3.8 [186].

$$
h=\frac{k}{D}\left(5+0.015 \operatorname{Re}^{0.83} \operatorname{Pr}^{0.66}\right)
$$




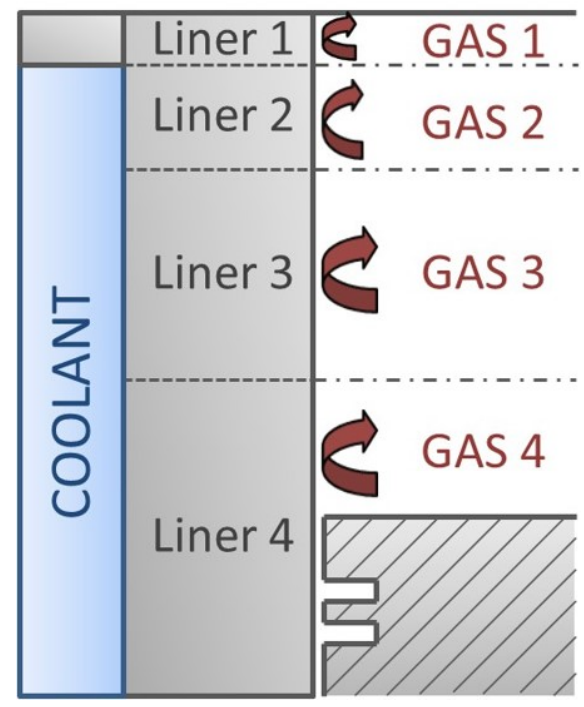

Figure 3.7: Gas nodes in contact with liner.

For the exhaust ports, two correlations are employed. The first one, Eq. 3.9, is applied during the period when exhaust valves are closed. It is due to Caton [187]. $\dot{m}$ is the average mass flow per engine cycle, $z$ is number of cylinders and $N_{v}$ is number of valves per cylinder.

$$
h=0.022 \frac{k}{D}\left(\frac{4 \dot{m}}{\mu z N_{v} \pi D}\right)^{0.8}
$$

During the part of the cycle when valves are open, there is gas flow through the ports. Instantaneous gas velocity of the exhaust flow can thus be obtained and used in Eq. 3.10 [188].

$$
h=0.00375 \frac{k \rho}{\mu} v
$$

Heat transfer coefficient calculated with Eq. 3.9 and Eq. 3.10 is finally averaged to obtain a mean value for the whole cycle. For the average, the relative portions of the cycle in which exhaust valves are open and closed are taken into consideration.

Heat transfer coefficients for convection between the engine structure and coolant are constant. There are two constant values: one for the liner galleries and one for the cylinder-head galleries. Both processes are independent. 
Oil heat transfer coefficients consider two processes: forced convection in one of the piston nodes and splash cooling at the underside. Forced convection is modeled with the DittusBoelter equation, Eq. 2.16. Oil splash is modeled with a correlation employed by Jarrier et al. [117], Eq. 3.11 , where $n$ is engine speed in revolutions per minute, $v$ is current dynamic viscosity and $v_{r e f}$ is reference dynamic viscosity at $90{ }^{\circ} \mathrm{C}$.

$$
h=350 \frac{n}{2000} \sqrt{\frac{v_{r e f}}{v}}
$$

In this thesis, the Nodal Model is thoroughly analyzed and improvement studies are conducted. Chapter 4 is entirely devoted to this task. The aim is to integrate the optimized heat transfer model in the Virtual Engine Model. The Nodal Model had been integrated in another code before: in CALMEC diagnosis tool. The version in CALMEC was used in this thesis to evaluate the initial heat transfer model, as reported in Section 4.2. There existed also an initially identical standalone version which was used for most of the research in Chapter 4. Model integration in CALMEC was mainly a source of advantages, since the model was adapted for exchanging data with other submodels. A drawback of integration was that it was adapted for simulating one single engine cycle, as CALMEC. The temporal scope of VEMOD is, in contrast, not limited to one cycle. During the reorganization of the model for VEMOD, one of the tasks was to make it work for the temporal range of the inputs, regardless of it being different from a complete cycle. A second important drawback was that the lumped model and CALMEC were in- 
tended for simulating steady-state operation. Consequently, modeling of thermal transients had to be added. In addition to those upgrades, many other improvements were implemented as explained in Chapter 4.

\subsection{Heat transfer in the turbocharger}

The turbocharger is a machine where complex heat transfer processes can be found. This is a thermal machine with a relevant role in the engine heat balance. Research has demonstrated the importance of heat transfer in turbochargers, specially at low load and under pulsating flow conditions [189]. Thus, it becomes necessary to take heat transfer into account to correctly predict enthalpy drops, outlet compressor temperature, which has influence on engine performance, and outlet turbine temperature, which can determine the operation of aftertreatment systems [190]. This justifies the existence of a dedicated model.
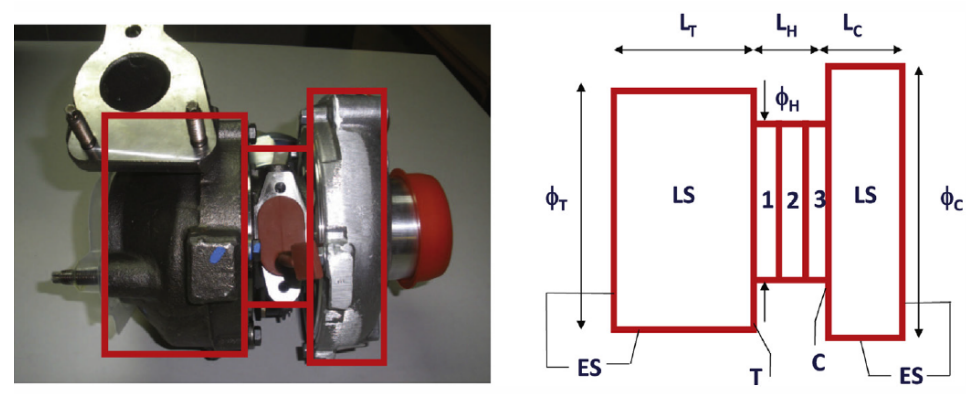

Figure 3.9: Simplification of turbocharger geometry in heat transfer model [191].

The development of the turbocharger heat transfer model is described in [192]. In the model, geometry is simplified to a set of coaxial cylinders, see Fig. 3.9. As in the engine, the lumped system approach is applied. The model considers heat conduction, internal and external convection, radiation to the exterior, thermal inertia and friction heat generation. Fig. 3.10 is a schema of the different heat transfer processes in the thermal network.

Conductive conductances and thermal capacitances are obtained for each turbocharger following the procedure explained in [193]. Radiation to the exterior and external convection are considered by the model but will not be reviewed in this section because they are not relevant for this 


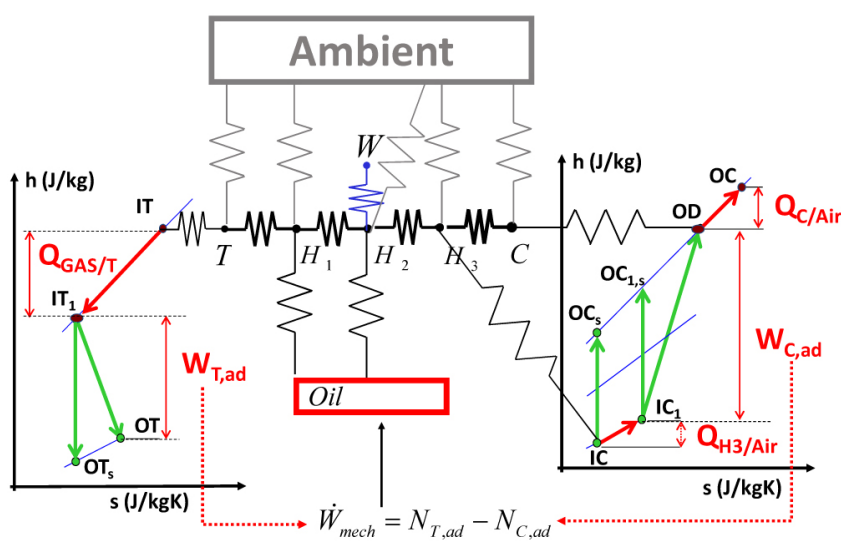

Figure 3.10: Lumped heat transfer model of the turbocharger [190].

thesis, which is focused on internal heat transfer. The detailed calculation procedure can be found in [191]. Internal convection processes are modeled with correlations based on Sieder-Tate equation, Eq. 2.17, or Dittus-Boelter equation, Eq. 2.16. Next, a brief account of the convective correlations is given. For full detail, the in-depth study can be consulted [194]. Correlations cited below obtain the Nusselt number, $N u$.

For convection between exhaust gas and turbine walls, Eq. 3.12, based on Sieder-Tate equation, is used. $C_{1}$ is a calibration constant, $\eta_{\max }$ is the maximum isentropic efficiency of the turbine for a determined opening, and $D_{\text {eff }}$ and $L_{\text {eff }}$ are characteristic lengths.

$$
N u=C_{1} \operatorname{Re}^{0.8} \operatorname{Pr}^{1 / 3}\left(\frac{\mu}{\mu_{w}}\right)^{0.14}\left(\frac{\eta_{\max } D_{e f f}}{L_{e f f}}\right)^{0.2178}
$$

For heat transfer between intake gas and compressor walls, Eq. 3.13, of the form of Dittus-Boelter equation, is employed. $C_{1}$ and $C_{2}$ are different when the gas is heating the wall and when the gas is cooling the wall. $C_{2}$ is equal to 0.3 in the former case and 0.4 in the latter.

$$
N u=C_{1} \operatorname{Re}^{0.8} \operatorname{Pr}^{C_{2}}
$$

The turbocharger can be cooled by oil, water or both. Oil cooling is modeled with Sieder-Tate equation and water cooling with Dittus-Boelter equation. Nevertheless, calibration constants $C_{1}$ are different from the original ones because they are adjusted for each turbocharger. 
This heat transfer model, the turbine and compressor models and the global turbocharger assembly have been tested and validated under different operating conditions [29]. The procedures for calibrating the heat transfer model have been gathered in [198]. The turbocharger also has a submodel of mechanical losses [199].

This model is part of VEMOD and was developed in parallel with this thesis. It was employed every time VEMOD was used in this thesis, as the modeled engine had a turbocharger.

\subsection{Heat transfer in gas ducts}

In VEMOD, the ducts of the gas circuit have a heat transfer submodel [195]. The submodel can take into account internal and external convection. The correlations used for internal heat transfer are relevant for this thesis. They are the correlations used to calculate heat transfer in the intake and exhaust ports of the cylinder-head and also in heat exchangers.

For ducts belonging to the intake system, a correlation proposed by Depcik and Assanis is used, Eq. 3.14 [196]. It is based on the relation between Reynolds and Nusselt numbers. $D_{\text {int }}$ is internal diameter.

$$
h=0.0694 R e^{0.75} \frac{k}{D_{\text {int }}}
$$

On the exhaust side, correlations developed in previous theses are employed. For exhaust ports, the equation is Eq. 3.15 [197]. This correlation is based on Sieder-Tate equation 2.17. Eq. 3.16, based on the relation between Reynolds and Nusselt numbers, is used in the rest of ducts of the exhaust system [188].

$$
\begin{gathered}
h=0.0709 R e^{0.8}\left(\frac{\mu}{\mu_{w}}\right)^{0.14} \frac{k}{D_{i n t}} \\
h=1.6 R e^{0.4} \frac{k}{D_{i n t}}
\end{gathered}
$$

External convection is considered by the model but will not be reviewed here because this thesis is focused on the internal heat transfer processes. Due to the existence of external boundary conditions, duct wall temperatures can be variable and can be calculated by the model. Alternatively, there is the possibility of setting fixed wall temperatures as boundary conditions. 
As the turbocharger model, the gas circuit model is part of VEMOD and thus it was active every time VEMOD was used in this thesis. 


\section{Heat transfer in the engine block}

4.1 Qualitative evaluation of the initial model . . . . . . . . . 94

4.2 Quantitative evaluation of the initial model . . . . . . . . 97

4.2 .1 Methods . . . . . . . . . . . . . . 97

4.2 .2 Results . . . . . . . . . . . . . . . 125

4.3 Multizone model of in-cilinder convection . . . . . . . . . 141

4.3 .1 In-cylinder gas motion . . . . . . . . . . . . . 143

4.3.2 Thermal analysis . . . . . . . . . . . . . . 170

4.4 Heat rejection to coolant and lubricant . . . . . . . . 188

4.5 Transient behavior . . . . . . . . . . . . . . . . . 193

4.6 Adaptation for integration into VEMOD . . . . . . . . . 201

This chapter covers the process of evaluation and improvement of the engine heat transfer model known as Nodal Model. The evaluation was both qualitative and quantitative. The qualitative part, reported in Section 4.1, consisted in a examination of the initial characteristics of the model, to contrast them with the target features. Particular attention was focused on prediction capabilities because VEMOD is a predictive tool. The quantitative evaluation was based on a experimental campaign in a diesel engine. It is thoroughly accounted in Section 4.2.

After evaluation, one of the proposed improvements was to include a multizone model to improve predictions of convective heat transfer to cylinder walls. Model implementation is described in Section 4.3, including related studies and the calibration, which were carried out with help of a computational fluid dynamics (3D-CFD) code. The predictive capacity 
of the multizone model was assessed using experimental measurements as reference.

The development of other improvements is reported in subsequent sections. Topics related to convection processes on the coolant and oil sides are covered in Section 4.4. Introduction of capacitances in the thermal network and mathematical methods to solve the transient response of the thermal system are discussed in Section 4.5. Other improvements and updates are described in Section 4.6. The final model underwent an additional validation to check the suitability of the new features.

\subsection{Qualitative evaluation of the initial model}

The initial engine heat transfer model was described in 3.3. It is a steadystate lumped element model called Nodal Model. The working principles of lumped element models were explained in 2.2.3. A survey of relevant models used for engine research was also undertaken. It was shown that lumped element models are non-dimensional tools that have advantages such as spatial resolution, consideration of the different heat transfer mechanisms and uncomplicated compatibility with other models. In the description of the initial Nodal Model in Section 3.3, its capabilities were discussed. Two previous research studies in which this model was the central subject were reviewed. Thereafter the scientific characteristics of the model were detailed. After examining the collected information, a qualitative evaluation of the model's capabilities could be accomplished. Initial features were:

- Broad spatial resolution with 35 nodes in the cylinder-head, 10 in the piston and a user-configurable number of nodes in the cylinder liner. Furthermore, the nodes corresponded to real elements or parts of the engine structure. Node shapes were fixed but dimensions were automatically scaled with input dimensions such as bore, stroke, bowl diameter and valve diameters.

- Modeling of convective processes was achieved through correlations that take into account the effect of engine parameters. A highly developed Woschni correlation was used in the cylinder walls. In addition, there were convective correlations for the intake and exhaust ports and for the oil. 
- The model could predict temperatures in the engine structure in steady state. Predictions were valid for different engine speeds, loads, coolant and oil temperatures and intake pressures.

- The model could predict temperatures of the solid as well as of the liquids (coolant and oil) during warm-up. This capability was not based on the consideration of the thermal capacity of the system, but on discrete quasi-steady estimations.

- The Nodal Model was already adapted to work within the combustion diagnosis tool CALMEC, coupled to other submodels.

This account portrays the Nodal Model as a suitable tool for its original application. The model had a solid predictive capacity of steady-state temperatures in the engine structure. It also provided considerable spatial resolution and physical foundation. Last, it was adapted to work integrated into a code of cylinder thermodynamics.

The presented account also allows to identify areas for improvement. Geometry was based on real engine dimensions and it was resized according to input parameters. However, engines can have very diverse geometries concerning piston head shape, cylinder-head configuration, number of elements such as valves, prechambers, etc. It was thus necessary to allow the possibility of manually defining the geometry so that any reciprocating machine could be defined.

Second, the quasi-steady approach used in the past to model transient behavior is not optimal. It was argued in Section 2.2.3 that the most rigorous way of simulating thermal inertia in lumped elements models is adding capacitances to the network.

The correlations used for convective heat transfer performed their function properly, but alternatives could be explored. In particular, the suitability of Woschni's correlation for in-cylinder heat transfer has been disputed, as was seen in Section 2.2.1. In addition, the initial Nodal Model had no correlation for convection on the coolant side. A physical correlation would allow to take into account important variables such as coolant mass flow.

Going deeper into in-cylinder heat rejection, it was found during the literature review in Section 2.2.4, that lumped models alone were unable to fully reproduce the temperature distribution on the cylinder walls. The assumption of a common heat transfer coefficient for the whole combustion chamber was unsatisfactory. Several researchers have proposed to use 
zonal heat transfer coefficients based on the flow distribution. This can be accomplished through non-dimensional modeling, by way of so-called multizone models. For the research of this thesis, it was interesting to assess whether the Nodal Model was able to predict the temperature distribution on the cylinder walls. Should it were incapable, a multizone model would be a promising add-in to be tested.

A related topic is swirl motion. Research studies about multizone heat transfer models give great importance to swirl. It is also known that swirl has a significant influence on engine performance [200]. However, the influence of swirl on engine heat transfer has not been thoroughly studied. Moreover, the Nodal Model had not been validated for different levels of swirl motion. That was therefore a subject of interest.

Finally, the integration of the Nodal Model into VEMOD implied that it would be coupled to various models. The Nodal Model had to be adapted to ensure compatibility and interaction among the constituents of the Virtual Engine Model. It was mentioned in Section 3.5 that VEMOD has heat transfer models for the intake and exhaust ports. The engine heat transfer model had to be made compatible with them. Friction and injector models should be able to exchange relevant data with the engine heat transfer model too. Another change was that, in VEMOD, thermodynamics are time-based instead of angle-based. The codes into which the engine was integrated initially are angle-based. That required adaptation as well. The last matter was the interaction of the Nodal Model with the ancillary systems models that were developed in this thesis, concerning the cooling and lubricating circuits.

In summary, the desired features were the following, enunciated in the order in which they were dealt with in this thesis:

- Ability to accept any user-defined geometry, not just the default one. This should be achieved by means of importing the lumped system data from an external source.

- Validated prediction of the influence of engine speed, load and swirl motion on wall temperatures. This could be fulfilled combining experimental tests and conventional simulations of the Nodal Model, as in previous studies.

- Validated prediction of the spatial distribution of wall temperatures. Experimental measurements should show the actual distribution. Predictions of the Nodal Model could be enhanced by implementing 
a multizone heat transfer model. This zonal model, in turn, would need to be calibrated and validated.

- Modeling of heat transfer coefficients on the coolant side. New correlations had to be included.

- Modeling of thermal transients by means of adding thermal capacitances into the network. A validation of the modeled thermal response was pertinent. The validation should be achieved through comparison with experimental measurements.

- Adaptation of the revised Nodal Model to VEMOD implementation. That should be attained through various modifications.

\subsection{Quantitative evaluation of the initial model}

\subsubsection{Methods}

The quantitative evaluation of the initial model of heat transfer in the engine block, i.e. of the initial version of the Nodal Model, should be accomplished through the validation of the predicted wall temperatures under different conditions of engine speed, load and swirl motion. These parameters alter in-cylinder conditions, allowing to analyze heat rejection to walls and thermal response of the engine structure. In the initial singlezone model, heat rejection was determined with Eq. 2.5. Three surfaces were considered: liner, firedeck and piston head. $T_{\text {wall }}$ and area are referred to these surfaces. Heat transfer coefficient and gas temperature were the same for the three surfaces. Thus, the evaluation of predicted temperatures had to cover $T_{\text {wall }}$ of the different walls, that is to say, an area-averaged temperature value for each wall. In addition, it was shown in Section 2.2.4 that another relevant matter was the temperature distribution on the cylinder walls. This had to be examined locally, without averaging whole surfaces. Thus, both area-averaged wall temperatures and non-averaged temperature distribution should be evaluated.

A combination of experiments and simulations could be employed to fulfill the validation of predicted temperatures. A method to realize the quantitative evaluation was proposed:

1. Set up an installation to measure temperatures of the engine structure experimentally. 
2. Carry out engine tests consisting in sweeps of relevant parameters: engine speed, load and swirl motion.

3. Supply a combustion diagnostics code, CALMEC, with data from measurements to replicate the experimental tests by simulating the cylinder.

4. Obtain predicted temperatures in the solid from the Nodal Model that is integrated into CALMEC.

5. Compare surface-averaged wall temperatures under the different operating conditions.

6. Compare temperature distributions locally, location by location.

\section{Test facility}

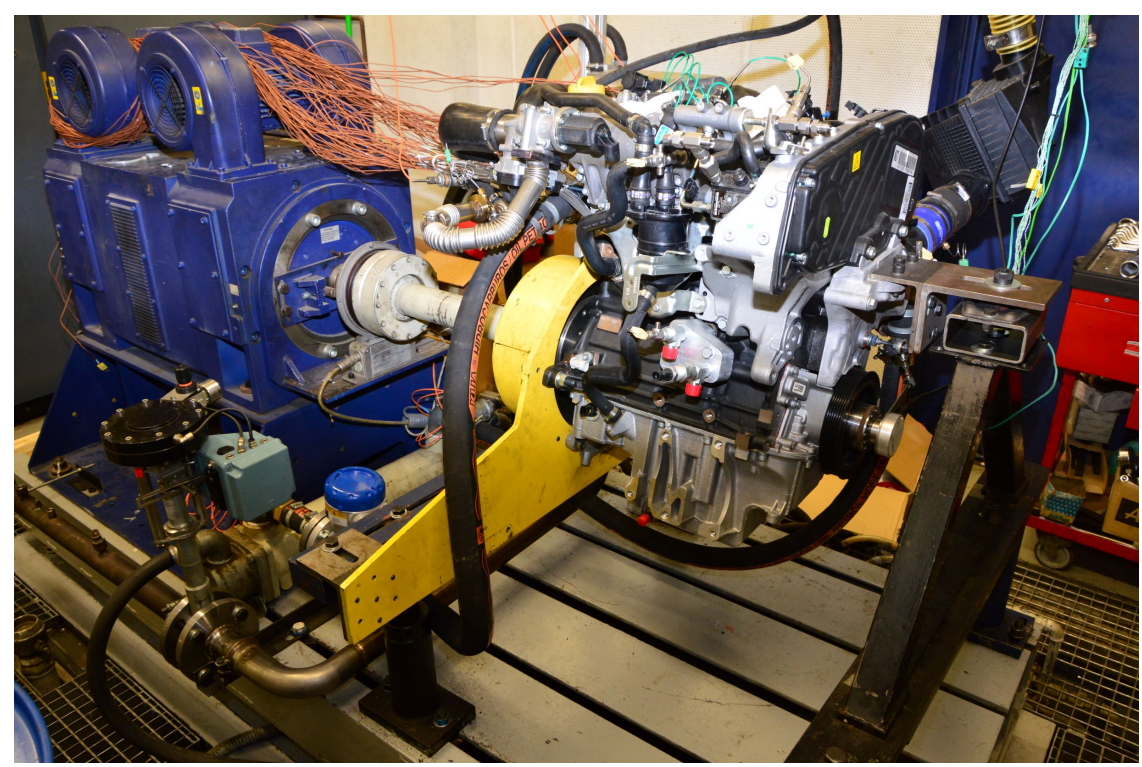

Figure 4.1: Photograph of Engine 1 in test facility.

The engine and the facility used to conduct the experimental research of this chapter are described here. The main feature of this facility was an installation of thermocouples embedded in the engine structure, devised to gain insight of the heat transfer processes inside the engine block. The engine is called Engine 1 to differentiate it from the engine used to do the 
experimental work of Chapter 6 . Engine 1 was a four-cylinder, four-stroke production diesel engine. It had four valves per cylinder, centrally located injectors and a re-entrant type piston bowl. The engine was equipped with a high pressure exhaust gas recirculation system and a variable-geometry turbocharger. Swirl control valves in helical intake ports allowed to set the swirl ratio to $1.4,2,2.5$ or 3 in every cylinder. The engine hardware complied with Euro 4 emissions regulations. Main engine specifications are given in Table 4.1.

\begin{tabular}{lc}
\hline Displaced volume & 1.9 liter \\
Bore & $82 \mathrm{~mm}$ \\
Stroke & $90.4 \mathrm{~mm}$ \\
Compression ratio & $17.5: 1$ \\
Torque (max.) & $320 \mathrm{Nm} \mathrm{@} \mathrm{2000-2750} \mathrm{rpm}$ \\
Power (max.) & $110 \mathrm{~kW} @ 4000 \mathrm{rpm}$ \\
\hline
\end{tabular}

Table 4.1: Main specifications of Engine 1.

\begin{tabular}{llcc}
\hline Variable & Sensor & Range & Accuracy \\
\hline Engine speed & Dynamometer & $0-7500 \mathrm{rpm}$ & $\pm 1 \mathrm{rpm}$ \\
Torque & Dynamometer & $0-500 \mathrm{~N} \mathrm{~m}$ & $0.5 \%$ \\
In-cylinder pressure & Kistler 6125C10 & $0-300 \mathrm{bar}$ & $\pm 1.25 \mathrm{bar}$ \\
Air mass flow & AVL Flowsonix Air & $0-1400 \mathrm{~kg} / \mathrm{h}$ & $1 \%$ \\
Fuel mass flow & AVL 733S & $0-41.67 \mathrm{~g} / \mathrm{s}$ & $0.12 \%$ \\
Temperature of & K-type & -200 to $1250{ }^{\circ} \mathrm{C}$ & $\pm 2{ }^{\circ} \mathrm{C}$ \\
gases and structure & thermocouples & & \\
Temperature of liquids & PT100 RTD & -30 to $350{ }^{\circ} \mathrm{C}$ & $\pm 0.01^{\circ} \mathrm{C}$ \\
\hline
\end{tabular}

Table 4.2: Main instrumentation used in Engine 1.

The facility included complete instrumentation to measure all relevant magnitudes. It is listed in Table 4.2. An electric dynamometer was directly coupled to the engine. The dynamometer allowed to set engine speed and measure torque. In-cylinder pressure was measured in each cylinder with Kistler $6125 \mathrm{C} 10$ piezoelectric transducers mounted in the glow-plug bores. The signal was then converted with Kistler 4603B10 charge amplifiers. A crank angle increment of 0.5 degrees was used for the in-cylinder pressure acquisition. A total of 25 consecutive cycles of in-cylinder pressure 
data were sampled. A DRIVVEN control module [201] recorded the pressure trace. In addition to this, DRIVVEN allowed to set and record all parameters related to injection, as well as the turbine configuration and the opening degree of the swirl and EGR valves. A picture of one of the swirl valves can be seen in Fig. 4.2. Signals of slow-response sensors were monitored and collected at a sample frequency of $100 \mathrm{~Hz}$ with a separated data acquisition system. In particular, temperatures of gases were measured with K-type thermocouples. Temperatures of liquids were measured with PT100 resistance temperature detectors. The complex installation to measure temperatures in the engine structure is described in detail in the section below. It featured an independent recording system. Four conditioning circuits controlled by PID were used to regulate coolant, oil, fuel and intake air temperature. The conditioning system is depicted in Fig. 4.3.

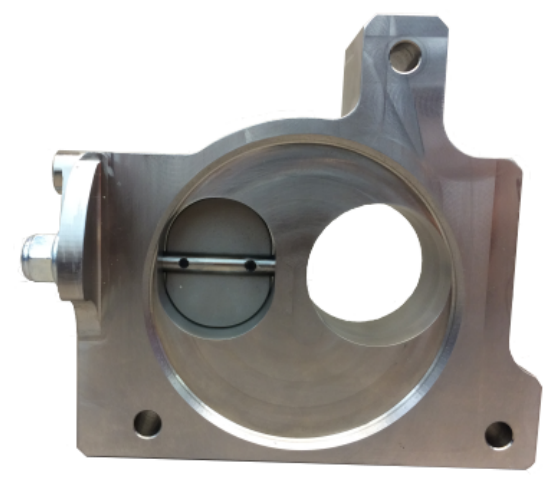

Figure 4.2: Photograph of a swirl valve of Engine 1 [144].

\section{Thermocouple installation}

Considering goal number 6 of the proposed method, it is clear that spatial resolution was a requirement. That dictated the need of a relatively high number of sensors. In Section 2.2.2, different options for temperature measurement in internal combustion engines were discussed. Among the less invasive techniques, fast-response sensors can provide high temporal resolution and some spatial resolution. However, it was shown that measurement with fast sensors is based on some assumptions that make the technique not fully reliable yet. On the contrary, slow-response sensors are free of questioning due to the technique's simplicity. They are un- 


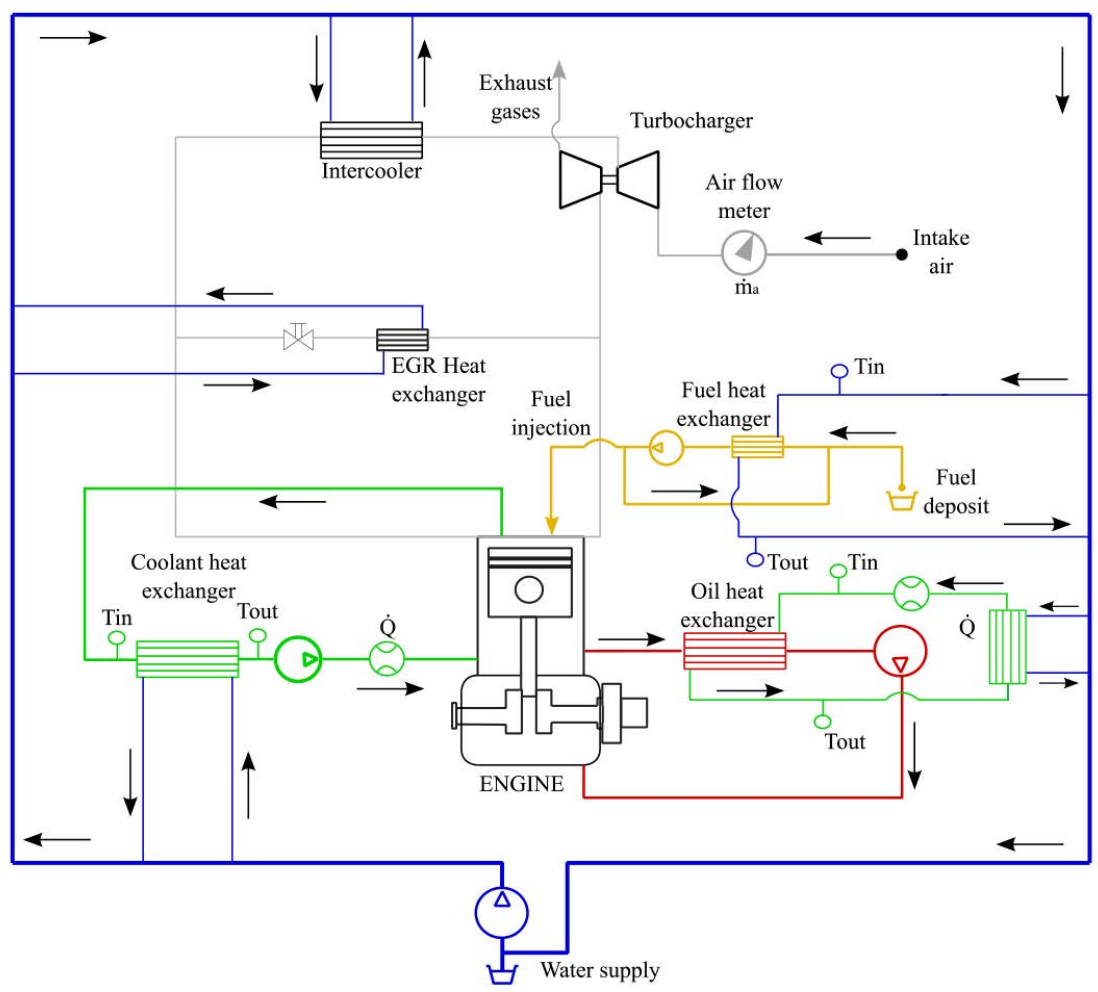

Figure 4.3: Temperature conditioning of fluid flows in Engine 1. 
able to provide sampling within an engine cycle but it is known that, in the solid, temperature changes become quasi-steady within a very short distance from the surface [133]. In summary, if one is willing to sacrifice temporal resolution, slow-response sensors are a suitable solution that have benefits in terms of reliability and can generally provide a higher spatial resolution than fast-response sensors because sensors are smaller. Moreover, sensor placement is more flexible for the same reason. For the particular application of this study, slow-response sensors were suitable to measure the steady-state response of wall temperatures to changes in engine parameters. Slow sensors were also useful for studying long transients, like warm-up or cool-down processes of the engine structure. The present section, however, is focused on steady-state analysis. Transient behavior is tackled later, in Section 4.5.

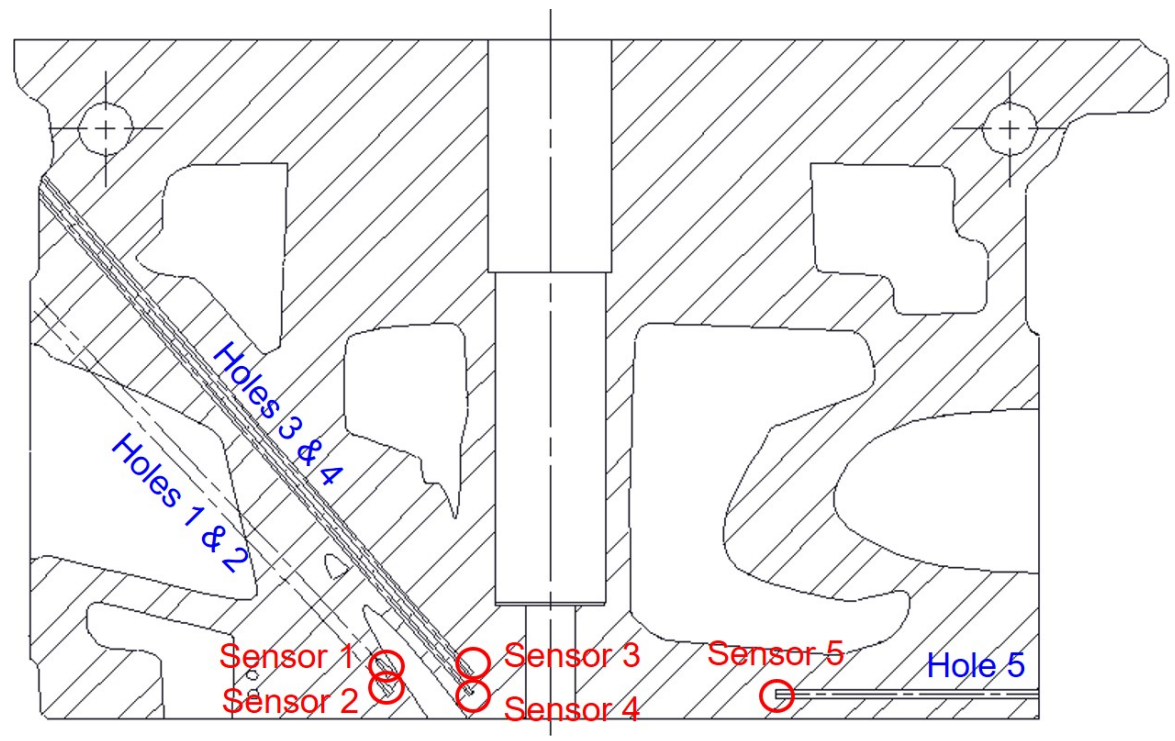

Figure 4.4: Drill holes and thermocouples in a section of the cylinder-head.

Embedded into the structure of Engine 1, there was the installation of slow-response thermocouples. In Fig. 4.4, some drill holes are depicted. Temperature sensors were $88 \mathrm{~K}$-type thermocouples located in the vicinity of the cylinder walls. In the cylinder-head, 54 thermocouples were installed. In the cylinder block, there were 34 thermocouples. The piston was not instrumented. As reported in Section 2.2.2, data transmission from sensors in the piston to a recorder is problematic due to the piston 
movement. Because of this, the reviewed techniques to measure piston temperature required a major overhaul of the engine structure. For this study, it was preferred to maintain engine integrity. This allowed to make tests in any operating condition, even at high engine speed and full load. In addition, the representativity of a production engine was conserved. In previous studies [183] [166], it was found that temperature prediction in the piston was as good as those in the cylinder-head and in the liner. In the present research it was assumed that if cylinder-head and liner predictions were accurate, piston predictions would be validated as well.

Location of the thermocouples was chosen to collect data from the closest areas to combustion chamber surfaces. Due to the fragility of the thermocouples and the adverse conditions found in the cylinder of a fired engine, the thermocouples were installed not flush to surface but within a short distance, embedded in the material. This allowed to measure temperature without exposing the sensors, thus ensuring high durability. This measure demonstrated to be effective, since no thermocouple stopped working after all experimental works with this engine.

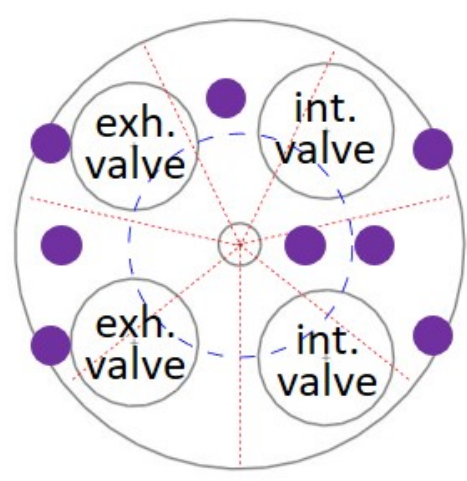

(a) Depth: $4 \mathrm{~mm}$

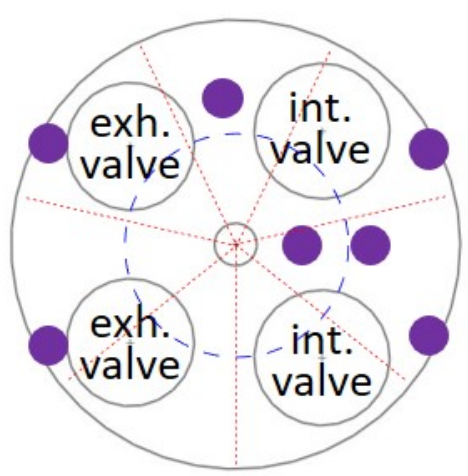

(b) Depth: $7 \mathrm{~mm}$

Figure 4.5: Location of thermocouples in the firedeck of cylinder 1 .

In the cylinder-head under study, there were 54 thermocouples distributed on two planes perpendicular to the cylinder axis. First section was located $4 \mathrm{~mm}$ above the firedeck surface and second section was at $7 \mathrm{~mm}$ above the surface. Location of thermocouples is indicated in Figs. 4.5 to 4.8 .

It can be seen that the distribution of thermocouples was very similar 


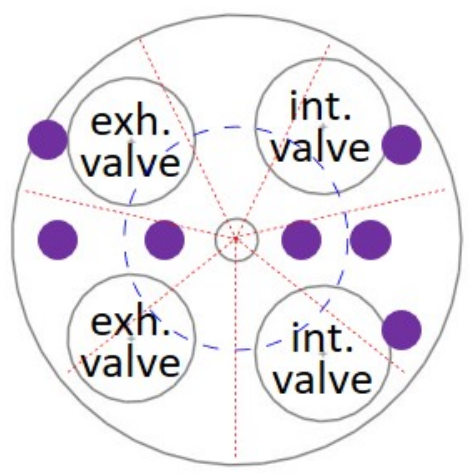

(a) Depth: $4 \mathrm{~mm}$

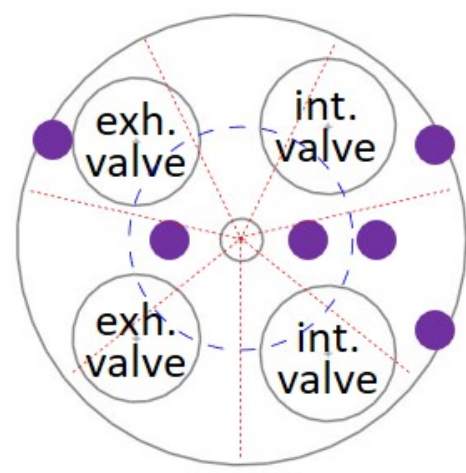

(b) Depth: $7 \mathrm{~mm}$

Figure 4.6: Location of thermocouples in the firedeck of cylinder 2.

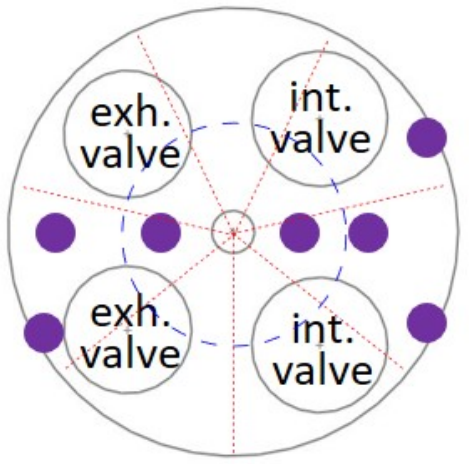

(a) Depth: $4 \mathrm{~mm}$

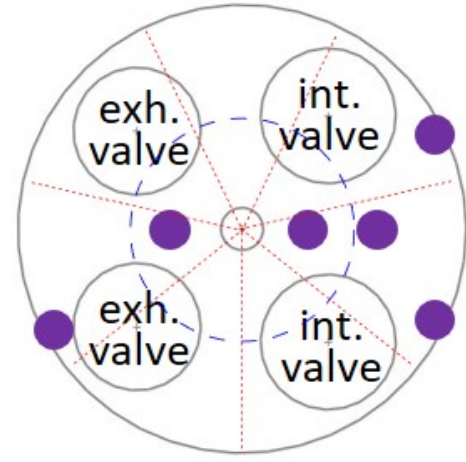

(b) Depth: $7 \mathrm{~mm}$

Figure 4.7: Location of thermocouples in the firedeck of cylinder 3. 


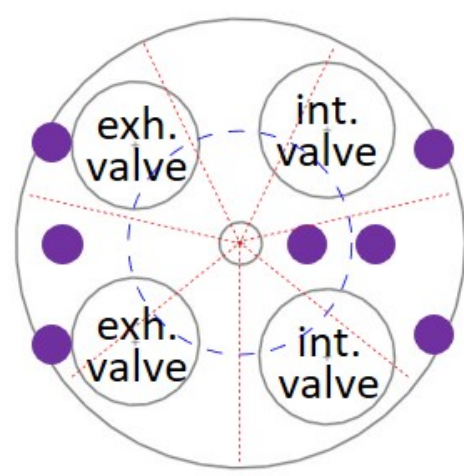

(a) Depth: $4 \mathrm{~mm}$

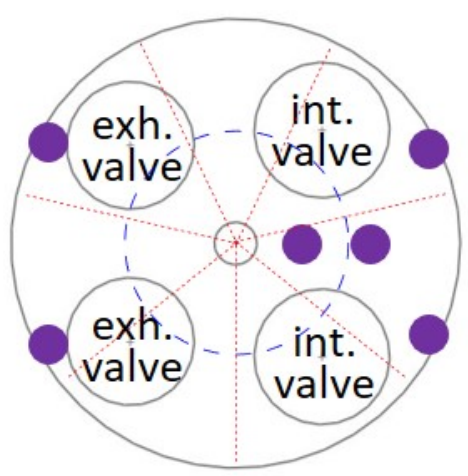

(b) Depth: $7 \mathrm{~mm}$

Figure 4.8: Location of thermocouples in the firedeck of cylinder 4.

on both planes, except for one thermocouple on the exhaust side. Piston bowl location and fuel spray estimated directions are also indicated in the figures, with dashed lines.

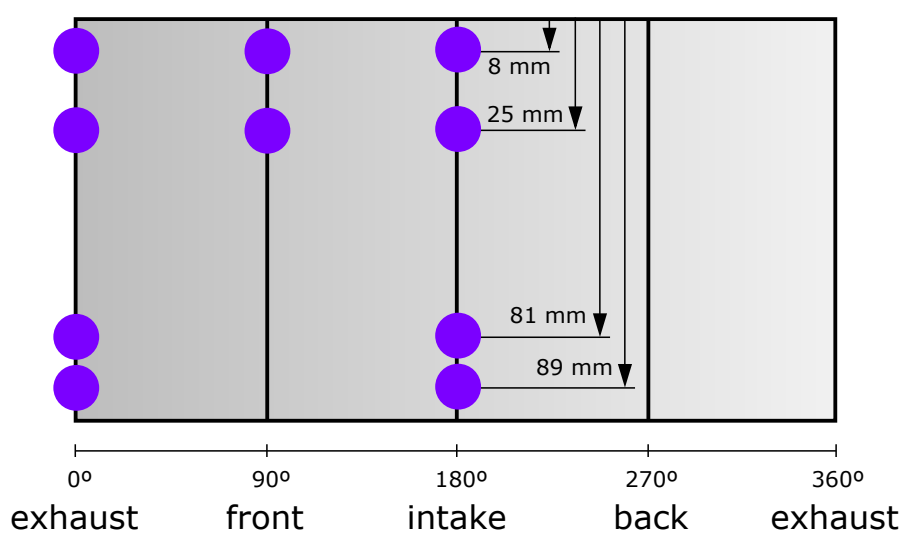

Figure 4.9: Location of thermocouples in the liner of cylinder 1.

Thermocouples in the cylinder block were installed $1.3 \mathrm{~mm}$ deep from the liner surface. They were located at different positions along the piston stroke. In each cylinder there were four vertical lines of thermocouples separated $90^{\circ}$ from each other. Each line had a different number of thermocouples. There were more thermocouples close to the cylinder top than near the bottom. The upper area was considered more interesting be- 


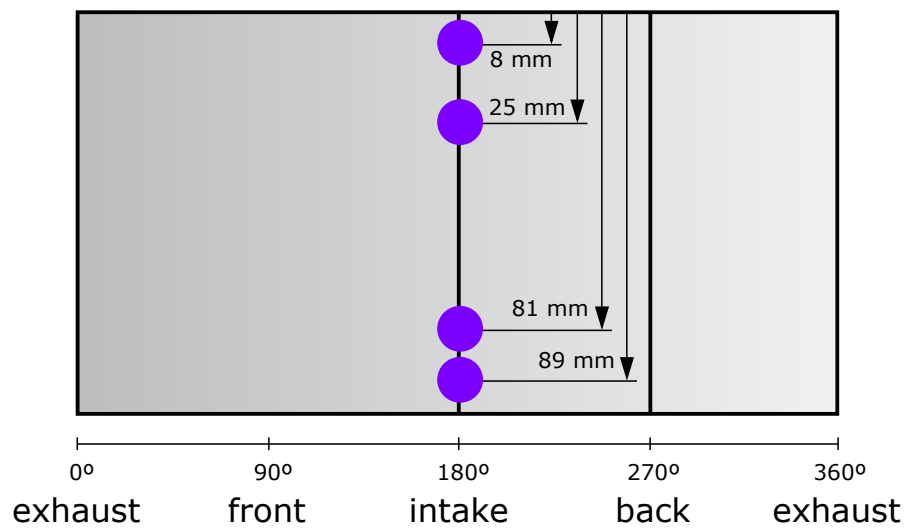

Figure 4.10: Location of thermocouples in the liner of cylinder 2.

cause, due to piston motion, it had a longer exposure time to in-cylinder gas. Along the vertical direction, thermocouples were installed on four planes at $8,25,81$ and $89 \mathrm{~mm}$ below the top of the block respectively. Figs. 4.9 to 4.12 show the location of thermocouples in the liner of the different cylinders. Thermocouples are marked as a purple dot. On the lower axis, the angle of the cylinder circumference is indicated, starting from the side of the engine where the exhaust system is. The vertical direction is cylinder height.

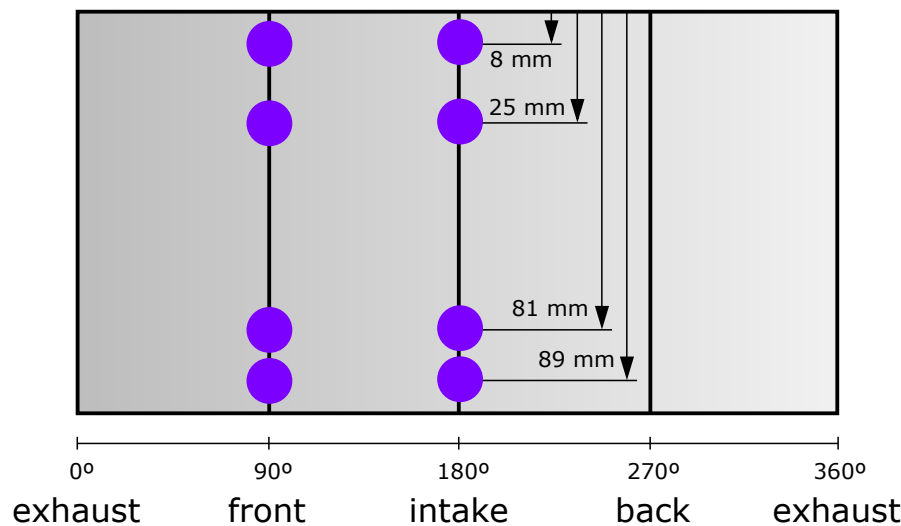

Figure 4.11: Location of thermocouples in the liner of cylinder 3.

In order to reduce temperature measurement errors with thermocouples, the following precautions were taken [202]: 


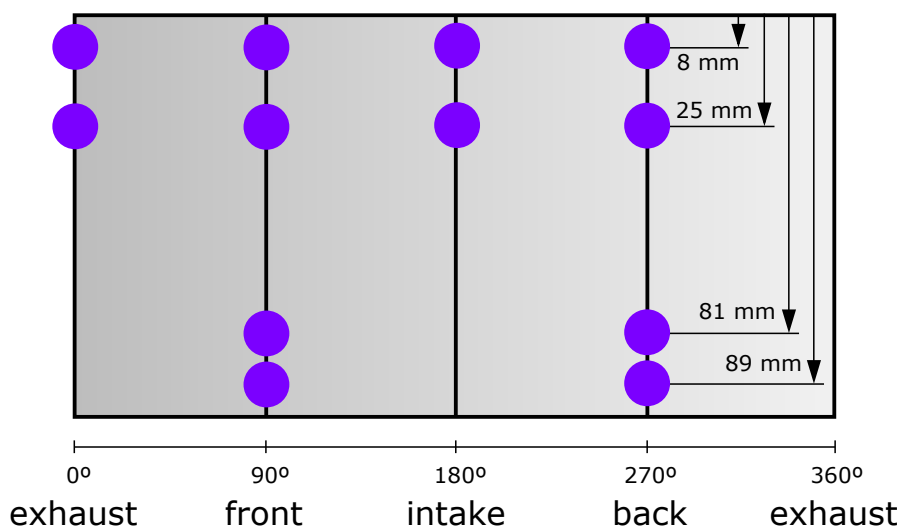

Figure 4.12: Location of thermocouples in the liner of cylinder 4.

- The inserted length of the thermocouple into the material was, at least, 5 times the thermocouple diameter.

- The shape of the bottom of the hole matched the shape of the end of the sensor and the sensor was pressed fully to the bottom.

Thermocouple signals were received by two data-loggers with 60 channels each and then sent to be recorded on a computer. After conducting the test campaign described below, the average standard deviation of thermocouple measurements was $0.35^{\circ} \mathrm{C}$. The median of the standard deviation was $0.29^{\circ} \mathrm{C}$. The maximum observed standard deviation was $0.83^{\circ} \mathrm{C}$ in one thermocouple of the liner while measuring the test point at the highest engine speed and load.

\section{Test campaign}

Step 2 of the proposed method consisted in a test campaign. The test campaign had to assess predictions of the Nodal Model under different conditions of engine speed, load and swirl motion. Engine speed and load were investigated in different operating points. Each operating point was a combination of engine speed and load. Besides, as pointed out by various authors cited in the literature survey of Section 2.2.4, swirl should play an important role in in-cylinder heat transfer. To investigate the effects of swirl, the test plan had to include sweeps of swirl ratio with the four levels of swirl allowed by the engine. To optimize the testing effort and keep the amount of data manageable, six operating points were selected in which swirl sweeps were performed. The operating points, or 
keypoints, were intended to cover varied operating conditions which belong to homologation cycles. They are shown in Fig. 4.13 and summarized in Table 4.3. Each operating point had a nickname formed by engine speed and brake mean effective pressure (BMEP) separated by the symbol @. The keypoints covered three levels of engine speed, different levels of load from very low to full load and four levels of swirl. In total, 24 operating conditions in steady state.

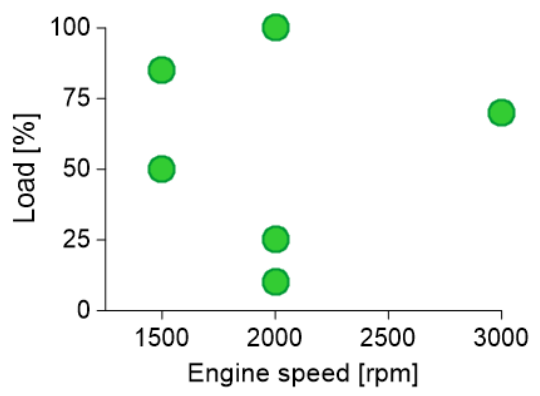

Figure 4.13: Operating points (keypoints).

\begin{tabular}{|c|c|c|c|c|c|c|}
\hline Nickname & $1500 @ 8$ & 1500@14 & $2000 @ 2$ & $2000 @ 5$ & $2000 @ 20$ & 3000@14 \\
\hline Engine speed [rpm] & 1500 & 1500 & 2000 & 2000 & 2000 & 3000 \\
\hline BMEP [bar] & 8 & 14 & 2 & 5 & 20 & 14 \\
\hline Torque $[\mathrm{N} \mathrm{m}]$ & 123 & 215 & 31 & 77 & 318 & 215 \\
\hline Load $[\%]$ & 50 & 85 & 10 & 25 & 100 & 70 \\
\hline Swirl ratio at IVC [-] & & & $1.4, \quad 2$, & 2.5 & & \\
\hline
\end{tabular}

Table 4.3: Test plan.

The test procedure was devised to keep constant the amount of fuel injected in the combustion chamber and conditions at intake valve closure in each swirl sweep. This way, the quantity of input energy was maintained constant under the four different swirl conditions. Thus, a rigorous back-to-back comparison among all measurements was made possible. Moreover, some settings were the same in all tests to facilitate direct comparison. They are summarized in the following list and their values can be consulted in Table 4.4 .

- Temperatures of intake gas, coolant, oil and fuel. 
- CA50: crank angle in which 50\% of fuel mass has burned.

- Fuel mass injected by pilot injections.

- Dwell among injections.

The injection profile was formed by two pilots and one main injection. Fuel mass injected by pilot injections and dwell among injections were the same for all tests. On the contrary, fuel mass injected by the main injection was adjusted in each test to reach the target torque, and injection timing was adjusted to keep the target CA50.

\begin{tabular}{lc}
\hline \multicolumn{3}{c}{ Injection/combustion settings } \\
CA50 [degrees after TDC] & 13 \\
Dwell: $1^{\text {st }}$ to $2^{\text {nd }}$ pilot $[\mu \mathrm{s}]$ & 800 \\
Dwell: $2^{\text {nd }}$ pilot to main $[\mu \mathrm{s}]$ & 800 \\
Fuel mass: $1^{\text {st }}$ pilot $[\mathrm{mg} / \mathrm{cycle}]$ & 1.5 \\
Fuel mass: $2^{\text {nd }}$ pilot $[\mathrm{mg} / \mathrm{cycle}]$ & 1.5 \\
\hline \multicolumn{2}{c}{ Fluid temperatures } \\
Intake temperature $\left[{ }^{\circ} \mathrm{C}\right]$ \\
Coolant outlet temperature $\left[{ }^{\circ} \mathrm{C}\right]$ & 87 \\
Oil outlet temperature $\left[{ }^{\circ} \mathrm{C}\right]$ & 95 \\
Fuel inlet temperature $\left[{ }^{\circ} \mathrm{C}\right]$ & 34 \\
\hline
\end{tabular}

Table 4.4: Common settings for all operating points.

Rail pressure changed depending on the operating point and was kept constant during each corresponding swirl sweep. Values are summarized in Table 4.5 for the different keypoints. In the same table, limits of in-cylinder pressure to ensure safe operation are shown. Both peak pressure and slope of the pressure curve had to be controlled. Those pressure restraints were defined by the engine manufacturer with the aim of guaranteeing mechanical integrity.

Finally, torque changed in every test, in response to the variations of swirl motion and other parameters.

After engine start up, various magnitudes were set for all tests in an 
Heat transfer in the engine block 4

\begin{tabular}{lcccccc}
\hline Keypoint & $1500 @ 8$ & $1500 @ 14$ & $2000 @ 2$ & $2000 @ 5$ & $2000 @ 20$ & $3000 @ 14$ \\
\hline Rail pressure [bar] & 850 & 1020 & 730 & 970 & 1230 & 1440 \\
Peak Firing Pressure [bar] & 130 & 130 & 160 & 160 & 160 & 160 \\
Pressure Rise Rate [bar/deg] & & \multicolumn{7}{c}{10} & & \\
\hline
\end{tabular}

Table 4.5: Pressure settings for each operating point.

operating point, as a first step.

- Engine speed.

- Rail pressure.

- Fuel mass injected by pilot injections.

- Dwell among injections.

First test was done under conditions of maximum swirl ratio $(\mathrm{SR}=3)$. When providing maximum swirl, the swirl valve was in the position that obstructed flow the most. That is to say, for maximum swirl, pressure loss in the intake ports was the highest. Accordingly, the configuration of the variable geometry turbine was adjusted to maximize intake pressure. Magnitudes that changed during the test, as injection timing and duration of main injection, were given preliminary values.

Next, the engine was warmed up until steady state was reached. Temperatures of coolant, oil and structure were monitored to check that temperature variation was lower than $0.5^{\circ} \mathrm{C}$ per minute. PID controllers regulated the water flows through intercooler, coolant cooler and oil cooler to maintain temperatures of intake gas, coolant and oil at the values shown in Table 4.4. Fuel was also conditioned (heated or cooled) before entering the engine. The temperature settings were chosen because they are found in typical engines during normal operation. Stabilization time was between 20 and 40 minutes depending on the measured point. During stabilization, injection duration and timing were gross tuned to get torque and CA50, respectively, closer to target values.

Once steady state was reached, the duration of the main injection was fine tuned to obtain nominal torque and BMEP. Injection timing was also tuned to keep CA50 at 13 degrees after top dead center. When all parameters were stable and nominal, mass flow rates of fuel and intake air were noted down, as they had to be kept during the whole swirl sweep. Afterwards, all relevant magnitudes were recorded, including temperatures of the engine structure. Three repetitions of the test were measured. In each repetition, thermocouple signals were scanned every four seconds for 
INITIAL TEST

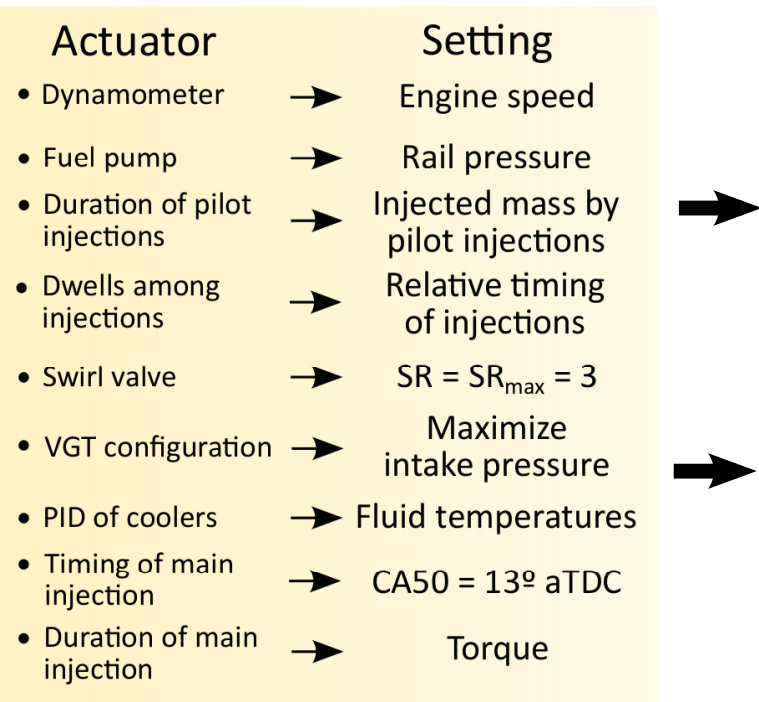

\section{Measurement}

Reference values

Intake mass flow

Fuel consumption

Figure 4.14: Procedure to measure the test at maximum swirl ratio.

one minute. Response time of thermocouples was below 2 seconds. Scans were then averaged for each thermocouple and repetition. Thereupon, the test of maximum swirl ratio was completed. A schema of the procedure to measure the first test of the swirl sweep, the one with the highest swirl ratio, is shown in Fig. 4.14.

Then, the swirl valve was operated to reduce swirl ratio to the next level $(\mathrm{SR}=2.5)$. When the swirl valve was operated, obstruction of the intake flow in the helical port changed. As explained previously, when swirl ratio was reduced, pressure loss in the valve decreased. Therefore, to keep the same intake mass flow when decreasing the swirl ratio, the turbine configuration had to be adjusted in order to provide lower intake pressure. Three parameters had to be iteratively adjusted to reach the reference values of intake mass flow rate, fuel consumption and CA50, as they had influence on all of them.

1. Turbine configuration.

2. Duration of main injection.

3. Timing of main injection.

Once all magnitudes converged, measurements were made. Among the measured variables was the new torque value. Temperatures of engine structure were measured as explained in the description of the first test. 
The process explained for the second swirl ratio was repeated for the two remaining swirl ratios. The whole procedure was repeated in all keypoints. Altogether, 54 measurements of 18 different operating conditions were collected. The procedure is depicted in Fig. 4.15.

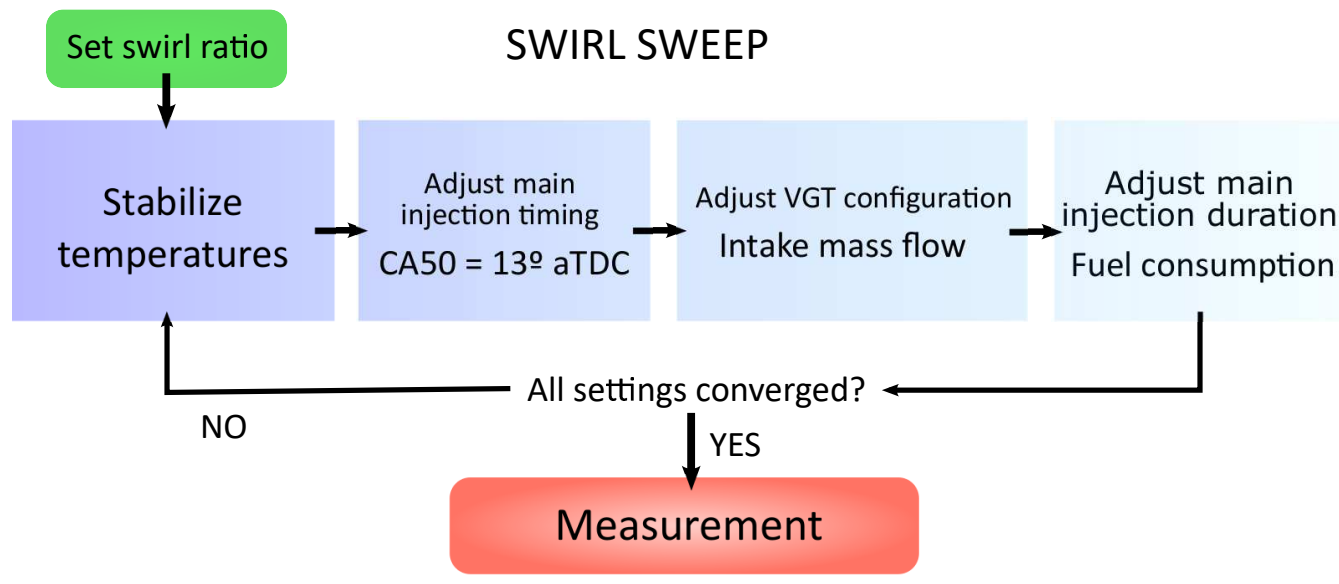

Figure 4.15: Procedure to measure the rest of the swirl sweep.

In addition to the research tests, a set of motoring tests was conducted to calibrate the cylinder model in CALMEC. Engine speed was varied between 1000 and $4500 \mathrm{rpm}$, every $500 \mathrm{rpm}$. All operating points were tested at two swirl levels, minimum $(\mathrm{SR}=1.4)$ and maximum $(\mathrm{SR}=3)$. Variables were measured as in the fired tests. In total, data of 14 operating conditions was obtained.

\section{Model calibration}

Apart from the thermocouple installation described above, the engine had additional instrumentation. Part of this instrumentation was useful to monitor engine operation, e.g. engine temperatures. However, most instrumentation was present to measure variables that were inputs of CALMEC. Examples of those measurements are the following:

- In-cylinder pressure trace.

- Crank angle degree.

- Fuel consumption.

- Coolant temperatures.

- Oil temperatures.

- Intake pressure and temperature. 
- Exhaust pressure and temperature.

- Blow-by rate.

Part of the data was used as a source of boundary conditions for CALMEC. Another part was used to calibrate models. The rest of the data was employed for later analyses. As explained in Sections 3.2 and 3.3, a very influential element that needs calibration is the modified Woschni correlation used to calculate heat rejection to cylinder walls, Eq. 3.3. It is shown again here due to its relevance. It was mentioned that the correlation includes constants that multiply mean piston speed $\left(C_{W 1}\right)$ and swirl velocity $\left(C_{W 2}\right)$ to adjust the effect of those parameters. Moreover, the calibrated values and the calibration process were discussed. Now, the calibration process can be related to the instrumentation and the test plan.

$$
\begin{aligned}
& h(\alpha)=0.012 D_{c y l}^{-0.2} p(\alpha)^{0.8} T(\alpha)^{-0.55} . \\
& \cdot\left[C_{W 1} c_{m}+C_{W 2} c_{u}(\alpha)+C_{2} \frac{V_{d} T_{I V C}}{p_{I V C} V_{I V C}}\left(p(\alpha)-p_{0}(\alpha)\right)\right]^{0.8}
\end{aligned}
$$

Motoring tests were used to adjust the cycle uncertainties, as described in Section 3.2, exploiting the fact that heat release from fuel was nonexistent. This procedure yielded the value of $C_{W 1}=2.17$ for the closed cycle. Since the motoring tests were done with two different swirl ratios, $C_{W 2}$ could be preliminary adjusted. In addition, in a closed volume and in absence of heat release, it is possible to apply the first law of thermodynamics to estimate heat losses to the cylinder walls. The method and its limitations were commented in Section 2.2.2. The equation to be used was Eq. 2.32. These curves of estimated heat flux to the cylinder walls were a result from CALMEC.

Later, the fired tests of the keypoints could be used to refine the calibration. The tuning constant of the combustion velocity term in Woschni correlation, $C_{2}$, could be adjusted to the value of $1.05 \times 10^{-3}$. The finetuned value of $C_{W 2}$ was 0.2 . To satisfy the global energy balance, the integrated curve of heat release had to match the energy of the fuel consumed in one engine cycle, which had been experimentally measured. During the open part of the cycle, constants $C_{W 1}$ and $C_{W 2}$ were set to 3.09 and 0.21 , respectively. These values are proportional to those proposed by Woschni $\left(C_{W 1}=6.18, C_{W 2}=0.417\right.$, see Section 3.3). A proportionality factor of 0.5 was selected to adjust the global energy balance during the open cycle. In particular, the fact that in-cylinder heat losses are dissipated into 
coolant and oil flows was exploited. Constants could be calibrated because all processes rejecting heat to coolant and oil were modeled and the results could be compared to experimental values of heat rejection to coolant and oil that had been measured. In the end, the global energy flows of mechanical power, chemical energy, heat and flow enthalpy were balanced for all tests and they were in agreement with experimental measurements. Beside the adjustment of the global energy balance, a thermocouple installation may allow to compare measured and predicted wall temperatures, which can be useful for calibration, as shown in Fig. 4.16. In this study, the later method was not employed because the thermocouple measurements were reserved to evaluate the Nodal Model predictions.

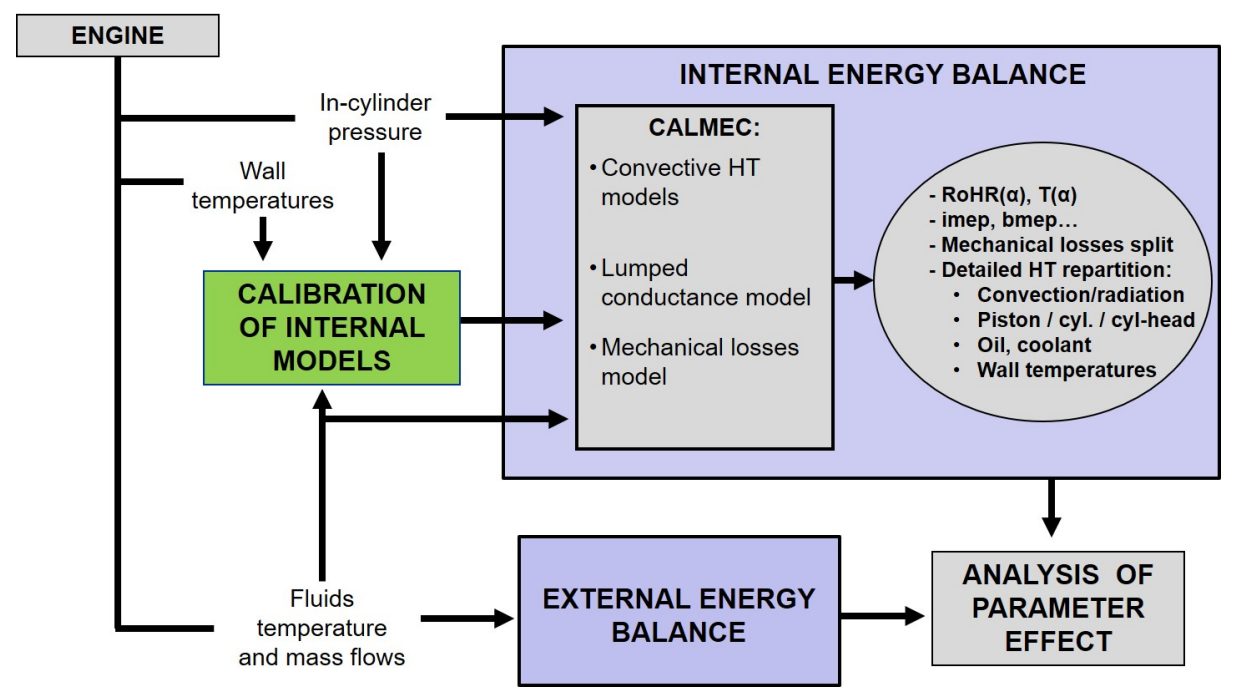

Figure 4.16: Workflow for studies with CALMEC.

\section{Adaptation of the engine heat transfer model}

The Nodal Model under evaluation was the one integrated into CALMEC. That permitted to reproduce the test conditions in the cylinder, which were boundary conditions for the Nodal Model. To replicate experimental conditions even better, model geometry was adapted to represent the real geometry obtained from engine drawings. In the cylinder-head, the configuration of nodes was rearranged in a way that made the geometrical center of the node match the thermocouple measurement location. Node shapes had to be modified and new nodes were added. The original 


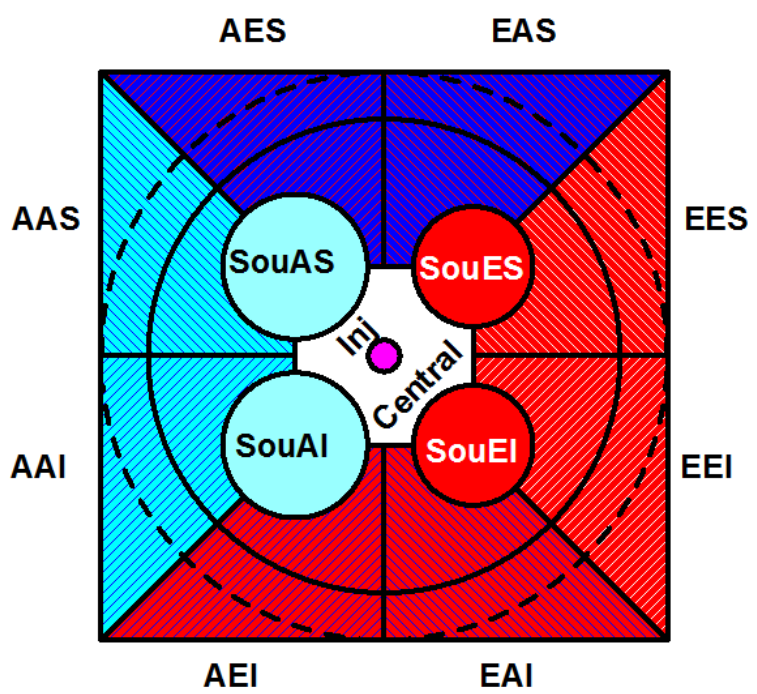

(a) Original configuration

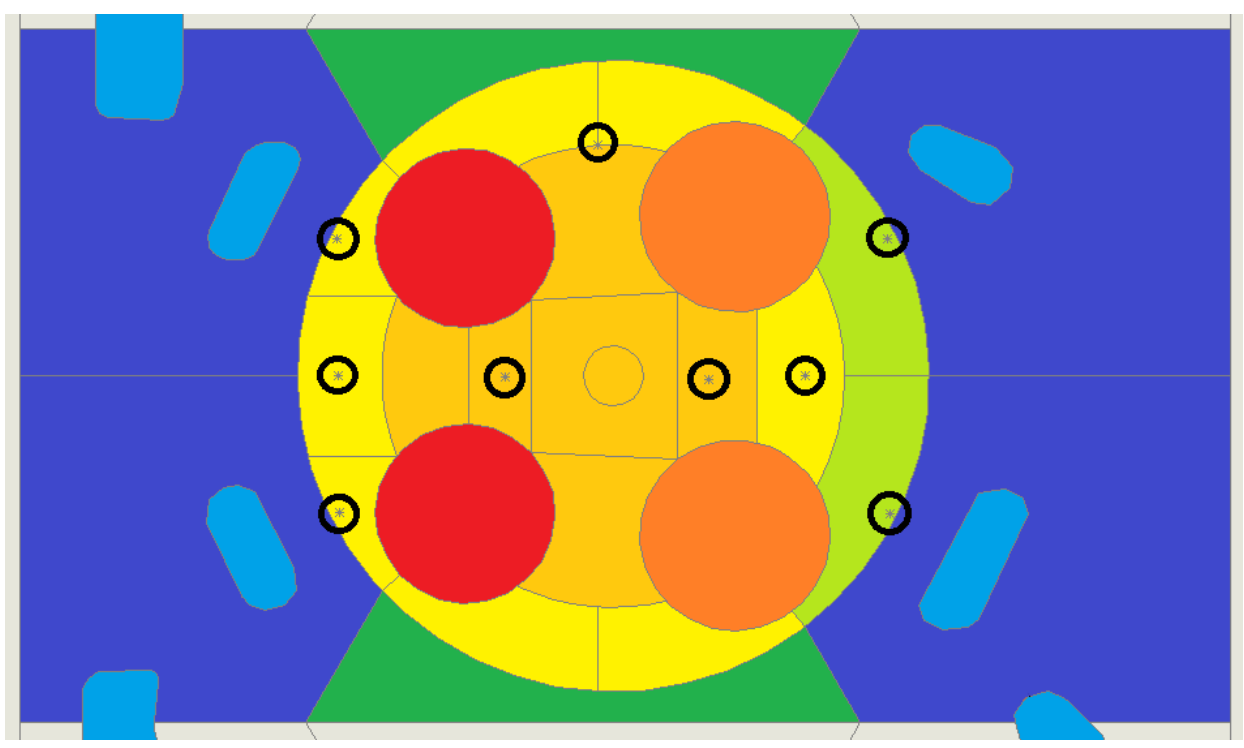

(b) Adapted configuration for Engine 1

Figure 4.17: Configuration of nodes in the firedeck. 


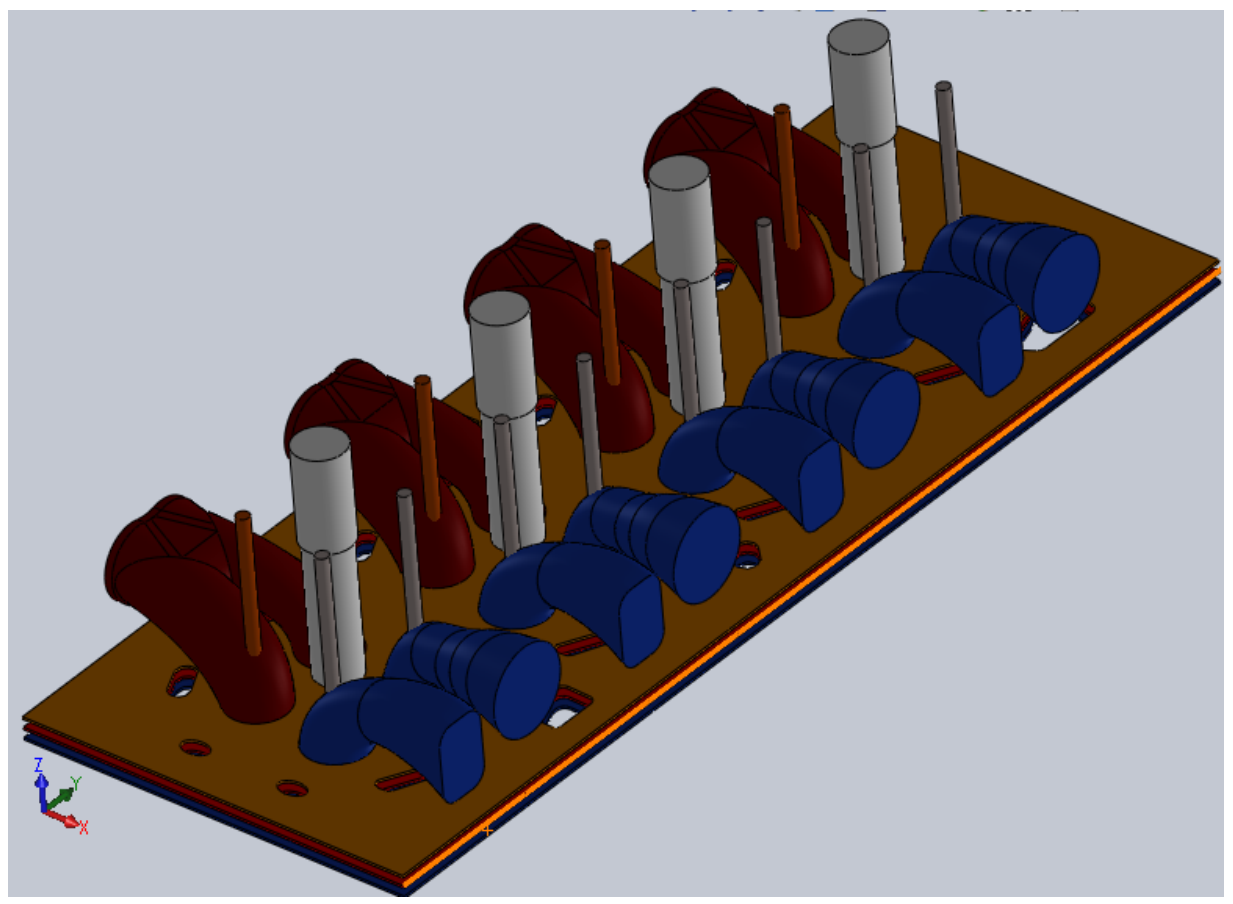

Figure 4.18: View from above of some elements of the new cylinder-head model 
and the new configurations of nodes in the firedeck can be compared Fig. 4.17. In that figure, the location of thermocouples is marked with a black circle. 3D CAD models of the cylinder-head were built using data from the engine drawings. Some elements can be seen from above in Fig. 4.18. Blue elements on the right side are intake ports. Red elements on the left side are exhaust ports. Stems of exhaust valves are visible in orange color and stems of intake valves in dark grey. Injectors are colored in light grey. The three planes at the bottom contained the firedeck nodes. Each of the three levels of nodes that formed the firedeck had a different thickness, as shown in Fig. 4.19. It must be noted that the nodes of the two upper levels had their geometrical centers at the same distance from the cylinder surface as the thermocouples: 4 and $7 \mathrm{~mm}$. The firedeck was crossed by coolant galleries, as was seen in Fig. 4.17(b). A lateral view of the cylinder-head is shown in Fig. 4.20. Intake ports are colored in dark blue, exhaust ports in dark red, valves in orange and the injector in green. Lighter blue elements represent cooling galleries and the surrounding areas. Light grey parts are oil galleries and the darker gray piece at the bottom is the firedeck. The solid material around all these elements and above the firedeck is not represented in the figure. The geometry of this material's nodes around the visible elements could be obtained by doing a negative of the model. Having the 3D CAD model of the cylinder head was very useful to measure node volumes, as well as contact areas and distances among nodes. As mentioned in Section 2.2.3, that data is required for defining the conductances and capacitances of the thermal network.

The 102 nodes in the new model of the cylinder-head could be classified as follows:

- 66 nodes formed the firedeck, which was divided into three levels. The total thickness was $8 \mathrm{~mm}$. All thermocouples coresponded with nodes in this area.

- For each valve there were two nodes for the plug and one node for the stem. In total, 12 nodes were used in the four-valve cylinder-head.

- One node for each of the four ports represented the walls in contact with intake or exhaust gas.

- Four nodes made up the injector.

- The solid bulk was represented by 8 nodes on each side of the cylinder-head (intake and exhaust). They were in contact with the 


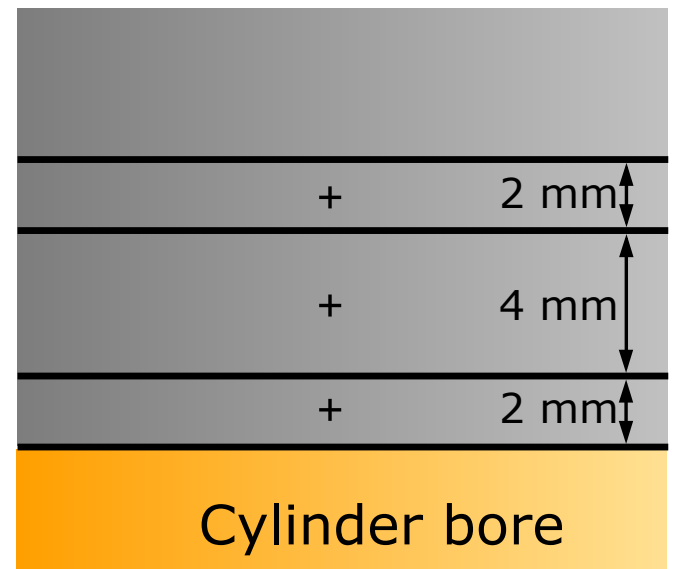

Figure 4.19: Thickness of firedeck nodes in the adapted model

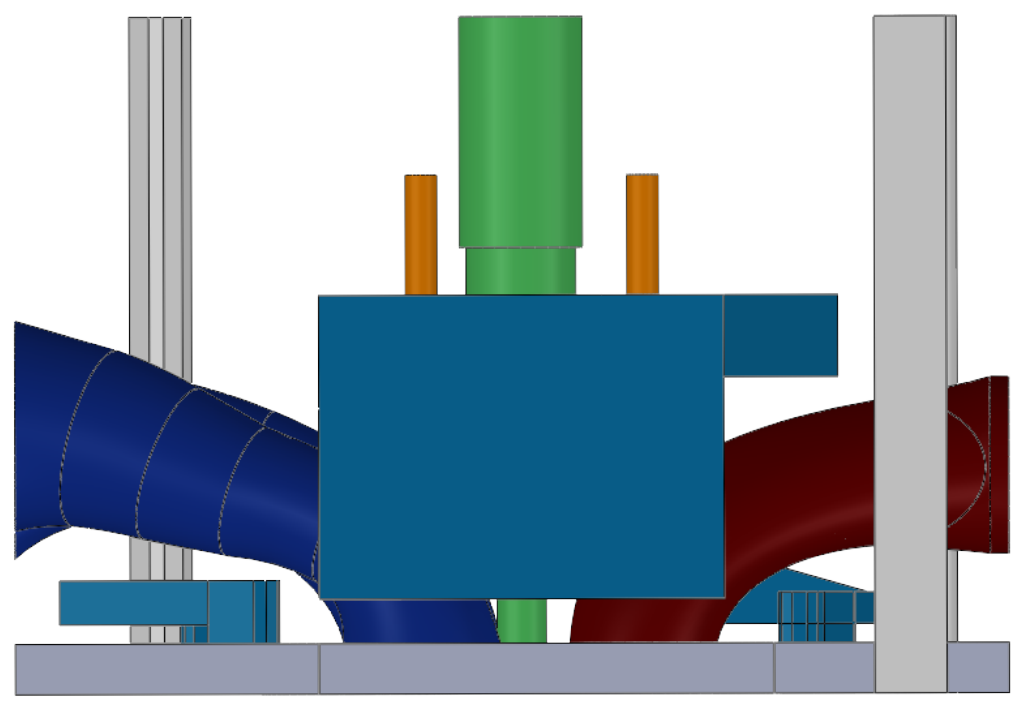

Figure 4.20: Lateral view of some elements in the new cylinder-head model 
coolant galleries that were shown in the Fig. 4.20. All coolant galleries were represented by a single node that acted as a boundary condition.

The liner has a much simpler geometry. The original geometry was kept, but an examination to find the optimal number of nodes was done. It was mentioned in Section 3.3 that the level of discretization in the liner model could be specified by the user. In the liner study, the automatic geometry was generated for different number of axial nodes. Number of radial nodes was kept in two and number of circumferential nodes in six. In Fig. 4.21 the locations of the thermocouples were superimposed to the nodal distribution. It was found that, in order to have one separated node for each of the two thermocouples at the bottom, it was necessary to use 9 axial nodes. Another option that matched the geometrical centers of the nodes with the thermocouples was to use 5 axial nodes. In that case, the two thermocouples at the bottom corresponded to the same node but they were near to the center. It had to be considered that twelve nodes had to be added for each additional axial level: six for the internal ring of nodes and six for the external ring, taking into account that the number of nodes in radial direction was two. Under those terms, using 9 axial nodes instead of 5 implied to add 48 new nodes to the system. Finally, the option of 5 axial nodes was considered accurate enough and was chosen, avoiding the penalty in computational cost and complexity of results.

Finally, the piston was modeled in 3D using the information from the engine drawings. The number of nodes, 10, was maintained, as shown in Fig 4.22. Geometry, notwithstanding, was altered to resemble the real piston.

For this engine, the geometry data of the nodes and the thermal conductances of the network were calculated manually. To achieve this, the 3D CAD models were instrumental. Location of the geometrical centers of the different nodes was necessary to determine the distances among nodes. Contact areas were obtained from the 3D geometry as well. With that data, conduction conductances were manually calculated. The data was then arranged in the format expected by the model. A function to read the data automatically and add it to the model was implemented. Both geometry data and conduction conductances were imported to the model. Conduction conductances were used directly by the model. The geometry data was required by the model to internally calculate the contact areas between solids and fluids, which were later used to obtain the 

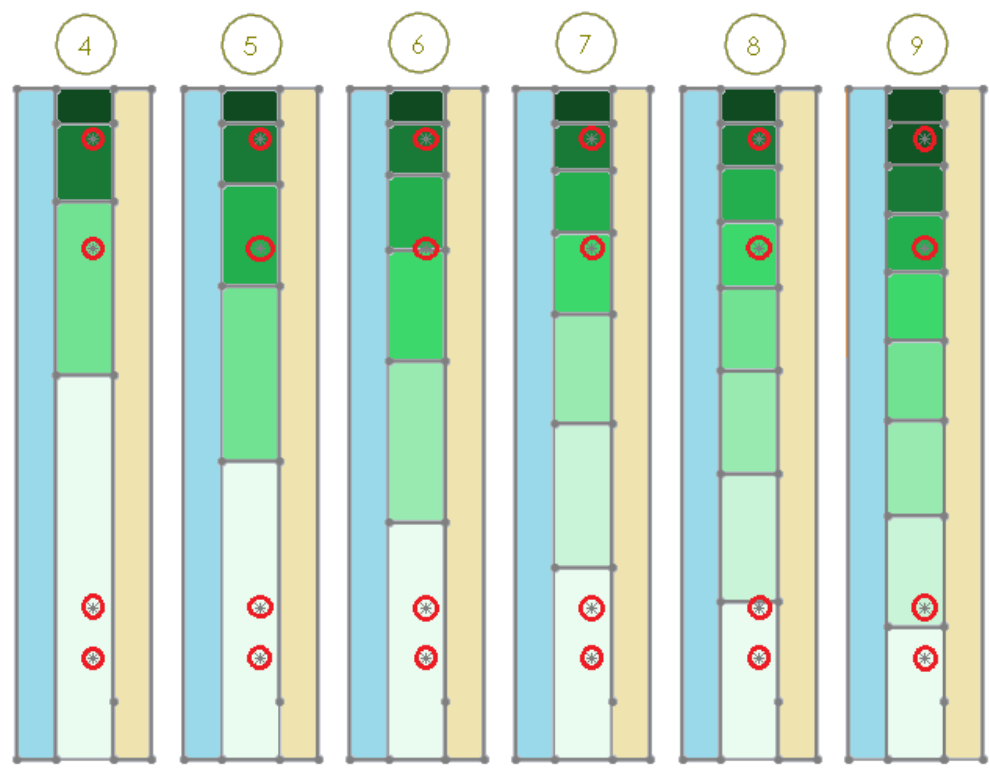

Figure 4.21: Study of number of axial nodes in the liner.

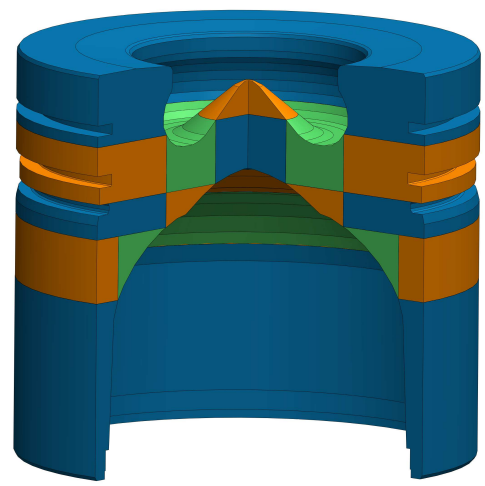

Figure 4.22: View of the piston's 3D reconstruction. 
convection conductances through Eq. 2.37.

\section{Obtaining mean wall temperatures}

The first analysis of the evaluation was focused on mean wall temperatures. This meant that an averaging process had to be performed on the experimental measurements, which were spatially discrete. The averaging method was based on considering that localized temperatures were representative of the region around the corresponding thermocouple.

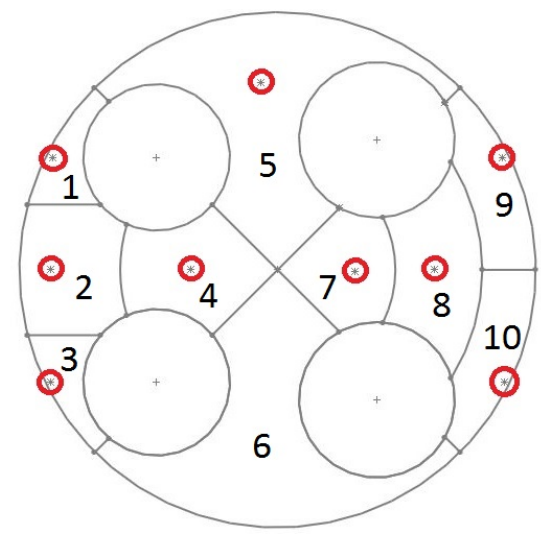

Figure 4.23: Obtaining mean wall temperature in firedeck [144].

In the cylinder-head, the averaging was done on the plane located 4 $\mathrm{mm}$ deep from the surface, the nearest one. The area division is displayed in Fig. 4.23. Thermocouple locations are marked with red circles. The method consisted in averaging the measured temperatures according to the respective weight of its area in the total area, Eq. 4.1. Valves did not have thermocouples and therefore their area was not included.

$$
\bar{T}=\frac{\sum_{i} T_{i} A_{i}}{\sum_{i} A_{i}}
$$

In Fig. 4.23, the highest possible number of thermocouples is shown, but, in reality, all firedecks had less thermocouples, as seen in Figs. 4.5 to 4.8. To deal with the sensors that were missing in areas 1, 2 and 4 of Fig. 4.23 , the following approximations were done: 


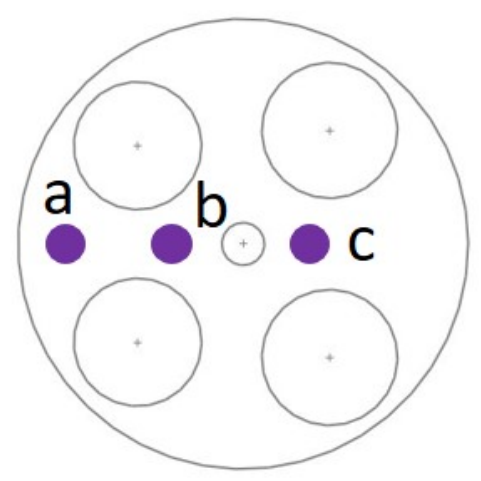

Figure 4.24: Notation used in Eq. 4.2.

- In cylinder 1 , to estimate the missing measurement in the exhaust valve bridge, the equivalence of Eq. 4.2 was assumed. Location notation is clarified in Fig. 4.24.

$$
\left(\frac{T_{b}-T_{c}}{T_{b}-T_{a}}\right)_{c y l 1}=\left(\frac{T_{b}-T_{c}}{T_{b}-T_{a}}\right)_{c y l 2}
$$

- In cylinder 2, the missing measurement next to the lower exhaust valve was replaced by the one next to the upper exhaust valve, under the symmetry assumption.

- In cylinder 3, the missing measurement next to the upper exhaust valve was replaced by the one next to the lower exhaust valve, under the symmetry assumption.

- In cylinder 4 , to estimate the missing measurement in the exhaust valve bridge, the same equation as in cylinder 1 was used, except that the equivalence was made between cylinders 3 and 4, instead of between 1 and 2 .

Temperatures of areas 5 and 6 in Fig. 4.23 underwent a different procedure because there were less sensors in those areas. First, two temperatures were calculated applying Eq. 4.1 to the quarters between intake valves and exhaust valves. Two temperatures were obtained, $\bar{T}_{\text {intake }}$ and $\bar{T}_{\text {exhaust }}$. The intake quarter was formed by areas $7,8,9$, and 10 of Fig. 4.23, while the exhaust quarter was formed by areas 1, 2, 3 and 4. Next, a factor, $\alpha$, was used to calculate temperature of areas 5 and 6 , employing equation 4.3. The criterion to choose the value of $\alpha$ was that, in the firedeck of cylinder $1, T_{5,6}$ was equal to the temperature measured by the 
only thermocouple in area 5.

$$
T_{5,6}=\bar{T}_{\text {intake }} \alpha+\bar{T}_{\text {exhaust }}(1-\alpha)
$$

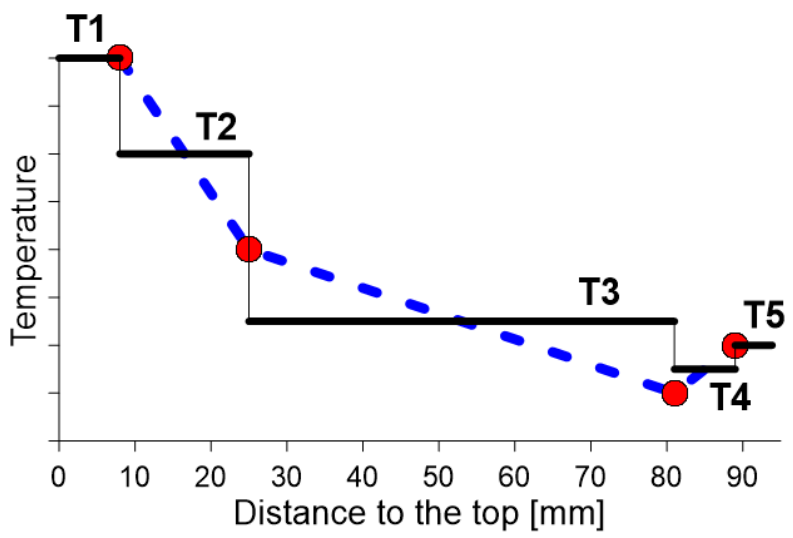

Figure 4.25: Obtaining mean wall temperature in cylinder liner.

An analogous method was employed to obtain the mean wall temperature of the cylinder liner. As thermocouples were arranged in vertical rows along the liner, linear distance instead of area was used as the basis for averaging. Fig. 4.25 illustrates the method. In the figure, the four red circles represent temperatures measured by thermocouples, as indicated by the vertical axis. The horizontal axis measures the distance along the liner from the top $(0 \mathrm{~mm})$ to the end of the piston stroke at the bottom dead center $(90.4 \mathrm{~mm})$. For the stretch between the top and the first thermocouple, located $8 \mathrm{~mm}$ below the firedeck, temperature $T_{1}$ was assumed constant and equal to the one measured by that thermocouple. Likewise, between the bottom and the thermocouple located $89 \mathrm{~mm}$ below the top, temperature $T_{5}$ measured by that thermocouple was assumed. For the other stretches, temperature was the mean of measurements at both ends of the stretch. In the example of Fig. 4.25, the resulting temperatures are marked with a thick black line and numbered. The different temperatures were averaged according to the length of the corresponding stretches, using Eq. 4.4. An average temperature, $\bar{T}_{\text {row }}$, was obtained for each row. Lastly, the average temperatures of the four rows were directly averaged to obtain the final mean wall temperature of the cylinder liner.

$$
\bar{T}_{\text {row }}=\frac{T_{1} \cdot 8+T_{2} \cdot 17+T_{3} \cdot 56+T_{4} \cdot 8+T_{5} \cdot 1.4}{90.4}
$$


In the liner, as in the firedeck, the number of thermocouples changed in each cylinder. Again, some approximations were done to make the averaging possible.

- In cylinder 1, the data of the row on the 'back' side (see Fig. 4.9) was replaced by the data of the 'exhaust' side. This was done after an empirical examination of similarities among the measurements at different locations in all cylinders. Moreover, two thermocouples on the 'front' side, at 81 and $89 \mathrm{~mm}$ below the top, were missing. To determine them, Eq. 4.5 was applied. In the equation, index 25 refers to the measurement $25 \mathrm{~mm}$ below the top and index x refers to the height of the temperature to be determined (in this case, 81 or $89 \mathrm{~mm}$ ).

$$
T_{\text {front }, x}=T_{\text {exh }, x} \frac{T_{\text {front }, 25}}{T_{\text {exh }, 25}}
$$

- In cylinder 2 , it has to be taken into account that the 'front' of cylinder 3 is the 'back' of cylinder 2 (see Figs. 4.10 and 4.11). Consistently with examinations, the data from this row was used for rows 'back', 'exhaust' and 'front'.

- In cylinder 3 , the row on the 'exhaust' side was replaced by the row on the 'front' side. For the 'back' side of cylinder 3, the 'front' row of cylinder 4 was employed because the measurement was done in the area between both cylinders.

- In cylinder 4 , the missing sensors were at the bottom of the 'exhaust' and 'intake' rows. For the bottom of the 'intake side', the data of the 'back' row was employed, after examination of the similarities on both sides. On the 'exhaust' row, the measurements of the 'front' row were used.

The method of area-averaging was also used to obtain mean wall temperatures from results of the Nodal Model. In that case, the application of Eq. 4.1 was straightforward because each node had one temperature and one defined area.

As previously mentioned, the campaign of fired tests consisted of 24 operating conditions in steady state. Accordingly, 24 simulations of the same operating conditions were completed to compare with the experimental tests. In the analysis of mean wall temperatures, results are presented divided by wall and by cylinder. As both the cylinder-head and the cylinder liner were instrumented, the analysis covered two walls, firedeck and liner. The engine had four cylinders. Consequently, the study of mean wall temperatures comprised 192 data points. 


\section{Temperature distribution maps}

The second analysis of the evaluation dealt with local temperature predictions and temperature distributions on the cylinder walls. In this study, the number of data points was much higher than in the study of mean wall temperatures, as for each of the 24 tests there were 88 measurements from thermocouples. That yielded a number of data points of 2112. Moreover, in this study, the physical location where temperature was measured mattered. Consequently, it was determined that an appropriate way to display the data would be to use temperature distribution maps. To produce this kind of maps, an auxiliary plotting tool was created. There was one map style for the firedeck and another one for the liner. In the report of the study, a sample set of representative results are shown with maps. However, for the sake of concision, most results are presented in the form of a joint statistical analysis.

\subsubsection{Results}

\section{Mean wall temperature}

In this section, predictions of mean wall temperature are analyzed from different points of view. The data was collected in the six engine operating points, with four levels of swirl ratio and at various locations. In all studies, data of the firedeck and data of the liner is presented separately. On the horizontal axis, there is experimental temperature, which was measured with the thermocouples and then averaged to obtain values of mean wall temperature, as explained in Section 4.2.1. On the vertical axis, there are the model results. The diagonal line indicates a perfect match of measured and modeled temperatures. Data points above the diagonal imply an overestimation of temperature by the model, while points below the diagonal indicate underestimation.

In the first analysis, all data points of the firedeck are plotted together in Fig. 4.26, but the markers allow to distinguish among operating points (see legend). Most data points are found slightly above the diagonal and concentrated by keypoints. This means that the model slightly overestimated mean wall temperature systematically. Moreover, the concentration of data points of the same operating point implied that engine speed and load had a bigger influence than swirl ratio or cylinder. Another interesting trend is that concentrations tend to be wider than tall. This means that modeled results were more similar among them than measurements. 


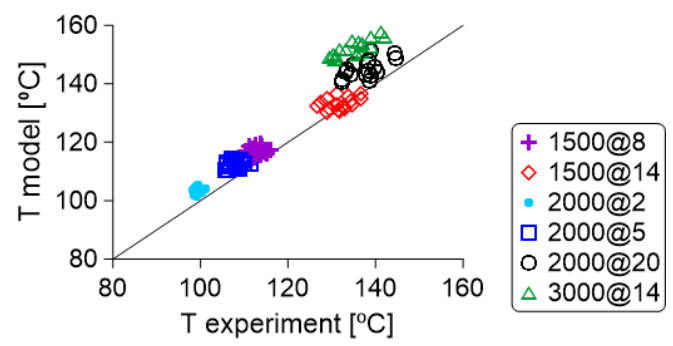

Figure 4.26: Comparison of predicted and measured mean cylinder-head temperatures classified by keypoint.

That is to say, the sensitivity of the model to changes in the parameters was slightly insufficient. Overall, however, the coefficient $\mathrm{R}^{2}$ was equal to 0.96, a high value that indicated that prediction was good. Only in one keypoint the temperature prediction was clearly unsatisfactory. This was the operating point at higher engine speed, identified as green triangles. In that keypoint, there was a systematical overestimation of temperature ranging between $10^{\circ} \mathrm{C}$ and $20^{\circ} \mathrm{C}$. A possible reason is the relation between engine speed and coolant flow rate. Since the coolant pump is impelled by the timing chain, when the engine shaft rotates at higher speed, flow rate increases. Thus, at high engine speed, cooling capacity is higher, which experimental measurements reflected. But, in the model, heat transfer coefficient on the coolant side was constant and equal for all keypoints. Therefore, the effect of increased coolant flow was not modeled. That misprediction should be solved by adding correlations for convection on the coolant side that take into account coolant flow rate. If the 3000@14 keypoint was excluded, the coefficient $\mathrm{R}^{2}$ went up to 0.98 .

Mean wall temperatures on the cylinder liner are shown in Fig. 4.27. On the left side, Fig. 4.27(a), it is possible to see a group of data points above the diagonal, separated from the majority of data points that are below the diagonal. This happened only in the liner (Fig. 4.27), not in the firedeck (Fig. 4.26). After examining the data, it was found that all the diverging points were measurements from cylinder 1. Fig. 4.27(b) shows the prediction when data points of cylinder 1 were removed. The likely cause of the deviating behavior of cylinder 1 was that the cylinder block had an aluminum piece clung to the area of the block that contained this cylinder. The sizable aluminum piece contributed to dissipate heat. Therefore, measured temperatures were lower than in the rest of the block. 


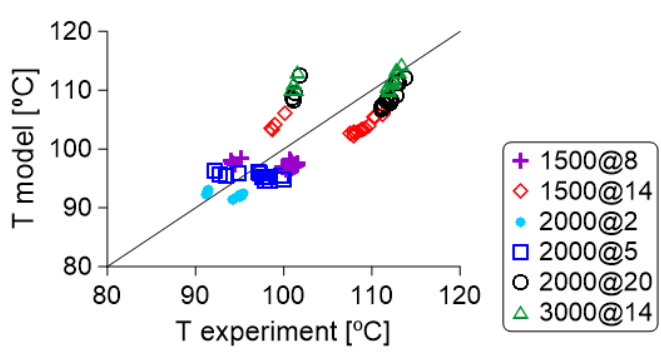

(a) All cylinders

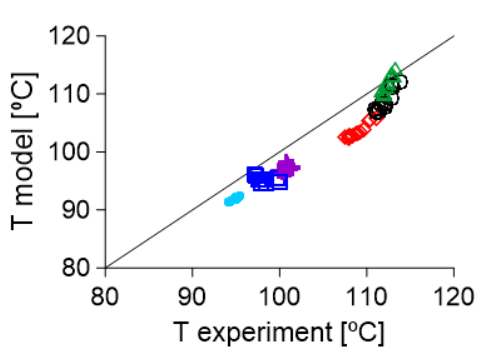

(b) Cylinders 2, 3 and 4

Figure 4.27: Comparison of predicted and measured mean liner temperatures classified by keypoint.

As a result, the model tended to overpredict wall temperatures of cylinder 1. Overall, the model predictions achieved a good compromise among all measured temperatures. The coefficient $\mathrm{R}^{2}$ was 0.83 . This was the result of an effective prediction capacity aided by a calibration based on the energy balance of the whole engine, considering the four cylinders as a group. Under normal conditions, a slight underestimation of mean wall temperatures on the cylinder liner, around $3^{\circ} \mathrm{C}$, could be expected.

To visualize the capability of the model to predict the influence of swirl on wall temperature, the data was rearranged. Data from each keypoint was plotted in a different graph. In Fig. 4.28 the predictions of the firedeck are shown. The data points are identified according to the swirl ratio of the test (see legend). Each swirl ratio contains four points, each one from one cylinder. From the examination of the plots, it can be concluded that all swirl ratios were predicted with the same accuracy. That implies that the model was able to correctly simulate the influence of swirl. In the last two graphs, corresponding to the keypoints 2000@20 and 3000@14, it can be observed that, for each swirl ratio, data points were divided in two groups of two. Further examination of the data revealed that temperatures measured in the inner cylinders, 2 and 3, were slightly higher than those measured in the external cylinders, 1 and 4 . The difference is due to the fact that cylinders 1 and 4 have better cooling because they have larger contact area with cooling galleries around them.

Data points of the cylinder liner are shown in Fig. 4.29. Again, the data points of cylinder 1 are separated from the rest. Focusing on the different swirl ratios, the prediction accuracy is similar and the effect of 

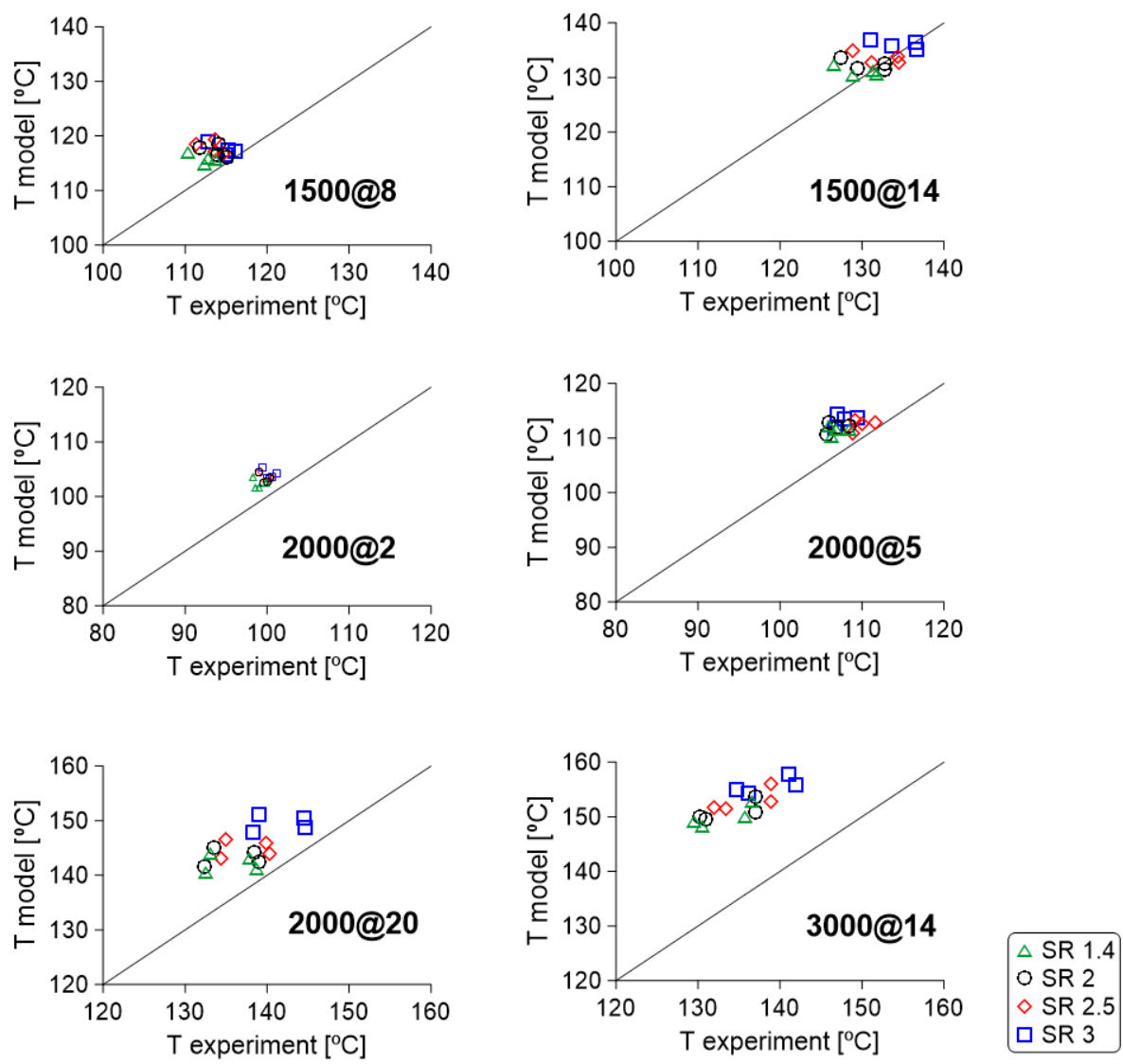

Figure 4.28: Comparison of predicted and measured mean firedeck temperatures in the swirl sweep. 

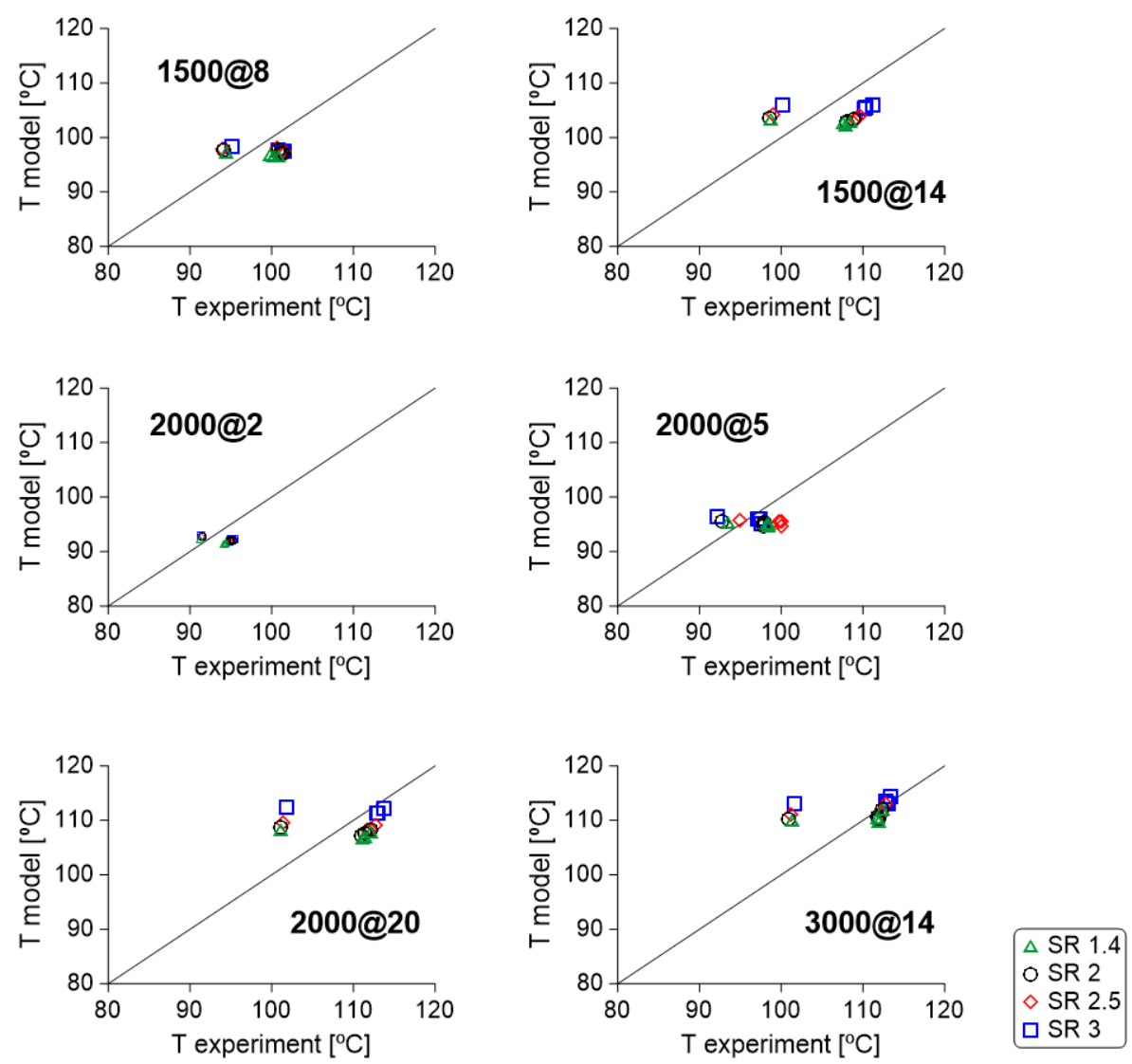

Figure 4.29: Comparison of predicted and measured mean liner temperatures in the swirl sweep. 
swirl is smaller than in the firedeck. Only in keypoints 2000@20 and $3000 @ 14$, it is possible to say that predictions at high swirl are slightly better. That could lead to slightly bigger underpredictions of temperature at low swirl ratio.
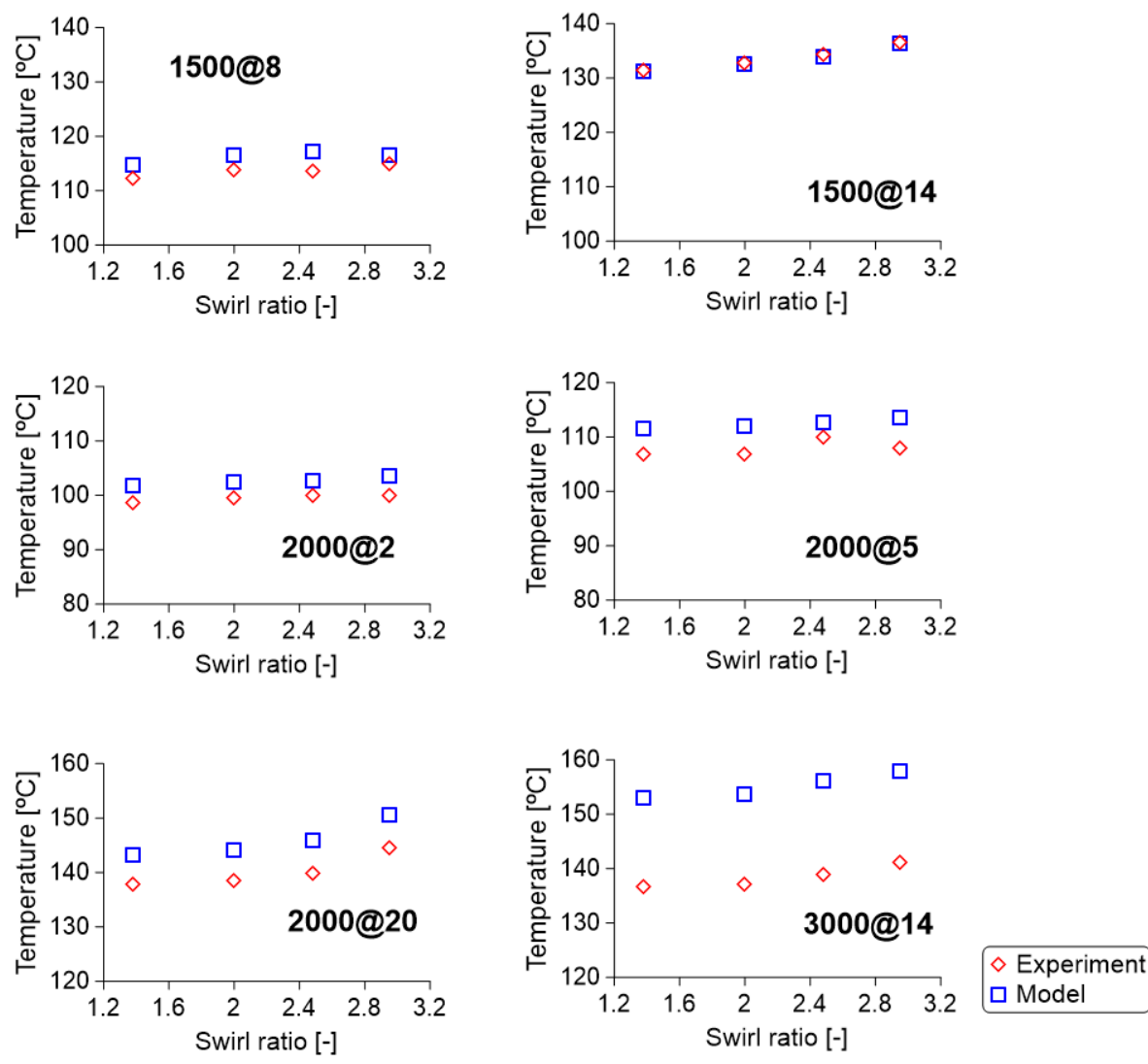

Figure 4.30: Measured cylinder-head temperature in the swirl sweep.

After analyzing the model predictions, it is pertinent now to visualize the influence of swirl on mean wall temperatures in a more convenient way. It could be seen that in some keypoints temperature changed significantly (around $10^{\circ} \mathrm{C}$ ) while in other the influence was negligible. To better study the effect of swirl, the horizontal axis of Figs. 4.30 and 4.31, is the swirl ratio of the test. Vertical axis indicates temperatures. Modeled and measured temperatures are signified by different curves (see legend). Only data of cylinder 3 is plotted, for the sake of clarity. 
Fig. 4.30 shows temperatures in the firedeck. Swirl tended to increase mean wall temperature, and the trend was not linear with swirl ratio. In the plots, it can be clearly seen that the model was able to capture the influence of swirl. Temperature prediction varied from a good fit in keypoint 1500@14 to a clear overprediction in 3000@14.
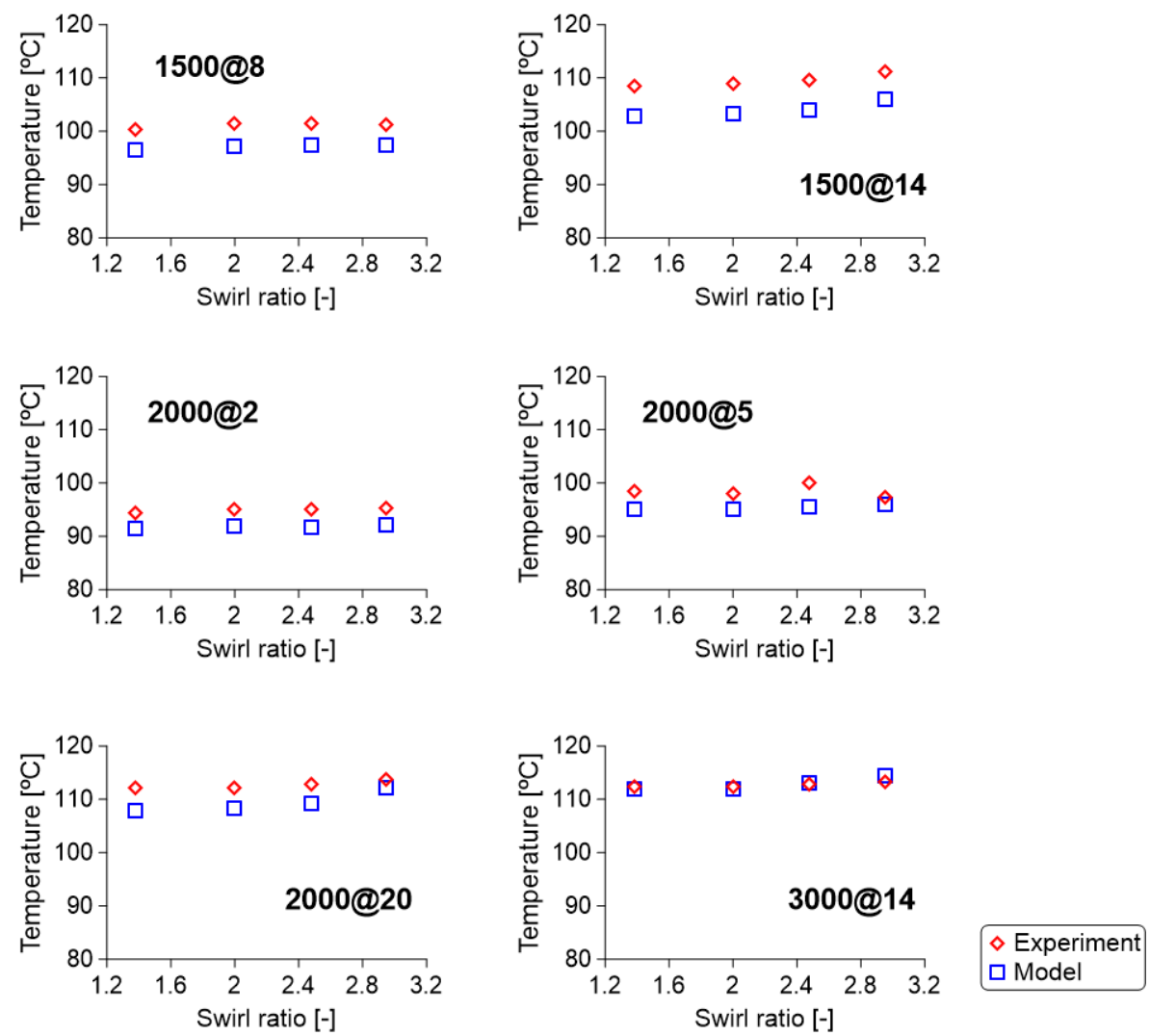

Figure 4.31: Measured cylinder liner temperature in the swirl sweep.

In Fig. 4.31, the influence of swirl on liner mean temperatures is assessed. In most cases, swirl tended to increase wall temperature, but less clearly than in the firedeck. Temperature trends were not linear with swirl ratio. In general, trends were captured by the model. In some operating conditions, wall temperature was slightly underpredicted, while in other the prediction was accurate.

In Table 4.6, the effect of swirl is summarized. They show the temper- 
ature difference between the highest and the lowest swirl ratio observed in the experimental measurements. In the second and fourth row, temperature difference was corrected with coolant temperature, which also influenced wall temperature and varied slightly among the tests. Standard deviation of coolant temperature is given in the fifth row of the table. The corrected values were calculated with Eq. 4.6. Comparing the lower standard deviation of coolant temperature with the higher temperature increments caused by swirl, it can be concluded that the influence of swirl was significant in all keypoints. However, in high load keypoints, the differences between low and high swirl could be better evidenced because heat rejection increased with engine load. Also, the impact of swirl was clearer in the firedeck than in the liner.

$$
\begin{aligned}
\Delta T_{\text {corr }}= & {\left[T_{\text {wall }}(S R=3)-T_{\text {cool }}(S R=3)\right]-} \\
& -\left[T_{\text {wall }}(S R=1.4)-T_{\text {cool }}(S R=1.4)\right]
\end{aligned}
$$

\begin{tabular}{lcccccc}
\hline Nickname & 1500@8 & 1500@14 & 2000@2 & 2000@5 & 2000@20 & 3000@14 \\
\hline$\Delta T$ firedeck $\left[{ }^{\circ} \mathrm{C}\right]$ & 2.7 & 5.2 & 1.4 & 1 & 6.6 & 4.5 \\
$\Delta T_{\text {corr } \text { firedeck }\left[{ }^{\circ} \mathrm{C}\right]}$ & 2.7 & 4.7 & 1.3 & 1.5 & 6.5 & 4.5 \\
$\Delta T$ liner $\left[{ }^{\circ} \mathrm{C}\right]$ & 1 & 2.7 & 0.9 & -1.1 & 1.6 & 0.9 \\
$\Delta T_{\text {corr } \text { liner }\left[{ }^{\circ} \mathrm{C}\right]}$ & 1 & 2.2 & 0.7 & -0.7 & 1.6 & 0.9 \\
$\mathrm{SD}(\mathrm{T}$ coolant $)\left[{ }^{\circ} \mathrm{C}\right]$ & 0.1 & 0.5 & 0.2 & 0.4 & 0.1 & 0.2 \\
\hline
\end{tabular}

Table 4.6: Influence of swirl ratio on wall temperature.

An additional analysis involving instantaneous curves of heat transfer to the cylinder walls was done. The curves where estimated from the pressure trace, as explained in Section 4.2.1. It is reminded that the method can only be applied under motoring conditions and during the closed part of the engine cycle. Therefore, data comes from the motoring tests, not from the fired keypoints. In Fig. 4.32, the heat flux curves for the lowest $(S R=1.4)$ and the highest $(S R=3)$ levels of swirl are plotted. Each graph shows the results for a different engine speed, but all the data comes from cylinder 3. In Fig. 4.32(a), it can be seen that heat flux was clearly higher for the test with highest swirl ratio. The difference was most prominent around the top dead center, when heat flux is higher. 


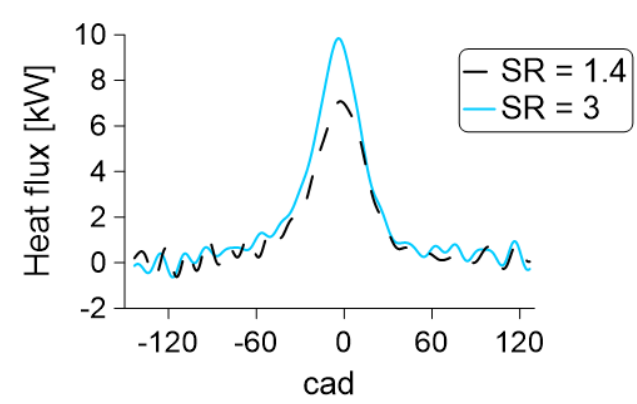

(a) $1000 \mathrm{rpm}$

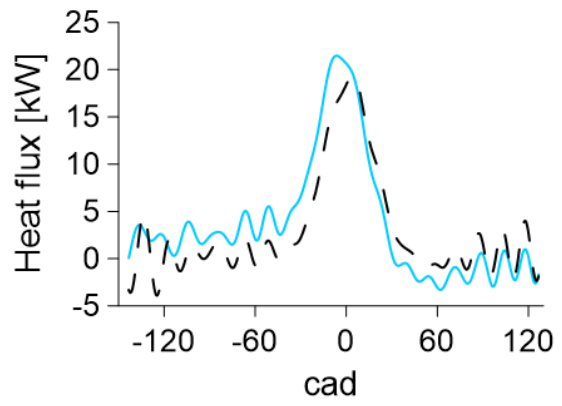

(b) $3000 \mathrm{rpm}$

Figure 4.32: Heat flux at different swirl ratios estimated from in-cylinder measurements.

That is coherent because during the rise of the piston in the compression stroke, there is a process of gas inflow to the piston bowl that increases swirl velocity. This effect is explained in much more detail in Section 4.3.1. Fig. 4.32(b) presents a smaller difference between the heat curves under low and high swirl conditions. Nevertheless, the curve of heat at high swirl still had a higher peak than the curve of heat at low swirl. In both cases, the curve of lowest swirl was clearly delayed with respect to the curve of high swirl. The same trends were observed in the other cylinders. Regarding the different operating points, the effect of swirl was more apparent at $1000 \mathrm{rpm}$. With increasing engine speed, the curves came closer to each other.

In summary, prediction of mean wall temperature by the Nodal Model was good. In general, the effects of engine speed, load and swirl motion were correctly predicted. An area of improvement was that firedeck temperature tended to be slightly overestimated by the model while liner temperature tended to be slightly underestimated. That suggests that the convective process was not exactly equal on both surfaces, and therefore the assumption of a single gas zone with a single heat transfer coefficient was not entirely satisfactory. Modeling of swirl influence, however, was accurate, since good agreement was observed regardless of swirl ratio. There were small differences among cylinders but the model was able to capture them, except in the liner of cylinder 1, where there was a heat-dissipating element that was not modeled. During the analyses, another subject of improvement was detected. There was an overprediction of wall temperature 
at high engine speed in the firedeck. This problem should be mitigated by the implementation of correlations for convection on the coolant side that take into account the coolant flow rate. To understand the influence of swirl ratio on in-cylinder heat rejection, both heat flux curves and wall temperatures from the experiments were analyzed. A clear increase of heat flux was observed when swirl ratio was higher. However, the difference decreased with increasing engine speed. Moreover, the assessment of wall temperatures revealed that in general, the effect of swirl was significant, particularly in the firedeck. Notwithstanding, the effect was not linear and it depended heavily on the keypoint. In some keypoints, the swirl influence was negligible, specially in the liner. Altogether, engine speed and load had a bigger influence on wall temperature than swirl ratio.

\section{Temperature distribution}

The study of local temperatures comprises three analyses: spatial distribution of temperature on the cylinder walls, assessment of local predictions of the Nodal Modal and localized influence of swirl on wall temperature.

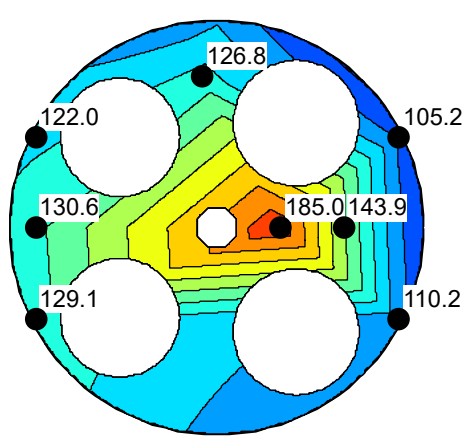

(a) Cylinder 1

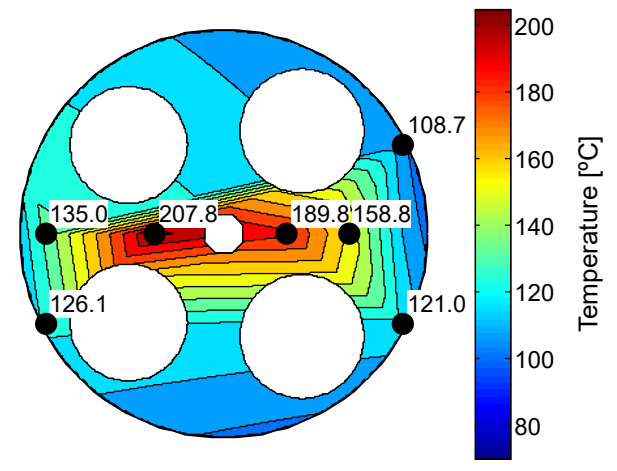

(b) Cylinder 3

Figure 4.33: Temperature distribution in the firedeck.

As previously mentioned, maps are a useful tool to study spatial distributions. In Figs. 4.33 and 4.34, temperature maps are shown. In this analysis, only experimental data is examined. Distributions correspond to keypoint 2000@20 with maximum swirl ratio. Since the distribution of thermocouples was heterogeneous throughout the engine, plots of different 
cylinders are presented in order to maximize the data in the plots. Black dots are locations that had sensors. Measured temperature is plotted next to each dot. In areas between dots, temperatures were interpolated. In external areas around dots, the temperature field was extrapolated.

In the firedeck, cylinder 1 and cylinder 3 had the most sensors. Moreover, the plane $4 \mathrm{~mm}$ deep from the surface had more sensors than the plane $7 \mathrm{~mm}$ deep. The maps of Fig. 4.33 contain the information of cylinder 1 (left) and 3 (right) on the plane $4 \mathrm{~mm}$ deep. Combining the information of both maps, a complete view of the temperature field could be obtained. It is clear that there were large temperature variations dependent on location. In cylinder 3 , temperature ranged almost $100^{\circ} \mathrm{C}$ from $208^{\circ} \mathrm{C}$ at the measurement point between exhaust valves to $109^{\circ} \mathrm{C}$ at the point between the edge of the firedeck and an intake valve. In cylinder 1 , the sensor between exhaust valves was missing but there was a sensor in the area between an intake and an exhaust valve, on the upper side of the map. The data from that sensor allows to speculate that the temperature field could be approximately radial. It can be seen that temperature decreases from the center to the periphery. However, the field was not axisymmetric, as modeled by LeFeubvre et al. or Dent and Suliaman (see Section 2.2.1), because temperatures on the exhaust side were significantly higher than temperatures on the intake side. Neither was the temperature distribution symmetrical with respect to a horizontal axis: temperatures on the upper and the lower side differed significantly.
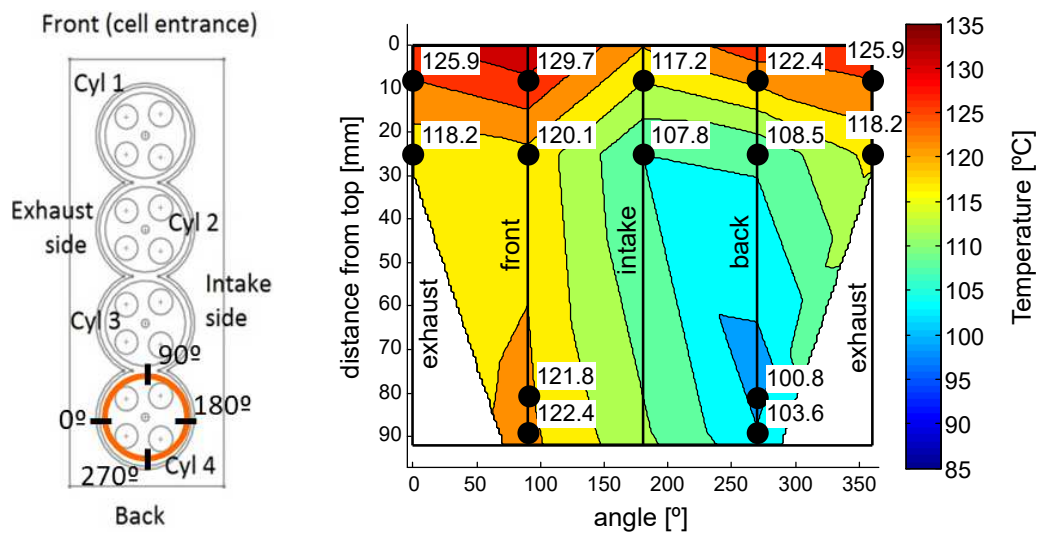

Figure 4.34: Temperature distribution in the liner.

Fig. 4.34 shows the measurements in the liner of cylinder 4 , the most instrumented cylinder. The representation uses the same principles com- 
mented in Figs. 4.9 to 4.12: the vertical direction is the cylinder height and the horizontal direction indicates the angle of the cylinder circumference, starting from the side of the engine where the exhaust system is. White masks cover the areas far from sensors where the extrapolation was not trustworthy. In the liner, as in the firedeck, the temperature field was very heterogeneous. A temperature drop of $30^{\circ} \mathrm{C}$ was observed between the hottest and the coldest spot. In general, temperature decreased from top to bottom. This is related to the fact that the upper area is exposed for longer time to in-cylinder gas during an engine cycle due to piston motion. However, there were also important temperature differences along the circumference. Temperature in the 'back' row was lower than temperature in the 'front' row because the former was well cooled while the latter is in the area between cylinders 3 and 4, where cooling was less effective. Moreover, temperature in the 'exhaust' row was higher than in the 'intake' row. It was seen that, in the firedeck, temperatures also followed that pattern. In addition, the exhaust side was the piston thrust side, which could produce heat by friction on that side.

Overall, temperature distribution was very heterogeneous in the firedeck and the liner. Some general trends were identified, but predicting such complex temperature fields with a model is a challenge.
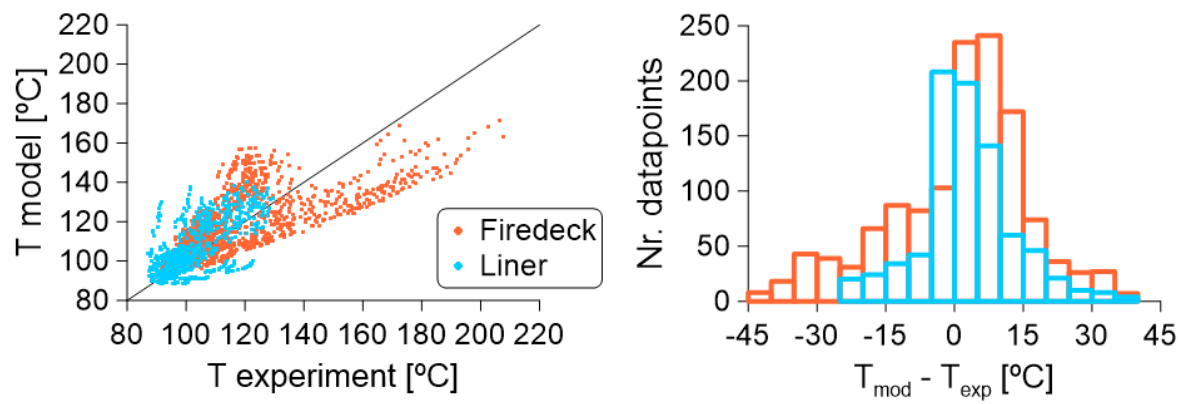

Figure 4.35: Prediction of local temperature at measurement locations.

After confirming that temperature was far from homogeneous on the cylinder walls, the capability of the Nodal Model to predict such local variations was assessed. In Fig. 4.35, predictions of all measurement locations (88) under all operating conditions (24) were examined. On the left side of Fig. 4.35, a direct comparison of measured and modeled temperatures is shown, with different colors for firedeck and liner. The plot shows an important concentration of data points at relatively low temperature 
around the diagonal. In general, the higher the temperature, the worse the prediction. In particular, the highest temperatures of the firedeck were systematically underpredicted by the model. Generally speaking, the amount of data points far from the diagonal was significant: the coefficient $\mathrm{R}^{2}$ was low, equal to 0.52 in the firedeck and equal to 0.42 in the liner. On the right side of Fig. 4.35, the histogram allows to split up predictions to analyze them more clearly. The studied magnitude was the difference between the modeled temperature of a node minus the temperature measured at the coincident location. That is the deviation of the prediction, where a positive value means that temperature was overpredicted and a negative value means it was underpredicted. The height of the column quantifies the number of data points in which the deviation is in a certain range. The closer to zero, the better the accuracy. It can be seen that most data points were in the range of deviation between $-5^{\circ} \mathrm{C}$ and $15^{\circ} \mathrm{C}$. In the firedeck, modeled temperatures tended to be above the measured ones. This fact agreed with the observation of slightly overpredicted mean wall temperatures that was reported in the previous section. It must be noted that, in the present study, areas with lower concentration of thermocouples were underrepresented. Also in the firedeck, the histogram shows a considerable amount of data points in the range of clear underestimations, on the left side of the horizontal axis. Overall, deviations around $30^{\circ} \mathrm{C}$ and $40^{\circ} \mathrm{C}$ were not infrequent in a local basis.

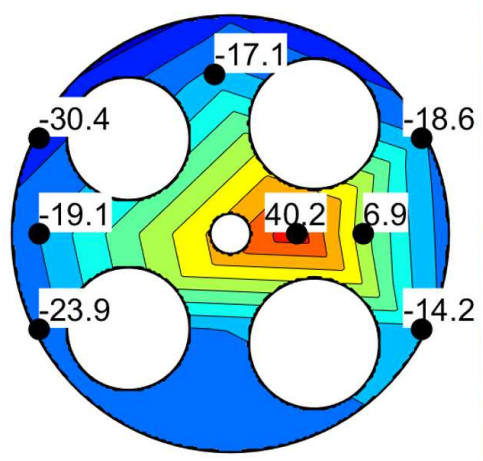

(a) Cylinder 1

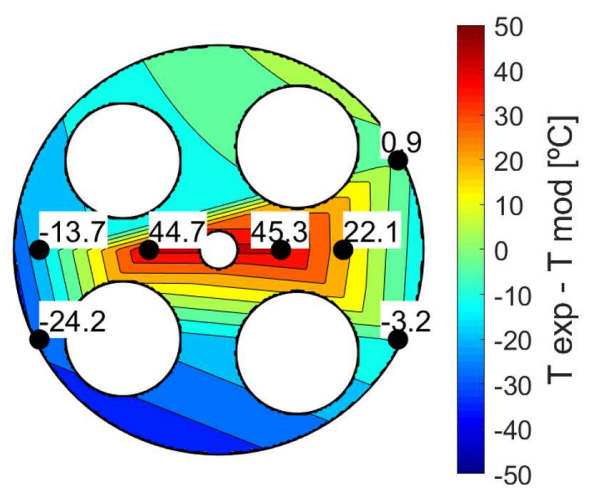

(b) Cylinder 3

Figure 4.36: Prediction map of the firedeck.

At this point, it was pertinent to examine temperature deviations us- 
ing a map, to identify areas where temperature was clearly overpredicted and underpredicted. The maps are shown in Figs. 4.36 and 4.37. In the maps, the plotted magnitude is not the modeled temperature minus the experimental temperature, as in previous studies. Instead, it is the other way around, experimental minus modeled. This was done because experimental temperatures were higher than modeled ones in the hotter areas. In such case, the difference was positive, and hues of red were used. In colder areas, where the model tended to overpredict temperature, the difference was negative and hues of blue were used. Therefore, hues of red mean that measured temperature is higher than modeled temperature, something that usually occurs in hot areas. Hues of blue are used when the model overpredicts temperature, something that is more frequent in cold areas. This way, maps can be interpreted more intuitively.

In Fig. 4.36, it is possible to see the that the distribution of deviations had approximately a radial shape. In the center, temperature was underpredicted by the model. Measured temperatures were up to $45^{\circ} \mathrm{C}$ higher than predicted. On the contrary, in the periphery of the firedeck, where temperatures were lower as seen in Fig. 4.33, overestimations of temperature were found. Discrepancy was up to $30^{\circ} \mathrm{C}$. Locally, prediction was accurate only at intermediate radii (green areas). The conclusion was that the distribution of deviations was similar to the temperature distribution, as seen in Fig. 4.33. That implies that the model yielded a more homogeneous temperature distribution than the one that was observed experimentally. According to the model, local temperatures did not vary much from the average wall temperature, but the experiment showed that was not true.

In Fig. 4.37, local predictions in the liner were analyzed. In general, the model overpredicted wall temperature near the top. On average, the overestimation was $10^{\circ} \mathrm{C}$, while the maximum deviation was $19^{\circ} \mathrm{C}$. At the bottom, accuracy depended on location. Prediction in the well-cooled 'back' area was good, with a slight underestimation up to $5^{\circ} \mathrm{C}$. In contrast, in the 'front' area, where cooling was worse, measured temperature was clearly higher than modeled temperature. Overall, in the liner, the field of the deviations map was clearly different from the temperature field of Fig. 4.34. This was opposite to the situation observed in the firedeck, where the deviation distribution and the temperature distribution were similar.

In summary, the model was unable to predict the local heat transfer processes on the cylinder walls satisfactorily. Such local processes had a larger influence on wall temperature than the Nodal Model anticipated. 

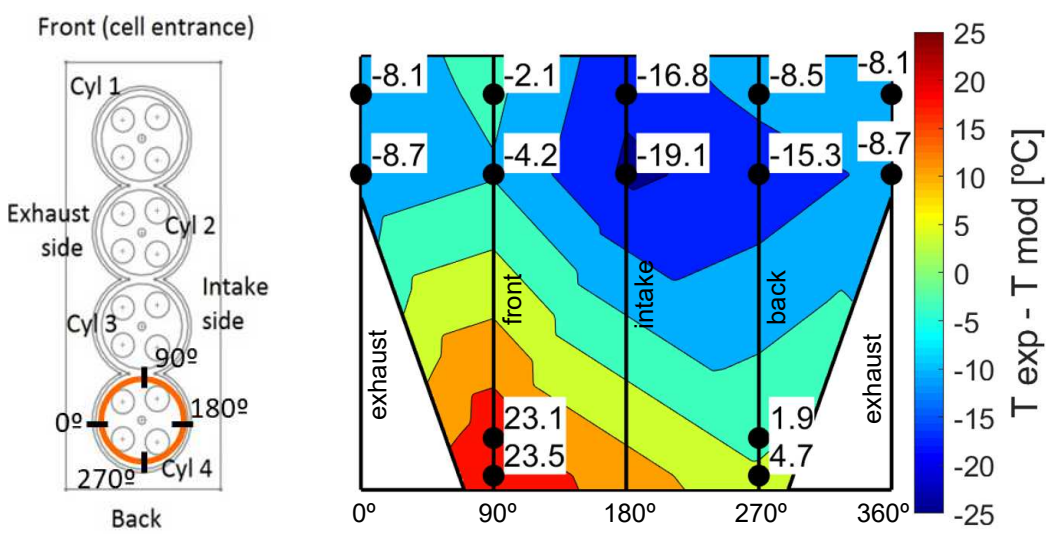

Figure 4.37: Prediction map of the liner.

Finally, the influence of swirl on wall temperature was analyzed locally. In this study, experimental measurements at three different locations were analyzed over a swirl sweep. Each plot includes six curves, corresponding to the six keypoints under study. That approach allowed to observe the effect of swirl, the influence of the operating point and the differences among various locations. The study covers only three locations for the sake of concision, but many other were examined and the same behavior was observed. The three reported locations were selected because they were representatives of the different areas. For consistency, the three locations belonged to cylinder 4 .

In the figures, a schema on the left shows the location of the sensor under study. The plotted magnitude in the graph on the right side, $\Delta T$, was calculated with Eq. 4.6. In this case, $T_{\text {wall }}$ was not an averaged temperature but the local measured temperature. Standard deviation of coolant temperature was reported for each keypoint in Table 4.6.

The first location studied here is the closest to the center of the firedeck. It is shown in Fig. 4.38 that swirl had an important influence on wall temperature. The effect varied widely among operating points and was not linear with swirl ratio. The keypoint in which the impact of swirl was the highest was 3000@14. It must be noted that, since $S R=\frac{\omega}{n}$, where $\omega$ is the swirling speed of the gas and $n$ is the engine speed, 3000@14 also had the strongest swirl motion. Temperature variation in the range of swirl ratios between 1.4 and 3 was $20^{\circ} \mathrm{C}$. In keypoint 2000@20, swirl influence was remarkable as well. In the other of keypoints, swirl had a significant role. The trends of temperature increment were not always monotonic 


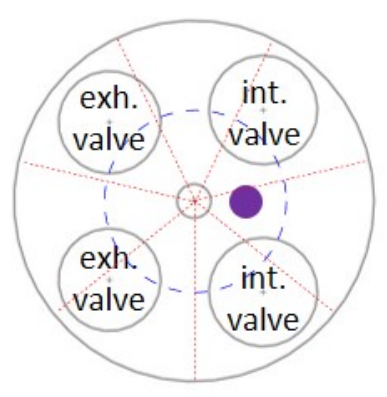

(a) Location

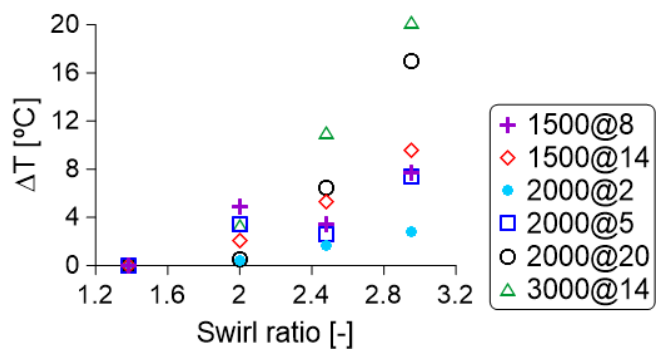

(b) Swirl sweep

Figure 4.38: Influence of swirl on a temperature near the center of the firedeck.

and they depended on the keypoint.

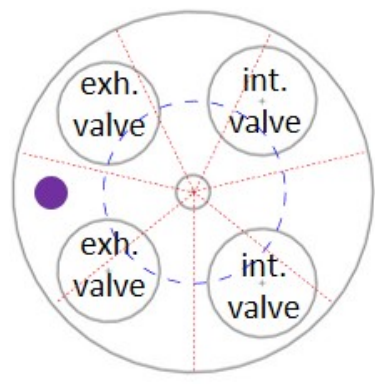

(a) Location

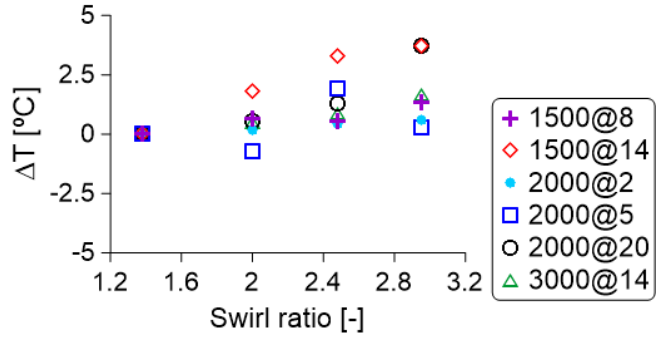

(b) Swirl sweep

Figure 4.39: Influence of swirl on a temperature away from the center of the firedeck.

The second sensor under examination was also located in the firedeck but away from the center. Fig. 4.39 shows that the influence of swirl at that location was much smaller than in the center. Note the scale change of the vertical axis with respect to Fig. 4.38. Keypoint 1500@14 presented the clearest variation, which was lower than $4^{\circ} \mathrm{C}$.

Last location was in the liner, $25 \mathrm{~mm}$ below the top of the cylinder. Results are shown in Fig. 4.40. Again, keypoint 1500@14 presented the clearest trend. The maximum temperature increment was lower than $3^{\circ} \mathrm{C}$. In keypoint 2000@20, there was also a monotonic temperature increase. In the other keypoints, trends were unclear. 


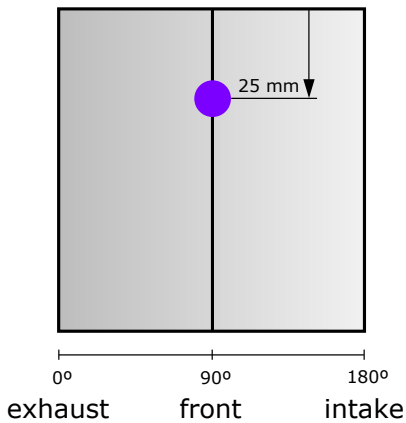

(a) Location

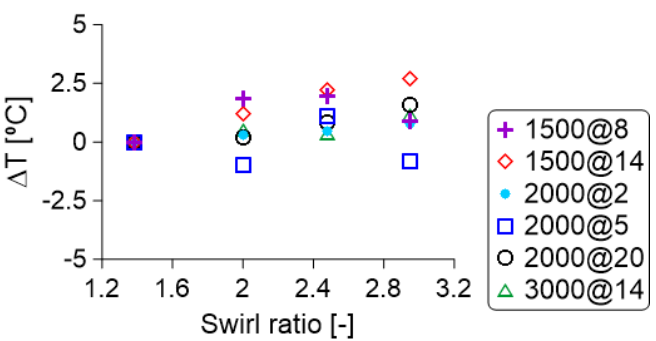

(b) Swirl sweep

Figure 4.40: Influence of swirl on a temperature of the liner.

In conclusion, it was found that swirl was influential in the center of the firedeck, but in the outer firedeck and in the liner, the effect was much less clear. Moreover, the operating point had a decisive role. Trends were not linear with swirl ratio and in many cases they were different from a monotonic increase of temperature.

\section{Concluding remarks}

The main takeaway of this section is that the heat transfer process on the cylinder walls is complex, leading to very profound local variations. The analysis of mean wall temperature led to the conclusion that the Nodal Model was able to predict wall temperatures under varying conditions of engine speed, load and swirl motion with reasonable accuracy. However, a sensor-by-sensor analysis showed that model predictions were not reliable at particular locations. Only average temperatures over relatively large areas could be correctly predicted. Therefore, in its initial form, the Nodal Model was suitable to obtain mean wall temperatures, but the local temperatures of the different nodes were not accurate, at least near the cylinder surfaces. The model could not benefit entirely from the resolution provided by the multiplicity of lumped elements.

\subsection{Multizone model of in-cilinder convection}

In Section 4.2, the capacity of the Nodal Model to predict local and spatially-averaged temperatures was assessed. It was found that the model was able to predict averaged magnitudes with good accuracy under dif- 
ferent operating conditions. However, even though the spatially-averaged temperatures were reliable, it was found that the model yielded a too uniform local temperature distribution. As a consequence, temperature was clearly overpredicted in some areas and clearly underpredicted in other areas. It was also seen that for swirl sweeps under fixed operating conditions, swirl effect on heat transfer depends on location. As discussed in Section 4.2.1, the geometry of the Nodal Model was adapted to resemble that of the real engine. Therefore, it is unlikely that local inaccuracies were caused by imprecisions in the definition of the engine structure. As pointed out in Section 2.2.4, inaccuracies were likely a consequence of assuming homogeneous in-cylinder conditions. In the same section, the validity of such assumption was discussed. It was seen that in-cylinder conditions are remarkably heterogeneous. After examining experiments and simulations from authoritative sources, it was concluded that a single-zone heat transfer approach has limited ability to capture local trends. This was confirmed and quantified during the present research in the final part of the evaluation reported in Section 4.2. In an attempt to solve this deficiency, researchers have modeled in-cylinder heat rejection by means of so-called multizone or zonal models. Zonal models are quasi-dimensional models that divide the combustion chamber into several volumes or zones. Each zone has a different temperature, composition or fluid motion. A thorough survey of zonal models reported in the literature was conducted in Section 2.2.4.

In this section, the implementation of a multizone model is proposed as an improvement that can lead to a better prediction of local temperatures. The zonal model relies on a submodel of gas motion to supply characteristic gas velocities in each zone. The first part of the section is committed to explain the works concerning the gas motion submodel. In the second part, an assessment of the potential of the zonal model to predict local temperatures is investigated.

To conduct studies that are necessary to implement a multizone heat transfer model, reference tests are required. Throughout Section 4.3, a set of motoring engine tests are used. Experimental tests were conducted in Engine 1, as part of the test campaign described in Section 4.2.1. These motoring tests were already used in the quantitative evaluation of Section 4.2 to calibrate uncertainties of the cylinder model, including the constant of the Woschni correlation. The tests covered the range of engine speeds found in typical diesel engine operation. At every engine speed, two swirl 
levels were evaluated: the minimum swirl ratio allowed by the engine (SR $=1.4$ ) and the maximum $(\mathrm{SR}=3)$. A set of 14 operating conditions was measured in total. They are summarized in Table 4.7.

\begin{tabular}{ccc}
\hline Nickname & Engine speed [rpm] & Swirl ratio [-] \\
\hline 1000low & 1000 & 1.4 \\
1000high & 1000 & 3 \\
1500low & 1500 & 1.4 \\
1500high & 1500 & 3 \\
2000low & 2000 & 1.4 \\
2000high & 2000 & 3 \\
2500low & 2500 & 1.4 \\
2500high & 2500 & 3 \\
3000low & 3000 & 1.4 \\
3000high & 3000 & 3 \\
3500low & 3500 & 1.4 \\
3500high & 3500 & 3 \\
4000low & 4000 & 1.4 \\
4000high & 4000 & 3 \\
\hline
\end{tabular}

Table 4.7: Motoring tests.

\subsubsection{In-cylinder gas motion}

The submodel of in-cylinder gas motion is the core of almost all zonal models reviewed in Section 2.2.4. In that section, it was commented how close related gas movement and heat transfer are. Also, from the review in Section 2.2.1, it is clear that flow conditions have a determining influence on convective heat transfer. As flow structure is clearly heterogeneous inside the cylinder, heat rejection is heterogeneous as well. In order to simulate in-cylinder convection, it is therefore necessary to model 
flow movement locally. In a quasi-dimensional model, at least the major mechanisms affecting the flow structure have to be simulated. Spatial resolution is ensured by dividing the combustion chamber into zones inside which gas movement is assumed uniform. Flow motion mechanisms allow momentum exchange among zones. When flow conditions are known in a zone, a zonal heat transfer coefficient can be obtained.

In order to develop a quasi-dimensional model, information about incylinder flow motion is needed. There are experimental techniques that allow to gain insight into in-cylinder flow processes, such as particle image velocimetry (PIV) or laser Doppler velocimetry (LDV) [56]. However, the application of such techniques require complex installations. A practical alternative is the 3D simulation of computational fluid dynamics (CFD). For the research of Section 4.3.1, the experimental tests of Table 4.7 were replicated in a CFD cylinder model. CFD results were used as a reference to assist development and to calibrate the gas motion model.

\section{Zones and characteristic velocity}

In the surveyed literature (Section 2.2.4), two kinds of zones are found in multizone models. One kind are flow regions, which are different parts of the in-cylinder flow structure. Each flow region represents gas movement in a particular area of the cylinder. The other kind of zones is related to combustion. The distinction between burned and unburned zones allows to have a different temperature and composition of gas in each zone. Most authors start their investigations using motoring tests. Motoring conditions allow to examine many aspects of engine operation while avoiding the complexity of fired operation. For the present study, motoring tests are used. According to results, it can be decided whether extending the study to fired operation is pertinent. For motored operation, only the first kind of zones, flow regions, was considered. Since there was no division between burned and unburned zones, temperature and composition of the gas were assumed homogeneous throughout the chamber in all studies. Therefore, the term 'zone' is in this thesis a synonym of flow region. Multizone models based on flow regions feature different characteristic velocities and flow conditions for different areas of the cylinder. That keeps away the assumption of flow uniformity throughout the combustion chamber and allows to spatially resolve the thermal effects of gas motion.

For the definition of flow regions, an approach proposed by Morel et al. [133] has been adopted by many subsequent authors (see Section 2.2.4). 
In general, reports were promising, although Hansen [142] noted that the application of this approach to fired operation failed because various injection and combustion effects were not modeled, even after considering radiation and burned zones. Morel's proposal to split the cylinder volume into flow regions was also followed in the present thesis. The approach was described in Section 2.2.4. In summary, three volumes and six surfaces were distinguished.

The three flow regions were numbered as follows:

1. Volume inside the piston bowl.

2. Inner volume directly above the piston bowl up to the firedeck. The shape of the region is a cylinder with one base being the entrance of the bowl and the second base being a projection of the bowl circumference on the firedeck.

3. Outer annular volume above the piston crown up to the firedeck. This is usually called squish region.

The six surfaces were numbered as follows:

1. Base of the piston bowl.

2. Lateral wall of the bowl.

3. Piston crown.

4. Liner wall.

5. Outer annular area of the firedeck.

6. Inner circular area of the firedeck.

Zonification can be graphically seen in Fig. 4.41, where volumes V1, V2 and V3 are flow regions and S1, S2, .. are surfaces. The characteristic velocity for each surface has to be calculated with Eq. 2.46, which is shown here again due to its relevance. Two of the velocity components, $v_{x}^{2}$ and $v_{y}^{2}$, are orthogonal and parallel to the surface. Later in the present section, the procedure to obtain each velocity component for each zone is described. The third component of Eq. 2.46 is the turbulent kinetic energy, whose calculation is also discussed later.

$$
v_{\text {char }}(t)=\sqrt{v_{x}^{2}(t)+v_{y}^{2}(t)+2 T K E(t)}
$$

There are several aspects to be commented regarding the two orthogonal velocity components parallel to the walls, $v_{x}^{2}$ and $v_{y}^{2}$. One parallel component is always swirl velocity. Indeed, the circumferential component of gas motion around the cylinder axis is parallel to every wall. In this thesis, a different swirl velocity curve was calculated in each zone. 


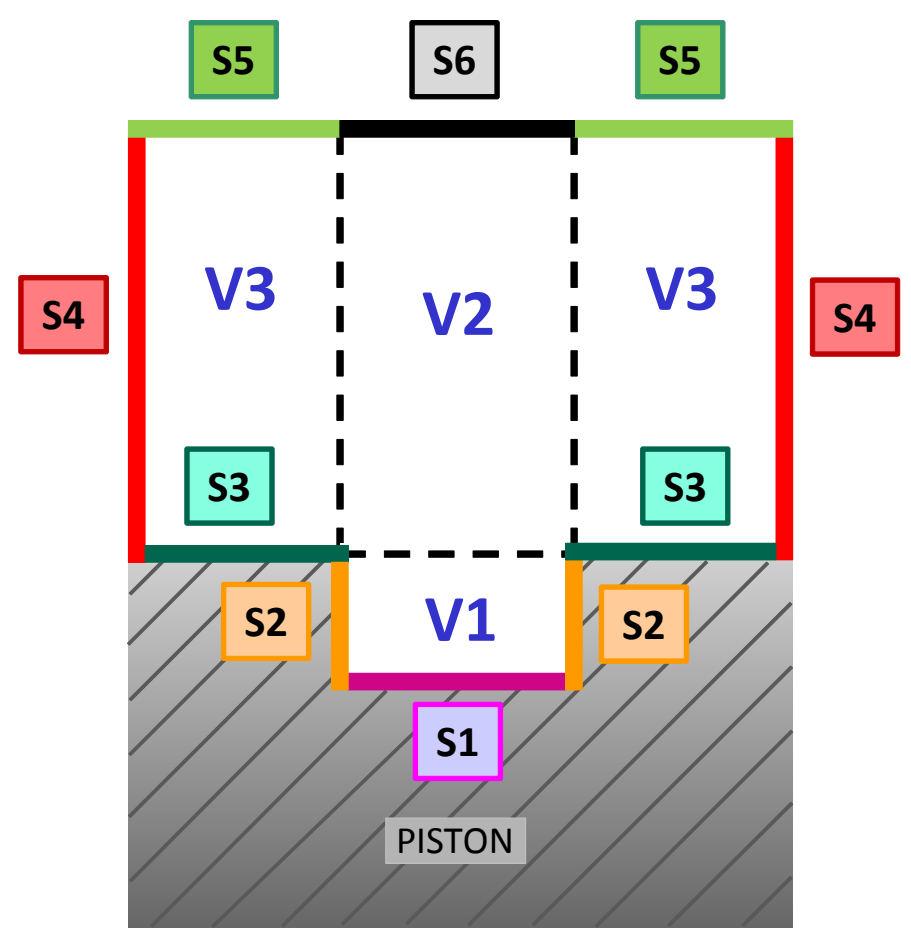

Figure 4.41: Split of flow regions and surfaces in the cylinder. 
The second orthogonal component varies depending on the surface. There are two mechanisms that generate gas motion inside the cylinder, apart from swirl and turbulence.

One is the movement of the piston relative to the gas. The piston pushes or pulls the gas. Immediately next to the piston surface, the gas being dragged has the same velocity as the piston. Gas in direct contact with the firedeck, on the contrary, is static. Between piston and firedeck, an axial gradient of velocity is found. This velocity component is parallel to vertical surfaces: liner and bowl walls. On the contrary, it is perpendicular to horizontal surfaces; thus, it is not employed in the calculation of convection on those surfaces.

The second mechanism of gas motion is called squish and it is also related to piston movement. When the piston moves upwards, the volume of the squish region becomes smaller. As a consequence, the gas moves out of the squish region. If valves are open, the gas can escape through the ports. If valves are closed, the gas moves inwards into the bowl, since the bowl volume does not change and is then relatively larger than the squish region. When the piston moves downwards, the inverse gas motion occurs. The squish is a movement in two directions. In radial direction because the gas moves inwards or outwards of the inner area where the bowl is. And in axial direction because it moves into and out of the bowl.

In summary, there are two sources of axial motion: the piston drag and the axial squish. In contrast, the radial component is only produced by the squish mechanism. In the model implemented for this thesis, it was considered that squish existed only when valves were closed. Therefore, when valves were open, the characteristic velocity on the horizontal surfaces had only two components: swirl and turbulence.

\section{CFD simulations}

CFD simulations of in-cylinder gas motion were conducted with the commercial CONVERGE ${ }^{\mathrm{TM}}$ code. The computational domain included a sector of $1 / 7$ of the combustion chamber with the real piston geometry. The simulated period was the whole closed cycle i.e. the extent of time between intake valves closing and exhaust valves opening. Base mesh size was determined by means of a mesh independence study which is reported below. CONVERGE $^{\mathrm{TM}}$ has a feature of automatic mesh refining to increase the resolution in areas with high gradients of velocity and temperature [203]. All tests included in Table 4.7 were simulated. Calibration of the CFD model was undertaken under the criterion of fitting the experimental pres- 
sure curve. The variable used for calibration was the clearance volume between piston and liner. Slight variations of the distance between the piston crown and the top of the fire ring led to small changes of compression ratio. This had considerable influence on in-cylinder pressure. Tests were not adjusted separately; instead, the same geometry was used for all tests. The results for tests 1500low and 3500low can be seen in Fig. 4.42. Good agreement was obtained in these two tests as well as in the rest. The optimal compression ratio for adjusting the pressure curve was almost equal to the one obtained from the characterization of uncertainties done in CALMEC (see Section 3.2). That fact provided extra consistency.

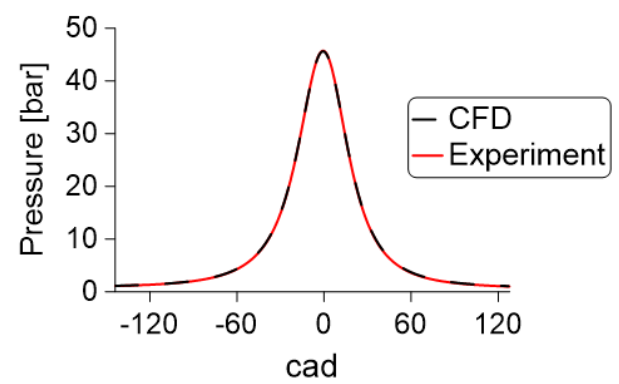

(a) $1500 \mathrm{rpm}$

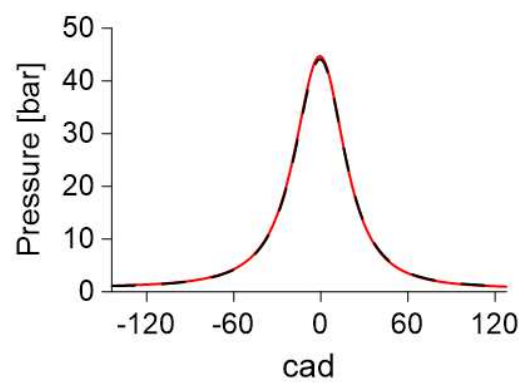

(b) $3500 \mathrm{rpm}$

Figure 4.42: Calibration of CFD model with pressure curve.

Simulation results were post-processed with MATLAB ${ }^{\circledR}$. During postprocessing, raw data of individual cells was classified in the three flow regions of the multizone model (see Fig. 4.41). Data of cells in each region was mass-averaged to obtain curves of relevant variables in each particular region.

Next, curves of velocity components in the three different regions are examined. Fig. 4.43 shows the velocity curves of one representative test, 2000low. Each graph contains curves of a different velocity component.

In the upper graph on the left side, the vertical velocity component parallel to the cylinder axis is plotted. Positive values indicate upwards motion while negative values indicate downwards motion referred to Fig. 4.41. The curves tend to follow the instantaneous piston speed. Notwithstanding, around top dead center $\left(0^{\circ}\right)$ there are fluctuations due to squish motion, specially in the inner regions, 1 and 2 . 

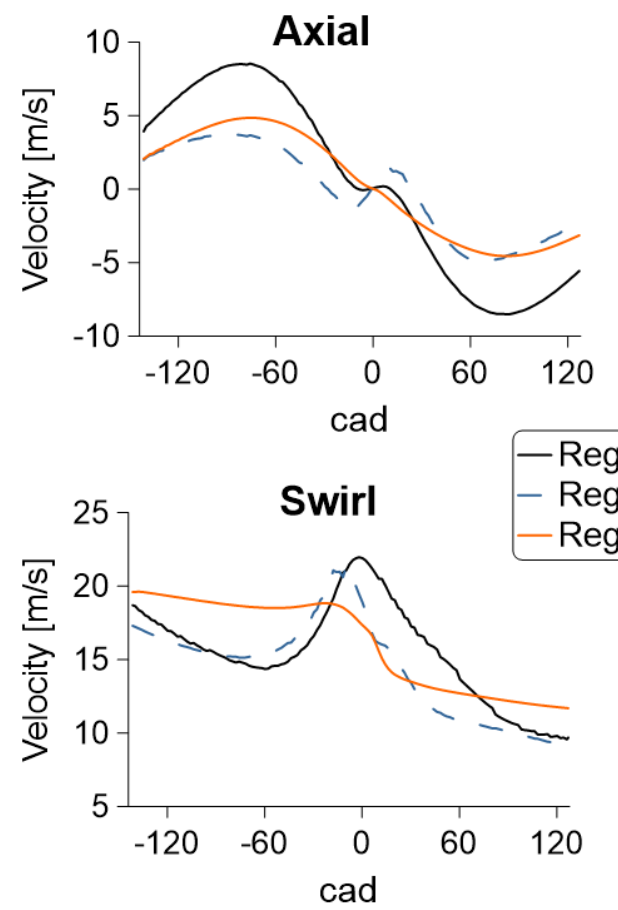

Radial
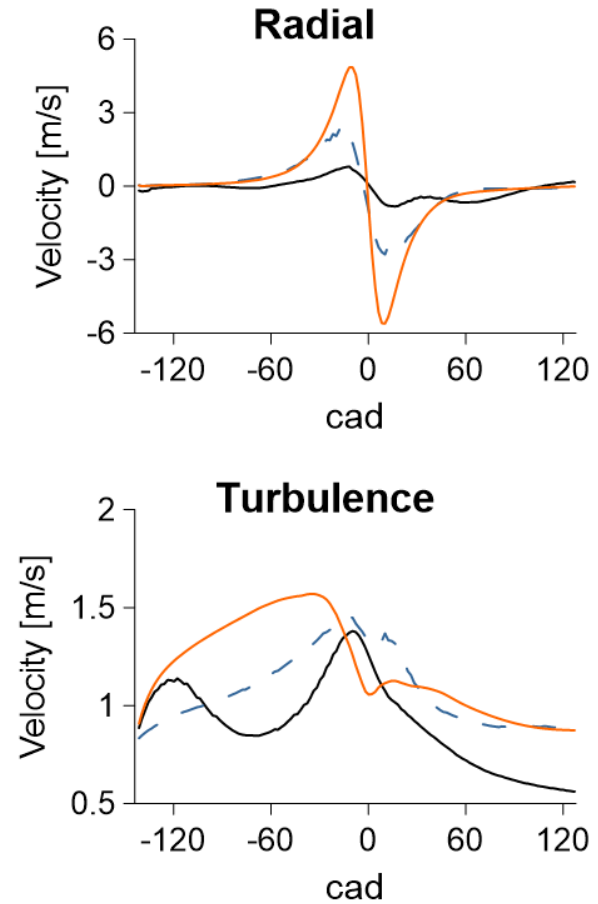

Figure 4.43: Gas velocities from CFD simulation. 
In the graph on the upper right, the radial velocity component is shown. That component is perpendicular to the cylinder axis, acting in inward or outward direction. In the graph, inwards motion is positive and outwards motion is negative. The dominant behavior is the inwards movement when the piston goes up and the outwards movement when the piston goes down, with absolute maximums near the top dead center.

The third graph shows swirl velocity curves. This magnitude is the linear velocity component around the cylinder axis, which was obtained as the product of angular velocity in each cell and radius of the cell relative to axis. The curves present an initial phase of deceleration due to wall friction. Then, around top dead center, velocity rises as a result of squish. When the gas is confined within the piston bowl, the radius of the rotating volume decreases and thus rotational speed increases due to conservation of angular momentum. After top dead center, swirl decays again. Swirl was the component with the highest velocity values, even though 2000low is a low swirl test. Therefore, an important influence of swirl could be expected from the model.

In the last plot, on the bottom right, curves of turbulent velocity are shown. The magnitude obtained from simulation was turbulent kinetic energy (TKE), which is the variable used in the characteristic velocity equation, Eq. 2.46. This is the energy per unit of mass associated to turbulent motion. Nevertheless, the plotted magnitude in the graph is turbulent velocity. It was obtained from TKE as $v_{t u r b}=\sqrt{2 T K E}$. Plotting turbulent velocity instead of TKE allowed for direct comparison with other velocity components. Temporal evolution of turbulence was complex and heterogeneous. In the inner regions, 1 and 2, turbulent velocity presented a peak due to turbulence generation by compression and squish effects. In the outer annular region, the maximum occurred earlier and at top dead center there was a local minimum. In comparison with other velocity components, turbulent velocity presented the lowest values and variations, on average.

It was observed that the curves of all velocity components were very different in the different zones. Thus, it can be concluded that considering a mean flow in the whole combustion chamber implies a significant loss of accuracy, specially if spatial resolution inside the cylinder is a requirement. Moreover, considering a constant velocity over time is also an erroneous approximation, since gas velocity changes significantly over the engine cycle. 
Mesh independence A mesh independence study was conducted to determine the optimal grid size for the CFD simulations. That is the base grid size because, as previously mentioned, CONVERGE ${ }^{\mathrm{TM}}$ dynamically refines the local grid size during the simulation. Notwithstanding, the base grid size is important because the refinement consists in gradual subdivisions of the base size. Sizes under study were $2 \mathrm{~mm}, 1.8 \mathrm{~mm}$ and $1.75 \mathrm{~mm}$. A grid size of $2 \mathrm{~mm}$ provides reasonable accuracy for typical engine simulations. Tests with smaller grid sizes allowed to check whether the results of gas velocity were affected by mesh size or were independent of this variable.
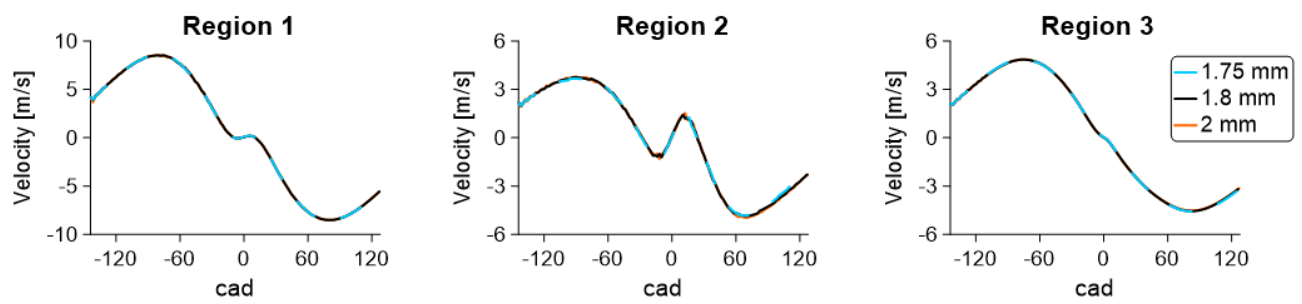

Figure 4.44: Mesh independence study of axial velocity.

The study is summarized in Figs. 4.44-4.47. The different velocity components of test 2000high are plotted for different grid sizes. Test 2000high was selected because it was an intermediate engine speed, results were representative of all tests and velocity variations were significant over time. First, the velocity component in axial direction, in Fig. 4.44, was examined. In all three regions under consideration, axial velocity presented the same temporal evolution regardless of grid size. Thus, axial velocity was independent of mesh size. Radial velocity, in contrast, showed
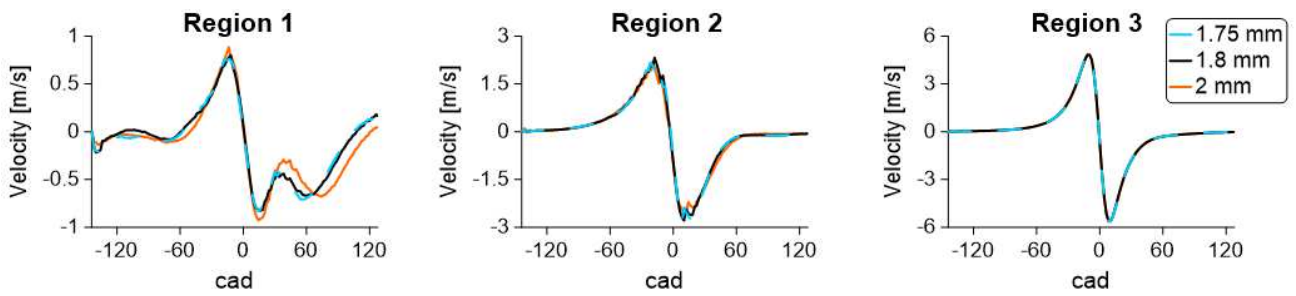

Figure 4.45: Mesh independence study of radial velocity.

a higher sensitivity to grid size, in particular in region 1, inside the piston bowl. This can be seen in Fig. 4.45. The curve from the test with a size of $2 \mathrm{~mm}$ was clearly different, but the tests with sizes of $1.8 \mathrm{~mm}$ and $1.75 \mathrm{~mm}$ 
were very similar. In region 2 , above the bowl, all three curves were very similar and in region 3, near the liner, curves overlapped. In Fig. 4.46,
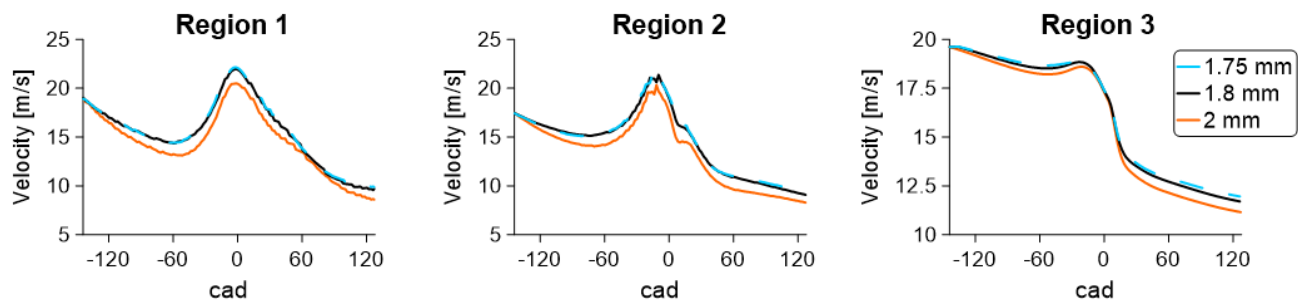

Figure 4.46: Mesh independence study of swirl velocity.

swirl velocity is analyzed. The test with a size of $2 \mathrm{~mm}$ presented lower swirl values with respect to the other two tests, which were very close. The same behavior was seen in the three flow regions. Lastly, turbulent
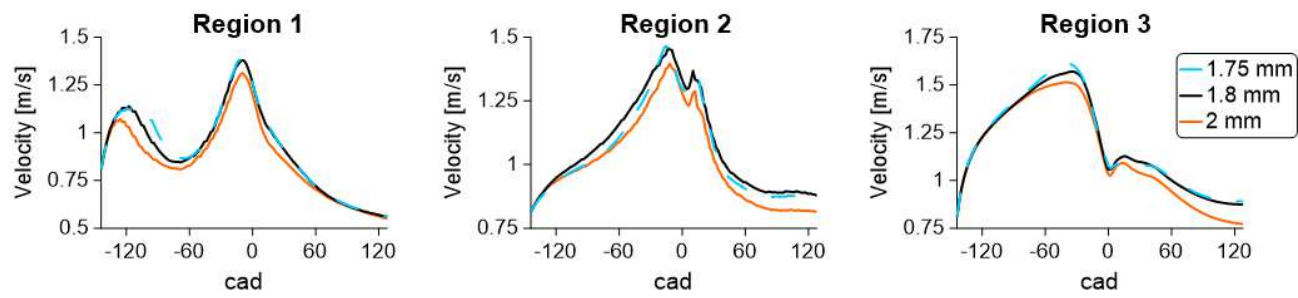

Figure 4.47: Mesh independence study of turbulent velocity.

velocity was examined in Fig. 4.47. Trends were similar to those seen in swirl velocity. The curve of the test with a size equal to $2 \mathrm{~mm}$ was lower in all flow regions. Tests with sizes of $1.8 \mathrm{~mm}$ and $1.75 \mathrm{~mm}$ presented a similar temporal evolution of the turbulent component, but they were slightly less close than in the case of swirl velocity.

In conclusion, the mesh independence study revealed that a reduction of grid size from $2 \mathrm{~mm}$ to $1.8 \mathrm{~mm}$ had a significant influence on various components of gas velocity. Swirl and turbulence were the most sensitive, while radial velocity was affected in the region inside the bowl. A further decrease of grid size from $1.8 \mathrm{~mm}$ to $1.75 \mathrm{~mm}$ had negligible effects overall. With those sizes, the simulation of fluid mechanics was mostly independent of mesh size, although computation time was higher with a grid size of $1.75 \mathrm{~mm}$. Therefore, a grid size of $1.8 \mathrm{~mm}$ was chosen for all subsequent simulations. For all grid sizes, calculation time was below 5 hours in tests at $2000 \mathrm{rpm}$ and below 10 hours in tests at $4000 \mathrm{rpm}$, the highest engine 
speed in the range. All simulations were run in a dedicated workstation using 8 cores. It is reminded that the simulation covered the closed part of the cycle, not the whole engine cycle.

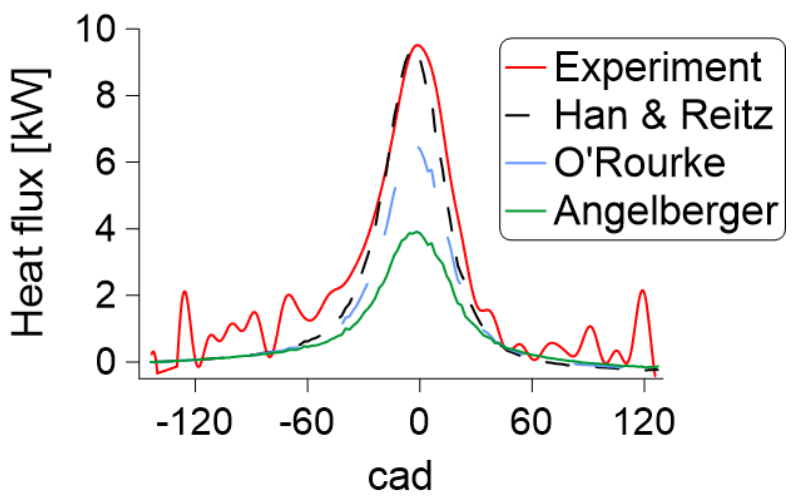

Figure 4.48: Heat flux with different laws of the wall.

Law of the wall Before running the final CFD simulations it was necessary to select a method to calculate convective heat transfer to the cylinder walls. That is determined by a so-called temperature wall function or law of the wall. CONVERGE ${ }^{\mathrm{TM}}$ has three models for the law of the wall, proposed by Han \& Reitz, O'Rourke and Angelberger. All three models were evaluated, mainly to clarify the influence they had on in-cylinder velocity, but also to determine the best model to be chosen. Curves of heat flux from the three models were compared with the experimental one, calculated with Eq. 2.32 as mentioned in Sections 2.2.2 and 4.2.1. The comparison was done in the closed part of the cycle since the method to calculate the experimental heat flux curve is only applicable to closed volumes. In Fig. 4.48, the comparison for test 1500low and cylinder 1 is shown. The law of the wall proposed by Han \& Reitz presented the best agreement with the curve of experimental heat flux, in particular with the less fluctuating central part. The models of O'Rourke and Angelberger systematically underpredicted heat flux. Later, the law of the wall from Han \& Reitz, being the most interesting, was evaluated for two levels of swirl. In Fig. 4.49, results of the simulations 1500low and 1500high are compared with the corresponding experimental curves. The curves presented good agreement. 


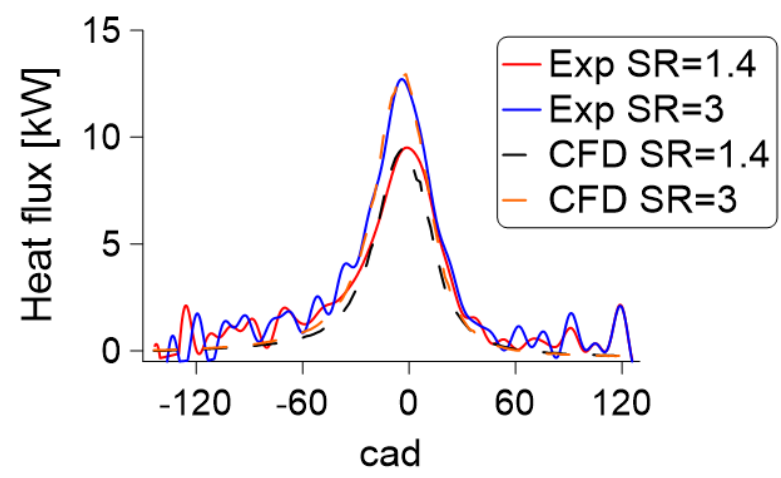

Figure 4.49: Heat flux using Han \& Reitz model at different swirl ratios.

In the following analysis, the curves of gas velocity were examined. It was found that no velocity component was affected by the change of law of the wall in any zone or operating condition. In Fig. 4.50, curves of two velocity components are shown as example. They were selected because the shape of the curves is relatively complex. On the left, the circumferential or swirl velocity in the upper inner volume is shown. On the right, a curve of turbulent velocity in the squish region above the piston crown is plotted. The graphs show that the curves of all laws of the wall overlap. The operating point is 1500 low and the cylinder is cylinder 1 again.

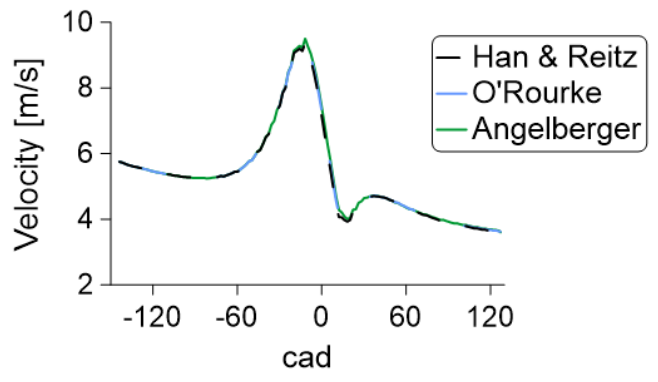

(a) Swirl velocity in the upper inner zone

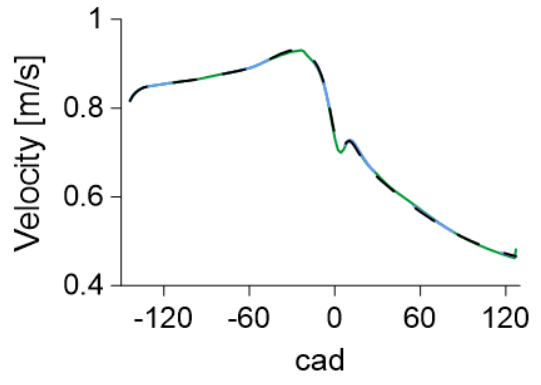

(b) Turbulence velocity in the outer zone

Figure 4.50: Gas velocities with different laws of the wall.

In conclusion, calculation of gas motion in CFD was independent of the law of the wall model selected by the user. Therefore, any model could be 
used in this application. Nevertheless, it was found during the study that the model proposed by Han \& Reitz clearly yielded a better agreement with experimental estimations than the other two models. Consequently, the model of Han \& Reitz was used for all CFD simulations in this thesis.

\section{Calculation of velocity components}

This section covers the description of methods to obtain the different velocity components needed in Eq. 2.46 for the multizone convection model.

First motion to be discussed is the velocity induced when the piston pushes up and pulls down the gas in the cylinder. That movement generates a velocity gradient in the gas because fluid molecules in immediate contact with a solid wall have the same velocity as the wall, and the piston surface has relative velocity with respect to the other cylinder walls. Since the piston moves only in axial direction, induced velocity and gradient in the gas can be assumed axial, although due to the complex geometry of the combustion chamber this is an approximation. Squish mechanism is calculated separately; that is thoroughly explained below. Focusing now only on the axial velocity gradient, the first aspect to be commented is that gas velocity is parallel to vertical surfaces. Among the surfaces defined in the previous section, schematized in Fig. 4.41, there are only two that are vertical: bowl wall and liner wall. Regarding the liner wall, the area affected by convection with gas reaches from the piston edge to the top of the cylinder. The velocity component involved in convective heat transfer is parallel to the surface. This velocity varies along the liner between zero at the top and the piston speed on the piston surface. It was assumed that velocity had a linear distribution. Consequently, the average axial velocity from the point of view of the liner is the instantaneous speed of the piston divided by two, as shown in Eq. 4.7.

$$
v_{\text {ax }, \text { iner }}=0.5 v_{\text {pis }}
$$

An analogous approach was followed to determine velocity on the bowl wall. Gas velocity on the ground of the bowl was assumed equal to piston speed. Under the assumption of a linear decrease of velocity in upward direction, an expression for gas velocity at the edge of the bowl could be found. The velocity for convection over the bowl wall was then the average of both values. The final expression can be seen in Eq. 4.8. $L_{\text {bowl }}$ is the bowl depth, $L_{0}$ is the squish height, or distance between the piston crown 
and the firedeck, and $s$ is the time-varying piston displacement.

$$
v_{a x, b o w l}=0.5\left(\frac{L_{0}+s}{L_{0}+s+L_{b o w l}}+1\right) v_{p i s}
$$

To model squish motion, the cylinder volume was split into two zones. Radial squish was obtained defining the zones indicated in Fig. 4.51, while for axial squish the division shown in Fig. 4.52 was employed. In both cases there was an internal zone, which included the piston bowl, and an external zone, which included the squish region. Calculation of squish flow was based on a mass flow balance among both zones. The balance is defined by Eq. 4.9, where $m_{\text {int }}$ is the mass of the internal volume, marked in Fig. 4.51 as Volume 1, and $m_{e x t}$ is the mass of the external volume, marked as Volume 2. It is noted that this equation does not take into account blow-by. Neither takes into account fuel injection because the model was meant for motored operation.

$$
\frac{\delta m_{i n t}}{\delta t}=-\frac{\delta m_{e x t}}{\delta t}
$$

Since pressure and temperature were assumed homogeneous throughout the combustion chamber, density $\rho$ was also spatially uniform. As $\rho=\frac{m}{V}$, Eq. 4.10 can be written.

$$
\frac{m_{\text {int }}}{V_{\text {int }}}=\frac{m_{\text {ext }}}{V_{\text {ext }}}
$$

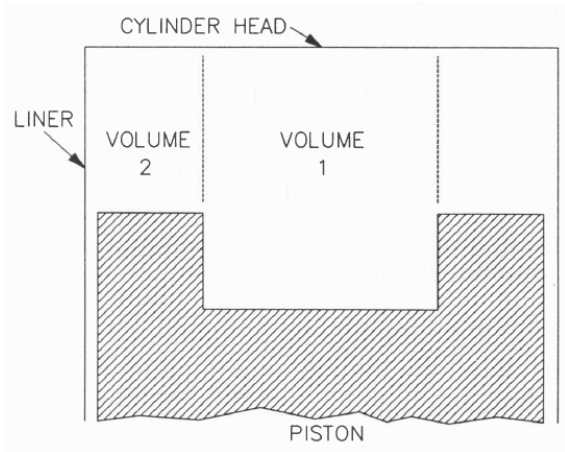

Figure 4.51: Control volumes for radial squish [139].

To calculate radial squish, the system of Fig. 4.51 had to be examined. The internal zone, or Volume 1, was formed by flow regions 1 and 2 of Fig. 4.41. Total volume of this region was $V_{\text {int }}=V_{\text {bowl }}+\frac{\pi}{4} D_{\text {bowl }}^{2}\left[L_{0}+s(t)\right]$. The 
external zone, Volume 2, matched flow region 3 in Fig. 4.41. Its volume was $V_{\text {ext }}=\frac{\pi}{4}\left(D_{\text {cyl }}^{2}-D_{\text {bowl }}^{2}\right)\left[L_{0}+s(t)\right]$. Introducing these expressions in Eq. 4.10, an expression for $\frac{\delta m_{i n t}}{\delta t}$ was obtained, Eq. 4.11. It had to be taken into account that $\frac{\delta s(t)}{\delta t}$ is piston speed, $v_{\text {pis }}$. Moreover, the expression of $V_{\text {ext }}$ was used to evaluate $m_{\text {ext }}=\rho V_{\text {ext }}$. Except masses and piston displacement, the rest of variables were constant.

$$
\begin{aligned}
& \frac{m_{\text {int }}(t)}{V_{\text {bowl }}+\frac{\pi}{4} D_{\text {bowl }}^{2}\left[L_{0}+s(t)\right]}=\frac{m_{\text {ext }}(t)}{\frac{\pi}{4}\left(D_{\text {cyl }}^{2}-D_{\text {bowl }}^{2}\right)\left[L_{0}+s(t)\right]} \\
& m_{\text {int }}(t)=\frac{V_{\text {bowl }} m_{\text {ext }}(t)+\frac{\pi}{4} D_{\text {bowl }}^{2}\left[L_{0}+s(t)\right] m_{\text {ext }}(t)}{\frac{\pi}{4}\left(D_{\text {cyl }}^{2}-D_{b o w l}^{2}\right)\left[L_{0}+s(t)\right]} \\
& \frac{\delta m_{\text {int }}}{\delta t}=\frac{V_{\text {bowl }}+\frac{\pi}{4} D_{\text {bowl }}^{2}\left(L_{0}+s\right)}{\frac{\pi}{4}\left(D_{\text {cyl }}^{2}-D_{\text {bowl }}^{2}\right)\left(L_{0}+s\right)} \frac{\delta m_{\text {ext }}}{\delta t}-\frac{\rho V_{\text {bowl }} v_{\text {pis }}}{L_{0}+s}
\end{aligned}
$$

It is noted that, according to Eq. $4.9, \frac{\delta m_{\text {ext }}}{\delta t}=-\frac{\delta m_{\text {int }}}{\delta t}$. Though substitution, $\frac{\delta m_{\text {ext }}}{\delta t}$ could be solved as a function dependent on piston displacement and speed but independent of gas mass, Eq. 4.12.

$$
\begin{aligned}
\frac{\delta m_{\text {ext }}}{\delta t} & =-\frac{V_{\text {bowl }}+\frac{\pi}{4} D_{\text {bowl }}^{2}\left(L_{0}+s\right)}{\frac{\pi}{4}\left(D_{c y l}^{2}-D_{\text {bowl }}^{2}\right)\left(L_{0}+s\right)} \frac{\delta m_{\text {ext }}}{\delta t}+\frac{\rho V_{\text {bowl }} v_{\text {pis }}}{L_{0}+s} \\
\frac{\delta m_{\text {ext }}}{\delta t} & =\frac{\rho V_{\text {bowl }} v_{\text {pis }}}{L_{0}+s}\left[1+\frac{V_{\text {bowl }}+\frac{\pi}{4} D_{\text {bowl }}^{2}\left(L_{0}+s\right)}{\frac{\pi}{4}\left(D_{\text {cyl }}^{2}-D_{\text {bowl }}^{2}\right)\left(L_{0}+s\right)}\right]^{-1}
\end{aligned}
$$

From the definition of mass flow rate, $\dot{m}=\rho A v$, squish velocity could be obtained, 4.13. This velocity was applicable to all horizontal surfaces. The area crossed by the gas was $A=\pi D_{\text {bowl }}\left(L_{0}+s\right)$ (see Fig. 4.51).

$$
v_{s q, \text { rad }}=\frac{1}{\rho A} \frac{\delta m}{\delta t}=\frac{V_{\text {bowl }}\left(D_{c y l}^{2}-D_{b o w l}^{2}\right)}{D_{\text {bowl }}\left(L_{0}+s\right)\left[4 V_{b o w l}+\pi D_{c y l}^{2}\left(L_{0}+s\right)\right]} v_{p i s}
$$

Radial velocity components used in the three flow regions, Eqs. 4.14, 4.15 and 4.16, were a correction of Eq. 4.13. Tuning constants were named $\alpha_{\text {rad }}$. A detailed explanation of the tuning procedure and outcome is shown in the next section.

$$
\begin{aligned}
& v_{\text {radial }, 1}=\alpha_{\text {rad }, 1} v_{s q, a x} \\
& v_{\text {radial }, 2}=\alpha_{\text {rad }, 2} v_{s q, a x} \\
& v_{\text {radial }, 3}=\alpha_{\text {rad }, 3} v_{s q, a x}
\end{aligned}
$$




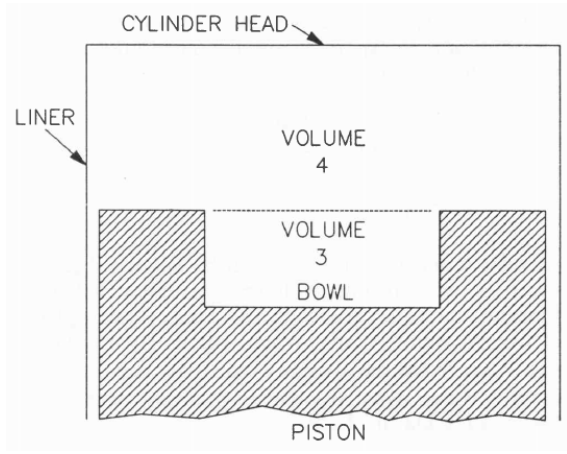

Figure 4.52: Control volumes for axial squish [139].

The volume split of Fig. 4.52 allowed to calculate axial squish. The internal zone, Volume 3, was the same as the flow region inside the bowl, zone 1 in Fig. 4.41. Its volume was $V_{\text {int }}=V_{\text {bowl }}$. The upper or external zone, Volume 2, contained the other flow regions, 2 and 3, which were located above the piston. Volume of the upper zone was $V_{e x t}=\frac{\pi}{4} D_{c y l}^{2}\left[L_{0}+\right.$ $s(t)$. The procedure to obtain axial squish velocity was analogous to the procedure to obtain radial squish velocity. Eq. 4.17 was obtained from Eq. 4.10, considering the volume expressions. Subsequently, Eq. 4.18 was deduced and then Eq. 4.19 could be finally obtained. In this case, the area crossed by the gas was $A=\pi D_{\text {bowl }}^{2}$, as indicated in Fig. 4.52. The same squish velocity was applied to all vertical surfaces.

$$
\begin{gathered}
\frac{m_{\text {int }}(t)}{V_{\text {bowl }}}=\frac{m_{\text {ext }}(t)}{\frac{\pi}{4} D_{c y l}^{2}\left[L_{0}+s(t)\right]} \\
m_{\text {int }}(t)=\frac{V_{\text {bowl }} m_{e x t}(t)}{\frac{\pi}{4} D_{c y l}^{2}\left[L_{0}+s(t)\right]} \\
\frac{\delta m_{\text {int }}}{\delta t}=\frac{V_{\text {bowl }}}{L_{0}+s}\left(\frac{4}{\pi D_{c y l}^{2}} \frac{\delta m_{\text {ext }}}{\delta t}-\rho v_{\text {pis }}\right) \\
\frac{m_{\text {ext }}}{\delta t}=\frac{\rho V_{b o w l} v_{\text {pis }}}{L_{0}+s}\left[1+\frac{V_{b o w l}}{\frac{\pi}{4} D_{c y l}^{2}\left(L_{0}+s\right)}\right]^{-1} \\
v_{s q, a x}=\frac{1}{\rho A} \frac{\delta m}{\delta t}=\left(\frac{D_{c y l}}{D_{b o w l}}\right)^{2} \frac{V_{b o w l} v_{p i s}}{V_{b o w l}+\pi D_{c y l}^{2}\left(L_{0}+s\right)}
\end{gathered}
$$


In the region inside the bowl, zone 1 , the resulting velocity component in axial direction was given by Eq. 4.20, the sum of Eq. 4.8 and Eq. 4.19 multiplied by an adjustment constant, $\alpha_{a x, 1}$.

$$
v_{a x i a l, 1}=v_{a x, b o w l}+\alpha_{a x, 1} v_{s q, a x}
$$

The internal region above the bowl only contained one surface and it was horizontal. Thus, no axial velocity had to be determined for zone 2 . In the outer region, zone 3 , total velocity in axial direction was the result of summing Eqs. 4.7 and 4.19. It is shown in Eq. 4.21. It is noted that Eq. 4.19 was corrected with the $\alpha_{a x}$ constant in both zones.

$$
v_{a x i a l, 3}=v_{a x, l i n e r}+\alpha_{a x, 3} v_{s q, a x}
$$

Eqs. 4.13 and 4.19 could be further simplified if an additional assumption had been introduced. This assumption was that the piston bowl was perfectly cylindrical [139]. That would allow to replace the variable $V_{\text {bowl }}$ by $\left(\frac{\pi}{4} D_{\text {bowl }}^{2} L_{\text {bowl }}\right)$. A test was done to compare results using the real volume of the bowl and assuming a perfectly cylindrical bowl. The difference in gas speed (axial and radial) was significant: from $3 \%$ to $20 \%$. The tests presented in this thesis were therefore conducted using the real bowl volume, but the other option was also implemented in the final model as an alternative for the user.

The method to model swirl was based on comprehensive descriptions from Murakami et al. [138] and Hansen [139]. They divided the cylinder volume into two subvolumes as in Fig. 4.51. Since three different flow regions were considered, that means that the two inner regions were assumed to have the same swirl velocity. The first task was to check the validity of that assumption. If the assumption were inaccurate, the method could be easily extended from two to three zones. The results from CFD simulations were used for the assessment. In Fig. 4.43 it was clearly observed that the temporal evolution of swirl was different in each zone. Thus, considering the swirl velocity in the two inner zones to be equal is not a good approximation. Consequently, the method was extended to three separate zones.

The swirl model relied on a balance of angular momentum in each zone. Each flow region contained a volume that rotated as a rigid body, i.e. angular swirl velocity was uniform in the whole region. The model considered momentum exchange between zones 1 and 2 and between zones 
2 and 3, according to the notation indicated in Fig. 4.41. The momentum exchange was caused by mass transfer, due to squish, and by viscous shear. In addition, there was continuous momentum decay as a result of wall friction. In summary, the differential equation that represented the variation of angular momentum in each region had the form of Eq. 4.22.

$$
\frac{d\left(I_{i} \omega_{i}\right)}{d t}=\sum_{j} \tau_{s q u i s h, j-i}+\sum_{j} \tau_{v i s c, j-i}+\sum_{k} \tau_{f r i c, k}
$$

$I$ is moment of inertia of the gas volume, $\omega$ is angular velocity of the gas, $\tau_{\text {squish }}$ is angular momentum flux transported between contacting regions by the squish flow, $\tau_{v i s c}$ is angular momentum flux transmitted between regions by viscous shear and $\tau_{\text {fric }}$ is friction moment between gas and wall. Subscript $i$ refers to the flow region under consideration, subscript $j$ refers to adjacent flow regions and subscript $k$ to surfaces in contact with flow region $i$. Since there were three flow regions, the system to be solved was formed by three equations.

The system was solved each time step, $\Delta t$, to determine the angular swirl acceleration, $\dot{\omega}_{i}$, in each region. $\dot{\omega}_{i}$ was obtained according to Eq. 4.23 .

$$
\begin{aligned}
& \frac{d\left(I_{i} \omega_{i}\right)}{d t}=\omega_{i} \frac{d I_{i}}{d t}+I_{i} \frac{d \omega_{i}}{d t} \\
& \dot{\omega}_{i}=\frac{d \omega_{i}}{d t}=\frac{1}{I_{i}} \frac{d\left(I_{i} \omega_{i}\right)}{d t}-\omega_{i} \frac{d I_{i}}{d t}
\end{aligned}
$$

In the following time step, Eq. 4.24 was applied to obtain the current $\omega_{i}$.

$$
\omega_{i}(t)=\omega_{i}(t-1)+\dot{\omega}_{i} \Delta t
$$

The procedure needed an initial condition, which was an initial swirl ratio specified by the user. In this thesis, the nominal swirl ratio measured by the manufacturer of the cylinder-head was used. Depending on the opening of the swirl valve, swirl ratio could vary between 1.4 and 3 .

In the following lines, the methods to obtain the different terms in Eqs. 4.22 and 4.23 are clarified. The moments of inertia depend on geometry of the different regions. They were given by the expressions of Eqs. 4.25, 4.26 and 4.27, where the volumes, $V_{i}$, are found in Eqs. 4.28, 4.29 and 4.30 .

$$
I_{1}(t)=\frac{R_{b o w l}^{2}}{2} m_{1}(t)=\frac{R_{b o w l}^{2}}{2} \rho(t) V_{1}
$$




$$
\begin{gathered}
I_{2}(t)=\frac{R_{\text {bowl }}^{2}}{2} m_{2}(t)=\frac{R_{\text {bowl }}^{2}}{2} \rho(t) V_{2}(t) \\
I_{3}(t)=\frac{1}{2} \frac{R_{c y l}^{4}-R_{\text {bowl }}^{4}}{R_{c y l}^{2}-R_{\text {bowl }}^{2}} m_{3}(t)=\frac{R_{c y l}^{2}+R_{\text {bowl }}^{2}}{2} \rho(t) V_{3}(t) \\
V_{1}=V_{\text {bowl }} \\
V_{2}(t)=\pi R_{\text {bowl }}^{2}\left[L_{0}+s(t)\right] \\
V_{3}(t)=\pi\left(R_{c y l}^{2}-R_{\text {bowl }}^{2}\right)\left[L_{0}+s(t)\right]
\end{gathered}
$$

To calculate derivatives of moments of inertia, the derivatives of mass and volume in each region were needed. The time derivative of volumes, Eqs. 4.31, 4.32 and 4.33, could be directly calculated from Eqs. 4.28, 4.29 and 4.30 .

$$
\begin{gathered}
\frac{d V_{1}}{d t}=0 \\
\frac{d V_{2}}{d t}=\pi R_{\text {bowl }}^{2} v_{\text {pis }}(t) \\
\frac{d V_{3}}{d t}=\pi\left(R_{c y l}^{2}-R_{\text {bowl }}^{2}\right) v_{\text {pis }}(t)
\end{gathered}
$$

To determine mass flow rates moving from one region to another, it was necessary to take into account the variation of cylinder volume. Uniform compression and expansion was assumed. Thus, the volume fraction of each region was used to calculate the relative share of global volume variation and subtract it from the volume variation of the zone. Eq. 4.34 evaluated the mass flow rate from zone 1 to zone $2, \frac{d m_{1 \rightarrow 2}}{d t}$. It was formulated as the loss of mass in zone 1. $\frac{d m_{2 \rightarrow 3}}{d t}$, in Eq. 4.35, evaluated the mass gain of zone 3, i.e. the mass flow rate from zone 2 to zone 3.

$$
\begin{aligned}
\frac{d m_{1 \rightarrow 2}}{d t} & =-\rho(t)\left[\frac{d V_{1}}{d t}-\left(\frac{d V_{1}}{d t}+\frac{d V_{2}}{d t}+\frac{d V_{3}}{d t}\right) \frac{V_{3}}{V_{1}+V_{2}+V_{3}}\right] \\
\frac{d m_{2 \rightarrow 3}}{d t} & =\rho(t)\left[\frac{d V_{3}}{d t}-\left(\frac{d V_{1}}{d t}+\frac{d V_{2}}{d t}+\frac{d V_{3}}{d t}\right) \frac{V_{3}}{V_{1}+V_{2}+V_{3}}\right]
\end{aligned}
$$

Time derivatives of the moments of inertia were defined by Eqs. 4.36, 4.37 and 4.38 .

$$
\begin{gathered}
\frac{d I_{1}}{d t}=-\frac{R_{\text {bowl }}^{2}}{2} \frac{d m_{1 \rightarrow 2}}{d t} \\
\frac{d I_{2}}{d t}=\frac{R_{\text {bowl }}^{2}}{2}\left(\frac{d m_{1 \rightarrow 2}}{d t}-\frac{d m_{2 \rightarrow 3}}{d t}\right)
\end{gathered}
$$




$$
\frac{d I_{3}}{d t}=\frac{R_{c y l}^{2}+R_{b o w l}^{2}}{2} \frac{d m_{2 \rightarrow 3}}{d t}
$$

Eq. 4.37 was divided in two to separate the variation of the moment of inertia in zone 2 caused by mass transfer from zone 1 (Eq. 4.39) and by mass transfer to zone 3 (Eq. 4.40).

$$
\begin{aligned}
\left(\frac{d I_{2}}{d t}\right)_{1 \rightarrow 2} & =\frac{R_{\text {bowl }}^{2}}{2} \frac{d m_{1 \rightarrow 2}}{d t} \\
\left(\frac{d I_{2}}{d t}\right)_{2 \rightarrow 3} & =-\frac{R_{\text {bowl }}^{2}}{2} \frac{d m_{2 \rightarrow 3}}{d t}
\end{aligned}
$$

One of the terms of Eq. 4.22, $\tau_{\text {squish }}$, could be obtained from the derivatives of the moments of inertia. It was the flux of angular momentum due to squish. During the compression stroke, the momentum flux from zone 2 to zone 1 was given by Eq. 4.41 and the momentum flux from zone 3 to zone 2 was calculated with Eq. 4.42. During the expansion stroke, the momentum flux from zone 2 to zone 1 was obtained with Eq. 4.43 and the momentum flux from zone 3 to zone 2 was calculated with Eq. 4.44. The different $\beta_{s q}$ are adjustment constants.

$$
\begin{gathered}
\left(\tau_{\text {squish }, 2 \rightarrow 1}\right)_{\text {comp }}=-\beta_{s q, c, 2 \rightarrow 1} \omega_{2}\left(\frac{d I_{2}}{d t}\right)_{1 \rightarrow 2} \\
\left(\tau_{\text {squish }, 3 \rightarrow 2}\right)_{\text {comp }}=-\beta_{s q, c, 3 \rightarrow 2} \omega_{3} \frac{d I_{3}}{d t} \\
\left(\tau_{\text {squish }, 2 \rightarrow 1}\right)_{\text {exp }}=\beta_{\text {sq }, e, 2 \rightarrow 1} \omega_{1} \frac{d I_{1}}{d t} \\
\left(\tau_{\text {squish }, 3 \rightarrow 2}\right)_{\text {exp }}=\beta_{\text {sq,e,3 } \rightarrow 2} \omega_{2}\left(\frac{d I_{2}}{d t}\right)_{2 \rightarrow 3}
\end{gathered}
$$

The transfer of angular momentum between flow regions due to viscous shear was modeled with Eqs. 4.45 and 4.46. The momentum flux from zone 3 to zone $2, \tau_{v i s c, 3 \rightarrow 2}$, was calculated as suggested by Murakami [138]. It was equal to the transmitted torque between two co-axial cylinders placed at the representative radii of each volume, $\bar{R}_{\text {inner }}$ and $\bar{R}_{\text {outer }}$. These radii were those that divided each volume into two equal volumes. In the equations there also appeared mean piston speed, $c_{m}$. Momentum flux from zone 2 to zone $1, \tau_{v i s c, 2 \rightarrow 1}$, was formulated by analogy as the transmitted torque between two rotating disks of the same radius, $R_{\text {bowl }}$. Constants $\beta_{v s}$ were included for adjustment.

$$
\tau_{v i s c, 2 \rightarrow 1}=\beta_{v s, 2 \rightarrow 1} 0.035 \sqrt{0.74} \pi c_{m} \rho R_{\text {bowl }}^{4}\left(\omega_{2}-\omega_{1}\right)
$$




$$
\begin{gathered}
\tau_{\text {visc }, 3 \rightarrow 2}=\beta_{v s, 3 \rightarrow 2} 0.28 \sqrt{0.74} \pi c_{m} \rho\left[L_{0}+s\right] . \\
\cdot R_{\text {cyl }} \frac{\bar{R}_{\text {outer }}^{2} \bar{R}_{\text {inner }}^{2}}{\bar{R}_{\text {outer }}^{2}-\bar{R}_{\text {inner }}^{2}}\left(\omega_{3}-\omega_{2}\right)
\end{gathered}
$$

The third term in Eq. 4.22 was momentum deceleration due to wall friction, $\tau_{\text {fric }}$. The proposal of Murakami et al. was followed once again for consistency. Friction moments on horizontal surfaces were modeled with the approximate solution of von Kármán for disk-like surfaces, see Eqs. 4.47, 4.49, 4.51 and 4.52. Friction moments on vertical surfaces were solved considering the local friction coefficient for a flat plate, see Eqs. 4.48 and 4.50. Murakami et al. added the constants $\beta_{f r}$ to correct these approximations. In this thesis, three constants were employed for calibration. Each $\beta_{f r, i}$ was shared by all surfaces inside the same flow region. It is remarked that all friction moments were negative, since they contributed to swirl decay.

Surface 1: $\tau_{f r i c, 1}=-\beta_{f r, 1} 0.0365 \rho R_{\text {bowl }}^{5}\left(\frac{v}{R_{\text {bowl }} \bar{R}_{\text {inner }} \omega_{1}}\right)^{0.2} \omega_{1}^{2}$

Surface 2: $\tau_{f r i c, 2}=-\beta_{f r, 1} 0.0592 \pi \rho L_{\text {bowl }} R_{\text {bowl }}^{2}$.

$$
\cdot\left(\frac{2 v}{R_{\text {bowl }} \bar{R}_{\text {inner }} \omega_{1}}\right)^{0.2}\left(\bar{R}_{\text {inner }} \omega_{1}\right)^{2}
$$

Surface 3: $\tau_{f r i c, 3}=-\beta_{f r, 3} 0.0365 \rho\left[R_{c y l}^{5}\left(\frac{v}{R_{c y l} \bar{R}_{\text {outer }} \omega_{3}}\right)^{0.2}-\right.$

$$
\left.-R_{\text {bowl }}^{5}\left(\frac{v}{R_{\text {bowl }} \bar{R}_{\text {inner }} \omega_{2}}\right)^{0.2}\right] \omega_{3}^{2}
$$

Surface 4: $\tau_{f r i c, 4}=-\beta_{f r, 3} 0.0592 \pi \rho\left[L_{0}+s\right] R_{c y l}^{2}$.

$$
\cdot\left(\frac{2 v}{R_{\text {cyl }} \bar{R}_{\text {outer }} \omega_{3}}\right)^{0.2}\left(\bar{R}_{\text {outer }} \omega_{3}\right)^{2}
$$

Surface 5: $\tau_{f r i c, 5}=\tau_{\text {fric }, 3}$ 
Surface 6: $\tau_{\text {fric }, 6}=-\beta_{f r, 2} 0.0365 \rho R_{\text {bowl }}^{5}\left(\frac{v}{R_{\text {bowl }} \bar{R}_{\text {inner }} \omega_{2}}\right)^{0.2} \omega_{2}^{2}$

Entering the expressions of moments into Eq. 4.22, the three equations that define the system were obtained, Eqs. 4.53, 4.54 and 4.55. During the period of the engine cycle when valves are open, it is assumed that there is no squish motion. Therefore, when valves were open, the momentum fluxes due to squish, $\tau_{\text {squish }}$, were made equal to zero.

Zone 1: $\frac{d\left(I_{1} \omega_{1}\right)}{d t}=\tau_{\text {squish }, 2 \rightarrow 1}+\tau_{\text {visc }, 2 \rightarrow 1}+\tau_{\text {fric }, 1}+\tau_{\text {fric }, 2}$

Zone $2: \frac{d\left(I_{2} \omega_{2}\right)}{d t}=\tau_{\text {squish }, 3 \rightarrow 2}-\tau_{\text {squish }, 2 \rightarrow 1}+$

$$
+\tau_{v i s c, 3 \rightarrow 2}-\tau_{v i s c, 2 \rightarrow 1}+\tau_{\text {fric }, 6}
$$

Zone $3: \frac{d\left(I_{3} \omega_{3}\right)}{d t}=-\tau_{\text {squish }, 3 \rightarrow 2}-\tau_{\text {visc }, 3 \rightarrow 2}+$

$$
+\tau_{\text {fric }, 3}+\tau_{\text {fric }, 4}+\tau_{\text {fric }, 5}
$$

The third component of characteristic velocity is turbulent kinetic energy (TKE). Turbulence is a complex process that concentrates many research efforts. Abundant studies about turbulence can be found in the literature, but studying the topic of turbulence in depth was out of the scope of this thesis. Instead, a less specialized point of view was kept. Reference works about multizone models were surveyed to understand the approaches employed to model turbulence. The authors of these works proposed methods based on thorough reviews of relevant works or on experiments. A standard approach was to use a pair of coupled equations. One equation modeled the variation of turbulence kinetic energy while the other equation modeled the dissipation rate. Morel et al. [133] used one pair of equations for each one of the three flow regions of their model. Hansen [140] reduced complexity by considering only an internal and external zone, as he did to model swirl. The internal zone included two flow regions as in Fig. 4.51. Schubert et al. [136] applied a global curve of turbulent kinetic energy for the whole cylinder. They calculated the variation of turbulence kinetic energy with a differential equation, but for dissipation they used a simplified formula. Despite the simplifications, 
they obtained reasonable agreement with CFD simulations. In Fig. 4.43, it was seen that turbulent velocities in the different zones were significantly dissimilar. Therefore, considering one global velocity is not a completely rigorous assumption. However, it was also seen that turbulent velocity was generally of lower magnitude than the other velocity components (see Figs. 4.43, 4.44-4.47 or 4.50). In addition, it will be shown later that the turbulence equations are formed by several terms; evaluating the different terms in each zone involves adding many uncertainties. On account of the presented reasoning, it was decided to follow the global approach of Schubert et al., although it entails a certain inaccuracy. The good results obtained by the authors make reasonable to rely on their proposal.

$$
\frac{d T K E}{d t}=\frac{1}{\rho}\left(T \dot{K} E_{c o m p}+T \dot{K} E_{\text {squish }} T \dot{K} E_{\text {int }}+T \dot{K} E_{\text {exh }}\right)-\varepsilon
$$

As mentioned, the model used a differential equation that represented the variation of turbulent kinetic energy over time. The implemented equation was Eq. 4.56. Among the terms, all mechanisms that generate significant turbulence were included. $T \dot{K} E_{\text {comp }}$ represents the production of turbulent kinetic energy by compression. It is given by Eq. 4.57 when density is increasing; otherwise it is equal to zero. $T \dot{K} E_{\text {squish }}$ is defined by Eq. 4.58 and it takes into account the turbulence generated by squish motion. The equation uses the squish velocities previously calculated in the model of radial and axial squish. $T \dot{K} E_{\text {comp }}$ and $T \dot{K} E_{\text {squish }}$ only apply when cylinder valves are closed. On the contrary, $T \dot{K} E_{\text {int }}$ is evaluated when intake valves are open, and $T \dot{K} E_{\text {exh }}$ is evaluated when exhaust valves are open. They represent the turbulence produced by the intake and exhaust processes and are defined by Eq. 4.59 and Eq. 4.60, respectively. Finally, $\varepsilon$ is the term responsible for turbulence dissipation, and it is approximated by Eq. 4.61 .

$$
\begin{gathered}
T \dot{K} E_{\text {comp }}=\gamma_{\Delta p} \frac{2}{3} T K E \frac{d \rho}{d t} \\
T \dot{K} E_{\text {squish }}=\gamma_{s q} 0.5\left[v_{\text {radial }, 1}^{2}+\left(\alpha_{a x, 1} v_{s q, a x}\right)^{2}\right]\left|\frac{d \rho}{d t}\right| \\
T \dot{K} E_{\text {int }}=\gamma_{\text {int }} 0.5 v_{\text {pis }}^{2}\left|\frac{d \rho}{d t}\right| \\
T \dot{K} E_{\text {exh }}=\gamma_{\text {exh }} 0.5 v_{\text {pis }}^{2}\left|\frac{d \rho}{d t}\right|
\end{gathered}
$$




$$
\varepsilon=\gamma_{\varepsilon} \frac{T K E^{1.5}}{\sqrt[3]{V}}
$$

In summary, the procedures to determine the different velocity components of Eq. 2.46 were explained in detail. The expressions obtained from the procedures were implemented in the multizone model. Finally, a different characteristic velocity was calculated for each surface inside the combustion chamber, Eqs. 4.62-4.67.

$$
\begin{aligned}
& \text { Surface 1: } v_{\text {char }, 1}=\sqrt{v_{\text {radial }, 1}^{2}+\omega_{1}^{2}+T K E^{2}} \\
& \text { Surface 2: } v_{\text {char }, 2}=\sqrt{v_{\text {axial }, 1}^{2}+\omega_{1}^{2}+T K E^{2}} \\
& \text { Surface 3: } v_{\text {char }, 3}=\sqrt{v_{\text {radial }, 3}^{2}+\omega_{3}^{2}+T K E^{2}} \\
& \text { Surface 4: } v_{\text {char }, 4}=\sqrt{v_{\text {axial }, 3}^{2}+\omega_{3}^{2}+T K E^{2}} \\
& \text { Surface 5: } v_{\text {char }, 5}=\sqrt{v_{\text {radial }, 3}^{2}+\omega_{3}^{2}+T K E^{2}} \\
& \text { Surface 6: } v_{\text {char }, 6}=\sqrt{v_{\text {radial }, 2}^{2}+\omega_{2}^{2}+T K E^{2}}
\end{aligned}
$$

\section{Calibration with CFD}

The in-cylinder gas motion submodel was calibrated using data from CFD simulations. All tests of Table 4.7 were simulated. Then, velocity curves obtained from post-processing were employed as reference for calibrating the gas motion model. A tuning constant had been included in each term used to determine the various velocity components. The calibration consisted in searching values of the tuning constants that produced velocity curves most similar to the references. Fitting was accomplished through the minimization of mean squared errors of all tests. Each velocity component was optimized with a separate MATLAB ${ }^{\circledR}$ script. The tuning constants had been named according to the velocity component.

Five so-called alpha constants were used to adjust squish influence. For radial squish, which affected surfaces in three flow regions, there were three constants included in Eqs. 4.14-4.16: $\alpha_{r a d, 1}, \alpha_{r a d, 2}$ and $\alpha_{r a d, 3}$. The other two constants were used to adjust axial squish, which is only parallel to two surfaces: bowl lateral wall, in region 1, and liner wall, in region 3. The constants were $\alpha_{a x, 1}$ and $\alpha_{a x, 3}$, from Eqs. 4.8 and 4.7, respectively. 
Swirl velocity was adjusted with nine beta constants. Four of them were present in the squish terms, Eqs. 4.41-4.44. They were $\beta_{s q, c, 2 \rightarrow 1}$ and $\beta_{s q, c, 3 \rightarrow 2}$ for the compression stroke and $\beta_{s q, e, 2 \rightarrow 1}$ and $\beta_{s q, e, 3 \rightarrow 2}$ for the expansion stroke. Each constant corrected momentum flux in one interface between flow regions. Analogously, there were two constants to adjust viscous shear, $\beta_{v s, 2 \rightarrow 1}$ and $\beta_{v s, 3 \rightarrow 2}$, in Eqs. 4.45 and 4.46, respectively. Moreover, three constants, one per flow region, were considered to adjust wall friction. They were $\beta_{f r, 1}, \beta_{f r, 2}$ and $\beta_{f r, 3}$, used in Eqs. 4.47-4.52.

Finally, five gamma constants were employed to adjust turbulent motion. Constants were included in the following terms: $\gamma_{\Delta p}$ for compression (Eq. 4.57), $\gamma_{s q}$ for squish (Eq. 4.58), $\gamma_{i n t}$ for intake (Eq. 4.59), $\gamma_{\text {exh }}$ for exhaust (Eq. 4.60) and $\gamma_{\varepsilon}$ for dissipation (Eq. 4.61). The curve of turbulent velocity was applied to the whole cylinder.

In total, the in-cylinder gas motion submodel has 19 calibration constants.

Next, results of the calibration of two tests are reported. Although the calibration was done for all tests simultaneously, only two tests, 1500low and 3500high, are covered for reasons of brevity. The tests were selected to show representative results of two engine speeds and two swirl ratios. The results of the other tests were similar to these; no different behaviors were observed. Figs. 4.53 to 4.56 assess the adjustment for the different velocity components, flow regions and the two mentioned tests. Each graph examines one flow region and contains four curves: velocity calculated by the gas motion model and velocity obtained from CFD simulations for both tests, during the closed cycle.
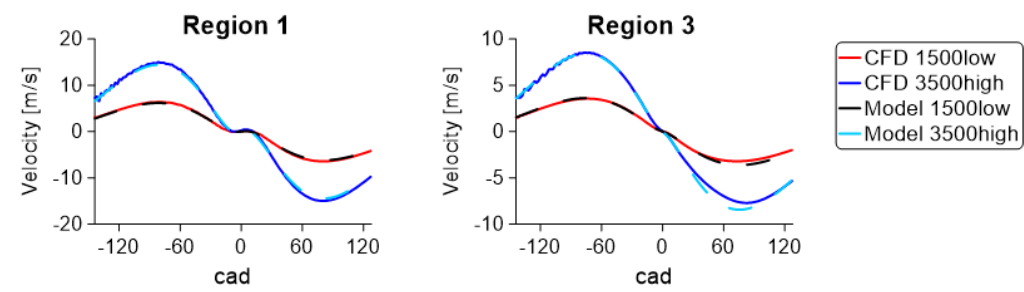

Figure 4.53: Adjustment of axial velocity component.

Results of the axial velocity component are shown in Fig. 4.53. The graph on the left contains the results of region 1, the piston bowl. The squish effect was adjusted to correct the curve around top dead center, when squish was more influential. A value of $\alpha_{a x, 1}$ equal to 0.45 provided 
the best fit. Region 3, the outer annular volume in contact with the piston crown, the liner and the external part of the firedeck, is examined in the graph on the right. The constant $\alpha_{a x, 3}$ was given a value of 0.15 . In general, there was good agreement between curves from CFD and from the model. Only in the middle of the expansion stroke, velocity was very slightly underpredicted by the model. In region 2, the inner volume above the bowl, the axial component was not parallel to any surface, as the only surface in flow region 2 was horizontal. Thus, this component was not even calculated in the model because it was irrelevant for convective heat transfer. Overall, very good agreement between velocity simulated with CFD and velocity calculated with the model was observed in axial direction.
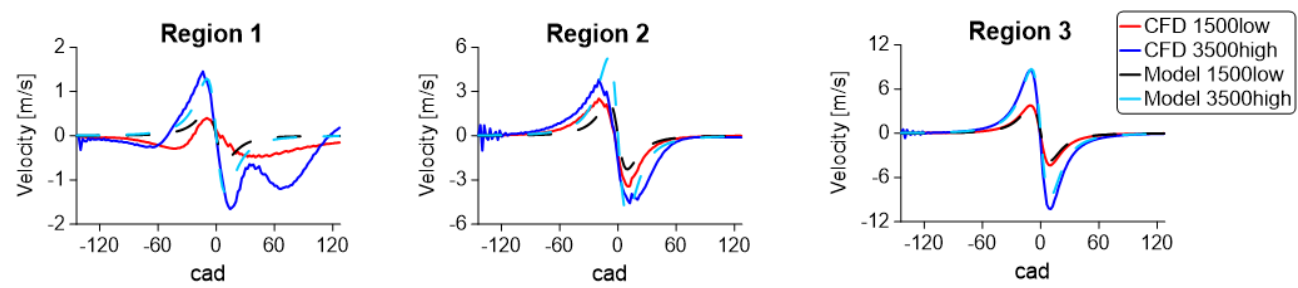

Figure 4.54: Adjustment of radial velocity component.

The radial velocity component consisted exclusively in squish motion. Results of the adjustment in the three flow regions are shown in Fig. 4.54. The values of the constants that best fitted the CFD curves were $\alpha_{\text {rad }, 1}=$ $0.17, \alpha_{r a d, 2}=0.7$ and $\alpha_{r a d, 3}=1.15$. In regions 1 and 2 , there were some behaviors that were not taken into account by the simple squish model. Curves obtained from the model were adjusted to follow the general trends, but presented limitations. In region 3, trends were much more predictable. The model achieved good agreement with CFD simulations in this region.
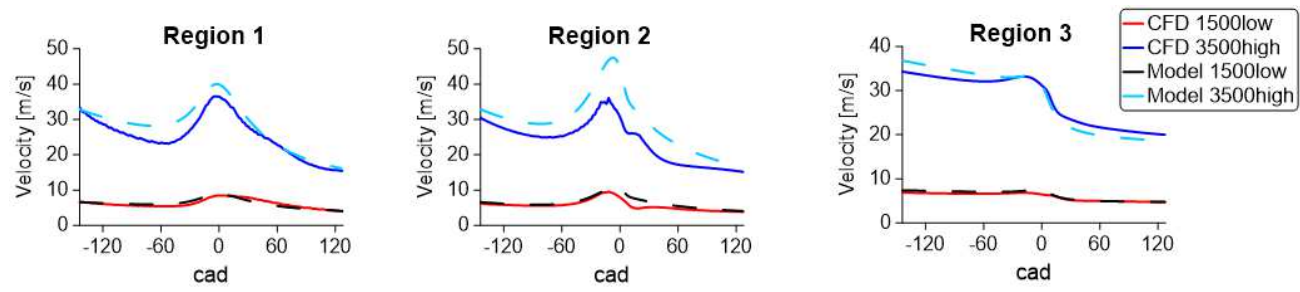

Figure 4.55: Adjustment of swirl velocity component.

In the model, swirl motion was controlled by three mechanisms. Due 
to the higher complexity of the swirl model in comparison with other velocity components, the number of tuning constants was relatively large. A total of nine constants were adjusted. To regulate the influence of squish during compression, $\beta_{s q, c, 2 \rightarrow 1}$ and $\beta_{s q, c, 3 \rightarrow 2}$ were given the values 1.1 and 0.8 , respectively. During expansion, $\beta_{s q, c, 2 \rightarrow 1}$ and $\beta_{s q, c, 3 \rightarrow 2}$ were equal to 1.2 and 1.4, respectively. The effect of squish was very important around the top dead center and could increase swirl motion considerably. The other mechanism producing momentum transfer among flow regions was viscous shear. The constants of the two interfaces, $\beta_{v s, 2 \rightarrow 1}$ and $\beta_{v s, 3 \rightarrow 2}$ were adjusted with the values of 1 and 0.5 , respectively. Finally, the three constants to calibrate swirl deceleration due to wall friction, $\beta_{f r, 1}, \beta_{f r, 2}$ and $\beta_{f r, 3}$, took the value 1.3. Trends observed in the CFD simulations were generally reproduced by the model, as shown in Fig. 4.55. However, swirl velocity was overpredicted in some cases. In particular, the test at $3500 \mathrm{rpm}$ and high swirl ratio presented a clear overestimation of swirl in flow region 2, specially in the peak near the top dead center. In general, the reliability of the model regarding swirl prediction was acceptable, but some deviations could be expected under certain conditions.

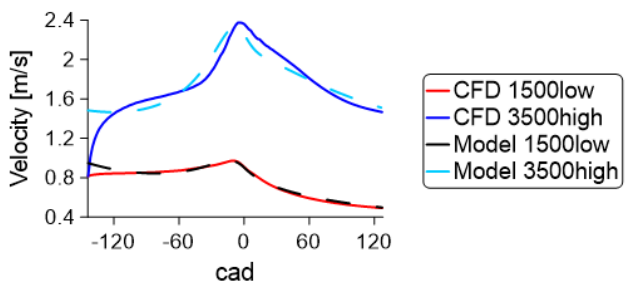

Figure 4.56: Adjustment of turbulent velocity component.

Lastly, the results of calibrating the turbulence model are presented in Fig. 4.56. There is only one graph because turbulence was modeled globally, encompassing all surfaces in the cylinder. Five constants were used to adjust turbulent kinetic energy. Among them, the model was found to be most sensitive to $\gamma_{s q}$. This constant regulated the effect of squish and was given a value of 0.013 after calibration. Another constant linked to turbulence production, $\gamma_{\Delta p}$, was set equal to 0.9 . The dissipation constant, $\gamma_{\varepsilon}$, took a value of 6.8. The other two tuning constants, $\gamma_{\text {int }}$ and $\gamma_{e x h}$, corrected the influence of the intake and exhaust processes on turbulence generation. As such, they could not be adjusted with CFD simulations because these were limited to the closed cycle. Instead, the constants were manually adjusted using curves of turbulent motion from 
the literature as reference [140] [136]. Their values were finally set to $\gamma_{\text {int }}=0.1$ and $\gamma_{\text {exh }}=0$.

The adjusted constants were entered in the model for all subsequent studies.

\subsubsection{Thermal analysis}

\section{Heat transfer coefficient calculation}

The multizone model was integrated into the thermal network that formed the Nodal Model. That implied the necessity to determine convective conductances in order to define the thermal connection between in-cylinder gas nodes and cylinder wall nodes. A convective conductance, Eq. 2.40, is the product of a contact area and a heat transfer coefficient. In the multizone model, the heat transfer coefficient was determined using a correlation directly derived from the Colburn analogy, Eq. 2.14, which is shown again here. Morel et al. proposed the usage of this correlation in the report of their multizone model [133]. Other authors of multizone models such as Hansen [141], Eiglmeier et al. [132] and Schubert et al. [136] followed this proposal. To evaluate the friction factor, $f$, Blasius' law, which is the expression given in Eq. 4.68, can be employed. Constant $C_{f}$ has the value 0.046 for flat plate boundary layers and 0.067 for fully developed pipe flow. Morel et al. and Hansen used the mean of both values, 0.0565 , and the same was done in this thesis. All fluid properties must be evaluated at film temperature, the mean of bulk gas temperature and wall temperature.

$$
\begin{gathered}
h=\rho C_{p} v \operatorname{Pr}^{2} / 3 \frac{f}{2} \quad(2.14) \\
f=C_{f} \operatorname{Re}^{-0.25}=C_{f}\left(\frac{\rho v L_{\text {char }}}{\mu}\right)^{-0.25}
\end{gathered}
$$

Variable $v$ is the characteristic gas velocity affecting a determined surface, solved with the gas motion submodel and with Eq. 2.46, as explained in Section 4.3.1. Therefore, a different heat transfer coefficient had to be calculated for each surface. Correspondingly, since the heat transfer coefficient is used to obtain gas temperature, six nodes representing the different gas temperatures had to be considered, one per heat transfer coefficient. The reason why the temperature of the gas nodes is dependent on the heat transfer coefficient was explained in Section 3.3, in particular 
with the demonstration of Eq. 3.6. The gas nodes were connected to the corresponding wall nodes through contact areas.

The Reynolds number of the friction factor has to be evaluated for a characteristic length, $L_{\text {char }}$. To maintain consistency, an approach proposed by Morel et al. and applied by Hansen was followed in this thesis. The approach consisted in using approximations of the boundary layer thickness on the different surfaces as characteristic lengths. They were the following:

1. Bowl ground: $L_{c h a r}(t)=0.05\left(L_{\text {bowl }}+\left[L_{0}+s(t)\right]\left(\frac{D_{\text {rim }}}{D_{\text {bowl }}}\right)^{2}\right)$

2. Bowl lateral wall: $L_{\text {char }}=0.05 D_{\text {bowl }}$

3. Piston crown: $L_{\text {char }}(t)=0.05\left[L_{0}+s(t)\right]$

4. Liner wall: $L_{\text {char }}=0.05 D_{\text {cyl }}$

5. Outer firedeck: $L_{\text {char }}(t)=0.05\left[L_{0}+s(t)\right]$

6. Inner firedeck: $L_{\text {char }}(t)=0.05\left(\left[L_{0}+s(t)\right]+L_{\text {bowl }}\left(\frac{D_{\text {rim }}}{D_{\text {bowl }}}\right)^{2}\right)$

$L_{\text {bowl }}$ is the bowl depth and $D_{\text {rim }}$ is the bowl diameter at the rim. $\left[L_{0}+\right.$ $s(t)]$ is the instantaneous distance between the piston top and the firedeck, a variable that has already been used in Section 4.3.1. Notation $L_{\text {char }}(t)$ indicates that thickness on a determined surface changes instantaneously over time, while $L_{\text {char }}$ indicates that it is constant. It is noted that the boundary layer thickness of surfaces 3 and 5 were assumed to be equal.

\section{Calibration and evaluation procedures}

The calibration procedure of the gas motion submodel was explained in the previous section, Section 4.3.1. The multizone convection model also 
underwent a calibration. In particular, the in-cylinder heat transfer coefficients were scaled with a tuning constant. The same value of the constant was used for all tests: all surfaces in the chamber, all cylinders, all engine speeds and the two swirl ratios. The adjustment was performed at the lowest swirl ratio, considering the seven tests at low swirl. Later, the adjusted value of the tuning constant was also employed for the tests at high swirl. This allowed to check whether the influence of swirl was well captured by the gas motion model alone, without a need for further adjustment. The aim was to evaluate the potential of the multizone model to predict wall temperatures under all conditions of the test campaign, after calibrating one single scaling constant. The prediction would be satisfactory if the model were able to capture the influences of engine speed and swirl at the different measurement locations. Engine speed and swirl are connected to in-cylinder heat transfer through gas motion. The capacity to predict local temperatures relies on the zonification of the heat transfer coefficient, which should achieve an improved prediction of the heat distribution throughout the cylinder walls.

To calibrate the tests at low swirl ratio, estimated curves of heat loss obtained with the energy balance method were used as reference. The method was described in Section 2.2.2 and the curves were employed in Sections 4.2 and 4.3.1. A simple optimization method was implemented to fit the value of the convection constant. The user had to enter an upper and a lower bound to specify the range in which the optimum had to be searched. Next, the range was divided into 10 sub-ranges. That provided 11 values of the tuning constant. The model had to be tested with those 11 values. For each value of the constant, all tests at low swirl were simulated. Since each cylinder was simulated separately, a total of 28 simulations were run. In each simulation, the instantaneous curve of heat flux from the gas to the cylinder walls was compared with the corresponding experimental curve and the mean absolute error was computed. For each value of the tuning constant, the mean errors of the 28 simulations were summed. This was the variable to be compared. After comparing the error for the 11 values of the constant, the range between the two values with lowest error was chosen as the optimal. Next, that optimal range was subdivided into 10 sub-ranges to gain further precision. After 11 evaluations in the new range, the optimal value of the tuning constant was found. Thus, each optimization process consisted of 616 simulations.

It must be noted that the experimental and the modeled heat flux curves were only compared in the closed part of the cycle, because that 
is the period when the energy balance method could be applied. When valves were open, there was no experimental data to be used as reference. Nevertheless, this was not considered a significant drawback because most heat rejection to cylinder walls occurs during the closed cycle, when the gas reaches higher temperatures.

Two correlations were optimized. The first correlation was the modified Woschni formula from the initial version of the Nodal Model. This approach used one common heat transfer coefficient for all surfaces inside the cylinder. The second correlation was the one discussed in the present section. This multizone correlation considered a different heat transfer coefficient for each of the six surfaces into which the cylinder walls were divided, because characteristic velocity is different for each surface. The values of the calibration constants that best fitted the reference heat curves were very similar: 1.795 for the global Woschni correlation and 1.735 for the multizone correlation.

\section{Heat flux study}

The first analysis of this section deals with the curves of heat flux from the gas to the cylinder walls. These heat flux curves were the data used for calibration. Therefore, the trends seen in these curves provided important information concerning what to expect from the model. In the graphs of Fig. 4.57, three curves are plotted: experimental heat flux calculated with the energy balance method, heat flux obtained with a Woschni correlation applied to the whole chamber (single-zone model, called global in the figure) and heat flux obtained with the multizone model described in this section, which considers six different heat transfer coefficients in different zones of the combustion chamber (called Zonal in Fig. 4.57). Each row of graphs is referred to a different engine speed and each column to a swirl ratio. Graphs on the left side are for the lowest value of swirl ratio $(\mathrm{SR}=1.4)$ while graphs on the right are for the highest $(\mathrm{SR}=3)$. Engine speeds shown in this figure are, from top to bottom, 1000, 2000 and 3000 rpm. All results are from cylinder 3, but the results shown in this figure are representative of all cylinders and are also representative of all tests in the campaign.

At low swirl, both models presented very similar results and good agreement with the experimental curve. The only exception was that, at $3000 \mathrm{rpm}$, the experimental curve had slightly higher slopes than those predicted by the models. Two conclusions can be extracted from the 

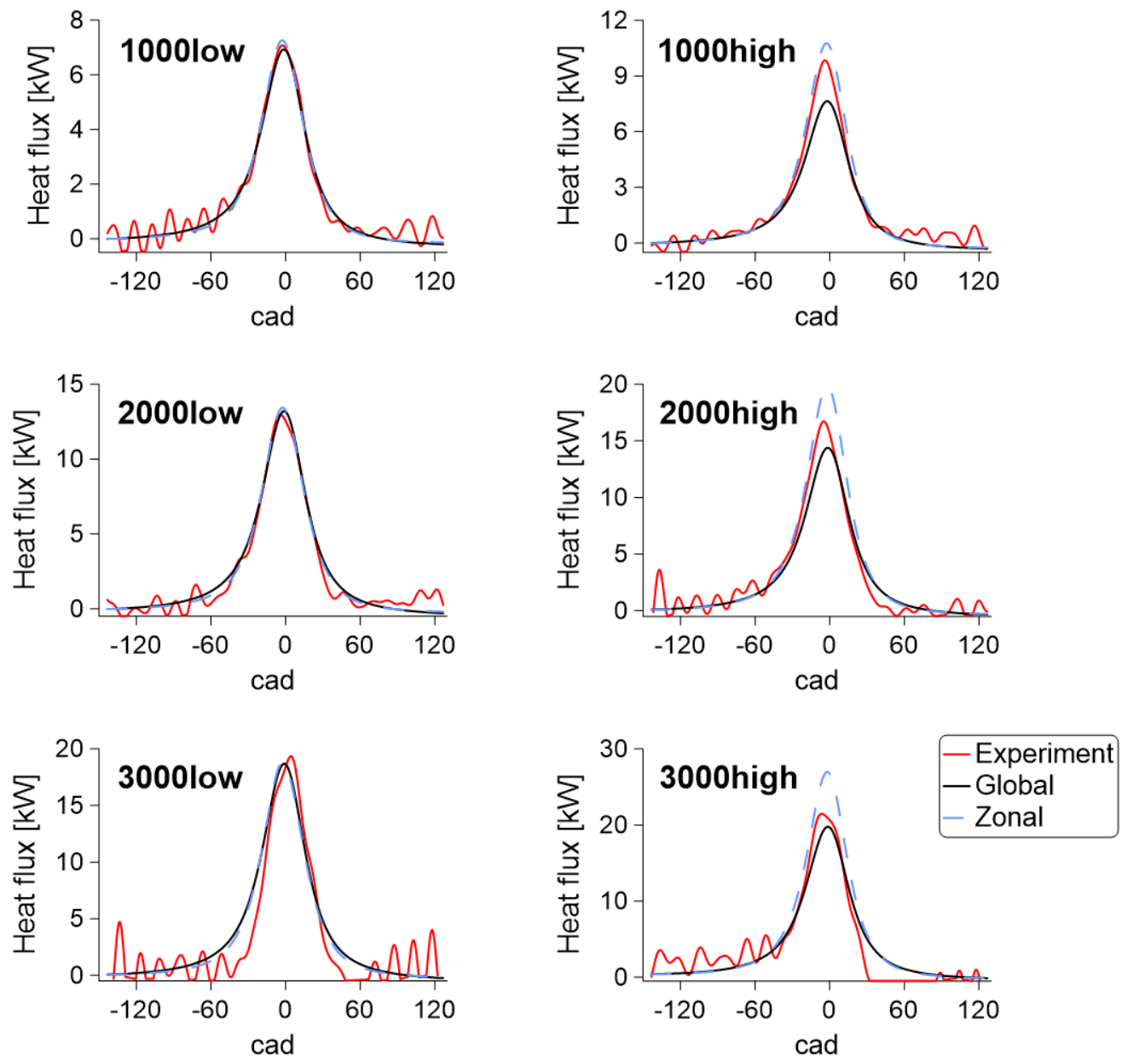

Figure 4.57: Comparison of heat flux obtained with different methods: experiment, global model and multizone model. 
graphs at low swirl. First, the results of both models were very similar, even though they used different correlations for calculating the in-cylinder heat transfer coefficient. That is not surprising, since both correlations are based on the same theory of the Colburn analogy. Regarding the multizone model, it should provide better prediction of the temperature distribution but that cannot be seen in this analysis; it will be assessed later, during the temperature study. Second, both the global and the multizone model were able to predict the heat flux for different engine speeds under motored operation. This seems a contradiction with results shown in Section 4.2, in which at $3000 \mathrm{rpm}$ there was an overestimation of mean wall temperatures. It was commented that a possible solution to this problem was to replace the constant heat transfer coefficients on the coolant side by a correlation that took into account coolant flow rate. The simulations reported in the present study already included the coolant correlation for both models (global Woschni and multizone). Therefore, it can be concluded that the correlation was effective in improving the predictions of the Nodal Model. All details about the correlation for convective heat transfer in the coolant are given in Section 4.4 .

In the tests at high swirl, on the right side of Fig. 4.57, there were clear differences among the curves. At $1000 \mathrm{rpm}$, during most of the compression and expansion strokes, heat flux obtained from both models agreed fairly well with the experimental curve. However, around top dead center $\left(0^{\circ}\right)$, the peak of heat flux was clearly underpredicted by the global model and slightly overpredicted by the multizone model. At $2000 \mathrm{rpm}$, the prediction of the global model improved but the peak was still underestimated. The prediction of the multizone model was worse than at $1000 \mathrm{rpm}$. At $3000 \mathrm{rpm}$, the slope during compression was well captured by the models but, during expansion, the heat flux dropped at a higher rate than predicted. At this engine speed, the global Woschni correlation slightly underpredicted the peak of heat flux while the multizone model clearly overpredicted the peak. Overall, it can be concluded that, during most of the closed cycle, predictions from the models were acceptable but, around the top dead center, the global model underpredicted heat flux and the multizone model overpredicted it. That is relevant because, due to the high gas temperature at the top dead center, a large portion of heat is rejected in this period. The issue was worse at high engine speed for the global model and at low engine speed for the zonal model. Since predictions at low swirl were arguably good for different engine speeds, the results at high swirl suggest that the influence of swirl and possible in- 
teractions between engine speed and swirl (e.g. squish) were not properly reproduced.

\section{Temperature prediction analysis}

The study of heat flux lacked spatial resolution, since only the total heat loss could be analyzed. The study could not provide details such as the distribution of heat to the different areas of the cylinder. A study of wall temperatures, in contrast, allows to assess the prediction accuracy at the different measurement locations on the firedeck and liner. It is reminded that the central motivation of testing a multizone model is to improve local predictions, which is the main deficiency of the Nodal Model. The study of wall temperatures is condensed in Fig. 4.58. The plotted magnitude, $\varepsilon_{T}$, is the difference between the modeled and the experimentally measured temperature at each location, i.e. $T_{\text {mod }}-T_{\text {exp }}$. The horizontal axis measures the error of the global convection model. In Section 4.2, it was found that the global model was far from perfect. Temperature in the firedeck was underpredicted near the center and overpredicted in the periphery. In the liner, temperature tended to be overpredicted near the top, while at the bottom a combination of underpredictions and correct estimations was observed. On the $\mathrm{Y}$ axis, the error of the multizone convection model is indicated. Ideally, all data points should be on a horizontal line that passes through $y=0$. That would mean that the multizone model predicts local temperatures with zero error. The diagonal lines indicate locations in the graph in which the errors of both models are equal. Therefore, data points in the upper and lower quarters mean that the error of the multizone model is higher than the error of the global model. On the contrary, data points in the lateral quarters indicate that the prediction of the multizone model is better than the prediction of the global model. A bigger concentration of data points in the lateral quarters would lead to the conclusion that the multizone model is superior to the global model in predictive power.

In the upper left graph, the locations on the firedeck are analyzed for the low swirl tests. Engine speeds or operating points are plotted in different hues of blue. Darker hues indicate higher engine speed and lighter hues mean lower engine speed. For each operating point, 54 data points are plotted, as there were 54 sensors in the firedeck (the experimental installation was thoroughly explained in Section 4.2.1). All data points are close to the diagonal, which means that both models predicted similar 

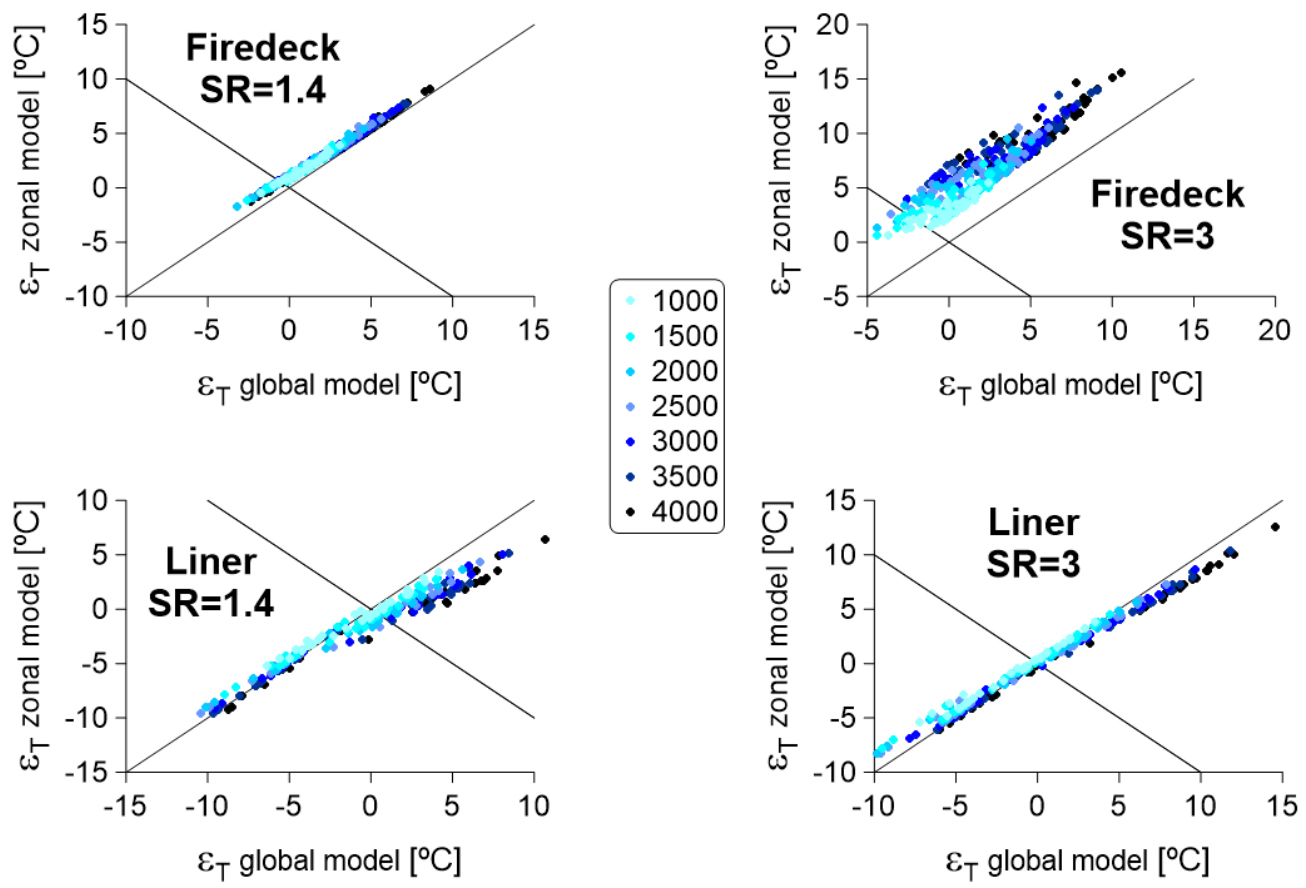

Figure 4.58: Temperature error comparison of a multizone and a global in-cylinder convection model in motored operation. 
temperatures. However, they are slightly above the diagonal, meaning that temperatures predicted by the multizone model were always slightly higher than those predicted by the global model. This helped to improve predictions at those locations were temperatures had been underpredicted by the global model, but was detrimental for most data points. It can also be observed that low engine speed data points presented lower error values.

The upper right graph shows the results for the firedeck and the tests with higher swirl ratio. All points are clearly above the diagonal. Thus, the multizone model calculated significantly higher temperatures than the global model. This is a clear indicator that the zonal model overestimated the effect of swirl, at least in the firedeck. The fact is consistent with the overestimation of the heat flux peak observed in Fig. 4.57. It was reported in Section 4.3.1 that swirl velocities were overpredicted by the gas motion model under concurring conditions of high swirl ratio and high engine speed in the inner cylinder regions. This is a likely error source in the center of the firedeck. A strong influence of engine speed can be observed, suggesting cross-interactions with swirl.

The graph on the lower left contains the 34 data points of the liner, from the tests at low swirl. In that case, most data points are found in the lateral quarters, on the left or on the right. That means that the multizone model improves the prediction of the global model. The results are distributed along a line, but this line has a slower slope than the diagonal, which is favorable to the predictions of the multizone model. In the multizone model, the whole liner had the same heat transfer coefficient, like in the global model. The advantage of the zonal model must be therefore in the improved consideration of gas velocity. In particular, the Woschni correlation of the Nodal Model used the mean piston speed as axial velocity, while the multizone approach considered an instantaneous gas velocity which additionally took into account the effect of squish.

Finally, in the graph on the lower right side, predictions for the liner in the high swirl tests can be assessed. As at low swirl, the data points are mostly found on the left and the right quarters, indicating a better prediction of the zonal model. However, they are close to the diagonal, which means that the results of the multizone model were very similar to those of the global model. It is noteworthy that, while the swirl effect was overestimated on the firedeck, the same did not happen in the liner. This fact may point to the inner zones as originators of the overestimation of 
swirl effect.

\section{Heat transfer coefficient analysis}

To shed light on the observed trends, the heat transfer coefficients obtained with the multizone model were analyzed. Fig. 4.59 shows curves of heat transfer coefficients for the different surfaces considered within the cylinder and for the two levels of swirl ratio. There is one graph for each surface. Each graph contains three curves. Two of them show the evolution of the heat transfer coefficient over the engine cycle at the two levels of swirl ratio. The third curve is the boundary layer thickness, used as characteristic length. The plotted curves are representative of all operating conditions. They were extracted from the test at $2000 \mathrm{rpm}$ and from cylinder 1 .

At all locations, the heat transfer coefficient curves are very similar for both swirl ratios over most of the engine cycle. The curve of high swirl is slightly above, indicating a higher heat transfer rate. An exception to the similarity of curves is the peak around the compression top dead center. The high swirl test presented a clearly higher heat transfer coefficient at that point. In all surfaces, the difference was similar, between $10 \%$ and $15 \%$, except in surface 2 , the bowl lateral wall, where the difference was $27 \%$. The later surface is in the inner region, but it is part of the piston, not the firedeck. Therefore, the singular increase of heat transfer coefficient in surface 2 does not help to explain why the firedeck presented an overestimation of swirl influence while the liner did not.

However, there is another finding that could be a likely responsible. In surfaces 3 and 5 , piston crown and outer firedeck respectively, it is apparent that there is a second peak at the exhaust top dead center, around $360^{\circ}$. This peak was not related to high gas velocity but to a low value of boundary layer thickness, used as characteristic length. Comparing the different graphs, it can be observed that the characteristic lengths for surfaces 3 and 5 reached the lowest values. They were obtained with Eqs. 4.71 and 4.73, following the proposal of Morel et al. [133]. In particular, the minimum value of $L_{c h a r}$ is $5 \%$ of the squish height. In the engine under study, the minimum was equal to $75 \mu \mathrm{m}$. Low values of $L_{\text {char }}$ promoted high peaks of heat transfer coefficient, as can be deduced from the equations presented at the beginning, of Section 4.3.2, Eqs. 2.14 and 4.68. Indeed the heat transfer coefficient reached higher values in surfaces 3 and 5 than in the rest. This behavior can explain the overestimation of temperatures in the outer area of the firedeck. 

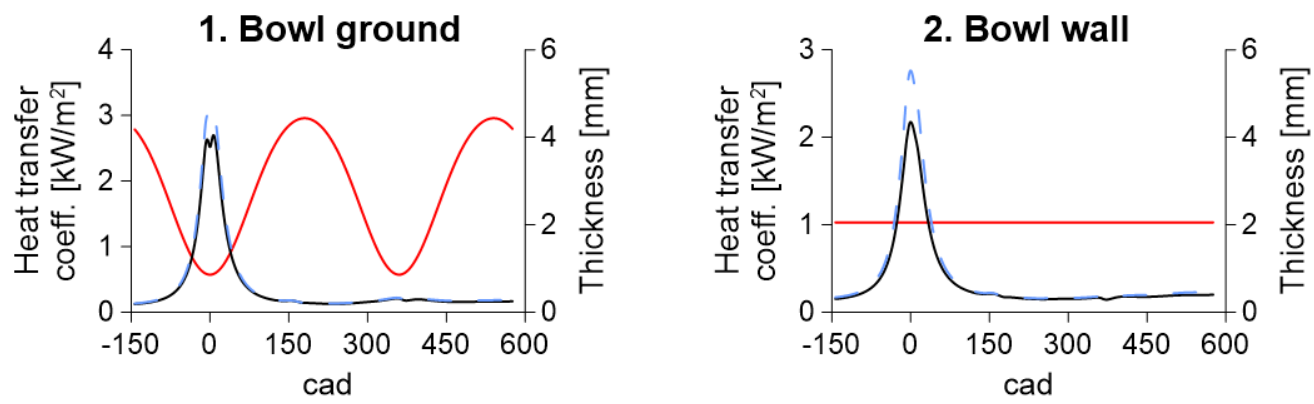

3. Piston crown

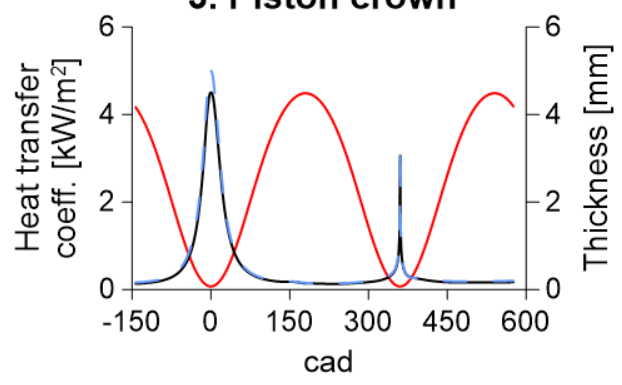

4. Liner wall
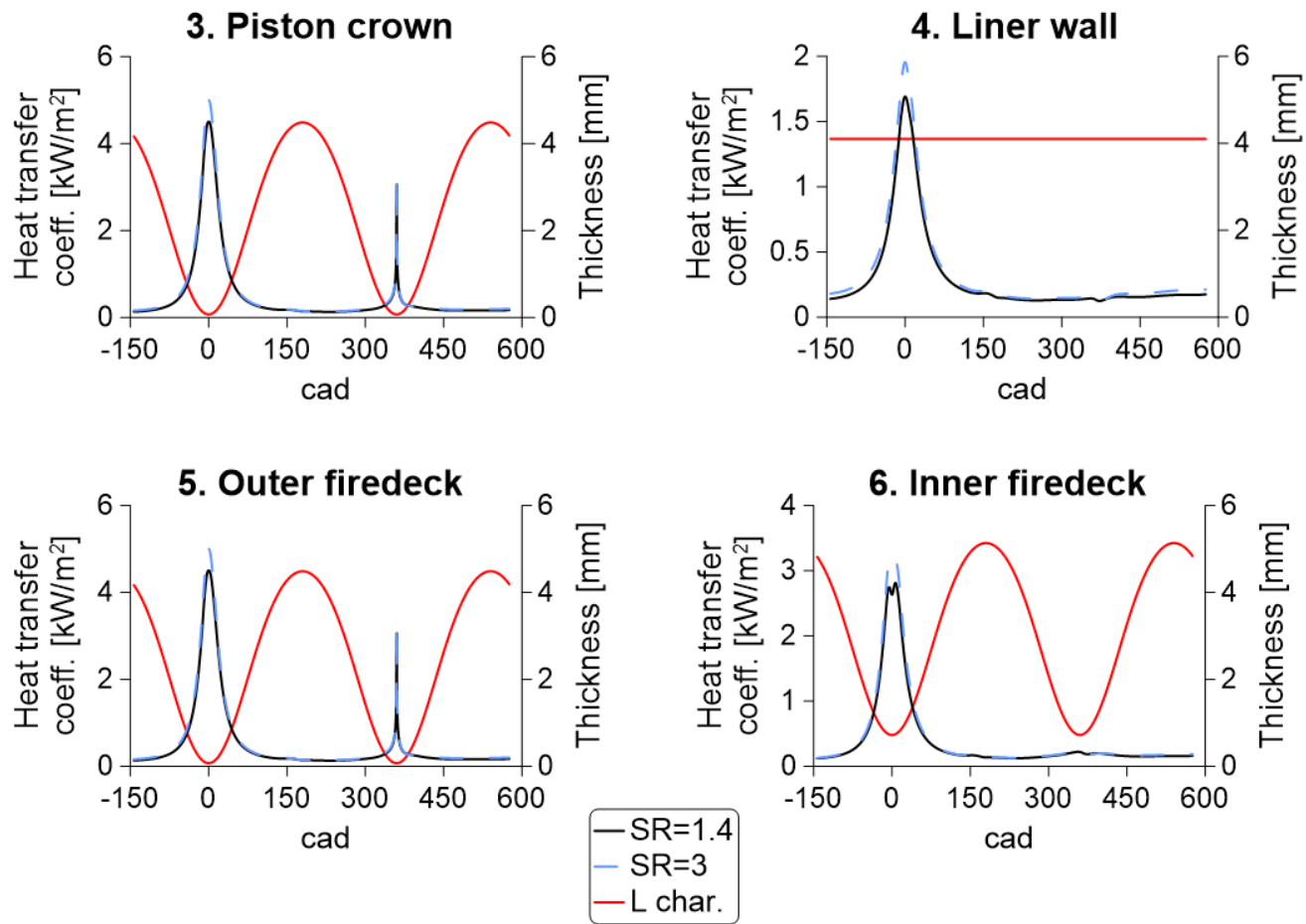

Figure 4.59: Heat transfer coefficient for the different surfaces and swirl ratios. 


\section{Temperature prediction maps}

Additional spatially-resolved information can be obtained from a study of distribution maps. The predictions of the global model and the multizone model were compared with each other. In the maps, the experimental minus the modeled temperature is plotted at the different measurement locations. The reason for plotting this magnitude, the reasons for choosing certain cylinders and many other details concerning these maps were thoroughly discussed in Section 4.2. All results are from the tests at 2000 rpm.

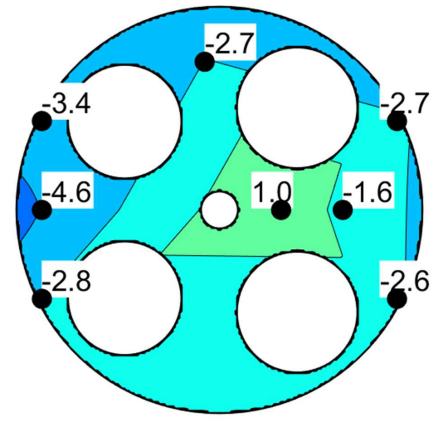

(a) Global

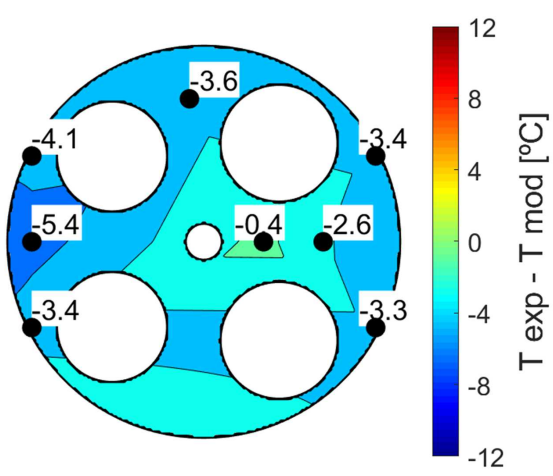

(b) Multizone

Figure 4.60: Prediction at low swirl in the firedeck of cylinder 1.

At low swirl, the multizone model worsened the prediction in the periphery of the firedeck with respect to the global model, as can be seen in Figs. 4.60 and 4.61. In particular, the model predicted higher temperatures that those experimentally measured. That fact could likely be related to the high peaks of heat transfer coefficient promoted by the small thickness of the boundary layer at the top dead center.

At high swirl, predictions were less accurate than at low swirl for both models, since they had been calibrated at low swirl. That can be noticed when Fig. 4.60 is compared with Fig. 4.62 or when Fig. 4.61 is compared with Fig. 4.63. However, the most remarkable observation is the important increase of modeled temperature in the multizone approach at high swirl and, consequently, the clear overpredictions with respect to experimental data. The global model presented considerable deficiencies as 


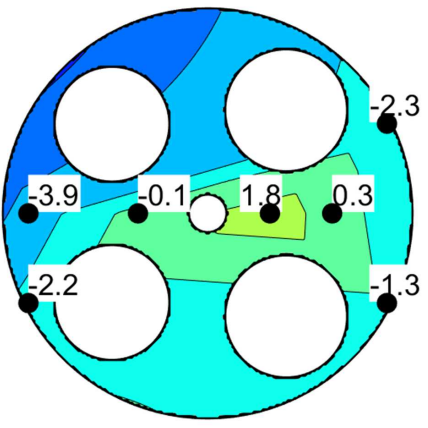

(a) Global

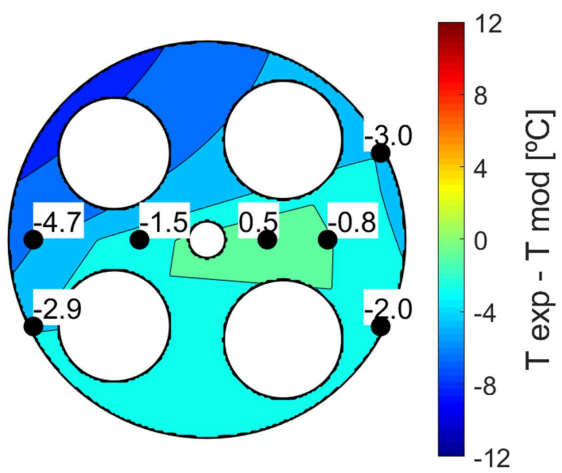

(b) Multizone

Figure 4.61: Prediction at low swirl in the firedeck of cylinder 3.

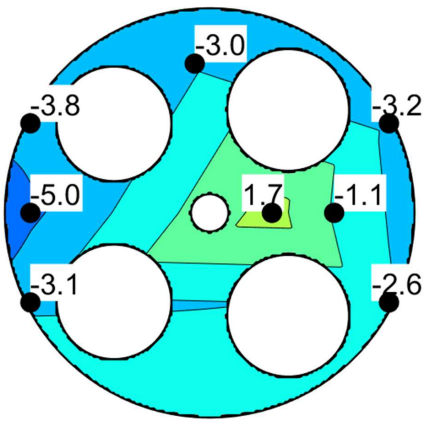

(a) Global

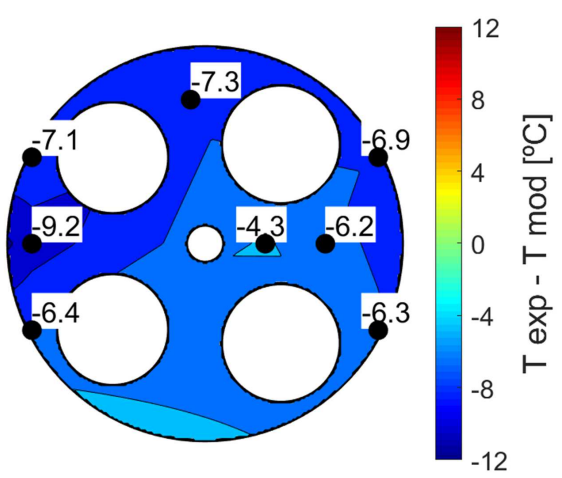

(b) Multizone

Figure 4.62: Prediction at high swirl in the firedeck of cylinder 1. 


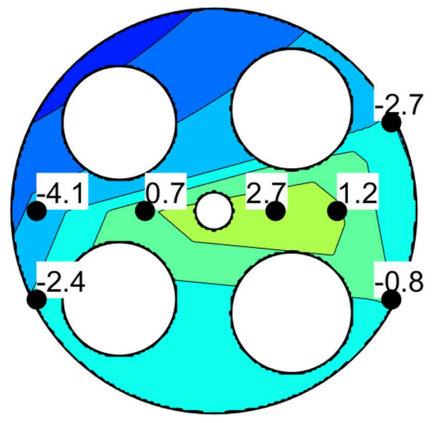

(a) Global

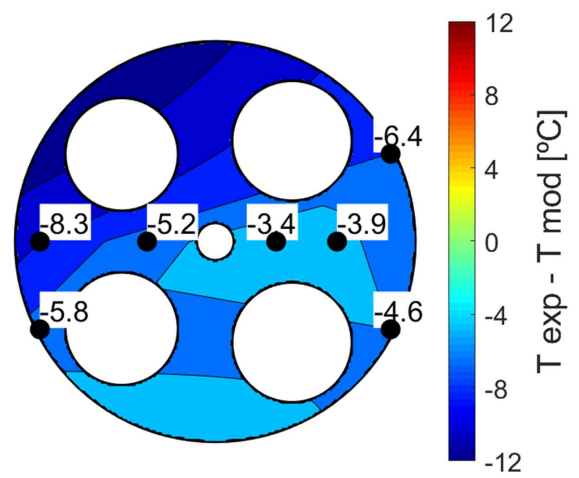

(b) Multizone

Figure 4.63: Prediction at high swirl in the firedeck of cylinder 3.

well but, from Figs. 4.62 and 4.63, it can be concluded that its predictions for the firedeck were better than those of the multizone model. Two likely causes for the unsatisfactory predictions of the multizone model in the firedeck have been commented: in the center, the overestimation of swirl velocity by the gas motion submodel and, in the outer area, the sharp peaks of heat transfer coefficient promoted by the reduction of the boundary layer thickness.

In the liner, some different trends were found. The multizone model slightly improved temperature prediction at some locations. This happened both at low swirl, Fig. 4.64, as at high swirl, Fig. 4.65. The consequence was a general but small improvement of predictions in the liner, as was seen in Fig. 4.58.

From a more fundamental point of view, Figs. 4.60 to 4.65 show that the multizone model is unable to provide an improved prediction of wall temperatures. An examination datapoint by datapoint leads to the conclusion that the multizone model, at best, achieves marginal prediction improvements at the cost of considerably increasing calculation complexity. At worst, the multizone model has flaws that produce worsened predictions with respect to a simpler global model.

\section{Post-calibration study}

As a final study, a calibration of the scaling constant was done with only the tests at high swirl ratio. The calibrated constants at high swirl can 


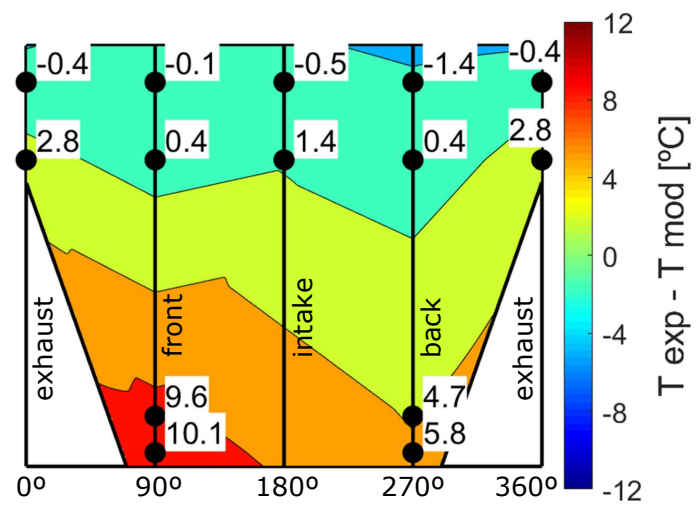

(a) Global

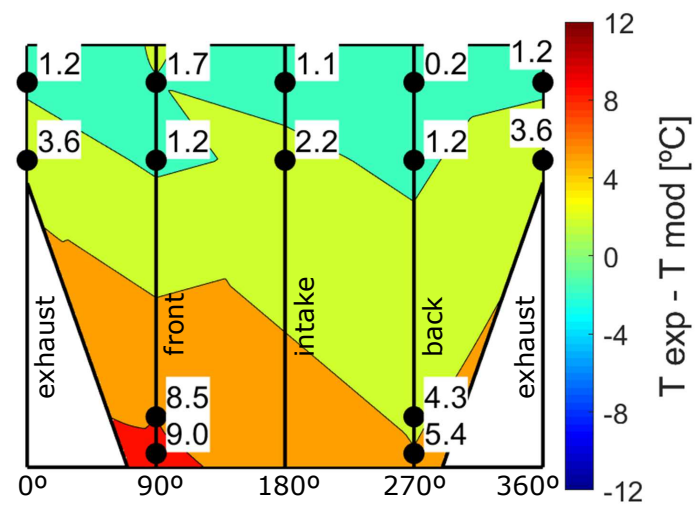

(b) Multizone

Figure 4.64: Prediction at low swirl in the liner of cylinder 4 . 


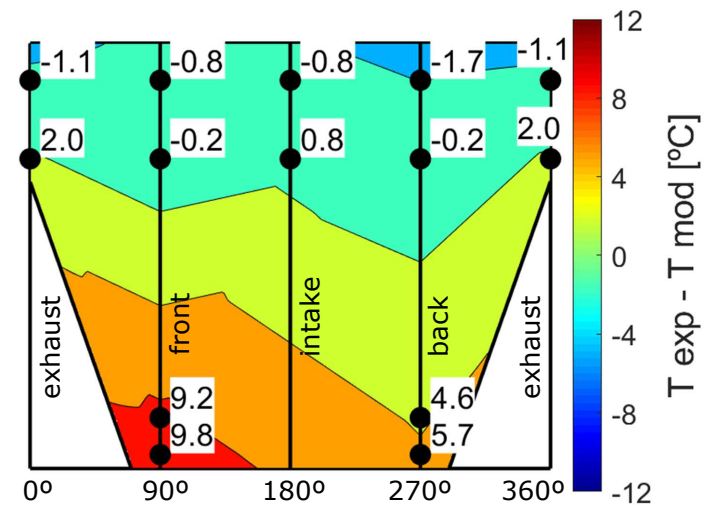

(a) Global

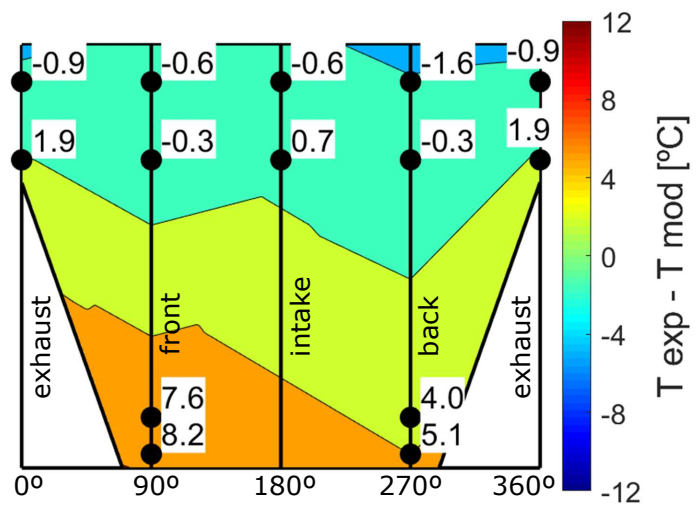

(b) Multizone

Figure 4.65: Prediction at high swirl in the liner of cylinder 4. 
be compared to those calibrated at low swirl to have a single numerical indicator of the accuracy of the model when predicting the swirl influence. The outcomes of the post-calibration and the old adjusted constants are summarized in Table 4.8. The global Woschni correlation would have provided more accurate heat flux curves with a constant equal to 1.988 , a value $10.7 \%$ higher than the optimum for low swirl. For the multizone model, a constant equal to 1.424 would be needed to decrease heat rejection to optimal levels. The value is $17.9 \%$ lower than the adjusted value for low swirl. This is consistent with previous observations indicating that the effect of swirl was clearly overpredicted by the multizone model. The results undoubtedly agree with the trends shown in Fig. 4.57 as well. Predicted wall temperatures from the multizone model adjusted for high

\begin{tabular}{lcc}
\hline Correlation & $\mathrm{SR}=1.4$ & $\mathrm{SR}=3$ \\
\hline Global Woschni & 1.795 & 1.988 \\
Multizone & 1.735 & 1.424 \\
\hline
\end{tabular}

Table 4.8: Adjusted constants for the two models and swirl ratios.

swirl (tuning constant $=1.424$ ) were also checked. Unsurprisingly, local predictions were still poor, because lowering the scaling constant decreased all temperatures similarly.

\section{Concluding remarks}

In summary, in Section 4.3 the potential of a multizone model to improve the prediction of wall temperatures was evaluated. The implementation of an in-cylinder convection model was thoroughly described. Methods and procedures used for calibration were reported and results were discussed. Lastly, predictions of the multizone model were compared with predictions of the global model that was employed in the initial Nodal Model.

It was found that, after calibration, both models presented good agreement with the experimental heat transfer rate over a wide range of engine speeds. However, at high swirl the multizone model tended to overpredict the heat flux peak at top dead center while the global model tended to underpredict it. Moreover, cross-interactions between swirl and engine speed were not fully captured by the models. 
An analysis of wall temperature prediction was performed next to provide spatial resolution to the study. In the firedeck, predictions of the multizone model were generally worse than those of the global model because the former predicted higher temperatures. Overprediction was specially prominent at high swirl ratio due to an overestimation of swirl influence. In the inner area of the firedeck, this could be caused by an overprediction of swirl motion, specially at high engine speed, as was reported in Section 4.3.1. In the outer area of the firedeck, a study focused on the heat transfer coefficient allowed to link temperature overpredictions to an unexpected behavior of the boundary layer thickness, used as characteristic length. Back to the analysis of temperature prediction, it was found that, in the liner, the multizone model slightly improved predictions and did not overestimate swirl influence. That was different from the behavior observed in the firedeck. Overall, however, the multizone model worsened predictions of wall temperature in many cases and, in the cases in which predictions improved, the gains were relatively small. These assertions were confirmed by an analysis datapoint by datapoint with temperature maps. Neither the global model nor the zonal model were able to properly predict the temperature distribution on the cylinder walls. Therefore, this study casts doubts on the potential of a 0D/1D zonal model to capture the highly localized heat transfer processes that take place within the engine cylinder.

From the research accounted so far in the present chapter (Sections 4.1, 4.2 and 4.3), it can be concluded that the Nodal Model has an undeniable capacity of predicting mean temperatures of the firedeck and the liner walls under different conditions of engine speed, load and swirl motion. That is a relevant capability for many applications, like the calculation of heat flux from in-cylinder gas to walls, the determination of heat rejection to coolant and oil or the estimation of temperatures in various parts of the engine structure. That can be achieved considering the same heat transfer coefficient throughout the combustion chamber. In the case of the Nodal Model, the heat transfer coefficient is obtained with a modified Woschni correlation that requires adjustment of the gas velocity terms. This kind of model is unable to provide a high spatial resolution of wall temperatures near the cylinder surfaces. A multizone model with different heat transfer coefficients for different areas of the combustion chamber seemed a promising solution to solve the temperature field on the cylinder walls. Notwithstanding, after the implementation and evaluation of such model, it was concluded that it was also incapable of accurately predicting 
local temperatures.

Although it would be a valuable feature of the model, a detailed spatial resolution of in-cylinder heat rejection is not a requirement for most applications of engine simulation. In particular, the thermal response of the global engine model, VEMOD, can be validated without this additional feature. Consequently, the developments of the Nodal Model focused on improving other areas unrelated to spatial resolution. The improvements were part of this thesis, and they involved considerable research efforts. Starting from the initial Nodal Model, the objectives for the rest of Chapter 4 are:

- To model heat rejection to coolant and oil circuits in order that the thermal evolution in those circuits can be accurately predicted.

- To model the transient thermal response of the engine structure.

- To make the necessary changes that ensure a perfect interaction with the other submodels of the Virtual Engine Model (VEMOD).

\subsection{Heat rejection to coolant and lubricant}

Various improvements were made to the way in which the hydraulic fluids of the engine were managed in the Nodal Model. The most notorious lack of the initial model was the absence of correlations for determining the heat transfer coefficients on the coolant side. Constant values were used, which did not take into account changes in flow rate or fluid properties. In Section 4.2, it was demonstrated that this led the model to overpredict engine temperature at high engine speed. The misprediction could be solved if coolant flow rate was taken into consideration by the heat transfer coefficient, by means of a correlation. To include new correlations, it had to be taken into account that coolant galleries in the cylinder block and in the cylinder-head were considered separately in the Nodal Model. Therefore, two correlations of forced convection and two of natural convection were implemented.

1. In the cylinder block, the proposal of Bohac et al. was imitated [40]. They used a correlation from Grimison [204] for flow past a bank of cylinders, Fig. 4.66, modified for fluids other than air, Eq. 4.75 [118]. $C_{2}$ for a single row of aligned cylinders is equal to 0.7 . $C_{1}$ and 


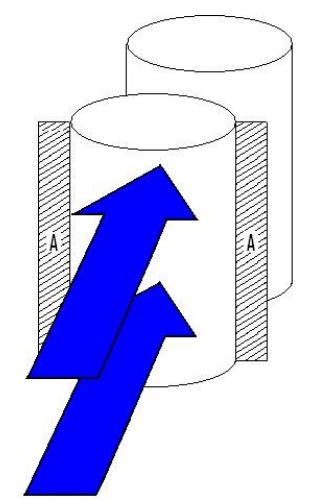

Figure 4.66: Flow past a single row of tubes.

$m$ are geometry-dependent constants whose values are 0.27 and 0.63 , respectively, for aligned cylinders, typical engine dimensions and the typical range of the Reynolds number found in engines. Flow rate was considered in the correlation through the Reynolds number, $R e$. This number can be expressed in terms of the mass flow rate, as shown in Eq. 4.76. As characteristic length, $L$, the bore diameter plus two times the thickness of the liner wall was used. Cross area $A$ is depicted in Fig. 4.66.

$$
\begin{gathered}
N u=1.13 C_{1} C_{2} \operatorname{Re}^{m} \operatorname{Pr}^{1 / 3} \\
R e=\frac{\dot{m} L}{A \mu}
\end{gathered}
$$

2. If coolant flow rate is zero in the liner galleries, natural convection is assumed. The correlation of Raithby and Hollands for natural convection inside concentric tubes was used, Eq. 4.77 [205] [118]. $r_{1}$ and $r_{2}$ are the internal and external radii of the gallery, respectively. $R a$ is the Rayleigh number, which is the product of the Prandtl number, $\operatorname{Pr}$ (see Eq. 2.13), and the Grashof number, Gr. The expression of the Grashof number is given in Eq. 4.78. $\beta$ is the coefficient of thermal expansion, which was calculated from density as $\beta=-\frac{d \ln (\rho(T))}{d T}$. Gravity acceleration is present in the formula as $g$. In this application, the characteristic length, $L$, is given by Eq. 4.79. For $\Delta T$, a value of $15^{\circ} \mathrm{C}$ typically observed in engine tests was 
chosen.

$$
h=\frac{k_{e f f}}{r_{1} \ln \frac{r_{2}}{r_{1}}}
$$

where,

$$
\begin{gathered}
k_{e f f}=0.386 k\left(\frac{P r R a}{0.861+P r}\right)^{0.25} \\
\text { if } k_{e f f}<k \text {, then } k_{e f f}=k \\
G r=\frac{\beta g \rho^{2} \Delta T L^{3}}{\mu^{2}} \\
L=\frac{2\left[\ln \left(\frac{r_{2}}{r_{1}}\right)\right]^{4 / 3}}{\left(r_{1}^{-3 / 5}+r_{2}^{-3 / 5}\right)^{5 / 3}}
\end{gathered}
$$

3. For forced convection in the cylinder-head, Dittus-Boelter correlation, Eq. 2.16, was employed. The Reynolds number, Re, was evaluated with Eq. 4.76 again. As characteristic length, $L$, the diameter of the cylinder-head galleries were selected. To find a suitable value, various cylinder-heads were measured and also typical values were surveyed from the literature [184]. A scalable typical value equal to $11 \%$ of the bore was accordingly implemented. The cross section, $A$ was assumed to be $8 \%$ of the bore section, after the same examinations done for the characteristic length.

4. For cylinder-head galleries when flow rate is zero, a correlation proposed by Churchill and Chu for natural convection in horizontal tubes was implemented, Eq. 4.80 [206] [207]. The gallery diameter, $D_{g a l}$, is the characteristic length and it is the same as in forced convection.

$$
h=\frac{k}{D_{\text {gal }}}\left(0.36+0.518\left[\frac{R a}{\left(1+\left[\frac{0.559}{P r}\right]^{9 / 16}\right)^{16 / 9}}\right]^{0.25}\right)
$$

In some versions of the initial Nodal Model, such as the one integrated in CALMEC, fluid nodes were simple boundary conditions with fixed temperature. No temperature variation, enthalpy variation or thermal inertia 
was modeled on the fluid side. In other versions, two nodes were considered: one represented the inlet and the other the outlet. In that case, enthalpy variation could then be taken into account. To model convective heat transfer, mean temperature of both nodes was considered. An example system is shown in Fig. 4.67, where $i$ is a solid node, in is a fluid inlet node, $f$ a fluid outlet node, $K_{c v}$ is convective conductance and $K_{f l}$ is flow conductance, which represents enthalpy variation between inlet and outlet as explained in Section 2.2.3. The equations resulting from the energy balance at this system's nodes are in Eq. 4.81 (see Section 2.2.3 for more details). The resulting system in matrix form can be seen in Eq. 4.82, with many terms in the conductance matrix. This approach was considered unsuitable to represent systems formed by large volumes of fluid with relatively small inlet and outlet flows. A clear example of this in an engine is the lubrication system, with a large oil pan and a small circulating flow. In the original approach, too much weight was given to inlet temperature. In relation with that, it was not clear where the fluid mass should be put for obtaining the capacitance: in one of the nodes or divided between inlet or outlet. The situation could get more complicated if there were multiple outlets with one common inlet.

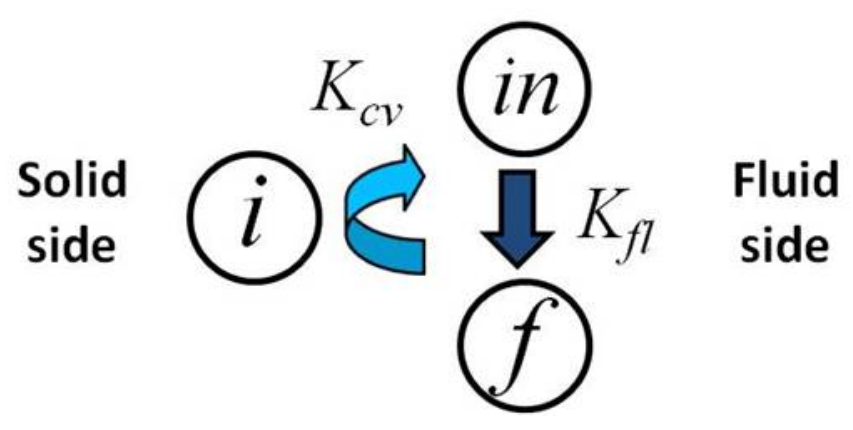

Figure 4.67: Convection between solid and fluid in the old configuration.
(I) $K_{c v}(t)\left[\left(\frac{T_{f}(t)+T_{i n}(t)}{2}\right)-T_{i}(t)\right]=0$
(II) $K_{c v}(t)\left[T_{i}(t)-\left(\frac{T_{f}(t)+T_{i n}(t)}{2}\right)\right]-K_{f l}(t)\left[T_{f}(t)-T_{i n}(t)\right]=0$
(III) $T_{i n}(t)-T_{i n}^{*}(t)=0$ 


$$
\left[\begin{array}{ccc}
-K_{c v} & \frac{K_{c v}}{2} & \frac{K_{c v}}{2} \\
K_{c v} & -\left(\frac{K_{c v}}{2}+K_{f l}\right) & \left(-\frac{K_{c v}}{2}+K_{f l}\right) \\
0 & 0 & 1
\end{array}\right]\left[\begin{array}{c}
T_{i}(t) \\
T_{f}(t) \\
T_{i n}(t)
\end{array}\right]+\left[\begin{array}{c}
0 \\
0 \\
-T_{i n}^{*}(t)
\end{array}\right]=0
$$

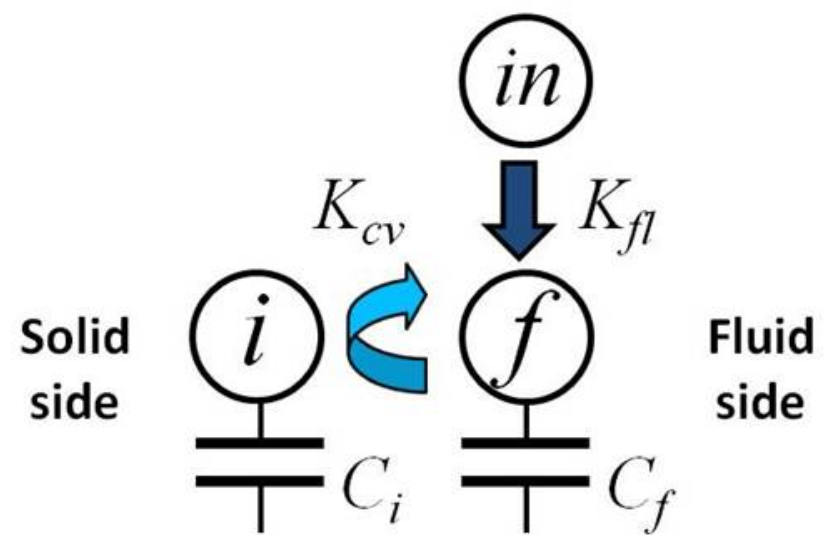

Figure 4.68: Convection between solid and fluid in the new configuration.

In the new Nodal Model, it was decided to consider inlet fluid nodes, in, as simple boundary conditions, while all fluid characteristics, like mass, were put in a node representing the fluid bulk, $f$. Then, heat convection had to take place between solid nodes, $i$, and fluid bulk. The inlet node was connected only to fluid bulk nodes, not to solid nodes. System definition had to be slightly different to represent the later fact. The same system of the previous example is defined under the new criterion in Fig. 4.68. Capacitances were added for transient modeling. The corresponding equations are gathered in Eq. 4.83 and the simpler matrix system is shown in Eq. 4.84 .

$$
\begin{aligned}
& \text { (I) } K_{c v}(t)\left[T_{f}(t)-T_{i}(t)\right]-C_{i}(t)\left[T_{i}(t)-T_{i}(t-1)\right]=0 \\
& \begin{array}{l}
\text { (II) } K_{c v}(t)\left[T_{i}(t)-T_{f}(t)\right]-K_{f l}(t)\left[T_{f}(t)-T_{i n}(t)\right]- \\
\quad-C_{f}(t)\left[T_{f}(t)-T_{f}(t-1)\right]=0 \\
\text { (III) } T_{i n}(t)-T_{i n}^{*}(t)=0
\end{array}
\end{aligned}
$$




$$
\begin{gathered}
{\left[\begin{array}{ccc}
-K_{c v} & K_{c v} & 0 \\
K_{c v} & -\left(K_{c v}+K_{f l}\right) & K_{f l} \\
0 & 0 & 1
\end{array}\right]\left[\begin{array}{c}
T_{i}(t) \\
T_{f}(t) \\
T_{i n}(t)
\end{array}\right]-\left[\begin{array}{ccc}
C_{i} & 0 & 0 \\
0 & C_{f} & 0 \\
0 & 0 & 0
\end{array}\right]\left[\begin{array}{c}
T_{i}(t) \\
T_{f}(t) \\
T_{i n}(t)
\end{array}\right]+} \\
+\left[\begin{array}{ccc}
C_{i} & 0 & 0 \\
0 & C_{f} & 0 \\
0 & 0 & 0
\end{array}\right]\left[\begin{array}{c}
T_{i}(t-1) \\
T_{f}(t-1) \\
T_{i n}(t-1)
\end{array}\right]+\left[\begin{array}{c}
0 \\
0 \\
-T_{i n}^{*}(t)
\end{array}\right]=0
\end{gathered}
$$

In addition to the mentioned improvements, the correlations to calculate physical properties of fluids, with focus on coolants, were reviewed. A literature and database survey allowed to update most correlations and also to add some fluids and mixtures. Real mixtures were considered, not ideal mixtures. Ultimately, density, conductivity, viscosity and specific heat could be determined for the following fluids:

- Air

- Water

- Steam

- Mixtures of water and ethylene glycol in any proportion

- Mixtures of water and diethylene glycol in any proportion

- Mixtures of water and triethylene glycol in any proportion

- Mixtures of water and propylene glycol in any proportion

- Mixtures of water and dipropylene glycol in any proportion

- Generic engine oil

Correlations of physical properties were included in diverse fluid submodels. The fluid submodels formed a generalized fluid model which was part of VEMOD.

\subsection{Transient behavior}

Initially, the lumped heat transfer model did not consider thermal inertia. However, this model had been previously used to predict the temperature evolution of coolant and oil during a New European Driving Cycle [166]. As the thermal network did not contain capacitances, a quasi-steady approach was used. In spite of the success of this approach, the inclusion of capacitances was imperative in order to provide a physical account of thermal inertia. That should enhance reliability in cases of fast transients and very dynamic conditions.

The first step to implement thermal capacitance in the Nodal Model was to determine the nodes' volumes. Node mass was considered as the 
product of density and volume, according to Eq. 2.42. In solids, density is fixed at the beginning of the test because mass must not change with temperature. In liquids, in contrast, the volume of the galleries is assumed constant and the density changes when temperature changes over time. The Nodal Model has a default geometry shown in Section 3.3. Formulas were implemented in the model to calculate the volume of each node according to the default geometry. In addition, the geometry of the model was adapted for Engine 1, as explained in Section 4.2. The adaptation included obtaining the volume of each node from 3D CAD models. That information was part of the geometry data and was imported with the rest.

To make the Nodal Model capable of simulating thermal transients, the mass of the whole engine block had to be considered. The initial model had a detailed geometry of the areas around the cylinder, but the external areas were not included. There were two nodes representing the bulk of the cylinder-head, but they did not account for the whole mass of the cylinder-head. The solution was to scale the size of those two nodes taking into consideration a user-specified value of cylinder-head mass and the mass of the rest of the nodes. Globally, the mass of the cylinder-head is equal to the value entered by the user. In the cylinder block, the liner between the cylinder wall and the coolant galleries was present, but the metal mass around the galleries was missing. A single node was added to each cylinder model to represent that mass. As in the cylinder-hear, a user-specified value of cylinder block mass was used to determine the mass of this node. Another matter of interest was that, in the initial Nodal Model, oil was in thermal contact with the piston but not with the block. The piston was in contact with the liner through the piston rings, but that thermal conductance was small. As a consequence, the oil node acted usually as a heat sink. However, studies of the Global Energy Balance found that heat transfer from oil to coolant through the structure of the engine block was not negligible, adding up to $25 \%$ of heat rejection to coolant under some operating conditions [101]. To model the entire engine it was necessary to include all paths through which oil can dissipate heat [152]. To achieve that, a thermal conductance between the the oil node and the block bulk node was added. The value of that conductance is a constant entered by the user. Another element for heat dissipation, the oil cooler, can be simulated with the model of heat exchangers described in Section 5.1. 
Once volumes and masses were determined, thermal capacitances could be obtained. The calculation was implemented in an independent function that can be called as frequently as needed. Specific heat capacity of each material is a function of temperature. Therefore, the model allows the possibility of recalculating capacitances taking into account the influence of temperature variations over time.

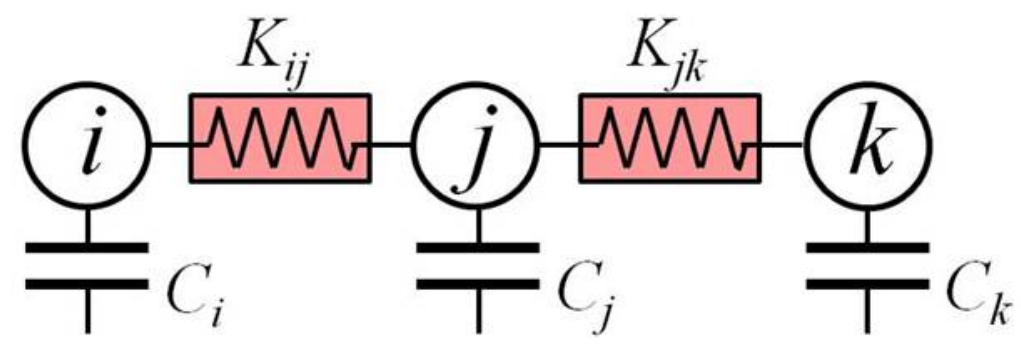

Figure 4.69: Example thermal system including capacitances.

The capacitances are introduced in the matrix system of equations that represents the thermal system in the lumped model. Under steadystate conditions, capacitances do not apply and the system has the form $[K][T(t)]+[B C]=0$, where $K$ represents the conductance matrix, $B C$ the vector of boundary conditions and source terms and $T(t)$ is the vector of temperatures, which is the only unknown to be solved (see Section 2.2.3). In that system, $T(t)$ can be determined by inverting the conductance matrix as $[T(t)]=-[K]^{-1}[B C]=0$. In transient, though, various finitedifference schemes can be used to set up and solve the linear system of equations. Three schemes were implemented in the Nodal Model and tested. Next, they are briefly described and applied to the simple example of Fig. 4.69. Fig. 4.70 illustrates the schemes. The vertical direction represents time, where $t$ is the current time step and $t-1$ is the previous one. The horizontal direction is spatial; $i, j$ and $k$ are the different nodes of Fig. 4.69. The blue area covers the data points included in the equation to solve the node $j$ in the time step $t$.

1. Explicit. To solve the state of the system in the current time step, only information of the previous time step is used, as schematized in Fig. 4.70(a). In node $j$, temperature in the previous time step, $T_{j}(t-1)$ is used to calculate heat accumulation. Also $T_{j}(t-1)$ and the temperatures of the surrounding nodes, $T_{i}(t-1)$ and $T_{k}(t-1)$ are used to calculate heat exchange. The resulting equation of node 


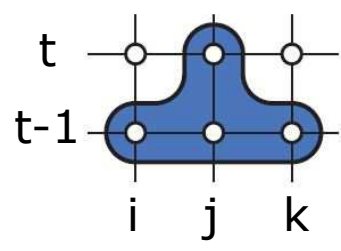

(a) Explicit

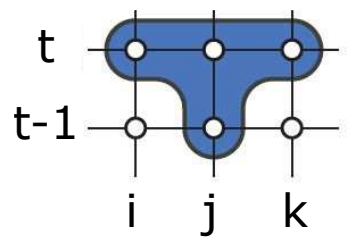

(b) Implicit

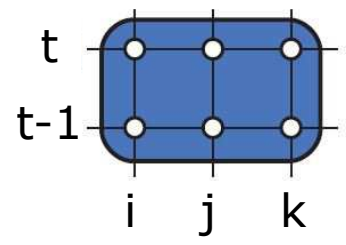

(c) Crank-Nicolson

Figure 4.70: Finite-difference schemes.

$j$ is Eq. 4.85. Note that the equation can be directly solved for $T_{j}(t)$, which is the only unknown. Therefore, in the explicit scheme, each equation is independent and can be solved separately. The matrix system has the form $-[C][T]+[K+C][T(t-1)]+[B C]=0$, where $C$ the capacitance matrix, $T(t-1)$ is the vector of temperatures in the previous time step and the other terms were clarified earlier. $C$ is a diagonal matrix. The explicit scheme is the fastest because no matrix inversion is needed. It is also very simple. The main practical drawback, however, is that the method is not unconditionally stable. Thus, the maximum viable time step has to be determined every call for every node and the system has to be solved within that time step, not the one requested by the caller. Moreover, the accuracy of the method is theoretically the lowest.

$$
\begin{gathered}
K_{i j}\left[T_{i}(t-1)-T_{j}(t-1)\right]+K_{j k}\left[T_{k}(t-1)-T_{j}(t-1)\right]- \\
-C_{j}\left[T_{j}(t)-T_{j}(t-1)\right]=0
\end{gathered}
$$

2. Implicit. This method is based on a balance in the current time step. Temperatures of the current time step, $T_{i}(t), T_{j}(t)$ and $T_{k}(t)$ are used to calculate heat exchange. From the previous time step, only $T_{j}(t-1)$ is used in the equation of node $j$ to determine the accumulated heat. That is illustrated in Fig. 4.70(b). The expression is Eq. 4.86. Note that the equations have to be solved together as a system due to the multiplicity of unknowns in each equation $\left(T_{i}(t), T_{j}(t)\right.$ and $\left.T_{k}(t)\right)$. The system has the form $[K-C][T(t)]+$ $[C][T(t-1)]+[B C]=0$. The key advantage of this scheme over the explicit scheme is that it is unconditionally stable, regardless of the time step. A drawback is the need for inverting the matrix $K-C$ to solve the system. However, this method is considered the most accurate of the three for relatively large time steps. The implicit 
scheme was used by Bohac et al. [40].

$$
\begin{gathered}
K_{i j}\left[T_{i}(t)-T_{j}(t)\right]+K_{j k}\left[T_{k}(t)-T_{j}(t)\right]- \\
-C_{j}\left[T_{j}(t)-T_{j}(t-1)\right]=0
\end{gathered}
$$

3. Crank-Nicolson. In this combined method, both the current state and the previous state are considered. To calculate heat exchange, $T_{i}(t), T_{j}(t)$ and $T_{k}(t)$ as well as $T_{i}(t-1), T_{j}(t-1)$ and $T_{k}(t-$ 1) are employed. Therefore, all data points of Fig. $4.70(\mathrm{c})$ are colored. The equation of node $j$ is Eq. 4.87 and, consequently, the matrix system has the form $[K / 2-C][T(t)]+[K / 2+C][T(t-$ $1)]+[B C]=0$. It can be deduced form this that, as in the implicit scheme, it is necessary to to invert the $K / 2-C$ matrix to solve for $T(t)$. Also as the implicit scheme, the Crank-Nicolson scheme is unconditionally stable. However, Crank-Nicolson scheme is more complex. Theoretically, this is the method that provides the highest accuracy among the three for small time steps.

$$
\begin{aligned}
K_{i j} & {\left[\frac{T_{i}(t)+T_{i}(t-1)}{2}-\frac{T_{j}(t)+T_{j}(t-1)}{2}\right]+} \\
& +K_{j k}\left[\frac{T_{k}(t)+T_{k}(t-1)}{2}-\frac{T_{j}(t)+T_{j}(t-1)}{2}\right]- \\
& -C_{j}\left[T_{j}(t)-T_{j}(t-1)\right]=\frac{K_{i j}}{2} T_{i}(t)+\frac{K_{i j}}{2} T_{i}(t-1)- \\
& -\frac{K_{i j}}{2} T_{j}(t)-\frac{K_{i j}}{2} T_{j}(t-1)+\frac{K_{j k}}{2} T_{k}(t)+\frac{K_{j k}}{2} T_{k}(t-1)- \\
& -\frac{K_{j k}}{2} T_{j}(t)-\frac{K_{j k}}{2} T_{j}(t-1)-C_{j}\left[T_{j}(t)-T_{j}(t-1)\right]=0
\end{aligned}
$$

As mentioned, the finite-difference schemes were put to test. The results are shown in Fig. 4.71. In the figure, the temperature of a node of the cylinder-head bulk is plotted. All schemes were tested with a time step of $0.1 \mathrm{~s}$. It was found that the explicit scheme caused temperature at this location to diverge after $1.5 \mathrm{~s}$ (purple curve). The implicit scheme (black) and the Crank-Nicolson scheme (green) are unconditionally stable, so they did not experience that problem. The test was run again with the explicit scheme and a time step of $0.01 \mathrm{~s}$. That time, the simulation did not diverge. Comparing the different curves, it can be concluded that 


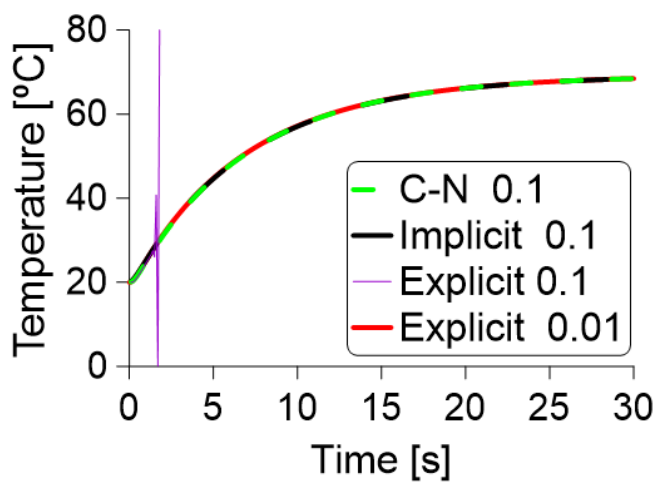

Figure 4.71: Comparison of different schemes.

the modeled evolution of temperature is the same. It was confirmed with the rest of nodal temperatures under several operating conditions that accuracy was not an issue in this application. Therefore, the implicit scheme was used for the rest of calculations of this thesis because it is simple and unconditionally stable.

Even though the solving procedure of the Nodal Model was modified to implement the transient finite-difference schemes, it was decided to keep the capability of steady-state solving. A steady-state solver is useful for pre-calculating temperatures in order to attain fast convergence to a thermal equilibrium. In VEMOD, the Nodal Model can be called in its transient or its steady form at any moment.

To finish this section and also to assess the suitability of the changes introduced in the previous section, a validation of the Nodal Model in transient operation is reported. This was a preliminary validation of the transient response considering only the model of heat transfer in the engine block. The validation is justified because the engine block is the most influencing component of the engine from the thermal point of view. A more global validation is presented later in this thesis, in Chapter 6. Since one of the main aims of this research was to have a physics-based transient thermal model, this preliminary validation was an opportunity to assess whether the model produced accurate results.

The Nodal Model was evaluated standalone after the discussed changes were introduced in a version that was not integrated into any other tool, and before its final integration into VEMOD. Because of this, there was no coupled model to simulate in-cylinder conditions (VEMOD or CALMEC). 
However, gas temperature was needed as a boundary condition. Another relevant variable that was typically obtained from CALMEC was instantaneous gas velocity in exhaust ports. To provide that data, CALMEC simulations were run at discrete time points of the tests. To supply CALMEC simulations, instantaneous variables (specially, in-cylinder pressure) were experimentally measured at those discrete time points. After the experimental tests, CALMEC simulations in steady-state mode were run separately for each time point. Then, in-cylinder gas temperature and exhaust gas velocity were delivered to the Nodal Model for standalone thermal simulations. Thermal simulations with the Nodal Model were not performed in a discrete way, but as single simulations of the same duration as the experimental test. Simulation time step was determined by the discrete time points because, at those points, inputs from CALMEC were available. As previously explained, at each time step, temperatures of the previous time step were used to calculate thermal inertia, constituting a single simulation. After the tests and simulations, mean temperatures of firedeck and liner were calculated as in Section 4.2.

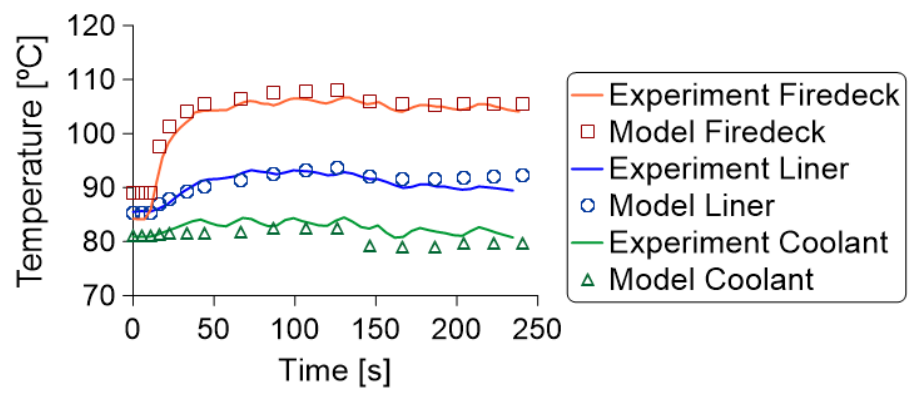

Figure 4.72: Transient validation: load change.

Two different tests were conducted in the facility of Engine 1. Description of the facility can be found in Section 4.2.1. In the first test, engine load was changed suddenly and then the engine was let to reach a new thermal equilibrium. Load changed from motored operation to $50 \%$ load. Engine speed was $1500 \mathrm{rpm}$. The final state corresponds to keypoint $1500 @ 8$ of Section 4.2. Coolant temperature was kept constant at $80^{\circ} \mathrm{C}$ during the test. This implied that the valve of the secondary cooling circuit was wide open after the test started, in order to cool down the engine coolant (see Fig. 4.3). Later, a PID was able to keep coolant temperature nominal. Oil and intake air temperatures were also kept constant at $87.5^{\circ} \mathrm{C}$ and $23^{\circ} \mathrm{C}$, respectively. Coolant flow rate was experimentally measured. 
This information and the rest of the test data were entered into the Nodal Model to replicate the test in a simulation. The test lasted 250 seconds. The first five modeled data points had a time step of 5.5 seconds. At that point, liner temperature was close to the stabilized value. The two following points were measured and simulated with a time step of 11 seconds. At that instant, firedeck temperature was close to stabilization. The rest of the test was sampled with a time step of 20 seconds. In Fig. 4.72, the results of cylinder 3 are shown. Experimental curves are plotted as lines and modeled data points are plotted as markers. It can be seen that coolant temperature (green) was maintained around the nominal value of $80^{\circ} \mathrm{C}$. Small fluctuations were captured by the model. Moreover, liner temperature (blue) increased around $5^{\circ} \mathrm{C}$ from the initial value of $85.5^{\circ} \mathrm{C}$. In contrast, firedeck temperature (orange) increased $20^{\circ} \mathrm{C}$ until stabilization. At the beginning, the modeled firedeck temperature was slightly higher than the measured one. Nonetheless, thermal evolution in the firedeck and the liner was predicted correctly and stabilized temperatures showed good agreement between model and experiment.

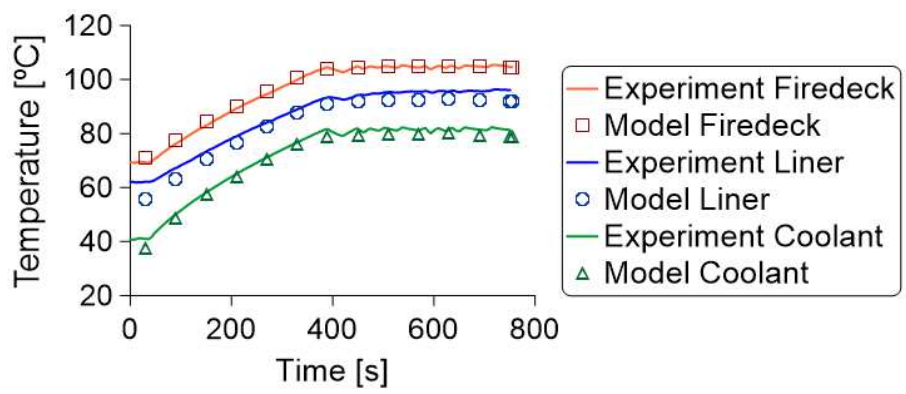

Figure 4.73: Transient validation: warm-up of structure, coolant and oil.

The second test was based on changing of coolant and oil temperatures. The engine was operated at $1500 \mathrm{rpm}$ and 50\% load, i.e. keypoint 1500@8 again. At the beginning, coolant and oil were cooled down to 40 and $60{ }^{\circ} \mathrm{C}$, respectively. This was achieved by entering those values as initial settings of the PID. The test started when settings were changed to the nominal values, $80^{\circ} \mathrm{C}$ for coolant and $87.5^{\circ} \mathrm{C}$ for oil. The engine entered in a process of a warm-up until it reached the new equilibrium. Since test 2 was longer than test 1, a time step of 60 seconds was used for the simulation. Fig. 4.73 shows the thermal evolution in cylinder 3 . Good agreement between model and experiment was obtained during the warm-up period and during the equilibrium period. Coolant temperature increased $40.5^{\circ} \mathrm{C}$ 
over the test while firedeck and liner temperatures increased around $35^{\circ} \mathrm{C}$.

It can be concluded that the thermal capacitances introduced in the Nodal Model were very effective in predicting the thermal response of engine structure and coolant during transients.

\subsection{Adaptation for integration into VEMOD}

As pointed out during the qualitative evaluation in Section 4.1, several actions were required to integrate the Nodal Model into VEMOD. The Nodal Model not only had to work in the VEMOD environment, but also had to be adapted to work coupled to other component models. The paradigm change introduced by VEMOD is that simulation is not focused on the thermodynamic cycle inside the cylinder anymore. Instead, engine processes are simulated in such a way that models can interact with other models. This means that the model structure has to separate initialization, data exchange and main execution. If the model has different modes, they have to be separated as well to be reachable from external calls. To comply with these requirements, all models underwent a process of standardization. In the Nodal Model, it was necessary to organize different tasks into modules: initialization, creation of invariable elements, data update from external sources, call to transient execution and call to steady-state execution. In addition, methods to supply results to external models had to be implemented. The schema of Fig. 4.74 is a grey-box representation of the Nodal Model.

Initialization consisted of three tasks.

1. To read the input data file in which the model was defined, typically coming from a graphical user interface.

2. To declare variables used throughout the model, e.g. number of nodes and system matrices.

3. To assign properties and initial values, e.g. materials and initial temperature.

The defining information of the engine heat transfer model had to be entered in a compatible way with the rest of engine submodels. For this reason, the highly standardized XML (Extensible Markup Language) format was chosen to specify the initial input data in VEMOD. The XML code is a schema of elements and attributes. It is contained in a XML file that can be generated with a graphical user interface created for VEMOD. 


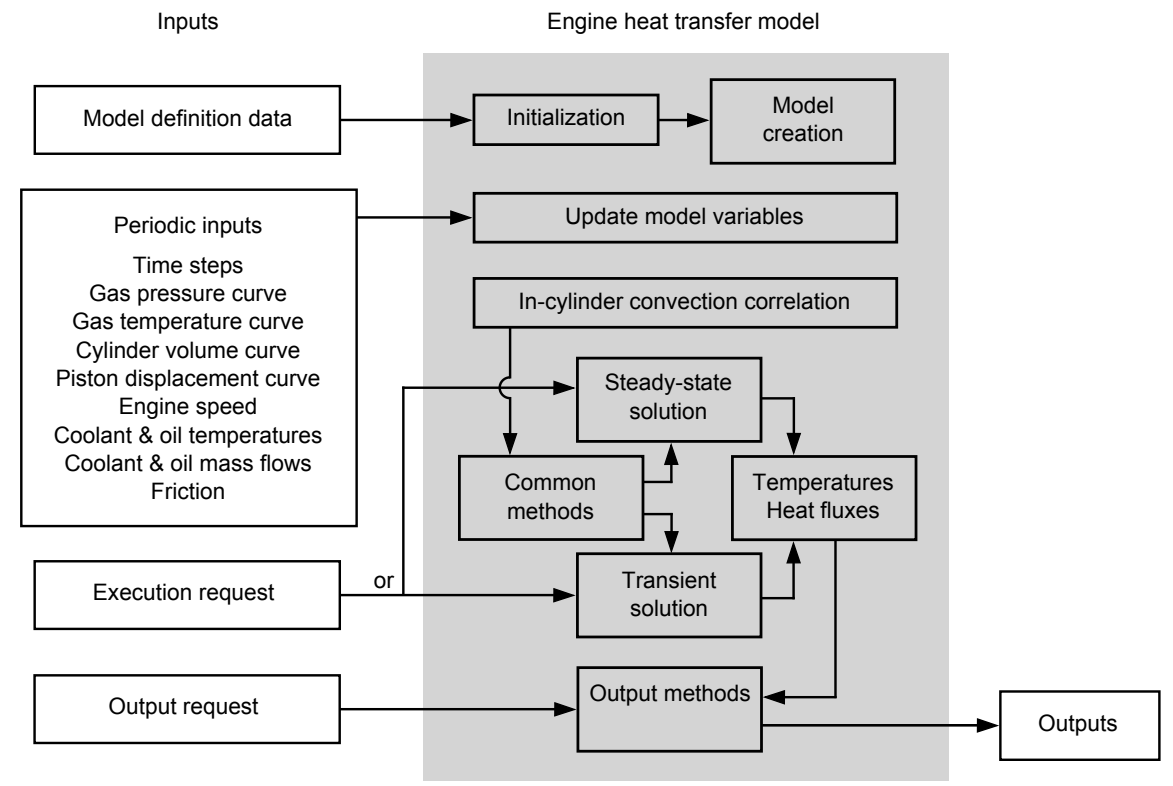

Figure 4.74: Grey-box diagram of the Nodal Model integrated in VEMOD.

The structure of the XML schema for the Nodal Model is shown in Fig. 4.75 .

One of the elements that contain information for the simulation is called 'General Data'. This element includes information about fluids and materials to be used, among other data. In Fig. 4.75, only the subelements that are relevant for the Nodal Model are shown for reasons of concision. Among the fluids, it is necessary to specify working fluid, lubricant and coolant. The working fluid is defined as a mix of chemical species. Therefore, it is necessary to specify the composition at the engine intake. The lubricant includes coefficients for the viscosity curve and also identification data to assign it to the lubricant circuit. The coolant contains identification data and weight fraction, to simulate a mix of water and antifreeze. Curves of physical properties are found in VEMOD's code, not entered as input data. Similarly, materials are listed under 'General Data' with their identification data. Note that the 'Material' element can be repeated as many times as necessary to include all necessary materials.

The other element that contains relevant information for the definition of the engine heat transfer model is 'Engine Block'. This element has many subelements, but only two are of interest for this thesis: 'Heat Transfer' 


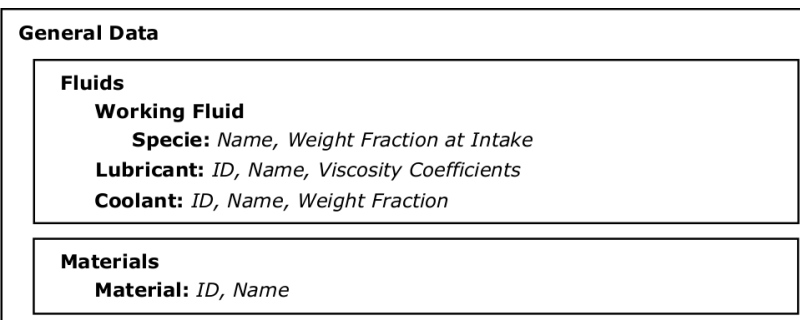

\section{Engine Block \\ Heat Transfer: Type, Mean Wall Temperatures $(t=0)$ of Liner, Firedeck and Piston, Multipliers in the Closed and the Open Periods of the Cycle \\ (CI Swirl): Correlation Coefficients, Swirl Ratio $(t=0)$ \\ (Swirl Table) \\ Swirl Data: Position, Swirl Ratio \\ (Nodal Model): Liner Thickness, Squish Height, Materials of Liner, Cylinder-Head, Piston, (Intake Valves, Exhaust Valves, Intake \\ Port Walls and Exhaust Port Walls), Mass of Cylinder Block, Mass of Cylinder-Head, Multipliers to Convection in Coolant and Oil, Conductance between Oil and Cylinder Block}

Coolant \& Oil: Split Cooling

Coolant: Inlet Temperature $(t=0)$, Mass Flow $(t=0)$, (Circuit ID), Internal Volume, (Hydraulic Resistance)

Coolant Cylinder-Head: Inlet Temperature $(t=0)$, Mass Flow

$(t=0)$, (Circuit ID), Internal Volume, (Hydraulic Resistance)

Oil: Inlet Temperature $(t=0)$, Mass Flow $(t=0)$, (Circuit ID), Internal Volume, (Hydraulic Resistance)
Bold: element

Italic: atribute

(brackets): optional

Figure 4.75: Structure of the XML code that contains the input data of the engine heat transfer model. 
and 'Coolant \& Oil'. One of the attributes of the 'Heat Transfer' element is 'Type'. 'Type' allows to select the in-cylinder convection model most suitable for each application. At the moment, the only available type is 'CI Swirl'. It is meant for compression ignition engines with swirl motion, and it is based on the modified Woschni correlation reviewed in Section 3.3. Depending on the type, the heat transfer model has properties included in the 'CI Swirl' subelement. Correlation constants are entered as attributes. The initial or nominal swirl ratio is also specified there. However, it is possible to enter a swirl table to determine swirl ratio as a function of the swirl valve position. That way, swirl ratio can vary during the simulation. Apart from data of the in-cylinder convection correlation, the 'Heat Transfer' element has other direct attributes:

- Mean wall temperatures on the cylinder walls are specified as initial or fixed values, because the usage of the Nodal Model to calculate wall temperatures is optional.

- A multiplier for the in-cylinder convection correlation during the period of the cycle in which valves are open and a multiplier for the closed period are present for additional tuning.

Under the subelement 'Nodal Model' some specific information is provided:

- Additional geometry, such as liner thickness and squish height.

- Material of each engine part.

- Multipliers for convective heat transfer on the coolant and the oil side.

- User-defined thermal conductance to consider heat exchange between the oil and the cylinder block, as mentioned in Section 4.5.

'Coolant \& Oil' is the second element of interest under 'Engine Block'. It contains information about the cooling and lubricating fluids present in the engine. The one attribute of the 'Coolant \& Oil' element is 'Split Cooling', which is true if cylinder block and cylinder-head are cooled by different circuits and false otherwise. Currently, the available sublements of 'Coolant \& Oil' are 'Coolant', 'Coolant Cylinder-Head' and 'Oil'. 'Coolant Cylinder-Head' is used only when 'Split Cooling' is true. The attributes of the fluid subelements are:

- Initial inlet temperature.

- Initial mass flow rate.

- ID of the corresponding circuit in the model of thermo-hydraulic circuits.

- Volume occupied by the fluid inside the engine block. 
- Hydraulic resistance of the engine block, which is defined as $R_{h}=$ $H / \dot{V}^{2}$, where $H$ is the hydraulic head drop in meter column fluid and $\dot{V}$ is the fluid flow rate. For more details, see Section 5.2.

After reading the input data file, part of the information is employed to assign properties and to initialize variables. Initial temperatures of solid nodes are taken respectively from the initial wall temperatures of liner, firedeck and piston. Gas nodes are initialized at ambient temperature. Initial temperatures and mass flow rates of coolant and oil nodes are taken from the 'Coolant \& Oil' element previously mentioned. VEMOD has dedicated models for fluids and materials that store and calculate physical properties such as density, thermal conductivity or specific heat as a function of temperature. Each fluid and material present in the engine block is linked to its corresponding fluid or material model. A new development was that valves and port walls can be assigned different materials from the cylinder-head material.

The next step during execution is to create a set of structures containing data that does not change during the simulation. Afterwards, some additional initializations are done. If geometry and conduction conductances have to be imported from files (see Section 4.2.1), the operation is done at this point. However, the most common case is to use the default geometry of the model seen in Section 3.3 and then obtain the corresponding conduction conductances. In that case, calculations are internally done by the model. In any case, all information is stored in the mentioned data structures.

In VEMOD, the Nodal Model is a member of another model, the Cylinder model. Each cylinder has a Nodal Model. The Cylinder model, in turn, is part of the Engine Block model and is also part of the model of Heat Exchangers that is described in Section 5.1. The Cylinder model can call the Nodal Model in two different ways: steady-state mode or transient mode. Most processes of both modes are common. However, the numerical solvers are different, as explained in Section 4.5. In addition to the solvers, in transient mode there is the need to calculate thermal capacitances and the storage term of heat flux, $C \frac{d T}{d t}$.

When the Cylinder model calls the Nodal Model, it supplies some inputs that are referred in Fig. 4.74 as periodic inputs. As VEMOD is a time-based model and not an angle-based model, two fundamental inputs are the time step and a vector with time increments. This vector 
has the same size as the vectors that contain instantaneous data of the thermodynamic cycle such as the gas temperature curve, and provides the time reference for each data point of the curve. Time step can be arbitrary, though by default the duration of an engine cycle is employed. That is to say, by default the Nodal Model is called at the end of each engine cycle and is supplied with the cycle's data (pressure, temperature, etc.).

The method to calculate in-cylinder convection is selected at the beginning, in the creation stage, and linked to the heat transfer model. Then, the in-cylinder convection submodel is fixed, and the same method is always applied throughout the simulation. As previously commented, at the moment there is only one available method, based on the modified Woschni correlation reviewed in Section 3.3. This method is suitable for compression ignition engines with swirl motion. The engines studied in this thesis are of that kind, but for future research, new models will have to be implemented. The procedure to obtain the heat transfer coefficient and the thermal conductances between gas and walls were only modified to allow a time-based calculation instead of an angle-based one. No other developments were introduced with respect to the original method.

Also in the topic of convection conductances, the methods related to the cooling and lubricating fluids were overhauled. Important modifications were introduced to adapt the fluid nodes, as discussed with examples in Section 4.4. Also in that section, the addition of new correlations was reported. The new correlations introduced the need for mass flow rate as a new input. The new Nodal Model works in close collaboration with the models of ancillary systems that were developed in this thesis and are described in Chapter 5. Data exchange between the Nodal Model and the Thermo-Hydraulic model is accomplished through the Heat Exchangers model. The Thermo-Hydraulic model provides inlet temperature and mass flow rate to the Cylinder model and reads heat rejection and outlet temperature, received by the Cylinder model from the Nodal Model. Another development was that new tuning constants were added to the convective correlations on the coolant and oil side in order to increase the degrees of freedom when adjusting the model.

An important change in the Nodal Model was the method for calculating heat transfer in the intake and exhaust ports. The models used in the initial Nodal Model, which were reviewed in Section 3.3, presented various drawbacks. 


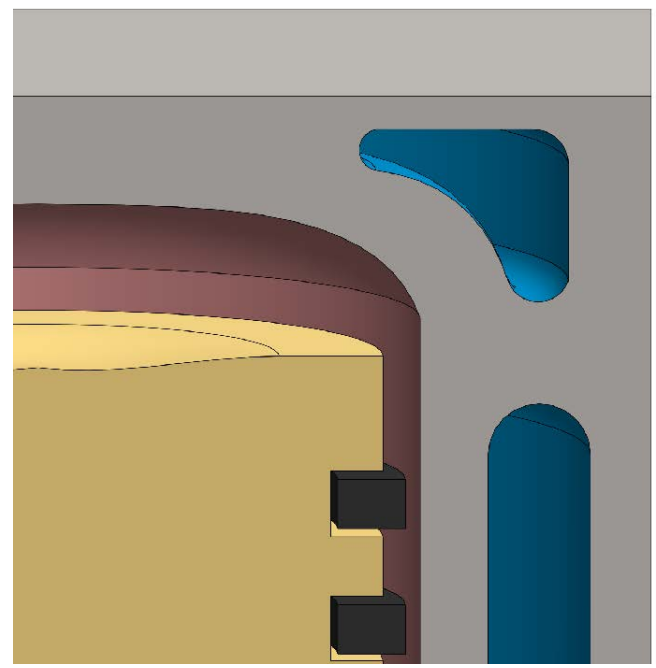

Figure 4.76: 3D cut of cylinder cooling galleries.

1. It depended on a simple emptying and filling model to supply gas flow conditions.

2. Gas properties were assumed equal to air properties.

3. A constant gas temperature value was used for the whole cycle. Temperature was experimentally measured with slow-response thermocouples in intake and exhaust manifolds.

4. In the exhaust ports, two correlations were used: one during the closed part of the cycle (Eq. 3.9) and a different one when exhaust valves were open (Eq. 3.10). Since Eq. 3.9 used mean mass flow rate and Eq. 3.10 used instantaneous velocity, the curve of heat transfer coefficient in the exhaust ports presented discontinuities at the instants of valve closure and opening. Moreover, the combination of mean and instantaneous magnitudes in Eq. 3.10 is questionable.

The Gas Dynamics model of VEMOD has heat transfer submodels for intake and exhaust ports that were reviewed in Section 3.5. VEMOD has a $1 \mathrm{D}$ model that can determine flow conditions accurately. Temperature is variable over the engine cycle and has spatial resolution. In addition, gas composition is dynamic and is coupled with the other models, being the Combustion model the most influential. Gas properties are a function of temperature and chemical composition. The correlations for determining the heat transfer coefficient, Eqs. 3.14 and 3.15, are continuous and they 
were recommended after substantial research, as reported in Section 3.5.

To use those heat transfer submodels, the Nodal Model was coupled with the Gas Dynamics model. Nodes representing the gas in the intake and exhaust ports were removed, since they were single nodes that did not provide any additional information. The new method that was implemented consists in retrieving heat data calculated by the Gas Dynamics model by means of a direct request and then introduce it in the equations of the port elements as a source term. The port elements are port walls and valves, including the upper face of the valve plugs and the stems. An example of an exhaust port is shown in Fig. 4.77. For each port, heat to wall, heat to valve and heat to stem can be read separately. The number of port ducts depends on the engine. The new Nodal Model is able to process one or two ports at the intake or at the exhaust. Wall temperatures of port elements are required by the Gas Dynamics model in order to calculate heat transfer. Every time the Nodal Model calculates engine temperatures, they are stored and made readable to the Gas Dynamics model. In addition to wall temperatures, the Gas Dynamics model needs the surface areas of each of the port elements in contact with gas to calculate heat transfer. This information is made accessible to the Gas Dynamics model when the Nodal Model geometry is created.

The original method is still allowed for cases in which the Nodal Model and the Gas Dynamics model are uncoupled. However, additional inputs are required: temperatures and mean mass flow rates of intake and exhaust gas. Moreover, at the beginning of the simulation, two nodes representing the intake and exhaust gas are created.

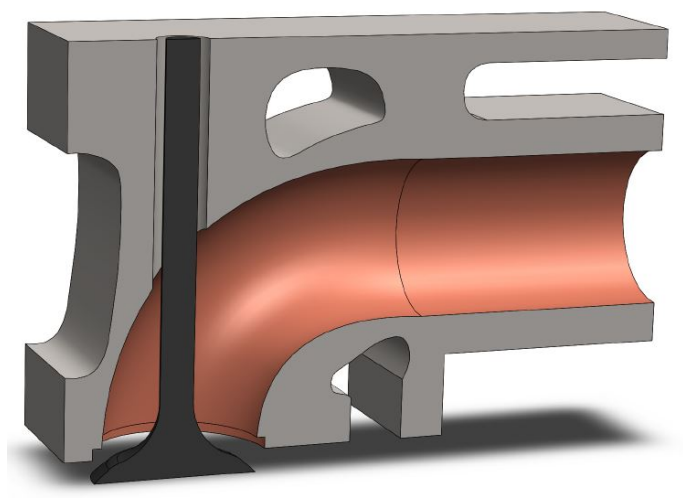

Figure 4.77: 3D cut of exhaust port. 
Friction is also contemplated as a heat source term in the new Nodal Model. Friction data is calculated by the Mechanical Losses models. However, friction heat is supplied to the Nodal Model by the Cylinder model as a periodic input. Two values are received: one is the heat dissipated in the piston rings due to friction with the liner and the other is the rest of heat dissipated by friction. Heat to piston rings is entered as a source term in the equation of one of the piston nodes, node 3 (see Fig. 3.6 in Section 3.3). Node 3 is assumed to contain the piston rings and receive all heat dissipated by friction. Regarding the second value, the rest of heat from friction, it is entered as a source term in the equation of the oil node.

Radiation was not considered in this model. According to modern experiments reviewed in Section 2.2.3, the contribution of radiation to in-cylinder heat rejection is small, even in diesel engines. Moreover, in Section 2.2.1 it was seen that many authors had modeled thermal radiation in a lumped form with convection, which is certainly the dominant heat transfer mechanism. In the case of the Woschni correlation used in the Nodal Model, radiation effects are considered in a lumped way by the combustion velocity term. However, it is possible to supply values of radiative heat calculated with another model to the Nodal Model with minor modifications. These inputs can be entered as heat source terms to the thermal system, an option commented in Section 2.2.3.

Convection conductances and source terms are recalculated every time step, because conditions on the fluid side are highly variable during engine operation. Thermal capacitances have to be recalculated every call as well because they use the time increment, $\Delta t$, which is variable (see Eq. 2.42). At the same time, specific heat capacities can be updated according to new node temperatures. Conduction conductances in the solid are by default calculated only during model creation and assumed invariable for the rest of the simulation. However, the calculation of conduction conductances is separated to allow being called at any time. The purpose of recalculating these conductances would be to update thermal conductivities of the solid using current temperatures. But, in general, temperatures in the solid do not vary much during engine operation and therefore the changes in thermal conductivity are not very important. Moreover, recalculating the conductances would come at the cost of slightly increasing computational demand. For those reasons, recalculation is deactivated by default.

All modifications introduced with respect to the initial Nodal Model 
had an impact on the definition of system matrices. The matrices constitute the system of equations that describe the thermal system to be solved. Solving methods were discussed in Section 4.5. Depending on an external call, a steady-state or a transient implicit method is employed. The solver's outcome is a vector that contains the temperature of every node. This is the fundamental result of the Nodal Model. Next, a post-processing procedure allows to obtain all relevant outputs. Temperatures are averaged to calculate wall temperatures or temperatures of certain elements. Heat fluxes among nodes are calculated by multiplying the conductance matrix and corresponding temperature differences. The result is a heat flux matrix. For instance, to obtain the element in row $i$ and column $j$, the operation is $Q_{i j}= \pm K_{i j}\left(T_{i}-T_{j}\right) . Q_{i j}$ is heat transfer between node $i$ and node $j$, which can be an inflow or an outflow depending on the sign of the thermal conductance $K$. Heat rejection to a particular node or group of nodes can be calculated summing all heat inflows from other nodes, plus the accumulated heat and the heat generated in it. Nonetheless, to obtain heat rejection to coolant and oil in VEMOD, a simpler method is used. From the heat balance, $\dot{Q}_{g e n}+\dot{Q}_{i n}-\dot{Q}_{\text {out }}=\dot{Q}_{\text {flow }}+\dot{Q}_{\text {accum }}$, where $\dot{Q}_{g e n}$ is generated heat, $\dot{Q}_{i n}$ is heat going to the fluid from other nodes, $\dot{Q}_{\text {out }}$ is heat flowing from the fluid to other nodes, $\dot{Q}_{\text {flow }}$ is enthalpy increment experienced by the fluid and $\dot{Q}_{a c c u m}$ is heat accumulated in the fluid. Therefore, total heat rejection to coolant and oil can be calculated as $\dot{Q}_{\text {fluid }}=\dot{Q}_{\text {flow }}+\dot{Q}_{\text {accum }}$.

Finally, the output of results is accomplished through a set of functions that can be externally called. Each function returns a different variable, which can be a temperature value, a temperature array or a heat flux value. Most results are requested by the parent Cylinder model, but other three models obtain data directly from the Nodal Model: the Gas Dynamics model receives temperature of valves and port walls in the cylinder-head, the Mechanical Losses model receives the piston rings temperature and the Injection model receives the injector temperature. Indirectly, the ThermoHydraulic model reads heat rejection and fluid outlet temperature from the Cylinder model, which is part of the Heat Exchangers model as explained in Section 5.1. Later, results can be written to VEMOD's output file. The writing functions are part of the Cylinder model and they were newly implemented as part of this thesis' works. 


\section{Heat transfer in ancillary systems}

5.1 Model of heat exchangers . . . . . . . . . . . . . . . 212

5.1 .1 Model structure . . . . . . . . . . . . . . . . 213

5.1 .2 Effectiveness-NTU method . . . . . . . . . . . 215

5.1.3 Definition of heat exchangers . . . . . . . . . . 218

5.2 Thermo-hydraulic model . . . . . . . . . . . . . . . 221

5.2 .1 Description of objects . . . . . . . . . . . . 223

5.2 .2 General workflow . . . . . . . . . . . . . . 227

5.2.3 Circuit definition . . . . . . . . . . . . . . . . 229

5.2 .4 Circuit creation . . . . . . . . . . . . . . 231

5.2.5 Hydraulic network analysis . . . . . . . . . . . . 242

5.2 .6 Thermal state of the circuit . . . . . . . . . . 255

This chapter describes two models that were developed from the ground up in order to extend the Virtual Engine Model. The models simulate ancillary systems of the engine where heat transfer processes occur, either as primary processes or as secondary ones.

One of the models, the model of heat exchangers, provides a common framework for all elements where heat transfer takes place. This allows all those elements to communicate with other models in a standard way. The model of heat exchangers also comprises methods for simulating heat transfer between two fluid flows.

The second model is a thermo-hydraulic model for fluid circuits. This model simulates the mass and energy transport throughout a hydraulic circuit. In a hydraulic circuit, flow is incompressible, i.e., density changes in the fluid are negligible. This is the case of liquids and of gases that 
do not go through significant compression and expansion processes. Together, the two models make possible to simulate a system like the cooling circuit of an engine, which exchanges heat with different components and moves the thermal energy from sources to sinks. Other systems whose main purpose is not to transfer heat, like the lubrication circuit, can be simulated with these models as well.

There were two purposes for modeling the ancillary systems of the engine. First, to capture more accurately the behavior of the engine as an assembly of multiple systems. Indeed, the ancillary systems have a significant influence on the thermal response of the engine. Second objective was to provide the capability of making thermal management studies.

Also, there were three criteria that were followed when the models were being developed. The first one was that every model had to be linkable to the other engine models in order to work like a network. The second criterion was that the models had to be general to allow simulating any kind of heat exchanger and any kind of hydraulic circuit. Lastly, the models had to be flexible enough to allow the study of diverse circuit layouts and a wide variety of hardware.

\subsection{Model of heat exchangers}

5.1 .1 Model structure . . . . . . . . . . . . . . . . . . 213

5.1 .2 Effectiveness-NTU method . . . . . . . . . . . . 215

5.1.3 Definition of heat exchangers . . . . . . . . . . 218

An internal combustion engine is a thermal machine in which many heat exchanges exist. Some of the thermal exchanges occur in devices whose purpose is to exchange heat between two fluids, called heat exchangers. Other heat exchanges occur in elements whose primarily purpose is not to exchange heat. For instance, the function of the engine cylinders is to convert one kind of energy into another kind. In the cylinders, fuel's chemical energy is converted into mechanical energy through changes in the thermal state of a working fluid. However, during this process, heat transfer processes take place. The turbocharger is another machine present in the engine whose purpose is not to exchange heat. In the turbocharger, thermal energy is recovered from the engine exhaust as mechanical energy and then this mechanical energy is used to increase the thermal state of the intake gas. As in the cylinders, heat transfer is present as a secondary effect. From the point of view of a computational model, 
all those heat exchanging elements can be simulated with a single model that is general enough, regardless of heat transfer being a primary or a secondary process. In this section, the name heat exchangers will be used to refer to the generalized element of the VEMOD code and the thermohydraulic model. That is, all engine components where heat transfer must be modeled will be heat exchangers. Computationally, they are objects of the heat exchanger model.

\subsubsection{Model structure}

To get a model that is general enough to consider all possible heat transfer processes, the structure of the model has to be relatively complex. The scope of the model is marked in grey color in Fig. 5.1. Heat exchanger types are classified as follows:

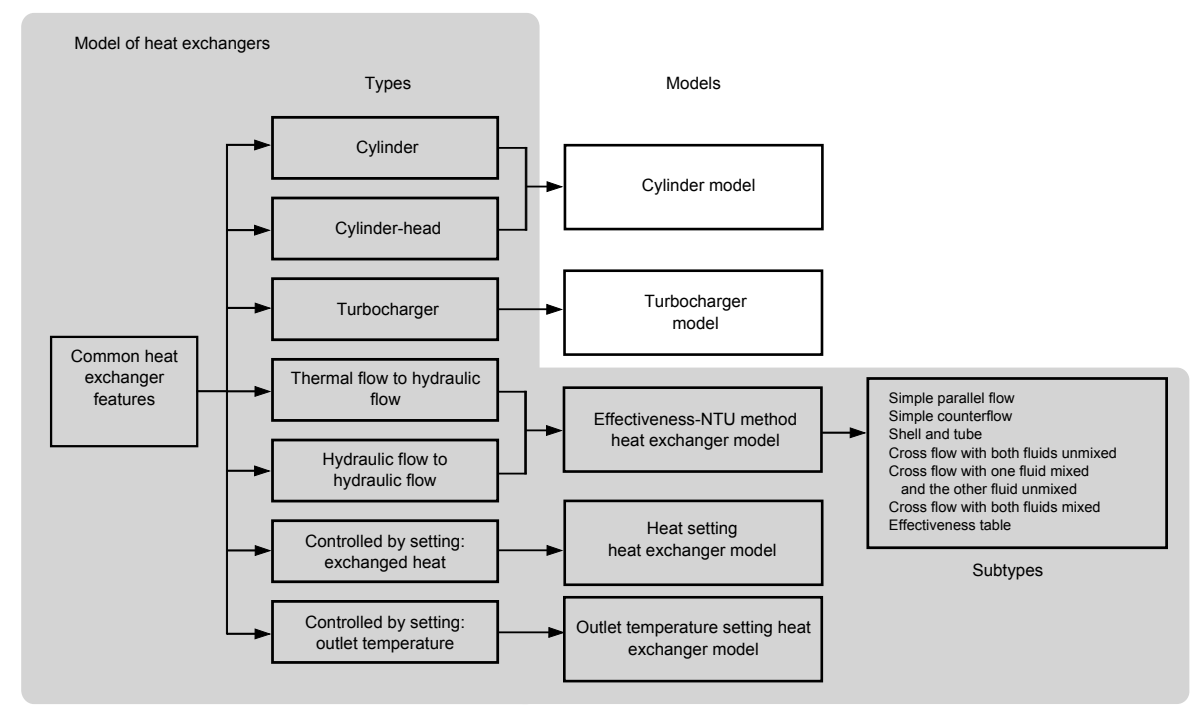

Figure 5.1: Structure of the model of heat exchangers.

1. Elements whose purpose is different from exchanging heat.

- Cylinder

- Cylinder-head

- Turbocharger

2. Elements whose main purpose is to exchange heat. 
- Thermal flow to hydraulic flow. Like heat transfer between a gas and a liquid through a solid. Example: from EGR gas to cooling fluid. In the code they are called Coolers.

- Hydraulic flow to hydraulic flow. Like heat transfer between two liquids or between a liquid and an incompressible gas flow through a solid. Example: heat rejection of the cooling fluid in the radiator. In the code they are called Liquid to liquid.

- Controlled by setting: exchanged heat. The exchanger is connected to only one circuit. The fluid receives the amount of heat specified by the user. Outlet temperature is calculated and stored. This is useful in case the user wants to prescribe the rejected energy, for instance because it was experimentally measured.

- Controlled by setting: outlet temperature. The exchanger is connected to only one circuit. The fluid goes out at the user-specified temperature. The necessary amount of exchanged heat is calculated and stored. This can be applied when the heat exchanger is programmed to keep a target outlet temperature.

The first group of heat exchanger elements (cylinder, cylinder-head and turbocharger) have dedicated models. For example, there is a cylinder model that simulates all the processes that take place in the cylinder: injection, combustion, gas flow, thermodynamic evolution, piston movement, friction and heat transfer. All processes are simulated by means of submodels. The submodel for heat transfer was extensively studied in Chapter 4. This dedicated model makes all calculations related to heat transfer. The cylinder model is, in turn, a submodel of the engine block model. But the cylinder model is also a submodel of the heat exchanger model that is being described in the present section. Therefore, all listed elements in both groups belong to the common part of the heat exchanger model. The functions of this common part are to exchange data with other models and to perform common operations such as calculating heat capacity of flows, but not to calculate heat transfer. The common part of the heat exchanger model also stores basic information like exchanged heat, flow conditions, fluid volumes or flow resistance curves.

Elements in group 2 have submodels inside the heat exchanger model. They determine the operation of the heat exchanger. Heat exchangers controlled by a setting are relatively simple. Setting (heat or temperature) is provided by a fixed value or by a table, as a function of engine speed 
and torque. The setting can be read at the beginning or be modified by an external object. In addition, these heat exchangers have a function to determine the exchanged heat or the outlet fluid temperature.

The remaining types are thermal-flow-to-hydraulic-flow and hydraulicflow-to-hydraulic-flow heat exchangers. Heat exchanges between two thermal flows (gas-gas) are out of the scope of this model and were not part of this thesis' works. The performance of the three mentioned types of heat exchanger is determined by the effectiveness-NTU method. This is described in Section 5.1.2. This method requires two design inputs: one is the subtype of heat exchanger and the other is the UA parameter. UA is called overall conductance and it is the product of the overall heat transfer coefficient and the heat transfer area. It is a measure of the capacity of the device for exchanging heat. UA can be entered as a fixed value or as a table which is a function of fluid mass flows. Regarding the subtype of heat exchanger, this second-level classification only has influence on the calculation of the effectiveness-NTU method. Subtypes are:

- Simple parallel flow.

- Simple counterflow.

- Shell and tube.

- Cross flow with both fluids unmixed.

- Cross flow with one fluid mixed and the other fluid unmixed.

- Cross flow with both fluids mixed.

- Effectiveness table.

A peculiarity of the heat exchange between a fluid flow and the ambient, like in a radiator, is that the heat exchanger is connected to only one circuit. Properties of the ambient side are obtained from global engine information.

\subsubsection{Effectiveness-NTU method}

The effectiveness-NTU (number of transfer units) method is used to calculate heat transfer between two fluid flows in a heat exchanger. This is a popular method widely reported in the literature [208] [209]. In this method, exchanged heat is calculated as the product of a parameter called 
effectiveness, $\epsilon$, and the maximum heat amount that can be exchanged theoretically between two flows, $\dot{Q}_{\max }$, as seen in Eq. 5.1.

$$
\dot{Q}_{\text {exch }}=\epsilon \dot{Q}_{\max }
$$

$\dot{Q}_{\max }$ is given by Eq. 5.2. $T_{i n}$ are the inlet temperatures of the fluids and $C_{\min }$ is the minimum heat capacity rate of both fluids. This maximum heat is based on a counterflow heat exchanger of infinite length and in absence of external heat losses. The largest temperature difference occurs in the fluid with lowest heat capacity rate. Therefore, the largest possible heat exchange happens when the outlet temperature of the fluid with the lowest heat capacity rate is equal to the inlet temperature of the other fluid. Indeed, the difference between inlet temperatures is the largest temperature difference in the system. This is illustrated in Fig. 5.2.

$$
\dot{Q}_{\max }=C_{\min }\left(T_{1, i n}-T_{2, i n}\right)
$$

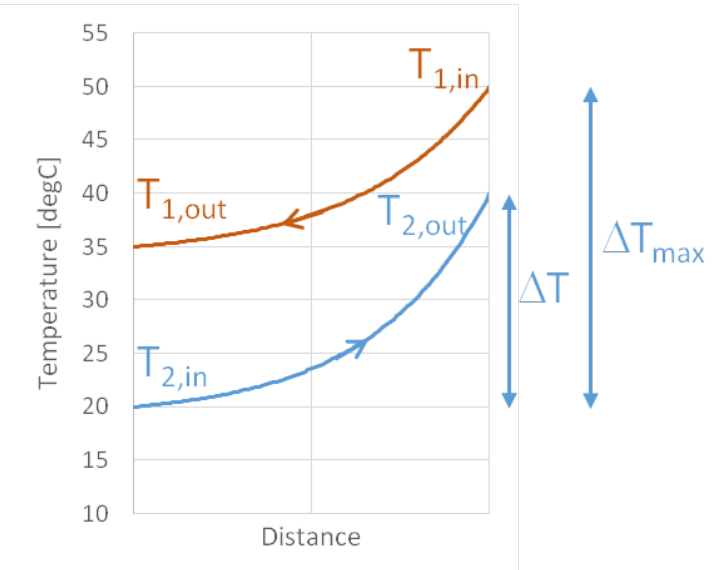

$$
\begin{aligned}
& \Delta T=T_{2, \text { out }}-T_{2, \text { in }} \\
& \Delta T_{\max }=T_{1, \text { in }}-T_{2, \text { in }} \\
& Q=C_{\text {min }} \Delta T \\
& Q_{\text {max }}=C_{\text {min }} \Delta T_{\max }
\end{aligned}
$$

Figure 5.2: Maximum temperature difference in counterflow heat exchanger.

To calculate effectiveness, two parameters are defined: $N T U$ and $C_{r}$. $N T U$ is number of transfer units and $C_{r}$ is heat capacity ratio. They are defined as follows:

$$
N T U=\frac{U A}{C_{\min }}
$$




$$
C_{r}=\frac{C_{\min }}{C_{\max }}
$$

The literature provides expressions to calculate effectiveness as a function of $N T U$ and $C_{r}$ for different subtypes of heat exchangers.

- Simple parallel flow.

$$
\epsilon=\frac{1-e^{-N T U\left(1+C_{r}\right)}}{1+C_{r}}
$$

- Simple counterflow.

$$
\epsilon=\frac{1-e^{-N T U\left(1-C_{r}\right)}}{1-C_{r} e^{-N T U\left(1-C_{r}\right)}}
$$

- Shell and tube.

One pass through shell

$$
\epsilon_{1}=2\left(1+C_{r}+\left(1+C_{r}^{2}\right)^{0.5} \frac{1+e^{-N T U\left(1+C_{r}{ }^{2}\right)^{0.5}}}{1-e^{-N T U\left(1+C_{r}{ }^{2}\right)^{0.5}}}\right)^{-1}
$$

$\mathrm{N}$ passes through shell

$$
\epsilon_{N}=\left[\left(\frac{1-\epsilon_{1} C_{r}}{1-\epsilon_{1}}\right)^{N}-1\right]\left[\left(\frac{1-\epsilon_{1} C_{r}}{1-\epsilon_{1}}\right)^{N}-C_{r}\right]
$$

- Cross flow with both fluids unmixed.

$$
\epsilon=1-e^{\frac{1}{C_{r}} N T U^{0.22}\left(e^{-C_{r} N T U^{0.78}}-1\right)}
$$

- Cross flow with one fluid mixed and the other one unmixed. $C_{\text {min }}$ fluid unmixed

$$
\epsilon=\frac{1}{C_{r}}\left[1-e^{-C_{r}\left(1-e^{-N T U}\right)}\right]
$$

$C_{\min }$ fluid mixed

$$
\epsilon=1-e^{\frac{1}{C_{r}}\left(1-e^{-C_{r} N T U}\right)}
$$

- Cross flow with both fluids mixed.

$$
\epsilon=\left(\frac{1}{1-e^{-N T U}}+\frac{C r}{1-e^{-C r N T U}}-\frac{1}{N T U}\right)^{-1}
$$


- Effectiveness table. $N T U$ and $C_{r}$ are independent variables of the table and effectiveness is the dependent variable.

- In any case, if $C_{r}=0$ :

$$
\epsilon=1-e^{-N T U}
$$

Once heat flux is known, outlet fluid temperatures can be calculated with Eq. 5.14.

$$
T_{\text {out }}=T_{\text {in }}+\frac{\dot{Q}_{\text {exch }}}{C}
$$

An assumption of this method is steady-state heat transfer, i.e., no thermal inertia.

\subsubsection{Definition of heat exchangers}

The information to define the heat exchangers was entered in XML format, the standard method in VEMOD. This way, the data and the reading methods were compatible with all VEMOD submodels. As previously mentioned, the XML code is a schema of elements and attributes. The structure of the schema for the model of heat exchangers is shown in Fig. 5.3. Block of Heat Exchangers is the main element that contains all heat exchangers. Subordinated to it, each heat exchanger constitutes a separate element. All heat exchangers contain identification data (ID and name) and type. Possible types are summarized in Section 5.1.1. Depending on the type, the element may contain further information in the form of attributes or subelements.

For the cylinders, cylinder-heads and turbochargers, only a reference ID of the object's model is stored (cylinder model or turbocharger model). This is enough to create an object of the common class for components that exchange heat.

For heat exchangers controlled by a setting, the heat exchanger element has two additional attributes. ReadSettingFrom tells whether the setting is entered as a constant value or as a table. In the first case, the value is specified in the Setting attribute. If the setting is defined as a table, a subelement representing the table is added. In the table, the independent variables are engine speed and torque and the dependent variable is the setting: heat or outlet temperature. Each row of the table is a subelement of the table with three attributes: engine speed, torque and heat or temperature. Moreover, the heat exchanger is connected to one fluid circuit. 


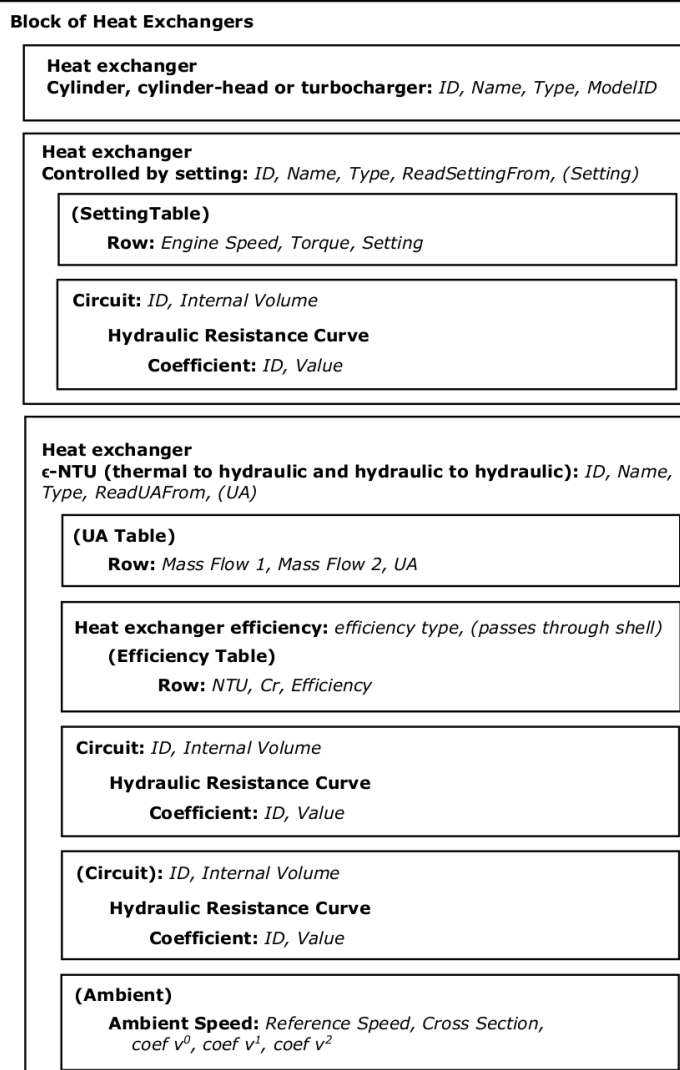

Bold:element

Italic: atribute

(brackets): optional

Figure 5.3: Structure of the XML code that contains the input data of the model of heat exchangers. 
It is necessary to provide as attributes the ID of the circuit and the volume of fluid in the heat exchanger. A necessary input for the circuit model is the hydraulic resistance curve of the heat exchanger. It is specified as a set of coefficients of a head loss curve in terms of temperature and flow rate. This is discussed in detail in Section 5.2.1 of the thermo-hydraulic circuit model.

Definition of heat exchangers that use the $\epsilon-N T U$ method, i.e., thermalflow-to-hydraulic-flow and hydraulic-flow-to-hydraulic-flow, is the most complex. The UA parameter can be specified as a value or as a table, as the setting of the previous group of heat exchangers. Attribute $U A$ is used to enter a constant value. The $U A$ table is a subelement formed by rows with three attributes: mass flow of the first fluid, mass flow of the second fluid and UA. Heat exchanger efficiency is a mandatory subelement that determines the efficiency subtype of the heat exchanger. In the shell and tube case, the number of passes through shell is also required to evaluate Eq. 5.8. Again, some information about the connected circuits must be provided. In general, the heat exchanger will be connected to two circuits. However, the heat exchanger can be connected to only one circuit if it is connected to the ambient on the other side. In the latter case, the Ambient subelement is required. This subelement contains fluid speed curves of the form of Eq. 5.15. They are necessary, along with ambient density, which is a global variable, to know air mass flow. For each speed curve, there are five attributes. Reference speed is $v_{\text {ref }}$ of Eq. 5.15. It can be Vehicle speed or Engine speed. Vehicle speed is a global variable accessible to the heat exchanger model. Engine speed can be read from the engine block model. The coefficients $c_{0}, c_{1}$ and $c_{2}$ are also entered as attributes. Finally, the cross section of the heat exchanger for the respective speed is requested.

$$
v_{a m b}=c_{0}+c_{1} v_{r e f}+c_{2} v_{r e f}^{2}
$$

The model of heat exchangers does not save any output by itself. Instead, the heat exchanger may be a component that has a dedicated model, such as the cylinder or the turbocharger. The dedicated model can save the exchanged heat as an output. On the other hand, the heat exchanger is part of at least one fluid circuit. In such case, the gas or liquid circuit can save the heat exchanged by the fluid as an output. 


\subsection{Thermo-hydraulic model}

5.2 .1 Description of objects . . . . . . . . . . . 223

5.2 .2 General workflow . . . . . . . . . . . . . . . 227

5.2 .3 Circuit definition . . . . . . . . . . . . . . . . . 229

5.2 .4 Circuit creation . . . . . . . . . . . . . . 231

5.2 .5 Hydraulic network analysis . . . . . . . . . . . 242

5.2 .6 Thermal state of the circuit . . . . . . . . . . . 255

To study engine thermal processes completely, it is necessary to take into consideration the incompressible flows that are present in the engine. Those flows are usually enclosed within closed circuits that interact with different engine elements. A hydraulic circuit can be seen as a network where a fluid flows and goes through connected elements. The most relevant incompressible flow from the point of view of thermal processes is the cooling flow, because its main function is to evacuate heat from the engine to prevent mechanical problems. The lubrication system can be seen as a hydraulic network as well. It also has a relevant role in thermal processes because the lubricant is the main cooling source of the piston and because it receives part of the heat produced by friction, which represents a significant share of the total heat rejection, specially during engine warm up [117] [160]. Another hydraulic circuit that can be present in the engine is the one used for liquid fuel distribution. However, thermal interactions of the fuel circuit circuit are usually of lesser importance in the engine [101].

To simulate the hydraulic circuits of the engine, the model described in the present section was developed. The thermo-hydraulic model was developed under the following specifications:

1. Network layout has to be defined with total freedom. This implies that the model must be able to solve any possible hydraulic circuit.

2. The model must analyze the hydraulic network to calculate flow rates and head losses over the whole network.

3. The model must be able to determine the temperature distribution of the fluid throughout the network.

4. The variety of objects that can be included in the network has to be enough to represent the different components that can be found in the hydraulic circuits of engines and the objects must behave realistically. 
5. The model has to be linkable to other submodels of engine components, in particular to the heat exchanging elements described in Section 5.1, including the cylinder heat transfer model of Chapter 4 as main heat source of the engine.

6. The model has to be able to work both stand-alone and integrated into the Virtual Engine Model.

7. Thermal inertia of fluids and temperature dependence of physical properties must be accounted for.

8. Characteristics of circuit components must be able to be defined with great flexibility.

9. Model structure has to be modular to allow future expansions.

The model is based on the simulation of a network. A network is a collection of nodes and branches. Prior to describing the model, some key concepts are briefly defined. They are related to the discipline of hydraulics and to the description of networks. These concepts are used regularly in this section.

- Flow rate: volume of fluid per unit of time.

- Hydraulic head or piezometric head: it is a measure of the potential of a fluid, taking into account elevation and fluid pressure. Definition of hydraulic head is $H=z+\frac{p}{\rho g}$, where $z$ is elevation and $p$ is pressure. In the thermo-hydraulic model, however, elevation is neglected since the influence is very low in automotive applications.

- Object: element that forms part of the circuit and has a determined function. Objects are described in Section 5.2.1.

- Node: junction point where any number of objects can be connected.

- Branch: linear path between two nodes. It is composed of a succession of objects. Flow rate is the same along a branch.

- Mesh: linear path that forms a closed loop. It is composed of a set of branches. 
This section is divided into several subsections. First, circuit components are described in detail. Second, an overview of the model's workflow is given. Next, it is explained how circuit information is specified and how a circuit is built using that information. The last two subsections deal with the calculations performed by the model to solve the hydraulic network and the thermal distribution.

\subsubsection{Description of objects}

A hydraulic circuit is made up of different elements. The thermo-hydraulic model has eight types of objects, each one with a different function. To fully define a circuit, it is necessary to specify the elements that are present in it, their type, their properties and the circuit layout. Next, object types and their properties are described.

\section{Node}

A node is a junction point where any number of elements can be connected. From the hydraulic point of view, nodes have zero volume and do not produce head losses. Thermally, nodes are considered adiabatic and the inflows undertake perfect mixing, as in [43].

\section{Pipe}

A pipe is a closed cylindrical tube. Flowing fluid does not have a free surface because it is assumed to fill the whole pipe volume. A pipe generates a head loss which is proportional to flow rate squared, as in Eq. 5.16. Hydraulic resistance of the pipe, $R_{h, 2}$, is obtained from the Darcy-Weisbach equation, Eq. 5.17. Combining Eqs. 5.16 and 5.17, and assuming that the tube is cylindrical, Eq. 5.18 is obtained. Therefore, for each pipe, it is necessary to know length, internal diameter and friction coefficient between fluid and wall. Friction coefficient is considered constant in this model. Pipes are assumed adiabatic, as in the reference work [153].

$$
\begin{aligned}
H & =R_{h, 2} \dot{V}^{2} \\
H & =f \frac{L}{D_{h}} \frac{v^{2}}{2 g} \\
R_{h, 2} & =\frac{8 f L}{\pi^{2} g D^{5}}
\end{aligned}
$$




\section{Heat exchanger}

A heat exchanger interacts thermally with the circuit fluid. Thermal properties of heat exchangers are thoroughly described in Section 5.1. Head loss is given by a curve in terms of flow rate and fluid temperature, as can be seen in Eq. 5.19. Temperature influence was accounted for because it can induce density changes that have an effect on head loss.

$$
\begin{array}{r}
H=\left(R_{h, 00}+R_{h, 01} T+R_{h, 02} T^{2}\right)+\left(R_{h, 10}+R_{h, 11} T+R_{h, 12} T^{2}\right) \dot{V}+ \\
+\left(R_{h, 20}+R_{h, 21} T+R_{h, 22} T^{2}\right) \dot{V}^{2}
\end{array}
$$

Curve coefficients $R_{h}$ are used to define an object which represents the curve. Curves belong to a class called Multivariate Polynomial. After a curve is created, it can be evaluated easily by calling the proper class method and providing the values of the parameters, in this case $\dot{V}$ and $T$. This simplifies the management of complex curves. All curves of the thermo-hydraulic model are defined like this.

\section{Pump}

A pump propels the fluid around the circuit. In addition, power consumed by the pump is rejected as heat to the fluid. Pump speed is updated dynamically by reference to an external rotational speed through a parameter called Speed Ratio, Eq. 5.20. For mechanical pumps in engine applications, reference speed is the engine speed.

$$
\text { SpeedRatio }=\frac{\text { PumpSpeed }}{\text { ReferenceSpeed }}
$$

Power consumption $\dot{W}$ is calculated with Eq. 5.21, once pump head and flow rate through the pump are known. Pressure difference is obtained from pump head. Pressure difference, flow rate and pump efficiency allow to obtain pump power.

$$
\begin{gathered}
\Delta p=\rho g H \\
\dot{W}=\frac{\Delta p \dot{V}}{\eta(\Delta p, \dot{V})}
\end{gathered}
$$

To define pump efficiency, a four-degree polynomial in terms of pressure difference and flow rate is employed, Eq. 5.22. 


$$
\begin{aligned}
\eta & =\left(a_{00}+a_{01} \Delta p+a_{02} \Delta p^{2}+a_{03} \Delta p^{3}+a_{04} \Delta p^{4}\right)+ \\
& +\left(a_{10}+a_{11} \Delta p+a_{12} \Delta p^{2}+a_{13} \Delta p^{3}+a_{14} \Delta p^{4}\right) \dot{V}+ \\
& +\left(a_{20}+a_{21} \Delta p+a_{22} \Delta p^{2}+a_{23} \Delta p^{3}+a_{24} \Delta p^{4}\right) \dot{V}^{2}+ \\
& +\left(a_{30}+a_{31} \Delta p+a_{32} \Delta p^{2}+a_{33} \Delta p^{3}+a_{34} \Delta p^{4}\right) \dot{V}^{3}+ \\
& +\left(a_{40}+a_{41} \Delta p+a_{42} \Delta p^{2}+a_{43} \Delta p^{3}+a_{44} \Delta p^{4}\right) \dot{V}^{4}
\end{aligned}
$$

There are two types of pumps:

- Positive displacement pump, also called volumetric pump. In this kind of pump, flow rate is independent of the discharge pressure, i.e. the head loss in the circuit. Instead, flow rate is determined by a curve dependent on two parameters: pump speed $N$ and fluid temperature T. Curve expression is provided in Eq. 5.23.

$$
\begin{aligned}
\dot{V}=\left(a_{00}+a_{01} N+a_{02} N^{2}\right) & +\left(a_{10}+a_{11} N+a_{12} N^{2}\right) T+ \\
& +\left(a_{20}+a_{21} N+a_{22} N 2\right) T^{2}
\end{aligned}
$$

Flow rate is limited by the maximum pressure allowed by the pump. Due to safety reasons, once the volumetric pump reaches the maximum head, flow is recirculated to a return line usually connected back to the supply line. The apparent consequence is that flow rate is "saturated" at a certain point. Pump pressure is calculated during network analysis. The thermo-hydraulic model takes into account the maximum pump pressure when solving the hydraulic network, as explained in section 5.2.5.

- Turbopump. In this type of pump, flow rate and pump head are interrelated. Pump head is given by a curve in terms of flow rate and pump speed. During network analysis, pump head and flow rate through the pump are determined simultaneously with the rest of flow rates and head differences of the circuit. Expression of pump head is provided in Eq. 5.24. Coefficients are noted as $R_{h}$ because they can be seen as hydraulic resistances. This is discussed in section 5.2.5. 


$$
\begin{aligned}
H=\left(R_{h, 00}+R_{h, 01} N+\right. & \left.R_{h, 02} N^{2}\right)+\left(R_{h, 10}+R_{h, 11} N+R_{h, 12} N^{2}\right) \dot{V}+ \\
& +\left(R_{h, 20}+R_{h, 21} N+R_{h, 22} N^{2}\right) \dot{V}^{2}
\end{aligned}
$$

This kind of pump does not require a value of maximum pressure because the pump curve is completely defined by Eq. 5.24.

\section{Valve}

A valve creates a localized hydraulic resistance. Valves are also considered adiabatic. There are three types of valves:

- Fixed head loss. General-purpose object which defines a constant hydraulic resistance. Head loss is calculated with Eq. 5.25. Three constants of hydraulic resistance can be specified: $R_{h, 0}$ is independent of flow rate and uses the unit m.c.f., $R_{h, 1}$ multiplies flow rate and its unit is m.c.f. $m^{-3} s$ and, finally, $R_{h, 2}$ multiplies flow rate squared and has the unit m.c.f. $m^{-6} s^{2}$. This is the only kind of valve that can have a volume different from zero.

$$
H=R_{h, 0}+R_{h, 1} \dot{V}+R_{h, 2} \dot{V}^{2}
$$

- Operable valve. This valve is able to change its opening from completely closed to wide open. Hydraulic resistance is variable and depends on the opening degree of the valve, $\theta$. Opening degree varies from 0 (fully closed) to 1 (wide open) and can be changed dynamically during the simulation. Head loss and hydraulic resistance are determined by means of Eq. 5.26. Head loss is always proportional to flow rate squared. Hydraulic resistance is determined by a polynomial.

$$
\begin{gathered}
H=R_{h, 2}(\theta) \dot{V}^{2} \\
R_{h, 2}(\theta)=a_{0}+a_{1} \theta^{-1}+a_{2} \theta^{-2}+a_{3} \theta^{-3}
\end{gathered}
$$

- Thermostat. Thermostats are special types valves whose opening degree depends on a fluid temperature. Temperature of any object of the circuit can be monitored. Opening curve as a function of temperature is a logistic curve which does not account for hysteresis. The curve is given in Eq. 5.27. 


$$
\begin{gathered}
\theta=\left(1+e^{-k\left(T-x_{0}\right)}\right)^{-1} \\
k=\frac{\ln (\gamma)}{T_{c}-x_{0}}
\end{gathered}
$$

$\theta$ is the opening degree, which varies from 0 (fully closed) to 1 (wide open), $T_{c}$ is the temperature at which the thermostat closes completely, $x_{0}$ is the mean between $T_{c}$ and the wide-opening temperature, $T$ is the monitored temperature and $\gamma$ is a shape factor which determines the curve slope. Once $\theta$ is known, hydraulic resistance is calculated with another curve of the form of Eq. 5.26. In summary, the thermostat object uses two curves: opening degree as a function of temperature and hydraulic resistance as a function of opening degree. Changes in opening degree are associated to variations of the monitored temperature, with some limitations. Limitations are due to two parameters: maximum opening degree and thermostat sensitivity. In each iteration, opening degree is updated by means of evaluating Eq. 5.27 at the current temperature. Then, it is compared to opening degree of the previous iteration to check whether opening speed is higher than the maximum. If it is higher, opening degree is recalculated so that opening speed is equal to maximum opening speed. Next, opening degree is compared with thermostat sensitivity which is, by default, $1 \%$ of total opening or an opening degree of 0.01. If it is lower, opening degree is overwritten with a zero.

\subsubsection{General workflow}

The thermo-hydraulic model starts reading the circuit definition explained in Section 5.2.3 and creating the circuit as described in Section 5.2.4. An arbitrary number of circuits can be created and they can thermally interact with each other through heat exchangers. Under the Virtual Engine Model environment, separated circuit models can be created for the different hydraulic circuits in the engine: cooling, lubrication, fuel, etc. Once the circuit is created, simulation can start. An iteration can be divided into three parts: an initial updating stage, a second stage during which the circuit is regarded as a plain hydraulic network, without thermal considerations, and a final phase in which the thermal state of the circuit fluid is determined. A schema of the global process can be found in Fig. 5.4 . 
Peliminary actions

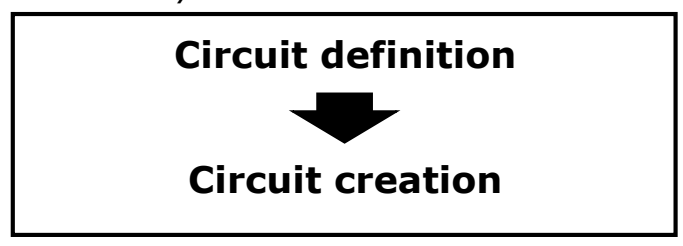

Simulation iterations

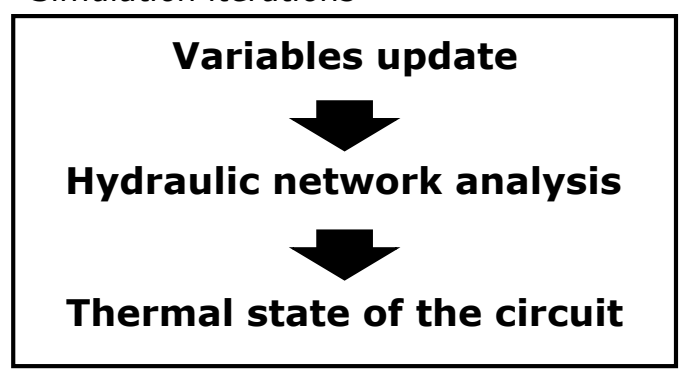

Figure 5.4: Global workflow of the thermo-hydraulic model.

Update of variables is performed at the beginning of each iteration. Time step is externally provided. By default, pump speed is calculated from a reference rotational speed using Eq. 5.20. Heat exchangers, managed by the heat exchangers model, are required to update transferred heat for the current time span. Depending on the heat exchanger type, this can trigger different tasks which are performed by the heat exchangers model. They are thoroughly explained in Section 5.1. Thermostats recalculate their opening degrees as mentioned in Section 5.2.1 during the updating stage as well.

The following stage analyses the hydraulic network to obtain flow rates and head losses in every element of the circuit. First, hydraulic resistances of all objects and branches are calculated. This is the main input to the network solver. For a detailed description of the network analysis, see Section 5.2.5. After solving the network, power consumed by the pump can be determined with Eq. 5.21.

The last part of the model deals with thermal considerations. The distribution of fluid temperature throughout the circuit is obtained. This is attained by way of a $0-\mathrm{D}$ approach which involves the discretization of the circuit into parcels of fluid. The method is comprehensively explained in Section 5.2.6. Temperature at locations of interest is stored. Inlet 
temperature of heat exchangers is determined and transferred to the heat exchanger model along with flow rate.

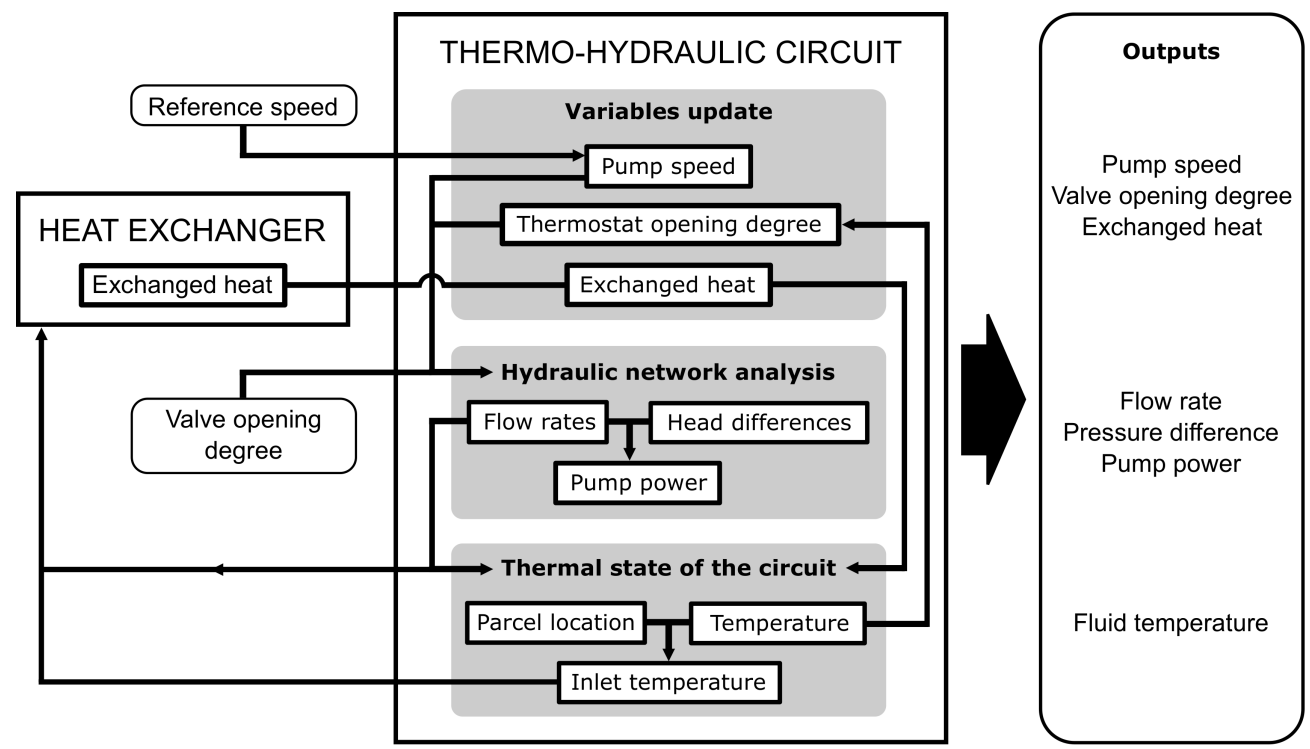

Figure 5.5: Flowchart of variables in the thermo-hydraulic model.

A summary of the commented procedure including the key variables is shown in Fig. 5.5.

\subsubsection{Circuit definition}

As in the model of heat exchangers, input data of the thermo-hydraulic circuit is entered in XML format to be compatible with the rest of engine submodels. Fig. 5.6 schematizes the structure of elements and attributes of the circuit definition. Block of Hydraulic Circuits is the main element that contains all hydraulic circuits. Subordinated to it, each circuit constitutes a separate element. For each circuit, the flowing fluid and the initial temperature must be indicated. A submodel provides all physical and chemical properties of each fluid. Information of each circuit's objects is organized in different blocks. First block is a list of the heat exchangers that are present in the circuit. Each heat exchanger is a new element listed simply with its ID (IDREF), since all heat exchangers data is included in the Block of Heat Exchangers, as explained in Section 5.1.3. Other simple blocks contain nodes and pipes. Blocks of pumps and valves 


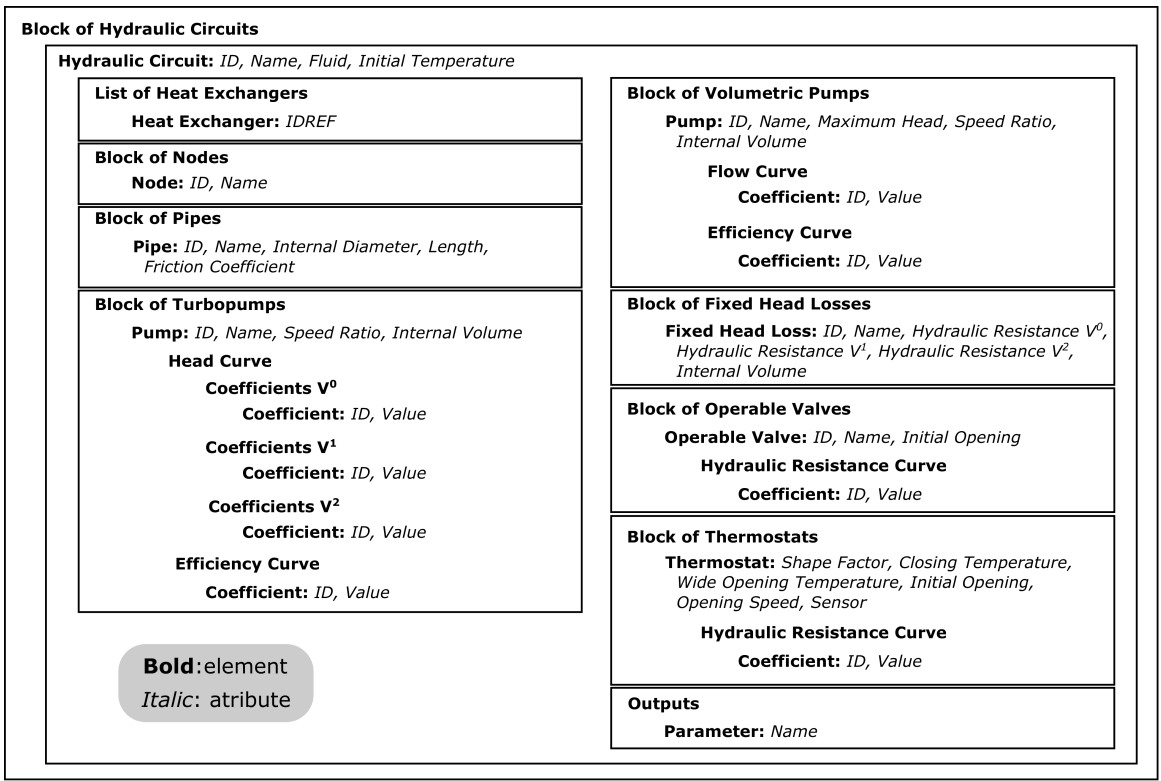

Figure 5.6: Structure of the XML code that contains the input data of the thermo-hydraulic model.

are more complex. There is a separate block for volumetric or positive displacement pumps and another one for turbopumps, as they use different information. Each element that represents a volumetric pump has two sub-elements: a Flow curve and an Efficiency curve. Curves are composed of coefficients. Each coefficient has two attributes, ID and Value. Curves of turbopumps are Head curve and Efficiency curve. Coefficients of Head curve are grouped in three sub-elements, in threes: coefficients that do not multiply flow rate, coefficients that multiply flow rate and coefficients that multiply flow rate squared. See the description of the turbopump for more details. In the case of valves, there are three sub-types and, therefore, three blocks. Each block has different attributes. The Block of Operable Valves and the Block of Thermostats have a Curve of Hydraulic Resistance which is a function of the opening degree.

A final block allows to specify the outputs to be written. The list of available outputs can be found in Table 5.1.

The circuit layout is defined by the connections among objects. Under the Virtual Engine Model environment, all elements are connected by pipes. Therefore, to define the layout is enough to specify two key at- 
Table 5.1: Outputs of the thermo-hydraulic model.

\begin{tabular}{ll}
\hline Variable & Object \\
\hline Flow Rate & every pump \\
Flow Rate & every heat exchanger \\
Pressure Difference & every pump \\
Pressure Difference & every valve \\
Pressure Difference & every thermostat \\
Pressure Difference & every heat exchanger \\
Temperature & every object \\
Inlet Temperature & every heat exchanger \\
Sensor Temperature & every thermostat \\
Heat & every heat exchanger \\
Rotational Speed & every pump \\
Power & every pump \\
Opening Degree & every valve \\
Opening Degree & every thermostat \\
\hline
\end{tabular}

tributes for each pipe: one is the ID of the element connected to the left end of the pipe, and the other one is the ID of the element on the right end. In general, notwithstanding, it is allowed to connect objects directly, without pipes. In such case, IDs of the adjoining elements are specified for each object except nodes, not only for pipes.

\subsubsection{Circuit creation}

Circuit creation involves different tasks. As a first step, information about components is read from a source with the format described in Section 5.2.3. This information is stored as needed by the network analysis and thermal state procedures. Once input information is gathered, it is necessary to identify all branches and meshes of the network. This is done by means of graph analysis using information about nodes and connections. Next, further information is systematically stored:

- Total volume of each branch.

- Location of each object's inlet.

- Location of each object's outlet. 
- Heat exchangers in each branch, sorted from the end of the branch to the beginning of the branch.

- Pumps in each branch, sorted from the end of the branch to the beginning of the branch.

- Base hydraulic resistance of each branch. There are two kinds of elements whose hydraulic resistance does not change dynamically during the simulation, i.e., their hydraulic resistance does not depend on variables such as fluid temperature, pump speed or valve opening degree. These static types are Pipe and Fixed head loss. To avoid repetitive calculations, the contribution of those objects to the hydraulic resistance of each branch is precalculated and stored at this point.

- For each thermostat, object whose temperature is monitored.

- For each thermostat, constants $x_{0}$ and $k$ are precalculated and stored (see Eq. 5.27).

- Thermal state vectors are initialized to the initial circuit temperature.

\section{Graph analysis}

Graph analysis allows the thermo-hydraulic model to interpret the circuit layout. The goals are to identify all branches and meshes of the circuit and to store their information as required by the model.

In graph theory, a graph is a collection of elements, called vertices, in which some pairs of vertices are related. Relations are called edges. In the case of a hydraulic network, the vertices are the objects that form the circuit. Edges are the physical connections among those objects. Moreover, a hydraulic network is an undirected graph because, in principle, the fluid can flow from one object to another in both directions. Thus, the first task is to determine the edges between the vertices, or connections between objects. To do this, the connections of each object are processed in order to create an adjacency matrix. The matrix indicates whether pairs of vertices are adjacent or not in the graph. An example can be seen in Fig. 5.7. 

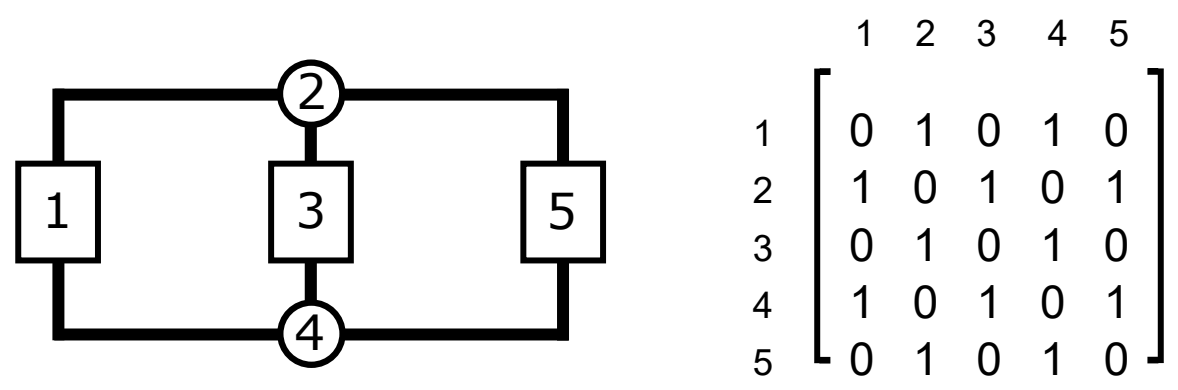

Figure 5.7: Example graph and associated adjacency matrix.

Second, the adjacency matrix is used to find all possible paths in the network. To achieve this, an original depth-first search algorithm was developed. The algorithm finds all paths starting from a given object. When a path is complete, the algorithm steps back one object and tries to find an alternative way from there, without going back through the explored path. If there are no alternatives, the algorithm keeps backtracking until it finds a new way. A clarifying schema can be found in Fig. 5.8. A depthfirst search finds paths in the order 1-2-3-4. The alternative would be a breadth-first search, which explores the neighbor objects first. This kind of search would find paths in the order 1-3-2-4. Both options are equally valid, and finally the depth-first search was chosen. In the algorithm, the starting point is the first stored node or, if the network has no nodes, the first stored object. Starting from a node simplifies the subsequent identification of branches and meshes. In the example of Fig. 5.7 and Figs. 5.9-5.15, the first node is object 2.

Fig. 5.9 shows the first move of the algorithm, both graphically and in the matrix. From object 2, the first found edge connects to object 1 . In the adjacency matrix, this is equivalent to search the first one from left to right in row 2. First one is found in the first column. Object 1 is added to the path.

When object 1 is stored, the crossed edge is removed from the adjacency matrix to prevent immediate backtracking. This is indicated in Fig. 5.10 along with the second move of the algorithm. A black circle marks the starting object. Red circles and arrow indicate the current search. To move from object 1 , the algorithm leaves row 2 and goes to row 1 . Then, it searches the first 1 . Since element in row 1 and column 2 is now zero, the next connection is with object 4 , which is a node.

In Figs. 5.11 and 5.12 it is possible to see the two following steps. 

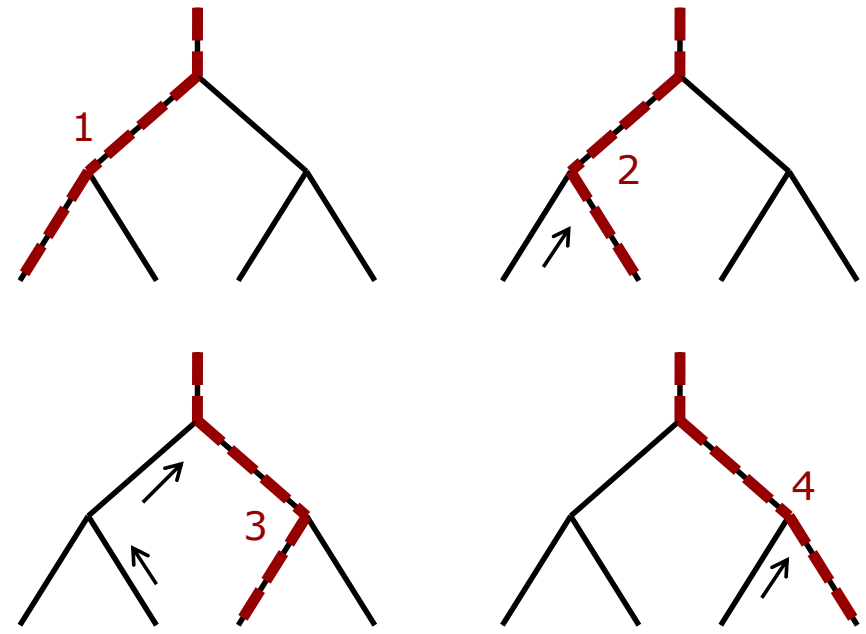

Figure 5.8: Depth-first search algorithm to find all possible paths from the top.
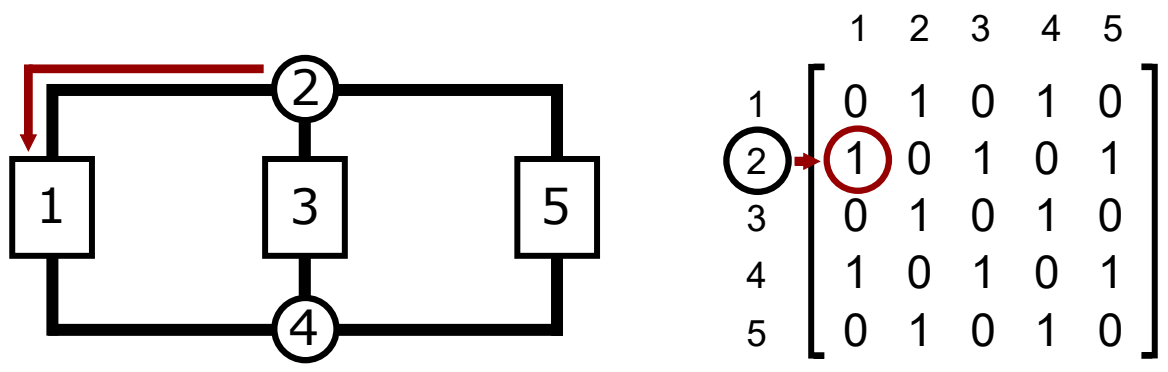

Figure 5.9: Path search. Step 1.
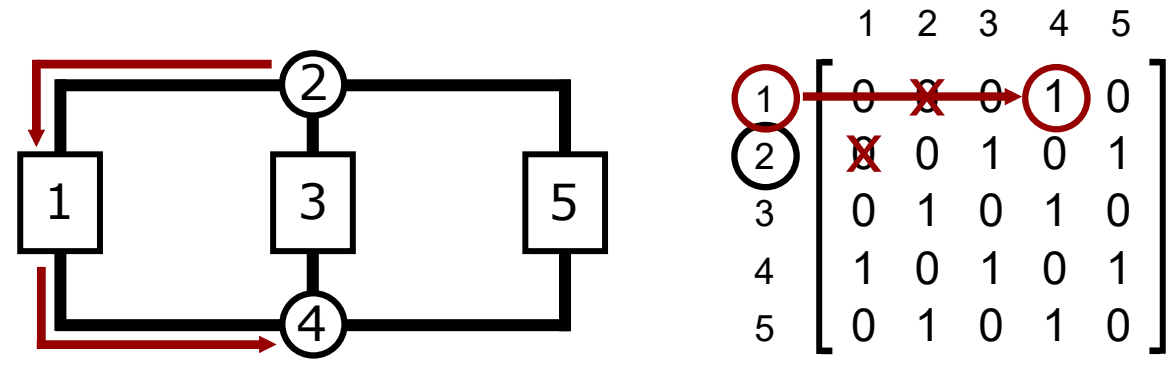

Figure 5.10: Path search. Step 2. 

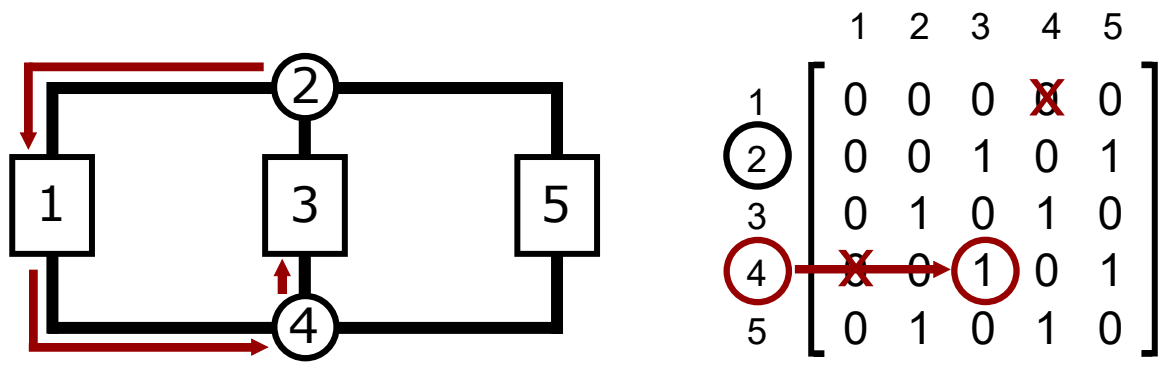

Figure 5.11: Path search. Step 3.

The algorithm continues searching through the rows and columns until it reaches object 2 . Then, it stops because object 2 is already part of the path.
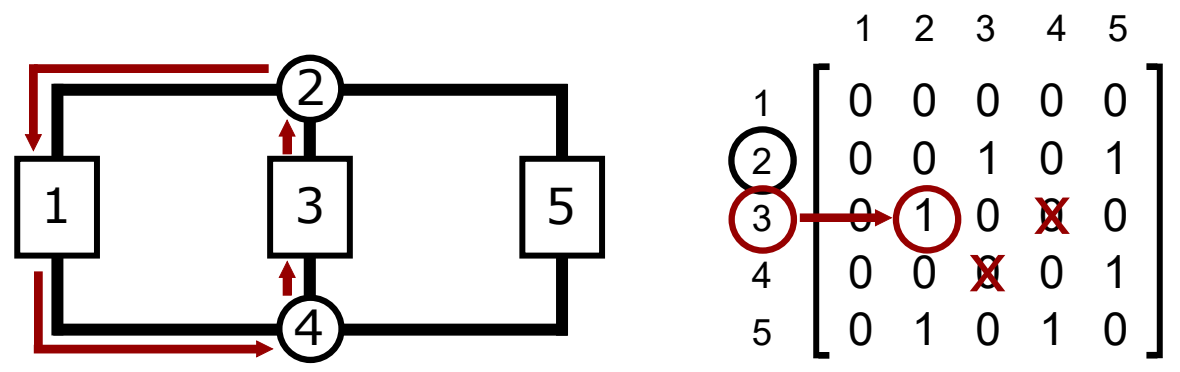

Figure 5.12: Path search. Step 4.

A path finishes when one of the following conditions is true:

(a) The path arrives to an object which was already in the path. It is the starting object.

(b) The path arrives to an object which was already in the path. It is not the starting object.

(c) The path arrives to a dead end. There are no more objects along the path.

In the example, case (a) happened. The obtained path connects objects 2-1-4-3-2.

In ending cases (b) and (c), the row of the final object is reset. In this manner, it will be possible to move to its adjacent objects when the object is reached again. In case (a), however, the final object is the starting 
object. Therefore, the already explored connections must not be explored again. Only the edge from the starting object to the previous object is restored. In this way, a new path can be started in the inverse direction in which the current one ended, i.e. from 2 to 3 in the example. The adjacency matrix at his point can be seen in Fig. 5.13.
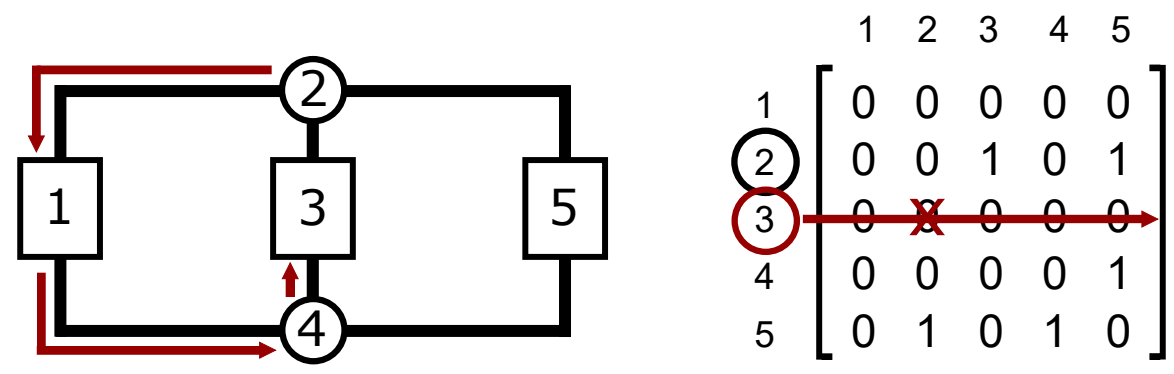

Figure 5.13: Path search. Step 5.

To search the next path, the algorithm must step back one object. The new path continues from 2-1-4-3-. Thus, the algorithm moves to row 3. According to Fig. 5.13, there are no ones in this row. This means that no new routes can be found from object 3 . When the end of a column is reached and no ones are found, a new process takes place. The following tasks are done:

1. Row of current object is restored. New paths that reach this object will then be able to access its adjacent objects.

2. The algorithm moves to the previous object in the path. In the example, it is object 4 . New row is row 4 and new path continues from 2-1-4-.

3. New column is that of the previous object. In the example, in row 4 , there is no need to check columns to the left of column 3 again because they were already processed along route 2-1-4-.

4. Edge from current object to previous object is removed. In the example, edge from 4 to 3 was already zero.

The result is the situation of Fig. 5.14. From object 4, it is possible to find a new way toward object 5. Next, as depicted in Fig. 5.15, algorithm will move from object 5 to object 2 . As this is the starting object, the path finishes there. Path is stored as 2-1-4-5-2. 

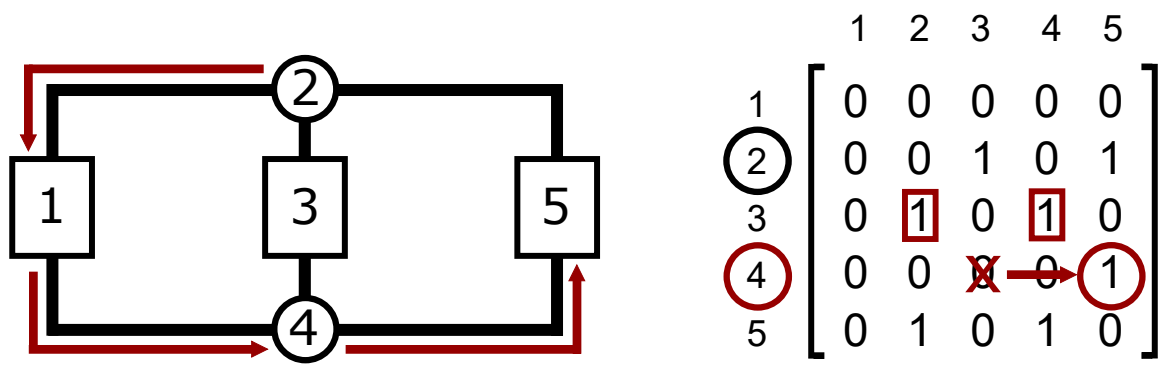

Figure 5.14: Path search. Step 6.
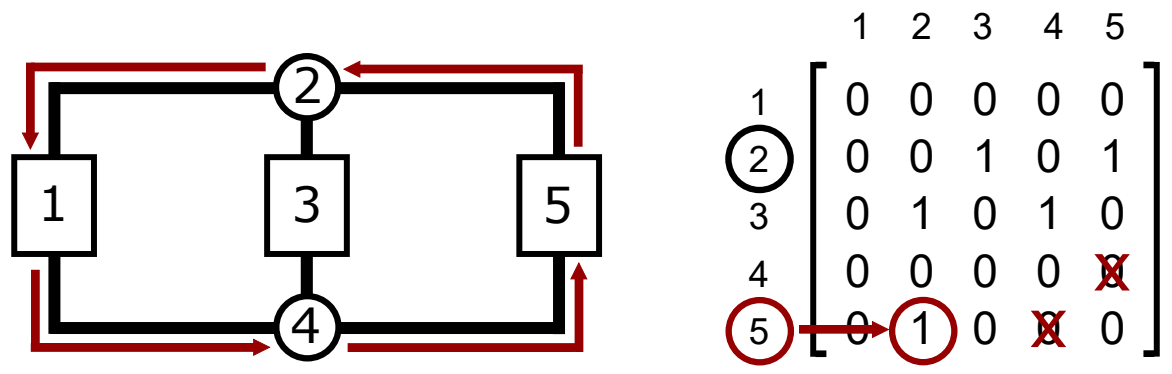

Figure 5.15: Path search. Step 7.

Now, following the rules that were previously explained, the algorithm will backtrack to object 2. Rows 1, 4 and 5 will be restored. From object 2 , new paths can be found toward objects 3 and 5 . The algorithm finishes when it reaches the end of the row of the starting object. This means that all paths from the starting object are explored.

In summary, the following six paths can be found in this circuit example. Note that the last three are the same as the first three in opposite direction.

- $2-1-4-3-2$

- $2-1-4-5-2$

- $2-3-4-5-2$

- $2-3-4-1-2$

- $2-5-4-1-2$

- $2-5-4-3-2$

Once paths are found, the model identifies those paths that are meshes. It is reminded that a mesh is a linear path that forms a closed loop. Some paths start and end at the starting object. They are already arranged as meshes and are stored without further processing. Other paths finish at a 
dead end. They can't be meshes. The third kind of paths end at an object which was previously read as part of the path but which is not the starting object. The latter case is graphically illustrated in Fig. 5.16. This hybrid type of path can be divided into two parts. One is open and goes from the starting object to the object where the cycle starts. In the example of Fig. 5.16 , the original path is $2-1-4-5-6-7-4$ and the new open path is $2-1$. The other part forms a closed cycle and is formed by all objects between the two appearances of the repeated object. In the example, the new closed path is 4-5-6-7-4. Due to the characteristics of the path-searching algorithm, it is impossible to have a path which has the closed, cyclic part at the beginning and the open part at the end. Those third-kind paths with an open and a closed part are split in the two paths previously mentioned. As a result, they are converted in a path which is purely a closed cycle and a path formed by the rest of elements. This effectively deletes the hybrid paths and leaves only two kinds of paths: open and closed. After removing all repeated paths, closed cycles are stored as meshes.

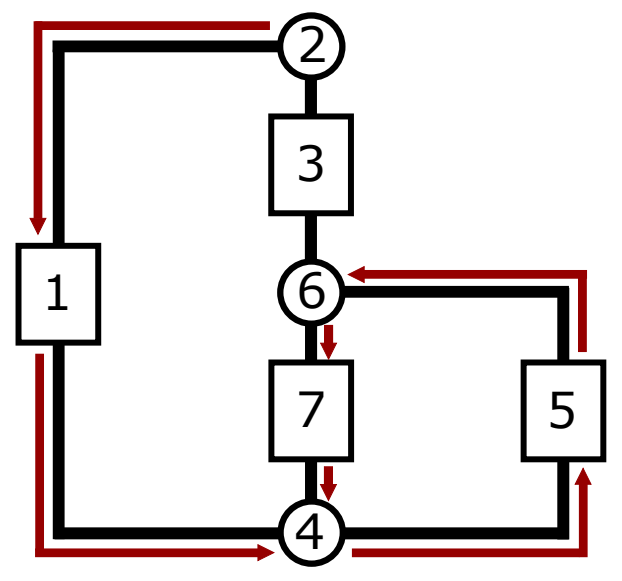

Figure 5.16: Split of a path into an open and a closed loop.

Next steps aim to identify and preprocess the branches of the network. Each path is parsed until reaching a node or the end of the path. Every time a node or an end is encountered, a branch is created and the starting point of the next branch is updated. In the example of Fig. 5.17, a path is split into three branches. Circles represent nodes while rectangles represent regular objects. First branch starts at the beginning of the path, object 1 (which is a node), and ends at the next node found, object 4. Hence, Branch 1 is 1-2-3-4. Starting point for the next branch is the 


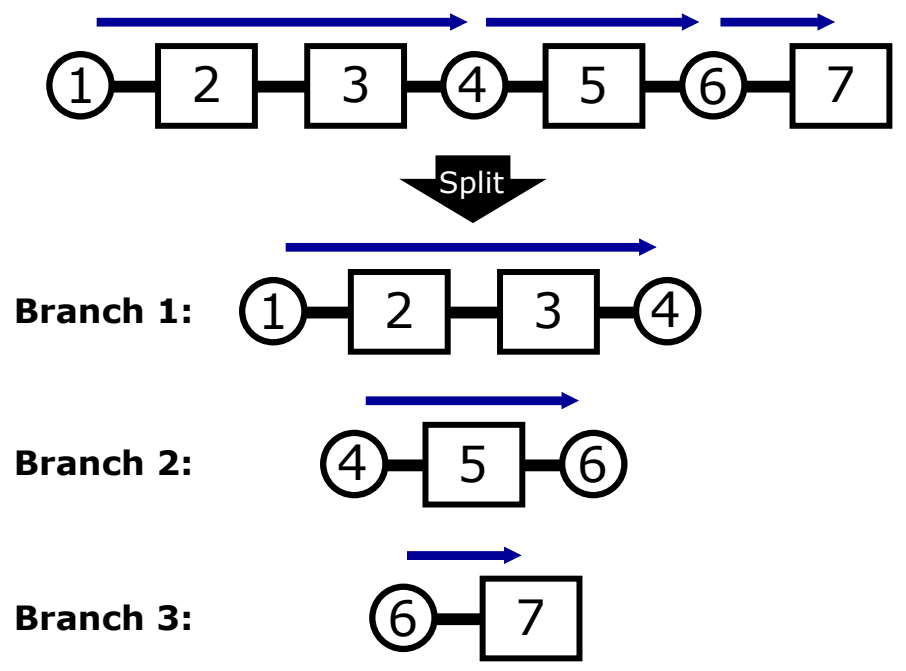

Figure 5.17: Split of a path into three branches.

last object of the previous branch, object 4 . Branch 2 ends at the next node, object 6 . It contains objects 4-5-6. Third branch starts from object 6 and continues until the end of the path. This branch is formed by 6 7. When a branch is identified, two additional pieces of information are stored: if it is part of a mesh and, if that is the case, the index of the mesh. This information is necessary to relate meshes and branches. Obtaining the data is straightforward because, in previous stages, it was determined whether each path was a closed or an open cycle. Splitting paths into branches potentially generates repeated branches, because different paths can share common segments, specially those segments located closest to the starting object. However, each closed path is a different mesh. When repeated branches are removed, the information of all the meshes that contain a certain branch is retained. Finally, for each branch, there is a list of meshes where the branch is included. For example, in Fig. 5.18, there are three branches and three meshes. Branch 1 is included in Mesh 1 and Mesh 2, but not in Mesh 3, which is formed by Branch 2 and Branch 3 .

A special case for identification of both paths and branches is when there are no nodes in the network. Since nodes are the only objects that can have more than two connections, only two configurations are possible without nodes: a series of objects with two unconnected ends or a succession of objects forming a closed loop. As previously noted, in the general 
Mesh 1

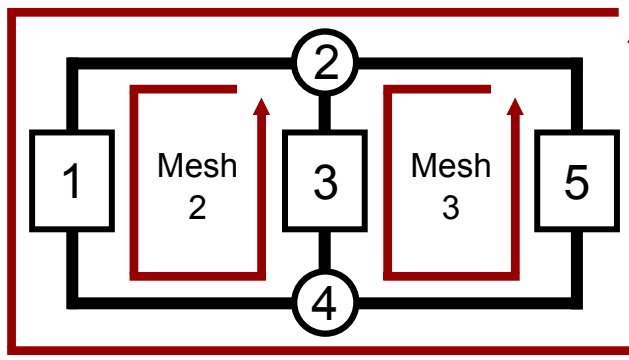

Mesh Branches

$1 \longrightarrow 1,3$

$2 \longrightarrow 1,2$

$3 \longrightarrow 2,3$

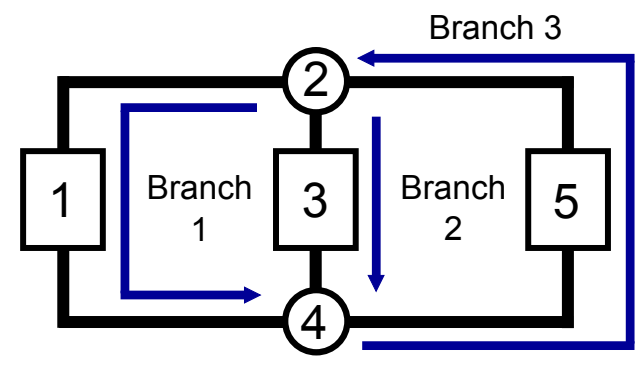
Branch Meshes
$1 \longrightarrow 1,2$
$2 \longrightarrow 2,3$
$3 \longrightarrow 1,3$

Figure 5.18: Relation between meshes and branches.

case, the starting point to find paths is the first node. If there are no nodes, the first object in the list of objects is used as starting point. If the network is a closed loop, one path is identified. This path is recognized as a mesh and a branch, without further complications. Nonetheless, if the ends are unconnected and the starting object is not at one end, two paths are found, as seen in Fig. 5.19. They form a branch, but the branch cannot be recognized because it is separated in two different paths, 3-2-1 and 3-4. Thus, two branches are identified. In this particular situation, the model is able to join the paths in the correct orientation to form the branch. In the example, branch 3-2-1 is reversed to 1-2-3. Then, branches 1-2-3 and 3-4 are joined to form a single branch, 1-2-3-4.

The next task related with branches is to examine the order in which the objects that form the branch are stored. For each object, it is possible to specify the nominal direction of the flow through that object. This is accomplished by indicating which object comes next, called outlet object. Under the Virtual Engine environment, the outlet object of each pipe and each element connected to a pipe is stored, except for nodes. An algorithm checks if the outlet object is located behind the object under examination in the branch. If that is the case, the order of the objects in the branch is 


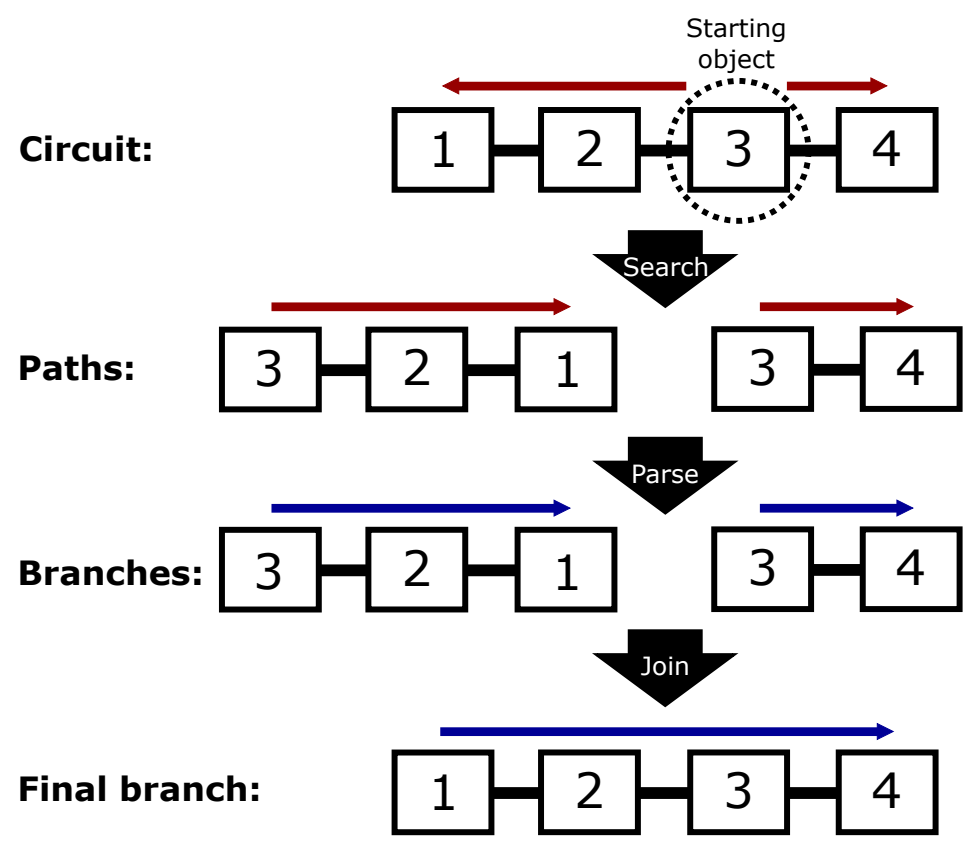

Figure 5.19: Union of two split branches.

reversed. This task is not critical because the thermo-hydraulic network is able to find out the correct direction of flow and invert the branch at any time. The topic of flow rates calculation is covered in Section 5.2.5.

The rest of the procedure is committed to collect and store information related to branches and meshes:

- For each node, branches connected to it.

- For each mesh, branches that form it. Branches are ordered forming a chain, i.e., branches that are adjacent are stored next to each other. In this manner, the first branch is the start of the mesh, the second one is connected to the first, the third to the second and so on. Adjacent branches are recognized because the object at one end of both branches is the same. Storing branches in this order simplifies the determination of the sign of mesh equations in Section 5.2.5.

- For each object, branch where it is located.

- For each branch, internal ID of each object contained in it. 
- For each branch, nodes at inlet and outlet of the branch, if they exist.

\subsubsection{Hydraulic network analysis}

One of the main features of the thermo-hydraulic model is the determination of flow rates and head differences in the circuit. Calculations are made under the quasi-steady assumption, which neglects transient responses of the hydraulic system. The non-linear system of equations that describes the network is obtained through Kirchhoff's circuit laws [210] [43]. A subset of equations may be solved by algebraic methods. Afterwards, the remaining equations are arranged in a matrix expression which is solved by a numerical method.

To solve the hydraulic network, flow rate in every branch and head of every positive displaced pump must be determined. Those are the unknowns of the network. Once all flow rates are known, head of turbopumps and head loss in all objects can be calculated by means of their curves or through expressions of the kind of Eq. 5.25. Each unknown is bound to a branch. Correspondingly, each branch has an associated unknown. For branches that contain volumetric pumps, the unknown is the pump head. This is because, in such case, flow rate can be obtained separately from the network, since it only depends on pump speed and fluid temperature (see curve 5.23). For the rest of branches, the unknowns are the flow rates.

Flow rates obtained after the network analysis may be negative. That means that the fluid flows in the opposite direction to the branch direction. A branch direction is determined by the order in which the objects that form the branch are stored. If flow rate is negative, the fluid flows from the object located at the end of the branch to the one located at the beginning. Therefore, when flow rate is negative, the model reverses the order of the objects in a branch and then changes the sign of flow rate to positive. This simplifies the rest of operations done by the model. Order reversal is performed every time it is necessary during the simulation, because flow direction may change dynamically.

At various points of the network analysis, a solution check is done. This check determines if all unknowns are solved. If they are, the remaining methods of the network analysis are skipped. 


\section{Preliminary tasks}

Some flow rates are known before the network analysis. They are boundary conditions of the system. Some may be specified by the user or by the model itself (see case described in Section Pressure limitation of volumetric pumps). A particular case are branches where flow rate is known to be zero. A reason for the absence of flow is that the branch has a dead end. This means that one end of the branch is connected to the circuit, but the other one is unconnected and closed. There is also the possibility that the end is open to the exterior and flow is going out of the circuit at a constant rate. Another reason for the absence of flow in a branch is that a valve is fully closed. Prior to the network analysis, the opening degree of every operable valve and thermostat is checked. If it is zero, flow rate in that branch is set to zero as new boundary condition. A first solution check is done at this point.

Flow rate of positive displacement pumps is independent of the circuit where they are, as long as the maximum discharge pressure of the pump is not reached. This allows to evaluate flow rate in the branch that contains the pump apart from the network. Flow rate is obtained by simply passing pump speed and fluid temperature as parameters to the pump curve. It is important to note that, even though the flow rate in the branch is determined, the branch is not solved yet: pump head is still an unknown of the branch. It is recalled that unknowns of the branches that contain volumetric pumps are the pumps heads, not flow rates. No solution check is done here for this reason.

\section{Network reduction}

A hydraulic circuit can be seen as a resistor network, where resistors are elements with hydraulic resistance. In a hydraulic network there are three types of resistances. One type, $R_{h, 0}$, is independent of flow rate. Another one, $R_{h, 1}$ multiplies flow rate. These two kinds of hydraulic resistance can be caused only by pumps or fixed head losses. The most common type of hydraulic resistance, $R_{h, 2}$, multiplies flow rate squared. Head difference in an object is calculated using Eq. 5.25. In the case of pumps, hydraulic resistance can be negative. A negative hydraulic resistance means that the object increases flow head, while a positive hydraulic resistance impedes flow pass producing a head loss. To simplify calculations, the network can be reduced by applying the rules for series and parallel circuits. 


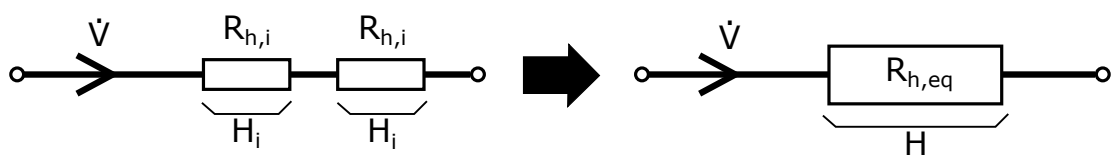

Figure 5.20: Simplification of series circuit.

Resistors located in the same branch are equivalent to resistors arranged in a chain in a series circuit. Total head difference between one end of the branch and the other is equal to the sum of head differences of the connected objects. Flow rate $\dot{V}$ is the same through all elements because they are in the same branch. Hence, resistors of the same type are equivalent to a single resistor of that type, as deduced in Eq. 5.28. Subscript $e q$ refers to the whole branch and $i$ represents individual objects. The equivalent resistance of the combined resistor $R_{h, e q}$ is the sum of resistances of the individual resistors $R_{h, i}$.

$$
\begin{gathered}
H=\sum_{i}\left(H_{i}\right) \\
R_{h, 0, e q}+R_{h, 1, e q} \dot{V}+R_{h, 2, e q} \dot{V}^{2}=\sum_{i}\left(R_{h, 0, i}+R_{h, 1, i} \dot{V}+R_{h, 2, i} \dot{V}^{2}\right) \\
R_{h, 0, e q}=\sum_{i}\left(R_{h, 0, i}\right) \quad R_{h, 1, e q}=\sum_{i}\left(R_{h, 1, i}\right) \quad R_{h, 2, e q}=\sum_{i}\left(R_{h, 2, i}\right)
\end{gathered}
$$

In conclusion, three separate values of hydraulic resistance are stored for each branch. Some hydraulic resistances are constant throughout the simulation, e.g., in pipes. To avoid repetitive operations, they are summed at the beginning of the simulation and stored as the base hydraulic resistance of each branch. Every time step, variable hydraulic resistances are evaluated by means of their curves and summed to the base value.
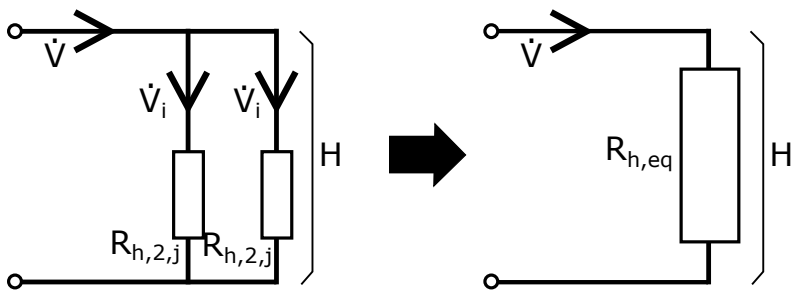

Figure 5.21: Simplification of parallel circuit. 
Rules for parallel circuits allow to further simplify the network. Parallel branches can be combined into a single one with an equivalent resistor. For this, flow direction must be the same in all of them. In addition, only resistances of the type that multiplies flow rate squared, $R_{h, 2}$ are combined. There are three reasons for this. First, only branches with resistances of a single type and with the same type can be combined, as it will be shown. In relation to this, $R_{h, 2}$ is the most common type of hydraulic resistance. Last reason is that hydraulic resistances of the kind $R_{h, 0}$ and $R_{h, 1}$ are usually associated to pumps; if there is a pump in one branch, flow direction can be different. In parallel branches, head difference $H$ between inlet and outlet of the branch is equal. Moreover, equivalent flow rate of the combined branch $\dot{V}$ is the sum of flow rates of the parallel branches $\dot{V}_{j}$. Equivalent resistance $R_{h, e q}$ can be found according to Eq. 5.29, where subscript $e q$ refers to the combined branch and $j$ represents the individual branches.

$$
\begin{aligned}
H & =R_{h, 2} \dot{V}^{2} \\
\dot{V} & =\sum_{j}\left(\dot{V}_{j}\right) \\
\sqrt{\frac{H}{R_{h, e q}}} & =\sum_{j}\left(\sqrt{\frac{H}{R_{h, 2, j}}}\right) \\
\frac{1}{R_{h, e q}} & =\sum_{j}\left(\frac{1}{R_{h, 2, j}}\right) \\
R_{h, e q} & =\frac{1}{\sum_{j}\left(\frac{1}{R_{h, 2, j}}\right)}
\end{aligned}
$$

Branches considered for combination need to fulfill the following conditions:

1. Flow rate through the branch must be initially unknown, i.e., must not be a boundary condition.

2. Hydraulic resistances $R_{h, 0}$ and $R_{h, 1}$ are equal to zero.

3. Hydraulic resistance $R_{h, 2}$ is not negative.

4. There are no pumps in the branch. 
First condition prevents losing information. Second condition forces the hydraulic resistance of the considered branches to be of the same type, plain $R_{h, 2}$. Last two conditions ensure that flow goes in the same direction in all parallel branches. Branches that fulfill the previous conditions are analyzed. Those branches that have the same inlet and outlet nodes are parallel and are combined into one. Once flow rates of the reduced circuit are solved, flow rates of the original branches can be found by applying the rules for parallel circuits again, Eq. 5.30.

$$
\begin{aligned}
H_{e q} & =H_{j} \\
R_{h, e q} \dot{V}_{e q}^{2} & =R_{h, j} \dot{V}_{j}^{2} \\
\dot{V}_{j} & =\dot{V} \sqrt{\frac{R_{h, e q}}{R_{h, j}}}
\end{aligned}
$$

\section{Kirchhoff's circuit laws}

The two Kirchhoff's laws allow to obtain the set of equations that describes a network.

1. First law states that the algebraic sum of all flow rates meeting at a junction is zero, Eq. 5.31. In the thermo-hydraulic model, flow rate sign is positive if flow is leaving the junction and negative if it goes in, as indicated in Fig. 5.22. This ensures the compliance of mass conservation, as junctions do not accumulate mass. In accordance, volume of nodes is zero. Only during the calculation of the thermal state of the circuit, temporary volumes associated to nodes are considered with the sole aim of averaging temperatures of the flows entering the node. However, that does not violate mass conservation because those volumes are entirely distributed to other elements, as explained in Section 5.2.6. Kirchhoff's first law allows to obtain a linear equation for each node of the circuit. Those equations are called node equations.

$$
\sum_{i}\left(\dot{V}_{i}\right)=0
$$

2. Second law is based on energy conservation. It states that, for a particular direction around a closed loop (mesh), the sum of head differences is zero. This is expressed by Eq. 5.32, where $i$ is the 


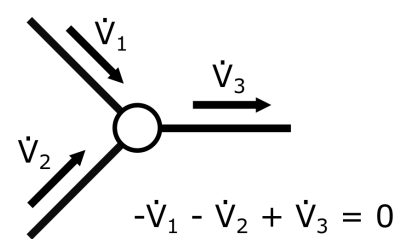

Figure 5.22: Kirchhoff's first law.

branch. Sign of head differences depends on the direction chosen as the mesh direction, which is arbitrary for each mesh. Head differences are calculated as explained in the Network reduction section. An example is provided in Fig. 5.23. The example consists of a single mesh with four branches. The circular arrow at the center indicates the mesh direction. Flow direction of Branch 3 is opposite to mesh direction. In consequence, sign of head difference is negative. The rest of branches have positive sign because flow direction is the same as the mesh direction. The system has as many mesh equations as the number of meshes in the circuit.

$$
\begin{gathered}
\sum_{i}\left(H_{i}\right)=0 \\
\sum_{i}\left(R_{h, 0, i}+R_{h, 1, i} \dot{V}_{i}+R_{h, 2, i} \dot{V}_{i}^{2}\right)=0 \\
\sum_{i}\left(R_{h, 0, i}\right)+\sum_{i}\left(R_{h, 1, i} \dot{V}_{i}\right)+\sum_{i}\left(R_{h, 2, i} \dot{V}_{i}^{2}\right)=0
\end{gathered}
$$

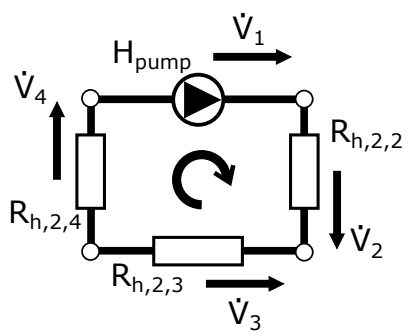

$$
\begin{aligned}
\mathrm{H}_{\text {pump }} & +\mathrm{R}_{\mathrm{h}, 2,2} \dot{\mathrm{V}}_{2}^{2}-\mathrm{R}_{\mathrm{h}, 2,3} \dot{\mathrm{V}}_{3}^{2} \\
& +\mathrm{R}_{\mathrm{h}, 2,4} \dot{\mathrm{V}}_{4}^{2}=0
\end{aligned}
$$

Figure 5.23: Kirchhoff's second law.

\section{Algebraic solver}

In view of the network equations, it is possible to devise methods to solve a subset of the equations. Depending on the flow rates that are known, in 
some node equations there may remain only one unknown. In the example of Fig. 5.22, $\dot{V}_{3}$ can be determined if $\dot{V}_{1}$ and $\dot{V}_{2}$ are known. Hence, the node equation can be solved separately from the system of equations. Not only node equations, but also mesh equations, can be solved if they have a single unknown. Moreover, algebraic solving methods must be iterative. In this way, a solution found in a certain iteration is available in the next iteration to solve equations that could not be solved previously.

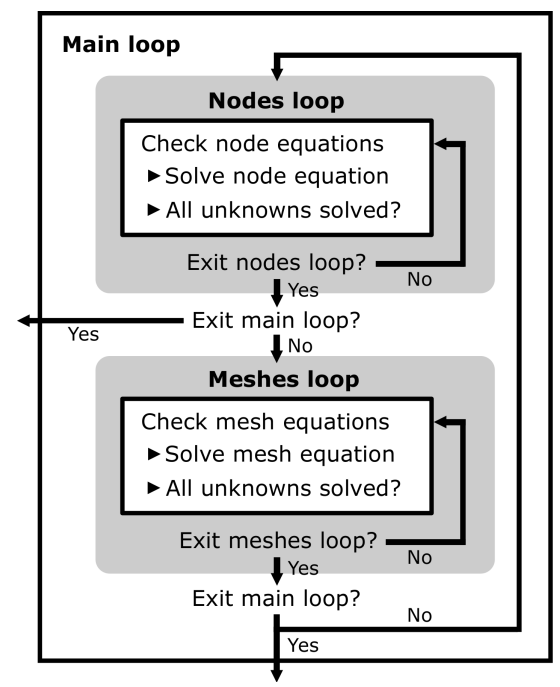

Figure 5.24: Algebraic solver.

Based on those ideas, the thermo-hydraulic model uses the following method, illustrated in Fig. 5.24. It consists of two loops inside a main loop. The two inner loops are a loop for solving node equations and a loop for solving mesh equations. In each inner loop, it is attempted to solve equations of the respective type. Equations are evaluated until no more equations of that type can be solved. Then, execution goes to the next loop: from the nodes loop to the meshes loop and vice versa by virtue of the main loop. Equation examination consists in checking if the equation has only one unknown.

For each node equation, branches connected to the node are examined. If only one of the branches is not marked as solved, the node equation is processed. Signs of flow rate are determined as explained in Kirchhoff's circuit laws section and, next, Eq. 5.31 is used. Then, the branch is marked as solved. A solution check is performed every time an equation 
is solved. The loop only ends after a complete iteration in which all node equations were examined and none of them could be solved. The meshes loop works in an analogous way to the nodes loop. In the meshes loop, the procedure to solve meshes and Eq. 5.32 are applied. A new solution check is performed at the end of the loop.

If this method does not accomplish the determination of all unknowns, the main loop has to be exited so that other methods can be executed. The criterion to leave the main loop is explained next. If the method has gone through an inner loop and no equation was solved, no change was made in the overall system of equations. If, in addition, the other inner loop did not solve any equation either, it is impossible to solve more equations with this method. The system of equations was not changed by the nodes loop neither by the meshes loop. Entering again those loops would not solve more equations. Under these circumstances, the algebraic solver is exited.

\section{System of equations setting}

When solving the hydraulic equations one by one is not possible, it is necessary to solve the system of equations as a whole. System size is equal to the number of unknowns that remain to be solved. The number of available equations can be higher than the number of unknowns. It is therefore necessary to follow a procedure to select linearly independent equations in order to prevent having an overdetermined system of equations. If all unsolved node equations are considered, one of them is always a linear combination of the other equations. Thus, the number of independent node equations is equal to the total number of node equations to be solved minus one. The number of independent mesh equations is equal to the number of branches to be solved minus the number of independent node equations.

The matrix expression is obtained from Kirchhoff's laws. $\dot{V}_{i}$ terms of the first law (see Eq. 5.31) are equivalent to $R_{h, 1, i} \dot{V}_{i}$ terms of the second law (see Eq. 5.32) with the proviso that $R_{h, 1, i}$ is equal to 1 or -1 . Hence, node equations can be expressed in the form of mesh equations with $R_{h, 0, i}=0, R_{h, 2, i}=0$ and $\left|R_{h, 1, i}\right|=1$. Moreover, determining the sign of each term is not trivial when flow is unknown, because, as seen in the example of Fig. 5.23, the sign of each head difference depends on flow direction. A robust way to overcome this issue is to replace $R_{h, 0, i}$ by $R_{h, 0, i} \frac{\dot{V}_{i}}{\left|\dot{V}_{i}\right|}$ and $R_{h, 2, i} \dot{V}_{i}^{2}$ by $R_{h, 2, i} \dot{V}_{i}\left|\dot{V}_{i}\right|$. Finally, the system must include 
independent terms. Those terms are the known values of flow rate (for node equations) and head difference (for mesh equations). Flow directions, and thus signs, are known. They are included in the system in a vector, $[C]$. The rest of coefficients, which can in general be expressed as $R_{h, i}$, are enclosed in matrices $\left[R_{h}\right]$ which multiply the vector of unknowns $[X]$. The resulting matrix expression is given in Eq. 5.35.

$$
\left[R_{h, 2}\right][X|X|]+\left[R_{h, 1}\right][X]+\left[R_{h, 0}\right]\left[\frac{X}{|X|}\right]+[C]=0
$$

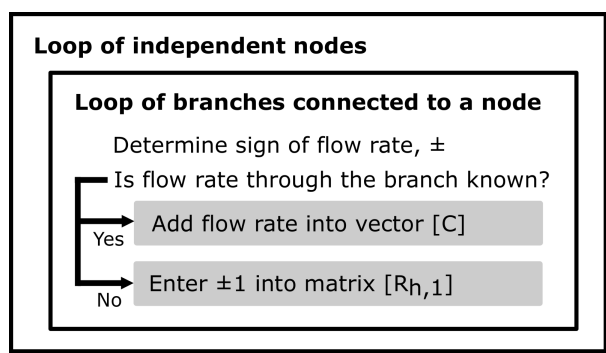

Figure 5.25: Formulation of node equations for the system solver.

As discussed, the first step to set the equations is to choose those that are linearly independent. For node equations, the election criterion is very simple: all unsolved equations are selected except the last one. There is no need for complex procedures since node equations are simple and linear, as Eq. 5.31 shows. Next, equation coefficients are added to the system. For each node, the connected branches are checked. Sign is changed if the branch ends at the current node. Branch flow rate can be known or not. If it is known, the flow rate value is added to the respective row of matrix $[C]$. Otherwise, a coefficient of 1 or -1 is entered in the proper position of matrix $\left[R_{h, 1}\right]$. In Fig. 5.25, equation formulation is outlined.

Regarding mesh equations, both selection of equations and determination of coefficients are more complex.

Selection of mesh equations is accomplished by means of a merit function. First, remaining branches are listed. Initially, they are the branches that are not solved. Mesh merit is equal to the number of remaining branches present in the mesh. In each iteration, the meshes are examined. After each iteration, the mesh with the highest merit is chosen for the system of equations and its branches are removed from the list of remaining 
Make a list of "remaining branches": branches with unknowns to be solved

\begin{tabular}{|c|}
\hline $\begin{array}{l}\text { Loop of independent meshes (to be selected) } \\
\text { Reset best mesh }\end{array}$ \\
\hline $\begin{array}{l}\text { Loop of meshes } \\
\text { Check that mesh is not selected yet }\end{array}$ \\
\hline $\begin{array}{l}\text { Loop of branches in a mesh } \\
\text { Count "remaining branches" of the mesh }\end{array}$ \\
\hline Compare current mesh with previous meshes \\
\hline Select best mesh \\
\hline $\begin{array}{l}\text { Remove branches of the best mesh from } \\
\text { "remaining branches" list }\end{array}$ \\
\hline
\end{tabular}

Figure 5.26: Selection of mesh equations for the system solver.

branches. As many meshes as the number of independent mesh equations are selected. Once a mesh is selected, its merit is not checked anymore. The procedure is illustrated in Fig. 5.26.

To enter the mesh equations into the matrix system, sign is determined with the criterion explained in Kirchhoff's circuit laws section. Mesh direction is arbitrarily set as the direction in which branches are sorted in the vector of meshes. Then, a loop goes over all branches in the mesh. Sequentially, the object at the end of the current branch is compared with the initial object of the next branch. If it is not the same, sign of the branch coefficients is changed. As for node equations, some flow rates may be known. If flow rate through the branch is known, flow rate value is used together with hydraulic resistances to calculate head difference in the branch. Then, it is added to the row of matrix $[C]$ that corresponds to the mesh equation. Additionally, if the branch contains a volumetric pump, a coefficient 1 or -1 should be included in the proper position of matrix $\left[R_{h, 1}\right]$, because pump head is an unknown. However, in order to accelerate the solving process, the coefficient is given the value of 10000 or -10000 instead of 1 or -1 . In this way, the unknown head to be solved has an order of magnitude close to that of flow rates in engine applications. When the system of equations is solved, head pump is multiplied by 10000 to obtain the correct result. Finally, in the case that branch flow rate remains to be calculated, branch hydraulic resistances $R_{h, 0}, R_{h, 1}$ and $R_{h, 2}$ are entered as coefficients into the matrices $\left[R_{h, 0}\right],\left[R_{h, 1}\right]$ and $\left[R_{h, 2}\right]$, respectively. Fig. 5.27 summarizes the overall process. 


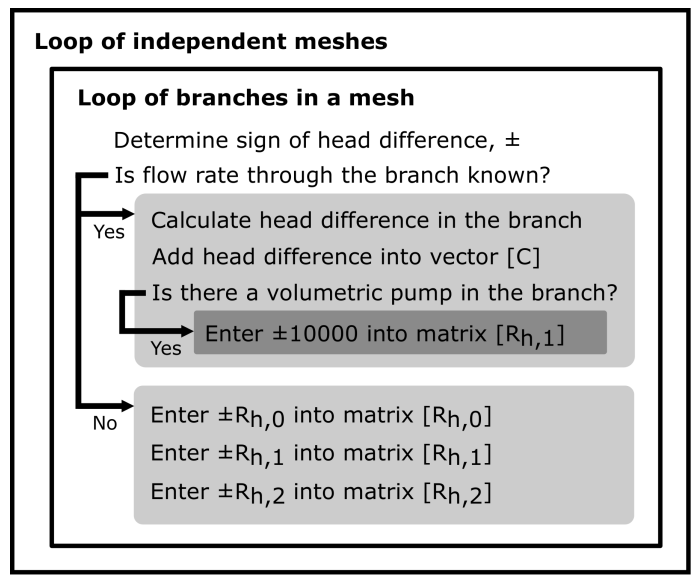

Figure 5.27: Formulation of mesh equations for the system solver.

Once the previous operations are done, the system is complete. The only remaining task before calling the numerical solver is to assign initial values to unknowns for the iterative algorithm. If the unknown is a flow rate, the value of flow rate in the previous instant is used. Nevertheless, if it is zero, a default value is given. The default value is currently set to 0.001. At the beginning of the simulation, all flow rates are initialized to the default value. Zero is avoided as initial guess because, after experimenting with the numerical method, it was found that it was unable to find the right solution when any initial value was zero. If the unknown is the head of a volumetric pump, the default value of 0.001 is also used. As mentioned above, the coefficient associated with this type of unknown is multiplied by 10000 .

\section{Numerical solver}

To solve the multivariable, nonlinear system of equations that represents the hydraulic network, Eq. 5.35, a numerical method is needed. The Virtual Engine Model uses an algebra C++ library called Eigen [211]. Eigen library was explored to find a suitable method. Among Eigen's unsupported modules [212], an algorithm to find zeros of nonlinear functions is provided. It is a modification of Powell's hybrid method ("dogleg") [213]. Originally, the algorithm formed part of a FORTRAN library called MINPACK. This library was developed at Argonne National Laboratory (USA) and published in 1980. MINPACK has been carefully tuned and tested 
for several decades [214]. The code was translated to $\mathrm{C}++$ by different authors and included in Eigen.

Powell's conjugate direction method [215] is an algorithm for finding a local minimum of a multivariate function. In the present application, the zero of the function is searched. An advantage of the method is that it has the property of quadratic convergence, which ensures a fast convergence rate for quadratic problems. Eq. 5.35 is a quadratic problem because it is a function expressed as a quadratic polynomial, i.e., a polynomial with integer powers and with no term having a degree greater than two. Convergence is efficient even when initial conditions are far from the final solution. This is relevant for hydraulic networks, where flow rates can change greatly from one instant to another through operation of valves or sharp changes of pump speed.

Powell's method is based on a simpler method called the taxi-cab method. In both methods, an initial point and a set of initial search vectors are given. Then, a bi-directional search is performed along each vector to find the minimum in that direction, one parameter at a time. In the taxi-cab method, directions are fixed. On the left side of Fig. 5.28, the path followed by the taxi-cab method is shown. In contrast, in Powell's method, one search vector is changed in each iteration. Direction of the new vector is the one that joins the two previous points of the path. A shape similar to a dog leg is generated, hence the algorithm's nickname. The path of Powell's method is shown on the right side of Fig. 5.28. This method is widely known and employed because of its efficiency [214]. MINPACK modifications to Powell's original algorithm are related to the updating method and the iterations, and were meant to increase efficiency [216].

In order to use the method, it was necessary to create an object which encloses the information of the system to be solved. Object's class is defined in the manner expected by the Eigen function that implements Powell's method. The class contains the values of the coefficients, the current guess of the unknowns and four subroutines. One of the subroutines returns the number of equations. Another one returns the number of unknowns. A key subroutine evaluates the function using the current guess of the unknown variables. The last subroutine evaluates the Jacobian of the function. Eigen allows the Jacobian to be either manually implemented or approximated using a forward-difference method. It was decided to manually implement the Jacobian due to its simplicity. It is 


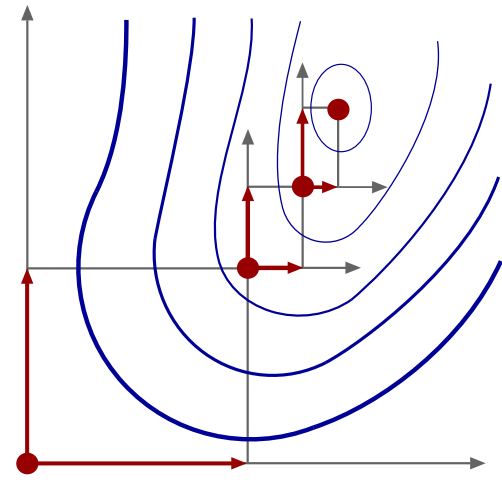

(a) Taxi-cab

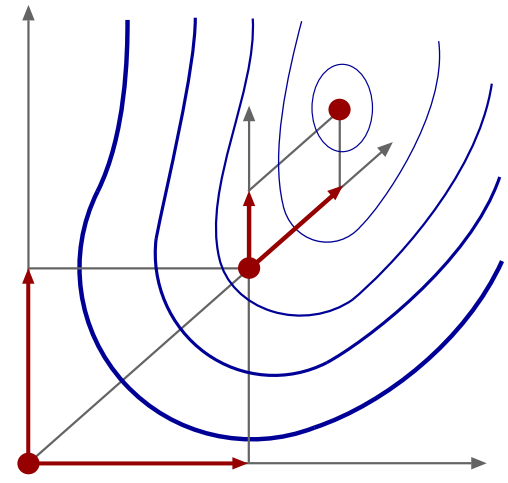

(b) Powell

Figure 5.28: Comparison of Taxi-cab and Powell solving methods.

shown in Eq. 5.36, where $n$ is the number of equations or rows and $m$ is the number of unknowns or columns. According to documentation, manually entering the Jacobian increases reliability because the algorithm is then much less sensitive to errors [216].

$$
\left[\begin{array}{ccc}
2\left(R_{h, 2}\right)_{1,1} X_{1}+\left(R_{h, 1}\right)_{1,1} & \cdots & 2\left(R_{h, 2}\right)_{1, m} X_{m}+\left(R_{h, 1}\right)_{1, m} \\
\vdots & \ddots & \vdots \\
2\left(R_{h, 2}\right)_{n, 1} X_{1}+\left(R_{h, 1}\right)_{n, 1} & \cdots & 2\left(R_{h, 2}\right)_{n, m} X_{m}+\left(R_{h, 1}\right)_{n, m}
\end{array}\right]
$$

\section{Pressure limitation of volumetric pumps}

It was mentioned in Section 5.2.1 that positive displacement pumps have a pressure limit. As discussed in that section, reaching the limit leads to an apparent "saturation" of flow rate through the pump. Pressure limitation is specified in the thermo-hydraulic model as a maximum head value of the pump. In the model, this value plays its role after the network has been solved. For each volumetric pump, the obtained pump head is compared with its head limit. If it is above the maximum (with a tolerance of $1 \%$ ), a new value of flow rate through the pump is estimated and imposed as boundary condition. This new flow rate is calculated with Eq. 5.37. That is an estimation of the correct flow rate which assumes that hydraulic resistance of branches is of the $R_{h, 2}$ kind, i.e., head losses in the branches are proportional only to flow rate squared. This is the most common situation. Under the new conditions, the network is recalculated. 
Recalculated pump head should be close to the maximum head. These actions are undertaken as part of a loop. If, in the previous iteration, pump head was above the limit, an additional check is enabled. In the previous correction, flow rate was reduced to lower pump head. Now, pump head can be unrealistically low, if it was overcorrected. Thus, if pump head is below the limit (with a convergence tolerance of $2 \%$ ), flow rate is corrected again and a new iteration is done. The process continues until pump head converges to its limit or until the maximum number of iterations is reached.

$$
\frac{R_{h, 2} \dot{V}^{2}}{H}=\frac{R_{h, 2} \dot{V}_{c o r r}^{2}}{H_{\max }} \rightarrow \dot{V}_{\text {corr }}=\dot{V} \sqrt{\frac{H_{\max }}{H}}
$$

An alternative to this process would be to impose the boundary condition that pump head is equal to the limit and recalculate the network once. This solution was not implemented because of the additional effort required to renovate the network solver.

It was also explained in Section 5.2.1 that when the pump reaches maximum head, outgoing flow is partially recirculated to a return line. Still, the whole flow passes through the pump impeller and contributes to energy consumption. For this reason, the original flow rate given by the flow curve is used, instead of the reduced one, for calculating pump power.

\section{Optimization of calculation}

The thermo-hydraulic model has a feature to prevent redundant calculations when conditions do not change. There are several flags which are set to true only if certain variables change from one iteration to the next. In particular, there are flags for pump speed, valve opening degree, thermostat opening degree and hydraulic resistance of branches. Only if any flag is true, the network analysis is executed. The network analysis is the most time-consuming method of the model because of the numerical procedure, described above, used to solve the system of non-linear equations representing the circuit.

\subsubsection{Thermal state of the circuit}

Together with the network analysis, the other main component of the thermo-hydraulic model is the calculation of the thermal state of the circuit. That means that the model determines fluid temperature at every point of the circuit over time. This is achieved through discretization of 
the circuit fluid in lumps or parcels. Each fluid parcel has a different temperature. A set of parcels is associated to each branch. Parcels undergo three processes on every call: they are displaced, they exchange heat with heat exchangers and pumps and they update their size and temperature to reflect the thermal state of the circuit.
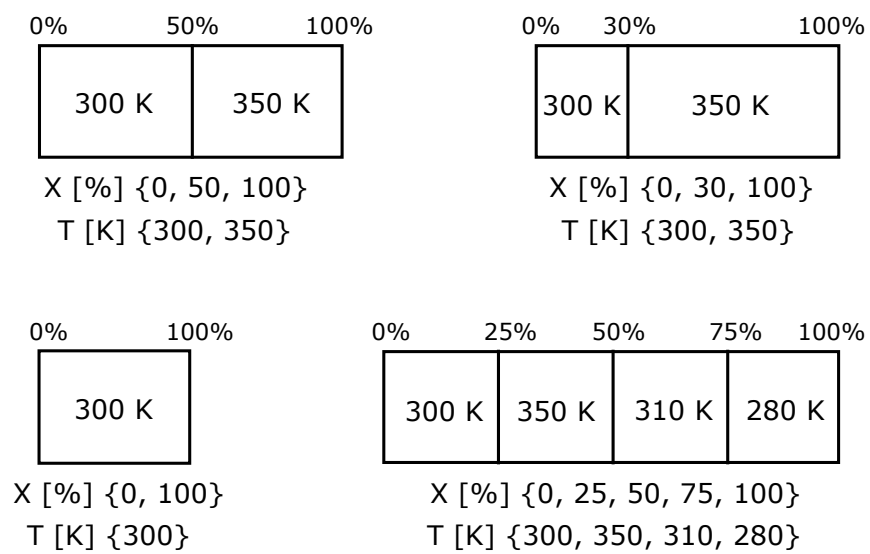

Figure 5.29: Example vector pairs defining the thermal states of branches.

Thermal state of a branch is defined by two vectors. One stores the location of each parcel's boundaries. Boundary location is indicated as percentage of the branch volume. For example, if a branch has two parcels of equal volume, the vector contains $\{0,50,100\}$. If total branch volume is 1 liter and first parcel occupies 0.3 liter, vector is $\{0,30,100\}$. If there is only one parcel, vector is $\{0,100\}$. Thus, the minimum size of this vector is 2 . The second vector stores each parcel's temperature. Its size is always equal to the size of the boundaries vector minus one. First value in the vector is temperature of the parcel located between the boundaries given by first and second position of the boundaries vector. Second value is the temperature of the parcel between the second and the third boundary, and so forth. For instance, given the boundaries vector $\{0,50,100\}$, an associated temperatures vector could be $\{300,350\}$. This indicates that the first parcel, which occupies the first half of the branch, is at 300 $\mathrm{K}$, while the second parcel makes the second half of the branch and its temperature is $350 \mathrm{~K}$. If there is only one parcel, the temperature vector has only one element. Some examples of vectors are given in Fig. 5.29. Definition of the vectors is dependent on flow direction. Boundary $0 \%$ is the end where the fluid enters the branch while $100 \%$ is the end where 
the fluid leaves the branch. If flow direction is reversed, i.e., flow rate is negative, both the boundaries vector and the temperatures vector invert their order. Other auxiliary vectors are inverted as well. Flow direction is checked every time flow rates are recalculated.

The section of code that accomplishes the calculation of the thermal state of the circuit can be divided into four parts: Part 1 updates the internal thermal state of branches, Part 2 deals with mass transfer among connected branches, Part 3 extracts and stores relevant information and Part 4 limits system complexity. Prior to the description of the different parts, some aspects of heat transfer in the thermo-hydraulic model are explained.

\section{Heat transfer}

Heat transfer occurs when there are heat exchangers or pumps in the branch. Heat exchangers store the value of energy exchanged with the circuit in the current time step. Pumps store current power consumption. In the thermo-hydraulic model, it is assumed that all power consumed by the pump is rejected to the circuit fluid as heat. Under this assumption, energy transferred from the pump to the fluid to increase pressure is dissipated into the fluid as heat trough friction in the ducts. Mechanical friction of the pump is also dissipated as heat to the fluid. The method to simulate the heat transfer process is the same for heat exchangers and pumps, except that pump power is multiplied by the time increment to convert power to energy. It must be noted that pipe heat loss is not explicitly modeled. If the user wants to take it into consideration, heat exchangers must be added to simulate pipe heat loss indirectly.

This part of the model makes frequent use of a dedicated function that creates parcels which can be inserted in branches. The function is called InsertVolume. Mandatory inputs to this function are the pair of boundaries and temperatures vectors of the concerned branch, location where the new parcel starts, location where the new parcel ends and action to be performed on the original volume. Three actions are possible.

1. First one, named Action 1, is to average temperatures of the original volume by mass, as in Eq. 5.38. To do that, existing parcels between the specified boundaries are read. Affected mass of each parcel, $m_{i}$, is calculated and used to average parcel temperatures, $T_{i}$. The result is a single parcel whose location is defined by the specified boundaries. 
Start location: $25 \%$

End location: $75 \%$

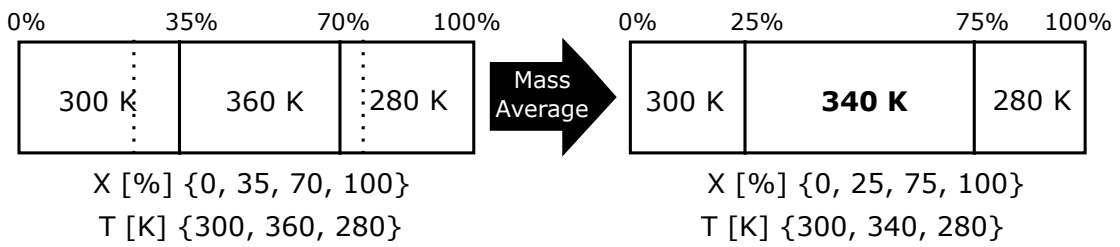

Figure 5.30: InsertVolume, Action 1.

Original parcels at both ends of the new parcel could be partially included in the new parcel. Thus, boundaries of those parcels are modified as well to match the new boundaries. The final outcome of the function is a new vector pair of boundaries and temperatures in the branch. The model can use those vectors to overwrite the existing ones. On the contrary, if the objective was just to determine the average temperature of the original volume, $T^{\prime}$, the temperature of the new parcel is received and the new vectors are dismissed. An example of thermal states before and after applying this method is shown in Fig. 5.30.

$$
T_{\text {parcel }}=T^{\prime}=\frac{\sum_{i}\left(m_{i} T_{i}\right)}{\sum_{i}\left(m_{i}\right)}
$$

2. Second available action of InsertVolume, Action 2, is to exchange energy with a determined portion of the branch. It needs, in addition to the stated mandatory inputs, the value of transferred energy and the branch volume. Initially, Action 1 is performed to obtain a single parcel which represents the affected volume. Then, heat is transferred to the new parcel and a new temperature is calculated according to Eq. 5.39, where $C_{p}$ is evaluated at $T^{\prime}$.

$$
T_{\text {parcel }}=T^{\prime}+\frac{Q}{m C_{p}\left(T^{\prime}\right)}
$$

3. Action 3 of InsertVolume is similar to Action 2 but it directly sets the temperature of the new parcel, as seen in Eq. 5.40. The only additional input required is the temperature value to be imposed, $T^{*}$. Again, Action 1 is performed first to create the parcel. Then, 
temperature of the new parcel is set to $T^{*}$. The outcome is always the vector pair which defines the thermal state of the branch.

$$
T_{\text {parcel }}=T^{*}
$$

\section{Thermal state determination, Part 1}

The goal of Part 1 is to update the internal thermal state of the different branches according to the fluid displacement obtained after solving the hydraulic network and according to heat transfer in discrete components. There are three possible cases, two of which are special cases while the third is the general case.

- The simplest case occurs when branch volume is zero. In that case, the branch is formed by one single parcel and there are no elements such as heat exchangers. Therefore, there is no parcel displacement nor heat exchange. In branches without volume, vectors of boundaries and temperatures of the current instant are made equal to those of the previous instant as an initialization. No more actions are done on these branches until Part 2.

- Another simple case is when flow rate in a branch is zero. There is no displacement of parcels, but there may be heat transfer. The applied process can be seen in steps 1) to 3) of Fig. 5.31. All heat exchangers are processed in order, starting from the one which is closest to the branch's end. First, function InsertVolume is called. A parcel is inserted which comprises the whole heat exchanger's volume, step 2). Hence, boundaries of the new parcel are the heat exchanger's inlet and outlet locations. Then, Action 2 is performed to simulate heat exchange, step 3). Vectors of the current branch are overwritten.

- In the general case, there is fluid displacement and heat transfer. Displaced fluid volume, $V_{d i s p}$, is calculated as flow rate multiplied by time increment, $V_{\text {disp }}=\dot{V} \Delta t$. Displacement divided by branch volume is the percentage advance of parcels within the branch, $\Delta x=V_{\text {disp }} / V_{\text {branch }} \cdot 100$. Thus, updating the vector of parcels' boundaries consists in adding up this percentage, $\Delta x$, to each existing value in the vector. Afterwards, some values are higher than 100\%, meaning that parcels boundaries are outside the branch. They represent the fluid that goes out of the branch. Boundaries 
1) Original state

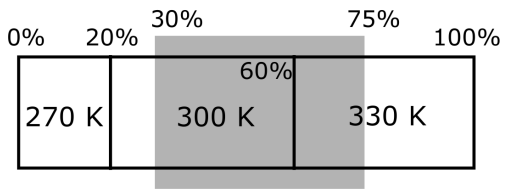

3) Heat transfer

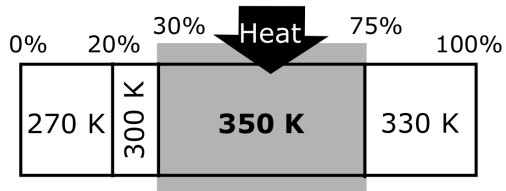

2) Parcel creation in heat exchanger

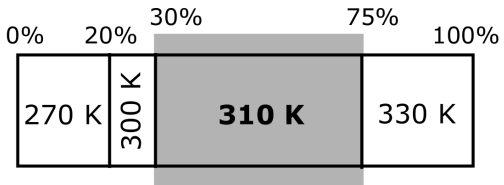

Figure 5.31: Procedure to model heat transfer in a heat exchanger. "Slow flow" case.

4) Displacement of parcels (15\%)

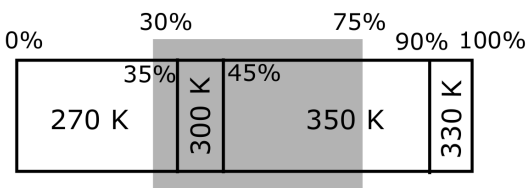

Volume displacement: $35 \%$ of branch volume

Original
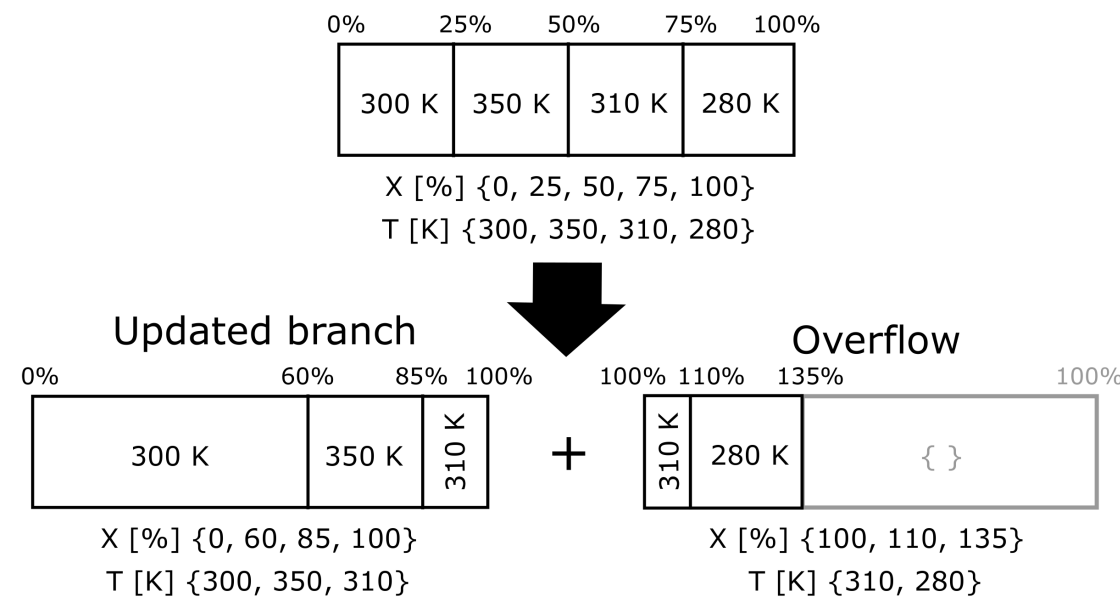

Figure 5.32: Parcel displacement inside and outside a branch. 
from $0 \%$ to $100 \%$ are stored in the original vector of parcels' boundaries while the rest are stored separately for later use. To do this, if the parcel that is currently located at the end of the branch is partly outside, it is divided in two parcels at location $100 \%$. The values of the vector of parcels' temperatures do not change, but the vector is also split in two (inside and outside of the branch). Parcels outside the branch simulate the overflow of the branch. The pair of overflow vectors (boundaries and temperatures) represent a temporary "phantom branch" with the same volume as the original branch and boundaries starting at $100 \%$. Fig. 5.32 displays an example in which the displaced volume is equal to $35 \%$ of the total branch volume.

For heat transfer in the general case, two situations are distinguished. In one of them, displaced volume is smaller than or equal to heat exchanger volume. This case is called "slow flow". On the contrary, when displaced volume is bigger than heat exchanger volume, the case is called "fast flow".

- The steps of the "slow flow" case are illustrated in Fig. 5.31. Heat is exchanged with the whole volume in the heat exchanger. Therefore, Action 2 of InsertVolume is applied between the heat exchanger boundaries, as in the case of flow rate equal to zero, steps 2) and 3) of Fig. 5.31. Once all "slow flow" heat exchangers are processed, the above-explained procedure for displacing parcels is undertaken. As a result, part of the volume that exchanged heat leaves the heat exchanger and part of the volume that was ahead of the heat exchanger enters in it. In step 4) of Fig. 5.31, the displaced volume is $15 \%$ of the branch volume.

- Only after all "slow flow" cases are solved, the heat exchangers under the "fast flow" situation are processed. In that case, the volume of fluid that passes through the heat exchanger is bigger than the volume of the heat exchanger itself. As in this case the heat exchanger transfers heat to a fluid volume that is bigger than its own, a different approach is needed. The procedure is summarized in Fig. 5.33. Heat is exchanged with the volume of fluid that starts at the heat exchanger outlet and is equal to the displaced volume. Possibly, part of the affected volume is outside the branch where the component is located. A fraction of heat proportional to the volume between the heat exchanger outlet and the end of the branch is exchanged with the fluid in that area. To represent this, a new parcel is generated with InsertVolume, left branch of step 2). The remaining heat 
1) State after displacement (40\%)

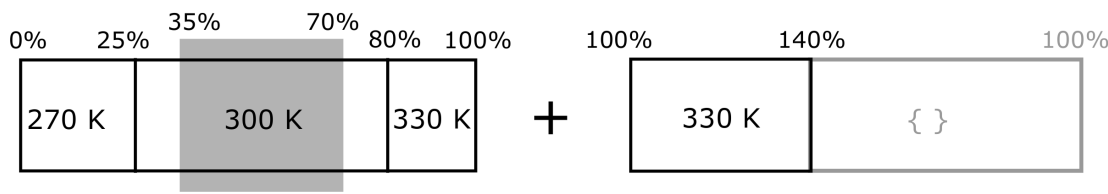

2) Parcel creation at heat exchanger outlet

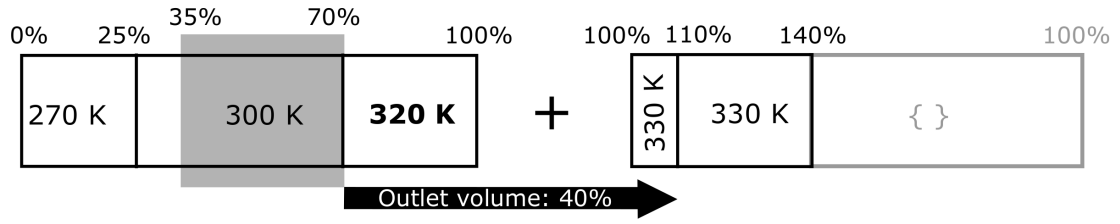

3) Heat transfer

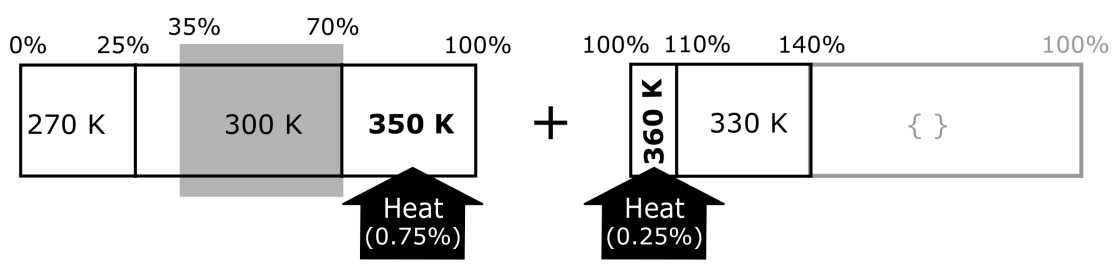

Figure 5.33: Procedure to model heat transfer in a heat exchanger. "Fast flow" case. 
is transferred to the overflow vectors. Insert Volume modifies them to insert a new parcel starting from the end of the branch, right branch of step 2). The right boundary of the new parcel is at the location $x_{F}$, given by Eq. 5.41, where $V_{b}$ is the branch volume and $x_{\text {HeatExchOut }}$ is the heat exchanger's outlet location.

$$
\begin{gathered}
V_{\text {disp }}=V_{b}\left(100-x_{\text {HeatExchOut }}\right)+V_{b}\left(x_{F}-100\right) \\
x_{F}=x_{\text {HeatExchOut }}+\frac{V_{\text {disp }}}{V_{b}}
\end{gathered}
$$

Every time InsertVolume is used, both the vectors of locations and temperatures are overwritten. It must be noted that, if outlet temperature instead of heat is to be imposed, Action 3 of InsertVolume can be used instead of Action 2.

To finish Part 1, information of the fluid that is outside the branch, in the temporary "phantom branch", is reduced. The vectors of parcels are used to make a mass-average of temperature. Mean temperature of the fluid volume leaving the branch, $T_{o}$, is thus obtained and stored. Volume that goes out of the branch is equal to the displaced volume in the branch. Total excess volumes of all branches, regardless of parcels, are stored as overflows. Thus, for each branch, two values are stored: volume and temperature of the branch's overflow.

\section{Thermal state determination, Part 2}

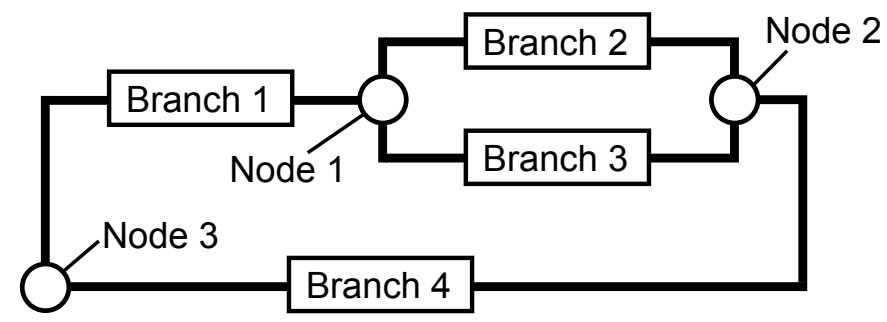

Figure 5.34: Mass and heat distribution among branches. Example circuit.

In Part 2, fluid volumes going out of branches as overflows are processed and allocated in the circuit. This is the case when flow rate is different from zero in any branch. An example of the procedure with the simple circuit of Fig. 5.34 is given in Figs. 5.35 to 5.39. 


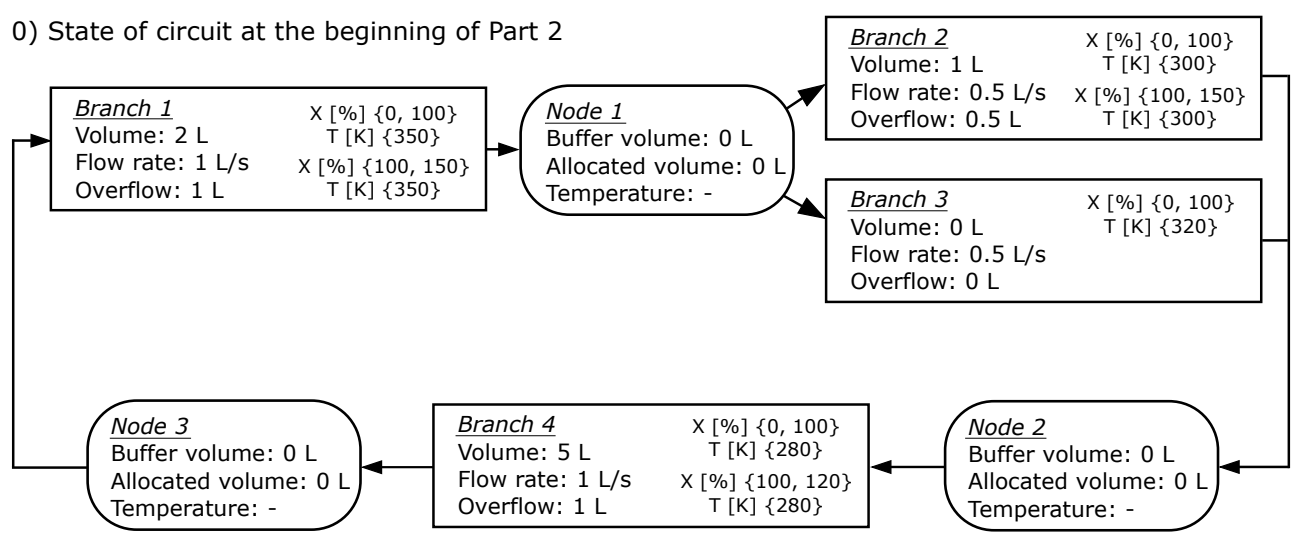

Figure 5.35: Mass and heat distribution among branches. Initial state.

Fig. 5.35 presents the state of the circuit at the start of Part 2. The circuit is formed by three nodes and four branches, one of them without volume. Time step is $1 \mathrm{~s}$. First, for each node, it must be identified which branches carry flow into the node (inlet branches) and out of the node (outlet branches). Therefore, in the same manner as branches have an inlet and an outlet node, nodes have inlet and outlet branches.

This information is used to accumulate overflows from the branches in so-called buffer volumes of the respective outlet nodes. The buffer volume associated to a node will receive all the overflows of its inlet branches. A temperature of the fluid in the buffer volume, $T_{n}$, is calculated by massaveraging temperatures of the inflows, $T_{o}$, assuming instantaneous mixing. In this manner, an overflow is an excess volume associated to a branch and a buffer volume is an excess volume associated to a node, since nodes have no volume. Fig. 5.36 shows the circuit after the branch overflows were transferred to buffer volumes of the nodes.

Next, buffer volumes accumulated in the nodes are distributed among the outlet branches. To do this, it is necessary to calculate the share of the buffer volume that goes out of a node into each outlet branch. For each particular branch, this volume share is determined by dividing the flow rate through the branch by the sum of flow rates through all the outlet branches. Each branch has only one inlet node. It is important to take into account that part of the volume that goes into a branch from the inlet node may go out of the branch at the same instant. This is always the case when the receiving branch has no volume. In addition, it is is 
1) Start loop. All overflows are equal to zero BUT node buffer volumes have to be allocated.

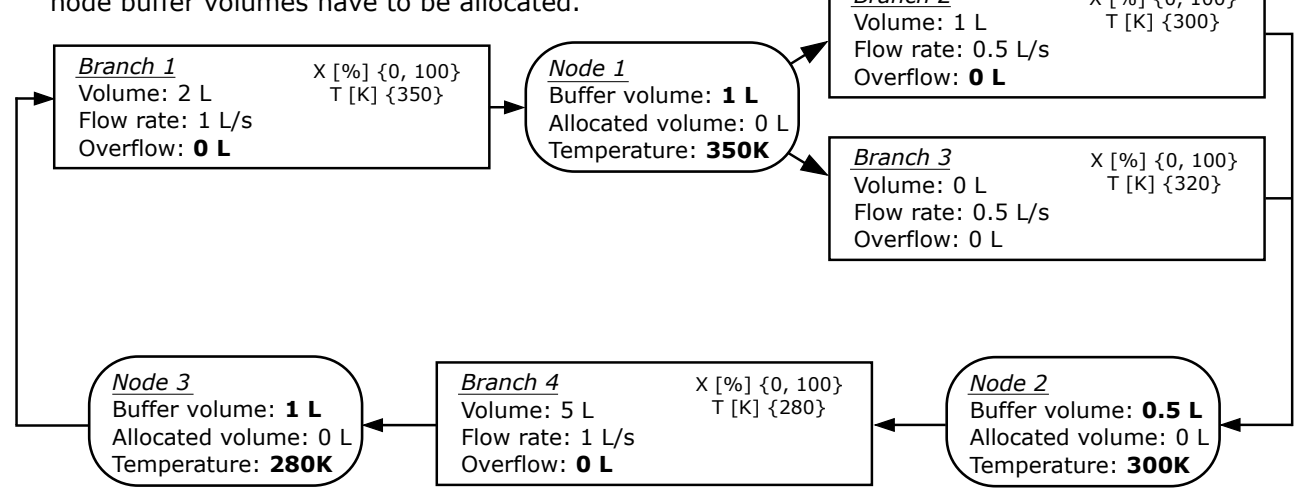

Figure 5.36: Mass and heat distribution among branches. State 1.

possible that branch volume is smaller than displaced volume. In such case, the part of the volume that does not fit into the branch becomes a new overflow associated to that branch. Then, as done previously, the overflow is sent into the buffer volume of the outlet node. When the new volume arrives to the node, temperature of the buffer volume, $T_{n}$, is recalculated by mass-averaging temperatures of the current and the new incoming volume. Again, instantaneous mixing is assumed. This is an iterative process which goes on until the whole excess volume is allocated. To prevent infinite iterations, the condition to exit the loop is that remaining overflows add up to less than 0.001 times the initial sum of overflows.

2) Allocated volumes are equal to buffer volumes BUT an overflow is different from zero. Go to next iteration.

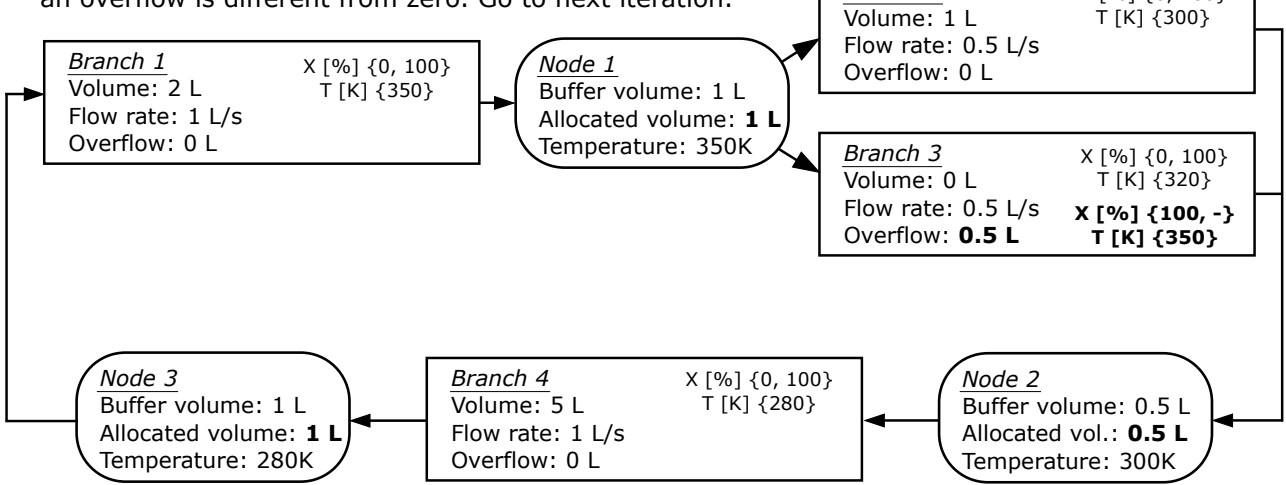

Figure 5.37: Mass and heat distribution among branches. State 2. 
In the example, half of the buffer volume of Node 1 goes into Branch 3. Since Branch 3 has no volume, the whole received volume of $0.5 \mathrm{~L}$ becomes an overflow of the branch. The other outgoing volumes fitted in the corresponding branches. All those allocations are accounted for in the variable allocated volume of each node, as can be seen in Fig. 5.37. Another iteration is needed to allocate the new overflow of Branch 3. Fig. 5.38 shows how the $0.5 \mathrm{~L}$ volume is incorporated to the buffer volume of Node 2, modifying its temperature. Current buffer volume of $1 \mathrm{~L}$ still fits into Branch 4. It is allocated without generating new overflows, thus ending the loop.

3) Second iteration. All overflows are equal to zero AND allocated volumes are equal to buffer volumes.
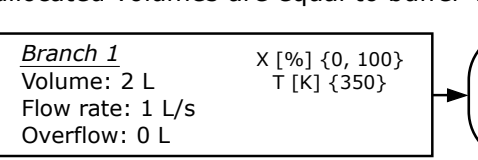

\section{Node 1}

Buffer volume: $1 \mathrm{~L}$

Allocated volume: 1

Temperature: $350 \mathrm{~K}$

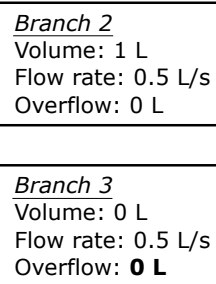

Overflow: $\mathbf{O} \mathbf{L}$

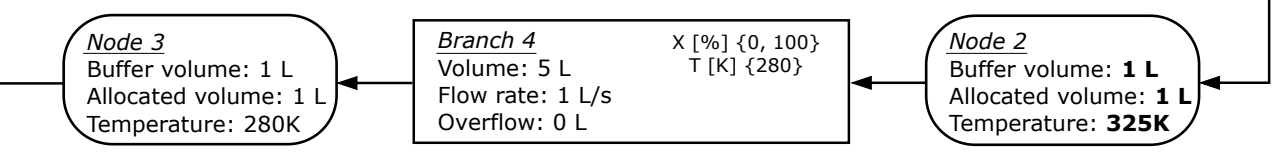

Figure 5.38: Mass and heat distribution among branches. State 3.

When the loop ends, overflows of all branches are zero and, in all nodes, buffer volumes are equal to allocated volumes. This is because all excess volumes are distributed in the circuit. Each node keeps a temperature which is the result of mixing the flows that passed through it. This temperature, $T_{n}$, is imposed to the outlet branches.

Two cases are possible. If the displaced volume is higher than or equal to the branch volume, a single parcel is created which has the temperature of the inlet node, $T_{n}$. On the contrary, if the branch volume is higher than the displaced volume, a parcel of the size of the displaced volume is created at the beginning of the branch. The parcel is at the inlet node temperature, $T_{n}$. A parcel of that size was created during the displacement of parcels inside the branch which was explained previously, but it is created again at this point because it may have been modified immediately afterwards, during the heat exchange operations. Fig. 5.39 shows 


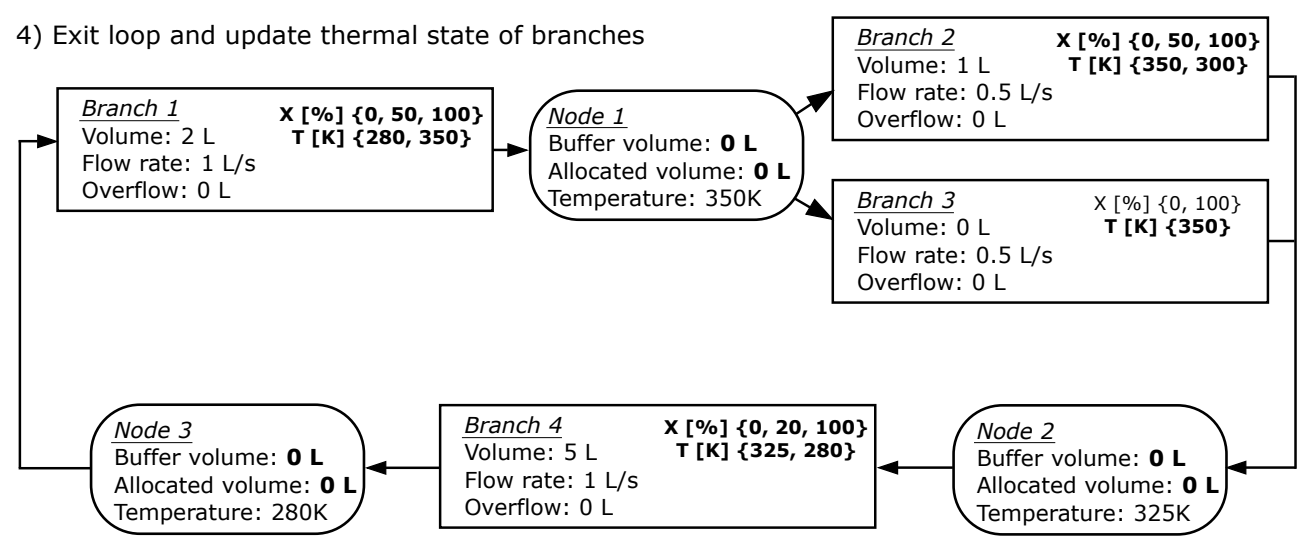

Figure 5.39: Mass and heat distribution among branches. Final state.

the final temperature distribution of the circuit after updating the state vectors. Both buffer and allocated volumes are finally set to zero.

\section{Thermal state determination, Part 3}

At this point, temperature distribution throughout the circuit is known. Tasks of Part 3 read temperatures at relevant locations. Those temperatures are required by other models or by the processor of outcomes.

An essential output of the thermo-hydraulic model is the fluid temperature at the inlet of each heat exchanger. There are different methods to obtain this temperature depending on the flow conditions. A common assumption is that the displaced volume on the next call will be the same as on the current call. Like in the methods for the displacement of parcels, three cases are considered: no flow, displaced volume smaller than or equal to heat exchanger's volume ("slow flow") and displaced volume bigger than heat exchanger's volume ("fast flow"). If there is no flow, then the whole heat exchanger volume is comprised into a single parcel. Temperature of that parcel is the inlet temperature.

- In the "slow flow" case, heat exchange was done first and later parcels were displaced. A volume of fresh fluid equal to the displaced volume entered the heat exchanger. That volume, stretching from the start of the heat exchanger to the the furthest point where the displaced volume arrived (still inside the heat exchanger), can be formed by one or more parcels or be a part of a bigger parcel. In any case, 


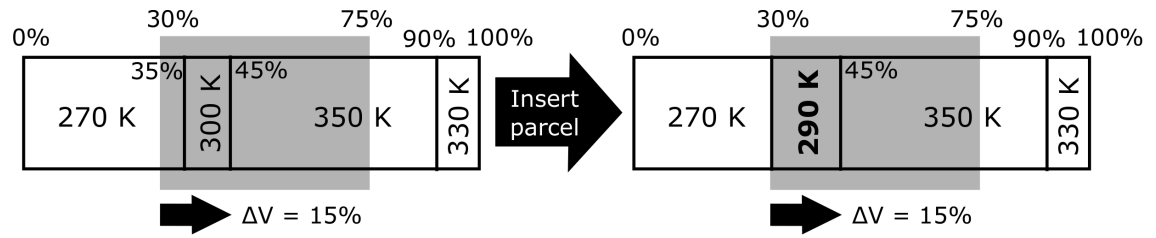

Figure 5.40: Calculation of inlet temperature of heat exchanger. "Slow flow" case.

temperature of the volume is obtained through mass-average and used as inlet temperature to the heat exchanger. In the example of Fig. 5.40 which continues the one of Fig. 5.31, inlet temperature is $290 \mathrm{~K}$.

- In the "fast flow" case, parcels were displaced first and then heat was applied to the displaced volume beyond the heat exchanger outlet. The incoming volume is, therefore, located immediately before the heat exchanger outlet. Inlet temperature is the temperature of a hypothetical parcel of the size of the displaced volume and the end at the heat exchanger outlet. The start of such parcel is outside (before) the heat exchanger, because the displaced volume is bigger that the volume of the heat exchanger. This case can be divided in two depending on whether the start of the parcel is in the same branch or in a previous branch.

Branch A: heat exchanger branch.

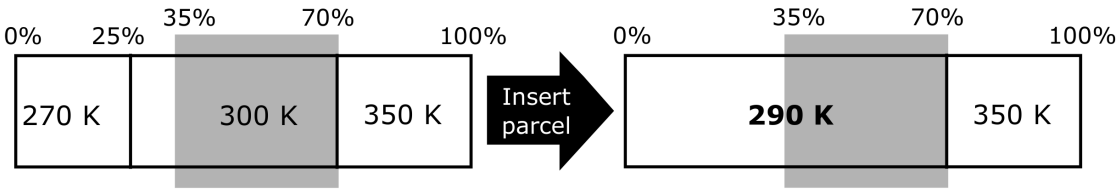

Figure 5.41: Calculation of inlet temperature of heat exchanger. "Fast flow" case. Heat exchanger's branch.

- If the start of the parcel is in the same branch, the method is to calculate the temperature of the hypothetical parcel through mass-average.

- If the start of the parcel is in the previous branch, it must be taken into account that there may exist several inlet branches. 


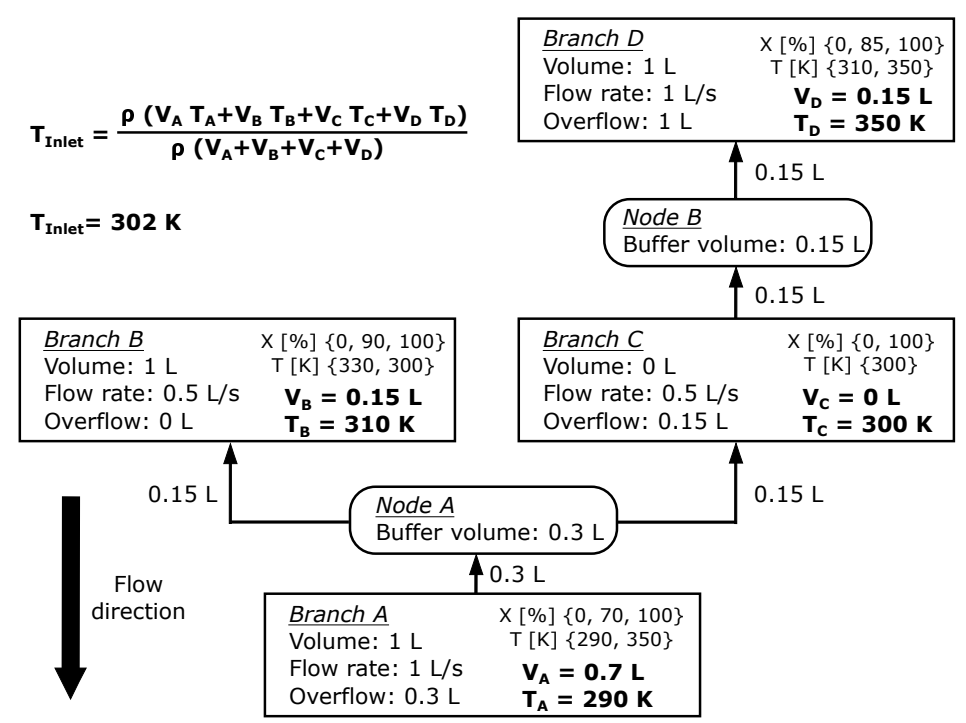

Figure 5.42: Calculation of inlet temperature of heat exchanger. "Fast flow" case. Previous branches.

The balance to calculate temperature is initiated with a massaverage of the volume between the start of the branch which contains the heat exchanger and the outlet of the heat exchanger. This is illustrated with an example in Fig. 5.41. The example is completed in Fig. 5.42. An overflow is generated at the branch start (not at the end) with volume equal to the displaced volume minus the volume between the branch start and the outlet of the heat exchanger, which was processed in the previous step. This overflow is converted into a buffer volume of the inlet node. Then, an iterative procedure similar to that of the distribution of overflows is undertaken to find the volumes that will pass through the heat exchanger on the next call. Volume share of each branch is recalculated attending to each node's inlet branches instead of its outlet branches. New overflows in the previous branches can be generated if the branch volume is small or zero. Every time a volume is processed, its temperature is mass-averaged and included into the main balance. Another difference is that, in this case, there is no need of calculating temperatures of buffer volumes. To prevent infinite iterations, the condition to exit the loop is that 
remaining overflows add up to less than 0.01 times the initial sum of overflows.

After setting inlet temperatures of heat exchangers, temperature of every object is read and stored. For elements that are not nodes, object's temperature is the temperature of the parcel in the middle of the element. In the case of nodes, temperature of the buffer volume, $T_{n}$, calculated during the volume distribution could be used directly. However, this temperature does not take into account connected branches where flow rate is zero. To include all connected branches, a temperature of each branch is considered. For inlet branches, it is temperature at the branch's end (last parcel), for outlet branches, it is temperature at the branch's start (first parcel). Those temperatures are averaged to obtain $\bar{T}$ of each node. As a compromise, final node temperature is the mean between $T_{n}$ and $\bar{T}$.

\section{Thermal state determination, Part 4}

A final task is done to prevent increasing complexity of the temperature distribution in the circuit. A maximum number of parcels per branch is allowed. Initially, the number of parcels in every branch is checked. If there are more parcels than a specified maximum, pairs of them are merged. First, the combined volume of every pair of adjacent parcels is calculated. The selected pair is the one with the lowest volume. An additional condition is that both parcels must be inside the same object (pipe, pump, heat exchanger, etc.) to avoid distorting object temperatures and inlet temperatures. The pair of selected parcels is then merged into one parcel. Temperature of the new parcel is calculated as the massaverage of the temperatures of the two original parcels. Every iteration merges a pair of parcels until the number of parcels is lower than or equal to the maximum. The loop also ends if there is no pair of parcels valid for merging, because each parcel belongs to a different element or because there is only one parcel in the branch. An example is given in Fig. 5.43.

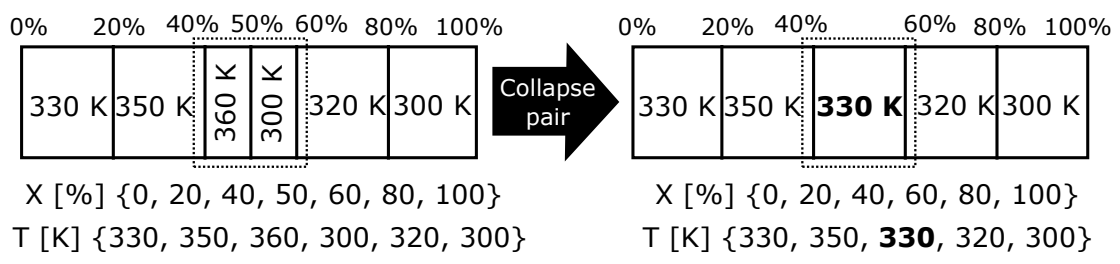

Figure 5.43: Simplification of parcels distribution by merging. 
A summary of the tasks undertaken in each of the four parts of the present section is displayed in Fig. 5.44.

Part 1. Update of internal thermal states.

Every branch

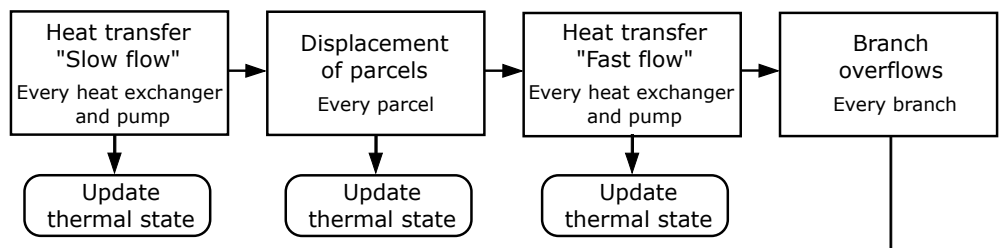

Part 2. Mass transfer among branches.

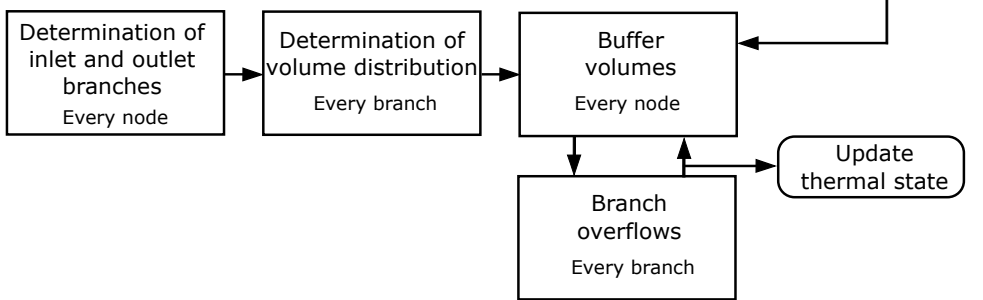

Part 3. Obtention of relevant information.

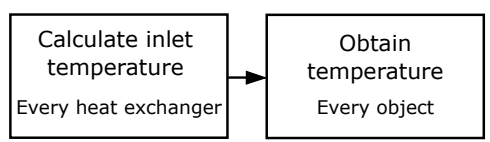

Part 4. Limitation of system complexity. Every branch

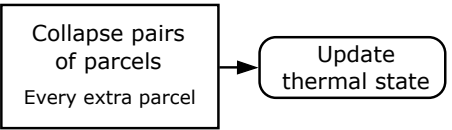

Figure 5.44: Summary of the procedure for thermal state determination. 



\section{Heat transfer in the global engine model}

6.1 Validation methods . . . . . . . . . . . . . . . . 274

6.1 .1 Experimental tests . . . . . . . . . . . . 275

6.1 .2 Simulation methods . . . . . . . . . . . . . 279

6.2 Validation results . . . . . . . . . . . . . . . . 287

6.2 .1 Validation in steady state . . . . . . . . . . 287

6.2.2 Validation in WLTP driving cycle . . . . . . . . 288

6.3 Concluding remarks . . . . . . . . . . . . . . 295

In the previous chapters, several models that simulate engine heat transfer processes were examined. The individual capabilities and limitations of each model were described. The problem of convective heat transfer to the cylinder walls was the most complex one to be dealt with in this thesis. This problem is not solved by the research community yet and only limited solutions can be implemented. The capacity of the model to provide reliable predictions of mean cylinder wall temperatures under diverse operating conditions was experimentally confirmed. The tendency of the model to overpredict temperature at high engine speed was corrected by introducing appropriate convective correlations on the coolant side. It was also seen that the temporal evolution of heat flux over the closed part of the engine cycle was correctly estimated under varying operating conditions. After the addition of new features to the model, a preliminary validation was pertinent. The transient thermal response of the engine block was analyzed. Good agreement between model and experiment was obtained regarding the temperature evolution of engine structure and outgoing coolant. The models of heat exchangers and 
thermo-hydraulic circuits deal with well-known physical processes and use widely accepted methods. In the corresponding sections, the limitations of the methods were reported to clarify which applications are suitable for these models.

In summary, the thermal models of engine block, heat exchangers and hydraulic circuits are applicable to the calculation of thermal transients under dynamic engine operation. The work presented up to this point motivated to test the capacity of the models in joint operation. This capacity was assessed through a validation of the thermal response of the Virtual Engine Model in complete driving cycle simulations. As mentioned in the introduction of this dissertation, a modern engine simulation tool should provide accurate results in a wide range of ambient temperatures; in particular, at low temperature. On the one hand, because it is common for an automotive engine to operate below nominal temperature, on the other hand, to comply with current homologation procedures and prepare for future ones. Therefore, all validations of this chapter were also conducted in cold ambient at $-7^{\circ} \mathrm{C}$.

The chapter is divided into several sections which cover the process of defining a validation procedure, collecting experimental data, setting a simulation procedure, setting up a suitable engine model for validation, calibrating the model, executing the validation and analyzing the outcome. A reduced version of this validation was published as one of the papers related to this thesis [217].

\subsection{Validation methods}

The validation of the thermal response of the Virtual Engine Model (VEMOD) was accomplished by means of a combination of simulations and test bench experiments. The reference test was the driving cycle of the Worldwide harmonized Light vehicles Test Procedure (WLTP) [24], which has replaced the New European Driving Cycle (NEDC) as standard emissions test in the European Union [21]. The driving cycle of the WLTP is called Worldwide harmonized Light vehicles Test Cycle (WLTC).

In Section 6.1.1, details about the test facility and the test campaign are explained. The test bench allowed to operate a production engine to follow a driving cycle. Regarding the experimental investigation, a preliminary validation in steady state was done. Steady-state operation is relevant for design, for investigating nominal operation and for model 
calibration. The second part of the validation was undertaken under dynamic engine operation. Dynamic operation is required for standard tests and also to reproduce real-life conditions; in particular, driving conditions are most relevant. As previously mentioned, the engine was operated in the test bench to follow the WLTP driving cycle. Since this is a very dynamic driving cycle, the transient behavior of the model could be properly evaluated.

For the simulation part of the research, a whole model of the real engine was built in the Virtual Engine Model environment. Simulations involved all submodels of the Virtual Engine Model, including those discussed in this dissertation. There are several aspects of the simulation procedure that require clarification. They are dealt with in Section 6.1.2.

The evaluation of the simulation tool's predictive performance is reported in Section 6.2. Analyses are focused on coolant and oil temperature. As mentioned, the validation was composed of different parts. Results of the steady-state preliminary validation are shown in Section 6.2.1. In that section, temperatures of coolant and oil are compared for the different operating points. Later, the results from WLTC tests are discussed throughout Section 6.2.2. There are three subsections, one for each test; since tests were done under three different conditions of initial engine temperature. Finally, observations and conclusions of the validation are summarized in Section 6.3.

\subsubsection{Experimental tests}

For the purposes of this investigation, the experimental facility needed to replicate a driving cycle in an engine and allow tests under cold ambient conditions. For this reason, the test bench was installed in a test cell capable of being conditioned from current ambient temperature up to a room temperature of $-7{ }^{\circ} \mathrm{C}$. The engine employed for this research was different from the engine used in Chapter 4 and thus it is called Engine 2. Engine 2 was a four-stroke production diesel engine compliant with Euro 5 emissions regulations. The engine had four cylinders and four valves per cylinder. A variable geometry turbocharger cooled by oil was coupled to the engine. Low pressure and high pressure exhaust gas could be recirculated into the cylinders. Engine thermal management was improved with an electrovalve which blocked coolant flow through the engine during warm-up. Main specifications of Engine 2 can be found in Table 6.1. The test bench was equipped with a test automation system which allowed to 


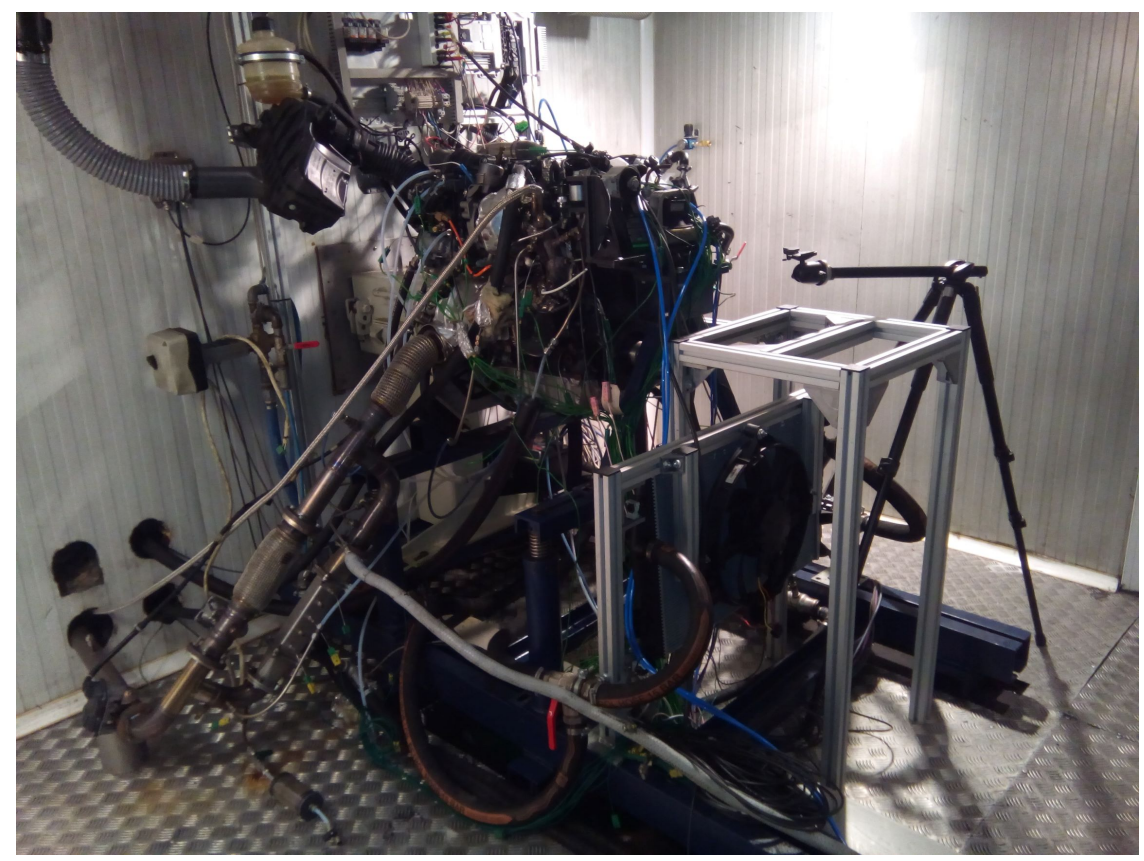

Figure 6.1: Photograph of Engine 2 in test facility.

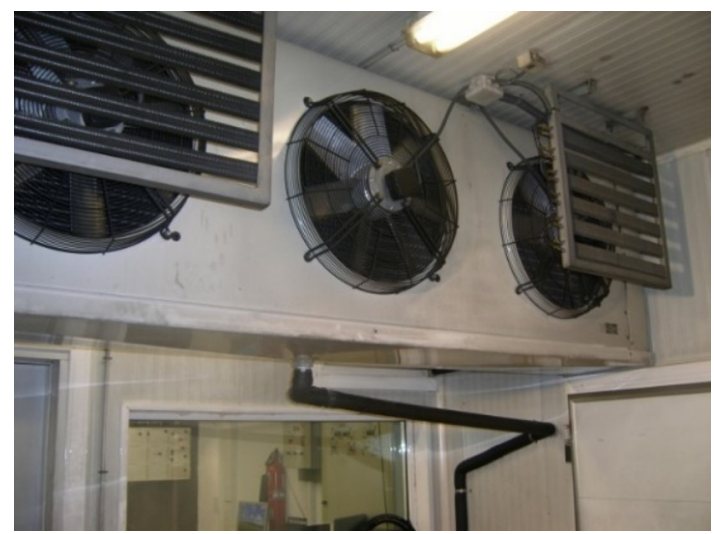

Figure 6.2: Photograph of room conditioning system. 
measure steady-state operating points as well as driving cycles. Data was recorded at a frequency of $10 \mathrm{~Hz}$. Except for the mentioned features, the characteristics of the measurement system were similar to those of Engine 1. Table 6.2 summarizes the instrumentation used in the studies presented in this work.

\begin{tabular}{lc}
\hline Displaced volume & 1.6 liter \\
Stroke & $79.5 \mathrm{~mm}$ \\
Bore & $80 \mathrm{~mm}$ \\
Compression ratio & $15.4: 1$ \\
Torque (max.) & $320 \mathrm{Nm} @ 1750 \mathrm{rpm}$ \\
Power (max.) & $96 \mathrm{~kW} @ 4000 \mathrm{rpm}$ \\
\hline
\end{tabular}

Table 6.1: Main specifications of Engine 2.

\begin{tabular}{llll}
\hline Variable & Instrument & Range & Accuracy \\
\hline Engine speed & Dynamometer & $0-7500 \mathrm{rpm}$ & $\pm 1 \mathrm{rpm}$ \\
Torque & Dynamometer & $0-400 \mathrm{Nm}$ & $0.5 \%$ \\
In-cylinder pressure & Kistler 6125C10 & $0-300 \mathrm{bar}$ & $\pm 1.25 \mathrm{bar}$ \\
Air mass flow & AVL Flowsonix Air & $0-1400 \mathrm{~kg} / \mathrm{h}$ & $1 \%$ \\
Fuel mass flow & AVL 733S & $0-41.67 \mathrm{~g} / \mathrm{s}$ & $0.12 \%$ \\
Temperature of & K-type & -200 to $1250{ }^{\circ} \mathrm{C}$ & $\pm 2{ }^{\circ} \mathrm{C}$ \\
fluids and structure & thermocouples & & \\
\hline
\end{tabular}

Table 6.2: Main instrumentation used in Engine 2.

The steady-state study was focused on a set of 22 operating points. They are summarized in Table 6.3. Some steady-state points were measured at a room temperature of $20^{\circ} \mathrm{C}$ and other at $-7^{\circ} \mathrm{C}$, with the aim of validating the model also under cold ambient conditions. The three operating points in bold font were measured at $20^{\circ} \mathrm{C}$ and $-7^{\circ} \mathrm{C}$, while the rest were measured at $20^{\circ} \mathrm{C}$. All operating points were measured three times to ensure repeatability. Combinations of engine speed and load were selected under two criteria: covering a large area of the engine map and being included in the WLTC range, or close to it. Fig. 6.3 shows the measurement points together with the engine map and the trace of operating points of the WLTC.

Engine operating parameters were set according to the calibration in- 


\begin{tabular}{ccccccc}
\hline Engine speed $[\mathrm{rpm}]$ & \multicolumn{5}{c}{ Load $[\%]$} \\
\hline 1000 & 21 & 44 & 66 & 88 & \\
1250 & 13 & 26 & 50 & 76 & 100 \\
1500 & 11 & $\mathbf{2 5}$ & 50 & $\mathbf{7 5}$ & 100 \\
2500 & 25 & 50 & 75 & 100 & \\
3500 & $\mathbf{2 5}$ & 50 & 75 & 100 & \\
\hline
\end{tabular}

Table 6.3: Steady-state operating points for validation.

cluded in the engine control unit (ECU). In particular, injection and charging settings were those of the ECU maps corresponding to the target operating point. The engine was warmed up until steady state was reached. Temperatures of coolant, oil and the external part of the engine block were monitored to check that temperature variation was lower than $0.5^{\circ} \mathrm{C}$ per minute. PID controllers regulated the water flows through intercooler and coolant cooler to maintain nominal engine temperatures. Stabilization time was between 20 and 40 minutes depending on the operating point. Once steady state was reached, three repetitions of each operating point were measured. All relevant magnitudes were recorded, including temperatures and operation settings.

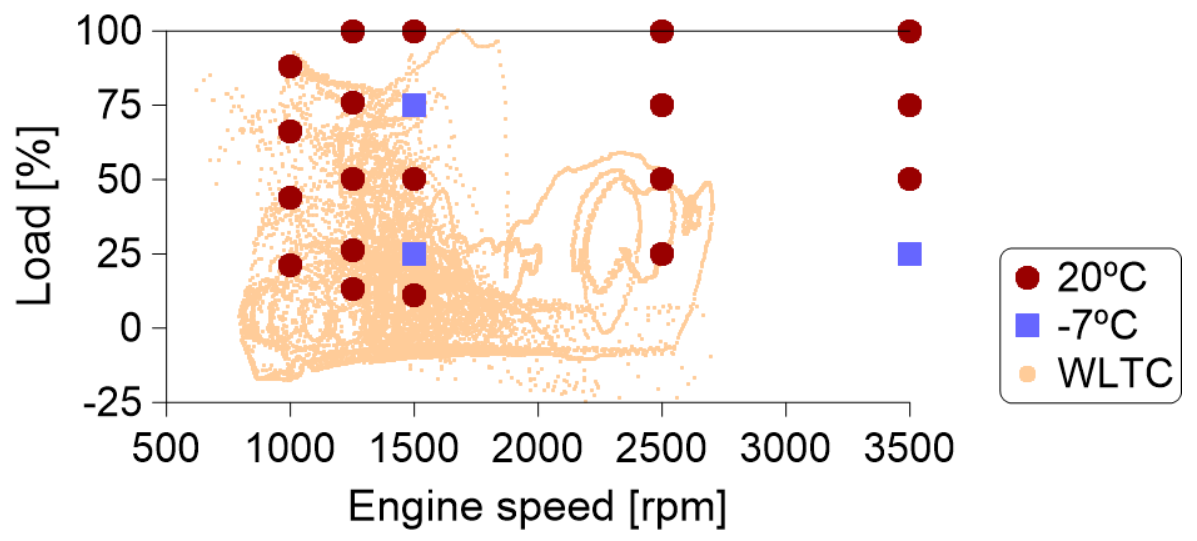

Figure 6.3: Steady-state operating points for validation and WLTC trace.

Regarding the transient tests following the WLTC, three different stud- 
ies were conducted. In the first transient study, the WLTC was measured starting from hot conditions, i.e., oil, coolant and engine structure were already warmed up. Starting from hot conditions allowed to have the thermostat actuating during the whole cycle. In the second study, the engine started the WLTC at a temperature of $20^{\circ} \mathrm{C}$ to examine the warmup process. The initial temperature of the final study was $-7^{\circ} \mathrm{C}$. That final study provided information concerning engine behavior under cold, transient, dynamic conditions. Each study was measured three times for repeatability. Tests were later replicated in the Virtual Engine Model to complete the validation.

\subsubsection{Simulation methods}

\section{Setup of validation model}

To run the simulations, it was necessary to fully define the engine in VEMOD. Most geometry parameters were obtained from the engine CAD. Dimensions of external circuits were measured in the test bench. ECU calibration maps were obtained from the engine manufacturer. Information about the performance of pumps, heat exchangers and thermostat was also requested to the manufacturer. Using this information, the input XML file could be filled with the necessary engine data. The input XML file was the same for all engine tests. A considerable amount of data was entered in the input file in the form of characteristic curves. The rest were constant values.

Circuit layouts were defined as indicated in Figs. 6.4 and 6.5. Coolant circuit was realistic, but oil circuit was a simplification. Thermocouples measuring temperatures used in the validation are depicted at the locations where they were installed. Circuit branches are numbered for internal reference. All branches are connected by nodes and all elements by pipes.

Looking at Fig. 6.4, the cooling fluid, after being impelled by the turbopump in branch 1, was split into three branches: 2, 3 and 4. Branch 1 contained the engine block, branch 2 contained the high pressure EGR cooler and branch 3 contained the low pressure EGR cooler. Thermal exchanges in the engine block were modeled with the Nodal Model, which was covered in Chapter 4. EGR coolers were modeled with the model of heat exchangers described in Section 5.1, as thermal-flow-to-hydraulic-flow heat exchangers. Branches 2 and 3 were connected at the outlet. At that location, the thermocouple used for validation was installed. That point 


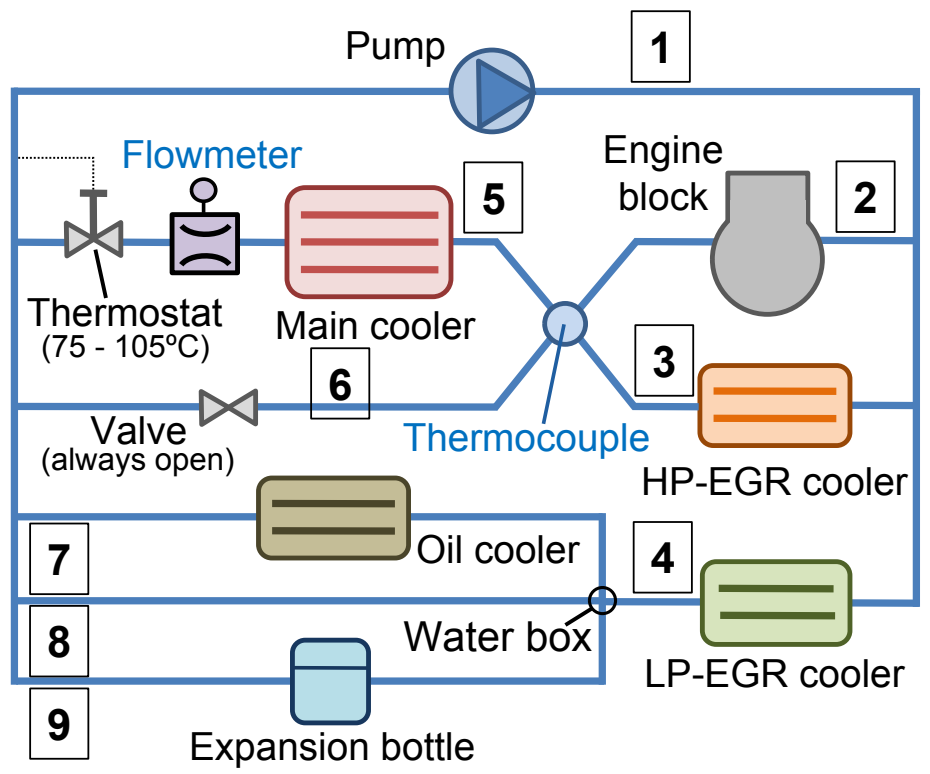

Figure 6.4: Diagram of cooling circuit model.

was connected to the return node through two branches, 5 and 6 . Branch 5 contained two long pipes that led to a backroom where the coolant cooler (main cooler) was located. The main cooler was modeled with the heat exchangers model but not with the effectiveness-NTU method, as the other heat exchangers. Instead, coolant temperature at the outlet of the main cooler was a given setting, because in the test bench target outlet temperature was always reached. Thus, from the modeling point of view, it was a heat exchanger controlled by a setting. All details about types of heat exchangers were explained in the corresponding section. After passing through the main cooler, coolant entered the flow meter. Before arriving at the return node, there was a thermostat to control flow in the branch of the main cooler. In the parallel branch 6 there was a valve that, in combination with the thermostat, allowed to stop coolant flow through the engine block completely. In all experiments and simulations of the present research, that engine valve was always wide open. Focusing on the lower part, in the water box flow from branch 4 split into three branches: 7, 8 and 9. Branch 7 contained the oil cooler (hydraulic-flowto-hydraulic-flow heat exchanger) and branch 9 the expansion bottle. All branches from 5 to 9 ended in the return node. From there, coolant entered again into the pump. Details about pump, valve and thermostat models, 
among other relevant information, can be found in Section 5.2.

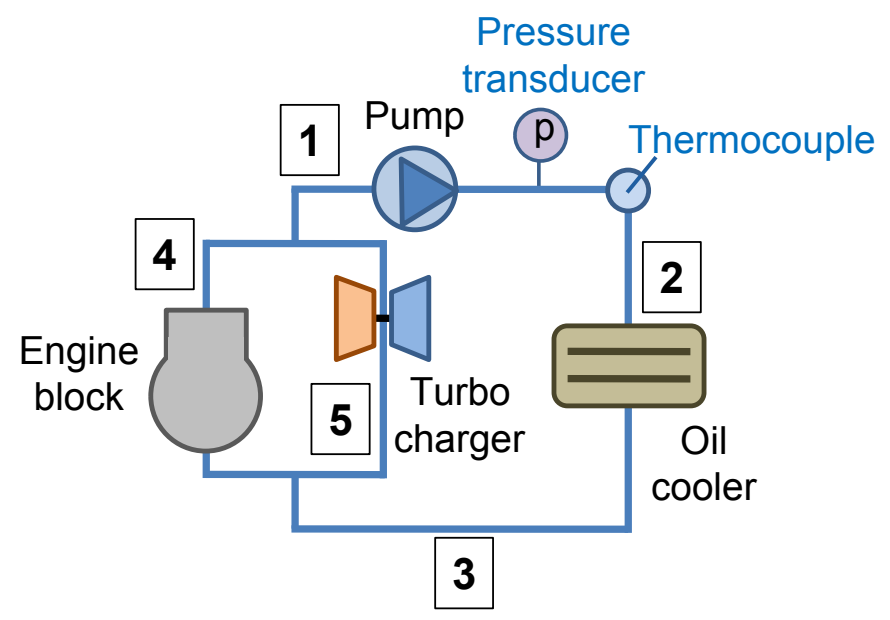

Figure 6.5: Diagram of lubrication circuit model.

Moving on to Fig. 6.5, the lubricating fluid (engine oil) was impelled by a positive-displacement pump. Oil first passed through the oil cooler mentioned in the description of the cooling circuit. Between pump outlet and cooler inlet there was a pressure transducer and a thermocouple that were used for validation. All those elements formed branch 1 . Due to the complexity of the oil path, a simplified approach was employed to model this circuit. It was considered that, after the oil cooler, flow was bifurcated into two branches, 4 and 5. Branch 4 lubricated the engine block and branch 5 lubricated the turbocharger. Like the engine block, the turbocharger had a dedicated model which was described in Section 3.4. Outlets of branches 4 and 5 were connected to inlet of branch 1 . The capacity of the simplified circuit to reproduce the behavior of the real circuit is assessed in Section 6.1.2.

Characteristic curves of pumps were defined as a set of coefficients. Coefficients were obtained by fitting to experimental data provided by the manufacturer. The curve equations were described in Section 5.2.1. To summarize, the coolant pump is a turbopump whose head curve is a function of pump speed and flow rate. The oil pump, on the contrary, is a positive-displacement pump with a flow rate curve dependent on pump speed and fluid temperature.

Thermal properties of the different materials were also defined as temperature-dependent curves. Again, curve coefficients were fitted to ex- 
perimental data. Available properties were thermal conductivity, specific heat and density. Material density was kept constant during the simulation to avoid variations of the engine mass when temperature changed.

Hydraulic resistance through heat exchangers was also obtained from experimental data supplied by the manufacturer. This allowed to define the corresponding curves by means of coefficients. Additional data regarding thermal behavior was used to calculate the overall heat transfer coefficient for the effectiveness-NTU model.

\section{Model calibration}

Part of the data from steady state experiments was used for calibrating the different models that compose the Virtual Engine Model. Other researchers have also used steady state tests to calibrate models for transient operation [117].

There are some similarities and some differences between the calibration of the cylinder model employed in the research of Chapter 4 and the calibration of the global engine model under study in the present chapter. The calibration of the former model relied on the CALMEC combustiondiagnosis tool (see Section 3.2) complemented with measurements in ancillary engine systems to provide further insights. A detailed account of the calibration procedure can be found in Section 4.2.1. It is reminded that the study was done with a different engine, described in the aforementioned section. In the present study, the measurement instrumentation and the simulation model were significantly different from those of the previous study. CALMEC was also used for preliminary calibration, but it did not contain a combustion model. In fact, the combustion model of the VEMOD tool was the most influential model in the final adjustment. Of particular relevancy for this thesis was the determination of optimal values for the constants of the modified Woschni correlation, Eq. 3.3, which calculates heat rejection from in-cylinder gas to walls. These heat losses significantly affect the combustion process. Therefore, an iterative process that is out of the scope of this thesis was followed to adjust different key parameters. The calibration ensured the agreement of pressure and heat release curves with experimental data.

For both the CALMEC simulations and the calibration of the combustion model, several magnitudes had to be experimentally measured. They were the following:

- In-cylinder pressure trace. 
- Crank angle degree.

- Fuel consumption.

- Intake pressure and temperature.

- Exhaust pressure and temperature.

- Blow-by rate.

Pressure and temperature were measured at other locations of the gas path to calibrate the gas dynamics model. Coolant and oil temperature were necessary for CALMEC, for the global engine model and for the validation covered in this chapter.

As in the research of Chapter 4, a set of motoring tests were carried out. In Section 3.2, it was explained how the measurement of indicated parameters and the absence of combustion allow to apply the first law of thermodynamics to perform a characterization of the engine cylinders. In this application, the analysis was necessary to know the actual compression ratio and the deformation constant of the piston assembly. Moreover, the constant $C_{W 1}$ of the Woschni correlation could be preliminary adjusted for the subsequent calibration of the combustion model. With the information of the fired tests in steady-state operation and the results of the combustion model calibration, the constants of the Woschni correlation were finally set. During the part of the engine cycle in which valves were closed, the fine-tuning of $C_{W 1}$ resulted in a value of 1.63. To find $C_{W 2}$, the formula $C_{W 2}=0.59 * C_{W 1}=0.96$ was applied. This formula was mentioned in Section 3.3; it is used when no tests with different swirl ratios are available and it is based on empirical studies. For the open part of the cycle, those values were divided by two, yielding $C_{W 1}=0.815$ and $C_{W 2}=0.48$. The optimal value for the combustion velocity constant, $C_{2}$ was 0.001. It is important to remark that the constants of the Woschni correlation were adjusted to achieve an agreement between experimental and modeled curves of in-cylinder pressure and heat release. They were not tuned to improve predictions of coolant and oil temperatures, which are the target magnitudes of the validation presented in this chapter.

Steady-state tests were also used to calibrate uncertainties of hydraulic circuits. Hydraulic resistance encountered by coolant and oil flow when passing through the engine block was unknown. In addition, the hydraulic resistance curve of the thermostat was also unknown. The combination of tests at different engine speeds and loads, with different thermostat openings, allowed to adjust hydraulic resistances. The measured magnitude in the cooling circuit was flow rate, while in the oil circuit it was pressure. 


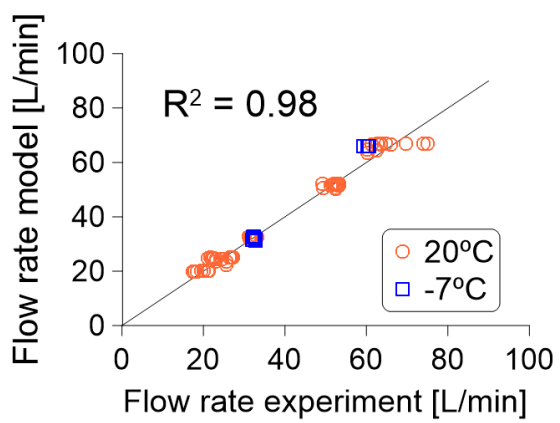

(a) Coolant flow rate

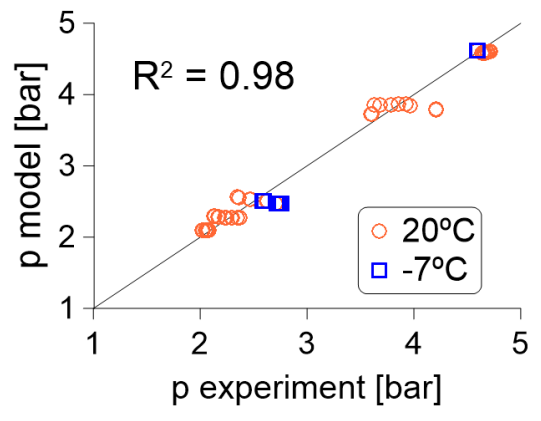

(b) Oil pressure

Figure 6.6: Coolant flow rate and oil pressure after calibration of engine and thermostat hydraulic resistances.

Results of the adjustment are shown in Fig. 6.6. Simulated magnitudes (vertical axis) are plotted against measured magnitudes (horizontal axis) for comparison. As commented in Section 6.1, some tests were measured at low temperature, $-7^{\circ} \mathrm{C}$. They are plotted in blue color. In the rest, in orange color, ambient temperature was $20^{\circ} \mathrm{C}$.

On the left side of Fig. 6.6, coolant flow rate is analyzed. Values are referred to Branch 5 of the cooling circuit, which contained the flow meter, the thermostat and the main cooler, see Fig. 6.4. Coefficient $\mathrm{R}^{2}$ of the comparison was high: 0.98. Mean error of all tests was 5\%. The group of tests with lower flow rates comprises operating points at engine speeds of 1000, 1250 and $1500 \mathrm{rpm}$, while the group with higher flow rates corresponds to engine speeds of 2500 and $3500 \mathrm{rpm}$.

On the right side of the figure, the comparison of oil pressure can be seen. Pressure was measured at the outlet of the oil pump, as shown in Fig. 6.5. Three data groups can be identified in the graph. The group with lower pressures comprises operating points at engine speeds of 1000, 1250 and $1500 \mathrm{rpm}$. The second group, with intermediate pressure, is formed by operating points at $2500 \mathrm{rpm}$. The group with the highest pressures comprise the tests at engine speed of $3500 \mathrm{rpm}$. Model predictions were in good agreement with measurements. Mean error was 3.8\%. Also for oil pressure, coefficient $\mathrm{R}^{2}$ was 0.98 . No systematic deviations were observed in low temperature points, neither in coolant flow rate nor in oil pressure. Prediction performance was similar at $-7^{\circ} \mathrm{C}$ and at $20^{\circ} \mathrm{C}$. That suggested that the hydraulic model is applicable regardless of fluid temperature. In conclusion, the calibration achieved good agreement between simulation 
and experiment regarding the hydraulic results.

Finally, another uncertainty was mass of the engine structure, which was not accurately known. Engine mass determined thermal capacity and thus influenced temperature evolution in the structure. In Section 4.5, it was discussed that the nodes that provide the highest thermal inertia to the engine heat transfer model do not represent the real engine shape. Starting values of mass could be obtained from the CAD, but not values of all components nor values of the whole engine mass. Therefore, mass of cylinder-head and mass of cylinder block were slightly adjusted to correct thermal inertia. Mass of engine fluids, on the contrary, depended on the volume of circuit components and ducts; thus, it was known and fixed by geometry.

\section{Simulation modes}

Simulations can be launched under different modes. Depending on the mode, the control model requests the input of certain variables as reference data. Inputs determine the operation of the ECU model, which is the control system. VEMOD allows the following modes:

1. Test bench. Values of most actuators over time are entered as inputs to the control system. The ECU model needs no maps, in part because actuator settings are supplied. Besides, the control system receives reference data of the gas path conditions (mass flow rate and pressure) and keeps those conditions in the simulation. During the simulation, the control system reads data from modeled sensors placed in the locations where reference data is provided. Then, it compares the sensor data with the reference data entered at the beginning and operates the model of the variable-geometry turbine to match both results. In summary, inputs are engine speed, injection parameters and reference data from the gas circuit. Usually, values come from experimental measurements in an engine test bench. This mode is employed when it is necessary to ensure that test conditions are replicated. Therefore, it is suitable for model testing and validation.

2. Test bench + calibration. Only engine speed and fuel mass flow rate are supplied as inputs. The ECU model contains maps as a function of those two parameters. Usually, the real ECU maps are 
used. Maps determine variables such as injection pressure, injection timing or position of the variable-geometry turbine.

3. Engine. Input variables are engine speed and torque curves. The control system regulates settings to reach the reference torque. Engine response is determined by ECU maps. This mode is useful to set a reference power output and then compare the performance of different alternatives.

4. Vehicle. Vehicle speed over time, i.e. a driving cycle, must be specified. The simulation contains a vehicle model that determines engine speed and torque for the engine model. The control system operates like in the Engine mode to complete the driving cycle.

In this thesis, the Test bench mode was used because the goal was to validate the thermal response of the engine model. Thus, the settings used in the real engine had to be used in the simulations. All inputs were measured during the experimental test campaign previously described.

\section{Other aspects of simulations}

In Section 6.1.2, it was mentioned that the same input XML file was used all engine tests, since engine data is the same. However, this is not completely true because, before being read by VEMOD, the simulation launcher makes some modifications in the XML file. Modifications introduce the experimental conditions of each particular test. In Test bench mode, explained in Section 6.1.2, experimental conditions for steady-state tests were:

- Engine speed.

- Injected mass, injection pressure and timing.

- Initial pressure and temperature of gas at various locations.

- Initial temperature of coolant and oil.

- Coolant temperature at the outlet of the main cooler, which was experimentally measured.

Experimental conditions for driving cycles were:

- Test duration.

- Initial pressure and temperature of gas at various locations.

- Initial temperature of coolant and oil.

- Coolant temperature at the outlet of the main cooler. It was experimentally measured during the tests. In each test, the mean value was used as setting in the model of the main cooler. 
- Traces of engine speed, injection settings and reference gas data over the time of the test.

The simulation launcher was a MATLAB ${ }^{\circledR}$ script. It controlled the reading of inputs and the modification of the XML input file, called the control system model and moved the generated results to a folder when the simulation ended. The control system was implemented in Simulink ${ }^{\circledR}$. It read sensor information, set actuator values and called the engine model. The engine model was supplied as a DLL (Dynamic Link Library) file.

All simulations were run in a desktop computer with a quad-core Intel ${ }^{\circledR}$ Core $^{\mathrm{TM}}$ i5-6600K processor using four threads. Simulation time heavily depended on engine speed because, for a given time, a higher number of engine cycles must be simulated at higher speed. As a reference order of magnitude, in WLTC tests, simulation duration was around 10 times higher than real duration. For tests in steady state, 5 minutes of engine operation were simulated. This ensured the stabilization of all variables.

\subsection{Validation results}

\subsubsection{Validation in steady state}

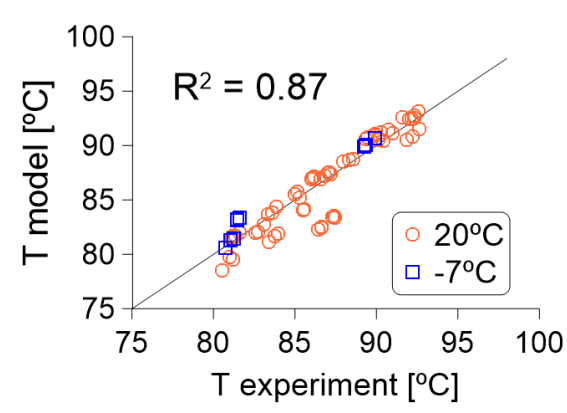

(a) Coolant temperature

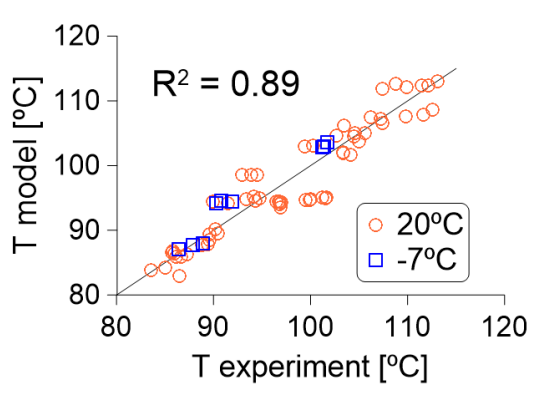

(b) Oil temperature

Figure 6.7: Steady-state validation.

The validation study under steady-state conditions is summarized in Fig. 6.7. The comparison of modeled and experimental coolant temperatures can be seen on the left side of the figure. All points were close to the diagonal line except three points corresponding to the three repetitions of a particular test, the one at $1500 \mathrm{rpm}$ and $50 \%$ load. The highest error was 
$4^{\circ} \mathrm{C}$, but $91 \%$ of the points had an error lower than $2^{\circ} \mathrm{C}$. Mean error was $1.1^{\circ} \mathrm{C}$. On the right side of Fig. 6.7, the oil temperature analysis is shown. Mean error was $2.2^{\circ} \mathrm{C}$. In $88 \%$ of the points error was below $4^{\circ} \mathrm{C}$. Tests done in cold environment were plotted in blue color. No specific limitations of prediction capability in the form of systematic errors were observed in cold environment tests, neither in coolant nor in oil temperature.

In conclusion, the steady-state validation revealed that the Virtual Engine Model was capable of providing reliable results with considerable precision. Overall, the model was able to capture the thermal influence of engine speed and load in steady-state operation. Also, cold temperature tests were simulated with similar performance. Next, model performance under transient conditions was studied.

\subsubsection{Validation in WLTP driving cycle}

The WLTC is a very dynamic driving cycle that encompasses diverse operating conditions. Before evaluating the ability of the global engine model to predict thermal responses, it was necessary to ensure that real engine operation was captured by the model after calibration. If engine operation were wrong, a good prediction of thermal evolution would be irrelevant, because thermal response is determined by engine operation. In Fig. 6.8, curves of engine speed and torque over the driving cycle are shown. As mentioned in Section 6.1.2, engine speed was an input to the model. Torque, on the contrary, was determined by the simulation. In the upper plot, a comparison between measured and modeled torque can be seen. Curves are colored red and blue respectively. The capacity of the engine model to reproduce the experimental torque was satisfactory throughout the cycle.

\section{Warmed-up conditions}

The first transient test was done warming up the engine prior to start. Temperatures in the engine (structure and fluids) were stable and nominal at the beginning of the driving cycle. During the 30 minutes of the cycle, temperatures changed as a response to varying operation. In this test, the warm-up process was therefore out of the study.

The first variable to be analyzed is coolant temperature. Modeled temperature is plotted as a blue curve. As three repetitions of the test were measured, experimental temperature is presented as a red band that 


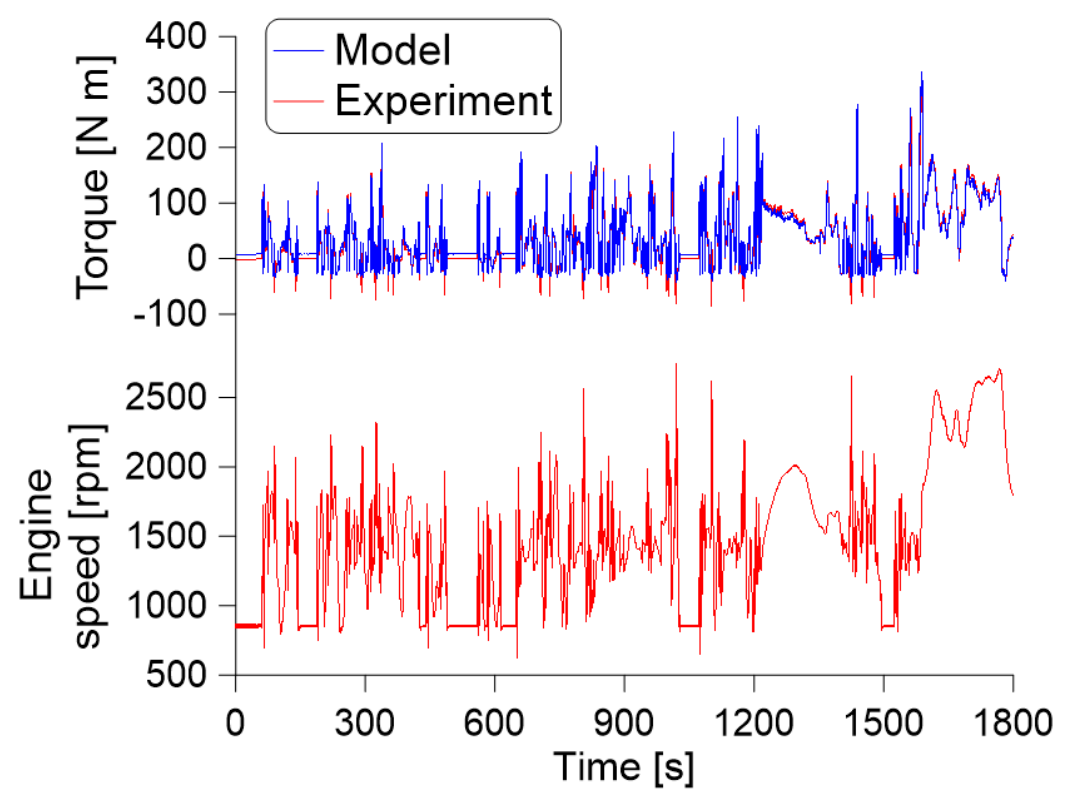

Figure 6.8: Engine speed and torque during WLTP driving cycle.

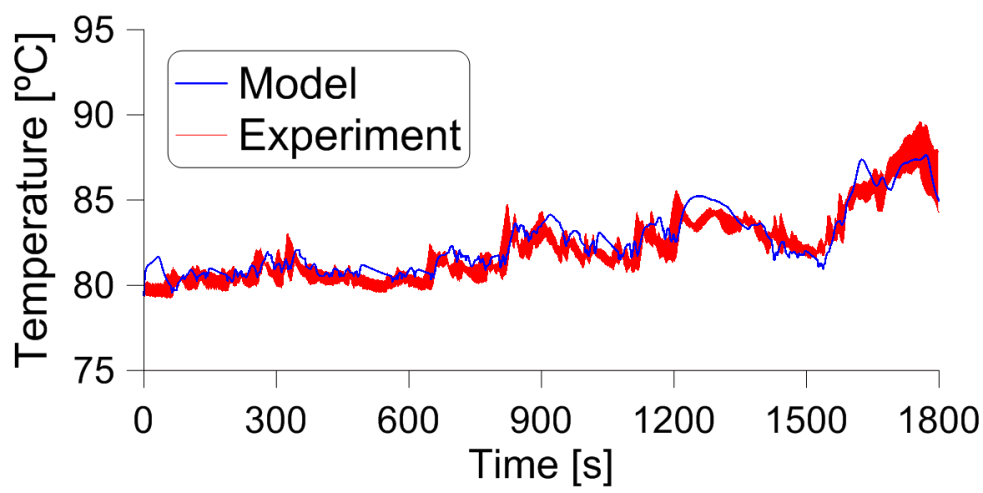

Figure 6.9: Coolant temperature during WLTC starting after warm-up. 
encompasses the values of the three traces. In Fig. 6.9, it can be seen that both the experimental traces and the simulation curve followed a similar evolution. There were minor discrepancies at high engine speed, but they were always lower than $1.7^{\circ} \mathrm{C}$ with respect to measurements. Overall, the prediction presented considerable accuracy, as mean error was $0.26^{\circ} \mathrm{C}$. For $93 \%$ of the cycle duration, error was below $1^{\circ} \mathrm{C}$.

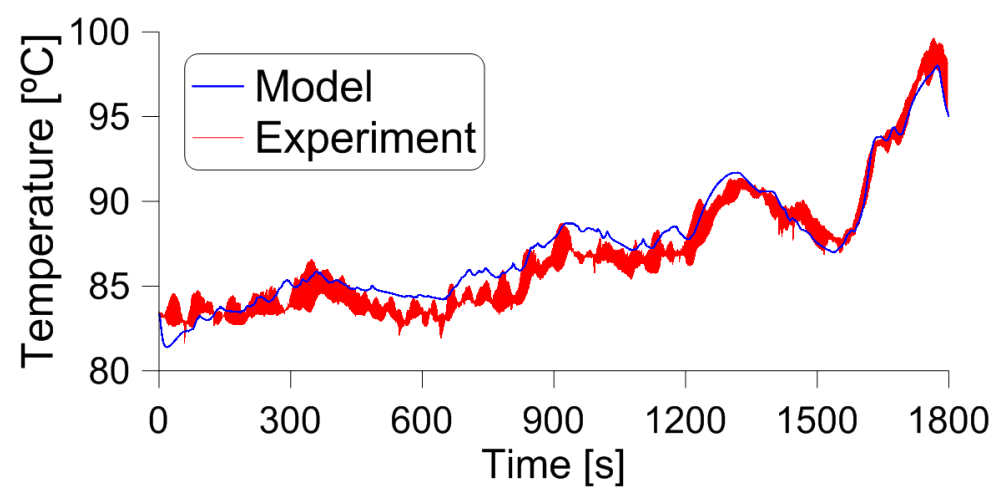

Figure 6.10: Oil temperature during WLTC starting after warm-up.

Regarding oil temperature, comparison between model and experiment is shown in Fig. 6.10. Oil temperature experienced larger variations than coolant temperature. The model reproduced the measured trend during most of the cycle. The maximum observed discrepancy was $2^{\circ} \mathrm{C}$. During $86 \%$ of the time, error was below $1^{\circ} \mathrm{C}$. In fact, mean error of the cycle was $0.41^{\circ} \mathrm{C}$. Inertia of the model was higher than in the experiment. This could be attributed either to the solid mass, to the oil or to the thermostat response time. Since temperature inertia on the coolant side was adequate, the issue might be likely caused by the simplified nature of the oil circuit. The modeled curve was above the average experimental trace $51 \%$ of the time, which comprised $85 \%$ of the error.

In summary, prediction of temperatures in this particular test was good. The accuracy of the predictions was demonstrated by the fact that mean errors were lower than $0.5^{\circ} \mathrm{C}$. Ensuing tests included the study of the warm-up process.

\section{Start at $20^{\circ} \mathrm{C}$}

In this test, temperature in the test cell was $20^{\circ} \mathrm{C}$. The engine was not warmed up before starting the test. Therefore, initial engine temperature 
at the beginning of the cycle was $20^{\circ} \mathrm{C}$. During the test, the engine warmed up until the coolant reached a temperature that triggered the thermostat opening. Then, part of the coolant flow passed through a heat exchanger where it was cooled down (branch 5 in Fig. 6.4). Throughout the driving cycle, changes in operation conditions determined the thermal evolution in the engine structure and fluids.

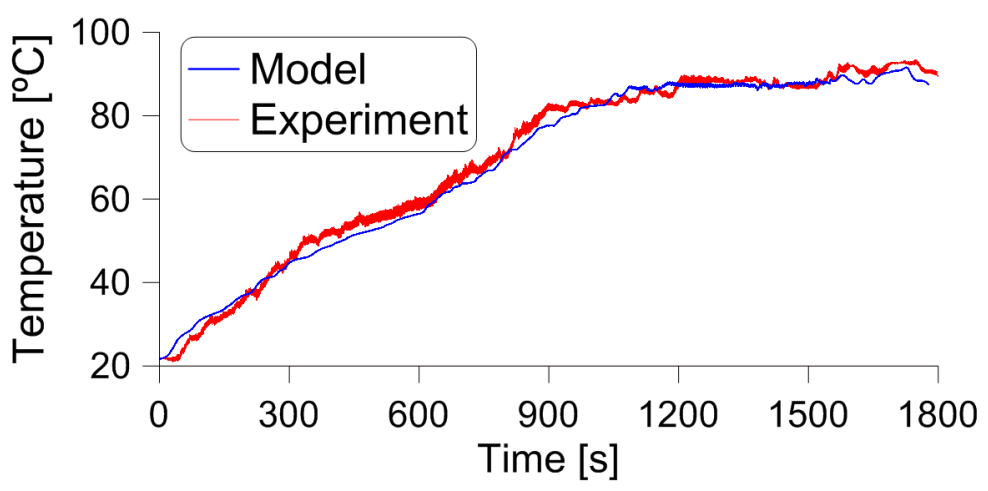

Figure 6.11: Coolant temperature during WLTC starting at $20^{\circ} \mathrm{C}$.

In the analysis of coolant temperature, shown in Fig. 6.11, it can be seen that very good agreement between model and experiment was obtained. Mean error was $1.15^{\circ} \mathrm{C}$. During $80 \%$ of the time, error was below $2^{\circ} \mathrm{C}$. Thermal inertia was well captured. Moreover, the model was able to correctly predict the slope change at around $600 \mathrm{~s}$. The effect of opening the thermostat and cooling the engine was also successfully modeled. The thermostat started opening upon reaching $75^{\circ} \mathrm{C}$, after approximately 900 s. The only noteworthy discrepancy was a small delay of warm-up at the beginning of the test that can be seen in the measured band but was not reproduced by the simulation. This caused a maximum error of $4^{\circ} \mathrm{C}$.

The evolution of oil temperature is shown in Fig. 6.12. During most of warm-up, agreement between simulation and experiment was very good. Thermal inertia was well captured by the model. Over the entire cycle, mean error was $1.24^{\circ} \mathrm{C}$. However, during the second half of the cycle, prediction deteriorated slightly. In that period, modeled oil temperature was a few degrees above the measured reference. Possible causes are an underestimation of the cooling capacity of the oil cooler or, also likely, an underprediction of heat transfer from oil to coolant through the engine block structure. The maximum error of the cycle was found at $1600 \mathrm{~s}$ 


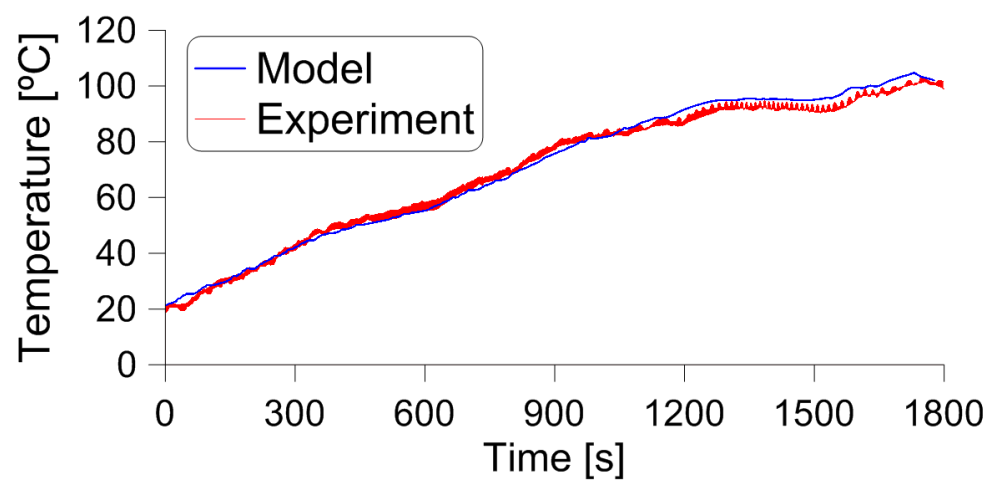

Figure 6.12: Oil temperature during WLTC starting at $20^{\circ} \mathrm{C}$.

and amounted to $5.8^{\circ} \mathrm{C}$. Still, for $89 \%$ of the cycle duration, error was below $3^{\circ} \mathrm{C}$. The misprediction at the beginning which was found in coolant temperature, was observed in oil temperature as well. Nevertheless, this discrepancy was smaller than $3.5^{\circ} \mathrm{C}$.

In sum, prediction of coolant and oil temperature was satisfactory throughout the driving cycle. Of particular noteworthiness was the performance during the warm-up process. That indicated that improvements reported in this thesis and aimed to model the transient response of the engine were successful. In addition, when the thermostat and cooler submodels started operating after warm-up, the simulated thermal response agreed with experimental observations.

\section{Cold environment}

The last test assessed prediction performance under cold ambient conditions, which is relevant for current developments of powertrain research. Temperature in the test cell was kept at $-7^{\circ} \mathrm{C}$. This was the engine temperature at the beginning of the driving cycle. As in the previous test, warm-up encompassed a substantial portion of the test duration.

In Fig. 6.13, the predictive performance of the model is shown for coolant temperature. In general, prediction was accurate and trends were reproduced. It was slightly poorer than in the previous test in the first part of the cycle, as coolant temperature was slightly overpredicted first (up to $6^{\circ} \mathrm{C}$ ) and slightly underpredicted later (maximum $8^{\circ} \mathrm{C}$ at $680 \mathrm{~s}$ ). Nonetheless, mean error considering the whole cycle was $2.52^{\circ} \mathrm{C}$. Temperature prediction after thermostat opening was very good. For $83 \%$ of the 


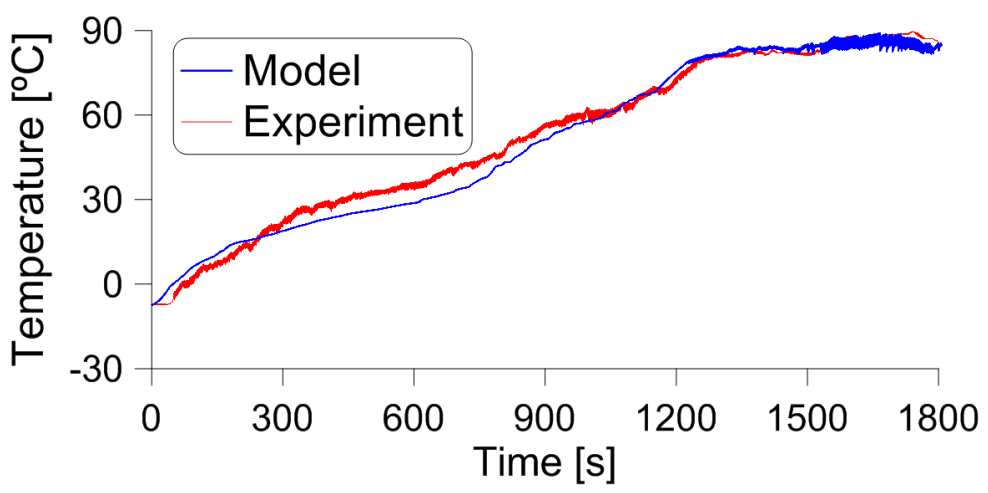

Figure 6.13: Coolant temperature during WLTC starting at $-7^{\circ} \mathrm{C}$.

cycle, error was below $5^{\circ} \mathrm{C}$.

Oil temperature is analyzed in Fig. 6.14. It is possible to see that the small discrepancies observed in the previous test were aggravated at low temperature. Causes that could lead to the overestimation of oil temperature in the late part of the cycle were mentioned in the previous section. The maximum error found in that period was $13.9^{\circ} \mathrm{C}$. Temperature overprediction at the beginning of the cycle, which had been a minor issue up to this point, was in contrast remarkable in the graph under current examination. Clearly, thermal inertia was underestimated in this period. That resulted in faster oil warm-up, producing again a discrepancy between model and experiment up to $14.7^{\circ} \mathrm{C}$. The small error observed in coolant temperature could be a consequence of the oil temperature misprediction. Several issues can be proposed as causes of this divergence:

- There are elements that significantly contribute to thermal inertia due to their mass, such as the connection rod, the crankshaft or the bearings, that are currently not being considered in the thermal model of the engine block [117] [160]. Those elements are in close contact with the lubricating fluid. Adding such elements would delay oil heating.

- As commented in the previous section, the amount of heat transferred from oil to coolant through the engine block structure could have been underestimated. Similarly, heat rejected from oil to coolant in the oil cooler could also be lower in the simulation than in the experiment. That would have accelerated oil warm-up and delayed coolant warm-up. It was seen in Fig. 6.13 that coolant warm-up was 


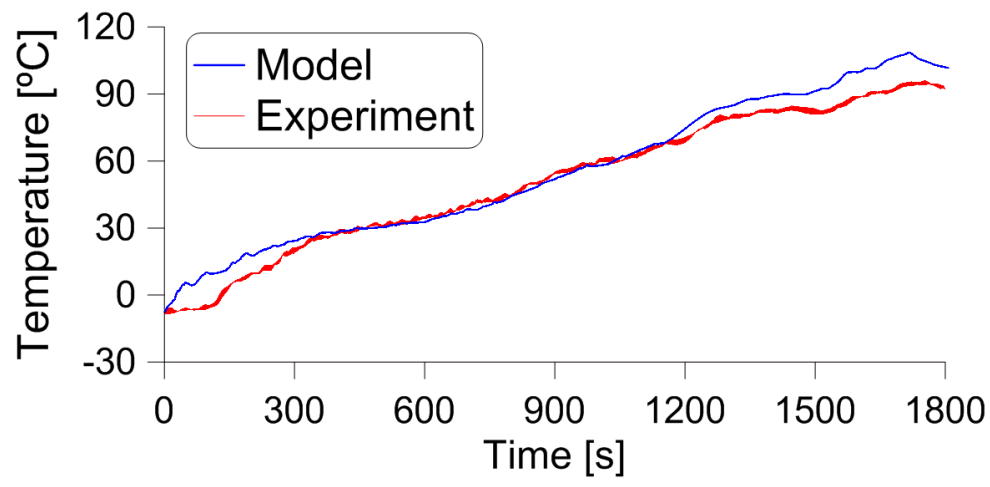

Figure 6.14: Oil temperature during WLTC starting at $-7^{\circ} \mathrm{C}$.

slightly faster than the experiment at the beginning and slightly slower afterwards. Therefore, this cause is only applicable to the second period. At start, the dominant cause was a different one.

- Thermal inertia of the oil cooler was neglected in the model of heat exchangers. The effect of that would be a faster warm-up.

- Heat loss to ambient was neglected in the engine block and the oil circuit. Results showed that this was not an issue in the previous test at $20^{\circ} \mathrm{C}$. However, as the current the test was done in a cold environment, the influence of heat losses to ambient could be significant. According to some authors, heat rejection to ambient during warm-up can be considerable [58].

These points can be used to propose future improvements for the thermal models in VEMOD, from adding missing components, to considering heat losses to ambient or thermal inertia of the oil cooler.

In spite of the problems observed at the beginning and the last part of the cycle, the excellent prediction during the middle part of the cycle kept the mean error over the whole cycle at a value of $4.57^{\circ} \mathrm{C}$. Considering this, it can be concluded that the results from the model can be trusted to a certain extent under cold environment conditions. Prediction of coolant temperature was robust and presented remarkable accuracy. However, oil temperature from the model should be considered an approximate value. Further improvements of the models can decisively increase prediction accuracy at low temperature. 


\subsection{Concluding remarks}

The present chapter has reported the validation of the models covered in this thesis. The validation was undertaken using a complete engine model. Heat transfer was co-simulated together with the other engine processes. The chapter did not only deal with the validation but also with the procedure to set up and calibrate a complex engine model. Several aspects of the simulation process with the Virtual Engine Model (VEMOD) tool were discussed as well. Moreover, a method to conduct the validation was proposed. The method comprised an experimental campaign of steadystate and transient tests at different temperatures. Results of coolant and oil temperature were examined to evaluate the prediction capacity of the models developed in this thesis. In general, good agreement was obtained between simulation results and experimental measurements from the tests. In steady-state tests, mean error was $1.1^{\circ} \mathrm{C}$ for the coolant and $2.2^{\circ} \mathrm{C}$ for the oil, over different operating points. Simulations at low temperature did not present specific issues. For the transient tests, the WLTC driving cycle was followed. Starting with a warmed-up engine, temperature predictions were very satisfactory, with mean errors below $0.5^{\circ} \mathrm{C}$. In the next test, in which the engine started at $20^{\circ} \mathrm{C}$, the warm-up process was correctly modeled. Overall, predictions were good, with mean errors of $1.2^{\circ} \mathrm{C}$ in coolant and oil temperature. The last test, performed at $-7^{\circ} \mathrm{C}$, yielded a mean error of $2.5^{\circ} \mathrm{C}$ in coolant temperature and $4.6^{\circ} \mathrm{C}$ in oil temperature. The temperature evolution curve was well captured in the case of the coolant. On the oil side, however, some improvements can still be made for low temperature applications. Some suggestions for improvement were given.

All things considered, the capability of doing thermal simulations with VEMOD was demonstrated in this chapter. Temperature predictions can be largely trusted over a wide range of operating conditions, no matter how dynamic. Only at very low temperature, the prediction of oil thermal evolution presented some specific limitations. 



\section{Conclusions and future work}

7.1 Main contributions . . . . . . . . . . . . . . . . . 298

7.2 Suggestions for future work . . . . . . . . . . . 302

This thesis is framed within the context of strict homologation procedures in the automotive industry and increasing reliance on simulation tools for research and development of powertrains. Global engine and vehicle simulation tools present many advantages over partial or simplified models. Such global simulation tools have become a fundamental resource for developing powertrains that are able to comply with new homologation standards. With the aim of completing an in-house integrated engine simulation tool, this thesis has focused on modeling heat transfer processes occurring in internal combustion engines. Throughout the document, works concerning the improvement of an existing heat transfer model, development of new models, models' integration into a global engine simulation tool and, finally, validation of thermal simulations, have been described. Three specific objectives were set for this thesis:

1. Modeling heat transfer processes in the engine block.

2. Modeling heat transfer processes in ancillary systems of the engine.

3. Validating the thermal behavior of a global engine model.

A thorough literature survey allowed to get acquainted with different approaches to model the diverse heat transfer processes of internal combustion engines. The topic of in-cylinder heat rejection was examined 
with special attention. Quasi-dimensional approaches of different complexity were found to be extensively used for system-level simulation of thermal processes due to their many advantages. In the last years, the trend towards integration intensified. It was argued that co-simulation of physics-based models is the most promising approach for the study of powertrains. In regard to this matter, the proposal of the institute in which this $\mathrm{PhD}$ research was conducted, CMT-Motores Térmicos, is called Virtual Engine Model (VEMOD). The main asset of this tool is the scientific soundness of the constituent models. The Virtual Engine Model combines the experience accumulated by years of research by integrating 0D and 1D models developed for different physical processes that take place in internal combustion engines. This tool aspires to be a research platform that generates knowledge to advance the state-of-the-art.

Next, the most relevant contributions and findings of this $\mathrm{PhD}$ investigation are highlighted. They are organized according to the mentioned particular objectives, following the same structure as the dissertation document. After those conclusions, some recommendations for future work are given, with the aim of continuing the development and improvement of the Virtual Engine Model, specially of thermal submodels. By means of those accounts, the present chapter summarizes the knowledge and experience obtained from the doctoral research.

\subsection{Main contributions}

\section{Heat transfer model of the engine block}

At the beginning of this doctoral research, a quasi-dimensional model of heat transfer in the engine block already existed. The initial task was to thoroughly examine this model to assess its suitability for integration into the Virtual Engine Model and for research studies of current interest. First, a qualitative evaluation was done, allowing to identify existing capabilities and aspects to improve. Second, basing on the qualitative evaluation, a method to quantitatively evaluate the model based on experimental tests was proposed and executed. The quantitative evaluation revealed that, in general, the model presented good prediction capacity of mean wall temperatures in steady state. That means that area-averaged temperatures of cylinder walls were correctly predicted under diverse conditions of engine speed, load and swirl motion. However, at high engine speed, the model overpredicted engine temperatures. This was one of the 
problems to be solved. Also important, prediction of local temperature at locations where sensors were installed was poor. Therefore, when wall area-averaged temperatures were compared, predictions matched experimental measurements, but this did not happen when particular locations were examined. The quantitative study also provided insights into the complex heat transfer distribution on the cylinder walls as well as into the influence of swirl on heat transfer, which were discussed. Subsequent activities to improve the model were based on the outcomes of this evaluation.

The issue of improving local modeling of heat transfer was dealt with next. According to literature, multizone approaches to model in-cylinder heat convection are better than single-zone approaches at predicting local temperatures. A detailed study assessed the potential of a multizone approach. First, the implementation of a multizone model was thoroughly described. Thereupon, a calibration and evaluation procedure based on experimental data and 3D-CFD simulation was proposed to analyze its performance. The investigation covered various aspects of heat transfer prediction. The conclusion of the study was that the multizone approach failed to improve local temperature predictions with respect to the original single-zone model. Gains in prediction accuracy were small and occurred only at few locations. Consequently, the multizone approach was discarded and subsequent work was carried out using the original model. This meant that wall temperature prediction was reliable only on an area-averaged basis.

Further areas of improvement had been identified during the aforementioned qualitative and quantitative evaluation. Heat rejection to coolant and oil was critically improved. First, semi-empirical correlations for determining the heat transfer coefficient on the coolant side were implemented, which solved the problem of temperature overprediction at high engine speed. Second, the method to represent fluids as elements of the quasi-dimensional model was modified in order to better simulate transient processes. Finally, the correlations to calculate the physical properties of fluids were updated, with focus on real mixtures used as coolants.

A crucial improvement of the heat transfer model of the engine block was the proper modeling of thermal transients. Introducing capacitances in the thermal network, thermal inertia could be physically modeled. This included extending the model to take into account areas of the engine block that had been neglected in the past and adding new connections. More- 
over, new solving schemes were implemented. After an examination of different alternatives and a comparison test, an implicit scheme was selected for all following studies. Next, all previous improvements were validated. Two experimental studies allowed to confirm the correct performance of the model at predicting the transient evolution of temperatures in the engine block.

Lastly, a number of tasks aimed at making integration into the VEMOD global simulation tool possible were accounted. The heat transfer model underwent a standardization process. Data input and interaction with other submodels were adapted to the requirements of a multi-physics code. A new coupling with the gas dynamics model was established in order to improve heat transfer calculations in the intake and exhaust ports. Another new coupling connected the mechanical losses model with the heat transfer model to exchange friction data. In addition, the code structure was optimized for computational efficiency and also to manage a steady and a transient solver. Moreover, some methods for updating variables and calculating outputs were improved.

\section{Heat transfer models of ancillary systems}

Unlike heat transfer in the engine block, there was no preexisting model of heat transfer in the ancillary systems of an engine. Therefore, those models had to be developed from the ground up. To complete the Virtual Engine Model, transport of hydraulic fluids and heat transfer in engine subsystems had to be modeled. This was achieved by means of two models. The first one, a model of heat exchangers that considered all heat transfer processes among different submodels, including heat rejection to fluid circuits. The second, a thermo-hydraulic model that simulated energy and mass transport in closed hydraulic circuits.

The model of heat exchangers was developed from the perspective that a generalized model had to manage all heat transfer processes among engine subsystems, i.e. among VEMOD submodels. The models of some components, like engine cylinder or turbocharger, already included heat transfer submodels that calculated heat fluxes inside the system and heat rejection to fluid circuits. However, in order to couple such components to the corresponding fluid circuits, a common framework needed to be used. This framework is the heat exchangers model, which stores and transfers data of thermal processes among submodels. Under this approach, the engine cylinder model or the turbocharger model are also members 
of the heat exchangers model. For heat exchangers like the radiator or the oil cooler, new generic methods were implemented, since they do not have dedicated models. Methods were based on the effectiveness-NTU approach. Effectiveness-NTU is a simple, fast and flexible method, and it is valid for a very large variety of heat exchangers. In this dissertation, the characteristics of the heat exchangers model and the calculation methods were thoroughly described.

The model of thermo-hydraulic circuits is formed by two main parts. The first one solves the hydraulic network to determine flow rates and pressure drops in the circuit. According to the literature, quasi-steady approaches are commonplace when modeling hydraulic flows in automotive applications. An efficient quasi-steady model was implemented. The model optimizes the network analysis process by applying circuit rules to reduce complexity, by using a preliminary algebraic solver and by preventing redundant calculations when hydraulic conditions do not change. This model was completed with a non-linear solver that uses a modified version of Powell's conjugate direction algorithm. Circuit definition is fully flexible and includes typical circuit components: pumps, pipes, valves (including thermostats) and heat exchangers. The procedure used by the code to interpret the circuit layout is an original contribution of this thesis based on graph analysis. A remarkable accomplishment is a depth-first search algorithm used to find all possible paths in the network. The second part of the thermo-hydraulic model comprises the thermal calculations. A quasi-dimensional approach based on isothermal parcels of fluid was implemented. In this model, parcels move around the circuit, exchange heat with heat exchangers and pumps and update their size and temperature to reflect the thermal state of the circuit. All those processes are modeled by original algorithms that are contributions of this thesis. Special attention was put on energy distribution in joints and also on determining the inlet temperature used by subsystems. The model was thoroughly described in the corresponding section of this document.

\section{Validation of the global thermal response}

The final validation was undertaken with the aforementioned models integrated into the Virtual Engine Model. The validation procedure was designed to incorporate dynamic driving conditions and cold conditions $\left(-7^{\circ} \mathrm{C}\right)$. Additionally, typical validation tests in steady state, normal room temperature $\left(20^{\circ} \mathrm{C}\right)$ and warmed-up conditions were also included. Ex- 
perimental tests over a wide range of operating conditions were used for validation. Regarding simulations, all procedural aspects were discussed in detail, from setting up the model to calibrating it and launching the computational studies. In steady state tests, simulation results presented good agreement with experimental measurements, even under cold ambient conditions. Transient tests were conducted following the Worldwide Harmonized Light-Duty Vehicles Test Cycle (WLTC), the reference driving profile of current homologation standards. In the first transient test, the engine was warmed up before start. Overall, the simulation tool was able to properly capture the temperature evolution. The second test was conducted at a room temperature of $20^{\circ} \mathrm{C}$, which was also the initial engine temperature. Again, good agreement between experiment and simulation was found. The model reproduced the trends observed during engine warm-up. The last test was undertaken at $-7^{\circ} \mathrm{C}$. Some discrepancies were observed in oil temperature during the early stages of warm-up but, in general, prediction of temperature evolution was satisfactory. What is more, measures to improve the thermal inertia of the lower parts of the cylinder block were proposed to fix the mentioned discrepancies. In conclusion, the capability to perform reliable thermal simulations with the Virtual Engine Model was therefore demonstrated.

\subsection{Suggestions for future work}

After accomplishing the goal of this doctoral investigation, a tool for simulating the complete engine operation is capable of taking into account thermal processes. Relevant research studies with this simulation tool can be proposed as future activities. Moreover, the development of the three thermal models covered in this dissertation can be continued. During research, ideas for improvement have arisen. Some improvements would allow to overcome current limitations of the models. Also, the models can be further expanded with new features. In this section, proposals for future work are summarized, organized by topic.

\section{In-cylinder heat rejection}

Although it was found that the multizone heat transfer model could not improve temperature prediction, some ideas of the underlying gas motion submodel can be adopted. Currently, the convective heat transfer correlation uses mean piston speed, a constant value, to take into account 
in-cylinder gas motion, as proposed by Woschni. For in-cycle applications like VEMOD, considering a curve of instantaneous speed like those calculated with the gas motion submodel would better reflect reality. Though swirl motion is already being considered by means of a curve, currently this curve has no physical foundation. The gas motion submodel, on the contrary, uses a physics-based approach to determine swirl motion.

In addition to the in-cylinder convection correlation, the modeling approach within the thermal network can be improved. Convective conductances between gas and wall nodes could be replaced by heat flux sources in each surface node of the wall, like in the intake and exhaust ports. That could provide important advantages. No gas nodes would be necessary, which would reduce the number and complexity of equations and prevent redundant gas temperature calculations. Consequently, system solving would be faster. From a scientific point of view, the main advantage would be the possibility to implement heat transfer correlations that are not based on Newton's law of cooling, Eq. 2.4, i.e. that do not assume that heat flux is directly proportional to the difference of temperature of the fluid and the wall. This would allow to consider non-linear and unsteady mechanisms, such as heat radiation or the thermal phase shift commented in Section 2.2.1. An example of heat flux equation that could be implemented is the widely known correlation due to Annand and Ma, Eq. 2.23.

\section{Heat transfer in the engine block}

The lower part of the engine can be better modeled. Nodes representing components with relevant thermal influence, such as the connecting rod, the crankshaft or the lower areas of the cylinder block, can be added to the thermal network. An improvement of thermal inertia account would follow. Another expected benefit is the availability of temperature data of the mentioned components. Such temperatures are important for calculations of heat rejection to oil, friction and thermal expansion. Moreover, no heat rejection from the block structure to ambient is currently considered. Including this mechanism could be specially beneficial in low ambient temperature situations.

\section{Model of heat exchangers}

The heat exchangers model implemented in this thesis successfully provides a comprehensive structure to all heat transfer processes among sub- 
systems. In that aspect, no further improvements are proposed. However, the effectiveness-NTU method used as generic way to calculate heat exchange between two flowing fluids is based on assumption that heat transfer is a steady process. Advanced approaches, capable of taking into account dynamic considerations and thermal inertia, are required.

\section{Model of thermo-hydraulic circuits}

The thermo-hydraulic model can be extended with additional features. The most relevant would be the following:

- Heat loss to ambient.

- Temperature-dependent friction coefficient in pipes.

- Tanks. Mixing processes should be modeled.

- Pressure boundary conditions to allow using absolute pressures and to enable open circuits.

- The thermostat submodel should be coupled to the control model and hysteresis should be considered.

In addition, a couple of areas have room for improvement:

- The non-linear solver and the solving procedure can be improved to increase stability and efficiency. For instance, the user should be allowed to enter initial values for the solver.

- Connection to the control model should be improved. Circuit objects must be more integrated with the system of sensors and actuators.

\section{Global engine model}

The Virtual Engine Model can be applied to many relevant studies. An immediate application is the investigation of engine performance and emissions in various scenarios. Simulation studies can be run under conditions of interest for homologation, specially under non-conventional test conditions (low temperature, low pressure, different driving profiles, etc.). The models developed in this thesis will simulate the thermal aspects of engine operation. As commented in the Introduction, thermal aspects are important for engine performance, including pollutant formation. Research studies assessing potential improvements in thermal management can be conducted using the Virtual Engine Model. Studies can be focused on hardware modifications, circuit layouts, operation strategies or innovative technologies. Nowadays, electrification is gaining momentum within the automotive industry. In hybrid or electric vehicles, thermal aspects also have an important influence on performance. Among the models devel- 
oped in this thesis, the thermo-hydraulic model and the heat exchangers model are not specific of conventional powertrains and can be used to simulate electrified vehicles. 



\section{Bibliography}

[1] J. R. Serrano, Imagining the Future of the ICE for Ground Transport in the Current Context, Applied Sciences 7 (10) (2017) 1001. doi: 10.3390/app7101001.

[2] Council Directive 70/220/EEC of 20 March 1970 the approximation of the laws of the Member States on measures to be taken against air pollution by emissions from motor vehicles.

[3] N. Hooftman, M. Messagie, J. Van Mierlo, T. Coosemans, A review of the European passenger car regulations - Real driving emissions vs local air quality, Renewable and Sustainable Energy Reviews 86 (2018) 1-21. doi:10.1016/j.rser.2018.01.012.

[4] G. Martini, Scientific evidence on vehicle's emissions, in: European Commission's stakeholder event 24 October 2018: Preparing for the future European Emission Standards for Light and Heavy-Duty Vehicles.

[5] G. Fontaras, V. Franco, P. Dilara, G. Martini, U. Manfredi, Development and review of Euro 5 passenger car emission factors based on experimental results over various driving cycles, Science of the Total Environment 468-460 (2014) 1034-1042. doi:10.1016/j. scitotenv.2013.09.043.

[6] G. Fontaras, N.-G. Zacharof, B. Ciuffo, Fuel consumption and CO2 emissions from passenger cars in Europe - Laboratory versus real- 
world emissions, Progress in Energy and Combustion Science 60 (2017) 97-131. doi:10.1016/j.pecs.2016.12.004.

[7] M. Weiss, P. Bonnel, R. Hummel, A. Provenza, U. Manfredi, OnRoad Emissions of Light-Duty Vehicles in Europe, Environmental Science \& Technology 45 (2011) 8575-8581. doi:10.1021/ es2008424.

[8] G. Kadijk, N. Ligterink, P. van Mensch, R. Smokers, NOx emissions of Euro 5 and Euro 6 diesel passenger cars - test results in the lab and on the road (2016).

[9] V. Heijne, G. Kadijk, N. Ligterink, P. van der Mark, J. Spreen, U. Stelwagen, NOx emissions of fifteen Euro 6 diesel cars: Results of the Dutch LD road vehicle emission testing programme 2016 (2016).

[10] R. Martinez-Botas, Vehicle emissions testing programme: data and conclusions, UK Department for Transport (2016).

[11] A. Moody, J. Tate, In Service CO2 and NOx Emissions of Euro 6/VI Cars, Light- and Heavy- dutygoods Vehicles in Real London driving: Taking the Road into the Laboratory, Journal of Earth Sciences and Geotechnical Engineering 7 (1) (2017) 51-62.

[12] Victor D. G., D. Zhou, E. H. M. Ahmed, P. K. Dadhich, J. G. J. Olivier, H-H. Rogner, K. Sheikho, and M. Yamaguchi, 2014: Introductory Chapter. In: Climate Change 2014: Mitigation of Climate Change. Contribution of Working Group III to the Fifth Assessment Report of the Intergovernmental Panel on Climate Change. Cambridge University Press.

[13] Sims R., R. Schaeffer, F. Creutzig, X. Cruz-Núñez, M. D’Agosto, D. Dimitriu, M. J. Figueroa Meza, L. Fulton, S. Kobayashi, O. Lah, A. McKinnon, P. Newman, M. Ouyang, J. J. Schauer, D. Sperling, and G. Tiwari, 2014: Transport. In: Climate Change 2014: Mitigation of Climate Change. Contribution of Working Group III to the Fifth Assessment Report of the Intergovernmental Panel on Climate Change. Cambridge University Press.

[14] H. He, L. Yang, China's Stage 6 Emission Standard for new LightDuty Vehicles (Final Rule) (2017). 
[15] R. Muncrief, NOx emissions from heavy-duty and light-duty diesel vehicles in the EU: Comparison of real-world performance and current type-approval requirements (2016).

[16] B. Giechaskiel, F. Riccobono, T. Vlachos, P. Mendoza-Villafuerte, R. Suarez-Bertoa, G. Fontaras, P. Bonnel, M. Weiss, Vehicle Emission Factors of Solid Nanoparticles in the Laboratory and on the Road Using Portable Emission Measurement Systems (PEMS), Frontiers in Environmental Science 3 (82) (2015). doi:10.3389/ fenvs.2015.00082.

[17] C. Brand, Beyond 'Dieselgate': Implications of unaccounted and future air pollutant emissions and energy use for cars in the United Kingdom, Energy Policy 97 (2016) 1-12. doi:10.1016/j.enpol. 2016.06 .036$.

[18] L. Bovens, The Ethics of Dieselgate, Midwest Studies in Philosophy XL (2016) 262-283.

[19] S. R. Barrett, R. L. Speth, S. D. Eastham, I. C. Dedoussi, A. Ashok, R. Malina, D. W. Keith, Beyond 'Dieselgate': Implications of unaccounted and future air pollutant emissions and energy use for cars in the United Kingdom, Environmental Research Letters 10 (2015) 114005. doi : 10.1088/1748-9326/10/11/114005.

[20] B. Degraeuwe, M. Weiss, Does the New European Driving Cycle (NEDC) really fail to capture the NOx emissions of diesel cars in Europe?, Environmental Pollution 222 (2017) 234-241. doi:10. 1016/j.envpol.2016.12.050.

[21] Regulation (EC) No 715/2007 of the European Parliament and of the Council of 20 June 2007 on type approval of motor vehicles with respect to emissions from light passenger and commercial vehicles (Euro 5 and Euro 6) and on access to vehicle repair and maintenance information, Official Journal of the European Union, June 2007.

[22] M. Williams, R. Minjares, A technical summary of Euro 6-VI vehicle emission standards (2016).

[23] J. Demuynck, C. Favre, D. Bosteels, H. Hamje, J. Andersson, RealWorld Emissions Measurements of a Gasoline Direct Injection Vehicle without and with a Gasoline Particulate Filter, SAE Technical Paper 2017-01-0985 (2017). doi : 10.4271/2017-01-0985. 
[24] ECE/TRANS/180/Add.15: Global technical regulation on Worldwide Harmonized Light Vehicles Test Pocedure, United Nations, 2014.

[25] J. Pavlovic, B. Ciuffo, G. Fontaras, V. Valverde, A. Marotta, How much difference in type-approval $\mathrm{CO} 2$ emissions from passenger cars in Europe can be expected from changing to the new test procedure (NEDC vs. WLTP)?, Transportation Research Part A 111 (2018) 136-147. doi:10.1016/j.tra.2018.02.002.

[26] J. Pavlovic, A. Marotta, B. Ciuffo, CO2 emissions and energy demands of vehicles tested under the NEDC and the new WLTP type approval test procedures, Applied Energy 177 (2016) 661-670. doi : 10.1016/j.apenergy. 2016.05.110.

[27] D. Tsokolis, S. Tsiakmakis, A. Dimaratos, G. Fontaras, P. Pistikopoulos, B. Ciuffo, Z. Samaras, Fuel consumption and CO2 emissions of passenger cars over the New Worldwide Harmonized Test Protocol, Applied Energy 179 (2016) 1152-1165. doi:10.1016/j. apenergy.2016.07.091.

[28] P. Dilara, Lessons learned from developing the latest emission standards, in: European Commission's stakeholder event 24 October 2018: Preparing for the future European Emission Standards for Light and Heavy-Duty Vehicles.

[29] M. Reyes, Contribution to the Experimental Characterization and 1-D Modelling of Turbochargers for IC Engines, Ph.D. thesis, Universitat Politècnica de València (2013).

[30] V. Franco, F. Posada Sánchez, J. German, M. Peter, Real-world exhaust emissions from modern diesel cars (2014).

[31] C. Dardiotis, G. Martini, A. Marotta, U. Manfredi, Extension of low temperature emission test to Euro 6 diesel vehicles (2012).

[32] G. Kadijk, N. Ligterink, R. Smokers, Review into the relation between ambient temperature and NOx emissions of a Euro 6 Mercedes C220 Bluetec with a diesel engine (2016).

[33] R. Suarez-Bertoa, C. Astorga, Impact of cold temperature on Euro 6 passenger car emissions, Environmental Pollution 234 (2018) 318329. doi:10.1016/j.envpol.2017.10.096. 
[34] AVL, Technical Committee of Motor Vehicles Meeting 2015.

[35] C. Daw, M. Kennel, C. Finney, F. Connolly, Observing and modeling nonlinear dynamics in an internal combustion engine, Physical Review E 57 (1998) 2811-2819. doi:10.1103/PhysRevE.57.2811.

[36] S.-L. Ding, E.-Z. Song, L.-P. Yang, G. Litak, Y.-Y. Wang, C. Yao, X.-Z. Ma, Analysis of chaos in the combustion process of premixed natural gas engine, Applied Thermal Engineering 121 (2017) 768778. doi:10.1016/j.applthermaleng. 2017.04.109.

[37] S. Tanov, Z. Wang, H. Wang, M. Richter, B. Johansson, Effects of Injection Strategies on Fluid Flow and Turbulence in Partially Premixed Combustion (PPC) in a Light Duty Engine, SAE Technical Paper 2015-24-2455 (2015). doi:10.4271/2015-24-2455.

[38] A. Torregrosa, P. Olmeda, A. García-Ricos, J. Natividad, C. Romero, A Methodology for the Design of Engine Cooling Systems in Standalone Applications, SAE Technical Paper 2010-01-0325 (2010). doi:10.4271/2010-01-0325.

[39] Z. Gao, J. Conklin, C. Daw, V. Chakravarthy, A proposed methodology for estimating transient engine-out temperature and emissions from steady-state maps, International Journal of Engine Research 11, 2 (2010) 137-151. doi:10.1243/14680874JER05609.

[40] S. Bohac, D. Baker, D. Assanis, A Global Model for Steady State and Transient S.I. Engine Heat Transfer Studies, SAE Technical Paper 960073 (1996). doi:10.4271/960073.

[41] T. Morel, R. Keribar, P. N. Blumberg, Cyclical Thermal Phenomena in Engine Combustion Chamber Surfaces, SAE Technical Paper 850360 (1985). doi:10.4271/850360.

[42] F. Millo, S. Caputo, C. Cubito, A. Calamiello, D. Mercuri, M. Rimondi, Numerical Simulation of the Warm-Up of a Passenger Car Diesel Engine Equipped with an Advanced Cooling System, SAE Technical Paper 2016-01-0555 (2016). doi:10.4271/2016-01-0555.

[43] T. Banjac, J. Wurzenberger, T. Katrašnik, Assessment of engine thermal management through advanced system engineering modeling, Advances in Engineering Software 71 (2014) 19-33. doi: 10.1016/j. advengsoft. 2014.01.016. 
[44] D. Chalet, M. Lesage, M. Cormerais, T. Marimbordes, Nodal modelling for advanced thermal-management of internal combustion engine, Applied Energy 190 (2017) 99-113. doi:10.1016/j. apenergy.2016.12.104.

[45] J. Martín, F. Arnau, P. Piqueras, A. Auñón, Development of an integrated Virtual Engine Model to simulate new standard testing cycles, SAE Technical Paper 2018-01-1413 (2018). doi:10.4271/ 2018-01-1413.

[46] A. Lowe, T. Morel, A New Generation of Tools for Accurate ThermoMechanical Finite Element Analyses of Engine Components, SAE Technical Paper 920681 (1992). doi: 10.4271/920681.

[47] J. Xin, S. Shih, E. Itano, Y. Maeda, et al., Theoretical Consideration to Improve Engine Cooling and Application of Coupling 3D Combustion Simulations with Heat Transfer in Water Jacket and Components, Honda R\&D technical review 15, 2 (2003) 117-125, nII:40005956609.

[48] Z. Memon, T. Sundararajan, V. Lakshminarasimhan, Y. Babu, V. Harne, Simulation and Experimental Evaluation of Air Cooling for Motorcycle Engine, SAE Technical Paper 2006-32-0099 (2006). doi:10.4271/2006-32-0099.

[49] E. Conte, K. Boulouchos, Unsteady wall heat flux in spark-ignited, homogeneous charge engines fuelled with reformer gas/gasoline blends, International Journal of Engine Research 9 (1) (2008) 29-44. doi:10.1243/14680874JER01907.

[50] S. Uppuluri, H. R. Khalane, Y. Umbarkar, A. Naiknaware, ColdAmbient Warm-Up Predictions: A Novel Approach Using 1D Computational Models, SAE Technical Paper 2016-01-0198 (2016). doi: 10.4271/2016-01-0198.

[51] B. Ciuffo, G. Fontaras, Models and scientific tools for regulatory purposes: The case of $\mathrm{CO} 2$ emissions from light duty vehicles in Europe, Energy Policy 109 (2017) 76-81. doi:10.1016/j.enpol. 2017.06 .057$.

[52] J. B. Heywood, Internal Combustion Engine Fundamentals, McGraw-Hill Education, 1988. 
[53] G. Borman, K. Nishiwaki, Internal-combustion engine heat transfer, Progress in Energy and Combustion Science 13 (1) (1987) 1-46. doi : 10.1016/0360-1285(87)90005-0.

[54] A. Torregrosa, P. Olmeda, J. Martín, B. Degraeuwe, Experiments on the influence of inlet charge and coolant temperature on performance and emissions of a DI Diesel engine, Experimental Thermal and Fluid Science 30 (7) (2006) 633-641. doi:10.1016/j. expthermflusci.2006.01.002.

[55] A. Broatch, J. M. Luján, S. Ruiz, P. Olmeda, Measurement of hydrocarbon and carbon monoxide emissions during the starting of automotive DI diesel engines, International Journal of Automotive Technology 9, 2 (2008) 129-140. doi:10.1007/s12239-008-0017-6.

[56] S. Soid, Z. Zainal, Spray and combustion characterization for internal combustion engines using optical measuring techniques. A review, Energy 36 (2) (2011) 724-741. doi:10.1016/j . energy . 2010. 11.022 .

[57] A. Roberts, R. Brooks, P. Shipway, Internal combustion engine coldstart efficiency: A review of the problem, causes and potential solutions, Energy Conversion and Management 82 (2014) 327-350. doi:10.1016/j.enconman.2014.03.002.

[58] J. Trapy, P. Damiral, An Investigation of Lubricating System Warmup for the Improvement of Cold Start Efficiency and Emissions of S.I. Automotive Engines, SAE Technical Paper 902089 (1990). doi : 10.4271/902089.

[59] C. Romero, A. Torregrosa, P. Olmeda, J. Martín, Energy Balance During the Warm-Up of a Diesel Engine, SAE Technical Paper 201401-0676 (2014). doi:10.4271/2014-01-0676.

[60] K. Choi, K. Kim, K. Lee, Investigation of emission characteristics affected by new cooling system in a diesel engine, J Mech Sci Technol 23, 7 (2009) 1866-1870. doi:10.1007/s12206-009-0616-9.

[61] H. Pang, C. Brace, S. Akehurst, Potential of a Controllable Engine Cooling System to Reduce NOx Emissions in Diesel Engines, SAE Technical Paper 2004-01-0054 (2004). doi: 10.4271/2004-01-0054. 
[62] M. Gumus, Reducing cold-start emission from internal combustion engines by means of thermal energy storage system, Applied Thermal Engineering 29, 4 (2009) 652-660. doi:10.1016/ j.applthermaleng. 2008.03.044.

[63] A. Osman, M. Muhammad Yusof, M. Rafi, Vehicle Testing and Development Involving a Simplified Split Cooling with Integrated Exhaust Heat Recovery and Reuse, SAE Technical Paper 2016-01-0647 (2016). doi:10.4271/2016-01-0647.

[64] H. Goettler, L. Vidger, D. Majkrzak, The effect of exhaust-tocoolant heat transfer on warm-up time and fuel consumption of two automobile engines, SAE Technical Paper 860363 (1986). doi: $10.4271 / 860363$.

[65] E. Cortona, C. Onder, Engine Thermal Management with Electric Cooling Pump, SAE Technical Paper 2000-01-0965 (2000). doi: 10.4271/2000-01-0965.

[66] D. Allen, M. Lasecki, Thermal Management Evolution and Controlled Coolant Flow, SAE Technical Paper 2001-01-1732 (2001). doi:10.4271/2001-01-1732.

[67] B. Luptowski, O. Arici, J. Johnson, G. Parker, Development of the Enhanced Vehicle and Engine Cooling System Simulation and Application to Active Cooling Control, SAE Technical Paper 2005-010697 (2005). doi: 10.4271/2005-01-0697.

[68] O. Taylor, R. Pearson, R. Stone, Reduction of CO2 Emissions through Lubricant Thermal Management During the Warm Up of Passenger Car Engines, SAE Technical Paper 2016-01-0892 (2016). doi:10.4271/2016-01-0892.

[69] F. Payri, P. Olmeda, J. Martín, A. García, A complete 0D thermodynamic predictive model for direct injection diesel engines, Applied Energy 88 (12) (2011) 4632-4641. doi : 10.1016/j . apenergy . 2011. 06.005 .

[70] A. Broatch, J. Martín, A. García, D. Blanco-Cavero, A. Warey, V. Domenech, Application of a zero-dimensional model to assess the effect of swirl on indicated efficiency, International Journal of Engine Research 1-12 (2018). doi:10.1177/1468087418779726. 
[71] J. Arrègle, J. López, J. Martín, E. Mocholí, Development of a Mixing and Combustion Zero-Dimensional Model for Diesel Engines, SAE Technical Paper 2006-01-1382 (2006). doi:10.4271/2006-01-1382.

[72] R. S. Subramanian, Heat transfer in Flow Through Conduits (2014).

[73] R. Brodkey, H. Hershey, Transport Phenomena: A Unified Approach, Brodkey Publishing, 2003.

[74] A. P. Colburn, A method of correlating forced convection heattransfer data and a comparison with fluid friction, International Journal of Heat and Mass Transfer 7 (12) (1964) 1359-1384, (reprinted). doi : 10.1016/0017-9310(64)90125-5.

[75] F. Dittus, L. Boelter, Heat transfer in automobile radiators of the tubular type, International Communications in Heat and Mass Transfer 12, 1 (1985) 3-22, (reprinted). doi:10.1016/ 0735-1933(85) 90003-X.

[76] R. Winterton, Where did the Dittus and Boelter equation come from?, Int. J. Heat Mass Transfer 41 (4-5) (1998) 809-810, pII:S00179310(97)00177-4.

[77] E. Sieder, G. Tate, Heat Transfer and Pressure Drop of Liquids in Tubes, Industrial and Engineering Chemistry 28 (12) (1936) 1429 1435 .

[78] V. Gnielinski, Neue Gleichungen für den Wärme- und den Stoffübergang in turbulent durchströmten Rohren und Kanälen, Forschung im Ingenieurwesen 41 (1) (1975) 8-16. doi:10.1007/ BF02559682.

[79] C. Taylor, T. Toong, Heat transfer in internal-combustion engines, ASME paper 57-HT-17 (1957).

[80] W. Annand, T. Ma, Instantaneous Heat Transfer Rates to the Cylinder Head Surface of a Small Compression-Ignition Engine, Proceedings of the Institution of Mechanical Engineers 185 (1970) 976-987. doi:10.1243/PIME_PROC_1970_185_110_02.

[81] B. Knight, The problem of predicting heat transfer in diesel engines, Proceedings of the Institution of Mechanical Engineers, Part C: Mechanical Engineering Science 179 (3C) (1964) 93-106. doi: 10.1243/PIME_CONF_1964_179_048_02. 
[82] W. Annand, Heat Transfer in the Cylinders of Reciprocating Internal Combustion Engines, Proceedings of the Institution of Mechanical Engineers 177 (36) (1963) 973-990. doi : 10.1243/PIME_PROC_1963_ 177_069_02.

[83] G. Woschni, A Universally Applicable Equation for the Intantaneous Heat Transfer Coefficient in the Internal Combustion Engine, SAE Technical Paper 670931 (1967). doi:10.4271/670931.

[84] G. Woschni, Die Berechnung der Wandverluste und der thermischen Belastung der Bauteile von Dieselmotoren, MTZ Motortechnische Zeitschrift 31 (12) (1970) 491-499.

[85] K. Sihling, G. Woschni, Experimental investigation of the instantaneous heat transfer in the cylinder of a high speed diesel engine, SAE Technical Paper 790833 (1979). doi:10.4271/790833.

[86] G. F. Hohenberg, Advanced Approaches for Heat Transfer Calculations, SAE Technical Paper 790825 (1979). doi:10.4271/790825.

[87] T. LeFeuvre, P. Myers, O. Uyehara, Experimental Instantaneous Heat Fluxes in a Diesel Engine and Their Correlation, SAE Technical Paper 690464 (1969). doi:10.4271/690464.

[88] J. Dent, S. Suliaman, Convective and Radiative Heat Transfer in a High Swirl Direct Injection Diesel Engine, SAE Technical Paper 770407 (1977). doi:10.4271/770407.

[89] W. Nusselt, Der Wärmeübergang in der Verbrennungskraftmachine, V.D.I.-Forschungsheft 264 (1923).

[90] B. Lawton, Effect of Compression and Expansion on Instantaneous Heat Transfer in Reciprocating Internal Combustion Engines, Proceedings of the Institution of Mechanical Engineers, Part A: Journal of Power and Energy 201 (3) (1987) 175-186. doi:10.1243/PIME_ PROC_1987_201_022_02.

[91] J. Chang, O. Güralp, Z. Filipi, D. N. Assanis, T.-W. Kuo, P. Najt, R. Rask, New Heat Transfer Correlation for an HCCI Engine Derived from Measurements of Instantaneous Surface Heat Flux, SAE Technical Paper 2004-01-2996 (2004). doi : 10.4271/2004-01-2996. 
[92] A. Kornhauser, J. Smith, Application of a Complex Nusselt Number to Heat Transfer During Compression and Expansion, Journal of Heat Transfer 116 (3) (1994) 536-542. doi:10.1115/1.2910904.

[93] R. Mathie, C. Markides, A. White, A framework for the analysis of thermal losses in reciprocating compressors and expanders, Heat Transfer Engineering 35 (16-17) (2014) 1435-1449. doi:10.1080/ 01457632.2014 .889460 .

[94] A. Catto, A. Prata, A numerical study of instantaneous heat transfer during compression and expansion in piston-cylinder geometry, $\mathrm{Nu}-$ merical Heat Transfer; Part A: Applications 38 (3) (2000) 281-303. doi:10.1080/10407780050136530.

[95] H. Park, D. Assanis, D. Jung, Development of an In-Cylinder Heat Transfer Model with Compressibility Effects on Turbulent Prandtl Number, Eddy Viscosity Ratio and Kinematic Viscosity Variation, SAE Technical Paper 2009-01-0702 (2009). doi:10.4271/ 2009-01-0702.

[96] D. R. Buttsworth, A. Agrira, R. Malpress, T. Yusaf, Simulation of Instantaneous Heat Transfer in Spark Ignition Internal Combustion Engines: Unsteady Thermal Boundary Layer Modeling, Journal of Engineering for Gas Turbines and Power 133 (2) (2010) 022802022802-5. doi:10.1115/1.4001080.

[97] A. Agrira, D. R. Buttsworth, M. A. Said, Instantaneous Heat Flux Simulation of a Motored Reciprocating Engine: Unsteady Thermal Boundary Layer With Variable Turbulent Thermal Conductivity, Journal of Heat Transfer 136 (3) (2013) 031703-031703-9. doi: 10.1115/1.4025639.

[98] D. Oude Nijeweme, J. Kok, C. R. Stone, L. Wyszynski, Unsteady in-cylinder heat transfer in a spark ignition engine: Experiments and modelling, Proceedings of the Institution of Mechanical Engineers, Part D: Journal of Automobile Engineering 215 (6) (2001) 747-760. doi:10.1243/0954407011528329.

[99] C. Rakopoulos, G. Mavropoulos, D. Hountalas, Measurements and analysis of load and speed effects on the instantaneous wall heat fluxes in a direct injection air-cooled diesel engine, International Journal of Energy Research 24 (2000) 587-604. doi:10.1002/ 1099-114X (20000610) $24: 7<587$ : :AID-ER604>3. 0. C0;2-F. 
[100] D. R. Buttsworth, R. Stevens, C. R. Stone, Eroding ribbon thermocouples: impulse response and transient heat flux analysis, Measurement Science and Technology 16 (2005) 1487-1494. doi:10.1088/ 0957-0233/16/7/011.

[101] R. Carreño, A comprehensive methodology to analyse the global energy balance in reciprocating internal combustion engines, Ph.D. thesis, Universitat Politècnica de València (2016).

[102] K.-W. Cho, D. Assanis, Z. Filipi, G. Szekely, P. Najt, R. Rask, Experimental investigation of combustion and heat transfer in a direct-injection spark ignition engine via instantaneous combustion chamber surface temperature measurements, Proceedings of the Institution of Mechanical Engineers, Part D: Journal of Automobile Engineering 222 (11) (2008) 2219-2233. doi:10.1243/ 09544070 JAUT0853.

[103] J. Desantes, A. Torregrosa, A. Broatch, P. Olmeda, Experiments on the influence of intake conditions on local instantaneous heat flux in reciprocating internal combustion engines, Energy 36 (1) (2011) 60-69. doi:10.1016/j. energy . 2010.11.011.

[104] I. Finlay, G. Gallacher, T. Biddulph, R. Marshall, The Application of Precision Cooling to the Cylinder-Head of a Small, Automotive, Petrol Engine, SAE Technical Paper 880263 (1988). doi:10.4271/ 880263.

[105] A. Alkidas, Effects of Operational Parameters on Structural Temperatures and Coolant Heat Rejection of a S.I. Engine, SAE Technical Paper 931124 (1993). doi:10.4271/931124.

[106] D. Bendersky, A special thermocouple for measuring transient temperatures, Mechanical Engineering 75 (1953) 117-121.

[107] A. Wimmer, R. Pivec, T. Sams, Heat Transfer to the Combustion Chamber and Port Walls of IC Engines - Measurement and Prediction, SAE Technical Paper 2000-01-0568 (2000). doi:10.4271/ 2000-01-0568.

[108] J. Demuynck, A Fuel Independent Heat Transfer Correlation for Premixed Spark Ignition Engines, Ph.D. thesis, Universiteit Gent (2012). 
[109] C. Arrighetti, M. Ballatari, E. Carnevale, Misure di temperature negli stantuffi di motore a combustione interna, La Termotecnica 28 (6) (1974) 299-308.

[110] T. Hendricks, D. Splitter, J. Ghandhi, Experimental investigation of piston heat transfer under conventional diesel and reactivity controlled compression ignition combustion regimes, International Journal of Engine Research 15 (6) (2014) 684-705. doi: 10.1177/1468087413512310.

[111] E. Mancaruso, L. Sequino, B. Vaglieco, F. Maroteaux, Experimental measurements of piston temperature and evaluation of heat flux in engine at transient conditions, in: THIESEL Conference on Thermoand Fluid Dynamic Processes in Direct Injection Engines, 2018.

[112] S. Kearney, R. Lucht, A. Jacobi, Temperature measurements in convective heat transfer flows using dual-broadband, pure-rotational coherent anti-Stokes Raman spectroscopy (CARS), Experimental Thermal and Fluid Science 19 (1999) 13-26. doi:10.1016/ S0894-1777 (99) 00004-7.

[113] P. Andresen, G. Meijer, H. Schlüter, H. Voges, A. Koch, W. Hentschel, W. Oppermann, E. Rothe, Fluorescence imaging inside an internal combustion engine using tunable excimer lasers, Applied Optics 29 (16) (1990) 2392-2404. doi:10.1364/AO.29. 002392.

[114] N. Fuhrmann, E. Baum, J. Brübach, A. Dreizler, High-speed phosphor thermometry, The Review of scientific instruments 82 (10) (2011) 104903. doi:10.1063/1.3653392.

[115] W. Bauer, C. Tam, J. Heywood, C. Ziegler, Fast Gas Temperature Measurement by Velocity of Sound for IC Engine Applications, SAE Technical Paper 972826 (1997). doi:10.4271/972826.

[116] J. Holman, Heat Transfer, 7th Edition, John Wiley \& Sons, 1990.

[117] L. Jarrier, J. Champoussin, R. Yu, D. Gentile, Warm-Up of a D.I. Diesel Engine: Experiment and Modeling, SAE Technical Paper 2000-01-0299 (2000). doi:10.4271/2000-01-0299.

[118] T. Bergman, A. Lavine, F. Incropera, D. DeWitt, Fundamentals of Heat and Mass Transfer, 7th Edition, John Wiley \& Sons, 2011. 
[119] F. Struwe, D. Foster, In-Cylinder Measurement of Particulate Radiant Heat Transfer in a Direct Injection Diesel Engine, SAE Technical Paper 2003-01-0072 (2003). doi:10.4271/2003-01-0072.

[120] P. Flynn, M. Mizusawa, O. Uyehara, P. Myers, An Experimental Determination of the Instantaneous Potential Radiant Heat Transfer Within an Operating Diesel Engine, SAE Technical Paper 720022 (1972). doi:10.4271/720022.

[121] S. Skeen, J. Manin, L. Pickett, Quantitative spatially resolved measurements of total radiation in high-pressure spray flames, SAE Technical Paper 2014-01-1252 (2014). doi : 10.4271/2014-01-1252.

[122] X. Li, J. Wallace, In-Cylinder Measurement of Temperature and Soot Concentration Using the Two-Color Method, SAE Technical Paper 950848 (1995). doi:10.4271/950848.

[123] P. Furmanski, J. Banaszek, T. Wisniewski, Radiative Heat Transfer in the Combustion Chamber of a Diesel Engine, International Journal of Computational Fluid Dynamics 11, 3-4 (1999) 325-339. doi:10.1080/10618569908940884.

[124] J. F. Wiedenhoefer, R. D. Reitz, Multidimensional Modeling of the Effects of Radiation and Soot Deposition in Heavy-duty Diesel Engines, SAE Technical Paper 2003-01-0560 (2003). doi:10.4271/ 2003-01-0560.

[125] J. Benajes, J. Martín, A. García, D. Villalta, A. Warey, In-cylinder soot radiation heat transfer in direct-injection diesel engines, Energy Conversion and Management 106 (2015) 414-427. doi:10.1016/j. enconman.2015.09.059.

[126] J. Kaplan, J. Heywood, Modeling the Spark Ignition Engine WarmUp Process to Predict Component Temperatures and Hydrocarbon Emissions, SAE Technical Paper 910302 (1991). doi:10.4271/ 910302.

[127] P. Shayler, S. Christian, T. Ma, A Model for the Investigation of Temperature, Heat Flow and Friction Characteristics During Engine Warm-Up, SAE Technical Paper 931153 (1993). doi:10.4271/ 931153. 
[128] A. Veshagh, C. Chen, A Computer Model for Thermofluid Analysis of Engine Warm-Up Process, SAE Technical Paper 931157 (1993). doi:10.4271/931157.

[129] D. Murrell, G. M. Lewis, D. M. Baker, D. N. Assanis, An EarlyDesign Methodology for Predicting Transient Fuel Economy and Catalyst-Out Exhaust Emissions, SAE Technical Paper 971838 (1997). doi: 10.4271/971838.

[130] J. Wagner, E. Marotta, I. Paradis, Thermal Modeling of Engine Components for Temperature Prediction and Fluid Flow Regulation, SAE Technical Paper 2001-01-1014 (2001). doi:10.4271/ 2001-01-1014.

[131] A. Noori, M. Rashidi, Computational Fluid Dynamics Study of Heat Transfer in a Spark-Ignition Engine Combustion Chamber, in: ASME Summer Heat Transfer Conference, 2003.

[132] C. Eiglmeier, H. Lettmann, G. Stiesch, G. Merker, A Detailed Phenomenological Model for Wall Heat Transfer Prediction in Diesel Engines, SAE Technical Paper 2001-01-3265 (2001). doi:10.4271/ 2001-01-3265.

[133] T. Morel, P. N. Blumberg, E. F. Fort, R. Keribar, Methods for heat transfer and temperature field analysis of the insulated diesel, Phase I, DOE/NASA/0342-1 NASA CR-174783 (1984).

[134] T. Morel, R. Keribar, A Model for Predicting Spatially and Time Resolved Convective Heat Transfer in Bowl-in-Piston Combustion Chambers, SAE Technical Paper 850204 (1985). doi:10.4271/ 850204.

[135] T. Morel, S. Wahiduzzaman, D. R. Tree, D. P. DeWitt, Effect of Speed, Load, and Location on Heat Transfer in a Diesel Engine Measurements and Predictions, SAE Technical Paper 870154 (1987). doi: 10.4271/870154.

[136] C. Schubert, A. Wimmer, F. Chmela, Advanced Heat Transfer Model for CI Engines, SAE Technical Paper 2005-01-0695 (2005). doi: 10.4271/2005-01-0695.

[137] T. Morel, R. Keribar, Heat Radiation in D.I. Diesel Engines, SAE Technical Paper 860445 (1986). doi:10.4271/860445. 
[138] A. Murakami, M. Arai, H. Hiroyasu, Swirl Measurements and Modelling in Direct Injection Diesel Engines, SAE Technical Paper 880385 (1988). doi: 10.4271/880385.

[139] A. C. Hansen, Modelling gas flow in a direct injection diesel engine: I - Squish and swirl, N\&O Joernaal 7 (2) (1991) 16-24.

[140] A. C. Hansen, Modelling gas flow in a direct injection diesel engine: II - Turbulence, N\&O Joernaal 7 (2) (1991) 25-31.

[141] A. C. Hansen, A Diagnostic Quasi-Dimensional Model of Heat Transfer Applied to a Motored Compression-Ignition engine, SAE Technical Paper 920542 (1992). doi:10.4271/920542.

[142] A. C. Hansen, A diagnostic quasi-dimensional model of heat transfer and combustion in compression-ignition engines, Ph.D. thesis, University of Natal (1989).

[143] N. Komninos, G. Kosmadakis, Heat transfer in HCCI multi-zone modeling: Validation of a new wall heat flux correlation under motoring conditions, Applied Energy 88 (2011) 1635-1648. doi: $10.1016 / j$. apenergy . 2010.11.039.

[144] A. Broatch, P. Olmeda, A. García, J. Salvador-Iborra, A. Warey, Impact of swirl on in-cylinder heat transfer in a light-duty diesel engine, Energy 119 (2017) 1010-1023. doi:10.1016/j . energy. 2016. 11.040 .

[145] S. Tanov, J. Salvador-Iborra, O. Andersson, P. Olmeda, A. García, Influence of the number of injections on piston heat rejection under low temperature combustion conditions in an optical compressionignition engine, Energy Conversion and Management 153 (2017) 335-345. doi:10.1016/j.enconman.2017.10.015.

[146] M. J. Jennings, T. Morel, A Computational Study of Wall Temperature Effects on Engine Heat Transfer, SAE Technical Paper 910459 (1991). doi:10.4271/910459.

[147] Z. Han, R. D. Reitz, A temperature wall function formulation for variable-density turbulent flows with application to engine convective heat transfer modeling, International Journal of Heat and Mass Transfer 40 (3) (1997) 613-625. doi:10.1016/0017-9310 (96) 00117-2. 
[148] C. Angelberger, T. Poinsot, B. Delhay, Advanced near-wall modeling for engine heat transfer, SAE Technical Paper 972881 (1997). doi: $10.4271 / 972881$.

[149] S. Šarić, B. Basara, Z. Žunič, Improving Near-Wall Combustion and Wall Heat Transfer Modeling in SI Engine Computations, International Journal of Heat and Fluid Flow 63 (2017) 205-211. doi:10.1016/j.ijheatfluidflow.2016.06.019.

[150] C. Rakopoulos, G. Kosmadakis, E. Patriotis, Critical evaluation of current heat transfer models used in CFD in-cylinder engine simulations and establishment of a comprehensive wall-function formulation, Applied Energy 87 (2010) 1612-1630. doi:10.1016/j. apenergy.2009.09.029.

[151] N. Fukushima, A. Tsunemi, M. Shimura, Y. Shim, M. Tanahashi, T. Miyauchi, DNS of Turbulent Premixed Flames and Heat Transfer in a Constant Volume Vessel, in: ASME/JSME 8th Thermal Engineering Joint Conference, 2011, pp. T20113-T20113-8. doi:10.1115/AJTEC2011-44473.

[152] S. Zoz, S. Strepek, M. Wiseman, C. Qian, Engine Lubrication System Model for Sump Oil T Prediction, SAE Technical Paper 200101-1073 (2001). doi:10.4271/2001-01-1073.

[153] K. Park, J. Won, H. Heo, Thermal flow analysis of vehicle engine cooling system, KSME International Journal 16, 7 (2002) 975-985. doi: 10.1007/BF02949727.

[154] A. Torregrosa, A. Broatch, P. Olmeda, C. Romero, Assessment of the influence of different cooling system configurations on engine warm-up, emissions and fuel consumption, International Journal of Automotive Technology 9, 4 (2008) 447-458. doi:10.1007/ s12239-008-0054-1.

[155] F. Caresana, M. Bilancia, C. Bartolini, Numerical method for assessing the potential of smart engine thermal management: Application to a medium-upper segment passenger car, Applied Thermal Engineering 31, 16 (2011) 3559-3568. doi:10.1016/j . applthermaleng. 2011.07 .017$. 
[156] R. Cipollone, C. Villante, A Fully Transient Model For Advanced Engine Thermal Management, SAE Technical Paper 2005-01-2059 (2005). doi:10.4271/2005-01-2059.

[157] R. Cipollone, D. Di Battista, A. Gualtieri, A novel engine cooling system with two circuits operating at different temperatures, Energy Conversion and Management 75 (2013) 581-592. doi:10.1016/j. enconman.2013.07.010.

[158] D. M. Baker, D. N. Assanis, A methodology for coupled thermodynamic and heat transfer analysis of a diesel engine, Applied Mathematical Modelling 18 (1994) 590-601.

[159] D. N. Assanis, J. B. Heywood, Development and Use of a Computer Simulation of the Turbocompounded Diesel System for Engine Performance and Component Heat Transfer Studies, SAE Transactions 95 (2) (1986) 451-476. doi : 10.4271/860329.

[160] C. Samhaber, A. Wimmer, E. Loibner, Modeling of Engine WarmUp with Integration of Vehicle and Engine Cycle Simulation, SAE Technical Paper 2001-01-1697 (2001). doi : 10.4271/2001-01-1697.

[161] F. Kitanoski, W. Puntigam, M. Kozek, J. Hager, An Engine Heat Transfer Model for Comprehensive Thermal Simulations, SAE Technical Paper 2006-01-0882 (2006). doi:10.4271/2006-01-0882.

[162] K. Kunze, S. Wolff, I. Lade, J. Tonhauser, A Systematic Analysis of CO2-Reduction by an Optimized Heat Supply during Vehicle Warm-up, SAE Technical Paper 2006-01-1450 (2006). doi: 10.4271/2006-01-1450.

[163] J. Galindo, J. Serrano, F. Arnau, P. Piqueras, Description of a Semi-Independent Time Discretization Methodology for a OneDimensional Gas Dynamics Model, Journal of Engineering for Gas Turbines and Power 131, 034504-5 (2009). doi: 10.1115/1.2983015.

[164] J. Galindo, A. Tiseira, R. Navarro, D. Tarí, et al., Compressor efficiency extrapolation for 0D-1D engine simulations, SAE Technical Paper 2016-01-0554 (2016). doi:10.4271/2016-01-0554.

[165] J. Serrano, F. Arnau, L. García-Cuevas, A. Dombrovsy, H. Tartoussi, Development and validation of a radial turbine efficiency and mass flow model at design and off-design conditions, Energy 
Conversion and Management 125 (2016) 281-293. doi:10.1016/j . enconman.2016.09.032.

[166] A. Torregrosa, P. Olmeda, J. Martín, C. Romero, A tool for predicting the thermal performance of a Diesel engine, Heat transfer engineering 32 (10) (2011) 891-904. doi:10.1080/01457632.2011. 548639 .

[167] F. Payri, P. Olmeda, J. Martín, R. Carreño, A new tool to perform global energy balances in DI Diesel engines, SAE Int. J. Engines 7 (1) (2014) 43-59. doi:10.4271/2014-01-0665.

[168] F. Payri, F. Arnau, P. Piqueras, M. Ruiz, Lumped Flow-Through and Wall-Flow Monolithic Reactors Modelling for Real-Time Automotive Applications, SAE Technical Paper 2018-01-0954 (2018). doi:10.4271/2018-01-0954.

[169] J. Martín, P. Piqueras, L. García-Cuevas, E. Sanchis, Lumped DOC modelling approach for fluid-dynamic simulation under engine dynamic operation, in: 15th EAEC European Automotive Congress, 2017.

[170] F. Payri, J. Luján, J. Martín, A. Abbad, Digital signal processing of in-cylinder pressure for combustion diagnosis of internal combustion engines, Mechanical Systems and Signal Processing 24 (6) (2010) 1767-1784. doi:10.1016/j.ymssp.2009.12.011.

[171] A. V. Bueno, J. A. Velásquez, L. F. Milanez, Internal Combustion Engine Indicating Measurements, Applied Measurement Systems. (2012). doi:10.5772/37889.

[172] M. Lapuerta, R. Ballesteros, J. R. Agudelo, Effect of the gas state equation on the thermodynamic diagnostic of diesel combustion, Applied Thermal Engineering 26 (14-15) (2006) 1492-1499. doi:10.1016/j . applthermaleng. 2006.01.001.

[173] M. Lapuerta, O. Armas, J. Hernández, Diagnosis of DI Diesel combustion from in-cylinder pressure signal by estimation of mean thermodynamic properties of the gas, Applied Thermal Engineering 19 (5) (1999) 513-529. doi:10.1016/S1359-4311(98)00075-1.

[174] F. Payri, J. Galindo, J. Martín, F. Arnau, A Simple Model for Predicting the Trapped Mass in a DI Diesel Engine, SAE Technical Paper 2007-01-0494 (2007). doi:10.4271/2007-01-0494. 
[175] F. Payri, S. Molina, J. Martín, O. Armas, Influence of measurement errors and estimated parameters on combustion diagnosis, Applied Thermal Engineering 26 (2-3) (2006) 226-236. doi:10.1016/ j.applthermaleng.2005.05.006.

[176] O. Armas, Diagnóstico experimental del proceso de combustión en motores Diésel de inyección directa, Ph.D. thesis, Universitat Politècnica de València (1999).

[177] J. Martín, Diagnóstico de la combustión en motores diesel de inyección directa, Ph.D. thesis, Universitat Politècnica de València (2012).

[178] J. Benajes, P. Olmeda, J. Martín, R. Carreño, A new methodology for uncertainties characterization in combustion diagnosis and thermodynamic modelling, Applied Thermal Engineering 71 (1) (2014) 389-399. doi:10.1016/j.applthermaleng.2014.07.010.

[179] A. Irimescu, C. Tornatore, L. Marchitto, S. S. Merola, Compression ratio and blow-by rates estimation based on motored pressure trace analysis for an optical spark ignition engine, Applied Thermal Engineering 61 (2013) 101-109. doi:10.1016/j.applthermaleng. 2013.07 .036 .

[180] G. Hohenberg, Definition und Eigenschaften des thermodynamischen Verlustwinkels von Kolbenmaschinen, Automobil-Industrie 4 (1976) 15-21.

[181] CALMEC FT v6.1.1 manuel scientifique.

[182] B. Degraeuwe, Contribution to the Thermal Management of DI Diesel Engines, Ph.D. thesis, Universitat Politècnica de València (2007).

[183] A. Torregrosa, P. Olmeda, B. Degraeuwe, M. Reyes, A concise wall temperature model for DI Diesel engines, Applied Thermal Engineering 26 (11-12) (2006) 1320-1327. doi:10.1016/j. applthermaleng.2005.10.021.

[184] F. Payri, J. M. Desantes, Motores de combustión interna alternativos, Reverté, 2011. 
[185] F. Payri, X. Margot, A. Gil, J. Martín, Computational Study of Heat Transfer to the Walls of a DI Diesel Engine, SAE Technical Paper 2005-01-0210 (2005). doi:10.4271/2005-01-0210.

[186] C. Sleicher, M. Rouse, A Convenient Correlation for Heat Transfer to Constant and Variable Property Fluids in Turbulent Pipe Flow, International Journal of Heat and Mass Transfer 18 (5) (1975) 677683. doi:10.1016/0017-9310(75)90279-3.

[187] J. Caton, Comparisons of Thermocouple, Time-Averaged and MassAveraged Exhaust Gas Temperatures for a Spark-Ignited Engine, SAE Technical Paper 820050 (1982). doi:10.4271/820050.

[188] M. Reyes, Modelo de transferencia de calor para colectores de escape de motores alternativos, Ph.D. thesis, Universitat Politècnica de València (1994).

[189] J. Serrano, P. Olmeda, F. Arnau, M. Reyes-Belmonte, A. Lefebvre, Importance of Heat Transfer Phenomena in Small Turbochargers for Passenger Car Applications, SAE Technical Paper 2013-01-0576 (2013). doi:10.4271/2013-01-0576.

[190] J. Serrano, P. Olmeda, F. Arnau, A. Dombrovsky, L. Smith, Turbocharger heat transfer and mechanical losses influence in predicting engines performance by using 1D simulation codes, Energy 86 (2015) 204-218. doi:10.1016/j. energy.2015.03.130.

[191] F. Payri, P. Olmeda, F. Arnau, A. Dombrovsky, L. Smith, External heat losses in small turbochargers: Model and experiments, Energy 71 (2014) 534-546. doi:10.1016/j.energy. 2014.04.096.

[192] A. Dombrovsky, Synthesis of the 1D modelling of turbochargers and its effects on engine performance prediction, Ph.D. thesis, Universitat Politècnica de València (2016).

[193] J. Serrano, P. Olmeda, A. Páez, F. Vidal, An experimental procedure to determine heat transfer properties of turbochargers, Meas. Sci. Technol. 21 (2010) 035109. doi:10.1088/0957-0233/21/3/ 035109.

[194] J. Serrano, P. Olmeda, F. Arnau, M. Reyes-Belmonte, H. Tartoussi, A study on the internal convection in small turbochargers. Proposal 
of heat transfer convective coefficients, Applied Thermal Engineering 89 (2015) 587-599. doi:10.1016/j . applthermaleng. 2015.06. 053.

[195] V. Dolz, Contribución al modelado de la transmisión de calor en los MCIA y su aplicación en el aprovechamiento energético de los gases de escape durante los transitorios de carga, Ph.D. thesis, Universitat Politècnica de València (2016).

[196] C. Depcik, D. Assanis, A Universal Heat Transfer Correlation for Intake and Exhaust Flows in an Spark-Ignition Internal Combustion Engine, SAE Technical Paper 2002-01-0372 (2002). doi:10.4271/ 2002-01-0372.

[197] R. Santos, Estudio del aprovechamiento de la energía de los gases de escape en motores diesel, Ph.D. thesis, Universitat Politècnica de València (1999).

[198] J. Serrano, P. Olmeda, F. Arnau, A. Dombrovsky, General Procedure for the Determination of Heat Transfer Properties in Small Automotive Turbochargers, SAE Technical Paper 2014-01-2857 (2014). doi:10.4271/2014-01-2857.

[199] J. Serrano, P. Olmeda, A. Tiseira, L. García-Cuevas, A. Lefebvre, Theoretical and experimental study of mechanical losses in automotive turbochargers, Energy 55 (2013) 888-898. doi:10.1016/j. energy. 2013.04.042.

[200] J. de la Morena, A. Vassallo, R. Peterson, V. Gopalakrishan, J. Gao, Influence of Swirl Ratio on Combustion System Performance of a 0.4L Single-Cylinder Diesel Engine, in: THIESEL Conference on Thermo-and Fluid Dynamic Processes in Direct Injection Engines, 2014, pp. 1-19.

[201] Drivven Inc., DRIVVEN stand alone direct injector driver system user's manual.

[202] P. R.N. Childs, Practical Temperature Measurement, ButterworthHeinemann, 2001. doi : 10.1016/B978-0-7506-5080-9.X5000-X.

[203] CONVERGE 2.2.0 Theory Manual.

[204] E. Grimison, Transactions of the ASME 59 (1937) 583. 
[205] G. Raithby, K. Hollands, A General Method of Obtaining Approximate Solutions to Laminar and Turbulent Free Convection Problems, Advances in Heat Transfer 11 (1975) 265-315.

[206] S. W. Churchill, H. H. Chu, Correlating equations for laminar and turbulent free convection from a vertical plate, International Journal of Heat and Mass Transfer 18 (11) (1975) 1323-1329. doi:10.1016/ 0017-9310 (75) 90243-4.

[207] S. K. Boetcher, Natural Convection Heat Transfer From Horizontal Cylinders, SpringerBriefs in Applied Sciences and Technology (2014) 3-22doi:10.1007/978-3-319-08132-8_2.

[208] W. Kays, A. London, Compact Heat Exchangers, 3rd Edition, McGraw-Hill, 1984.

[209] R. Shah, D. Sekulic, Fundamentals of Heat Exchanger Design, John Wiley \& Sons, 2003.

[210] P. Boulos, K. Lansey, B. Karney, Comprehensive Water Distribution Systems Analysis Handbook for Engineers and Planners, Second Edition, MWH Soft, 2006.

[211] Eigen, eigen.tuxfamily.org, accessed Dec. 2017.

[212] Eigen's unsupported modules, https://eigen.tuxfamily.org/ dox/unsupported/index.html, accessed Dec. 2017.

[213] Eigen's HybridNonLinearSolver class template reference, https://eigen.tuxfamily.org/dox/unsupported/classEigen_ 1_1HybridNonLinearSolver.html, accessed Dec. 2017.

[214] Eigen's Non Linear Optimization module, https: //eigen.tuxfamily.org/dox/unsupported/group_ _NonLinearOptimization__Module.html, accessed Dec. 2017.

[215] M. J. D. Powell, An efficient method for finding the minimum of a function of several variables without calculating derivatives, Computer Journal 7 (2) (1962) 155-162. doi:10.1093/comjnl/7.2.155.

[216] J. J. Moré, B. S. Garbow, K. E. Hillstrom, User guide for MINPACK-1, ANL-80-74, Argonne National Laboratory. 
[217] A. Broatch, P. Olmeda, J. Martín, J. Salvador-Iborra, Development and Validation of a Submodel for Thermal Exchanges in the Hydraulic Circuits of a Global Engine Model, SAE Technical Paper 2018-01-0160 (2018). doi:10.4271/2018-01-0160. 
